UNITED STATES DEPARTMENT OF THE INTERIOR

Harold L. Ickes, Secretary

GEOLOGICAL SURVEY

W. C. Mendenhall, Director

Water-Supply Paper 938

\title{
WATER LEVELS AND ARTESIAN PRESSURE \\ IN OBSERVATION WELLS IN THE UNITED STATES IN 1941
}

Part 3. NORTH-CENTRAL,STATES

BY $\because$

O. E. MEINZER, L. K. WENYZSL and others

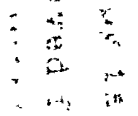

Prepared in cooperation with the States of

IOWA, KANSAS, MINNESOTA, MISSOURI, NEBRASKÄ, NORTH DAKOTA SOUTH DAKOTA, and WISCONSIN and other agencies
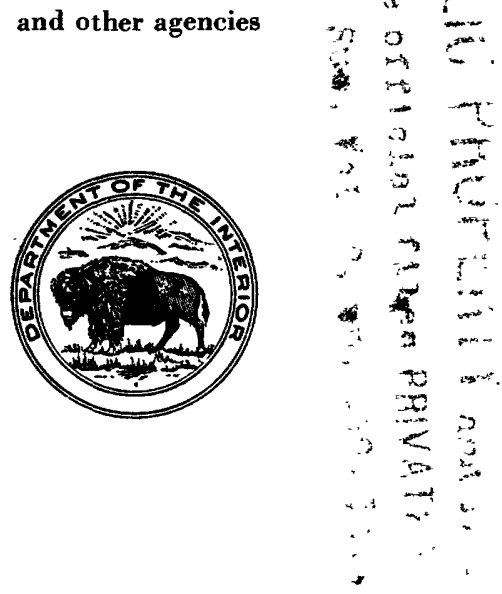

UNITED STATES

GOVERNMENT PRINTING OFFICE

WASHINGTON : 1943

For sale by the Superintendent of Documents, U. S. Goverament Printing Office Washington, D. C. 
Introduction, by 0. E. Meinzer and L. K. Wenzel. . . . . . . .

Iowa... . . . . . . . . . . . . . . . . . . .... . 6

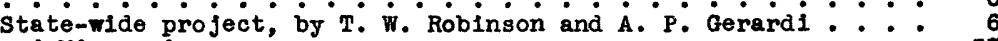

Iowa and Missouri. . . . . . . . . . . . . . . . 37

Tarkio Creek area of Soll Conservation Service, by . . . 37

T. W. Robinson. . . . . . . . . . . . . . . . . 37

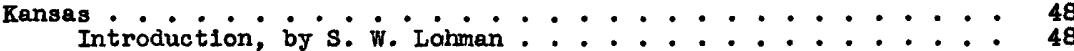

Berber County, by C. C. Wliliams................. 52

Clark County, by J. C. Frje . : : : : : : : : : : : : : 55

Comanche County, by J. C. Frye. . . . . . . . . . . . . . . 57

Ellis County, by J. C. Prye . . . . . . . . . . . . . . . . 59

Finney County, by B. F. Latta................. . 60

Ford County, by H. A. Walte .................... 66

Grant county, by T. G. Yclaughilin: : : : : : : : : : 68

Gray County, by B. F. Latta . . . . . . . . . . . . . . . . 71

Hamilton County, by T. G. McLaughlin. : : : : : : . : . 76

Harvey County, by C. C. Williams and G. H. von Hein ...... 79

Haskell County, by T. G. Mclaughlin.............. 108

Hodgeman County, by H. A. Walte.................. 110

Jewell County, by V. C. Fishel. ................. . 111

Kearny county, by T. G. Mclaughlin. . . . . . . . . . . I15

Klowa county, by B. F. Latta. . . . . . . . . . . . . . II9

McPherson County, by C. C. Wililiams and $\dot{G}$. H. von Hein. . . . 120

Meade County, by J. C. Frye . . . . . . . . . . . . . . . 122

Morton County, by T. G. McLaughlin. . . . . . . . . . . . 127

Ness County, by H. A. Walte ................... 128

Pamee County, by H. A. Walte.................. . 129

Russell County, by J. C. Frye . . . . . . . . . . . . . 130

scott County, by H. A. Walte. . . . . . . . . . . . . . 132

Sedgrick County, by C. C. Williams and G. H. von Heln . : : : 139

Seward County, by C. C. W1lllams. . . . . . . . . . . . . . 146

Stanton County, by B. F. Latta. . . . . . . . . . . . . . 148

Minnesota, by A. C. Byers. . . . . . . . . . . . . . . . . 150

Kebraska, by H. A. Walte and I. P. Buck. . . . . . . . . . . . . . . 154

North Dakota ........................ . . . . . 184

State-wide project, by w. C. Rasmussen. . . . . . . . . . . 184

Cass County (Fargo area), by A. C. Byers............. 190

South Dakota . . . . . . . . . . . . . . . . . . . . . . 224

Southeastern South Dakota, by T. W. Robinson. . . . . . . . 224

Clty of Huron, by T. W. Robinson. . . . . . . . . . . . . 231

wisconsin. . . : . . . . . . . . . . . . . . . . . . 232

Coon Creek area by L. K. Wenzel. . . . . . . . . . . 232

\section{IILUSTRATIONS}

Figure 1. Outline map of the United States, showing sections of the country covered by the six water-supply papers on water levels and artesian pressure in obaervation wells in $1941 . .$. ........... 
Figure 2. Hydrograph showing fluctuation of water level in well

Page at Ames, Iowa....

3. Graph showing average water level in 9 to 12 wells and precipitation at Shenandoah in the Tarkio Creek

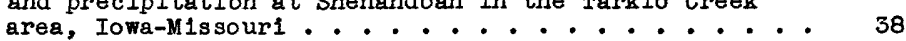

4. Graphs showing the average of water levels in weils

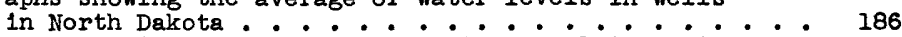

5. Graphs showing fluctuations of water level in typical wells in southeastern South Dakota. . . . . . . . 225 


\section{INTRODUCTION}

By O. E. Meinzer and L. K. Wenzel

The rock formations of the earth are great natural underground reservoirs in which a part of the water derived from rain and snow is stored to supply wells and springs and to maintain the flow of streams during periods of fair weather. Water levels in wells register the stages of these natural reservoirs; they show the extent to which water supplies are depleted by drought or by heavy pumping for public waterworks, for irrigation, or for industrial uses and the extent to which they are replenished in seasons of abundant rainfall or melting snow. The changes in pressure recorded on flowing wells may indicate depletion or replenishment of the artesian reservoirs.

The regular publication of records of water levels and artesian pressure in the United States was begun by the Geological Survey in 1935, and from that year through 1939 one volume containing these data was published each year. The volumes were issued as Water-Supply Papers 777, 817, 840,845 , and 886. The number of observation wells and the quantity of records on water levels and artesian pressure obtained from them have increased gradually from year to year. As a result the records for 1940 were published in six volumes, Water-Supply Papers 906-911 inclusive. WaterSupply Paper 906 contains the records for the northeastern states, 907 for the southeastern states, 908 for the north-central states, 909 for the south-central states, 910 for the northwestern states, and 911 for the southwestern states and Hawa11. Records for 1941 are being published in six volumes also, each volume covering a section of the United states corresponding to that covered by one of the volumes containing records for 1940. (See fig. 1.) This series of reports is in a sense an inventory, year by year, of the ground-water supplies of those parts of the country that are covered. 
This volume covers the north-central section and gives records of water levels or artesian pressure in about 1,350 observation wells of the Geological Survey and cooperating agencies in Iowa, Kansas, Minnesota, Missour1, Nebraska, North Dakota, South Dakota, and Wisconsin. Of these wells 27 are equipped with automatic water-stage recorders. For some wells for which records had not heretofore been published, complete records of water levels are given in this report, including those for years before 1941. For wells whose previous records have been published, however, this volume gives only current records. If complete descriptions of the wells were given in one of the previous reports, only the well numbers or the well mumbers and brief ldentifying descriptions are given in this report. The report includes about 17,400 individual measurements of water levels or artesian pressure.

The water levels in this report are given with reference to datum planes of different kinds. Some are given in depths below measuring point-that 1s, below the recognized reference mark, at or near the top of the well, from which the depth to water level is usually measured; and some are given in helght above an assumed datum plane. As the measuring points on some of the wells were changed In 1941, the records may not be directly comparable with those in previous annual volumes, but changes in measuring points are recorded in this report. Water levels given in helght above seo level or above assumed datum planes are generally comparable with those given in the previous volumes. Unless otherwise stated, the depth of wells is usualif the measured depth below the measuring point.

Acknowledgments for effective services in the preparation of this report are due Miss Dorothy M. Ireland, Mrs. Roxie Lou Dav1s, and Mrs. Margaret F. Monk, who typed the offset copy; and to Rodney Hart, who prepared the 1llustrations and gave other assistance in preparing the copy. GENERAL SUMMARY OF CHANGES IN GROUND-WATER LEVELS IN 1941

IN THE NORTH-CENTRAL PART OF THE UNITED STATES

In 1941 the precipitation in all the north-central states was above normal, and in most parts of the region the water levels in wells rose appreciably. The fluctuations of the water levels and artesian pressure In wells depend, however, on many factors besides the amount of precipitation. Consequently, it is usually not possible to find a simple relation 
between the changes in water level or artesian pressure and the departures from normal precipitation.

The following statements are taken chlefly from the interpretative text of the several state sections in this volume. They summarize the changes in ground-water levels and artesian pressure that occurred in 1941

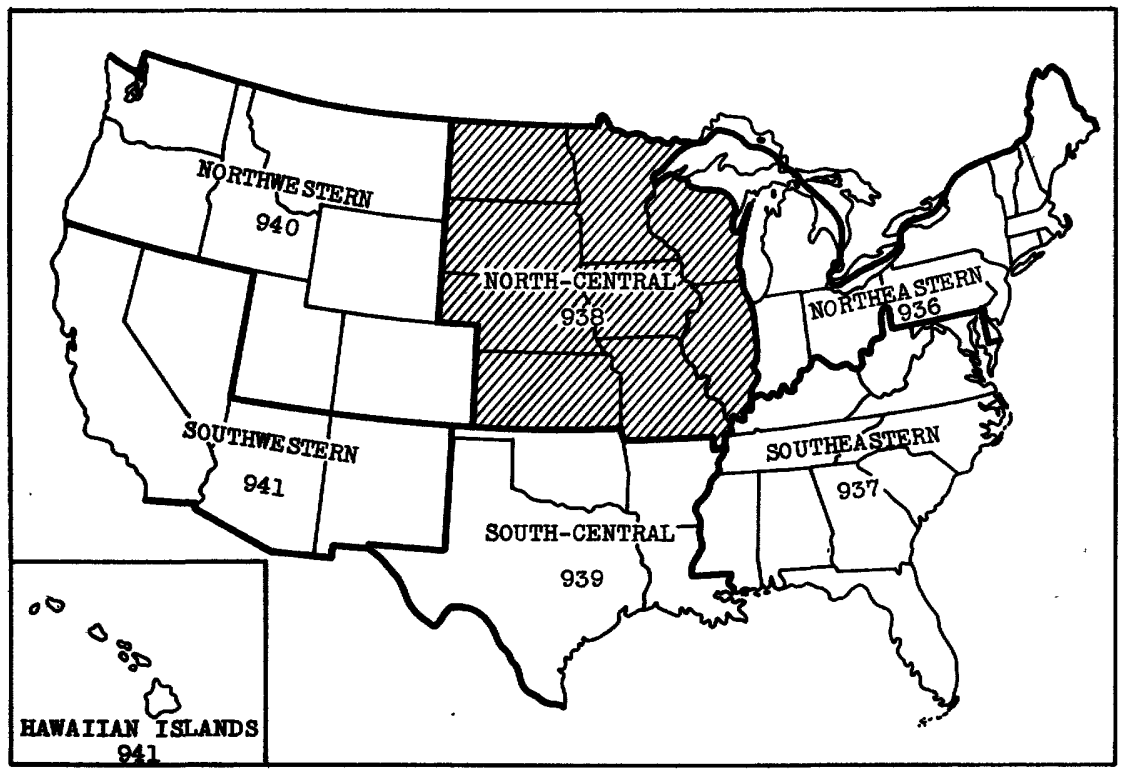

Figure 1.--outline map of the United States, showing sections of the country covered by the six water-supply papers on water levels and artesian pressure in observation wells in 1941. The shaded section represents the part of the country covered by this volume.

In the parts of the underground reservoirs in the north-central states that are tapped by the observation wells.

Iowa.--Owing to coplous rains during the late summer and early fall, the water levels in shallow observation wells over the state were on the average higher in December 1941 than in December 1940. The average rise in 46 wells in 15 counties was 2.7 feet. In many of the wells the rise in water level was 10 feet or more, but in a few wells there was a small decline.

The water levels in wells in the Tark10 Creek area, in Iowa and adjacent parts of M1ssour1, rose to the highest stages since measurements were begun In 1934 as the result of the heavy precipltation, which at Shenandoah amounted 
to 48.07 inches--14.07 inches above normal. The average of the water levels in the wells stood about 7.7 feet higher in December 1941 than in December 1940. A large part of the total rise took place in the months of September and October, when the rainfall at Shenandoah amounted to 23.11 inches.

Kansas.--In response to unusually abundant precipitation the water levels in most of the observation wells reached in 1941 the highest stages during their perlods of record, and in most of the wells there was an appreciable net rise in water level during the year. In 18 of 21 counties for which records of a jear or more are available, there was a net rise in water level during the year in 60 to 100 percent of the wells. In the 3 counties in which the percentage of wells showing net rises was only 50 percent or less, most of the observation wells were pumped or were affected noticeably by nearby pumping during the year. Water-level trends in these wells, therefore, reflect the extent of nearby pumping rather than the amount of recharge.

Nebraska.--In 1941 the change in average water level in 118 key wells distributed over the State was a rise of 0.55 foot; for the period 1934 through 1941 there was a net decline in the same wells of 0.08 foot. During the 7-year period there has been, therefore, essentially no appreciable net average change of water level in the wells despite the fact that precipitation in the State in the same period was about 22.5 inches below normal. Water levels in'wells in all parts of Nebraska rose in 1941, but the rises were most pronounced in the northeast and southeast sections. It is estimated that the water stored in the underground reservoirs of the state increased about 4,000,000 acre-feet in the year.

North Dakota.--The average of the water levels in 22 key wells situated in different parts of the State rose slightly more than 1.3 feet in 1941. This is the only annual net rise that has occurred since observations were begun in september 1937. In the first 3 t years of record the average water levels in corresponding months show, in general, decreases in each succeedIng year, but in May 1941 the water levels began a recovery that brought them to a new high average stage by october. The jear closed with the average water level above that at the close of any previous year since the observations were begun. 
South Dakota.--A comparison of the average water levels in a group of 22 wells in southeastern South Dakota shows that in 1941 there was a net rise in 7 wells, a net decline in 14 wells and almost no net change in one well. The average net change in these wells was, however, a net rise of 0.23 foot. 
The cooperative program of water-level measurements in observation wells in Iowa; $1 /$ begun in 1938, was continued in 1941 by the Federal Geological Survey in cooperation with the Iowa Geological Survey.

The observation-well program at the end of 1941 included 208 wells, of which 8 were equipped with automatic water-level recorders. Six wells were dropped during the year and 108 added. Approximately 1,400 individual measurements of water level were made during the year. The water levels in six wells equipped with 8-day automatic water-level recorders were measured weekly. Some wells were measured once a month and others less frequently. A few were measured only once during the year. In addition to the regular observation-well measurements, numerous measurements of water level and pressure-gage readings were made on individual wells whenever the opportunity was afforded.

As a result of coplous rains during the late summer and early fall, the water levels in the shallow wells were on the average higher in December 1941 than in December 1940. The average rise in 46 shallow wells located in 15 counties was 2.7 feet during the year. In many of the wells the rise in water level was 10 feet or more, but in a few wells there was a small decline.

The following table gives the average rise of water levels in the shallow wells, by counties, from December 1940 to December 1941, the precipitation at the nearest United States Weather Bureau station during 1941, and departure from normal. The normal of the precipitation for 1940 was used to obtain the departure.

1 See Water-Supply Papers 886 and 908. 


\begin{tabular}{|c|c|c|c|c|}
\hline County & $\begin{array}{l}\text { No. of } \\
\text { wells }\end{array}$ & $\begin{array}{l}\text { Average net } \\
\text { change in } \\
\text { water levels } \\
\text { in } 1941 \text {, } \\
\text { in feet }\end{array}$ & $\begin{array}{l}\text { Precipitation in } \\
\text { I94l, In inches } \\
\text { (by U.S.Weather } \\
\text { Bureau at nearest } \\
\text { station) }\end{array}$ & $\begin{array}{l}\text { Departure } \\
\text { from } \\
\text { normal } \\
\text { precipitation, } \\
\text { in inches }\end{array}$ \\
\hline $\begin{array}{l}\text { Adalr } \\
\text { Buena Vista } \\
\text { Calhoun } \\
\text { Cerro Gordo } \\
\text { Clay - Palo Alto } \\
\text { Johnson } \\
\text { Linn } \\
\text { Lyon } \\
\text { Madison } \\
\text { Marion } \\
\text { Page } \\
\text { Polk } \\
\text { Sac } \\
\text { Story }\end{array}$ & $\begin{array}{l}4 \\
5 \\
5 \\
6 \\
6 \\
1 \\
5 \\
2 \\
1 \\
5 \\
1 \\
1 \\
3 \\
1\end{array}$ & $\begin{array}{r}+3.00 \\
+2.65 \\
+1.91 \\
+1.25 \\
+2.35 \\
+1.91 \\
+3.52 \\
+.04 \\
+.19 \\
+6.67 \\
+4.76 \\
+1.44 \\
+10.71 \\
+103\end{array}$ & $\begin{array}{l}43.06 \\
29.24 \\
27.93 \\
35.24 \\
34.21 \\
39.33 \\
39.44 \\
25.03 \\
42.52 \\
36.67 \\
43.72 \\
37.11 \\
21.75 \\
41.52\end{array}$ & $\begin{array}{r}+10.77 \\
+.27 \\
-4.73 \\
+4.41 \\
+6.45 \\
+4.48 \\
+8.90 \\
-1.48 \\
+10.59 \\
+2.88 \\
+10.00 \\
+2.01 \\
-7.22 \\
+5.04\end{array}$ \\
\hline
\end{tabular}

An average of the precipitation in the area in which the wells are located was about 36 inches, or approximately 5 inches above the 68-year average for the state. The water levels rose even in some areas in which the precipitation for the year was below the annual normal, as in calhoun and Iyon counties. In sac county, where the precipitation was 7.22 inches below normal, the water levels declined in all of the 3 observation wells, the average being 0.71 foot. In general the water levels in the shallow wells showed a definite rise during the winter and spring, a sag in the summer, a sharp $r i s e$ in the fall, and a small decline during December.

Most of the deep observation wells, in which the water is under artesian pressure, are located close to or in areas of heavy pumping. The water level in these wells shows the effect of seasonal pumpage. In general the water levels were highest during the winter and spring and lowest during the mid-summer months of July and August, when pumping is the heaviest.

The fluctuation of the water level in a well 2,250 feet deep, at Ames, is shown in figure 2 , as obtalned from recorder charts. This well taps the Jordan sandstone. There is no pumping from this aquifer for a distance of about 10 miles from the well, and there has been no pumping from the well since about a year prior to the beginning of the record. The water level is affected by changes in atmospheric pressure and also apparently by pumping at distant points and possibly by variations in the rate of recharge. The water level is highest during late winter and early spring 


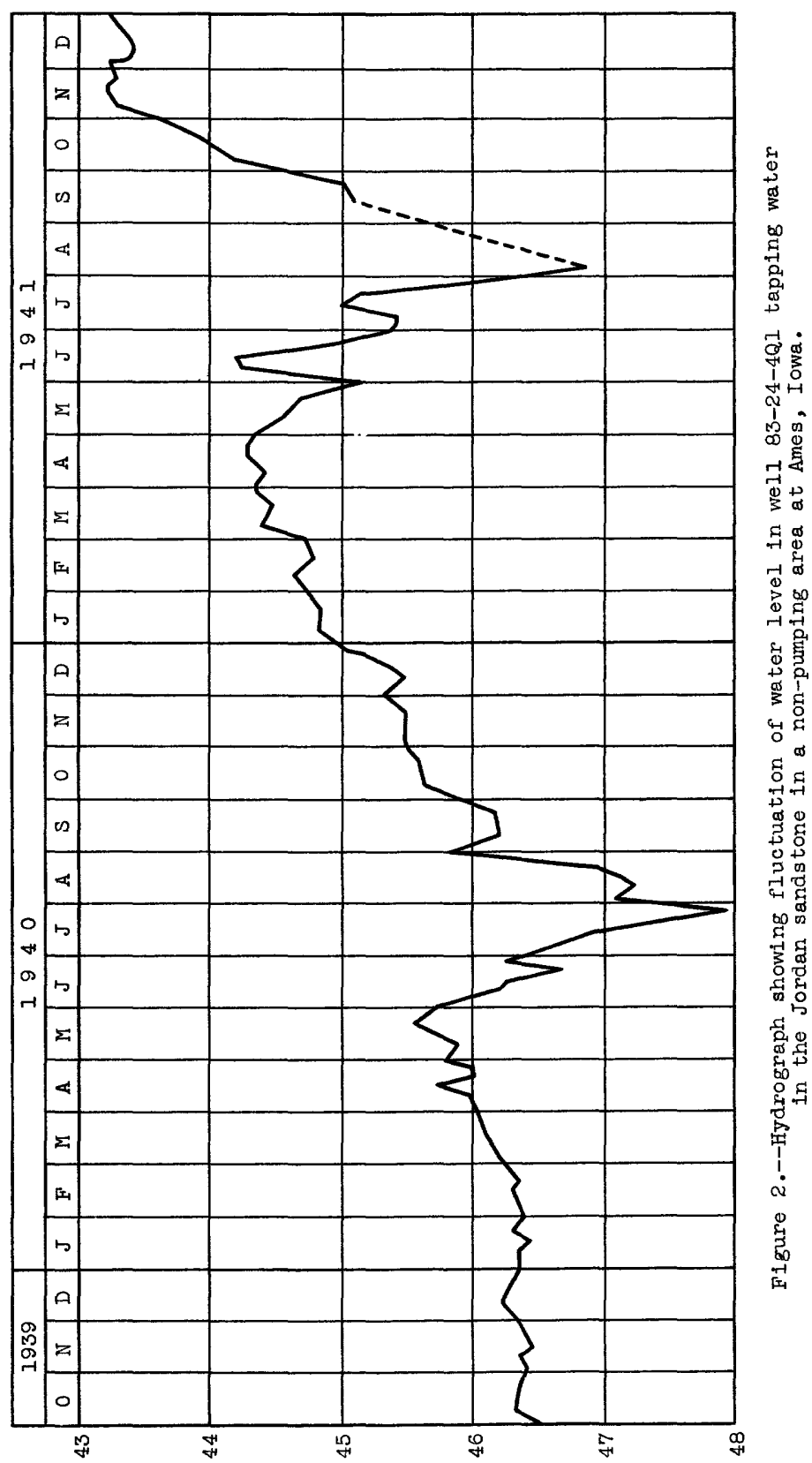

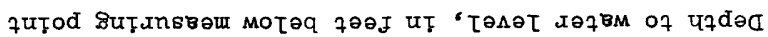


and lowest in mId-summer, which corresponds to variations in seasonal pumpage. The general trend of the water levels during the period of record 1s upward. The lowest water level observed was 47.97 feet below the measuring point on July 27, 1940, and the highest was 42.98 feet below the measuring point on December 4, 1941.

Progress was made in the collection of records of pumpage from underground sources, especlally in Cerro Gordo county, where pumpage records or estimates were obtalned from all of the municipalities and for most private users of ground water. During the year many pumping tests were made by the state and Federal surveys to determine the water-ylelding capacities of wells and the transmissibilities of water-bearing formations.

Records of the observation wells are 11sted alphabetically by counties on the following pages. All water levels are expressed in feet below the measuring point. If descriptions of wells and measuring points are not given in this report they will be found in Water-supply Paper 886 or 908 .

\section{Adair county}

76-3l-2lRl. John Breheny. Water levels, in feet below measuring point, 1941: Feb. 11, 22.61; Mar. 29, 19.73; Apr. 29, 18.65; May 28, 19.00. Measurements temporarily discontinued.

76-31-25RI. Harold Bochart.

Water level, in feet below measuring point, 1941

\begin{tabular}{|c|c|c|c|c|c|c|c|}
\hline Date & $\begin{array}{l}\text { Water } \\
\text { level }\end{array}$ & Date & $\begin{array}{l}\text { Water } \\
\text { level }\end{array}$ & Date & $\begin{array}{l}\text { Water } \\
\text { level }\end{array}$ & Date & $\begin{array}{l}\text { Water } \\
\text { level }\end{array}$ \\
\hline $\begin{array}{l}\text { Feb. } 11 \\
\text { Mar. } 29 \\
\text { Apr. } 29 \\
\end{array}$ & $\begin{array}{l}6.55 \\
6.81 \\
6.10 \\
\end{array}$ & $\begin{array}{ll}\text { May } & 28 \\
\text { June } & 30 \\
\text { July } & 29 \\
\end{array}$ & $\begin{array}{l}6.64 \\
6.29 \\
7.70 \\
\end{array}$ & $\begin{array}{l}\text { Aug. } 28 \\
\text { Sept. } 30 \\
\text { Oct. } 28 \\
\end{array}$ & $\begin{array}{l}8.07 \\
6.04 \\
4.98 \\
\end{array}$ & $\begin{array}{l}\text { Nov. } 28 \\
\text { Dec. } 21\end{array}$ & $\begin{array}{l}4.99 \\
5.40\end{array}$ \\
\hline
\end{tabular}

75-31-15BI. John E. Soderberg.

Water level, in feet below measuring point, 1941

\begin{tabular}{ll|ll|ll|ll}
\hline Feb. 11 & 7.79 & May 28 & 5.02 & Aug. 28 & 8.04 & Nov. 28 & 2.67 \\
Mar. 29 & 5.13 & June 28 & 4.53 & Sept.30 & 7.27 & Dec. 21 & 3.28 \\
Apr. 29 & 4.29 & July 28 & 6.05 & oct. 28 & 2.14 & & \\
\hline
\end{tabular}

75-31-18Bl. Charles Gilham.

Water level, in feet below measuring point, 1941

\begin{tabular}{lr|lr|rr|rr}
\hline Feb. 11 & 9.38 & May 28 & 9.21 & Aug. 28 & 11.29 & Nov. 28 & 8.83 \\
Mar. 29 & 9.30 & June 30 & 9.23 & Sept.30 & 9.71 & Dec. 21 & 9.49 \\
Apr. 29 & 8.96 & July 29 & 10.59 & Oct. 28 & 8.26 & & \\
\hline
\end{tabular}


Adair County--Continued

75-30-8Al. Edward Snlthen and Ernest M1ller.

Water level, in feet below measuring point, 1941

\begin{tabular}{lr|lr|lr|lr}
\hline Date & $\begin{array}{r}\text { Water } \\
\text { level }\end{array}$ & Date & $\begin{array}{l}\text { Water } \\
\text { level }\end{array}$ & Date & $\begin{array}{r}\text { Water } \\
\text { level }\end{array}$ & Date & $\begin{array}{r}\text { Vater } \\
\text { level }\end{array}$ \\
\hline Feb. 11 & 6.16 & May 28 & 8.13 & Aug. 28 & 9.16 & Nov. 28 & 5.67 \\
Mar. 29 & 11.73 & June 30 & 6.74 & Sept.30 & 7.61 & Dec. 21 & 6.20 \\
Apr. 29 & 8.68 & July 29 & 7.84 & Oct. 28 & 5.49 & & \\
\hline
\end{tabular}

\section{Benton county}

85-10-16M3. Owner's number 3. Clty of vinton. Measurements by R. G. Milier, water superintendent. Water level affected by pumping. Nater levels, in feet below measuring point, 1941: Jan. 13, a/ 29.12; b/ 49.0 ; Dec. 22 , c/ 26.17 ; b/ 45.25 .

Buena Vista County

(Vicinity of Storm Lake)

91-37-32E1.

Water level, in feet below measuring point, 1941

\begin{tabular}{ll|ll|ll|lll}
\hline Feb. 12 & 3.66 & Apr. 18 & 4.53 & June 17 & 4.36 & Aug. 27 & 6.93 \\
Mar. 20 & 4.85 & Maj 20 & 4.77 & JuIy 23 & 5.44 & Dec. 11 & 4.47 \\
\hline
\end{tabular}

90-37-3El. Emil Schmitz.

Water level, in feet below measuring point, 1941

\begin{tabular}{ll|ll|llr|rr}
\hline Feb. 12 & 14.01 & Apr. 18 & 12.62 & June 17 & 7.44 & Aug. 27 & 13.66 \\
Mar. 20 & 13.64 & May 20 & 10.70 & July 23 & 11.48 & Dec. 11 & $\mathbf{8 . 9 0}$ \\
\hline
\end{tabular}

90-37-3MI. L. B. Watt.

Water level, in feet below measuring point, 1941

\begin{tabular}{ll|ll|lll|ll}
\hline Feb. 12 & 22.45 & Apr. 18 & 22.08 & June & 17 & 18.66 & Aug. 27 & 19.88 \\
Mar. 20 & 22.27 & May 20 & 19.47 & July & 23 & 18.58 & Dec. 11 & 16.37 \\
\hline
\end{tabular}

90-37-11J1. United States Geological Survey.

Water level, in feet below measuring polnt, 1941

\begin{tabular}{ll|ll|lll|ll}
\hline Feb. 12 & 4.27 & Apr. 18 & 3.54 & June 17 & 3.88 & Aug. 27 & (d) \\
Mar. 20 & 4.47 & May 20 & 4.55 & July 23 & 5.44 & Dec. 11 & 5.15 \\
\hline
\end{tabular}

90-37-22J1. William Monteful.

Water level, in feet below measuring point, 1941

\begin{tabular}{ll|ll|lll|ll}
\hline Feb. 12 & 34.82 & Apr. 18 & 35.15 & June 17 & 35.06 & Aug. 27 & 34.69 \\
Mar. 20 & 35.04 & May & 20 & 35.18 & JulJ 23 & 34.80 & Dec. 11 & 33.96 \\
\hline
\end{tabular}

90-37-23D1. Biggins Bros.

Water level, in feet below measuring point, 1941

\begin{tabular}{|c|c|c|c|c|c|c|c|c|}
\hline $\begin{array}{l}\text { Feb. } 12 \\
\text { Mar. } 20\end{array}$ & $\begin{array}{l}27.23 \\
27.07\end{array}$ & $\begin{array}{l}\text { Apr. } \\
\text { May }\end{array}$ & $\begin{array}{l}18 \\
20\end{array}$ & $\begin{array}{l}26.87 \\
26.81\end{array}$ & $\begin{array}{l}\text { June } 1 \\
\text { July }\end{array}$ & $\begin{array}{l}26.69 \\
27.72\end{array}$ & $\begin{array}{ll}\text { Aug. } 27 \\
\text { Dec. } 11\end{array}$ & $\begin{array}{l}28.29 \\
25.80\end{array}$ \\
\hline
\end{tabular}

a Pump shut down $1 \frac{1}{4}$ hours.

b Pumping.

c Pump shut down 3 hours.

d Dry. 
Buena Vista County--Continued

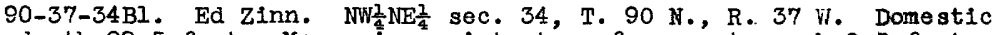
well, depth 28.5 feet. Measuring point, top of concrete curb 2.5 feet above land surface. Equipped with lift pump.

Water level, in feet below measuring point, 1940-41

\begin{tabular}{ll|ll|lr}
\hline Date & $\begin{array}{l}\text { Water } \\
\text { level }\end{array}$ & Date & $\begin{array}{l}\text { Water } \\
\text { level }\end{array}$ & Date & $\begin{array}{r}\text { Water } \\
\text { level }\end{array}$ \\
\hline June 26, 1940 & 17.34 & Apr. 18, 1941 & 17.70 & July 23, 1341 & 17.59 \\
Feb. 12, 1941 & 18.37 & May 20 & 16.86 & Aug. 27 & 20.32 \\
Mar. 20 & 18.04 & June 17 & 15.98 & Dec. 11 & 18.10 \\
\hline
\end{tabular}

Calhoun County

(Vicinity of Twin Lakes)

$89-32-28 \mathrm{~N} 1$.

Water level, in feet below measuring point, 1941

\begin{tabular}{|c|c|c|c|c|c|c|c|}
\hline Date & $\begin{array}{l}\text { Water } \\
\text { level }\end{array}$ & Date & $\begin{array}{l}\text { Water } \\
\text { level }\end{array}$ & Date & $\begin{array}{l}\text { Water } \\
\text { level }\end{array}$ & Date & $\begin{array}{l}\text { Water } \\
\text { level }\end{array}$ \\
\hline $\begin{array}{l}\text { Feb. II } \\
\text { Mar. } 20\end{array}$ & $\begin{array}{l}7.43 \\
6.10\end{array}$ & $\begin{array}{ll}\text { Apr. } & 18 \\
\text { May } 27\end{array}$ & $\begin{array}{l}5.76 \\
8.04\end{array}$ & $\begin{array}{ll}\text { June } & 18 \\
\text { July } & 22\end{array}$ & $\begin{array}{l}5.50 \\
7.72\end{array}$ & $\begin{array}{l}\text { Aug. } 27 \\
\text { Dec. } 11\end{array}$ & $\begin{array}{l}\text { (a) } \\
6.98\end{array}$ \\
\hline
\end{tabular}

89-32-3IRl. E. F. Leg8.

Water level, in feet below measuring point, 1941

\begin{tabular}{ll|ll|ll|ll}
\hline Feb. 11 & 11.27 & May 27 & 11.58 & July 22 & 11.14 & Dec. 11 & 12.06 \\
Apr. 18 & 11.26 & June 18 & 10.88 & Aug. 27 & 12.43 & & \\
\hline
\end{tabular}

89-32-35N1. Ben Burns.

Water level, in feet below measuring point, 1941

\begin{tabular}{ll|ll|ll|ll}
\hline Feb. II & 8.13 & Apr. 18 & 4.72 & June 18 & 5.15 & Aug. 27 & 8.28 \\
Mar. 20 & 5.54 & May 27 & 5.45 & July 22 & 7.47 & Dec. 11 & 4.80 \\
\hline
\end{tabular}

88-33-1Bl. -- Burns.

Water level, in feet below measuring point, 1941

\begin{tabular}{rr|rr|lr|rr}
\hline Feb. 11 & 10.94 & Apr. 18 & 9.21 & June 18 & 9.45 & Aug. 27 & 14.04 \\
Mar. 20 & 9.60 & May 27 & 10.46 & July 22 & 11.46 & Dec. 11 & 13.09 \\
\hline
\end{tabular}

88-33-DI. George Voss.

Water level, in feet below measuring point, 1941

\begin{tabular}{ll|ll|ll|ll}
\hline Feb. 11 & 10.51 & Apr. 18 & 9.26 & June 18 & 9.42 & Aug. 27 & 11.22 \\
Mar. 20 & 10.57 & May 27 & 9.96 & July 22 & 9.28 & Dec. 11 & 10.85 \\
\hline
\end{tabular}

Carroll County

85-35-18DI. City of Breda.

Water level, in feet below measuring point, 1941

\begin{tabular}{ll|l|ll|lll|ll}
\hline Feb. $\begin{array}{ll}11 \\
\text { Mar. } 19\end{array}$ & 193.64 & Apr. 17 & 192.74 & June 17 & 193.64 & Aug. 27 & 193.53 \\
May 27 & b 207.35 & July 29 & 193.06 & Dec. 11 & 194.39 \\
\hline $\begin{array}{l}\text { b Dry. } \\
\text { New city well, } 25 \text { feet south, pumping. }\end{array}$
\end{tabular}


Carroll County--Continued

84-34-25Fl. Owner's test hole 1. City of Carroil.

Water level, in feet below measuring point, 1941

\begin{tabular}{ll|ll|ll|ll|l}
\hline Date & $\begin{array}{l}\text { Water } \\
\text { level }\end{array}$ & Date & $\begin{array}{l}\text { Water } \\
\text { level }\end{array}$ & Date & $\begin{array}{l}\text { Water } \\
\text { level }\end{array}$ & Date & $\begin{array}{r}\text { Water } \\
\text { level }\end{array}$ \\
\hline Feb. 11 & a 44.24 & Apr. 17 a 48.66 & June 17 & a 46.52 & Aug. 27 & 48.68 \\
Mar. 10 a 43.09 & May 27 a 50.02 & July 28 & a 51.74 & Dec. 12 & 44.05 \\
\hline
\end{tabular}

\section{Cerro Gordo County}

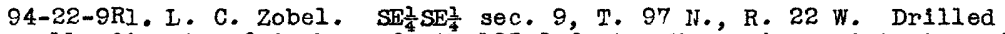
stock well, diameter 6 inches, depth 123.0 feet. Measuring point, top of concrete curb at pump base, 2.1 feet above land surface. Equipped with lift pump and windmill. Water level, in feet below measuring point, 194l: Qct. $7,35.78$.

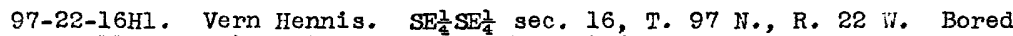
domest1c wel1, diameter 12 inches, depth 33.8 feet. Heasuring point, top of tile casing, 1.0 foot above land surface. Taps water in glacial arift. Equipped with ift pump. Water level, in feet below measuring point, 1941: oct. $7,18.61$.

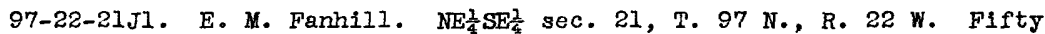
feet south of well 97-22-21J2. Drilled stock weil, diameter 5 inches, depth 106.0 feet. Measuring point, top of casing flange, 1.0 foot above land surface and 1.4 feet (by hand level) below measuring point of well 97-22-21J2. Equipped with lift pump and windmill. Water level, in feet below measuring point, 1941: 0ct. 7, 16.12.

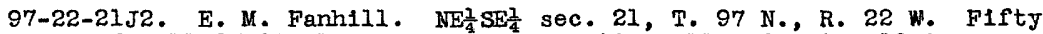
feet north of well 97-22-2lJ1. Drilled domestic well, diameter 12 inches, depth 53.0 feet. Measuring point, top of 1 -inch board, pump support, 0.5 foot above land surface and 1.4 feet, (by hand level) above measuring point of well 97-22-J1. Taps water in glacial drift. Equipped with lift pump. Water level, in feet below measuring point, 1941: 0ct. 7, 12.85 .

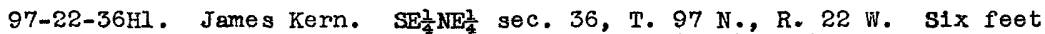
north of well 97-22-36H2. Abandoned bored weil, diameter 18 inches, depth 36 feet. Measuring point, top of tile casing at north side, at land surface and 1.26 feet below measuring point of well 97-22-36H2. Taps water in glacial drift. Water level, in feet below measuring point, 194l: 0ct. 8 , 22.37 .

97-22-36H2. James Kern. SE $\frac{1}{4} \operatorname{SE} \frac{1}{4}$ sec. 36, T. 97 N., R. 22 . Six feet south of 97-22-36Hl. Drilled domestic and stock well, diameter 4 inches, reported depth 112 feet. Measuring point, top of casing, 1.0 foot above land surface and 1.26 feet above measuring point of weli'97-22-36H1. Water level, in feet below measuring point, 1941: 0ct. 8, 23.90 .

97-21-9El. E. H. Phillips. SWI NW sec. 9, T. 97 N., R. 21 W. Drilled domestic and stack we11, diameter 6 inches, depth 206.0 feet. Measuring point, top of casing, 3.4 feet below land surface. Taps water in limestone. Equipped with automatic pressure system, operated by one horsepower electric motor. Water level, in feet below measuring point, 1941: Nov. 11, 91.20 .

97-21-9E2. E. H. Ph1llips, sW $\frac{3}{4}$ W $\frac{x}{4}$ sec. 9, T. 97 N., R. 21 W. Abandoned dug and bored well, diameter 5 feet to 1 foot, depth 37.0 feet. Measuring point, top of concrete casing, 0.8 foot above 1 and surface. Taps water in glacial drift. Water level, in feet below measuring point, 1941: Nov. 11, 31.80 .

a Nearby wells pumping. 
Cerro Gordo County--Continued

97-21-18MI. W. D. Hurd. NW $\frac{2}{4} S W^{2}$ sec. 18, T. $97 \mathrm{~N}$, , R. 21 W. Drilled domestic and stock well, diameter 5 inches, depth 63 feet. Drilied in bottom of well 97-21-18M2. Measuring point, common to well 97-21-18M2, top of wood pump platform and concrete curb, 1.2 feet above land surface. Taps water in limestone. Equipped with Ifft pump and windmill. Water level, in feet below measuring point, 1941: Nov. 11, 12.3.

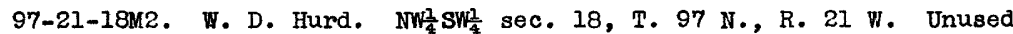
dug well, diameter 33 inches, depth 13 feet. Measuring point common to well 97-21-18M1, top of wood pump platform and concrete curb, 1.2 feet above land surface. Water level, in feet below measuring point, 194l: Nov. $11,10.22$.

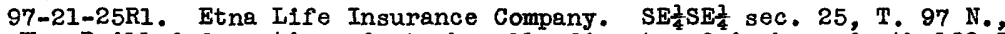
R. 21 W. Drilled domest1c and stock well, diameter 6 inches, depth 189.5 feet. Measuring point, top of 2 -inch plank well platform, 0.2 foot above land surface. Taps water in limestone. Water level, in feet below measurIng point, 1941: Nov. 13, 27.03.

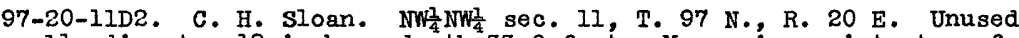
bored well, diameter 18 inches, depth 33.0 feet. Measuring point, top of plank cover at land surface. Equipped with lift pump. Water level, in feet below measuring point, 1941: Nov. 18, 7.74 .

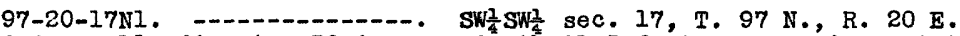
Unused dug well, diameter 36 inches, depth 21.5 feet. Measuring point, top of casing level with concrete curb, 0.3 foot above land surface. Water level, in feet below measuring point, 1941: Nov. 19, 10.10.

97-20-24HI. Mrs. Vinnie Shanks. SE $\frac{1}{4} \mathrm{NE} \frac{1}{4}$ sec. 24, T. 97 N., R. 20 E. Unused dug well, diameter 36 inches, depth 33.0 feet. Measuring point, top of concrete weli platform, 0.4 foot above land surface. Water level, in feet below measuring point, 1941: Nov. 14, 5.55.

97-20-24H2. Mrs. Vinnie Shanks. SE $\frac{1}{4} \mathrm{NE}_{\frac{1}{4}}^{\frac{1}{4}}$ sec. 24, T. 97 N., R. $20 \mathrm{E}$. Drilled domestic and stock well, diameter 5 inches, reported depth 280 feet. Measuring point, top of casing, 4.2 feet below land surface. Equipped with lift pump and electric motor. Water level, in feet below measuring point, 1941: Nov. 14, 48.81 .

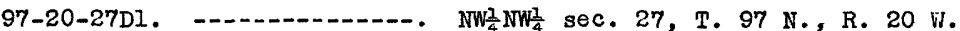
Unused drilled well, diameter 6 inches, depth 66.4 feet. Meásuring point, bottom of crack in pump housing, 0.3 foot above land surface. Equipped with lift pump. Water level, in feet below measuring point, 194l:

Nov. $19,13.68$.

97-20-32H1. Unused drilled well, diameter $4 \frac{1}{2}$ inches, depth 41.6 feet. Méasuring point, top of casing and concrete curb, 0.3 foot above land surface. Water level, in feet below measuring point, 194l: Nov. $21,15.58$.

97-19-5Nl. Chicago, Milwaukee, st. Paul and Pacific Railroad. SW $W_{4}^{\frac{1}{4}}$ SW sec. 5, T. $97 \mathrm{~N}$., R. $19 \mathrm{~W}$. Dug domestic well, diameter 5 feet, depth 22 feet. Measuring point, top of concrete platform, 1.0 foot above land surface. Equipped with iff pump. Water level, in feet below messuring point, 1941: Nov. 14, 18.00 .

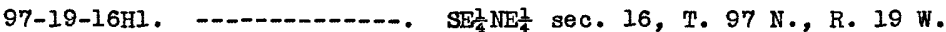
Unused drilled well, diameter 6 inches, depth 62.0 feet. Measuring point, top of casing, 2.0 feet above land surface. Water level, in feet below measuring point, 1941: Nov. 13, 6.15 .

97-19-21H2. Mrs. Oscar Engstrom. SE $\mathrm{NE}_{4}^{\frac{1}{4}}$ sec. 21, T. 97 N., R. 19 W. Dug domestic well, diameter 36 inches, depth 11.0 feet. Measuring point, top of plank cover, 0.8 foot above land surface. Equipped with suction pump. Water level, in feet belok measuring point, 1941: Nov. 13, 5.78.

$5110050-48-2$ 
Cerro Gordo County--Continued

97-19-23Hl. Jas. Senior. $\mathrm{SE}_{\frac{1}{4}} \mathrm{NE}_{\frac{1}{4}}^{\frac{1}{4}}$ sec. 23, T. $97 \mathrm{~N} ., \mathrm{R}, 19 \mathrm{~W}$. Drilled domestic and stock well, diameter 5 inches, reported depth 239 feet. Measuring point, top of west bolt hole in casing, 0.7 foot above land surface. Taps water in limestone. Equipped with lift pump and windmill. Water level, in feet below measuring point, 1941: Nov. 14, 19.20.

97-19-30RI. E. Stebens. SE⿺ SEI sec. 30, T. 97 N., R. 19 W. Dug domestic well, diameter 36 inches, depth 16.0 feet. Measuring point, top of plank cover, 0.75 foot above land surface. Taps water in sand. Equipped with lift pump and pressure system operated by electric motor. Water level, in feet below measuring point, 1941: Nov. 13, 9.18.

97-19-30R2. E. Stebens, $\mathrm{SE}_{4}^{\frac{1}{4}} \mathrm{SE}_{\frac{1}{4}}^{\mathrm{z}}$ sec. 30, T. $97 \mathrm{~N}$, , R. $19 \mathrm{~W}$. Drilled stock well, diameter 5 inches, depth 60.0 feet. Measuring point, top of casing, 5.0 feet below land surface. Equipped with lift pump and windmill. Water level, in feet below measuring point, 1941: Nov. 13, 11.50 .

96-22-7QI. W. S. Overgaard. SW $\frac{1}{4} S E \frac{1}{4}$ sec. 7, T. $96 \mathrm{~N} .$, R. $22 \mathrm{~W}$. Drilled domestic and stock well, depth 101.2 feet. Measuring point, top of casing, 1.0 foot above land surface and 0.25 foot above measuring point of well 97-22-7Q2. Equipped with lift pump and windmill. Water level, in feet below measuring point, 1941: 0ct. 14, 20.66 .

96-22-7Q2. W. S. Overgaard. SW $\frac{1}{4} \mathrm{SE}_{4}^{\frac{1}{4}}$ sec. 7, T. $96 \mathrm{~N}$, , R. $22 \mathrm{~W}$. Abandoned dug well, diameter 15 inches, depth 18.6 feet. Measuring point, top of concrete curb, 0.3 foot above land surface and 0.25 foot below measuring point of well 97-22-7Q1. Water level, in feet below measuring point, 1941: oct. 14, 8.72.

96-22-12P1. Daughters of the American Revolution Camp.

Water level, in feet below measuring point, 1941

\begin{tabular}{ll|ll|ll}
\hline Date & $\begin{array}{l}\text { Water } \\
\text { level }\end{array}$ & Date & $\begin{array}{l}\text { Water } \\
\text { level }\end{array}$ & Date & $\begin{array}{r}\text { Water } \\
\text { level }\end{array}$ \\
\hline Mar. 22 & 44.45 & June 19 & 43.65 & Aug. 20 & 43.37 \\
May 21 & 44.37 & July 22 & 43.20 & 0ct. 14 & 43.11 \\
\hline
\end{tabular}

96-22-14BI. A. A. Adams.

Water level, in feet below measuring point, 1941

\begin{tabular}{ll|ll|ll|lr}
\hline Date & $\begin{array}{l}\text { Water } \\
\text { level }\end{array}$ & Date & $\begin{array}{l}\text { Water } \\
\text { level }\end{array}$ & Date & $\begin{array}{l}\text { Water } \\
\text { level }\end{array}$ & Date & $\begin{array}{r}\text { Water } \\
\text { level }\end{array}$ \\
\hline Feb. 13 & 28.10 & Apr. 22 & 27.12 & June 19 & 26.40 & Aug. 20 & 27.47 \\
Mar. 22 & 27.29 & May 21 & 27.34 & July 22 & 26.47 & Oct. 14 & 26.90 \\
\hline
\end{tabular}

96-22-14Cl. Fred stephens.

Water level, in feet below measuring point, 1941

\begin{tabular}{ll|lll|ll|ll}
\hline Feb. 13 & 33.64 & May 25 & 33.44 & July 22 & 33.05 & Oct. 14 & 33.21 \\
Mar. 22 & 33.69 & June 19 & 32.98 & Aug. 20 & 33.63 & Dec. 10 & 32.61 \\
Apr. 22 & 33.55 & & & & & & & \\
\hline
\end{tabular}

96-22-2001. The Willow Inn.

Water level, in feet below measuring point, 1941

\begin{tabular}{ll|ll|ll|lll}
\hline Feb. 13 & 6.10 & May 21 & 4.84 & July 22 & 5.44 & Oct. 14 & 4.35 \\
Mar. 22 & 5.05 & June 19 & 3.75 & Aug. 20 & 6.47 & Dec. 12 & 4.20 \\
Apr. 22 & 2.40 & & & & & & & \\
\hline
\end{tabular}


Cerro Gordo County--Continued

96-22-20L1.

Water level, in feet below measuring point, 1941

\begin{tabular}{ll|ll|ll|ll}
\hline Date & $\begin{array}{l}\text { Water } \\
\text { level }\end{array}$ & Date & $\begin{array}{l}\text { Water } \\
\text { level }\end{array}$ & Date & $\begin{array}{l}\text { Water } \\
\text { level }\end{array}$ & Date & $\begin{array}{r}\text { Water } \\
\text { level }\end{array}$ \\
\hline Feb. 13 & 32.51 & Maj 21 & 32.14 & July 22 & 41.73 & Oct. 14 & 32.45 \\
Mar. 22 & 32.41 & June 19 & 37.38 & Aug. 20 & 36.47 & Dec. 10 & 31.48 \\
Apr. 22 & 32.27 & & & & & &
\end{tabular}

96-22-23Q1.

Water level, in feet below measuring point, 1941

\begin{tabular}{ll|ll|lll|ll}
\hline Feb. 13 & 23.27 & May 21 & 21.72 & July 22 & 17.30 & Oct. 14 & 21.28 \\
Mar. 22 & 23.37 & June 19 & 19.80 & Aug. 20 & 18.51 & Dec. 10 & 20.67 \\
Apr. 22 & 22.50 & & & & & & & \\
\hline
\end{tabular}

96-22-25D2. United States Geological Survey.

Water level, in feot below measuring point, 1941

\begin{tabular}{ll|ll|ll|ll}
\hline Feb. 13 & 7.47 & Apr. 22 & 5.57 & June 19 & 5.70 & Aug. 20 & 7.45 \\
Mar. 22 & 6.40 & May 21 & 6.61 & July 22 & 6.63 & Dec. 10 & 6.25 \\
\hline
\end{tabular}

96-22-30H1. Mrs. Francis skene. SE $\frac{1}{4} \mathrm{NE}_{\frac{1}{4}}$ sec. 30, T. 96 N., R. 22 W. Bored and driven domestic well, diameter 24 inches, depth 19.5 feet. Measuring point, top of casing, at land surface. Taps water in glacial drift. Equipped with lift pump. Water level, in feet below measuring point, 1941: oct. 15, 11.67 .

96-22-30H2. Mrs. Francis Skene. SE $\frac{1}{4} \mathrm{NE} \frac{1}{4}$ sec. 30, T. $96 \mathrm{~N} ., \mathrm{R} .22 \mathrm{~W}$. Drilled stock well, diameter 5 inches, depth 125 feet. Measuring point, top of casing 6.4 feet below concrete pit curb which is 1.0 foot above land surface. Equipped with force pump and windmill. Water level, in feet below measuring point, 1941: oct. 15, 12.03.

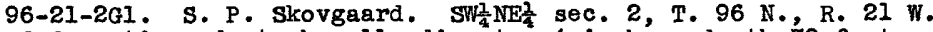
Drilled domestic and stock well, diameter 4 inches, depth 79 feet. Measuring point, top of casing 2.6 feet above land surface. Taps water in limestone. Equipped with lift pump and windmill. Water level, in foot below measuring point, 1941: Nov. 12, 12.64.

96-21-5Gl. Farmers Nat1onal Iife Insurance Company. SW $\mathbb{1}_{4} \sqrt{\frac{1}{4}}$ sec. 5 , T. 96 N., R. 21 W. Abandoned dug and driven well, diameter 3 feet to 2 inches, depth 34.0 feet. Measuring point, top of concrete curb, 1.5 feet above land surface. Taps water in glacial drift. Water level, in feet below measuring point, 1941: Nov. 13, 24.35.

96-21-7Pl. -- Water level, in feet below measuring point, 1941: Feb. 13, 19.5. Measurements discontinued.

96-21-13El. Mason City and Clear Lake Railway.

Water level, in feet below measuring point, 1941

\begin{tabular}{ll|ll|ll|ll}
\hline Feb. 13 & 6.42 & Apr. 22 & 5.40 & June 19 & 5.32 & Nov. 19 & 5.40 \\
Mar. 22 & 5.64 & May 22 & 6.25 & Aug. 20 & 6.56 & & \\
\hline
\end{tabular}

96-21-17Cl. Clear Lake Sand and Gravel Company. (Incorrectly shown as 96-21-17Bl in Water-Supply Paper 908).

Water level, in feet below measuring point, 1941

\begin{tabular}{ll|ll|ll|lll}
\hline Feb. 13 & 11.65 & May 21 & 11.72 & July 22 & 10.98 & Nov. 18 & 11.03 \\
Mar. 22 & 11.17 & June 19 & 11.09 & Aug. 20 & 11.50 & Dec. 10 & 11.17 \\
Apr. 22 & 11.35 & & & & & & & \\
\hline
\end{tabular}


Cerro Gordo County--Continued

96-2I-18HI. Sam Kennedy.

Water level, in feet below measuring polnt, 194 I

\begin{tabular}{|c|c|c|c|c|c|c|c|}
\hline Date & $\begin{array}{l}\text { Water } \\
\text { level }\end{array}$ & Date & $\begin{array}{l}\text { Water } \\
\text { level }\end{array}$ & Date & $\begin{array}{l}\text { Water } \\
\text { level }\end{array}$ & Date & $\begin{array}{l}\text { Vater } \\
\text { level }\end{array}$ \\
\hline $\begin{array}{ll}\text { Feb. } 13 \\
\text { Mar. } 22 \\
\text { Apr. } 22\end{array}$ & $\begin{array}{l}11.45 \\
11.07 \\
11.12\end{array}$ & $\begin{array}{cc}\text { May } & 21 \\
\text { June } & 19\end{array}$ & $\begin{array}{l}11.20 \\
10.24\end{array}$ & $\begin{array}{l}\text { July } 22 \\
\text { Aug. } 20\end{array}$ & $\begin{array}{l}10.40 \\
10.67\end{array}$ & $\begin{array}{l}\text { Nov. } 19 \\
\text { Dec. } 10\end{array}$ & $\begin{array}{r}9.98 \\
10.20\end{array}$ \\
\hline
\end{tabular}

96-2I-17MI.

Water level, in feet below measuring point, 1941

\begin{tabular}{ll|ll|lll|ll}
\hline Feb. 13 & 4.25 & May 21 & 3.22 & July 22 & 4.39 & Nov. 19 & 4.02 \\
Mar. 22 & 3.88 & June 19 & 2.31 & Aug. 20 & 4.57 & Dec. 10 & 4.33 \\
Apr. 22 & 2.43 & & & & & & & \\
\hline
\end{tabular}

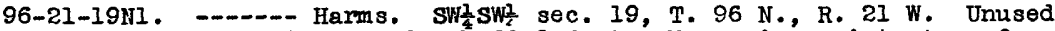
bored well, diameter 12 inches, depth 60.8 feet. Measuring point, top of casing 0.3 foot above land surface. Equipped with lift pump. Water level, in feet below measuring point, 194l: Nov. 18, 6.56 .

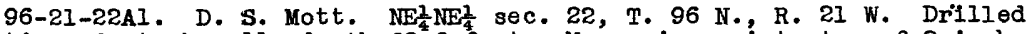
domestic and stock well, depth 68.6 feet. Measuring point, top of 2 -inch plank pit cover, 0.2 foot above land surface. Equipped with lift pump and Findmili. Water level, in feet below measuring point. 1941: Nov. 18, 17.15.

96-2l-23Rl. Elmer and Willard Thrams. SW $\frac{1}{4}$ SW $\frac{1}{4}$ sec. 23, T. 96 N., R. 21 W. Abandoned dug and driven well, diameter 4 feet to $1 \frac{1}{4}$ inches, depth 35.5 feet. Measuring point, top of 2 -inch plank pit cover level with concrete floor 1.0 foot above land surface. Water level, in feet below measuring point, 1941: Nov. 19, 5.94.

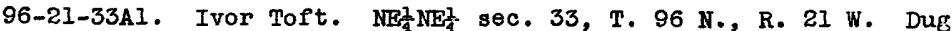
domestic and stock well, diameter 36 inches, depth 40.0 feet. Measuring point, top of 2-inch plank well cover level'with concrete floor and land surface. Taps water in glacial drift. Equipped with lift pump and electric motor. Water level, in feet below measuring point, 194l: Nov. 19, 17.17 .

96-20-3L2. Owmer No. 8. City of Mason City. NE $\frac{1}{4} \mathrm{SW}_{4}^{\mathrm{z}}$ sec. 3, T. $96 \mathrm{~N}$, R. $20 \mathrm{~W}$. Drilled well, diameter 20 inches, depth 1,219 feet. Measuring point, top of steel plate, over casing at hole, 4.8 feet below land surface. Equipped with air lift pump, and used as standby for city supply. Taps water in Jordan sandstone. Water levels affected by pumping from nearby wells. Water-level recorder maintained on well since Nov. 5, 1941 .

Water level, in feet below measuring point, 1941

\begin{tabular}{|c|c|c|c|c|c|}
\hline Date & Hour & $\begin{array}{l}\text { Water } \\
\text { level }\end{array}$ & Date & Hour & $\begin{array}{l}\text { Water } \\
\text { level }\end{array}$ \\
\hline $\begin{array}{lr}\text { July } & 9 \\
& 16 \\
\text { Nov. } & 5\end{array}$ & $\begin{array}{l}4: 15 \text { p.m. } \\
2: 50 \text { p.m. } \\
3: 10 \text { p.m. }\end{array}$ & $\begin{array}{l}184.84 \\
189.78 \\
183.35\end{array}$ & $\begin{array}{ll}\text { Nov. } 20 \\
\text { Dec. } 12\end{array}$ & $\begin{array}{l}\text { 2:00 p.m. } \\
4: 10 \text { p.ms }\end{array}$ & $\begin{array}{l}180.65 \\
187.20\end{array}$ \\
\hline
\end{tabular}

96-20-3PI. Minneapolis and St. Louls Railroad. SE $\frac{1}{4} \mathrm{SW} \frac{1}{4}$ sec. 3 ,

T. 96 N., R. $20 \mathrm{~W}$. Drilled rallroad well, diameter 12 inches, depth 805 feet. Measuring point, top of casing, 6.3 feet below land surface. Taps water in st. Peter sandstone. Equipped with 15 horsepower turbine pump.

Water level, in feet below measuring point, 1941: 35.92 feet.

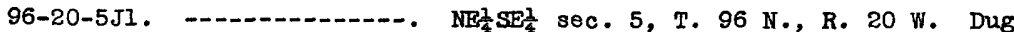
stock well, diameter 24 inches, depth 11.5 feet. Measuring point, top of 2-inch pump support platform, 1.7 feet above land surface. Taps water in glacial drift. Equipped with lift pump. Water level, in feet below measuring point, 1941: Nov. $21,8.57$. 
Cerro Gordo County--Continued

96-20-10N1. Swift and Company. SW $\mathrm{SW}_{4}^{\frac{1}{4}}$ sec. 10, T. 96 N., R. 20 W. Drilled Industrial well, diameter 12 inches, depth 462 feet. Measuring point, horizontal hole in pump base, at west side 0.8 foot above land surface. Taps water in limestone. Equipped with 10 horsepower turbine pump. Water levels, in feet below measuring point, 1941: June, a/ 50.70; Nov. $20,37.50$.

96-20-16J1. Owner's number 11. City of Mason City.

Water level, in feet below measuring point, 1941

\begin{tabular}{lrr|lr|lrr}
\hline Date & $\begin{array}{r}\text { Water } \\
\text { level }\end{array}$ & Date & $\begin{array}{l}\text { Water } \\
\text { level }\end{array}$ & Date & $\begin{array}{r}\text { Water } \\
\text { level }\end{array}$ \\
\hline May 22 & $\begin{array}{l}194.50 \\
\text { June } 4\end{array}$ & $\begin{array}{l}\text { June } 11 \\
\text { 192.70 }\end{array}$ & Dct. 9 & $\begin{array}{l}189.95 \\
207.83\end{array}$ & Nov. 5 & 200.94 \\
\hline
\end{tabular}

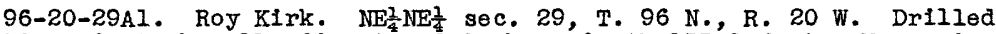
domestic and stock well, diameter 6 inches, depth 155.8 feet. Measuring point, top inside rim of manhole cover 0.1 foot above land surface. Equipped with automatic pressure system. Water level, in feet below measuring point, 1941: Nov. 21, 13.05.

96-20-36NI. - Sin Sill sec, 36, T. $96 \mathrm{~N}$, , R. $20 \mathrm{~W}$. Bored domestic well, diameter 6 inches, depth 16.0 'feet. Mésuring point, top of casing, 0.7 foot above land surface. Equipped with lift pump and windmill. Water level, in feet below measuring point, 1941: 0ct. 10, 1.45.

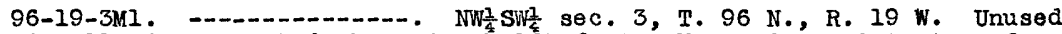
drilled well, diameter 5 inches, depth 165 feet. Measuring point, top of casing, 1.8 feet above land surface. Taps water in limestone. Water level, in feet below measuring point, 1941: Nov. 6, 60.68 .

96-19-18Rl. Chlcago, Milwaukee, St. Paul, and Pacific Rallroad. Se $\frac{1}{4}$ SE $\frac{1}{4}$ sec. 18 , T. 96 N., R. 19 W. Unused drilied well, diameter 6 inches, depth 120 feet. Measuring point, top plank cover, 0.8 foot above land surface. Taps water in limestone. Equipped with lift pump. Water levels, in feet below measuring point, 1941: Aug. 14, 10.53; Nov. 11, 6.28.

96-19-18R2. R. L. Billings, SEl SEl sec. 18, T. 96 N., R. 19 W. Drilled domestic well, dlameter 5 inches, depth 25.0 feet. Measuring point, top of casing at land surface. Equipped with lift pump. Water level, in feet below measuring point, 1941: Nov. 11, 5.60 .

96-19-27El. Independent order of Foresters. sw $\frac{1}{4} \mathrm{NW} \frac{2}{4}$ sec. 27, T. 96 N., R. 19 W. Drilled domestic and stock well, diameter 6 inches, depth 67.2 feet. Measuring point, top of casing, 1.3 feet above land surface. Equipped with lift pump. Water level, in feet below measuring point, 1941: Nov. $7,6.39$.

96-19-31P2. Fay Thompson. SE $\mathrm{SW}_{\mathrm{I}}^{\mathrm{I}}$ sec. 31, T. $96 \mathrm{~N}, \mathrm{R}, 19 \mathrm{~W}$. Abandoned well, diameter 5 inches, depth 117.0 feet. Measuring point, top of casing 5.0 feet below land surface. Water level, in feet below measuring point, 1941: Nov. 11, 84.46 feet.

95-22-3Bl. Knut 0lson. NW $\mathrm{NEE}_{\frac{1}{4}}^{\frac{1}{4}}$ sec. 3, T. $95 \mathrm{ll}, \mathrm{R} .22 \mathrm{~W}$. Drilled domestic and stock well, diameter 4 inches, depth 134.2 feet. Measuring point, top of casing, 1.4 feet above land surface. Taps water in limestone. Equipped with suction pump and electric motor. Water level, in feet below measuring point, 1941: 0ct. 15, 18.03.

95-22-5MI. School District. NW/ $\mathrm{NWW}_{\frac{3}{4}}$ sec. 5, T. $95 \mathrm{~N},, \mathrm{R} .22 \mathrm{~W}$. Unused jetted well, diameter 4 inches, depth 29.3 feet. Measuring point, top. of casing, 1.3 feet above land surface. Taps water in glacial drift. Equipped with lift pump. Water level, in feet below measuring point, 1941: 0ct. 15, 5.42 .

a After extensive pumping. 


\section{Cerro Gordo County--Continued}

95-22-8C1. Jurgenson Bros. NE $\frac{1}{4} N W \frac{1}{4}$ sec. 8, T. 95 N., R. 22 W. Drilled domestic and stock well, diameter $43 / 4$ inches, depth 152.5 feet. Measuring point, top of concrete p1t cover, 0.5 foot above land surface. Taps water in limestone. Equipped w1th automatic pressure system. Water level, in feet below measuring point, 1941: oct. 16, 14.43.

95-22-19R1. … $\operatorname{SE} \frac{1}{4} \mathrm{SE}_{\frac{1}{4}}$ sec. 19, T. $95 \mathrm{~N} ., \mathrm{R} .22 \mathrm{~W}$. Drilled domestic and stock well, dismeter 5 inches, depth 70.9 feet. Measuring point, top of casing, 0.85 foot above land surface. Equipped with lift pump and windmill. Water level, in feet below measuring point, 1941: 0ct. 16, 4.05.

95-22-22CI. John M1les, NE $\mathrm{N}_{4}^{2}$ sec. 22, T. 95 N., R. $22 \mathrm{~W}$. Abandoned bored well, diameter 18 inches, depth 21.5 feet. Measuring point, top of concrete curb, 0.3 foot above land surface. Water level, in feet below measuring point, 1941: 0ct. 16, 10.50.

95-22-34E1. J. G. Lindon. SWT $\mathrm{NW} \frac{1}{4}$ sec. 34, T. $95 \mathrm{~N}$, , R. 22 W. Dr1lled stock well, dlameter 4 inches, depth 132 feet. Measuring point, top of casing, 1.1 feet above land surface. Taps water in Iimestone. Equipped with lift pump and windmill. Water level, in feet below measuring point, 1941: 0ct. 16, 30.29 .

95-21-2HI. Amy J. Houck. $\operatorname{SE}_{\frac{1}{2}} \mathrm{NE}_{\frac{1}{4}}^{\frac{1}{2}}$ sec. 2, T. $95 \mathrm{~N} ., \mathrm{R}, 21 \mathrm{~W}$. Drilled domest1c and stock well, dlameter 5 inches, depth 138.3 feet. Measuring point, top of plank cover, 2.2 feet above land surface. Taps water in imestone. Equipped with lift pump and windmill. Water level, in feet below measuring point, 1941: Sept. 23, 38.01.

95-21-7DI. Commissioner of Insurance of Iowa. NWW NWT sec. 7, T. 95 N., R. $21 \mathrm{~W}$. Dug and drilled domestic and stock well, diameter 42 inches, depth 40 feet. Measuring point, top of 2-inch plank cover, 2.2 feet above land surface. Taps water in giacial drift. Equipped with lift pump. Water level, in feet below measuring point, 1941: Sept. 23, 16.57.

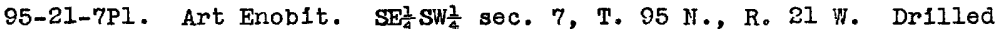
domestic and stock well, diameter 5 inches, depth over 100 feet. Measuring point, top of casing 1.0 foot above land surface. Taps water in limestone. Equipped with ift pump. Water level, in feet below measuring point, 1941: Sept. 24, 13.66

95-21-12D2. № NW Bored domestlc well, diameter 5 inches, depth 25.0 feet. Measuring point, top of casing, 0.3 foot above land surface. Taps water in glacial drift. Equipped with pitcher pump. Water level, in feet below measuring point, 1941: Sept. 23, 6.85 .

95-21-27Q1. John Hancock Insurance Company. SW $\frac{1}{4}$ SE $\frac{1}{4}$ sec. 27, T. 95 N., R. $21 \mathrm{~W}$. Drilled domestic well, diameter 5 inches, depth 114.1 feet. Measuring point, top of casing, 2.4 feet above land surface. Equipped with Ift pump. Water level, in feet below measuring point, 1941: Sept. 25, 21.76 .

95-20-3BI. Farmers Co-op Soclety. NW $\frac{1}{4} \mathrm{NE} \frac{2}{4}$ sec 3, T. 95 N., R. 20 W. Drilled domestic well, diameter 5 inches, depth 60.8 feet. Measuring point, top of wood platform 2.2 feet above land surface. Water level, in feet below measuring point, 1941: oct. 15, 11.36 .

95-20-5J2. W11l Hunt. NE $\frac{1}{4}$ SE $\frac{1}{4}$ sec. 5, T. 95 N., R. 20 W. Dug and drilled domestic well, diameter 18 inches to 5 inches, depth 43.3 feet. Equipped with lift pump. Water level, in feet below measuring point, 194l: oct. $15,7.70$.

95-20-13N1. School District. SW Swi sec. 13, T. 95 N., R. 20 W. Used drilled well, diameter 4 inches, depth 50.5 feet. Measuring point, top of casing 0.5 foot above land surface. Equipped with lift pump. Water level, in feet below measuring point, 1941: oct. 16, 3.24 . 
Cerro Gordo County--Continued

95-20-20Cl. Unused drilled well, diameter 5 inches, depth 40.5 feet. Measuring point, top of casing 2.0 feet above land surface. Equipped with lift pump. Water level, in feet below measuring point, 1941: 0ct. 15, 7.02.

95-20-27Q1. Bored stock well, diameter 6 inches, depth 16.5 feet. Measuring soint, top of casing, 2.0 feet above land surface. Equipped with lift pump. Water level, in feet below measuring point, 1941; 0ct. 16, 4.09 .

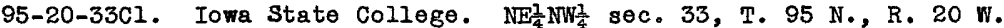
Drilled domestic and stock well, diameter 5 inches, depth 54.0 feet. Measuring point, top of wood platform, 1.0 foot above land surface. Equipped with lift pump. Water level, in feet below measuring point, 1941: 0ct. 15, 10.35 .

95-19-9Hב. SE $\frac{1}{4} N_{\frac{1}{4}}^{\frac{1}{4}}$ sec. 9, T. $95 \mathrm{~N} .$, R. $19 \mathrm{~W}$. Drilled stock well, diameter 5 inches, depth 44.0 feet. Measuring point, top of casing, 0.3 foot above land surface. Equipped with ift pump and windmill. Water level, in feet below measuring point, 1941: 0ct. 10, 10.58 .

95-19-18M1. NWT $S W \frac{1}{4}$ sec. 18, T. $95 \mathrm{~N} ., \mathrm{R} .19 \mathrm{~W}$. Drilled stock well, diameter 5 inches, depth 30.5 feet. Measuring point, top of casing 1.2 feet above land surface. Equipped with lift pump and windmill. Water level, in feet below measuring point, 1941: Oct. 10, 2.49.

95-19-26D2. H. F. Coyle. NW NW sec. 26, T. 95 N., R. 19 W. Drilled domestic well, diameter 5 inches, depth 64.0 feet. Measuring point, top of casing, 1.0 foot above land surface. Equipped with lift pump. Waier level, in feet below measuring point, 194l: oct. 14, 26.09 .

95-19-30P1. Drilled domestic well, diameter 5 inches, depth 35.0 feet. Measuring point, top of casing, 0.2 foot above land surface, and 3.2 feet above measuring point of well 95-19-30P2. Equipped with lift pump. Water level, in feet below measuring point, 1941: oct. 10, 4.91.

95-19-30P2. Bored stock well, diameter 8 inches, depth 23.5 feet. Measuring point, top of wood platform, 1.0 foot above land surface, and 3.2 feet below measuring point of well 95-19-30Pl. Equipped with lift pump and windmill. Water level, in feet below measuring point, 1941: oct. 10, 3.74 .

94-22-8Dl. -..-- Dugan. NW/ $N W \frac{1}{4}$ sec. 8, T.' 94 N., R. 22 W. Bored domestic well, diameter 8 inches, depth 74.0 feet. Measuring point, top of casing, 1.5 feet above land surface. Equipped with lift pump. Water level, in feet below measuring point, 1941: 0ct. 21, 13.90.

94-22-20Dl. Pete Wohler. NW $\frac{1}{4}$ W sec. 20, T. 94 N., R. 22 W. Drilled stock well, diameter 5 inches, depth 104 feet. Measuring polnt, top of $\frac{1}{2}$-inch hole in pump base, 1.0 foot above land surface. Equipped with lift pump and windmill. Water level, in feet below measuring point, 194l: 0ct. $21,3.71$.

94-22-24J1. Abandoned bored well, diameter 10 inches, depth 34.0 feet. Measuring point, top of concrete curb, 1.0 foot above land surface. Equipped with lift pump. Water level, in feet below measuring point, 1941: Sept. 26, 12.24.

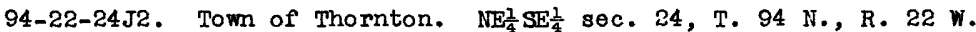
Drilled municipal well, diameter 8 inches, depth 290 feet. Measuring point, top of breather hole in pump base, 1.8 feot above land surface. Equipped with 7.5 horsepower turbine pump. Taps water in limestone. Town supply. Water level, in feet below measuring point, 194l: Sept. 26, 74.75 .

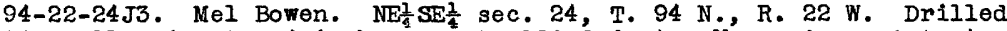
damestic well, diameter 4 inches, depth 120.6 feet. Measuring point, top of casing at land surface. Equipped with lift pump. Water level, in foet below measuring point, 1941: Sept. 26, 20.72. 


\section{Cerro Gordo County--Continued}

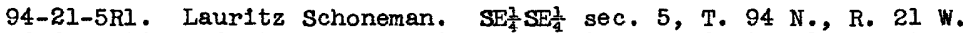
Drilled domestic and stock well, diameter 5 inches, depth 58.6 feet. Measuring point, top of hole in pump base, 0.9 foot above land surface. Equipped with lift pump and windmili. Viater levels, in feet below measuring point, 1941: June 13, 15.44; Oct. 24, 16.40.

94-21-10D2. - NW/ $N W_{4}^{\frac{2}{4}}$ sec. 10, T. $94 \mathrm{~N} .$, R. 21 W. Unused drilled well, dlameter'5 inches, depth 56.0 feet. Measuring point, top of casing, 0.4 foot above land surface. Equipped with lift pump. Water level, in feet below measuring point, 194l: Nov. 4, 17.05 .

94-21-24Al. Titus Management Company. NE $\frac{1}{4} \mathrm{NE}_{\frac{1}{4}}^{\mathrm{l}}$ sec. 24, T. $94 \mathrm{~N}$., R. $21 \mathrm{~W}$. Drilled stock well, diameter 5 inches, depth 35.5 feet. Measuring point; top of casing 2.0 feet above land surface. Equipped with ilft pump and windmill. Water level, in feet below measuring point, 1941: 0ct. 24, 7.62 .

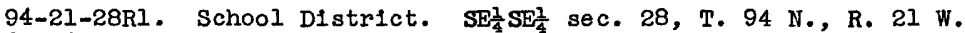
Unused drilled well, diameter 6 inches, depth 40.0 feet. Measuring point, top of casing, 0.3 foot above land surface. Water level, in feet below measuring point, 1941: 0ct. $23,3.83$.

94-20-3Kl. City of Rockwell. NW/ $\mathrm{SE}_{\frac{1}{4}}^{1}$ sec. 3, T. $94 \mathrm{~N}$, , R. $20 \mathrm{~W}$. Abandoned drilied well, diameter 8 inches, depth 230 feet. 'Measuring point, top of casing, 2.0 feet above land surface. Taps water in limestone. Water level, in feet below measuring point, 1941: Nov. 19, 7.76.

94-20-5P1. James P. Conrin. $\mathrm{SE}_{\frac{1}{4}} \mathrm{SW}_{4}^{\frac{1}{4}}$ sec. 5, T. $94 \mathrm{~N}$, , R. $20 \mathrm{~W}$. Domestic and stock well, diameter 6 inches, depth 42.8 feet. Measuring point, top of casing, 5.8 feet below land surface. Equipped with ift pump and windmill. Water level, in feet below measuring point, 1941: 0ct. 23, 12.56 .

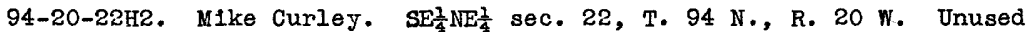
drilled well, diameter 5 inches, depth 39.0 feet. Measuring point, top of casing, 0.5 foot above land surface. Equipped with lift pump. Water level, in feet below measuring point, 1941: 0ct. 22, 16.35 .

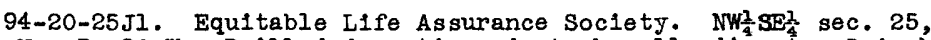
T. $94 \mathrm{~N} .2$ R. $20 \mathrm{~W}$. Drilled domestic and stock well, diameter 5 inches, reported depth 278 feet. Measuring point, bottom of hole in casing, 0.9 foot above land surface. Taps water in limestone. Equipped with ilft pump and windmill. Water level, in feet below measuring point, 1941: oct. $23,19.20$.

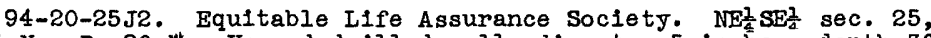
T. 94 N., R. 20 W. Unused drilled well, diameter 5 inches, depth 36.0 feet. Measuring point, top of casing, 1.3 feet above land surface. Water level, in feet below measuring point, 1941: oct. 23, 6.28 .

94-19-3NI. -Drilled domestic and stock well, diameter 5 inches, depth 41.0 feet. Heasuring point, top of casing, 0.2 foot above land surface. Equipped with ilft pump and gasoline engine. Water level, in feet below measuring point, 1941: oct. 6, 8.20.

94-19-16Rl. Edmond Kelsh. $\operatorname{SE} \frac{1}{4} \operatorname{SE} \frac{1}{4}$ sec. 16, T. 94 N., R. $19 \mathrm{~W}$. Drilled domestic and stock well, dlameter 5 inches, depth 30.8 feet. Measuring point, top of casing, 1.25 feet above land surface. Water level, In feet below land surface, 1941: oct. 7, 13.00.

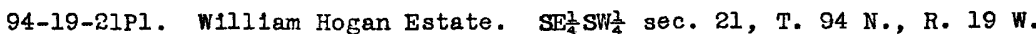
Drilled domestic and stock well, diameter 6 inches, depth 55.8 feet. Measuring point, top of casing, 1.2 feet above land surface. Equipped with lift pump and windmill. Water level, in feet below measuring point, 194l: oct. $7,31.97$. 


\section{Cerro Gordo County--Continued}

94-19-25N

SW $\frac{1}{4}$ SW $\frac{2}{4}$ sec. 25, T. 94 N., R. 19 W. Bored domestic well, diameter 6 inches, depth 19.2 feet. Equipped with lift pump. Water level, in feet below measuring point, 1941: 0ct. 8, 2.96.

\section{Cherokee County}

92-40-26P1. Owner's number 3 south. City of Cherokee. Water level measurements by D. Kennedy, Cherokee Water Works.

Water level, in feet below measuring point, 1941

\begin{tabular}{lc|lr|lr}
\hline Date & $\begin{array}{l}\text { Water } \\
\text { level }\end{array}$ & Date & $\begin{array}{r}\text { Water } \\
\text { level }\end{array}$ & Date & $\begin{array}{r}\text { Water } \\
\text { level }\end{array}$ \\
\hline Feb. I2 & a 34.07 & Apr. 5 & b 37.67 & July 30 & b 45.5 \\
Apr. 5 & 17.5 & July 30 & 23.75 & & \\
\hline
\end{tabular}

\section{Clay County}

96-35-2P1. Eva D. Monselle. Water level, In feet below measuring point, 1941: Feb. 12, 30.44. Measurements discontinued.

96-35-3Rl. Allis Wilson.

Water level, in feet below measuring point, 1941

\begin{tabular}{lr|lr|lr|lr}
\hline Date & $\begin{array}{l}\text { Water } \\
\text { level }\end{array}$ & Date & $\begin{array}{r}\text { Water } \\
\text { level }\end{array}$ & Date & $\begin{array}{r}\text { Water } \\
\text { level }\end{array}$ & Date & $\begin{array}{r}\text { Water } \\
\text { level }\end{array}$ \\
\hline Feb. 12 & 6.17 & Apr. 22 & 4.47 & June 18 & 5.15 & Aug. 20 & 7.39 \\
Mar. 21 & 4.85 & May 21 & 6.59 & July 22 & 6.05 & Dec. 11 & 4.30 \\
\hline
\end{tabular}

\section{clayton county}

$94-4-4 \mathrm{LI}$ No measurements made in 1941.

\section{C] inton County}

81-6E-22H1. Owner's number 2. E. I. duPont de Nemours \& Company. Water level, in feet above mean sea level, 1941

\begin{tabular}{|c|c|c|c|c|c|c|c|c|c|}
\hline May & $\begin{array}{r}8 \\
13 \mathrm{~d} \\
20 \\
27 \\
4 \\
10\end{array}$ & $\begin{array}{l}561.25 \\
550.4 \\
558.9 \\
559.6 \\
558.0 \\
557.1\end{array}$ & $\begin{array}{l}\text { June } \\
\text { JuIy }\end{array}$ & $\begin{array}{r}17 \\
23 \\
1 \\
8 \\
15 \\
22\end{array}$ & $\begin{array}{l}555.2 \\
553.6 \\
555.7 \\
553.2 \\
552.5 \\
552.5\end{array}$ & $\begin{array}{lr}\text { July } 29 \\
\text { Aug. } 5 \\
12 \\
20 \\
26 \\
\text { Sept. } 2 \text { e }\end{array}$ & $\begin{array}{c}550.6 \\
549.9 \\
(e) \\
548.3 \\
549.5 \\
548.1\end{array}$ & $\begin{array}{l}\text { Sept. } 9 \\
\text { oct. } 10 \\
17 \\
24 \\
31 \\
\text { Nov. } 14\end{array}$ & $\begin{array}{l}\text { f } 548.1 \\
\text { f } 547.6 \\
549.9 \\
549.5 \\
550.1 \\
\text { d } 545.7\end{array}$ \\
\hline
\end{tabular}

81-7E-6Kl. National Biscuit Company. Water levels, in feet below measuring point, 1941: Mar. 20, 50.35; Dec. 23, 58.55.

a Pump stopped a short time before measurement.

b Pumping.

c All mea surements made by E. I. duPont de llemours :- Company.

d Pump in operation.

e Water level below bottom of air line.

f Pump shut down for 5 minutes. 


\section{Dickinson County}

99-36-6G1.

SW $\frac{1}{4} \mathrm{NE}_{4}^{1}$ sec. 6 , T. $89 \mathrm{~N} .$, R. $36 \mathrm{~W}$.

Unused bored we1l, diameter 16 inches, depth 34.4 feet. Measuring point, top of east curb, 2.0 feet above land surface. Equipped with pitcher pump.

Water level, in feet below measuring point, 1940-41

\begin{tabular}{|c|c|c|c|c|c|}
\hline Date & $\begin{array}{l}\text { Water } \\
\text { level }\end{array}$ & Date & $\begin{array}{l}\text { Water } \\
\text { level }\end{array}$ & Date & $\begin{array}{l}\text { Water } \\
\text { level }\end{array}$ \\
\hline $\begin{array}{l}\text { Dec. 20, } 1940 \\
\text { Feb. 12, } 1941 \\
\text { Mar. 21. }\end{array}$ & $\begin{array}{l}8.50 \\
5.84 \\
5.70\end{array}$ & $\begin{array}{l}\text { Apr. 22, } 1941 \\
\text { May } 20\end{array}$ & $\begin{array}{l}3.96 \\
5.66\end{array}$ & $\begin{array}{l}\text { July 22, } 1941 \\
\text { Dec. } 10\end{array}$ & $\begin{array}{l}6.10 \\
4.60\end{array}$ \\
\hline
\end{tabular}

\section{Decatur County}

69-25-29R1. No measurement made in 1941.

\section{Emmet County}

100-32-11Rl. Okamanpedan state Park. Water level, in feet below measuring point, 1941: Dec. 10,62.48.

99-34-14Bl. No measurements made in 1941.

99-32-10El. C. E. Birney.

Water level, in feet below measuring point, 1941

\begin{tabular}{ll|ll|ll}
\hline Mar. 21 & 6.00 & May 21 & 5.32 & Dec. 10 & 4.09 \\
Apr. 18 & 3.96 & Aug. 20 & 7.61 & & \\
\hline
\end{tabular}

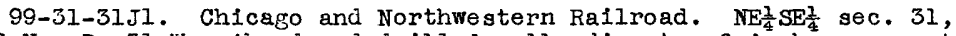
T. 99 N., R. 31 W. Abandoned drilled well, diameter 6 inches, reported depth 321 feet. Measuring point, top of plank covering over well pit. Water level, in feet below measuring point, 1941: Aug. 20, 84.15 .

\section{Hamilton County}

86-25-5El. No measurements made in 1941.

\section{Hardin County}

89-20-7El. Wm. H. Gilbert. SW/ NW $\frac{1}{4}$ sec. 7, T. 89 N., R. 20 W. Bored domestic and stock well, diameter 16 inches, depth 46.5 feet. Measuring point, top of wood plank pump platform, 0.2 feet above land surface.

Equipped with lift pump. Water levels, in feet below measuring point, 1941: Dec. 13, 18.02; Dec. 21, 17.5; Dec. 28, 16.75.

\section{Harrison County}

80-42-11Q1. No measurements made in 1941 .

80-41-20M1. Mutual Benefit Life Insurance Company. Water level, in feet below measuring point, 1941: Aug. 28, 76.03. 


\section{Harrison County--Continued}

79-41-34-N1. Mutual Benefit Life Insurance Company. Water levels, In feet below measuring point, 1941: Mar. 18, 55.70; Apr. 17, 61.14; Juig 28, 58.10; Aug. 28, 54.15.

78-42-12Q1. Mutual Benefit Iife Insurance Company. Water levels, in feet below messuring point, 1841: Feb. 12, 22.60; July 28, 24.16; Aug. a8, 26.51; Dec. 20, 25.07 .

78-42-11A1. Mutual Benefit Life Insurance Company.

Water level, in feet below measuring point, 1941

\begin{tabular}{|c|c|c|c|c|c|c|}
\hline Date & & $\begin{array}{l}\text { Water } \\
\text { level }\end{array}$ & Date & $\begin{array}{l}\text { Water } \\
\text { level }\end{array}$ & Date & $\begin{array}{l}\text { Water } \\
\text { level }\end{array}$ \\
\hline $\begin{array}{l}\text { Feb. } 12 \\
\text { Mar. } 18\end{array}$ & - & $\begin{array}{l}27.91 \\
29.09\end{array}$ & $\begin{array}{l}\text { Apr. } 17 \\
\text { Aug. } 28\end{array}$ & $\begin{array}{l}27.88 \\
35.18\end{array}$ & Dec. 20 & 27.75 \\
\hline
\end{tabular}

\section{Ida county}

89-40-35DI. No measurements made in 1941 .

89-39-12L1. Measurements discontinued.

\section{Jasper County}

80-18-31Cl. Union Central Life Insurance Company (former owner); Maynard Lust (new owner). Water levels, in feet below measuring point, 1941: Feb. 11, 21.72; Sept. 18, 22.36; Dec. 5, 22.90.

\section{Johnson County}

80-5-9KI. Measurements discontinued.

80-5-9K2. United States Geological Survey. Equipped with water-level recorder. Highest water level observed from recorder charts for 1941 was 2.34 feet below measuring point on Nov. 1 , and lowest observed water level was 7.52 feet below measuring point on sept. 7 .

Water level, in feet below measuring point, 1941

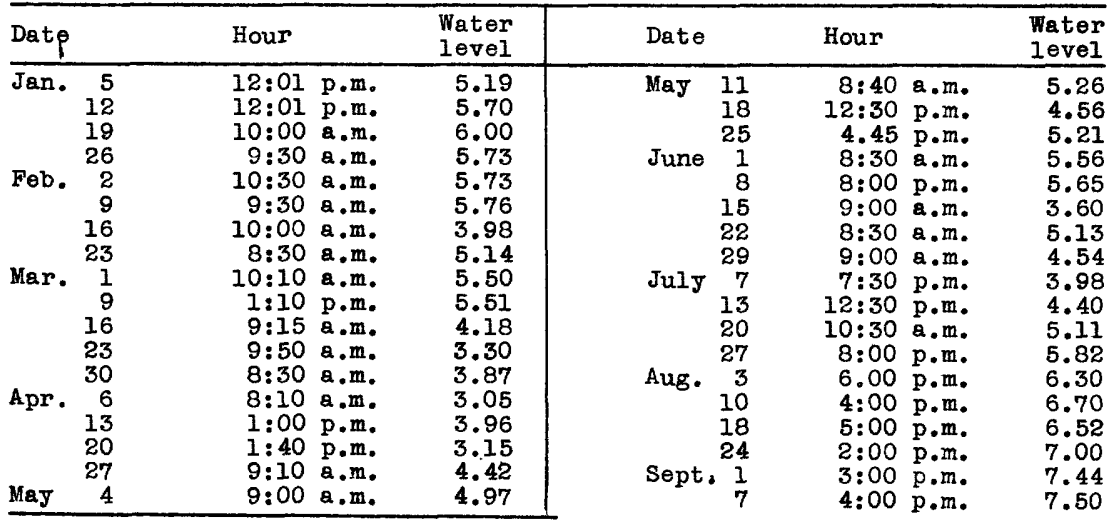


Johnson County--Continued

80-5-9K2.--Continued.

Water level, in feet below measuring point, 1941

\begin{tabular}{|c|c|c|c|c|c|}
\hline Date & Hour & $\begin{array}{l}\text { Water } \\
\text { level }\end{array}$ & Date & Hour & $\begin{array}{l}\text { Water } \\
\text { level }\end{array}$ \\
\hline 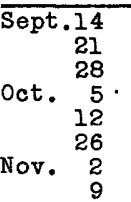 & $\begin{aligned} 1: 00 & \text { p.m. } \\
1: 10 & \text { p.m. } \\
1: 20 & \text { p.m. } \\
1: 30 & \text { p.m. } \\
1: 50 & \text { p.m. } \\
\text { 4:10 } & \text { p.m. } \\
11: 00 & \text { a.m. } \\
2: 35 & \text { p.m. }\end{aligned}$ & $\begin{array}{l}4.65 \\
5.45 \\
5.89 \\
3.45 \\
3.20 \\
2.52 \\
2.50 \\
2.85\end{array}$ & $\begin{array}{rr}\text { Nov. } & 16 \\
23 \\
30 \\
\text { Dec. } 7 \\
14 \\
21 \\
28\end{array}$ & $\begin{array}{ll}1: 50 & \mathrm{p} \cdot \mathrm{m} . \\
2: 00 & \mathrm{p} . \mathrm{m} . \\
1: 55 & \mathrm{p} . \mathrm{m} . \\
2: 05 & \mathrm{p} . \mathrm{m} . \\
1: 50 & \mathrm{p} . \mathrm{m} . \\
2: 15 & \mathrm{p} \cdot \mathrm{m} . \\
1: 40 & \mathrm{p} . \mathrm{m} .\end{array}$ & $\begin{array}{l}3.50 \\
3.77 \\
4.05 \\
4.35 \\
4.72 \\
4.68 \\
3.40\end{array}$ \\
\hline
\end{tabular}

80-5-22Ml. Chicago, Rock Island, and Pacific Railroad. NW $\frac{1}{4}$ SW $\frac{1}{4}$ sec. 22 , T. 80 N., R. 5 i. Abandoned dug well, diameter 4 feet, depth 19.5 . Measuring point, top of concrete curb, 0.3 foot above land surface. waterlevel recorder maintained on well since Dec. 7, 1941. Measurements by Maurice Albertson, State University of Iowa.

Water level, in feet below measuring point, 1941

\begin{tabular}{lrr|lr|ll|ll}
\hline Date & $\begin{array}{l}\text { Water } \\
\text { level }\end{array}$ & Date & $\begin{array}{l}\text { Water } \\
\text { level }\end{array}$ & Date & $\begin{array}{l}\text { Water } \\
\text { level }\end{array}$ & Date & $\begin{array}{r}\text { Water } \\
\text { level }\end{array}$ \\
\hline Oct. 24 & 6.25 & Dec. $\begin{array}{r}7 \\
10\end{array}$ & $\begin{array}{l}12.08 \\
12.43\end{array}$ & Dec. 13 & 12.43 & Dec. 18 & 12.75 \\
Dec. 6 & 12.13 & & 14 & 12.56 & 26 & 11.56 \\
\hline
\end{tabular}

80-5-22M2. Chicago, Rock Island, and Pacific Railroad. NW $W_{\frac{1}{4}}$ sec. 22, T. 80 N., R. 5 W. Abandoned drilled well, diameter $43 / 4$ inches, depth 82.5 feet. Measuring point, top of concrete curb, 0.3 foot above land surface. Measurements by Maurice Albertson, State University of Iowa. Water level, in feet below measuring point, 1841

\begin{tabular}{|c|c|c|c|c|c|c|c|c|}
\hline Dec. & $\begin{array}{l}6 \\
7\end{array}$ & $\begin{array}{l}16.60 \\
16.28\end{array}$ & Dec. $\frac{1}{1}$ & $\begin{array}{l}16.73 \\
16.63\end{array}$ & Dec. $\frac{1}{1}$ & $\begin{array}{l}17.25 \\
16.74\end{array}$ & Dec. 26 & 15.73 \\
\hline
\end{tabular}

79-6-10N1. Measurements discontinued.

\section{Linn County}

(Indian Creek Project of the Soll Conservation Service)

85-6-19A1. United States Geological Survey.

Water level, in feet below measuring point, 1941

\begin{tabular}{|c|c|c|c|c|c|c|c|c|c|}
\hline $\begin{array}{rr}\text { Jan. } 27 \\
\text { Feb. } 7 \\
\\
26 \\
\text { Mar. } 18 \\
31 \\
\end{array}$ & $\begin{array}{l}5.26 \\
5.30 \\
5.51 \\
5.20 \\
4.89\end{array}$ & $\begin{array}{l}\text { Apr. } \\
\text { May } \\
\text { June }\end{array}$ & $\begin{array}{r}14 \\
28 \\
12 \\
26 \\
9\end{array}$ & $\begin{array}{l}5.09 \\
5.17 \\
5.57 \\
6.05 \\
4.24\end{array}$ & $\begin{array}{l}\text { June } \\
\text { July } \\
\text { Aug. }\end{array}$ & $\begin{array}{r}23 \\
7 \\
21 \\
11 \\
29 \\
\end{array}$ & $\begin{array}{l}5.43 \\
5.10 \\
5.50 \\
6.67 \\
7.74 \\
\end{array}$ & $\begin{array}{l}\text { Sept. } 18 \\
29 \\
\text { oct. } 27 \\
\text { Nov. } 25 \\
\text { Dec. } 22 \\
\end{array}$ & $\begin{array}{l}5.74 \\
5.27 \\
4.10 \\
4.85 \\
4.87\end{array}$ \\
\hline
\end{tabular}

85-6-26D1. United States Geological survey.

Water level, in feet below measuring point, 1941

\begin{tabular}{|c|c|c|c|c|c|c|c|c|}
\hline $\begin{array}{lr}\text { Jan. } 27 \\
\text { Feb. } 7 \\
\\
26 \\
\text { Mar. } 18 \\
31\end{array}$ & $\begin{array}{l}4.17 \\
4.28 \\
4.06 \\
4.07 \\
1.23\end{array}$ & $\begin{array}{l}\text { Apr. } \\
\text { May } \\
\text { June }\end{array}$ & $\begin{array}{l}14 \\
12 \\
26 \\
23\end{array}$ & $\begin{array}{l}1.37 \\
2.82 \\
3.14 \\
3.09\end{array}$ & $\begin{array}{l}\text { July } 21 \\
\text { Aug. } 11 \\
29 \\
\text { sept. } 18\end{array}$ & $\begin{array}{l}4.38 \\
5.40 \\
6.40 \\
3.37\end{array}$ & $\begin{array}{l}\text { Sept.29 } \\
\text { Oct. } 27 \\
\text { Nov. } 25 \\
\text { Dec. } 22\end{array}$ & $\begin{array}{l}4.75 \\
1.36 \\
1.50 \\
1.75\end{array}$ \\
\hline
\end{tabular}


Linn County--Continued

85-6-29Bl. Earl Balderson.

Water level, in feet below measuring point, 1941

\begin{tabular}{|c|c|c|c|c|c|c|c|}
\hline Date & $\begin{array}{l}\text { Water } \\
\text { level }\end{array}$ & Date & $\begin{array}{l}\text { Water } \\
\text { level }\end{array}$ & Date & $\begin{array}{l}\text { Water } \\
\text { level }\end{array}$ & Date & $\begin{array}{l}\text { Water } \\
\text { level }\end{array}$ \\
\hline $\begin{array}{lr}\text { Jan. } & 27 \\
\text { Feb. } & 7 \\
& 26 \\
\text { Mar. } & 18 \\
& 31\end{array}$ & $\begin{array}{l}64.83 \\
65.02 \\
62.73 \\
64.52 \\
64.20\end{array}$ & $\begin{array}{lr}\text { Apr. } & 14 \\
& 28 \\
\text { May } & 12 \\
& 26 \\
\text { June } & 9\end{array}$ & $\begin{array}{l}64.04 \\
63.52 \\
62.70 \\
62.55 \\
62.35\end{array}$ & $\begin{array}{lr}\text { June } & 23 \\
\text { July } & 7 \\
& 21 \\
& 11 \\
\text { Aug. } & 29 \\
& 29\end{array}$ & $\begin{array}{l}62.41 \\
62.30 \\
62.57 \\
63.39 \\
64.09\end{array}$ & $\begin{array}{l}\text { Sept. } 18 \\
29 \\
\text { Oct. } 27 \\
\text { Nov. } 25 \\
\text { Dec. } 22\end{array}$ & $\begin{array}{l}63.55 \\
63.75 \\
62.62 \\
60.17 \\
59.85\end{array}$ \\
\hline
\end{tabular}

84-7-13El. Alfred Rinderknecht.

Water level, in feet below measuring point, 1941

\begin{tabular}{|c|c|c|c|c|c|c|c|c|}
\hline $\begin{array}{rr}\text { Jan. } & 27 \\
\text { Feb. } & 7 \\
& 26 \\
\text { Mar. } & 18 \\
& 31\end{array}$ & $\begin{array}{l}8.00 \\
7.80 \\
7.32 \\
7.21 \\
6.00\end{array}$ & $\begin{array}{l}\text { Apr. } \\
\text { May } \\
\text { June }\end{array}$ & $\begin{array}{r}14 \\
28 \\
12 \\
26 \\
9\end{array}$ & $\begin{array}{l}5.26 \\
4.72 \\
5.18 \\
5.00 \\
5.12\end{array}$ & $\begin{array}{l}\text { June } \\
\text { Jul } \bar{y} \\
\text { Aug. }\end{array}$ & $\begin{array}{l}5.00 \\
5.17 \\
5.71 \\
6.89 \\
8.06\end{array}$ & $\begin{array}{l}\text { Sept. } 18 \\
29 \\
\text { oct. } 27 \\
\text { Nov. } 25 \\
\text { Dec. } 22\end{array}$ & $\begin{array}{l}5.67 \\
6.81 \\
4.40 \\
3.90 \\
4.45\end{array}$ \\
\hline
\end{tabular}

84-6-20N1. United States Geological Survey.

Water level, in feet below measuring point, 1941

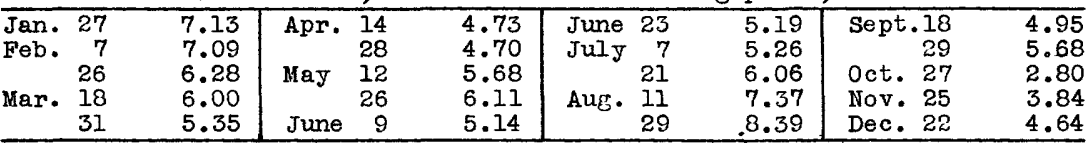

84-6-22F1. C. A. Wissler.

Water level, in feet below measuring point, 1941

\begin{tabular}{|c|c|c|c|c|c|c|c|c|}
\hline $\begin{array}{lr}\text { Jan. } & 27 \\
\text { Feb. } & 7 \\
& 26 \\
\text { Mar. } & 18 \\
& 31\end{array}$ & $\begin{array}{l}8.88 \\
8.83 \\
8.15 \\
8.15 \\
6.54\end{array}$ & $\begin{array}{ll}\text { Apr. } & \frac{1}{2} \\
\text { May } & 1 \\
\text { June } & 2\end{array}$ & $\begin{array}{l}14 \\
28 \\
12 \\
26 \\
23\end{array}$ & $\begin{array}{l}5.02 \\
4.59 \\
5.32 \\
5.90 \\
5.61\end{array}$ & $\begin{array}{ll}\text { JulJ } & 2 \\
\text { Aug. } & 1 \\
& 2\end{array}$ & $\begin{array}{l}6.27 \\
6.50 \\
7.79 \\
8.56\end{array}$ & $\begin{array}{l}\text { Sept. } 18 \\
29 \\
\text { Nov. } 25 \\
\text { Dec. } 22\end{array}$ & $\begin{array}{l}7.64 \\
7.57 \\
4.49 \\
5.37\end{array}$ \\
\hline
\end{tabular}

(Cedar Rapids Project)

83-7-1B1 City of Marion. NE $\frac{1}{4} N W \frac{1}{4}$ sec. 1, T. 83 N., R. 7 W. Drilled well, diameter 12 inches, reported depth, 437 feet. Measuring point, top of casing, 0.8 foot above land surface, Feb. 25 to Aug. 19, 1941. Beginning sept. 18, 1941, measuring point is top of $\frac{1}{2}$-inch hole in pump base, 0.30 foot above top of casing. Equipped with turbine pump.

Water level, in feet below measuring point, 1941

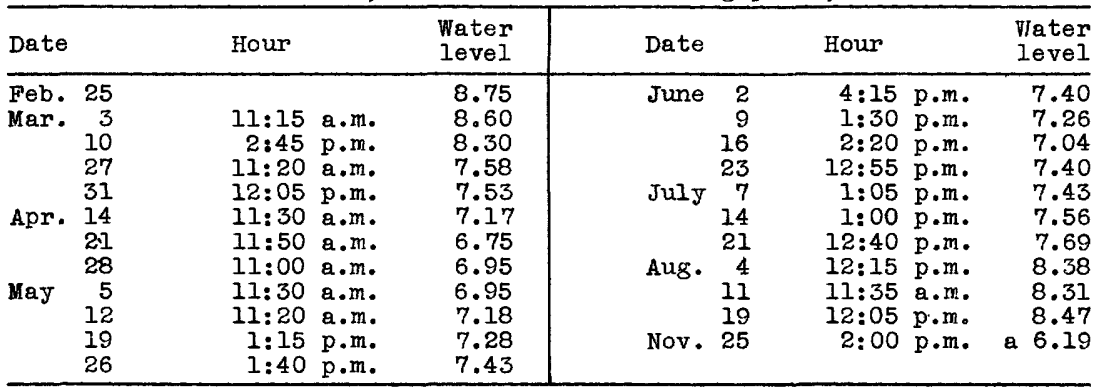

New Measuring point. 
Linn County--Continued

83-7-2PI. Hollenbeck.

Water level, in feet below measuring point, 1941

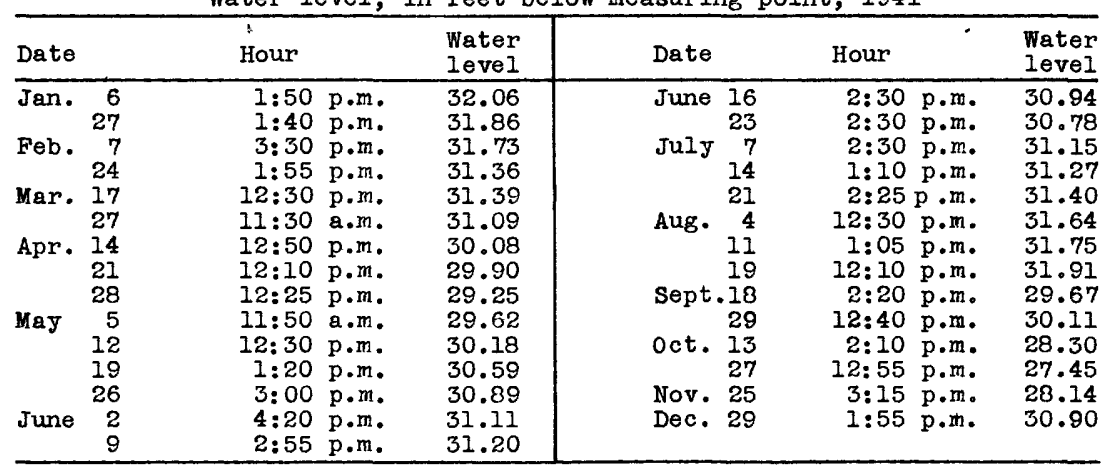

83-7-6BI. Schrimper Estate.

Water level, in feet above measuring point, 1941

\begin{tabular}{|c|c|c|c|c|c|c|c|c|}
\hline $\begin{array}{l}\text { Jan. } \\
\text { Feb. } \\
\text { Mar. } \\
\text { Apr. } \\
\text { May }\end{array}$ & $\begin{array}{r}6 \\
3 \\
7 \\
24 \\
27 \\
14 \\
21 \\
28 \\
5 \\
12 \\
19 \\
26 \\
2 \\
9\end{array}$ & $\begin{array}{r}1: 25 \\
11: 45 \\
1: 15 \\
1: 20 \\
11: 10 \\
11: 15 \\
11: 30 \\
10: 45 \\
11: 15 \\
11: 05 \\
12: 55 \\
1: 20 \\
4: 05 \\
1: 05\end{array}$ & $\begin{array}{l}\text { p.m. } \\
\text { a.m. } \\
\text { p.m. } \\
\text { p.m. } \\
\text { a.m. } \\
\text { a.m. } \\
\text { a.m. } \\
a \cdot m . \\
a \cdot m . \\
a \cdot m . \\
\text { p.m. } \\
\text { p.m. } \\
\text { p.m. } \\
\text { p.m. }\end{array}$ & $\begin{array}{l}70.67 \\
69.70 \\
69.26 \\
68.09 \\
65.75 \\
65.33 \\
62.74 \\
62.26 \\
60.73 \\
60.75 \\
62.51 \\
64.04 \\
63.34 \\
64.32\end{array}$ & $\begin{array}{l}\text { June } \\
\text { July } \\
\text { Aug. } \\
\text { Sept. } \\
\text { oct. } \\
\text { Nov. }\end{array}$ & $\begin{array}{r}2: 05 \\
12: 45 \\
12: 35 \\
12: 50 \\
12: 30 \\
12: 05 \\
11: 15 \\
11: 50 \\
12: 15 \\
11: 00 \\
1: 50 \\
11: 25 \\
1: 50\end{array}$ & $\begin{array}{l}\text { p.m. } \\
\text { p.m. } \\
\text { p.m. } \\
\text { p.m. } \\
\text { p.m. } \\
\text { p.m. } \\
\text { a.m. } \\
\text { a.m. } \\
\text { p.m. } \\
\text { a.m. } \\
\text { p.m. } \\
\text { a.m. } \\
\text { p.m. }\end{array}$ & $\begin{array}{l}62.76 \\
60.75 \\
61.65 \\
60.56 \\
63.00 \\
65.86 \\
66.52 \\
67.14 \\
66.70 \\
66.80 \\
62.33 \\
61.05 \\
57.80\end{array}$ \\
\hline
\end{tabular}

83-7-IIEl. Louis Maresh. (On 36th street N. E., midway between C Ave. N. E. and lst Ave. E.). SW $\frac{2}{4} N W_{4}^{\frac{1}{4}}$ sec. 11, T. 83 N., R. 7 W. Unused drilled well, diameter 10 inches, depth 195.0 feet. Measuring point, top of casing, at land surface. Water level affected by pumping. Water-level rocorder maintained on well since April 7, 1941. Highest water level

observed from recorder charts for 1941 was 71.56 feet below measuring point on June 26 and lowest observed water level was 86.85 feet below measuring point on April 30, during a pumping test on a well about 3,000 feet distant.

Water level, in feet below messuring point, 1941

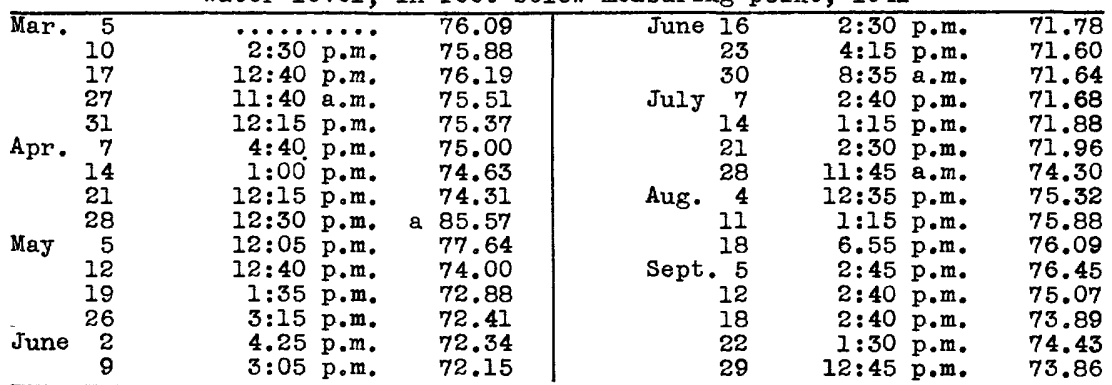

a Pumping test on well about 3,000 feet distant in progress. 
Linn County--Continued

83-7-11E1.--Continued.

Water level, in feet below measuring point, 1941

\begin{tabular}{|c|c|c|c|c|c|c|}
\hline Date & Hour & $\begin{array}{l}\text { Water } \\
\text { level }\end{array}$ & Date & Hour & & $\begin{array}{l}\text { Water } \\
\text { level }\end{array}$ \\
\hline $\begin{array}{rr}\text { Oct. } & 6 \\
13 \\
20 \\
27 \\
\text { Nov. } 3 \\
10 \\
17\end{array}$ & $\begin{aligned} 10: 45 & \text { a.m. } \\
2: 15 & \text { p.m. } \\
3: 35 & \text { p.m. } \\
3: 00 & \text { p.m. } \\
6: 30 & \text { p.m. } \\
12: 45 & \text { p.m. } \\
3: 00 & \text { p.m. }\end{aligned}$ & $\begin{array}{l}72.89 \\
71.99 \\
71.39 \\
70.77 \\
69.55 \\
68.79 \\
68.14\end{array}$ & $\begin{array}{l}\text { Nov. } 25 \\
\text { Dec. } \\
2 \\
8 \\
15 \\
22 \\
29 \\
29\end{array}$ & $\begin{array}{r}11: 25 \\
11: 00 \\
4: 00 \\
1: 15 \\
10: 45 \\
2: 05\end{array}$ & $\begin{array}{l}\text { a.m. } \\
\text { a.m. } \\
p \cdot m_{.} \\
p \cdot m . \\
\text { a.m. } \\
p \cdot m .\end{array}$ & $\begin{array}{l}67.84 \\
67.95 \\
67.93 \\
67.91 \\
68.03 \\
68.20\end{array}$ \\
\hline
\end{tabular}

83-7-16D1. City of Cedar Rapids (Shaver Park).

Water level, in feet below measuring point, 1941

\begin{tabular}{|c|c|c|c|c|c|c|c|c|}
\hline $\begin{array}{l}\text { Jan. } \\
\text { Keb. } \\
\text { Mar. } \\
\text { Apr: } \\
\text { May } \\
\text { June }\end{array}$ & $\begin{array}{r}6 \\
3 \\
7 \\
24 \\
17 \\
27 \\
14 \\
21 \\
28 \\
5 \\
12 \\
19 \\
26 \\
2 \\
9\end{array}$ & $\begin{array}{r}12: 55 \\
10: 40 \\
12: 50 \\
12: 45 \\
11: 15 \\
10: 50 \\
10: 45 \\
11: 10 \\
10: 30 \\
11: 00 \\
10: 50 \\
12: 45 \\
1: 05 \\
3: 55 \\
12: 40\end{array}$ & $\begin{array}{l}\text { p.m. } \\
\text { a.m. } \\
\text { p.m. } \\
\text { p.m. } \\
\text { a.m. } \\
\text { a.m. } \\
\text { a.m. } \\
\text { a.m. } \\
\text { a.m. } \\
\text { a.m. } \\
\text { a.m. } \\
\text { p.m. } \\
\text { p.m. } \\
\text { p.m. } \\
\text { p.m. }\end{array}$ & $\begin{array}{l}91.61 \\
91.74 \\
91.33 \\
91.20 \\
91.30 \\
90.56 \\
90.52 \\
89.36 \\
89.19 \\
89.10 \\
89.44 \\
89.56 \\
89.44 \\
89.54 \\
89.40\end{array}$ & $\begin{array}{ll}\text { June } & \frac{1}{2} \\
\text { July } & 1 \\
& 2 \\
\text { Aug. } & 1 \\
& 1 \\
\text { Sept. } & 1 \\
& 2 \\
\text { oct. } & 1 \\
& 2 \\
\text { Nov. } & 2 \\
\text { Dec. } & 2\end{array}$ & $\begin{array}{r}1: 55 \\
12: 30 \\
12: 25 \\
12: 40 \\
12: 20 \\
11: 55 \\
11: 05 \\
11: 40 \\
12: 05 \\
10: 50 \\
1: 35 \\
11: 10 \\
1: 30 \\
11: 55\end{array}$ & $\begin{array}{l}\text { p.m. } \\
\text { p.m. } \\
\text { p.m. } \\
\text { p.m. } \\
\text { p.m. } \\
\text { a.m. } \\
\text { a.m. } \\
\text { a.m. } \\
\text { p.m. } \\
\text { a.m. } \\
\text { p.m. } \\
\text { a.m. } \\
\text { p.m. } \\
\text { a.m. }\end{array}$ & $\begin{array}{l}89.37 \\
89.52 \\
89.90 \\
90.24 \\
90.25 \\
90.29 \\
90.54 \\
91.02 \\
90.12 \\
90.48 \\
89.10 \\
87.59 \\
86.82 \\
87.67\end{array}$ \\
\hline
\end{tabular}

83-7-16J1. City of Cedar Rapids (Daniel Park).

Water level, in feet below measuring point, 1941

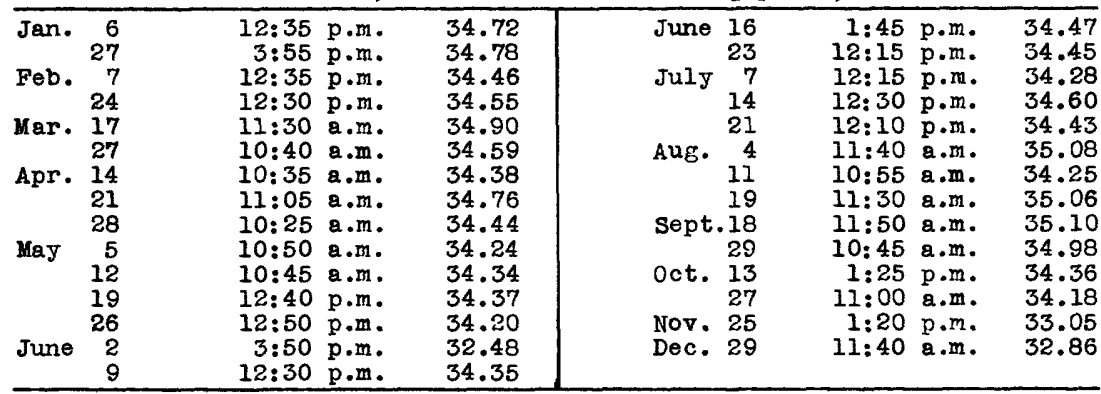

83-7-17L1. City of Cedar Rapids (Ellis Park).

Water level, in feet below measuring point, 1941

\begin{tabular}{|c|c|c|c|c|c|c|c|c|}
\hline $\begin{array}{l}\text { Jan. } \\
\text { Feb. } \\
\text { Mar. } \\
\text { Apr. } \\
\text { May } \\
\text { June }\end{array}$ & $\begin{array}{r}6 \\
27 \\
7 \\
24 \\
17 \\
27 \\
14 \\
21 \\
28 \\
5 \\
12 \\
19 \\
26 \\
2 \\
9\end{array}$ & $\begin{array}{r}9: 50 \\
10: 50 \\
11: 00 \\
11: 45 \\
11: 00 \\
10: 15 \\
10: 25 \\
10: 50 \\
10: 05 \\
10: 25 \\
10: 30 \\
12: 05 \\
12: 10 \\
3: 40 \\
12: 10\end{array}$ & $\begin{array}{l}\text { a.m. } \\
\text { a.m. } \\
\text { a.m. } \\
\text { a.m. } \\
\text { a.m. } \\
\text { a.m. } \\
\text { a.m. } \\
\text { a.m. } \\
\text { a.m. } \\
\text { a.m. } \\
a \cdot m . \\
p \cdot m \cdot \\
p \cdot m . \\
\text { p.m. } \\
\text { p.m. }\end{array}$ & $\begin{array}{l}20.56 \\
20.87 \\
20.69 \\
20.56 \\
19.98 \\
19.55 \\
19.58 \\
19.65 \\
19.62 \\
20.80 \\
20.27 \\
20.49 \\
20.43 \\
20.28 \\
20.02\end{array}$ & $\begin{array}{l}\text { June } \\
\text { July } \\
\text { Aug. } \\
\text { sept. } \\
\text { oct. } \\
\text { Nov. } \\
\text { Dec. }\end{array}$ & $\begin{array}{r}1: 15 \\
11: 35 \\
11: 55 \\
11: 00 \\
11: 40 \\
11: 35 \\
10: 45 \\
11: 10 \\
10: 55 \\
10: 25 \\
1: 00 \\
10: 50 \\
1: 10 \\
11: 15\end{array}$ & $\begin{array}{l}\text { p.m. } \\
\text { a.m. } \\
\text { a.m. } \\
\text { a.m. } \\
\text { a.m. } \\
\text { a.m. } \\
\text { a.m. } \\
\text { a.m. } \\
\text { a.m. } \\
\text { a.m. } \\
\text { p.m. } \\
\text { a.m. } \\
\text { p.m. } \\
\text { p.m. }\end{array}$ & $\begin{array}{l}19.88 \\
20.16 \\
20.30 \\
20.70 \\
20.88 \\
21.27 \\
21.26 \\
21.41 \\
20.47 \\
20.85 \\
19.79 \\
19.65 \\
19.42 \\
19.83\end{array}$ \\
\hline
\end{tabular}




\section{Linn County--Continued}

83-7-2lLl. City of Cedar Raplds. Equipped with water-level recorder Water level affected by pumping from nearby industrial wells. Highest water level observed from recorder charts for 1941 was 18.91 feet below measuring point on Jan. 13 and lowest was 46.6 feet below measuring point (extrapolated from partial record) on Aug. 14.

Water level, in feet below measuring point, 1941

\begin{tabular}{|c|c|c|c|c|c|}
\hline Date & Hour & $\begin{array}{l}\text { Water } \\
\text { level }\end{array}$ & Date & Hour & $\begin{array}{l}\text { Water } \\
\text { level }\end{array}$ \\
\hline $\begin{array}{lr}\text { Jan. } & 6 \\
& 13 \\
20 \\
\\
27 \\
\text { Feb. } \\
\\
\\
\text { Mar. } \\
\text { May } & 5 \\
\end{array}$ & $\begin{array}{r}10: 10 \text { a.m. } \\
9: 47 \text { a.m. } \\
3: 00 \text { p.m. } \\
11: 05 \text { a.m. } \\
1: 25 \text { p.m. } \\
11: 05 \text { a.m. } \\
1: 25 \text { p.m. } \\
4: 10 \text { p.m. }\end{array}$ & $\begin{array}{l}23.02 \\
22.19 \\
24.40 \\
23.46 \\
24.44 \\
24.28 \\
23.36 \\
27.17\end{array}$ & $\begin{array}{lr}\text { July } 21 \\
28 \\
\text { Sept. } 5 \\
18 \\
\text { oct. } 5 \\
27 \\
\text { Nov. } 10 \\
\text { Dec. } 15\end{array}$ & $\begin{array}{r}12: 01 \text { p.m. } \\
12: 20 \text { p.m. } \\
3: 40 \text { p.m. } \\
11: 20 \text { a.m. } \\
10: 30 \text { a.m. } \\
4: 20 \text { p.m. } \\
12: 30 \text { p.m. } \\
1: 45 \text { p.m. }\end{array}$ & $\begin{array}{l}36.64 \\
37.71 \\
42.18 \\
33.68 \\
31.52 \\
32.42 \\
30.27 \\
32.92\end{array}$ \\
\hline
\end{tabular}

83-7-21P1. Kresge Company. (Southwest corner of lst Ave. S. F. and 3rd street N.E.). SE⿺辶 SW sec. 21, T. 83 N., R. 7 W. Drilled alrconditioning well. Measuring point, top of $3 / 8-1$ nch hole in pump base, 6.41 feet below manhole cover at street level, 0.60 foot above top of concrete curb. Equipped with 25 horsepower electric turbine pump. Water levels, in feet below measuring point, 1941: Mar. 6, 27.14; Apr. 7, 12:01 p.m., 25.89; May 5, 2:45 p.m., al 35.95; Dec. 29, 3:55 p.m., 34.70.

83-7-21Q1. Iowa Theatre (Southeast comer of 1 st Ave. S.E. and $3 \mathrm{rd}$ Street N.E.). SW SE $\frac{1}{4}$ sec. 21, T. 83 N., R. 7 W. Drilled industrial well, diameter 10 inches, reported depth 428 feet. Measuring point, top of pump base, 5.65 feet below manhole cover at street level. Equipped with 20 horsepower electric turbine pump. Water levels, in feet below measuring point, 194l: Jan. 13, 4:00 p.m., 24.32; Mar. 5, 24.89; Apr. 7, 11:15 a.m., 24.24; Dec. 29, 4:20 p.m., 32.30.

83-7-28G2. Cedar Rapids Gas Company. Equipped with water-level recorder. Water level affected by pumping from nearby wells, particularly a group of Industrial wells 1,000 to 1,500 feet distant. Highest water level observed from recorder charts for 1941 was 32.93 feet below measuring point on Apr. 21 and lowest was 78.91 feet below measuring point on Aug. 1 . Maximum recorded dally fluctuation was 17.67 feet on July 7,1941 , and maximum estimated daily fluctuation extrapolated from partial record was 21.4 feet on June 30, 1941. Maximum weekly fluctuation was 29.85 feet for the week ending July 14, 1941.

Water level, in feet below measuring point, 1941

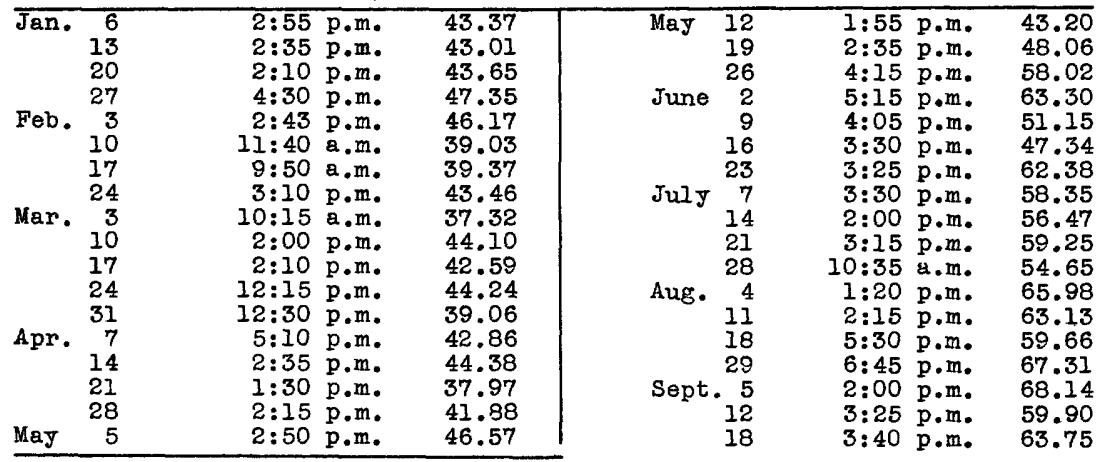

a Pumped heavily recently. 
Linn County--Continued

83-7-28G2,--Continued.

Water level, in feet below measuring point, 1941

\begin{tabular}{|c|c|c|c|c|c|c|}
\hline Date & Hour & $\begin{array}{l}\text { Water } \\
\text { level }\end{array}$ & Date & Hour & & $\begin{array}{l}\text { Water } \\
\text { level } \\
\end{array}$ \\
\hline $\begin{array}{l}\text { Sept. } 22 \\
29 \\
\text { Oct. } 6 \\
13 \\
20 \\
27 \\
\text { Nov. } 3 \\
10\end{array}$ & $\begin{aligned} 12: 15 & \text { p.m. } \\
2: 15 & \text { p.m. } \\
10: 00 & \text { a.m. } \\
4: 30 & \text { p.m. } \\
3: 05 & \text { p.m. } \\
2: 20 & \text { p.m. } \\
6: 00 & \text { p.m. } \\
12: 10 & \text { p.m. }\end{aligned}$ & $\begin{array}{l}56.33 \\
56.26 \\
53.64 \\
54.57 \\
50.94 \\
49.85 \\
61.22 \\
50.88\end{array}$ & $\begin{array}{r}\text { Nov. } 17 \\
25 \\
\text { Dec. } 2 \\
8 \\
15 \\
22 \\
29\end{array}$ & $\begin{array}{r}3: 00 \\
11: 45 \\
10: 45 \\
3: 30 \\
1: 50 \\
10: 15 \\
10: 35\end{array}$ & $\begin{array}{l}\text { p.m. } \\
\text { a.m. } \\
\text { a.m. } \\
\text { p.m. } \\
\text { p.m. } \\
\text { a.m. } \\
\text { a.m. }\end{array}$ & $\begin{array}{l}50.62 \\
48.98 \\
48.94 \\
59.95 \\
52.85 \\
50.19 \\
50.12\end{array}$ \\
\hline
\end{tabular}

83-7-23Gl. City of Cedar Rapids (Bever Park).

Water level, in feet.below measuring point, 1941

\begin{tabular}{|c|c|c|c|c|c|c|c|c|}
\hline $\begin{array}{l}\text { Jan. } \\
\text { Feb. } \\
\text { Mar. } \\
\text { Apr. } \\
\text { May }\end{array}$ & $\begin{array}{r}6 \\
27 \\
7 \\
24 \\
17 \\
27 \\
14 \\
21 \\
28 \\
5 \\
12 \\
19 \\
26 \\
2 \\
9\end{array}$ & $\begin{array}{r}2: 00 \\
1: 20 \\
3: 45 \\
2: 05 \\
12: 55 \\
11: 50 \\
1: 50 \\
12: 45 \\
1: 25 \\
1: 10 \\
1: 20 \\
2: 00 \\
3: 40 \\
5: 00 \\
3: 25\end{array}$ & $\begin{array}{l}\text { p.m. } \\
\text { p.m. } \\
\text { p.m. } \\
\text { p.m. } \\
\text { p.m. } \\
\text { a.m. } \\
\text { p.m. } \\
\text { p.m. } \\
\text { p.m. } \\
\text { p.m. } \\
\text { p.m. } \\
\text { p.m. } \\
\text { p.m. } \\
\text { p.m. } \\
\text { p.m. }\end{array}$ & $\begin{array}{l}4.76 \\
4.80 \\
4.77 \\
4.73 \\
4.64 \\
4.57 \\
4.53 \\
4.42 \\
4.47 \\
4.49 \\
4.55 \\
4.70 \\
4.68 \\
4.73 \\
4.57\end{array}$ & $\begin{array}{ll}\text { June } & \frac{1}{2} \\
\text { July } & 1 \\
& 2 \\
\text { Aug. } & 1 \\
& 1 \\
\text { Sept. } 1 \\
\\
\text { Oct. } \\
\\
\text { Nov. } \\
\text { Dec. } \\
2 \\
2\end{array}$ & $\begin{array}{r}2: 55 \\
2: 50 \\
3: 00 \\
1: 45 \\
2: 45 \\
12: 50 \\
1: 30 \\
12: 20 \\
2: 55 \\
1: 35 \\
2: 30 \\
1: 30 \\
3: 35 \\
2: 30\end{array}$ & $\begin{array}{l}\text { p.m. } \\
\text { p.m. } \\
\text { p.m. } \\
\text { p.m. } \\
p \cdot m . \\
p \cdot m . \\
p \cdot m . \\
p \cdot m . \\
p \cdot m . \\
p \cdot m= \\
p \cdot m . \\
p \cdot m . \\
p \cdot m . \\
\text { p.m. }\end{array}$ & $\begin{array}{l}4.72 \\
5.00 \\
5.02 \\
5.15 \\
5.24 \\
5.44 \\
5.70 \\
5.68 \\
5.20 \\
5.17 \\
4.91 \\
4.60 \\
4.44 \\
4.30\end{array}$ \\
\hline
\end{tabular}

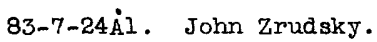

Water level, in feet below measuring point, 1941

\begin{tabular}{|c|c|c|c|c|c|c|c|c|}
\hline $\begin{array}{l}\text { Jan. } \\
\text { Feb. } \\
\text { Mar. } \\
\text { Apr. } \\
\text { May }\end{array}$ & $\begin{array}{r}6 \\
27 \\
7 \\
24 \\
17 \\
27 \\
14 \\
21 \\
28 \\
5 \\
12 \\
19 \\
26 \\
2 \\
9\end{array}$ & $\begin{aligned} & 2:: 15 \\
& 12: 30 \\
& 4: 15 \\
& 2: 20 \\
& 1: 10 \\
& 12: 01 \\
& 2: 00 \\
& 12: 55 \\
& 1: 40 \\
& 1: 20 \\
& 1: 30 \\
& 2: 15 \\
& 3: 50 \\
& 5: 05 \\
& 3: 30\end{aligned}$ & $\begin{array}{l}\text { p.m. } \\
\text { p.m. } \\
\text { p.m. } \\
\text { p.m. } \\
\text { p.m. } \\
\text { p.m. } \\
\text { p.m. } \\
\text { p.m. } \\
\text { p.m. } \\
\text { p.m. } \\
\text { p.m. } \\
\text { p.m. } \\
\text { p.m. } \\
\text { p.m. } \\
\text { p.m. }\end{array}$ & $\begin{array}{l}30.32 \\
30.37 \\
30.46 \\
29.94 \\
30.09 \\
29.50 \\
29.47 \\
28.54 \\
28.92 \\
29.23 \\
29.97 \\
29.77 \\
29.56 \\
29.35 \\
29.60\end{array}$ & $\begin{array}{ll}\text { June } & \frac{1}{2} \\
\text { July } & 1 \\
& 2 \\
\text { Aug. } & 1 \\
& 1 \\
\text { Sept. } \\
\text { oct. } \\
\text { Nov. } \\
\text { Dec. } \\
2 \\
2 \\
\text { Don }\end{array}$ & $\begin{array}{r}3: 00 \\
2: 50 \\
3: 15 \\
1: 55 \\
2: 55 \\
1: 00 \\
1: 40 \\
12: 30 \\
3: 15 \\
1: 45 \\
2: 40 \\
1: 40 \\
3: 45 \\
2: 55\end{array}$ & $\begin{array}{l}\text { p.m. } \\
\text { p.m. } \\
\text { p.m. } \\
\text { p.m. } \\
\text { p.m. } \\
\text { p.m. } \\
\text { p.m. } \\
\text { p.m. } \\
\text { p.m. } \\
\text { p.m. } \\
\text { p.m. } \\
\text { p.m. } \\
\text { p.m. } \\
\text { p.m. }\end{array}$ & $\begin{array}{r}29.63 \\
29.40 \\
29.64 \\
30.62 \\
30.24 \\
30.38 \\
30.50 \\
30.80 \\
29.35 \\
29.29 \\
28.97 \\
28.29 \\
27.71 \\
\text { a } 33.36\end{array}$ \\
\hline
\end{tabular}

83-7-32G1. Floyd Felter.

Water level, in feet below measuring point, 1941

\begin{tabular}{|c|c|c|c|c|c|c|c|c|}
\hline $\begin{array}{lr}\text { Jan. } & 6 \\
& 27 \\
\text { Feb. } & 7 \\
& 24 \\
\text { Mar. } & 17 \\
& 27 \\
\text { Apr. } & 14 \\
& 21 \\
& 28 \\
\text { May } & 5\end{array}$ & $\begin{array}{r}9: 35 \\
10: 25 \\
10: 30 \\
11: 30 \\
10: 30 \\
10: 05 \\
10: 10 \\
10: 40 \\
9: 55 \\
10: 10\end{array}$ & $\begin{array}{l}\text { a.m. } \\
\text { a.m. } \\
\text { a.m. } \\
\text { a.m. } \\
\text { a.m. } \\
\text { a.m. } \\
\text { a.m. } \\
\text { a.m. } \\
\text { a.m. } \\
\text { a.m. }\end{array}$ & $\begin{array}{l}81.38 \\
81.97 \\
81.70 \\
81.46 \\
81.96 \\
81.66 \\
81.49 \\
81.56 \\
81.32 \\
81.25\end{array}$ & $\begin{array}{l}\text { May } \\
\text { June } \\
\text { July }\end{array}$ & $\begin{array}{r}12 \\
19 \\
26 \\
2 \\
9 \\
16 \\
23 \\
7 \\
14 \\
21\end{array}$ & $\begin{array}{r}10: 25 \\
11: 50 \\
12: 01 \\
3: 30 \\
12: 05 \\
1: 05 \\
11: 20 \\
11: 35 \\
10: 50 \\
11: 30\end{array}$ & $\begin{array}{l}\text { a.m. } \\
\text { a.m. } \\
\text { p.m. } \\
\text { p.m. } \\
\text { p.m. } \\
\text { p.m. } \\
\text { a.m. } \\
\text { a.m. } \\
\text { a.m. } \\
\text { a.m. }\end{array}$ & $\begin{array}{l}81.34 \\
81.51 \\
81.72 \\
82.27 \\
82.27 \\
81.81 \\
81.86 \\
81.97 \\
82.14 \\
82.13\end{array}$ \\
\hline
\end{tabular}

a Pumped a short time before measurement. 


\section{Iinn County--Continued}

83-7-32GI.--Continued.

Water level, in feet below measuring point, 1941

\begin{tabular}{|c|c|c|c|c|c|}
\hline Date & Hour & $\begin{array}{l}\text { Water } \\
\text { level }\end{array}$ & Date & Hour & $\begin{array}{l}\text { Water } \\
\text { level }\end{array}$ \\
\hline $\begin{array}{r}\text { Aug. } 4 \\
11 \\
19 \\
\text { Sept. } 18 \\
29\end{array}$ & $\begin{array}{l}11: 25 \text { a.m. } \\
10: 35 \text { a.m. } \\
11: 00 \mathrm{a} . \mathrm{m} . \\
10: 45 \text { a.m. } \\
10: 15 \text { a.m. }\end{array}$ & $\begin{array}{l}82.96 \\
83.48 \\
82.93 \\
82.19 \\
81.89\end{array}$ & $\begin{array}{ll}\text { Oct. } & 13 \\
& 27 \\
\text { Nov. } 25 \\
\text { Dec. } 29\end{array}$ & $\begin{array}{l}12: 45 \text { p.m. } \\
10: 40 \text { a.m. } \\
12: 55 \text { p.m. } \\
10: 25 \text { a.m. }\end{array}$ & $\begin{array}{l}80.05 \\
78.47 \\
77.25 \\
77.60\end{array}$ \\
\hline
\end{tabular}

83-7-33F1.

Water level, in feet below measuring point, 1941

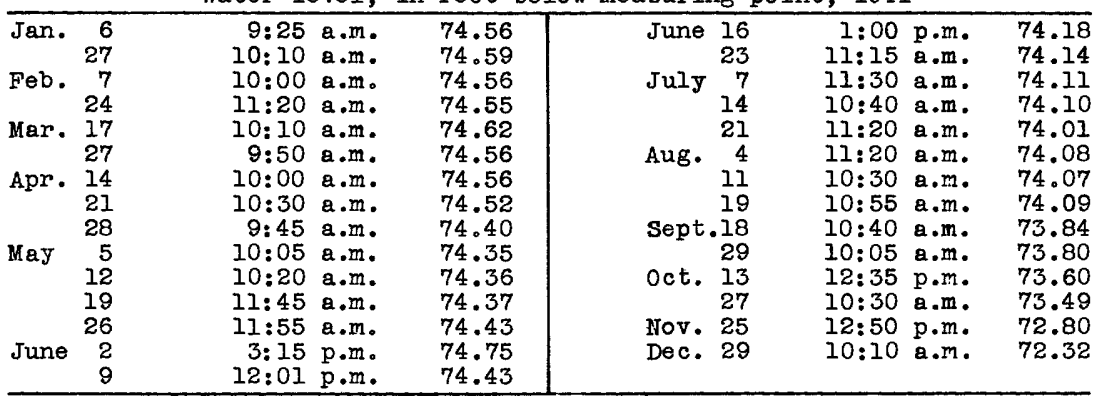

83-6-28Bl. Measurements discontinued.

83-6-30BI. -..-- Katz.

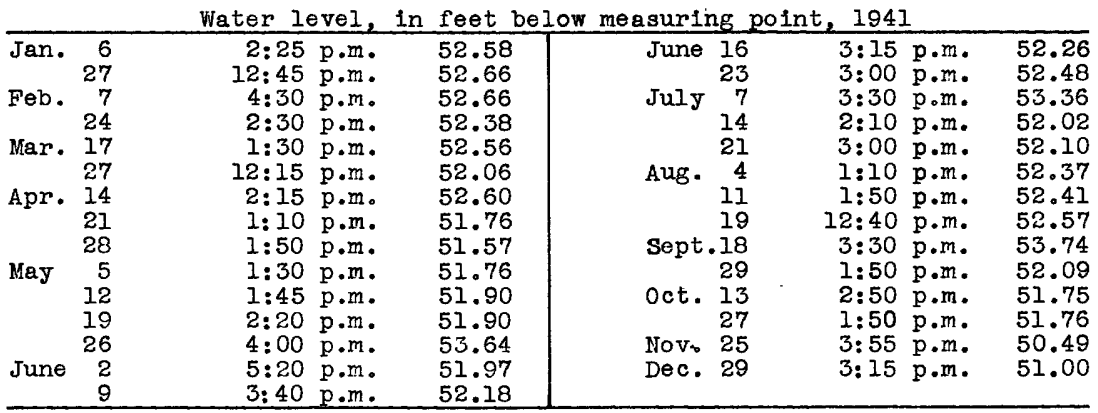

\section{Lyon County}

$99-44-26 \mathrm{RI}$.

Water level, in feet below measuring point, 1941

\begin{tabular}{|c|c|c|c|c|c|c|c|}
\hline Date & $\begin{array}{l}\text { Water } \\
\text { level }\end{array}$ & Date & $\begin{array}{l}\text { Water } \\
\text { level }\end{array}$ & Date & $\begin{array}{l}\text { Water } \\
\text { level }\end{array}$ & Date & $\begin{array}{l}\text { Water } \\
\text { level }\end{array}$ \\
\hline $\begin{array}{l}\text { Feb. } 12 \\
\text { Mar. } 21\end{array}$ & $\begin{array}{l}10.46 \\
10.86\end{array}$ & $\begin{array}{ll}\text { Apr. } & 18 \\
\text { May } & 20\end{array}$ & $\begin{array}{r}9.90 \\
10.28\end{array}$ & $\begin{array}{l}\text { July } 22 \\
\text { Aug. } 20\end{array}$ & $\begin{array}{l}10.78 \\
11.30\end{array}$ & Dec. 10 & 11.36 \\
\hline
\end{tabular}


Lyon County--Continued

99-43-11Hב.

Water level, in feet below measuring point, 1941

\begin{tabular}{lr|lr|lr|lr}
\hline Date & $\begin{array}{r}\text { Water } \\
\text { level }\end{array}$ & Date & $\begin{array}{r}\text { Water } \\
\text { level }\end{array}$ & Date & $\begin{array}{r}\text { Water } \\
\text { level }\end{array}$ & Date & $\begin{array}{r}\text { Water } \\
\text { level }\end{array}$ \\
\hline Feb. 12 & 3.56 & Apr. 18 & 2.43 & July 22 & 3.98 & Dec. 10 & 3.65 \\
Mar. 21 & 2.80 & May 20 & 3.44 & Aug. 20 & 4.30 & & \\
\hline
\end{tabular}

98-48-24M1. A. C. Hanson.

Water level, in feet below measuring point, 1941

\begin{tabular}{ll|lll|lll|lll}
\hline Feb. 28 a 18.58 & Apr. 19 & a 15.00 & July 14 & a 16.03 & Dec. 23 & c 19.75
\end{tabular}

\begin{tabular}{ll|ll|l|l|l} 
Mar. 27 a 15.77 & June 4 & a 15.00 & Aug. 20 & b 18.83 \\
\hline
\end{tabular}

Madison County

76-28-2Bl. Glen Newton.

Water level, in feet below measuring point, 1941

\begin{tabular}{ll|lll|ll|ll}
\hline Feb. 11 & 18.03 & May & 28 & 18.12 & Aug. 28 & 20.63 & Oct. 28 & 18.80 \\
Mar. 29 & 17.95 & June 30 & 18.64 & Sept.30 & 19.81 & Nov. 28 & 18.85 \\
Apr. 29 & 17.50 & July 29 & 18.99 & & & & \\
\hline
\end{tabular}

\section{Marion county}

77-18-34c1. No measurements made in 1941.

75-20-22HI. Union Central Iife Insurance Company.

Water level, in feet below measuring point, 1941

\begin{tabular}{lr|lr|ll|ll}
\hline Jan. 28 & 13.07 & Apr. 29 & 9.87 & July 29 & 10.57 & Oct. 28 & 5.71 \\
Feb. 10 & 13.87 & May 28 & 11.27 & Aug. 29 & 11.50 & Nov. 28 & 5.22 \\
25 & 13.58 & June 30 & 9.57 & Sept.30 & 11.21 & Dec. 21 & 5.44 \\
Mar. 28 & 8.80 & & & & & & \\
\hline
\end{tabular}

75-20-29Kl. J. D. Cleair.

Water level, in feet below measuring point, 1941

\begin{tabular}{ll|ll|lll|lll}
\hline Jan. 28 & 12.05 & Mar. 28 & 12.25 & June 30 & 11.80 & Sept.30 & 13.06 \\
Feb. 10 & 11.72 & Apr. 29 & 12.00 & July 29 & 12.87 & Oct. 28 & d 11.35 \\
25 & 12.12 & May 28 & 12.05 & Aug. 29 & 14.10 & & \\
\hline
\end{tabular}

75-20-31Cl. Miss Amanda Elliot. Water levels, in feet below measuring point, 194l: Jan. 28, 2:15 p.m., 24.93; Feb. 10, 4.05 p.m., 23.76; Feb. 25, 11.35 a.m., 22.48; Apr. 29, 10:35 a.m.,e/21.20.

75-20-3lc2. Miss Amanda Elliot.

Water level, in feet below measuring point, 1941

\begin{tabular}{lll|ll|ll|ll}
\hline Jan. 28 & 14.15 & Apr. 29 & 15.00 & July 29 & 15.98 & Oct. 28 & 7.35 \\
Feb. 10 & 13.68 & May 28 & 18.47 & Aug. 29 & 16.22 & Nov. 28 & 7.44 \\
& 25 & 11.17 & June 30 & 17.05 & Sept.30 & 15.83 & Dec. 21 & 8.47 \\
Mar. 28 & 12.50 & & & & & & &
\end{tabular}

a No pumping since summer of 1940.

b Pumping 30 gallons per day.

c No pumping since fall of 1941 .

d Well destroyed. Measurements discontinued on oct. 28.

e Measurements discontinued on Apr. 29. 


\section{Marion County--Continued}

74-21-26El. Griesbaum Estate.

Water level, in feet below measuring point, 1941

\begin{tabular}{lr|lr|ll|lr}
\hline Date & $\begin{array}{l}\text { Water } \\
\text { level }\end{array}$ & Date & $\begin{array}{r}\text { Water } \\
\text { level }\end{array}$ & Date & $\begin{array}{r}\text { Water } \\
\text { level }\end{array}$ & Date & $\begin{array}{r}\text { Water } \\
\text { level }\end{array}$ \\
\hline Jan. 28 & 12.88 & Apr. 29 & 9.14 & July 29 & 11.32 & Oct. 28 & 5.60 \\
Feb. 10 & 11.65 & May 28 & 10.23 & Aug. 29 & 13.08 & Nov. 29 & 5.45 \\
25 & 10.68 & June 30 & 10.06 & Sept.30 & 12.44 & Dec. 21 & 6.33 \\
Mar. 28 & 9.57 & & & & & &
\end{tabular}

74-20-2MI.

Water level, in feet below measuring point, 1941

\begin{tabular}{ll|ll|ll|lll}
\hline Jan. 28 & 5.86 & Apr. 29 & 4.80 & July 29 & 4.28 & Oct. 28 & 4.95 \\
Feb. 10 & 5.59 & May 28 & 4.75 & Aug. 29 & 4.43 & Nov. 29 & 3.40 \\
25 & 5.41 & June 30 & 3.91 & Sept.30 & 3.65 & Dec. 21 & 2.57 \\
Mar. 28 & 4.80 & & & & & & & \\
\hline
\end{tabular}

74-20-16MI. C. Wendall.

Water level, in feet below measuring point, 1941

\begin{tabular}{ll|ll|ll|l|l}
\hline Jan. 28 & 30.66 & Feb. 25 & 31.16 & Apr. 29 & 32.66 & June 30 ab 33.00 \\
Feb. 10 & 30.90 & Mar. 28 & 32.00 & May 28 & 33.30 & \\
\hline
\end{tabular}

74-20-33Dl. T. V. Beebout.

Water level, in feet below measuring point, 1941

\begin{tabular}{ll|ll|ll|ll}
\hline Jan. 28 & 27.15 & Apr. 29 & 27.10 & July 29 & 26.94 & Oct. 28 & 26.67 \\
Feb. 10 & 27.15 & May 28 & 27.07 & Aug. 29 & 26.94 & Nov. 29 & 26.52 \\
25 & 27.17 & June 30 & 27.06 & Sept.30 & 26.75 & Dec. 21 & 26.38 \\
Mar. 28 & 27.14 & & & & & & \\
\hline
\end{tabular}

\section{Muscatine county}

76-2-14Dl. Owner's test well 4. City of Muscatine. Water level, in feet below measuring point, 1941: Dec. 23, 13.30 .

76-2-15Al. Owner's test well 5. City of Muscatine. Water level, in feet below measuring point, 1941: Dec. 23, 11.03.

\section{o'Brien County}

94-40-22J1. Illinois Central Railroad. Abandoned drilled well, diameter 10 to 6 inches, reported depth 520 feet. Measuring point, top of iron pump base, 0.10 foot above concrete floor and 0.30 foot below top of rail of main line. Taps water in Dakota sandstone. Water levels, in feet below measuring point, 1941: Mar. 28, 235.95; Aug. 26, 233.49; Dec. 10, 244.54 .

a Measurement probably inaccurate.

b Measurements temporarily discontinued. 
Osceola County

99-41-18Cl. City of Sibley.

Water level, in feet below measuring point, 1941

\begin{tabular}{lrr|llr}
\hline Date & Hour & $\begin{array}{c}\text { Water } \\
\text { level }\end{array}$ & Date & Hour & $\begin{array}{r}\text { Water } \\
\text { Ievel }\end{array}$ \\
\hline Feb. 12 & $1: 20$ p.m. & 23.37 & Aug. 20 & $4: 55$ p.m. & 17.77 \\
Apr. 18 & $12: 55$ p.m. & 18.30 & Dec. 10 & $3: 00$ p.m. & 21.00 \\
May 20 & a $3: 45$ p.m. & 18.06 & & & \\
\hline
\end{tabular}

99-41-18C2. City of Sibley. Water levels, in feet below measuring point, 1941: Feb. 12, 1:30 p.m., 16.30; Mar. 21, 11:15 a.m., 15.99; Apr. 18, 1:00 p.m., 15.85; May 20, a/ .

\section{Page County}

69-36-3IKI. City of Clarinda. Equipped with water-level recorder. Highest water level observed from recorder charts for 1941 was 14.58 feet below the measuring point. on Nov. 2 , and lowest observed water level was 24.15 feet below the measuring point on Sept. 1. Measurements by F. E. Allison, Water superintendent.

Water level, in feet below measuring point, 1941

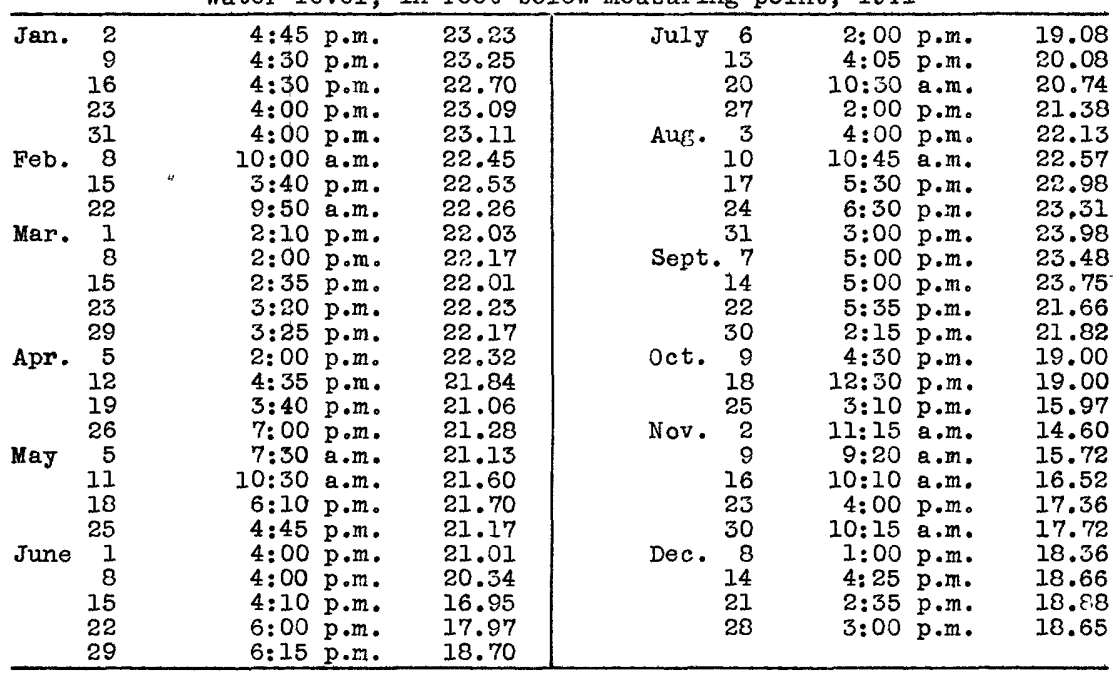

Palo Alto county

(Vicinity of Lost Island Lake)

$97-34-29 \mathrm{~N} 1$.

Water level, in feet below measuring point, 1941

\begin{tabular}{lrr|rrr}
\hline Feb. 12 & $5: 30 \mathrm{p} . \mathrm{m}$. & 2.34 & July 22 & $2: 40 \mathrm{p} . \mathrm{m}$. & 2.60 \\
Mar. 21 & $4: 25 \mathrm{p.m}$. & 2.28 & Aug. 20 & $12: 40 \mathrm{p} . \mathrm{m}$. & 4.55 \\
May 21 & $12: 50 \mathrm{p.m}$. & 2.70 & Dec. 11 & $11: 20 \mathrm{a.m}$. & 2.25 \\
June 18 & $2: 35 \mathrm{p.m}$ & 2.44 & & & \\
\hline
\end{tabular}


Palo Alto County--Continued

$97-34-29$ N2.

Water level, in feet below measuring point, 1941

\begin{tabular}{|c|c|c|c|c|c|}
\hline Date & Hour & $\begin{array}{l}\text { Water } \\
\text { level }\end{array}$ & Date & Hour & $\begin{array}{l}\text { Water } \\
\text { level }\end{array}$ \\
\hline $\begin{array}{ll}\text { Feb. } & 12 \\
\text { Mar. } & 21 \\
\text { May } 21 \\
\text { June } 18\end{array}$ & $\begin{aligned} 5: 30 & \text { p.m. } \\
4: 25 & \text { p.m. } \\
12: 55 & \text { p.m. } \\
2: 35 & \text { p.m. }\end{aligned}$ & $\begin{array}{r}0.76 \\
1.04 \\
(a) \\
\text { (a) }\end{array}$ & $\begin{array}{l}\text { July } 22 \\
\text { Aug. } 20 \\
\text { Dec. } 11\end{array}$ & $\begin{array}{r}2: 40 \mathrm{p} . \mathrm{m} \\
12: 40 \mathrm{p} . \mathrm{m} \\
11: 20 \mathrm{a.m} .\end{array}$ & $\begin{array}{l}\text { (a) } \\
.77 \\
(a)\end{array}$ \\
\hline
\end{tabular}

97-34-30Q1. Norman Broadwel1.

Water level, in feet below measuring point, 1941

\begin{tabular}{|c|c|c|c|c|c|c|c|c|}
\hline $\begin{array}{ll}\text { Feb. } & 1 \\
\text { Mar. } & 2 \\
\text { May } & 2 \\
\text { June } & \end{array}$ & $\begin{array}{l}12 \\
21 \\
21 \\
18\end{array}$ & $\begin{array}{l}5: 35 \\
4: 40 \\
1: 10 \\
2: 40\end{array}$ & $\begin{array}{l}p \cdot m . \\
p \cdot m . \\
p \cdot m . \\
p \cdot m .\end{array}$ & $\begin{array}{l}19.13 \\
18.72 \\
18.31 \\
18.05\end{array}$ & $\begin{array}{ll}\text { July } 22 \\
\text { Aug. } 20 \\
\text { Dec. } 11\end{array}$ & $\begin{array}{r}2: 50 \\
12: 45 \\
11: 30\end{array}$ & $\begin{array}{l}\mathrm{p} \cdot \mathrm{m} . \\
\mathrm{p} \cdot \mathrm{m} . \\
\mathrm{a} . \mathrm{m} .\end{array}$ & $\begin{array}{l}18.29 \\
19.01 \\
17.50\end{array}$ \\
\hline
\end{tabular}

97-34-32P1: Lost Island state Park.

Water level, in feet below measuring point, 1941

\begin{tabular}{lrr|rrr}
\hline Feb. 12 & $5: 20$ p.m. & 11.86 & June 18 & $2: 20$ p.m. & 3.59 \\
Mar. 21 & $4: 10$ p.m. & 7.21 & July 22 & $2: 30$ p.m. & 4.39 \\
Apr. 22 & $2: 30$ p.m. & 4.45 & Aug. 20 & $12: 20$ p.m. & 5.78 \\
May 21 & $12: 35$ p.m. & 5.19 & Dec. 11 & $10: 30$ a.m. & 5.05 \\
\hline
\end{tabular}

96-34-6J1. "Electric Park".

Water level, in feet below measuring point, 1941

\begin{tabular}{|c|c|c|c|c|c|c|c|c|c|}
\hline $\begin{array}{l}\text { Feb. } \\
\text { Mar. } \\
\text { Apr. } \\
\text { May }\end{array}$ & $\begin{array}{l}12 \\
21 \\
22 \\
21\end{array}$ & $\begin{array}{r}5: 05 \\
3: 50 \\
2: 15 \\
12: 30\end{array}$ & $\begin{array}{l}p \cdot m . \\
p \cdot m . \\
p \cdot m . \\
p \cdot m .\end{array}$ & $\begin{array}{l}5.36 \\
5.04 \\
4.66 \\
4.83\end{array}$ & $\begin{array}{l}\text { June } \\
\text { July } \\
\text { Aug. } \\
\text { Dec. }\end{array}$ & $\begin{array}{l}18 \\
22 \\
20 \\
11\end{array}$ & $\begin{array}{r}2: 10 \\
2: 20 \\
12: 10 \\
10: 20\end{array}$ & $\begin{array}{l}\text { p.m. } \\
\text { p.m. } \\
\text { p.m. } \\
\text { a.m. }\end{array}$ & $\begin{array}{l}4.56 \\
4.71\end{array}$ \\
\hline
\end{tabular}

Plymouth county

91-48-19M1. No measurements made in 1941.

\section{Polk county}

79-22-22Al. J. G. Reed. Water levels, in feet below measuring point, 1941: Feb. 11, 5.50; Sept. 18, 6.74; Dec. 5, 3.57

Sac county

89-38-26A2. City of Schaller.

Water level, in feet below measuring point, 1941

\begin{tabular}{lr|lr|lr}
\hline Date & $\begin{array}{r}\text { Water } \\
\text { level }\end{array}$ & Date & $\begin{array}{r}\text { Water } \\
\text { level }\end{array}$ & Date & $\begin{array}{r}\text { Water } \\
\text { level }\end{array}$ \\
\hline Mar. 20 & 221.85 & May 27 & 221.46 & Aug. 27 & 222.01 \\
Apr. 17 & 221.40 & June 17 & 221.97 & Dec. 12 & 221.84 \\
\hline
\end{tabular}

a Flowing. 
Sac County--Continued

86-36-2Cl. John Christian.

Water level, in feet below measuring point, 1941

\begin{tabular}{|c|c|c|c|c|c|c|c|}
\hline Dete & $\begin{array}{l}\text { Water } \\
\text { leveI }\end{array}$ & Date & $\begin{array}{l}\text { Water } \\
\text { level }\end{array}$ & Date & $\begin{array}{l}\text { Water } \\
\text { level }\end{array}$ & Date & $\begin{array}{l}\text { Water } \\
\text { level }\end{array}$ \\
\hline $\begin{array}{l}\text { Jan. } 17 \\
\text { Feb. } 11 \\
\text { Mar. } 20\end{array}$ & $\begin{array}{l}3.70 \\
4.44 \\
4.05\end{array}$ & $\begin{array}{lr}\text { Apr. } & 7 \\
& 17 \\
\text { May } & 27\end{array}$ & $\begin{array}{l}3.50 \\
3.61 \\
4.95\end{array}$ & $\begin{array}{ll}\text { June } 17 \\
\text { July } 29\end{array}$ & $\begin{array}{l}2.31 \\
6.26\end{array}$ & $\begin{array}{l}\text { Aug. } 27 \\
\text { Dec。 } 12\end{array}$ & $\begin{array}{l}8.38 \\
6.32\end{array}$ \\
\hline
\end{tabular}

86-36-2El. Albert Kulver, Jr.

Water level, in feet below measuring point, 1941

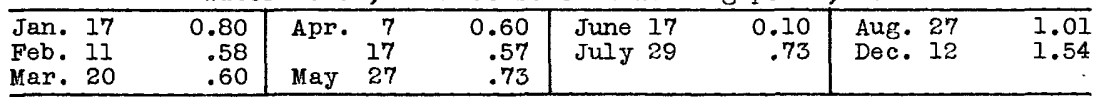

86-36-4N1. Iowa state Conservation Commission.

Water level, in feet below measuring point, 1941

\begin{tabular}{lr|rrr|rr|rr}
\hline Jan. 17 & a 6.80 & Apr. & 7 & a 7.90 & June 17 & 6.93 & Aug. 27 & 8.87 \\
Feb. 11 & 8.08 & & 17 & 7.66 & July 29 & 8.33 & Dec. 12 & 8.62 \\
Mar. 20 & 7.97 & May & 27 & 8.27 & & & & \\
\hline
\end{tabular}

Sioux County

95-45-5Al. City of sioux center. Water levels, in feet below measuring point, 1941: Aug. 25, 263.50; Dec. 10, 264.21.

94-45-17Al. City of Maurice. Water level, in feet below measuring point, 1941: Aug. 26, 122.0.

\section{Story county}

83-24-4Q1. Iowa State college. Water levels affected by changes in atmospheric pressure. Equipped with water-level recorder. Highest water level observed from recorder charts for 1941 was 42.98 feet below measuring point on Dec. 4, and lowest observed water level was 47.10 feet below measuring point on August 4. Measurements after Sept. 17 by J. B. Codlin. Water level, in feet below measuring point, 1941

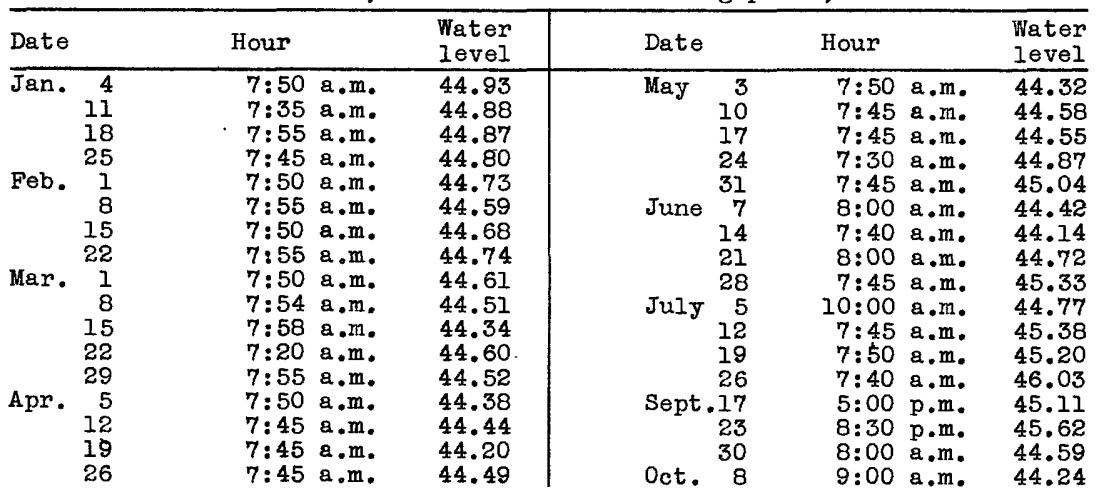

a Measurement made by Iowa State Conservation Commission. 
Story County--Continued

83-24-4Q1.--Continued.

Water level, in feet below measuring point, 1941

\begin{tabular}{|c|c|c|c|c|c|c|}
\hline Date & Hour & $\begin{array}{l}\text { Water } \\
\text { level }\end{array}$ & Date & Hour & & $\begin{array}{l}\text { Water } \\
\text { level }\end{array}$ \\
\hline 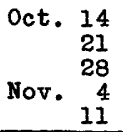 & $\begin{aligned} 11: 00 & \text { a.m. } \\
\text { 4:30 } & \text { p.m. } \\
11: 00 & \text { a.m. } \\
11: 15 & \text { a.m. } \\
11: 30 & \text { a.m. }\end{aligned}$ & $\begin{array}{l}44.11 \\
43.94 \\
43.92 \\
43.35 \\
43.34\end{array}$ & $\begin{array}{r}21 \\
\text { Nov. } \\
25 \\
\text { Dec. } 3 \\
9 \\
19\end{array}$ & $\begin{array}{r}11: 15 \\
11: 00 \\
9: 00 \\
10: 00 \\
11: 30\end{array}$ & $\begin{array}{l}\mathrm{a} \cdot \mathrm{m} . \\
\mathrm{a} \cdot \mathrm{m} . \\
\mathrm{a} \cdot \mathrm{m} . \\
\mathrm{a} \cdot \mathrm{m} . \\
\mathrm{a} \cdot \mathrm{m} .\end{array}$ & $\begin{array}{l}43.20 \\
44.16 \\
43.25 \\
43.26 \\
43.36\end{array}$ \\
\hline
\end{tabular}

83-24-17Rl. No measurements made in 1941.

83-24-20J1. Agricultural Engineering Experiment Station.

Water level, in feet below measuring point, 1941

\begin{tabular}{|c|c|c|c|c|c|}
\hline Date & $\begin{array}{l}\text { Water } \\
\text { level }\end{array}$ & Date & $\begin{array}{l}\text { Water } \\
\text { level }\end{array}$ & Date & $\begin{array}{l}\text { Water } \\
\text { level }\end{array}$ \\
\hline $\begin{array}{ll}\text { Mar. } 18 \\
\text { Apr. } 16\end{array}$ & $\begin{array}{l}20.14 \\
19.33\end{array}$ & $\begin{array}{l}\text { May } 19 \\
\text { Sept. } 18\end{array}$ & $\begin{array}{l}21.20 \\
18.01\end{array}$ & Nov. 4 & 8.40 \\
\hline
\end{tabular}

\section{Warren county}

76-25-8Q1. Iowa state college. Water levels, in feet below measuring point, 1941: Feb. 11, 14.53; Sept. 18, 13.87 .

\section{Wayne county}

67-23-20Q1. No measurements made in 1941.

\section{Woodbury county}

89-48-23Bl. Owner's Riverside station west well. City of sioux City. Water level measurements by Ed. Harbeck, Sioux City Water Works.

Water level, in feet below measuring point, 1941

\begin{tabular}{lrr|rr|rrr|rr}
\hline Date & $\begin{array}{r}\text { Water } \\
\text { level }\end{array}$ & Date & $\begin{array}{r}\text { Water } \\
\text { level }\end{array}$ & Date & $\begin{array}{r}\text { Water } \\
\text { level }\end{array}$ & Date & $\begin{array}{r}\text { Water } \\
\text { level }\end{array}$ \\
\hline Jan. 2 & $\mathbf{3 . 0 0}$ & Apr. & 2 & 6.92 & July 2 & 6.08 & Oct. 2 & 7.50 \\
Feb. 2 & 7.92 & May & 2 & 7.50 & Aug. 2 & 7.08 & Nov. 2 & 7.46 \\
Mar. 2 & 7.79 & June 2 & 6.79 & Sept. 2 & 7.50 & Dec. 2 & 7.54 \\
\hline
\end{tabular}

89-47-22Bl. Owner's Lowell 4. City of sioux city. Water level measurements by N. L. Nelson, Chief Engineer, Sloux City Water Works.

Water level, in feet below measuring point, 1941

\begin{tabular}{|c|c|c|c|c|c|c|c|c|c|c|c|c|c|}
\hline $\begin{array}{l}\text { Jan. } \\
\text { geb. }\end{array}$ & $\begin{array}{l}2 \\
2 \\
2\end{array}$ & & $\begin{array}{l}35.50 \\
35.17 \\
36.25\end{array}$ & $\begin{array}{l}\text { Apr. } \\
\text { May } \\
\text { June }\end{array}$ & & $\begin{array}{l}\text { a } 36 \\
\text { a } 34 \\
\text { a } 36\end{array}$ & $\begin{array}{l}\text { July } \\
\text { Aug. } \\
\text { Sept. }\end{array}$ & $\begin{array}{l}2 \\
2 \\
2\end{array}$ & & $\begin{array}{l}7 \\
2\end{array}$ & & & $\begin{array}{l}\text { a } 36 \\
\text { a } 35 \\
\text { a } 36\end{array}$ \\
\hline
\end{tabular}

a Nearby wells pumping. 


\section{IOWA AND MISSOURI}

\section{TARKIO CREEK AREA OF SOII COISERVATION SERVICE}

By T. W. Robinson

The observation-well program in the Tarklo Creek area, $1 /$ In Iowa and an adjacent part of Missouri, was continued in 1941. Of the 75 wells under observation at the beginning of 1941, 4 wells $(37,43 \mathrm{~A}, 69$, and 77 ) were dropped during the jear. The water-level measurements were made monthly, usually between the 25 th and the end of the month. A total of 709 individual measurements of water levels was made during the year. The measurements were made by $W$. M. Mulnix untIl July 1 and by D. W. Knox for the remainder of the year.

Water-level measurements in 9 to 12 wells $(1,2,5,6,7,10,11,12$, 14, 15, 16, 17) were used in computing the average water levels, in 1941, given in the following table.

- Average water levels, in feet above assumed datum planes, in 9 to i2 observation wells, 1941

\begin{tabular}{ll|ll|ll}
\hline Date & $\begin{array}{l}\text { Water } \\
\text { level }\end{array}$ & Date & $\begin{array}{l}\text { Water } \\
\text { level }\end{array}$ & Date & $\begin{array}{l}\text { Water } \\
\text { level }\end{array}$ \\
\hline Jan. 30-31 & 10.65 & May 26-27 & 12.17 & Sept.24-25 & 12.51 \\
Feb. 24-25 & 11.47 & June 27-28 & 14.79 & Oct.27-28 & 18.26 \\
Mar. 27-28 & 11.55 & July 24-25 & 13.90 & Nov.25-26 & 18.60 \\
Apr. 24-25 & 11.72 & Aug. 26-27 & 11.64 & Dec. 26-29 & 18.30 \\
\hline
\end{tabular}

The precipitation for the year at shenandoah was 48.07 Inches, which was 14.07 inches above normal. In response to this heavy precipltation, the greatest rise in water levels occurred since measurements were begun in May 1934. The average water level stood 7.71 feet higher in December 1941 than in December 1940. The water levels rose 4.20 feet from the end of December 1940 to the end of June 1941, and then declined 3.15 feet during July and August. During the extremely wet season from the end of August to the end of November, there was a rise of 6.96 feet, which was followed in December by a decline of 0.30 foot. A large part of the total rise took place in the months of september and october, when,according to the records of the United States Weather Bureau, a total of 23.11 Inches of rain fell at Shenandoah. 
Figure 3 shows the average fluctuation of the water levels and the monthly and annual precipitation since August 1934. The precipitation at Shenandoah has been below normal in each year since measurement of water levels was begun in that area except in 1941. The lowest water level during

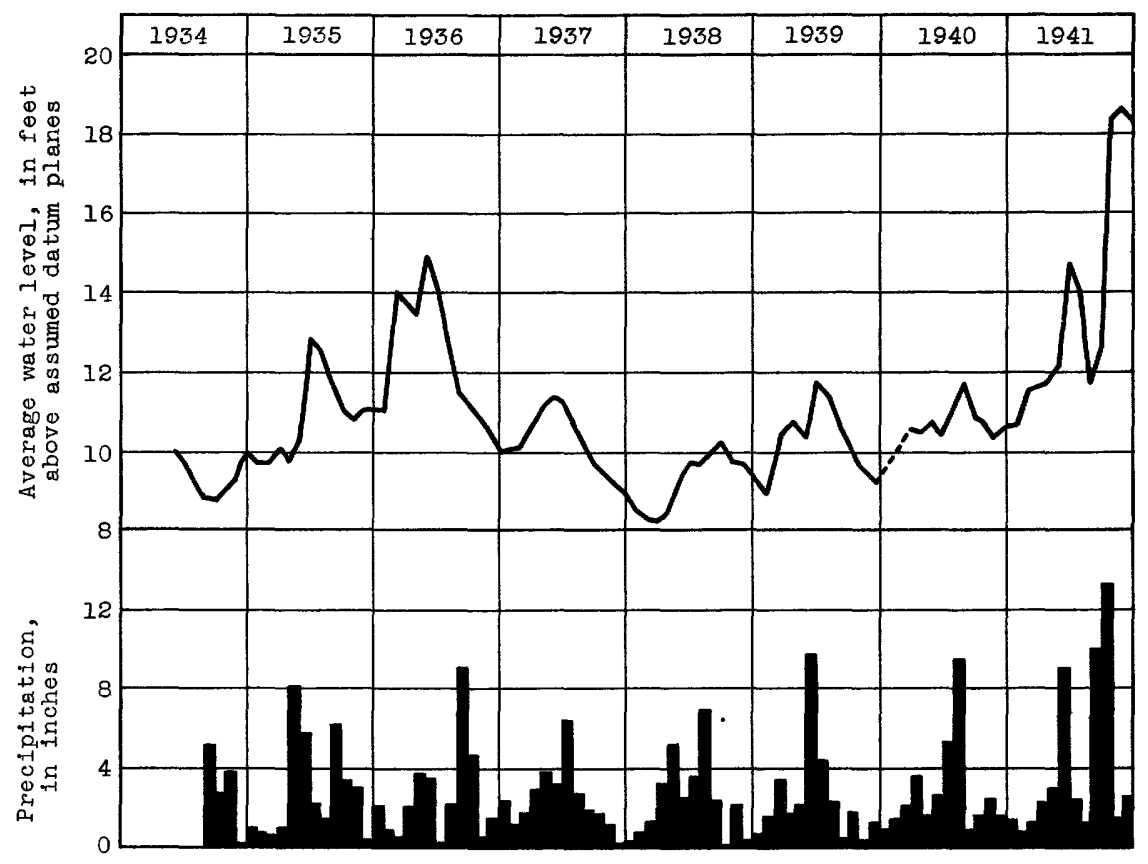

Figure 3.--Graph showing average water level in 9 to 12 wells and precipitation at Shenandoah in the Tarkio Creek area, Iowa-Missouri.

the period of record occurred in March 1938. Beginning in April 1938 there has been a persistent upward trend, which reached a climax with the high water level of November 1941. The average net rise from the low level of March 1938 to the high level of lovember 1941 amounted to 10.36 feet.

The following table gives the average stage of water levels in the observation wells in the Tarkio Creek area for each month since May 1934. 
Average monthly water levels in 9 to 12 observation wells in the Tarkio Creek area, Iowa - Missouri, 1934-1941.

(Elevations are given in feet above assumed datum plane)

\begin{tabular}{|c|c|c|c|c|c|c|c|c|c|c|c|c|}
\hline & Jan. & Feb. & Mar. & Apr. & May & June & July & Aug. & Sept. & - oct. & Nov. & Dec. \\
\hline $\begin{array}{l}1934 \\
1935 \\
1936 \\
1937 \\
1938 \\
1939 \\
1940 \\
1941\end{array}$ & $\begin{array}{r}0.01 \\
11.06 \\
10.10 \\
8.52 \\
9.03 \\
10.06\end{array}$ & $\begin{array}{r}9.72 \\
14.09 \\
10.14 \\
8.29 \\
8.95 \\
11.94\end{array}$ & $\begin{array}{r}10.12 \\
13.74 \\
10.61 \\
8.24 \\
10.47 \\
10.53 \\
11.55\end{array}$ & $\begin{array}{r}9.79 \\
9.79 \\
13.43 \\
11.19 \\
8.48 \\
10.79 \\
10.43 \\
11.72\end{array}$ & $\begin{array}{r}10.04 \\
10.45 \\
15.01 . \\
11.41 \\
9.31 \\
10.33 \\
10.71 \\
12.17\end{array}$ & $\begin{array}{r}9.72 \\
12.89 \\
14.16 \\
11.24 \\
9.75 \\
11.77 \\
10.37 \\
14.79\end{array}$ & $\begin{array}{r}9.16 \\
12.52 \\
12.69 \\
10.82 \\
9.68 \\
11.40 \\
13.00\end{array}$ & $\begin{array}{r}8.79 \\
11.63 \\
11.56 \\
10.18 \\
10.02 \\
10.66 \\
11.71 \\
11.64\end{array}$ & $\begin{array}{r}8.76 \\
11.02 \\
11.09 \\
9.70 \\
10.28 \\
10.16 \\
10.82 \\
12.51\end{array}$ & $\begin{array}{r}9.02 \\
10.82 \\
10.90 \\
9.51 \\
9.75 \\
9.60 \\
10.69 \\
18.26\end{array}$ & $\begin{array}{r}9.28 \\
11.07 \\
10.61 \\
9.17 \\
9.69 \\
9.34 \\
10.34 \\
18.60\end{array}$ & $\begin{array}{r}10.02 \\
11.14 \\
10.05 \\
8.93 \\
9.35 \\
9.18 \\
10.59 \\
18.30\end{array}$ \\
\hline
\end{tabular}

1. W. R. Marshall.

Water level, in feet above assumed datum, 1941

\begin{tabular}{lr|ll|ll|ll}
\hline Date & $\begin{array}{l}\text { Water } \\
\text { level }\end{array}$ & Date & $\begin{array}{l}\text { Water } \\
\text { level }\end{array}$ & Date & $\begin{array}{l}\text { Water } \\
\text { level }\end{array}$ & Date & $\begin{array}{l}\text { Water } \\
\text { level }\end{array}$ \\
\hline Jan. 30 & 9.95 & Apr. 24 & 11.31 & July 24 & $\ldots . .34$ & Oct. 27 & 16.33 \\
Feb.24 & 10.87 & May 26 & 10.93 & Aug. 26 & i3.84 & Nov. 25 & 20.41 \\
Mar.27 & 10.71 & June 27 & 15.83 & Sept.24 & 14.03 & Dec. 26 & 19.42 \\
\hline
\end{tabular}

2. H. W. Klutas.

Water level, in feet above assumed datum, 1941

\begin{tabular}{ll|ll|ll|ll}
\hline Jan. 30 & 10.89 & Apr. 24 & 11.33 & July 24 & 11.57 & Oct. 27 & 16.13 \\
Feb. 24 & 11.23 & May 26 & 11.10 & Aug. 26 & 10.33 & Nov.25 & 12.51 \\
Mar.27 & 10.98 & June 27 & 12.22 & Sept.24 & 10.97 & Dec. 26 & 11.06 \\
\hline
\end{tabular}

5. John Toft.

Water level, in feet above assumed datum, 1941

\begin{tabular}{ll|ll|ll|ll}
\hline Jan. 30 & 10.53 & Apr. 24 & 15.81 & July 24 & 13.75 & Oct. 27 & 18.40 \\
Feb. 24 & 12.90 & May 26 & 14.09 & Aug. 26 & 10.06 & IJov. 25 & 18.16 \\
Mar. 27 & 13.10 & June 27 & 17.56 & Sept.24 & 11.46 & Dec. 26 & 20.04 \\
\hline
\end{tabular}

6. T. Slickerveer.

Water level, in feet above assumed datum, 1941

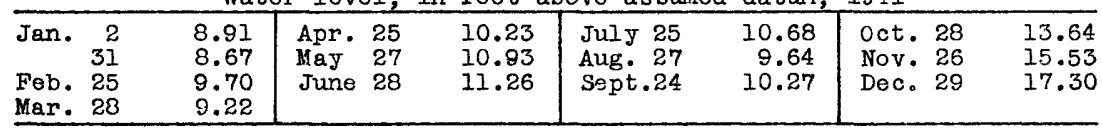

7. E. F. Holquist.

Water level, in feet above assumed datum, 1941

\begin{tabular}{|c|c|c|c|c|c|c|c|c|}
\hline 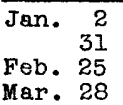 & $\begin{array}{r}9.69 \\
9.94 \\
10.38 \\
12.37\end{array}$ & $\begin{array}{l}\text { Apr } \\
\text { May } \\
\text { Jun }\end{array}$ & $\begin{array}{l}25 \\
27 \\
28\end{array}$ & $\begin{array}{r}9.20 \\
14.44 \\
18.95\end{array}$ & $\begin{array}{l}\text { July } 25 \\
\text { Aug. } 27 \\
\text { Sept. } 25\end{array}$ & $\begin{array}{l}15.48 \\
11.55 \\
14.17\end{array}$ & $\begin{array}{ll}\text { Oct. } & 28 \\
\text { Nov. } & 26 \\
\text { Dec. } & 29\end{array}$ & $\begin{array}{l}19.22 \\
22.23 \\
23.83\end{array}$ \\
\hline
\end{tabular}

10. R. Palmquist.

Water level, in feet above assumed datum, 1941

\begin{tabular}{lrr|ll|ll|ll}
\hline Jan. & 2 & 10.67 & Apr. 25 & 12.17 & July 25 & 15.11 & Oct. 28 & 19.47 \\
31 & 10.99 & May 27 & 12.17 & Aug. 27 & 12.42 & INov. 26 & 20.70 \\
Feb. 25 & 11.42 & June 28 & 18.31 & Sept.25 & 12.89 & Dec. 29 & 21.92 \\
Mar. 28 & 11.93 & & & & & & & \\
\hline
\end{tabular}


11. R. Palmquist.

Water level, in feet above assumed datum, 1941

\begin{tabular}{|c|c|c|c|c|c|c|c|}
\hline Date & $\begin{array}{l}\text { Water } \\
\text { level }\end{array}$ & Date & $\begin{array}{l}\text { Water } \\
\text { level }\end{array}$ & Date & $\begin{array}{l}\text { Water } \\
\text { level }\end{array}$ & Date & $\begin{array}{l}\text { Water } \\
\text { level }\end{array}$ \\
\hline $\begin{array}{lr}\text { Jan. } & 2 \\
& 31 \\
\text { Feb. } 25 \\
\text { Mar. } 28\end{array}$ & $\begin{array}{l}\text { (a) } \\
\text { (a) } \\
\text { (a) }\end{array}$ & $\begin{array}{ll}\text { Apr. } & 25 \\
\text { May } & 11 \\
\text { June } 28\end{array}$ & $\begin{array}{c}\left(\begin{array}{l}\text { a } \\
\text { a }\end{array}\right) \\
12.16\end{array}$ & $\begin{array}{l}\text { July } 25 \\
\text { Aug. } 27 \\
\text { Sept.25 }\end{array}$ & $\begin{array}{r}10.45 \\
9.58 \\
10.26\end{array}$ & $\begin{array}{l}\text { Oct. } 28 \\
\text { Nov. } 26 \\
\text { Dec. } 29\end{array}$ & $\begin{array}{l}13.72 \\
13.39 \\
13.64\end{array}$ \\
\hline
\end{tabular}

12. Amil Windhorst.

Water level, in feet above assumed datum, 1941

\begin{tabular}{|c|c|c|c|c|c|c|}
\hline $\begin{array}{lr}\text { Jan. } & 2 \\
& 31 \\
\text { Feb. } & 25 \\
\text { Mar. } 28\end{array}$ & $\begin{array}{l}13.47 \\
13.57 \\
13.82 \\
13.67\end{array}$ & $\begin{array}{ll}\text { Apr. } 24 \\
\text { May } 27 \\
\text { June } 28\end{array}$ & $\begin{array}{l}14.38 \\
14.38 \\
\text { b } 22.47\end{array}$ & $\begin{array}{l}\text { July } 25 \\
\text { Aug. } 27 \\
\text { Sept.25 }\end{array}$ & $\begin{array}{l}\text { b } 22.20 \\
\text { b } 18.61 \\
\text { b } 23.68\end{array}$ & $\begin{array}{lll}\text { oct. } 28 & \text { b } & 32.13 \\
\text { Nov. } 26 & \text { b } 27.96 \\
\text { Dec. } 29 & \text { b } 27.59\end{array}$ \\
\hline
\end{tabular}

13. Amil Windhorst.

Water level, in feet above assumed datum, 1941

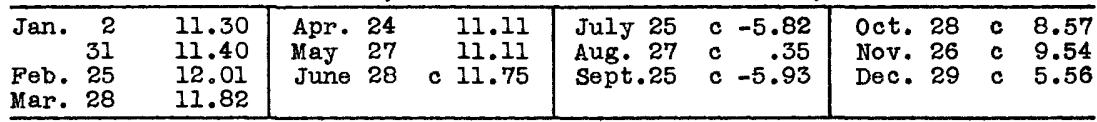

14. Floyd Hoskins.

Water level, in feet above assumed datum, 1941

\begin{tabular}{lrr|rr|ll|lll}
\hline Jan. & 2 & 12.93 & Apr. 24 & 13.96 & July 24 & 17.46 & 0ct. 27 & 20.00 \\
30 & 13.21 & May 26 & 15.10 & Aug. 26 & 14.28 & Nov.25 & 25.52 \\
Feb. 24 & 15.00 & June 27 & 17.31 & Sept.24 & 14.35 & Dec. 24 & 20.52 \\
Mar. 27 & 15.04 & & & & & & & \\
\hline
\end{tabular}

15. Metropolitan Life Insurance Co.

Water level, in feet above assumed datum, 1941

\begin{tabular}{ll|l|l|l|l|l|l|}
\hline Jan. 30 & 10.61 & Apr. 24 & 11.42 & July 24 & 12.74 & Oct. 27 & 16.58 \\
Feb. 24 & 11.14 & May 26 & 11.15 & Aug. 26 & 9.70 & Nov. 25 & 16.78 \\
Mar. 27 & 10.22 & June 27 & 13.28 & Sept.24 & 6.76 & Dec. 26 & 13.09 \\
\hline
\end{tabular}

- 16. Metropolitan Iife Insurance Co.

Water level, in feet above assumed datum, 1941

\begin{tabular}{ll|ll|ll|lll}
\hline Jar. 30 & (d) & Apr. 24 & (d) & July 24 & (d) & Dct. 27 & (d) \\
Feb. 24 & (d) & May 26 & (d) & Aug. 26 & (d) & Nov. 25 & (d) \\
Mar. 27 & (d) & June 27 & 8.21 & Sept.24 & (d) & Dec. 26 & (d) \\
\hline
\end{tabular}

17. Albert Nordholm.

Water level, in feet above assumed datum, 1941

\begin{tabular}{ll|ll|ll|ll}
\hline Jan. 30 & 8.11 & Apr. 24 & 7.40 & July 24 & 9.57 & Oct. 27 & 15.27 \\
Feb. 24 & 8.21 & May 26 & 7.45 & Aug. 26 & 7.98 & Nov. 25 & 11.40 \\
Mar. 27 & 8.25 & June 27 & 9.90 & Sopt.24 & 8.81 & Dec. 26 & 12.92 \\
\hline
\end{tabular}

20.

Water level, in feet above assumed datum, 1941

\begin{tabular}{ll|ll|ll|lll}
\hline Jan. 30 & 11.44 & Apr. 24 & 10.23 & July 24 & 13.14 & Oct. 27 & 15.63 \\
Feb. 24 & 12.98 & May 26 & 11.67 & Aug. 26 & 11.03 & Nov. 25 & 13.87 \\
Mar.27 & 10.85 & June 27 & 16.04 & Sept.24 & 11.93 & Dec. 26 & 12.44 \\
\hline
\end{tabular}

a Dry.

b Weil No. 13, 65 feet distant pumping.

c Pumping.

d Well bridged above water level. 
21 .

Water level, in feet above assumed datum, 1941

\begin{tabular}{lr|ll|ll|ll}
\hline Date & $\begin{array}{l}\text { Water } \\
\text { level }\end{array}$ & Date & $\begin{array}{l}\text { Water } \\
\text { level }\end{array}$ & Date & $\begin{array}{l}\text { Water } \\
\text { level }\end{array}$ & Date & $\begin{array}{r}\text { Water } \\
\text { level }\end{array}$ \\
\hline Jan. 30 & 7.92 & Apr. 24 & (a) & July 24 & 10.03 & 0ct. 27 & 15.07 \\
Feb. 24 & 7.58 & May 26 & 7.16 & Aug. 26 & 8.77 & Nov. 25 & 18.17 \\
Mar.27 & 6.67 & June 27 & 9.77 & Sept.24 & 10.11 & Dec. 26 & 17.15 \\
\hline
\end{tabular}

22. J. A. McAllister.

Water level, in feet above assumed datum, 1941

\begin{tabular}{ll|ll|ll|lll}
\hline Jan. 30 & 10.90 & Apr. 24 & 10.26 & July 24 & 12.46 & Oct. 27 & 15.61 \\
Feb.24 & 10.86 & May 26 & 10.93 & Aug. 26 & 10.04 & Nov. 25 & 17.51 \\
Mar. 27 & 10.20 & June 27 & 12.18 & Sept.24 & 11.51 & Dec. 26 & 13.45 \\
\hline
\end{tabular}

23. No measurements made in 1941.

24.

Water level, in feet above assumed datum, 1941

\begin{tabular}{|c|c|c|c|c|c|c|c|}
\hline $\begin{array}{ll}\text { Jan. } 30 \\
\text { Feb. } 24 \\
\text { Mar. } 27\end{array}$ & $\begin{array}{l}9.77 \\
9.54 \\
9.67\end{array}$ & $\begin{array}{l}\text { Apr. } 2 \\
\text { May } \\
\text { June } 2\end{array}$ & $\begin{array}{r}9.68 \\
10.18\end{array}$ & $\begin{array}{l}\text { July } 24 \\
\text { Aug. } 26 \\
\text { Sept. } 24\end{array}$ & $\begin{array}{r}10.28 \\
9.64 \\
10.86\end{array}$ & $\begin{array}{l}\text { Oct. } \\
\text { Nov. } \\
\text { Dec. }\end{array}$ & $\begin{array}{l}11.72 \\
12.06 \\
10.65\end{array}$ \\
\hline
\end{tabular}

25. Edwin Rolf.

Water level, in feet above datum of well 1,1941

\begin{tabular}{ll|ll|ll|ll|l}
\hline Jan. 30 & 30.10 & Apr. 24 & 30.84 & July 24 & 36.72 & 0ct. 27 & 52.31 \\
Feb. 24 & 30.92 & May 26 & 30.65 & Aug. 26 & 32.60 & Nov. 25 & 42.33 \\
Mar. 27 & 30.34 & June 27 & 36.17 & Sept.24 & 36.29 & Dec. 26 & 38.25 \\
\hline
\end{tabular}

26. No measurements made in 1941.

27. Edwin Rolf.

Water level, in feet above datum of well 1, 1941

\begin{tabular}{ll|ll|ll|ll}
\hline Jan. 30 & 29.00 & Apr. 24 & 28.78 & July 24 & 31.97 & 0ct. 27 & 33.85 \\
Feb.24 & 28.74 & May 26 & 28.69 & Aug. 26 & 30.61 & Nov. 25 & 34.82 \\
Mar. 27 & 28.62 & June 27 & 31.13 & Sept.24 & 31.36 & Dec. 26 & 40.35 \\
\hline
\end{tabular}

28. Edwin Rolf.

Water level, in feet above datum of well 1,1941

\begin{tabular}{ll|lr|lll|ll}
\hline Jan. 30 & 48.19 & Apr. 24 & -49.58 & July 24 & 57.07 & Oct. 27 & 60.54 \\
Feb. 24 & 48.87 & May 26 & 49.74 & Aug. 26 & 49.42 & Nov. 25 & 60.95 \\
Mar. 27 & 49.42 & June 27 & 57.68 & Sept.24 & 52.79 & Dec. 26 & 54.68 \\
\hline
\end{tabular}

29. Edwin Rolf.

Water level, in feet above datum of well 1, 1941

\begin{tabular}{ll|ll|ll|lll}
\hline Jan. 30 & 31.89 & Apr. 24 & 32.56 & July 24 & 36.26 & Oct. 27 & 39.54 \\
Feb. 24 & 32.81 & May 26 & 32.46 & Aug. 26 & 32.14 & Nov. 25 & 38.08 \\
Mar. 27 & 32.62 & June 27 & 36.69 & Sept.24 & 32.16 & Dec. 26 & 35.72 \\
\hline
\end{tabular}

30. W. F. Marshall.

Water level, in feet above datum of well 1,1941

\begin{tabular}{ll|ll|ll|ll}
\hline Jan. 30 & 15.21 & Apr. 24 & 16.32 & July 24 & 21.75 & Oct. 27 & 25.15 \\
Feb. 24 & 16.62 & May 26 & 15.86 & Aug. 26 & 20.04 & Nov. 25 & 23.84 \\
Mar. 27 & 15.88 & June 27 & 24.07 & Sept.24 & 21.87 & Dec. 26 & 24.44 \\
\hline
\end{tabular}

a Dry. 
31. W. F. Marshall.

Water level, in feet above datum of well 1, 1941

\begin{tabular}{lr|lr|ll|ll}
\hline Date & $\begin{array}{r}\text { Water } \\
\text { level }\end{array}$ & Date & $\begin{array}{r}\text { Water } \\
\text { level }\end{array}$ & Date & $\begin{array}{l}\text { Water } \\
\text { level }\end{array}$ & Date & $\begin{array}{r}\text { Water } \\
\text { level }\end{array}$ \\
\hline Jan. 30 & $\mathbf{8 . 9 7}$ & Apr. 24 & 9.61 & July 24 & 14.35 & Oct. 27 & 18.56 \\
Feb. 24 & 10.10 & May 26 & 9.55 & Aug. 26 & 13.51 & Nov. 25 & 17.81 \\
Mar. 27 & 9.66 & June 27 & 12.45 & Sept.24 & 13.83 & Dec.26 26 & 19.07 \\
\hline
\end{tabular}

32. W. F. Marshall.

Water level, in feet above datum of well 1, 1941

\begin{tabular}{ll|lr|lr|lr}
\hline Jan. 30 & 1.79 & Apr. 24 & 5.57 & July 24 & 7.68 & 0ct. 27 & 9.44 \\
Feb. 24 & 4.85 & May 26 & 2.56 & Aug. 26 & 4.65 & Nov. 25 & 11.44 \\
Mar. 27 & 3.94 & June 27 & 10.61 & Sept.24 & 7.74 & Dec. 26 & 11.62 \\
\hline
\end{tabular}

33. W. F. Marshall.

Water level, in feet, with reference to datum of well 1, 1941

\begin{tabular}{lr|lr|lr|rrr}
\hline Jan. 30 & -1.53 & Apr. 24 & -1.17 & July 24 & 1.93 & Oct. 27 & 6.31 \\
Feb. 24 & -.45 & May 26 & -1.70 & Aug. 26 & -2.14 & Nov. 25 & 3.99 \\
Mar. 27 & -2.55 & June 27 & 4.16 & Sept.24 & 4.04 & Dec. 26 & 4.33 \\
\hline
\end{tabular}

34. W. F. Marshall.

Water level, in feet above datum of well 1, 1941

\begin{tabular}{ll|lr|rr|rr}
\hline Jan. 30 & 6.90 & Apr. 24 & 10.12 & July 24 & 11.39 & Oct. 27 & 11.87 \\
Feb. 24 & 7.83 & May 26 & 7.54 & Aug. 26 & 8.23 & Nov. 25 & 14.97 \\
Mar. 27 & 7.64 & June 27 & 15.08 & Sept.24 & 12.99 & Dec. 26 & 15.19 \\
\hline
\end{tabular}

35. W. F. Marshall.

Water level, in feet above datum of well 1,1941

\begin{tabular}{ll|ll|lll|ll}
\hline Jan. 30 & 50.16 & Apr. 24 & 51.56 & July 24 & 52.92 & Oct. 27 & 52.72 \\
Feb. 24 & 51.35 & May 26 & 51.18 & Aug. 26 & 50.60 & Nov. 25 & 56.10 \\
Mar. 27 & 50.52 & June 27 & 54.94 & Sept.24 & 51.11 & Dec. 26 & 56.90 \\
\hline
\end{tabular}

36. George Rolf.

Water level, in feet above datum of well 1,1941

\begin{tabular}{ll|ll|ll|ll}
\hline Jan. 30 & 86.92 & Apr. 24 & 86.76 & July 24 & 88.15 & 0ct. 27 & 92.26 \\
Feb. 24 & 86.97 & May 26 & 86.81 & Aug. 26 & 86.90 & Nov. 25 & 92.26 \\
Mar. 27 & 86.70 & June 27 & 86.96 & Sept.24 & 90.89 & Dec. 26 & 97.68 \\
\hline
\end{tabular}

37. Well destrojed, measurements discontinued.

38. Elsie Nordstrom.

Water level, in feet above assumed datum, 1941

\begin{tabular}{lrr|cc|cc|cc}
\hline Jan. 2 & 72.04 & Apr. 25 & 71.14 & July 24 & 74.02 & Oct. 27 & 79.58 \\
30 & 72.32 & May 27 & 71.20 & Aug. 26 & 73.86 & Nov. 25 & 78.25 \\
Feb. 24 & 70.70 & June 27 & 73.30 & Sept.24 & 73.67 & Dec. 26 & 81.99 \\
Mar. 27 & 70.76 & & & & & & & \\
\hline
\end{tabular}

39. Elsie Nordstrom.

Water level, in feet above datum of well 38,1941

\begin{tabular}{lr|ll|l|ll|ll}
\hline Jan. 2 & 70.08 & Apr. 25 & 69.42 & July 24 & 71.58 & Oct. 27 & 73.48 \\
& 30 & 70.17 & May 27 & 69.16 & Aug.26 & 71.14 & Nov.25 & 78.74 \\
Feb. 24 & 68.57 & June 27 & 70.66 & Sept.24 & 71.19 & Dec. 26 & 77.71 \\
Mar.27 & 68.71 & & & & & & & \\
\hline
\end{tabular}


40. Elsie Nordstrom.

Water level, in feet above datum of well 38, 1941

\begin{tabular}{lcc|cc|cc|cr}
\hline Date & $\begin{array}{c}\text { Water } \\
\text { level }\end{array}$ & Date & $\begin{array}{c}\text { Water } \\
\text { level }\end{array}$ & Date & $\begin{array}{c}\text { Water } \\
\text { level }\end{array}$ & Date & $\begin{array}{r}\text { Water } \\
\text { level }\end{array}$ \\
\hline Jan. 2 & (a) & Apr. 25 & (a) & July 24 & 68.94 & 0ct. 27 & 72.15 \\
30 & (a) & May 27 & (a) & Aug. 26 & 68.27 & Nov. 25 & 80.99 \\
Feb. 24 & (a) & June 27 & 69.32 & Sept.24 & (a) & Dec. 26 & 77.71 \\
Mar. 27 & (a) & & & & & & &
\end{tabular}

41. Elsie Nordstrom.

Water level, in feet above datum of well 38, 1941

\begin{tabular}{rrr|ll|ll|ll}
\hline Jan. & 2 & 67.77 & Apr. 25 & 67.42 & JuIy 24 & 70.07 & Oct. 27 & 71.07 \\
30 & 67.85 & May 27 & 67.06 & Aug. 26 & 67.70 & Nov. 25 & 78.67 \\
Feb. 24 & 70.21 & June 27 & 71.11 & Sept.24 & $\ldots . .$. & Dec. 26 & 76.41 \\
Mar. 27 & 69.92 & & & & & & & \\
\hline
\end{tabular}

42. Elsie Nordstrom.

Water level, in feet above datum of well 38, 1941

\begin{tabular}{lrr|ll|ll|ll}
\hline Jan. 2 & 66.34 & Apr. 25 & 66.34 & July 24 & 68.58 & Oct. 27 & 73.19 \\
30 & 66.46 & May 27 & 65.95 & Aug. 26 & 66.81 & Nov. 25 & 80.31 \\
Feb. 24 & 68.38 & June 27 & 68.89 & Sept.24 & 68.07 & Dec. 26 & 77.45 \\
Mar. 27 & 68.71 & & & & & & & \\
\hline
\end{tabular}

43. Elsie Nordstrom.

Water level, in feet above datum of well 38, 1941

\begin{tabular}{lrr|ll|ll|ll}
\hline Jan. 2 & 67.35 & Apr. 25 & 66.63 & July 24 & 67.85 & Oct. 27 & 70.73 \\
30 & 67.30 & May 27 & 66.50 & Aug. 26 & 67.52 & Nov. 25 & 79.93 \\
Feb. 24 & 66.19 & June 27 & 66.96 & Sept.24 & 67.63 & Dec. 26 & 75.66 \\
Mar.27 & 66.34 & & & & & & & \\
\hline
\end{tabular}

43A. Well destrojed, measurements discontinued.

44. Elsie Nordstrom.

Water level, in feet above datum of well 38,1941

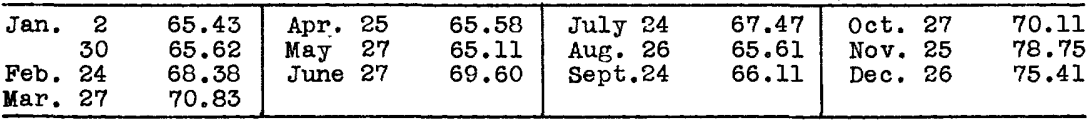

44A. Elsie Nordstrom.

Water level, in feet above datum of well 38, 1941

\begin{tabular}{lrr|ll|ll|ll}
\hline Jan. 2 & 63.89 & Apr. 25 & 64.68 & July 24 & 66.14 & Oct. 27 & 69.36 \\
30 & 64.05 & May 27 & 64.32 & Aug. 26 & 64.13 & Nov. 25 & 76.60 \\
Feb. 24 & 69.16 & June 27 & 69.29 & Sept.24 & 64.57 & Dec. 26 & 74.62 \\
Mar. 27 & 70.42 & & & & & & & \\
\hline
\end{tabular}

45. Elsie Nordstrom.

Water level, in feet above datum of well 38,1941

\begin{tabular}{lrr|ll|ll|lll}
\hline Jan. & 2 & 63.26 & Apr. 25 & 67.56 & July 24 & 65.85 & Oct. 27 & 70.20 \\
30 & 63.39 & May 27 & 66.85 & Aug. 26 & 63.63 & Nov. 25 & 75.98 \\
Feb. 24 & 69.50 & June 27 & 68.97 & Sept.24 & 64.18 & Dec.26 & 75.31 \\
Mar. 27 & 70.10 & & & & & & & \\
\hline
\end{tabular}

a Dry. 
46. Elsie Nordstrom.

Water level, in feet above datum of well 38,1941

\begin{tabular}{lr|ll|ll|ll}
\hline Date & $\begin{array}{l}\text { Water } \\
\text { level }\end{array}$ & Date & $\begin{array}{l}\text { Water } \\
\text { level }\end{array}$ & Date & $\begin{array}{l}\text { Water } \\
\text { level }\end{array}$ & Date & $\begin{array}{r}\text { Water } \\
\text { level }\end{array}$ \\
\hline Jan. 2 & 58.51 & Apr. 25 & 61.54 & July 24 & 62.52 & Oct. 27 & 66.19 \\
30 & 58.43 & May 27 & 60.31 & Aug. 26 & 59.25 & Nov.25 & 71.99 \\
Feb. 24 & 64.48 & June 27 & 62.76 & Sept.24 & 59.80 & Dec. 26 & 71.62 \\
Mar. 27 & 65.90 & & & & & &
\end{tabular}

47. Elsie Nordstrom.

Water level, in feet above datum of well 38, 1941

\begin{tabular}{lrr|cc|cc|cc}
\hline Jan. 2 & 56.23 & Apr. 25 & 56.68 & July 24 & 58.35 & Oct. 27 & 61.21 \\
30 & 56.30 & May 27 & 56.43 & Aug. 26 & 56.65 & Nov.25 & 65.04 \\
Feb. 24 & 58.08 & June 27 & 58.61 & Sept.24 & 57.11 & Dec. 26 & 66.73 \\
Mar. 27 & 58.35 & & & & & & & \\
\hline
\end{tabular}

48. Elsie Nordstrom.

Water level, in feet above datum of well 38, 1941

\begin{tabular}{ll|ll|ll|l|l}
\hline Jan. 2 & (a) & Apr. 25 & (a) & July 24 & (a) & Oct. 27 & (a) \\
30 & (a) & May 27 & (a) & Aug. 26 & (a) & Nov.25 & 50.83 \\
Feb. 24 & (a) & June 27 & (a) & Sept.24 & (a) & Dec.26 & (a) \\
Mar. 27 & (a) & & & & & &
\end{tabular}

49. Elsie Nordstrom.

Water level, in feet above datum of well 38, 1041

\begin{tabular}{rrr|ll|lll|ll}
\hline Jan. 2 & 71.71 & Apr. 25 & 71.88 & July 24 & 77.09 & Oct. 27 & 79.07 \\
30 & 71.84 & May 27 & 71.48 & Aug. 26 & 76.77 & Nov.25 & 80.14 \\
Feb. 24 & 72.08 & June 27 & 77.03 & Sept.24 & 70.23 & Dec. 26 & 82.04 \\
Mar. 27 & 72.23 & & & & & & & \\
\hline
\end{tabular}

50. Elsie Nordstrom.

Water level, in feet above datum of well 38, 1941

\begin{tabular}{rr|ll|lll|ll}
\hline Jan. 2 & 61.29 & Apr. 25 & 61.53 & July 24 & 67.65 & Oct. 27 & 71.99 \\
30 & 61.35 & May 27 & 61.33 & Aug. 26 & 64.09 & Nov. 25 & 75.28 \\
Feb. 24 & 63.11 & June 27 & 68.61 & Sept.24 & 77.38 & Dec. 26 & 77.74 \\
Mai. 27 & 63.45 & & & & & & & \\
\hline
\end{tabular}

51. Elsie Nordstrom.

Water level, in feet above datum of well 38,1941

\begin{tabular}{ll|ll|ll|ll}
\hline Jan. 2 & (b) & Apr.25 & (b) & July 24 & (b) & Oct. 27 & (b) \\
30 & (b) & May 27 & (b) & Aug. 26 & (b) & Nov. 25 & 69.67 \\
Feb. 24 & (b) & June 27 & (b) & Sept.24 & (b) & Dec. 26 & 72.78 \\
Mar. 27 & (b) & & & & & &
\end{tabular}

52. Elsie Nordstrom.

Water level, in feet above datum of well 38,1941

\begin{tabular}{ll|ll|ll|ll}
\hline Jan. 2 & (b) & Apr. 25 & (b) & July 24 & (b) & Oct. 27 & (b) \\
30 & (b) & May 27 & (b) & Aug. 26 & (b) & Nov. 25 & 69.79 \\
Feb. 24 & (b) & June 27 & (b) & Sept.24 & (b) & Dec. 26 & 73.57 \\
Mar. 27 & (b) & & & & & &
\end{tabular}

54. Elsie Nordstrom.

Water level, in feet above datum of well 38, 1941

\begin{tabular}{|c|c|c|c|c|c|c|c|c|}
\hline $\begin{array}{l}\text { Jan. } \\
\text { Feb. } \\
\text { Mar. }\end{array}$ & $\begin{array}{r}2 \\
30 \\
24 \\
27\end{array}$ & $\begin{array}{l}\text { (b) } \\
(\mathrm{b}) \\
(\mathrm{b}) \\
(\mathrm{b})\end{array}$ & $\begin{array}{ll}\text { Apr. } & 25 \\
\text { May } & 27 \\
\text { June } & 27\end{array}$ & $\begin{array}{l}\text { (b) } \\
(\mathrm{b}) \\
(\mathrm{b})\end{array}$ & $\begin{array}{l}\text { July } 24 \\
\text { Aug. } 26 \\
\text { Sept. } 24\end{array}$ & $\begin{array}{l}(\mathrm{b}) \\
(\mathrm{b}) \\
(\mathrm{b})\end{array}$ & $\begin{array}{ll}\text { Oct. } 27 \\
\text { Nov. } 25 \\
\text { Dec. } 26\end{array}$ & $\begin{array}{c}\text { (b) } \\
74.29 \\
75.84\end{array}$ \\
\hline
\end{tabular}

a Well bridged above water level.

b Dry. 
55. Elsie Nordstrom.

Water level, in feet above datum of well 38, 1941

\begin{tabular}{|c|c|c|c|c|c|c|c|}
\hline Date & $\begin{array}{l}\text { Water } \\
\text { level }\end{array}$ & Date & $\begin{array}{l}\text { Water } \\
\text { level }\end{array}$ & Date & $\begin{array}{l}\text { Water } \\
\text { level }\end{array}$ & $D_{\text {ate }}$ & $\begin{array}{l}\text { Water } \\
\text { level }\end{array}$ \\
\hline $\begin{array}{l}\text { Jan. } \\
\text { Feb. } \\
\text { Mar. }\end{array}$ & $\begin{array}{l}\text { (a) } \\
(a) \\
(a) \\
(a)\end{array}$ & $\begin{array}{ll}\text { Apr. } & 25 \\
\text { May } & 27 \\
\text { June } & 27\end{array}$ & $\begin{array}{l}\text { (a) } \\
\text { (a) } \\
\text { (a) }\end{array}$ & $\begin{array}{l}\text { July } 24 \\
\text { Aug. } 26 \\
\text { Sept. } 24\end{array}$ & $\begin{array}{l}\text { (a) } \\
\text { (a) } \\
\text { (a) }\end{array}$ & $\begin{array}{l}\text { Oct. } 27 \\
\text { Nov. } 25 \\
\text { Dec. it }\end{array}$ & $\begin{array}{c}\text { (a) } \\
72.93 \\
74.43\end{array}$ \\
\hline
\end{tabular}

56. Elsie Nordstrom.

Water level, in feet above datum of well 38, 1941

\begin{tabular}{ll|ll|lll|ll}
\hline Jan. & 2 & (a) & Apr. 25 & (a) & July 24 & (a) & Oct. 27 & (a) \\
& 30 & (a) & May 27 & (a) & Aug. 26 & (a) & Nov. 25 & 72.62 \\
Feb. 24 & (a) & June 27 & (a) & Sept.24 & (a) & Dec. 26 & 77.54 \\
Mar. 27 & (a) & & & & & & &
\end{tabular}

57. Elsie Nordstrom.

Water level, in feet above datum of well 38, 1941

\begin{tabular}{lrr|ll|ll|l|l}
\hline Jan. 2 & 45.91 & Mar. 27 & 46.36 & June 27 & 47.26 & Sept.24 & 47.33 \\
& 30 & 446 & Apr. 25 & 45.29 & July 24 & 47.26 & Nov.25 & 56.69 \\
Feb. 24 & 46.25 & May 27 & 44.73 & Aug. 26 & 46.55 & Dec. 26 & 59.72 \\
\hline
\end{tabular}

58. Elsie Nordstrom.

Water level, in feet above datum of well 38, 1941

\begin{tabular}{lrr|ll|lll|ll}
\hline Jan. 2 & 38.74 & Mar. 27 & 40.01 & June 27 & 44.98 & Sept.24 & 39.99 \\
& 30 & 39.11 & Apr. 25 & 43.52 & July 24 & 42.70 & Nov.25 & 44.26 \\
Feb. 24 & 39.87 & May 27 27 & 42.90 & Aug. 26 & 39.36 & Dec. 26 & 47.28 \\
\hline
\end{tabular}

59. Frank Goodner.

Water level, in feet above datum of well 38, 1941

\begin{tabular}{ll|ll|ll|ll}
\hline Jan. 2 & (a) & Apr. 25 & (a) & July 24 & (a) & Oct. 27 & 56.07 \\
30 & (a) & May 27 & (a) & Aug. 26 & 39.57 & Nov. 25 & 51.31 \\
Feb. 24 & a) & June 27 & (a) & Sept.24 & (a) & Dec. 26 & 42.29 \\
Mar. 27 & (a) & & & & &
\end{tabular}

69. Well destroyed, measurements discontinued.

70. John snyder.

Water level, in feet above assumed datum, 1941

\begin{tabular}{lrr|ll|ll|ll}
\hline Jan. 2 & 8.50 & Apr. 24 & 13.65 & July 25 & 11.16 & Oct. 28 & 15.56 \\
30 & 8.24 & May 27 & 11.63 & Aug. 26 & .8 .96 & Iov. 25 & 15.86 \\
Feb. 24 & 10.81 & June 27 & 13.60 & Sept.24 & 9.59 & Dec. 29 & 12.77 \\
Mar. 27 & 10.50 & & & & & & & \\
\hline
\end{tabular}

71. John Snyder.

Water level, in feet above assumed datum, 1941

\begin{tabular}{lrr|ll|ll|ll}
\hline Jan. 2 & 10.19 & Mar. 27 & 12.72 & June 27 & 13.30 & Sept.24 & 10.69 \\
30 & 10.59 & Apr. 24 & 13.00 & July 24 & 10.72 & Nov. 25 & 14.14 \\
Feb. 24 & 9.96 & May 27 & 11.17 & Aug. 27 & 10.14 & & \\
\hline
\end{tabular}

72. 0. A. Milner.

Water level, in feet above assumed datum, 1941

\begin{tabular}{lrr|lll|ll|ll}
\hline Jan. 2 & 12.38 & Apr. 25 & 15.80 & July 25 & 19.05 & Oct. 28 & 20.37 \\
31 & 12.42 & May 27 & 14.91 & Aug. 27 & 13.39 & Nov. 26 & 20.59 \\
Feb. 25 & 14.08 & June 28 & 20.29 & Sept.25 & 18.00 & Dec. 29 & 20.37 \\
Mar. 28 & 14.32 & & & & & & &
\end{tabular}

a Dry.

$5110050-43-4$ 
73.

Water level, in feet above assumed datum, 1941

\begin{tabular}{|c|c|c|c|c|c|c|c|}
\hline Date & $\begin{array}{l}\text { Water } \\
\text { level }\end{array}$ & Date & $\begin{array}{l}\text { Water } \\
\text { level }\end{array}$ & Date & $\begin{array}{l}\text { Water } \\
\text { level }\end{array}$ & Date & $\begin{array}{l}\text { Water } \\
\text { level }\end{array}$ \\
\hline $\begin{array}{lr}\text { Jan. } & 2 \\
& 31 \\
\text { Feb. } & 25 \\
\text { Mar. } 28\end{array}$ & $\begin{array}{l}10.11 \\
10.21 \\
12.12 \\
12.32\end{array}$ & $\begin{array}{ll}\text { Apr. } 25 \\
\text { May } 27 \\
\text { June } 28\end{array}$ & $\begin{array}{l}13.56 \\
13.16 \\
14.48\end{array}$ & $\begin{array}{l}\text { July } 25 \\
\text { Aug. } 27 \\
\text { Sept.25 }\end{array}$ & $\begin{array}{l}13.16 \\
11.22 \\
13.91\end{array}$ & $\begin{array}{l}\text { oct. } 28 \\
\text { Nov. } 26 \\
\text { Dec. } 29\end{array}$ & $\begin{array}{l}18.03 \\
17.51 \\
18.66\end{array}$ \\
\hline
\end{tabular}

74. Fred Miller.

Water level, in feet above assumed datum, 1941

\begin{tabular}{ll|ll|ll|ll}
\hline Jan. 30 & 10.08 & Apr.24 & 13.75 & July 24 & 19.22 & Oct. 27 & 23.71 \\
Feb. 24 & 10.57 & May 26 & 15.68 & Aug. 26 & 18.89 & Nov. 25 & 24.68 \\
Mar. 27 & 11.63 & June 27 & 19.30 & Sept.24 & 19.31 & Dec. 26 & 14.28 \\
\hline
\end{tabular}

75. I. W. Runyon.

Water level, in feet above assumed datum, 1941

\begin{tabular}{ll|ll|ll|ll}
\hline Jan. 30 & 11.97 & Apr. 24 & 21.33 & July 24 & 19.86 & Oct.27 & 31.84 \\
Feb. 24 & 13.38 & May 26 & 17.44 & Aug. 26 & 10.95 & Nov. 25 & 29.43 \\
Mar. 27 & 13.08 & June 27 & 25.64 & Sept.24 & 13.33 & Dec. 26 & 17.11 \\
\hline
\end{tabular}

76. Metropolitan Life Insurance Co.

Water level, in feet above assumed datum, 1941

\begin{tabular}{lll|ll|ll|ll}
\hline Jan. 30 & 10.44 & Apr. 24 & 12.41 & July 24 & 11.74 & Oct. 27 & 13.18 \\
Feb. 24 & 11.11 & May 26 & 12.18 & Aug. 26 & 10.58 & Nov. 25 & 15.61 \\
Mar. 27 & 11.25 & June 27 & 12.70 & Sept.24 & 12.23 & Dec. 26 & 16.18 \\
\hline
\end{tabular}

77. Well destroyed, measurements discontinued.

78. Mainquist.

Water level, in feet above assumed datum, 1941

\begin{tabular}{ll|ll|l|ll|ll}
\hline Jan. 2 & 10.08 & Apr. 25 & 11.77 & July 25 & 12.00 & 0ct. 28 & 14.15 \\
31 & 10.13 & May 27 & 10.73 & Aug.27 & 10.26 & Nov. 26 & 14.26 \\
Feb. 25 & 10.48 & June 28 & 12.72 & Sept.25 & 11.29 & Dec. 29 & 14.34 \\
Mar. 28 & 10.17 & & & & & & & \\
\hline
\end{tabular}

79.

Water level, in feet above assumed datum, 1941

\begin{tabular}{lll|ll|ll|ll}
\hline Jan. & 2 & 10.51 & Apr. 25 & 17.53 & July 25 & 14.71 & 0ct. 28 & 23.85 \\
31 & 10.76 & May 27 & 14.21 & Aug. 27 & 11.57 & Nov. 26 & 24.17 \\
Feb. 25 & 11.87 & June 28 & 19.70 & Sept.25 & 23.03 & Dec. 29 & 25.11 \\
Mar. 28 & 13.75 & & & & & & & \\
\hline
\end{tabular}

80. Burton.

Water level, in feet above assumed datum, 1941

\begin{tabular}{rr|ll|l|ll|ll}
\hline Jan. 2 & 11.53 & Apr. 24 & 15.95 & July 24 & 16.24 & Oct. 28 & 16.64 \\
31 & 11.93 & May 27 & 16.53 & Aug.27 & 15.22 & Nov.26 & 15.42 \\
Feb. 24 & 14.59 & June 28 & 17.83 & Sept.25 & 18.39 & Dec. 29 & 15.09 \\
Mar. 28 & 14.87 & & & & & & & \\
\hline
\end{tabular}

81. L. G. Bergen.

Water level, in feet above assumed datum, 1941

\begin{tabular}{ll|l|l|lll|ll}
\hline Jan. 2 & 10.56 & Apr. 25 & 12.68 & July 25 & 11.48 & 0ct. 28 & 15.45 \\
31 & 10.68 & May 27 & 11.18 & Aug. 27 & 10.52 & Nov. 26 & 15.62 \\
Feb. 25 & 11.11 & June 28 & 12.93 & Sept.25 & 12.20 & Dec. 29 & 15.90 \\
Mar. 28 & 11.13 & & & & & & & \\
\hline
\end{tabular}


82.

Water level, in feet above assumed datum, 1941

\begin{tabular}{|c|c|c|c|c|c|c|c|}
\hline Date & $\begin{array}{l}\text { Water } \\
\text { level }\end{array}$ & Date & $\begin{array}{l}\text { Water } \\
\text { level }\end{array}$ & Date & $\begin{array}{l}\text { Water } \\
\text { level }\end{array}$ & Date & $\begin{array}{l}\text { Water } \\
\text { level }\end{array}$ \\
\hline $\begin{array}{lr}\text { Jan. } & 2 \\
& 31 \\
\text { Feb. } 25 \\
\text { Mar. } 28\end{array}$ & $\begin{array}{l}13.51 \\
13.77 \\
13.72 \\
15.61\end{array}$ & $\begin{array}{ll}\text { Apr. } & 25 \\
\text { May } & 27 \\
\text { June } & 28\end{array}$ & $\begin{array}{l}18.66 \\
17.87 \\
20.71\end{array}$ & $\begin{array}{l}\text { July } 25 \\
\text { Aug. } 27 \\
\text { Sept.25 }\end{array}$ & $\begin{array}{l}18.35 \\
14.27 \\
18.47\end{array}$ & $\begin{array}{ll}\text { Oct. } 28 \\
\text { Nov. } 26 \\
\text { Dec. } 29\end{array}$ & $\begin{array}{l}23.99 \\
21.43 \\
24.12\end{array}$ \\
\hline
\end{tabular}

83. Elsie Nordstrom.

Water level, in feet above assumed datum, 1941

\begin{tabular}{lrr|ll|ll|ll}
\hline Jan. 2 & 70.09 & Apr. 25 & 70.21 & July 24 & 71.81 & Oct. 27 & 78.75 \\
30 & 70.16 & May 27 & 70.03 & Aug. 26 & 71.81 & Nov.25 & 80.97 \\
Feb. 24 & 70.14 & June 27 & 71.97 & Sept.24 & 72.24 & Dec. 29 & 82.18 \\
\hline
\end{tabular}

84. Elsie Nordstrom.

Water level, in feet above datum of well 83, 1941

\begin{tabular}{lrr|ll|ll|ll}
\hline Jan. 2 & 69.66 & Apr. 25 & 70.06 & July 24 & 71.68 & Oct. 27 & 73.80 \\
30 & 69.69 & May 27 & 69.74 & Aug. 26 & 70.67 & Nov. 25 & 73.03 \\
Feb. 24 & 70.25 & June 27 & 72.16 & Sept.24 & 72.30 & Dec. 29 & 73.00 \\
Mar. 27 & 70.55 & & & & & & & \\
\hline
\end{tabular}

85. Elsie Nordstrom.

Water level, in feet above datum of well 83, 1941

\begin{tabular}{lr|ll|ll|lll}
\hline Jan. 2 & 70.24 & Apr. 25 & 69.93 & July 24 & 71.47 & Oct. 27 & 73.15 \\
30 & 69.31 & May 27 & 69.59 & Aug. 26 & 70.67 & Nov. 25 & 74.27 \\
Feb. 24 & 70.19 & June 27 & 72.37 & Sept.24 & 72.07 & Dec. 29 & 76.15 \\
Mar. 27 & 70.35 & & & & & & &
\end{tabular}

86. Elsie Nordstrom.

Water level, in feet above datum of well 83, 1941

\begin{tabular}{lr|ll|l|ll|ll}
\hline Jan. 2 & 69.09 & Apr. 25 & 69.85 & July 24 & 71.41 & Oct. 27 & 77.68 \\
30 & 69.18 & May 27 & 69.46 & Aug. 26 & 70.86 & Nov. 25 & 73.33 \\
Feb. 24 & 70.57 & June 27 & 72.59 & Sept.24 & 72.21 & Dec. 29 & 76.44 \\
Mar. 27 & 70.71 & & & & & & & \\
\hline
\end{tabular}

87. Elsie Nordstrom.

Water level, in feet above datum of well 83, 1941

\begin{tabular}{lrr|ll|ll|ll}
\hline Jan. 2 & 70.31 & Apr. 25 & 71.37 & July 24 & 73.00 & Oct. 27 & 80.68 \\
30 & 70.35 & May 27 & 70.83 & Aug. 26 & 72.43 & Nov. 25 & 75.47 \\
Feb. 24 & 71.85 & June 27 & 74.72 & Sept.24 & 73.40 & Dec. 29 & 79.56 \\
Mar. 27 & 72.05 & & & & & & & \\
\hline
\end{tabular}




\section{KANSAS}

\section{INTRODUCTION}

By s. w. Lohman

The Kansas section of the 1940 water-level report (Water-Supply Paper 908) contained records for 21 counties. The present section contains records for 25 counties and includes for the first time records for ElI1s, Grant, Haskell, and Russell Counties. Observation well programs in each of the 25 countles are being conducted by the State Geological Survey of Kansas and the Geological Survey, United States Department of the Interior, in cooperation with the Division of Water Resources of the Kansas State Board of Agriculture and the Division of Sanitation of the Kansas state Board of Health. The program in Jewell county is being carried on in cooperation also with the Soll Conservation Service, United States Department of Agriculture; and the programs in Harvey, McPherson, and Sedgwick Counties are being carried on in cooperation also with the city of wichita.

At the beginning of 1941 perlodic water-level measurements were being made in 437 observation wells. (Erroneously given as 486 wells at end of 1940 in Water-Supply Paper 908.) During the year 30 wells were discontinuad and perlodic water-level measurements were begun or resumed in 60 other wells. The descriptions of all wells in which measurements of water level were made for the first time during 1941 are given in this report under the county in which they are situated. The descriptions of the other wells were given in the reports for previous jears. (Water-supply Papers 777, 817, $840,845,886$, and 908). At the end of 1941 perlodic water-level measurements were being made in 467 wells, including 7 wells observed quarterlj. 339 wells observed monthly, 110 wells observed weekly, and 11 wells equipped with automatic water-stage recorders. A total of 7,246 wettedtape water-level measurements was made during the year. These data are classifled by counties in the following table. 
Names of observers, changes in number of wells observed for all observation wells in Kansas, classifled by counties, during 1941

\begin{tabular}{|c|c|c|c|c|c|}
\hline \multirow[b]{2}{*}{ County } & \multirow[b]{2}{*}{$\begin{array}{c}\text { Name } \\
\text { of } \\
\text { observer }\end{array}$} & \multicolumn{4}{|c|}{ Number of observation wells } \\
\hline & & $\begin{array}{l}\text { Measured } \\
\text { at be- } \\
\text { ginning } \\
\text { of } 1941\end{array}$ & $\begin{array}{l}\text { Discon- } \\
\text { tinued } \\
\text { during } \\
1941\end{array}$ & $\begin{array}{l}\text { Added } \\
\text { during } \\
1941\end{array}$ & $\begin{array}{l}\text { Measured } \\
\text { at end } \\
\text { of } 1941\end{array}$ \\
\hline $\begin{array}{l}\text { Barber } \\
\text { Clark } \\
\text { Comanche } \\
\text { Ellis } \\
\text { Finney } \\
\text { Ford } \\
\text { Grant } \\
\text { Gray } \\
\text { Hamilton } \\
\text { Harvey } \\
\text { Haskell } \\
\text { Hodgeman } \\
\text { Jewell } \\
\text { Kearny } \\
\text { Kiowa } \\
\text { McPherson } \\
\text { Meade } \\
\text { Morton } \\
\text { Ness } \\
\text { Pawnee } \\
\text { Russell } \\
\text { Scott } \\
\text { Sedgwick } \\
\text { Seward } \\
\text { Stanton }\end{array}$ & 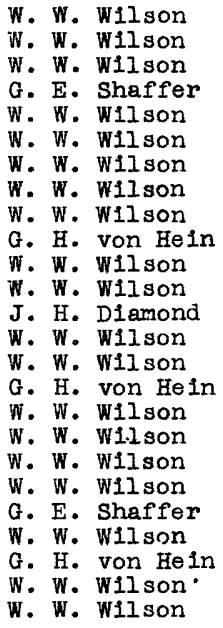 & $\begin{array}{r}12 \\
13 \\
8 \\
0 \\
27 \\
15 \\
0 \\
24 \\
21 \\
118 \\
0 \\
3 \\
38 \\
23 \\
7 \\
10 \\
28 \\
6 \\
2 \\
3 \\
0 \\
32 \\
31 \\
11 \\
5\end{array}$ & $\begin{array}{l}0 \\
3 \\
2 \\
0 \\
2 \\
0 \\
0 \\
2 \\
5 \\
1 \\
0 \\
0 \\
3 \\
4 \\
2 \\
1 \\
1 \\
0 \\
0 \\
0 \\
0 \\
1 \\
0 \\
3 \\
0\end{array}$ & $\begin{array}{r}2 \\
1 \\
0 \\
6 \\
0 \\
0 \\
14 \\
0 \\
0 \\
1 \\
14 \\
0 \\
1 \\
0 \\
1 \\
0 \\
0 \\
0 \\
0 \\
0 \\
19 \\
0 \\
0 \\
0 \\
1\end{array}$ & $\begin{array}{r}14 \\
11 \\
6 \\
6 \\
25 \\
15 \\
14 \\
22 \\
16 \\
118 \\
14 \\
33 \\
36 \\
19 \\
6 \\
9 \\
27 \\
6 \\
2 \\
3 \\
19 \\
31 \\
31 \\
8 \\
6\end{array}$ \\
\hline Total & & 437 & 30 & 60 & 467 \\
\hline
\end{tabular}

Frequency of measurement and number of wetted-tape measurements for all observation wells in Kansas, classifled by counties, during 1941

\begin{tabular}{|c|c|c|c|c|c|}
\hline \multirow[b]{2}{*}{ County } & \multicolumn{5}{|c|}{ Number of observation wells } \\
\hline & $\begin{array}{l}\text { observed } \\
\text { quarterly }\end{array}$ & $\begin{array}{l}\text { Observed } \\
\text { monthly }\end{array}$ & $\begin{array}{c}\text { Observed } \\
\text { weekly }\end{array}$ & $\begin{array}{l}\text { Equipped } \\
\text { with } \\
\text { recorders }\end{array}$ & $\begin{array}{l}\text { Number of } \\
\text { measure- } \\
\text { ments made } \\
\text { in } 1941\end{array}$ \\
\hline Barber & 1 & 13 & 0 & 0 & 129 \\
\hline Clark & 0 & 11 & 0 & 0 & 106 \\
\hline Comanche & 0 & 6 & 0 & 0 & 63 \\
\hline Ellis & 0 & 6 & 0 & 0 & 12 \\
\hline Finney & 0 & 24 & 0 & 1 & 240 \\
\hline Ford & 0 & 15 & 0 & $\overline{0}$ & 161 \\
\hline Grant & 0 & 14 & 0 & 0 & 102 \\
\hline Gray & 0 & 22 & 0 & 0 & 213 \\
\hline Hamil ton & 0 & 16 & 0 & 0 & 153 \\
\hline Harvey & 0 & 13 & 102 & 3 & 3,498 \\
\hline HaskeII & 0 & 14 & 0 & 0 & 88 \\
\hline Hodgeman & 0 & 3 & 0 & 0 & 33 \\
\hline Jewell & 0 & 36 & 0 & 0 & 450 \\
\hline Kearny & 0 & 19 & 0 & 0 & 173 \\
\hline Klowa & 0 & 6 & 0 & 0 & 53 \\
\hline McPherson & 5 & 3 & 1 & 0 & 82 \\
\hline Meade & 1 & 25 & $\overline{0}$ & 1 & 310 \\
\hline Morton & 0 & 6 & 0 & 0 & 68 \\
\hline Ness & 0 & 2 & 0 & 0 & 22 \\
\hline
\end{tabular}


Frequency of measurement and number of wetted-tape measurements for all observation wells in Kansas, classified by counties, during 1941--Continued

\begin{tabular}{lccccc}
\hline & \multicolumn{5}{c}{ Number of observation wells } \\
\cline { 2 - 6 } County & $\begin{array}{l}\text { Observed } \\
\text { quarterly }\end{array}$ & $\begin{array}{c}\text { observed } \\
\text { monthly }\end{array}$ & $\begin{array}{c}\text { observed } \\
\text { weekly }\end{array}$ & $\begin{array}{c}\text { Equipped } \\
\text { with } \\
\text { recorders }\end{array}$ & $\begin{array}{c}\text { Number of } \\
\text { measure- } \\
\text { ments made } \\
\text { in }\end{array}$ \\
\hline Pawnee & 0 & 3 & 0 & 0 & 321 \\
Russell & 0 & 19 & 0 & 0 & 76 \\
Scott & 0 & 28 & 0 & 3 & 288 \\
Sedgw1ck & 0 & 21 & 7 & 3 & 741 \\
Seward & 0 & 8 & 0 & 0 & 65 \\
Stanton & 0 & 6 & 0 & 11 & 7,246 \\
\hline Total & 7 & 339 & 110 & 1 & 0 \\
\hline
\end{tabular}

During 1941 cooperative ground-water reports on the following areas of Kansas were published by the State Geological Survey of Kansas: Stanton County, Atchison County, Lawrence and vicinity,,$\frac{3}{2}$ and southeastern Kansas. In addition an article explaining water-level trends in scott County 5 was published. During 1941 ground-water investigations were made In the following areas of Kansas by the authors named: Grant and Haskell Counties by Thad G. McLaughlin; Klowa County, by Bruce F. Latta; Barber County, by Frank Byrne; Jewell County, by V. C. Fishel and H. A. Waite; and Russell and Ellis Counties, by J. C. Frye and J. J. Brazll.

The following table summarizes the trends in ground-water levels and in precipitation during 1941 in the 21 counties of Kansas in which waterlevel records are avallable for a full year or longer. Detalled information on the highest and lowest water levels of record, the net changes in water level in 1941, and the net changes in water level during the entire period of record is given in tables that begin each of the 21 county chapters. No such information is included for wells in the 4 counties in which waterlevel measurements were begun in 1941.

I/ Latta, Bruce F., Geology"and ground-water resources of stanton Count $\bar{y}$, Kansas: Kansas Geol. Survey Bull. 37, 119 pp., 9 pls., 6 flgs., 1941 .

2/ Frye, John C., Reconnaissance of ground-water resources in Atchison County, Kansas: Kansás Geol. Survey Bull. 38, pt. 9, pp. 237-260, pls. 1-3, flgs. $1-6,1941$.

3/ Lohman, Stanley W., Ground-water conditions in the vicinity of Lawrence, Kansas: Kansas Geol. Survey Bull. 38, part 2, pp. 17-64, pls. 1, 2, flgs., 1-6, 1941 .

4 Abernathy, G. E., Ground-water resources of Mississippian and older rocks in Bourbon, Crawford, Cherokee, and Labette Counties, southeastern Kansas: Kansas Geol. Survey Bull. 38, part 8, pp. 221-236, fig. 1, 1941. 5 Waite, H. A., Factors producing a nine-year decline in ground-water levels in Scott County, Kansas: Am. Geophysical Union Trans. 1941, pp. 772775, flgs. 1, 2, 1941 . 
Relation between the percentage of wells in which highest water levels of record were recorded in 1941, the percentage of wells in which there was a net rise in water level during 1941, and the precipitation during 1941, classified by counties

\begin{tabular}{|c|c|c|c|c|c|c|}
\hline County & $\begin{array}{l}\text { Number } \\
\text { of wells } \\
\text { observed } \\
\text { entire } \\
\text { year }\end{array}$ & $\begin{array}{l}\text { Percentage } \\
\text { of wells } \\
\text { in which } \\
\text { highest } \\
\text { water } \\
\text { levels of } \\
\text { record were } \\
\text { reached }\end{array}$ & $\begin{array}{l}\text { Percent- } \\
\text { age of } \\
\text { welis } \\
\text { having a } \\
\text { net rise } \\
\text { in water } \\
\text { level }\end{array}$ & $\begin{array}{l}\text { Precipit } \\
\text { nearest } \\
\text { Bureau s } \\
\text { Percent- } \\
\text { age of } \\
\text { normal }\end{array}$ & $\begin{array}{l}\text { tation at } \\
\text { Weather } \\
\text { station } \\
\text { station }\end{array}$ & Remarks \\
\hline $\begin{array}{l}\text { Barber } \\
\text { Clark } \\
\text { Comanche }\end{array}$ & $\begin{array}{r}14 \\
10 \\
6\end{array}$ & $\begin{array}{r}100 \\
90 \\
67\end{array}$ & $\begin{array}{r}100 \\
90 \\
67\end{array}$ & $\begin{array}{l}142 \\
145 \\
125\end{array}$ & $\begin{array}{l}\text { Medicine Lodge } \\
\text { Ashland } \\
\text { Coldwater }\end{array}$ & \\
\hline Ellis & $\cdots$ & $\cdots$ & $\therefore \therefore$ & 119 & Hays & $\begin{array}{l}\text { Record begun } \\
\text { in } 1941 \text {. }\end{array}$ \\
\hline $\begin{array}{l}\text { Finney } \\
\text { Ford }\end{array}$ & $\begin{array}{l}25 \\
15\end{array}$ & $\begin{array}{l}56 \\
87\end{array}$ & $\begin{array}{l}76 \\
93\end{array}$ & $\begin{array}{l}132 \\
147\end{array}$ & $\begin{array}{l}\text { Garden city } \\
\text { Dodge city }\end{array}$ & $\begin{array}{l}\text { Some wells } \\
\text { pumped for } \\
\text { irrigation. }\end{array}$ \\
\hline Grant & $\cdots$ & $\cdots$ & $\cdots$ & 162 & Ulysses & $\begin{array}{l}\text { Record begun } \\
\text { in } 1841 \text {. }\end{array}$ \\
\hline $\begin{array}{l}\text { Gray } \\
\text { Hamilton }\end{array}$ & $\begin{array}{l}22 \\
16\end{array}$ & $\begin{array}{l}68 \\
69\end{array}$ & $\begin{array}{l}64 \\
62\end{array}$ & $\begin{array}{l}127 \\
149\end{array}$ & $\begin{array}{l}\text { Cimarron } \\
\text { Syracuse }\end{array}$ & \\
\hline Harvey & 25 & 92 & 100 & 123 & Newton & $\begin{array}{l}94 \text { wells pumpe } \\
\text { or affected by } \\
\text { pumping not } \\
\text { included. }\end{array}$ \\
\hline Haske11 & -. & $\cdots$ & $\cdots$ & 129 & sublette & $\begin{array}{l}\text { Record begun } \\
\text { in } 1941 \text {. }\end{array}$ \\
\hline Hodgeman & 3 & 100 & 100 & 119 & Jetmore & $\begin{array}{l}\text { All wells } \\
\text { pumped for } \\
\text { irrigation. }\end{array}$ \\
\hline Jewell & 34 & 59 & 97 & 143 & Burr Oak (Near) & \\
\hline Kearny & 19 & 42 & 47 & 176 & Lakin (riear) & $\begin{array}{l}\text { Some wells } \\
\text { pumped or } \\
\text { affected by } \\
\text { pumping. }\end{array}$ \\
\hline $\begin{array}{l}\text { Klowa } \\
\text { McPherson } \\
\text { Meade } \\
\text { Morton }\end{array}$ & $\begin{array}{r}5 \\
9 \\
26 \\
6\end{array}$ & $\begin{array}{l}60 \\
56 \\
42 \\
83\end{array}$ & $\begin{array}{r}60 \\
67 \\
65 \\
100\end{array}$ & $\begin{array}{l}117 \\
115 \\
154 \\
173\end{array}$ & $\begin{array}{l}\text { Greensburg } \\
\text { McPherson } \\
\text { Plains } \\
\text { Elkhart }\end{array}$ & \\
\hline Ness & 2 & 100 & 50 & 122 & Ness Gity & $\begin{array}{l}\text { All wells } \\
\text { pumped for } \\
\text { irrigation. }\end{array}$ \\
\hline Pawnee & 3 & 100 & 100 & 143 & Lamed & $\begin{array}{l}\text { All wells } \\
\text { pumped for } \\
\text { Irrigation. }\end{array}$ \\
\hline RusseII & $\cdots$ & $\cdots$ & $\cdots$ & 140 & Fussell & $\begin{array}{l}\text { Record begun } \\
\text { in } 1941 \text {. }\end{array}$ \\
\hline Scott & 31 & 16 & 29 & 124 & scott city & $\begin{array}{l}\text { Half of wells } \\
\text { pumped or } \\
\text { affected by } \\
\text { pumping. }\end{array}$ \\
\hline $\begin{array}{l}\text { Sedgwick } \\
\text { Seward }\end{array}$ & $\frac{31}{8}$ & $\begin{array}{r}77 \\
100\end{array}$ & $\begin{array}{r}100 \\
38\end{array}$ & $\begin{array}{l}110 \\
143\end{array}$ & $\begin{array}{l}\text { Wichita } \\
\text { Liberal }\end{array}$ & $\begin{array}{l}\text { Most wel1s } \\
\text { were pumped. }\end{array}$ \\
\hline Stanton & 6 & 67 & 67 & 165 & Johnson & $\begin{array}{l}\text { One well } \\
\text { pumped. }\end{array}$ \\
\hline
\end{tabular}


As indicated in the above table, the precipitation during 1941 was substantially above normal in each of the 25 counties, and ranged from 110 percent of normal in Sedgwick county to 176 percent of normal in Kearny County. In response to the unusually abundant preclpitation the water levels in most of the wells reached in 1941 the highest stages during their periods of record, and in most of the wells there was an appreciable net rise in water level during the year. In 18 of the 21 counties for which records of a year or more are available, there was a net rise in water level during the year in 60 to 100 percent of the wells. In the three counties in which the percentage of wells showing net rises was only 50 percent or less, most of the observation wells were pumped or were affected noticeably by nearby pumping duping the year. Water-level trends in these wells, therefore, reflect the extent of nearby pumping rather than the amount of recharge.

BARBER COUNTY

By C. C. W11liams

Highest and lowest water levels, in feet below measuring point,

\begin{tabular}{|c|c|c|c|c|c|}
\hline Well & $\begin{array}{l}\text { Highest } \\
\text { recorded } \\
\text { water level } \\
\text { (feet) }\end{array}$ & & Date & $\begin{array}{l}\text { Lowest } \\
\text { recorded } \\
\text { water level } \\
\text { (feet) }\end{array}$ & Date \\
\hline $\begin{array}{l}1 \\
2 \\
3 \\
4 \\
5 \\
6 \\
7 \\
8 \\
9 \\
10 \\
11 \\
12 \\
13 \\
15 \\
\end{array}$ & $\begin{array}{r}77.64 \\
13.00 \\
12.34 \\
16.45 \\
25.29 \\
38.05 \\
19.43 \\
9.37 \\
5.47 \\
103.90 \\
46.62 \\
5.95 \\
11.33 \\
39.85\end{array}$ & $\begin{array}{l}\text { Dec. } \\
\text { May } \\
\text { Dec. } \\
\text { Nov. } \\
\text { Nov. } \\
\text { Nov. } \\
\text { July } \\
\text { Nov. } \\
\text { May } \\
\text { Nov. } \\
\text { Nov. } \\
\text { Nov. } \\
\text { Nov. } \\
\text { Aug. }\end{array}$ & $\begin{array}{r}22,1841 \\
8,1941 \\
22,1941 \\
20,1941 \\
20,1941 \\
20,1941 \\
14,1941 \\
21,1941 \\
8,1941 \\
21,1941 \\
21,1941 \\
20,1941 \\
20,1941 \\
14,1941\end{array}$ & $\begin{array}{r}83.99 \\
14.22 \\
18.42 \\
18.19 \\
31.65 \\
40.06 \\
20.32 \\
17.98 \\
7.26 \\
104.35 \\
48.72 \\
13.18 \\
18.49 \\
40.88\end{array}$ & $\begin{array}{l}\text { Oct. } 17,1940 \\
\text { Sept. } 24,1941 \\
\text { Oct. } 21,1940 \\
\text { Nov. } 26,1940 \\
\text { Sept.24, } 1941 \\
\text { Mar. } 22,1941 \\
\text { Oct. 21, } 1940 \\
\text { Mar. 21, } 1941 \\
\text { Oct. 22, } 1940 \\
\text { Oct. 22, } 1940 \\
\text { Oct. } 22,1940 \\
\text { Oct. 22, } 1940 \\
\text { Oct. } 22,1940 \\
\text { AuE. } 9,1941\end{array}$ \\
\hline
\end{tabular}


Barber County--Continued

Net changes in water level in 1941, and net changes in water level for the period of record in 14 wells in Barber County

\begin{tabular}{|c|c|c|c|c|}
\hline Well & $\begin{array}{l}\text { Length } \\
\text { of record } \\
\text { (years) }\end{array}$ & $\begin{array}{l}\text { Difference } \\
\text { between highest } \\
\text { and lowest } \\
\text { water levels } \\
(\text { feet) }\end{array}$ & $\begin{array}{l}\text { Net rise (t) } \\
\text { or net decline } \\
(-) \text { in } 1941 \\
(\text { feet) }\end{array}$ & $\begin{array}{l}\text { Net rise ( }+) \\
\text { or net decline } \\
\text { (-) for perlod } \\
\text { of record } \\
\text { (feet) }\end{array}$ \\
\hline $\begin{array}{r}1 \\
2 \\
3 \\
4 \\
5 \\
6 \\
7 \\
8 \\
9 \\
10 \\
11 \\
12 \\
13 \\
15\end{array}$ & $\begin{array}{l}1 \\
1 \\
1 \\
1 \\
1 \\
1 \\
1 \\
1 \\
1 \\
1 \\
1 \\
1 \\
1 \\
1\end{array}$ & $\begin{array}{l}6.35 \\
1.22 \\
6.08 \\
2.54 \\
6.06 \\
2.01 \\
.89 \\
8.61 \\
1.79 \\
.45 \\
2.10 \\
7.23 \\
7.16 \\
1.03\end{array}$ & $\begin{array}{r}+0.99 \\
+.05 \\
+2.73 \\
+1.05 \\
+4.86 \\
+2.01 \\
+.73 \\
+8.06 \\
+.09 \\
+.19 \\
+1.38 \\
+7.03 \\
+6.05 \\
+.02\end{array}$ & $\begin{array}{r}+6.35 \\
+.65 \\
+6.08 \\
+1.33 \\
+4.38 \\
+1.67 \\
+.73 \\
+7.93 \\
+1.37 \\
+.43 \\
+1.94 \\
+7.04 \\
+6.78 \\
\text { (a) }\end{array}$ \\
\hline
\end{tabular}

Well descriptions and water-level measurements

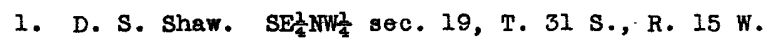

Water level, in feet below measuring point, 1941

\begin{tabular}{|c|c|c|c|c|c|c|c|}
\hline Date & $\begin{array}{l}\text { Water } \\
\text { level }\end{array}$ & Date & $\begin{array}{l}\text { Water } \\
\text { level }\end{array}$ & Date & $\begin{array}{l}\text { Water } \\
\text { level }\end{array}$ & Date & $\begin{array}{l}\text { Water } \\
\text { level }\end{array}$ \\
\hline $\begin{array}{lr}\text { Jan. } & 23 \\
\text { Mar, } & 22 \\
\text { May } & 8\end{array}$ & $\begin{array}{l}78.63 \\
78.63 \\
78.71\end{array}$ & $\begin{array}{ll}\text { June } & 21 \\
\text { July } & 14\end{array}$ & $\begin{array}{l}78.80 \\
78.88\end{array}$ & $\begin{array}{l}\text { Aug. } 9 \\
\text { Sept.24 }\end{array}$ & $\begin{array}{l}78.76 \\
78.55\end{array}$ & $\begin{array}{l}\text { Nov. } 20 \\
\text { Dec. } 22\end{array}$ & $\begin{array}{l}78.96 \\
77.64\end{array}$ \\
\hline
\end{tabular}

2. Russe11 Lake. SW SW $_{\frac{1}{4}}^{\frac{1}{4}}$ sec. 14, T. 31 S., R. 14 W.

Water level, in feet below measuring point, 1941

\begin{tabular}{|rr|lr|rrr|rr}
\hline Jan. 23 & 13.30 & June 21 & 13.02 & Aug. 9 & 13.61 & Nov. 20 & 13.10 \\
Mar. 22 & 13.32 & July 14 & 13.35 & Sept.24 & 14.22 & Dec. 22 & 13.25 \\
May 8 & 13.00 & & & & & & & \\
\hline
\end{tabular}

3. Mr. Griever. $\operatorname{sW}_{\frac{1}{4}}^{\frac{1}{4}} \mathrm{NE}_{\frac{1}{4}}^{\frac{1}{4}}$ sec. 12, T. $32 \mathrm{~S} ., \mathrm{R} .12 \mathrm{~W}$.

Water level, in feet below measuring point, 1941

\begin{tabular}{|rr|lr|lrr|rr}
\hline Jan. 23 & 15.07 & June 21 & 14.44 & Sept.24 & 16.48 & Nov. 20 & 12.50 \\
Mar. 22 & 13.90 & July 14 & 15.18 & Oct.22 & 15.21 & Dec. 22 & 12.34 \\
May 8 & 13.65 & Aug. 9 & 16.11 & & & & & \\
\hline
\end{tabular}

4. Madge Evans. SW $\frac{1}{4} \mathrm{SE}_{\frac{1}{4}}^{\frac{1}{3}}$ sec. $4, \mathrm{~T}, 32 \mathrm{~S} ., \mathrm{R} .12 \mathrm{~W}$.

Water level, in feet below measuring point, 1941

\begin{tabular}{lr|ll|l|ll|ll}
\hline Jan. 23 & 17.82 & June 21 & 17.17 & Aug. 9 & 17.68 & Nov. 20 & 16.45 \\
Mar. 22 & 17.60 & July 14 & 17.45 & Sept.24 & 17.59 & Dec. 22 & 16.77 \\
May 8 & 17.35 & & & & & & & \\
\hline
\end{tabular}

5. R. Kenney. $\operatorname{NE}_{\frac{1}{4}} N W_{\frac{1}{4}}^{\frac{1}{4}}$ sec. 1, T. 33 S., R. 12 W.

Water level, in feet below measuring point, 1941

\begin{tabular}{|rr|lr|lr|ll}
\hline Jan. 23 & 30.83 & June 21 & 30.85 & Aug. 9 & 31.28 & Nov. 20 & 25.29 \\
Mar. 22 & 31.00 & July 14 & 30.83 & Sept.24 & 31.65 & Dec. 22 & 25.97 \\
May 8 & 30.83 & & & & & & \\
\hline
\end{tabular}

a Record begun in 1941 . 
Barber County--Continued

6. F. H. BogEs and Ben Barthlow. NW $W_{1}$ NW sec. 4, T. 33 S., R. 12 W. Water level, in feet below measuring point, 1941

\begin{tabular}{lr|ll|lr}
\hline Date & $\begin{array}{r}\text { Water } \\
\text { level }\end{array}$ & Date & $\begin{array}{l}\text { Water } \\
\text { level }\end{array}$ & Date & $\begin{array}{r}\text { Water } \\
\text { level }\end{array}$ \\
\hline Mar. 22 & 40.06 & June 21 & 39.96 & Aug. 9 & 39.60 \\
May 8 & 40.00 & July 14 & 39.45 & Nov. 20 & 38.05 \\
\hline
\end{tabular}

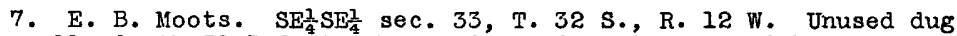
stock well, depth 32.5 feet. Measuring point, top of brick and concrete curb, west side, 2.5 feet above land surface. No pump in well.

Water level, in feet below measuring point, 1940-41

\begin{tabular}{ll|lll|ll}
\hline Oct. 21, 1940 & 20.32 & June 21, 1941 & 19.61 & Sept.24, 1941 & 20.29 \\
Jan. 23, 1941 & 20.08 & July 14 & 19.43 & Nov. 20 & 19.87 \\
Mar. 22 & 19.73 & Aug. 9 & & 19.76 & Dec. 22 & 19.59 \\
May 8 & 19.54 & & & & & \\
\hline
\end{tabular}

8. P. Brock. SE $\frac{1}{4} N E \frac{1}{2}$ sec. 17, T. 34 S., R. 15 W. Water level, in feet below measuring point, 1941

\begin{tabular}{|c|c|c|c|c|c|c|c|}
\hline Date & $\begin{array}{l}\text { Water } \\
\text { level }\end{array}$ & Date & $\begin{array}{l}\text { Water } \\
\text { level }\end{array}$ & Date & $\begin{array}{l}\text { Water } \\
\text { level }\end{array}$ & Date & $\begin{array}{l}\text { Water } \\
\text { level }\end{array}$ \\
\hline $\begin{array}{lr}\text { Jan. } & 22 \\
\text { Mar. } & 21 \\
\text { May } & 8\end{array}$ & $\begin{array}{l}17.74 \\
17.98 \\
16.08\end{array}$ & $\begin{array}{lr}\text { June } & 21 \\
\text { July } & 14 \\
\text { Aug. } & 9\end{array}$ & $\begin{array}{l}13.95 \\
14.34 \\
13.94\end{array}$ & $\begin{array}{l}\text { Sept.24 } \\
\text { Oct. } 21\end{array}$ & $\begin{array}{l}14.72 \\
14.12\end{array}$ & $\begin{array}{l}\text { Nov. } 21 \\
\text { Dec. } 23\end{array}$ & $\begin{array}{l}9.37 \\
9.68\end{array}$ \\
\hline
\end{tabular}

9. V. D. Wells. $\mathrm{SE}_{\frac{1}{4}}^{\frac{1}{4}} \mathrm{SE}_{\frac{1}{4}}^{\frac{1}{4}}$ sec. 18, T. 34 S., R. $15 \mathrm{w}$.

Water level, in feet below measuring point, 1941

\begin{tabular}{lr|ll|lr|ll}
\hline Jan. 22 & 5.98 & June 21 & 6.37 & Aug. 9 & 5.99 & Nov. 21 & 5.71 \\
Mar. 31 & 5.76 & July 14 & 6.88 & Sept.24 & 6.56 & Dec. 23 & 5.89 \\
\hline
\end{tabular}

10. G. H. Davis. NW/ $S W_{\frac{1}{4}}$ sec. 11, T. 35 S., R. 15 W. Water level, in feet below measuring point, 1941

\begin{tabular}{lrr|lr|rr|rr}
\hline Jan. 22 & 104.11 & June 21 & 104.16 & Sept.24 & 104.17 & Nov. 21 & 103.90 \\
Mar. 21 & 104.02 & July 14 & 104.25 & oct. 21 & 103.99 & Dec. 23 & 103.92 \\
May 8 & 103.95 & Aug. 9 & 104.17 & & & & & \\
\hline
\end{tabular}

11. A. Achenbach. $\mathrm{NE}_{\frac{1}{4}}^{\frac{1}{4}} \mathrm{SE}_{\frac{1}{4}}^{\frac{1}{4}}$ sec. 9, T. $35 \mathrm{~S}, \mathrm{R}, 12 \mathrm{~W}$. Water level, in feet below measuring point, 1941

\begin{tabular}{|c|c|c|c|c|c|c|c|c|}
\hline $\begin{array}{l}\text { Jan. } \\
\text { Mar. }\end{array}$ & $\begin{array}{l}22 \\
21\end{array}$ & $\begin{array}{l}48.16 \\
47.65\end{array}$ & $\begin{array}{l}\text { July } 14 \\
\text { Aug. } 9\end{array}$ & $\begin{array}{l}47.80 \\
48.09\end{array}$ & $\begin{array}{l}\text { Sept.24 } \\
\text { oct. } 21\end{array}$ & $\begin{array}{l}48.30 \\
48.06\end{array}$ & $\begin{array}{l}\text { Nov. } 21 \\
\text { Dec. } 23\end{array}$ & $\begin{array}{l}46.62 \\
46.78\end{array}$ \\
\hline & 12 & \multicolumn{7}{|c|}{$\begin{array}{l}\text { B. Mills. SW } \frac{1}{4} S W \frac{1}{4} \text { sec. } 34, T .33 \text { S., R. } 10 \text { W. } \\
\text { Water level, in feet below measuring polnt }\end{array}$} \\
\hline $\begin{array}{l}\text { Jan. } \\
\text { Mar. } \\
\text { May }\end{array}$ & $\begin{array}{r}22 \\
22 \\
8\end{array}$ & $\begin{array}{l}13.17 \\
12.78 \\
11.82\end{array}$ & $\begin{array}{rr}\text { June } & 21 \\
\text { July } & 14 \\
\text { Aug. } & 9\end{array}$ & $\begin{array}{r}7.84 \\
8.58 \\
10.60\end{array}$ & $\begin{array}{l}\text { Sept.24 } \\
\text { Oct. } 22\end{array}$ & $\begin{array}{r}10.96 \\
9.02\end{array}$ & $\begin{array}{l}\text { Nov. } 20 \\
\text { Dec. } 22\end{array}$ & \\
\hline
\end{tabular}

13. J. A. Hrencher. SW $\frac{1}{4} \mathrm{SE} \frac{1}{4}$ sec. 17, T. 32 S., R. 10 W. Water level, in feet below measuring point, 1941

\begin{tabular}{lrr|lr|ll|l|l}
\hline Jan. 22 & 17.76 & June 21 & 15.99 & Sept.24 & 17.07 & Nov. 20 & 11.33 \\
Mar. 22 & 16.94 & July 14 & 17.24 & Oct. 22 & 12.96 & Dec. 22 & 11.71 \\
May 8 & 16.80 & Aug. 9 & 17.87 & & & & & \\
\hline
\end{tabular}

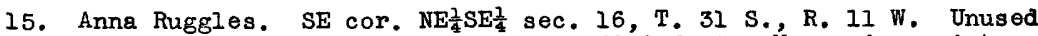
drilled stock well, diameter 4 inches, depth 49.2 feet. Measuring point, top of casing, north side, 1.0 foot above land surface. No pump in well; windmill tower over well.

Water level, in feet below measuring point, 1941

\begin{tabular}{lrr|lr|rr|rr}
\hline Mar. 22 & 39.90 & June 21 & 39.88 & Aug. 9 & 40.88 & Nov. 20 & 39.87 \\
May 8 & 39.90 & JuIy 14 & 39.85 & Sept.24 & 39.87 & Dec. 22 & 39.88 \\
\hline
\end{tabular}


CLARK COUNTY

By J. C. Frye

Highest and lowest water levels, in feet below measuring point, in 10 wells in Clark County, 1941

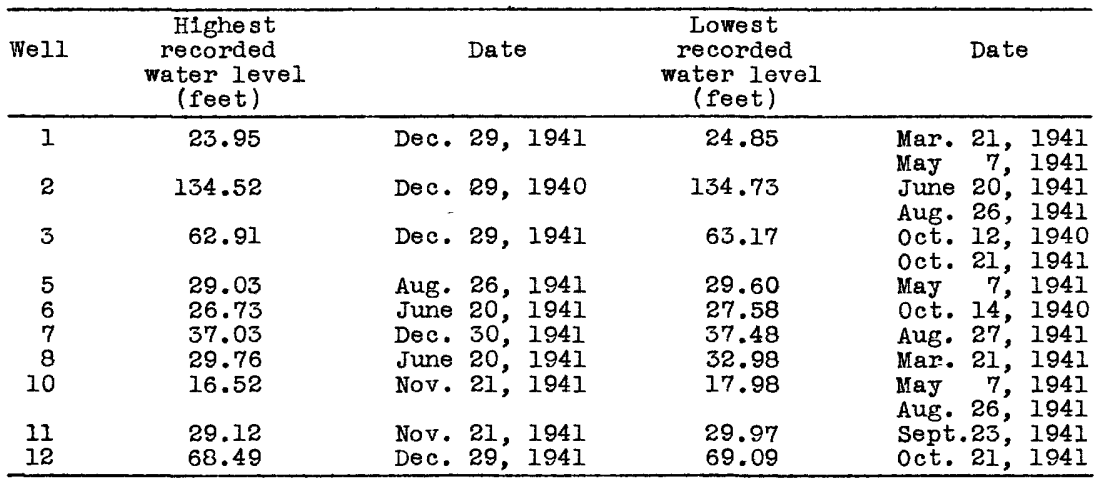

Net changes in water level in 1941, and net changes in water level for the perlod of record in 10 wells in Clark County

\begin{tabular}{rcccc}
\hline Well & $\begin{array}{c}\text { Length } \\
\text { of record } \\
\text { (jears) }\end{array}$ & $\begin{array}{c}\text { Difference } \\
\text { between highest } \\
\text { and lowest } \\
\text { water levels } \\
\text { (feet) }\end{array}$ & $\begin{array}{c}\text { Net rise (t) } \\
\text { or net decline } \\
(-) \text { in } 1941 \\
\text { (feet) }\end{array}$ & $\begin{array}{c}\text { Net rise }(+) \\
\text { or net decline } \\
\text { for period } \\
\text { of record } \\
\text { (feet) }\end{array}$ \\
\hline 1 & 1 & 0.90 & +0.77 & +0.64 \\
2 & 1 & .21 & -.03 & +.10 \\
3 & 1 & .26 & +.13 & +.26 \\
5 & 1 & .57 & +.13 & +.0 \\
6 & 1 & .69 & +.41 & +.80 \\
7 & 1 & .45 & +.18 & +.22 \\
8 & 1 & +1.95 & +1.57 \\
10 & 1 & 1.42 & +1.30 & +1.23 \\
11 & 1 & .35 & +.35 & +.19 \\
12 & 1 & +60 & +.21 & +.21 \\
\hline
\end{tabular}

Well.descriptions and water-level measurements

1. Central Iife Assurance Co. $\mathrm{SE}_{\frac{1}{4}}^{1} \mathrm{NW}_{\frac{1}{4}}$ sec. 17, T. 34 S., R. $25 \mathrm{~W}$.

Water level, in feet below measuring point, 1941

\begin{tabular}{ll|ll|ll|ll}
\hline Date & $\begin{array}{l}\text { Water } \\
\text { level }\end{array}$ & Date & $\begin{array}{l}\text { Water } \\
\text { level }\end{array}$ & Date & $\begin{array}{l}\text { Water } \\
\text { level }\end{array}$ & Date & $\begin{array}{r}\text { Water } \\
\text { level }\end{array}$ \\
\hline Jan. 22 & 24.72 & June 20 & 24,66 & Sept.23 & 24.30 & Nov. 22 & 24.32 \\
Mar. 21 & 24.85 & July 22 & 24.71 & Oct.21 & 24.32 & Dec. 29 & 23.95 \\
May 7 & 24.85 & Aug. 26 & 24.33 & & & & \\
\hline
\end{tabular}

2. George F. Batt. NW $\frac{1}{4} \mathrm{SW} \frac{2}{4}$ sec. 5, T. $30 \mathrm{s.,}$ R. 23 W.

Water level, in feet below measuring point, 1941

\begin{tabular}{lrr|ll|ll|ll}
\hline Jan. 21 & 134.62 & June 20 & 134.73 & Aug. 26 & 134.73 & Nov. 22 & 134.70 \\
Mar. 21 & 134.64 & July 22 & 134.71 & Sept.23 & 134.67 & Dec. 29 & 134.65 \\
\hline May & 7 & 134.69 & & & & & & \\
\hline
\end{tabular}


Clark County--Continued

3. T. L. Blair. SW $\mathrm{NW}_{\frac{1}{2}}^{\frac{1}{2}}$ sec. 17, T. $30 \mathrm{~S}, \mathrm{R}, 24 \mathrm{~W}$.

Water level, in feet below measuring point, 1941

\begin{tabular}{ll|ll|ll|ll}
\hline Date & $\begin{array}{l}\text { Water } \\
\text { level }\end{array}$ & Date & $\begin{array}{l}\text { Water } \\
\text { level }\end{array}$ & Date & $\begin{array}{l}\text { Water } \\
\text { level }\end{array}$ & Date & $\begin{array}{r}\text { Water } \\
\text { level }\end{array}$ \\
\hline Jan. 21 & 63.04 & June 20 & 63.08 & Sept.23 & 63.14 & Nov. 22 & 63.12 \\
Mar. 21 & 63.05 & July 22 & 63.13 & Oct.21 & 63.17 & Dec. 29 & 62.91 \\
May 7 & 63.07 & Aug. 26 & 63.15 & & & & \\
\hline
\end{tabular}

4. N. B. Estes. $N E \frac{1}{4} \mathrm{NE} \frac{1}{4}$ sec. 5, T. 31 S., R. 25 W.

Water level, in feet below measuring point, 1941

\begin{tabular}{ll|ll|ll}
\hline Date & $\begin{array}{l}\text { Water } \\
\text { level }\end{array}$ & Date & $\begin{array}{l}\text { Water } \\
\text { level }\end{array}$ & Date & Water \\
\hline Jan. 21 & 92.99 & May 7 & 93.01 & July 22 & 97.80 \\
Mar. 21 & 92.97 & June 20 & 92.95 & Iov. 22 & a 93.10 \\
\hline
\end{tabular}

5. Winnie Floyd. NWT NW sec. 12, T. 33 S., R. $25 \mathrm{~W}$.

Water level, in feet below measuring point, 1941

\begin{tabular}{lll|ll|ll|ll}
\hline Date & $\begin{array}{l}\text { Water } \\
\text { level }\end{array}$ & Date & $\begin{array}{l}\text { Water } \\
\text { level }\end{array}$ & Date & $\begin{array}{l}\text { Water } \\
\text { level }\end{array}$ & Date & $\begin{array}{r}\text { Water } \\
\text { level }\end{array}$ \\
\hline Mar. 21 & 29.36 & Aug. 26 & 29.03 & Oct. 21 & 29.19 & Dec. 29 & 29.23 \\
May 7 & 29.60 & Sept.23 & 29.27 & Nov.22 & 29.25 & & \\
\hline
\end{tabular}

6. District School. $\mathrm{NE}_{\frac{1}{4}}^{\frac{1}{4} \mathrm{NE} \frac{1}{4}}$ sec. 7, T. 35 S., R. $21 \mathrm{~W}$.

Water level, in feet below measuring point, 1941

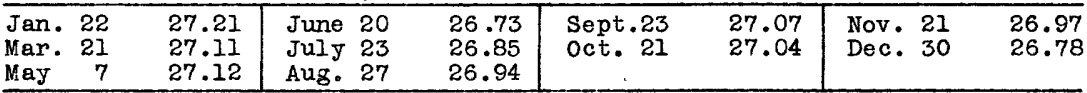

7. M. C. Harper. $S W_{\frac{1}{4}} \mathrm{SE} \frac{1}{4} \mathrm{sec} .32, \mathrm{~T} .33 \mathrm{~S} ., \mathrm{R} .21 \mathrm{~W}$.

Water level, in feet below measuring point, 1941

\begin{tabular}{lrr|ll|ll|ll}
\hline Jan. 22 & 37.21 & June 20 & 37.28 & Sept.23 & 37.37 & Nov. 21 & 37.42 \\
Mar. 21 & 37.28 & July 23 & 37.36 & Oct.21 & 37.39 & Dec. 30 & 37.03 \\
May & 7 & 37.39 & Aug. 27 & 37.48 & & & & \\
\hline
\end{tabular}

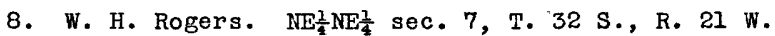

Water level, in feet below measuring point, 1941

\begin{tabular}{lrr|ll|ll|l|l|l}
\hline Jan. 22 & 32.53 & June 20 & 29.76 & Sept.23 & 30.61 & Nov. 21 & 30.62 \\
Mar. 21 & 32.83 & July 23 & 30.60 & Oct. 21 & 30.64 & Dec. 30 & 30.58 \\
May & 7 & 32.98 & Aug. 27 & 30.66 & & & & & \\
\hline
\end{tabular}

9. F. Balley. SW $\frac{1}{4} W_{4}^{1}$ sec. $34, T .30 \mathrm{~S},,^{-} \mathrm{R} .21 \mathrm{~W}$. Water levels, in feet below measuring polnt, 1941: Jan. 22, 13.56; Mar. 21, 13.48. Measurements discontinued after liar. 21 owing to excessive pumping.

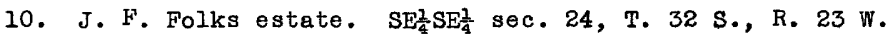
Water level, in feet below measuring point, 1941

\begin{tabular}{|c|c|c|c|c|c|c|c|}
\hline $\begin{array}{l}\text { Jan. } 22 \\
\text { Mar. } 21 \\
\text { May }\end{array}$ & $\begin{array}{l}17.91 \\
17.97 \\
17.98\end{array}$ & $\begin{array}{l}\text { June } 20 \\
\text { July } 23 \\
\text { Aug. } 26\end{array}$ & $\begin{array}{l}17.92 \\
17.75 \\
17.98\end{array}$ & $\begin{array}{l}\text { Sept.23 } \\
\text { Oct. } 21\end{array}$ & $\begin{array}{l}17.57 \\
17.73\end{array}$ & $\begin{array}{l}\text { Nov. } 21 \\
\text { Dec. } 30\end{array}$ & $\begin{array}{l}16.52 \\
16.61\end{array}$ \\
\hline
\end{tabular}

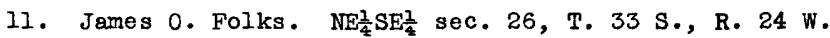

Water level, in feet below measuring point, 1941

\begin{tabular}{lll|ll|ll|ll}
\hline June 20 & 29.85 & Aug. 26 & 29.68 & Oct. 21 & 29.88 & Dec. 29 & 29.26 \\
July 22 & 29.86 & Sept.23 & 29.97 & Nov. 21 & 29.12 & & \\
\hline
\end{tabular}

a Measurements discontinued after Nov. 22. 
Clark County--Continued

12. Ralph Gardner. swa $s w_{4}^{2}$ sec. 11, T. 33 S., R. 24 W.

Water level, in feet below measuring point, 1941

\begin{tabular}{ll|lr|ll|ll}
\hline Date & $\begin{array}{l}\text { Water } \\
\text { level }\end{array}$ & Date & $\begin{array}{l}\text { Water } \\
\text { level }\end{array}$ & Date & $\begin{array}{l}\text { Water } \\
\text { level }\end{array}$ & Date & $\begin{array}{r}\text { Water } \\
\text { level }\end{array}$ \\
\hline Jan. 22 & 68.70 & May 7 & 68.72 & 0ct. 21 & 69.09 & Dec. 29 & 68.49 \\
Mar. 21 & 68.81 & June 20 & 68.75 & Nov. 21 & 68.71 & & \\
\hline
\end{tabular}

13. W. H. Shattock. NE $\frac{1}{4} N E \frac{1}{4}$ sec. 16, T. 31 S., R. 21 W. Unused bored domestic and stock well, diameter 6 inches, depth not known. Measuring point, top of galvanized-iron casing, east side, 0.1 foot above land surface. No pump on well.

Water level, in feet below measuring point, 1941

\begin{tabular}{lrr|ll|ll|ll}
\hline May 7 & 33.39 & July 23 & 33.15 & Sept.23 & 33.19 & Nov. 21 & 32.97 \\
June 20 & 33.06 & Aug. 27 & 33.16 & Oct. 21 & 33.17 & Dec. 30 & 32.94 \\
\hline
\end{tabular}

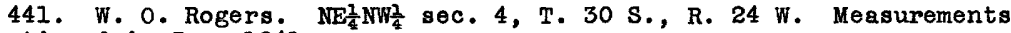
discontinued in Jan. 1941.

COMANCHE COUNTY

By J. C. Frye

Highest and lowest water levels, in feet below measuring point, in 6 wells in Comanche County, 1941

\begin{tabular}{ccccc}
\hline We11 & $\begin{array}{c}\text { Highest } \\
\text { recorded } \\
\text { water level } \\
\text { (feet) }\end{array}$ & Date & $\begin{array}{c}\text { Lowest } \\
\text { recorded } \\
\text { water level } \\
\text { (feet) }\end{array}$ & Date \\
\hline 1 & 40.34 & Dec. 30, 1941 & 41.52 & June 20, 1941 \\
2 & 17.54 & June 21, 1941 & 17.92 & Nov. 20, 1940 \\
3 & 84.67 & Dec. 27, 1940 & 86.70 & June 21, 1941 \\
6 & 79.67 & Dec. 30, 1941 & 80.36 & Nov. 27, 1940 \\
7 & 40.32 & May 7, 1941 & 59.53 & Jan. 22, 1941 \\
9 & 89.10 & Dec. 28, 1940 & 90.59 & Sept.23, 1941 \\
\hline
\end{tabular}

Net changes in water level in 1941, and net changes in water level for the perlod of record in 6 wells in Comanche County

\begin{tabular}{|c|c|c|c|c|}
\hline Well & $\begin{array}{l}\text { Length } \\
\text { of record } \\
\text { (years) }\end{array}$ & $\begin{array}{c}\text { Difference } \\
\text { between highest } \\
\text { and lowest } \\
\text { water levels } \\
\text { (feet) }\end{array}$ & $\begin{array}{l}\text { Net rise (t) } \\
\text { or net decline } \\
(-) \text { in } 1941 \\
\text { (feet) }\end{array}$ & $\begin{array}{l}\text { Net rise (+) } \\
\text { or net decline } \\
(-) \text { for period } \\
\text { of record } \\
\text { (feet) }\end{array}$ \\
\hline $\begin{array}{l}1 \\
2 \\
3 \\
6 \\
7 \\
9\end{array}$ & $\begin{array}{l}1 \\
1 \\
1 \\
1 \\
1 \\
1\end{array}$ & $\begin{array}{r}1.18 \\
.38 \\
2.03 \\
.69 \\
19.21 \\
1.49\end{array}$ & $\begin{array}{r}+0.88 \\
+.12 \\
-1.75 \\
+.57 \\
+12.19 \\
-.65\end{array}$ & $\begin{array}{r}+0.83 \\
+.23 \\
-1.30 \\
+.50 \\
+4.05 \\
-.74\end{array}$ \\
\hline
\end{tabular}


Comanche County--Continued

Water-level measurements

1. A. A. Carpenter. $\mathrm{NE}_{\frac{1}{4}}^{\frac{1}{4}} \mathrm{NE}_{\frac{1}{4}}^{\frac{1}{4}}$ sec. 8, T. 33 S., R. $20 \mathrm{~W}$. Water level, in feet below measuring point, 1941

\begin{tabular}{ll|ll|ll|ll}
\hline Date & $\begin{array}{l}\text { Water } \\
\text { level }\end{array}$ & Date & $\begin{array}{l}\text { Water } \\
\text { level }\end{array}$ & Date & $\begin{array}{l}\text { Water } \\
\text { level }\end{array}$ & Date & $\begin{array}{r}\text { Water } \\
\text { level }\end{array}$ \\
\hline Jan. 22 & 41.22 & June 20 & 41.52 & Sept.23 & 41.35 & Nov.21 & 40.45 \\
Mar. 21 & 41.47 & July 23 & 41.51 & Oct.21 & 41.14 & Dec. 30 & 40.34 \\
May 7 & 41.50 & Aug. 27 & 41.48 & & & & \\
\hline
\end{tabular}

2. Nina Clark. SW $\frac{1}{4} S W \frac{1}{4}$ sec. 27, T. 31 S., R. 30 W.

Water level, in feet below measuring point, 1941

\begin{tabular}{lrr|lc|cc|cr}
\hline May & 8 & 17.76 & July 23 & 17.71 & Sept.23 & 17.68 & Nov. 21 & 17.64 \\
June 21 & 17.54 & Aug. 27 & 17.66 & oct. 21 & 17.67 & & & \\
\hline
\end{tabular}

3. E. Deewall. SW $\frac{1}{4} \mathrm{NE}_{\frac{1}{4}}^{\frac{1}{4}}$ sec. 19, T. 31 S., R. $18 \mathrm{~W}$.

Water level, in feet below measuring point, 1941

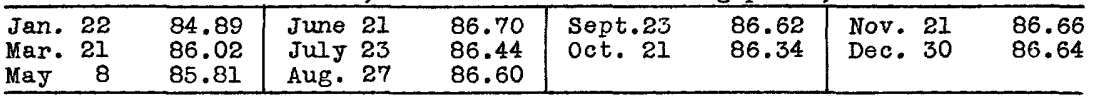

4. E. G. Thorp. SW $\frac{1}{4}$ SW $\frac{1}{4}$ sec. 35, T. 34 S., R. 20 w. Water levels, in feet below measuring point, 1941: Jan. 22, 10.24; Mar. 21, 9.96; May 7 , 9.67; June 20,9.83. Measurements discontinued after June 20 .

6. Christopher Nickolson. NW $\frac{1}{4} N E \frac{1}{4}$ sec. 6, T. 35 S., R. 18 W. Water level, in feet below measuring point, 1941

\begin{tabular}{|c|c|c|c|c|c|c|c|c|}
\hline $\begin{array}{l}\text { Jan. } 22 \\
\text { Mar. } 21 \\
\text { May } 7\end{array}$ & $\begin{array}{l}80.24 \\
80.22 \\
80.18\end{array}$ & $\begin{array}{l}\text { June } 2 \\
\text { July } 2 \\
\text { Aug. }\end{array}$ & $\begin{array}{l}20 \\
23 \\
27\end{array}$ & $\begin{array}{l}80.05 \\
80.16 \\
80.09\end{array}$ & $\begin{array}{l}\text { Sept.23 } \\
\text { oct. } 21\end{array}$ & $\begin{array}{l}80.18 \\
79.86\end{array}$ & $\begin{array}{l}\text { Nov. } 21 \\
\text { Dec. } 30\end{array}$ & $\begin{array}{l}79.92 \\
79.67\end{array}$ \\
\hline
\end{tabular}

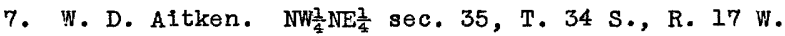

Water level, in feet below measuring point, 1941

\begin{tabular}{|c|c|c|c|c|c|c|c|}
\hline $\begin{array}{l}\text { Jan. } 22 \\
\text { Mar. } 21 \\
\text { May }\end{array}$ & $\begin{array}{l}59.53 \\
58.60 \\
40.32\end{array}$ & $\begin{array}{l}\text { June } 2 \\
\text { July } 2 \\
\text { Aug. } 2\end{array}$ & $\begin{array}{l}50.54 \\
57.52 \\
56.28\end{array}$ & $\begin{array}{l}\text { sept.23 } \\
\text { oct. } 21\end{array}$ & $\begin{array}{l}59.10 \\
51.23\end{array}$ & $\begin{array}{l}\text { Nov. } 2 \\
\text { Dec. } 3\end{array}$ & $\begin{array}{l}55.33 \\
47.34\end{array}$ \\
\hline
\end{tabular}

8. Christopher Beltler. NWT $\mathrm{NE}_{4}^{\frac{1}{4}}$ sec. 34, T. 33 S., R. 17 W. Water levels, in feet below measuring point, 1941: Jan. 22, 34.78; Dec. 30, 41.16. Observations discontinued.

9. H. R. Burnette. NW/ $\mathrm{lJE}_{\frac{1}{4}}^{\frac{1}{4}}$ sec. 13, T. $32 \mathrm{~S} ., \mathrm{R} .17 \mathrm{~W}$. Water level, in feet below measuring point, 1941

\begin{tabular}{lll|ll|ll|ll}
\hline Jan. 22 & 89.23 & June 20 & 89.17 & Sept.23 & 90.59 & Nov. 21 & 89.77 \\
Mar. 21 & 89.21 & July 23 & 89.34 & Oct.21 & 89.48 & Dec. 30 & 89.88 \\
May & 7 & 89.27 & Aug. 27 & 89.28 & & & & \\
\hline
\end{tabular}


ELLIS COUNTY

By J. C. Frye

An Investigation of the ground-water resources of northeastern Ellis County and western and sopthern Russell County, Kansas, was started in 1941 by the Federal Geological Survey and the Division of Sanitation of the Kansas State Board of Health, with the cooperation of the state Geological Survey of Kansas and the Division of Water Resources of the Kansas state Board of Agriculture. The work comprises a field and laboratory study of the Cretaceous sandstones that underlie oil flelds in these counties, with reference to the quantity and quality of the ground water in the sandstones and the suitability of the sandstones as reservoirs for disposition of oilfield brines. The Tertiary and Quaternary deposits of the area were also studied. This work is being done by J. J. Brazil, of the State Board of Health, and the writer, under the supervision of $S$. W. Lohman, Federal geologist in charge of ground-water investigations in Kansas, and ogden $S$. Jones, Geologist of the 0il-Field Waste Disposal Section of the Division of Sanitation of the Kansas State Board of Health. Assistance was rendered during the period August to December 1941 by Gordon Shaffer.

Ellis County is in the central part of the Plains Border section of the Great Plains province. It is drained by the Saline and Smoky Hill Rivers and by Big Creek, a tributary of the Smoky Hill. All of Ellis County is underlain by Cretaceous rocks, although in certain areas they are overlain by Tertiary deposits. Pleistocene terrace deposits occur along the major valleys. Most of the domestic, stock, and municipal supplies in the county are obtained from wells.

At the end of 1941 monthly water-level measurements were being made in six wells in Ellis County by the wetted-tape method. All measurements were made by Gordon Shaffer. A total of 12 individual water-level measurements was made from october to the end of the year. 


\section{Ellis County--Continued}

Well descriptions and water-level measurements

182. Polcyn et al. $\mathrm{NE}_{\frac{1}{4}}^{\frac{1}{4}} \mathrm{NE}_{\frac{1}{4}}^{\frac{1}{2}}$ sec. 10, T. $14 \mathrm{~S} ., \mathrm{R}, 16 \mathrm{~W}$. Unused drilled stock well, dlameter 6 inches, depth 153.7 feet. Water is obtained from a Cretaceous sandstone. Measuring point, top of 6-inch galvanized-iron casing, north side, 0.3 foot below land surface. No pump on well. Water levels, In feet below measuring point, 1941: 0ct. 16, 151.05; Dec. 2, 149.52.

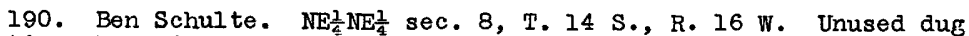
domest1c well, diameter 24 Inches, depth 17.9 feet. Water is obtalned from Tertiary deposits. Measuring point, top edge of casing, west side, 0.2 foot above land surface. Equipped with hand-operated lift pump. Water levels, In feet below measuring point, 1941: 0.t. 16, 14.00; Dec. 2, 14.02.

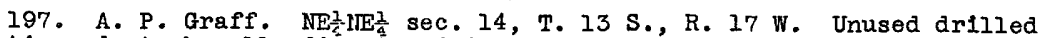
domestic and stock well, diameter 6 inches, depth' 29.6 feet. Water is outained from Codell sandstone. Measuring point, top of galvanized-iron casing, east side, 0.6 foot above land surface. No pump on well. Water levels, in feet bélow measuring point, 1941: 0ct. 17, 26.30; Dec. 2, 26.42.

215. A. H. Romine. NE $\frac{1}{c} N W_{\epsilon}^{1}$ sec. 7, T. 11 S., R. 16 W. Dug stock well, diameter 24 Inches, depth 20 feet. Water is obtained from Plelstocene deposits. Measuring point, top of rock casing, south side, 2.1 feet above land surface. Equipped with lift pump and windmill. Water levels, in feet below measuring point, 1941: Nov. 13, 16.42; Dec. 2, 16.14 .

218. W. W. Bemis. $\mathrm{NE}_{\frac{1}{4}} \mathrm{NE}_{\frac{1}{4}}^{\frac{1}{3}}$ sec. 16, T. $12 \mathrm{~S} ., \mathrm{R} .17 \mathrm{~W}$. Unused dug stock well, diameter 24 inches, depth 82.6 feet. Water is obtained from Codell sandstone. Measuring point, top of rock curbing, south side, 0.3 foot above land surface. Equipped with lift pump and windmill. Water levels, in feet below measuring point, 1941: Nov. 14, 54.24; Dec. 2, 52.10.

225. Ray Smith. NW $\mathrm{NWP}_{\mathrm{a}}$ sec. 30, T. $12 \mathrm{~S} ., \mathrm{R} .17 \mathrm{~W}$. Unused drilled domestic and stock well, dlameter 6 inches, depth 61.0 feet. Water is obtained from Codell sandstone. Measuring point, top of 6-inch galvanizedIron casing, west side, 0.3 foot above land surface. No pump on well. Water levels, in feet below measuring point, 1941: Nov. 15, 54.22; Dec. 2, 54.10 .

FINIEY COUNTY

By B. F. Latta

Highest and lowest water levels, in feet below measuring point, in 25 wells in Finney County, 1941

\begin{tabular}{|c|c|c|c|c|}
\hline Well & $\begin{array}{l}\text { Highest } \\
\text { recorded } \\
\text { water level } \\
\text { (feet) }\end{array}$ & Date & $\begin{array}{l}\text { Lowest } \\
\text { recorded } \\
\text { water level } \\
\text { (feet) }\end{array}$ & Date \\
\hline $\begin{array}{l}1 \\
2 \\
3 \\
4 \\
5\end{array}$ & $\begin{array}{r}11.12 \\
109.31 \\
44.64 \\
34.36 \\
23.12\end{array}$ & $\begin{array}{l}\text { Aug. } 6,1937 \\
\text { Oct. } 11,1941 \\
\text { Mar. 22, } 1940 \\
\text { Aug. 5, } 1941 \\
\text { June 21, } 1941 \\
\text { July 24, } 1940 \\
\text { Dec. 11, } 1941\end{array}$ & $\begin{array}{r}8.14 \\
109.71 \\
61.78 \\
36.53 \\
23.54\end{array}$ & $\begin{array}{l}\text { Mar. } 8,1941 \\
\text { Oct. 26, } 1939 \\
\text { Aug. 21, } 1940 \\
\text { Sept.20, } 1939 \\
\text { Jan. 28, } 1940\end{array}$ \\
\hline $\begin{array}{r}6 \\
7 \\
8 \\
9 \\
10 \\
12 \\
\end{array}$ & $\begin{array}{r}17.25 \\
77.59 \\
75.92 \\
72.86 \\
11.20 \\
107.49 \\
\end{array}$ & $\begin{array}{l}\text { June } 21,1940 \\
\text { Nov. } 1,1941 \\
\text { Sept.20, } 1940 \\
\text { Oct. 28, } 1940 \\
\text { Nov. 2, } 1941 \\
\text { Apr. 24, } 1941\end{array}$ & $\begin{array}{r}19.65 \\
78.22 \\
76.75 \\
73.74 \\
\\
14.73 \\
\text { b } 108.13\end{array}$ & $\begin{array}{l}\text { Apr. 24, } 1941 \\
\text { June 22, } 1940 \\
\text { June 21, } 1940 \\
\text { Aug. 22, } 1940 \\
\text { Sept.20, } 1940 \\
\text { Nov. 3, } 1941\end{array}$ \\
\hline
\end{tabular}


Finney County--Continued

Highest and lowest water levels, in feet below measuring point, in 25 wells in Finney County, 1941--Continued

\begin{tabular}{|c|c|c|c|c|}
\hline We11 & $\begin{array}{c}\text { Highest } \\
\text { recorded } \\
\text { water level } \\
\text { (feet) }\end{array}$ & Date & $\begin{array}{c}\text { Lowest } \\
\text { recorded } \\
\text { water level } \\
\text { (feet) }\end{array}$ & Date \\
\hline $\begin{array}{l}13 \\
14 \\
15 \\
16 \\
17 \\
18 \\
19 \\
20 \\
21 \\
22\end{array}$ & $\begin{array}{r}3.11 \\
46.89 \\
13.62 \\
37.05 \\
5.48 \\
10.32 \\
30.62 \\
67.60 \\
100.27 \\
120.14\end{array}$ & $\begin{array}{l}\text { Dec. } 11,1941 \\
\text { Dec. } 11,1941 \\
\text { Nov. 30, } 1941 \\
\text { Dec. 7, } 1941 \\
\text { Dec. 7, } 1941 \\
\text { Aug. } 5,1941 \\
\text { Oct. 2, } 1939 \\
\text { Jan. 30, } 1941 \\
\text { Jan. 30, } 1941 \\
\text { Sept.28, } 1941 \\
\text { Oct. 2, } 1939\end{array}$ & $\begin{array}{r}5.63 \\
47.65 \\
15.40 \\
43.42 \\
9.81 \\
12.31 \\
32.66 \\
68.99 \\
100.82 \\
120.96\end{array}$ & $\begin{array}{l}\text { Sept.23, } 1939 \\
\text { May 23, } 1940 \\
\text { Sept.20, } 1940 \\
\text { May 19, } 1941 \\
\text { Oct. 26, } 1939 \\
\text { Feb. 17, } 1940 \\
\text { June 3, } 1941 \\
\text { Oct. 2, } 1941 \\
\text { Oct. 2, } 1941 \\
\text { Oct. 2, } 1941\end{array}$ \\
\hline $\begin{array}{l}23 \\
26 \\
27\end{array}$ & $\begin{array}{l}44.98 \\
69.95 \\
76.07\end{array}$ & $\begin{array}{l}\text { July } 24,1940 \\
\text { June } 21,1940 \\
\text { Mar. 22, } 1940 \\
\text { Sept.20, } 1940\end{array}$ & $\begin{array}{l}45.80 \\
71.60 \\
77.19\end{array}$ & $\begin{array}{l}\text { Feb. } 17,1940 \\
\text { Apr. } 24,1941 \\
\text { Aug. } 4,1941\end{array}$ \\
\hline 28 & 36.59 & $\begin{array}{l}\text { Oct. } 28,1940 \\
\text { Dec. } 24,1940\end{array}$ & 37.06 & $\begin{array}{l}\text { Apr. 24, } 1940 \\
\text { May } 23,1940\end{array}$ \\
\hline
\end{tabular}

Net changes in water level in 1941, and net changes in water level for the period of record in 25 wells in Finney County

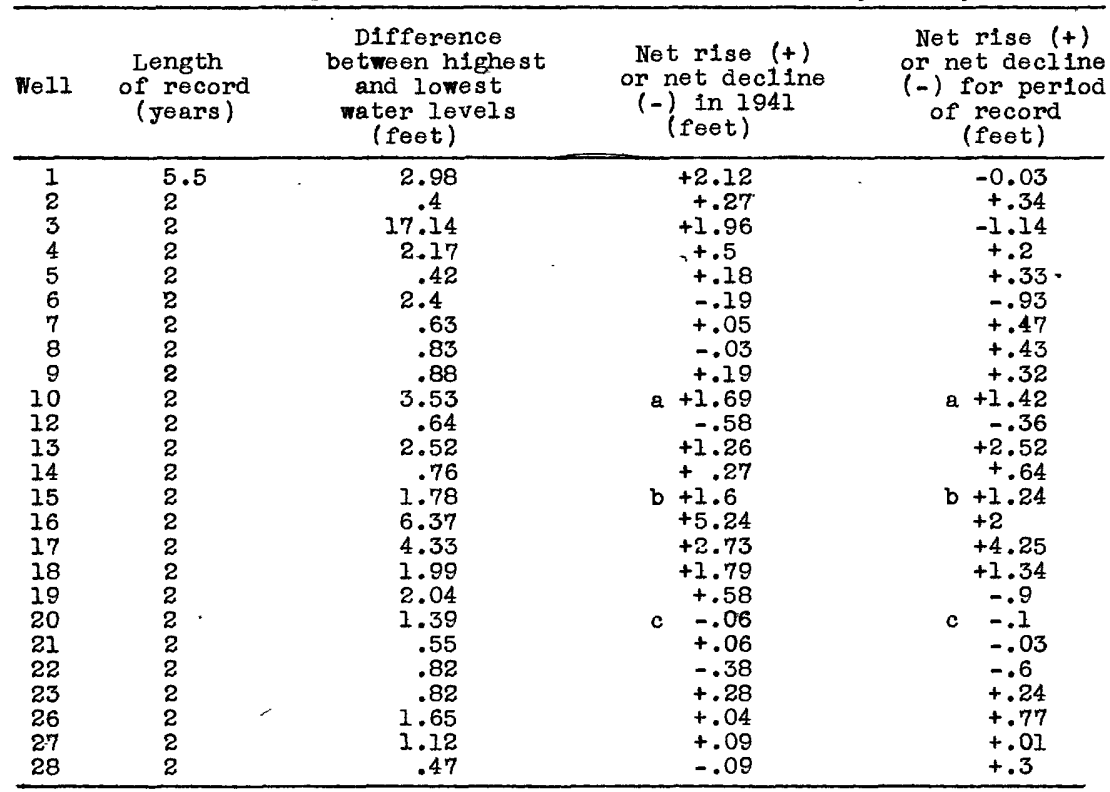

a To Nov, 2, 1941 .

b To Nov. 30, 1941 .

c To Nov. 13, 1941 .

$5110050-48-5$ 


\section{Finney County--Continued}

Water-level measurements

1. Mrs. A. M. Re1d. NE $\mathrm{NE}_{\frac{2}{4}}^{\frac{2}{4}}$ sec. 9, T. 24 S., R. 33 W. Measurements supplied through courtesy of the Division of Water Resources of the Kansas state Board of Agriculture.

Yean dally water level, in feet above datum, 1941

\begin{tabular}{|c|c|c|c|c|c|c|c|c|c|c|c|c|}
\hline & & & & & & & & . & & t. & v. & \\
\hline $\begin{array}{l}1 \\
2 \\
3 \\
4 \\
5 \\
6 \\
7 \\
8 \\
9 \\
10 \\
11 \\
12 \\
13 \\
14 \\
15 \\
16 \\
17 \\
18 \\
19 \\
20\end{array}$ & $\begin{array}{l}8.20 \\
8.20 \\
8.19 \\
8.19 \\
8.19 \\
8.19 \\
8.18 \\
8.18 \\
8.18 \\
8.18 \\
8.18 \\
8.18 \\
8.17 \\
8.18 \\
8.17 \\
8.17 \\
8.16 \\
8.17 \\
8.17 \\
8.17 \\
8.17 \\
8.17 \\
8.17 \\
8.17 \\
8.17 \\
8.16\end{array}$ & $\begin{array}{l}8.17 \\
8.17 \\
8.17\end{array}$ & $\begin{array}{l}8 . \\
8 . \\
8 . \\
8 . \\
8 . \\
8 .\end{array}$ & $\begin{array}{l}8 \\
8 \\
8 \\
8 \\
8 \\
8\end{array}$ & ? & $\begin{array}{l}8 . \\
8 \\
8 . \\
9 \\
9 \\
9 \\
9 \\
9 \\
9 \\
9 \\
9 \\
9 \\
9 \\
9 \\
9 \\
9 \\
9\end{array}$ & & & & & & \\
\hline
\end{tabular}

2. Maggle B. Smith. NE cor. NW/ $\mathrm{NE}_{\frac{1}{4}}$ sec. 30, T. 26 S., R. $32 \mathrm{~W}$. Measuring polnt, $2,913.3$ feet above sea level.

Water level, in feet below measuring point, 1941

\begin{tabular}{|c|c|c|c|c|c|c|c|}
\hline Date & $\begin{array}{l}\text { Water } \\
\text { level }\end{array}$ & Date & $\begin{array}{l}\text { Water } \\
\text { level }\end{array}$ & Date & $\begin{array}{l}\text { Water } \\
\text { level }\end{array}$ & Date & $\begin{array}{l}\text { Water } \\
\text { level }\end{array}$ \\
\hline $\begin{array}{ll}\text { Jan. } 29 \\
\text { Mar. } 19 \\
\text { Apr. } 24\end{array}$ & $\begin{array}{l}109.53 \\
109.49 \\
109.50\end{array}$ & $\begin{array}{lr}\text { May } & 15 \\
\text { June } & 3 \\
\text { July } & 2\end{array}$ & $\begin{array}{l}109.45 \\
109.44 \\
109.44\end{array}$ & $\begin{array}{l}\text { Aug. } 4 \\
\text { Sept. } 2 \\
\text { oct. } 11\end{array}$ & $\begin{array}{l}109.37 \\
109.37 \\
109.31\end{array}$ & $\begin{array}{l}\text { Nov. } 1 \\
\text { Dec. Il }\end{array}$ & $\begin{array}{l}109.33 \\
109.33\end{array}$ \\
\hline
\end{tabular}

3. Nora Will. SW $\frac{7}{4} S W_{4}^{\frac{7}{4}}$ sec. 35, T. 23 S., R. 33 W. Measuring point, $2,893.9$ feet above sea ìevel.

Water level, in feet below measuring point, 1941

\begin{tabular}{ll|lr|lrr|rrr}
\hline Jan. 29 & 47.77 & Apr. 24 & 46.78 & July & 2 & 45.48 & Oct. & 1 & a 55.19 \\
Feb. 22 & 47.54 & May 19 & 46.45 & Aug. & 5 & 45.17 & Nov. 1 & 47.23 \\
Mar. 19 & 47.19 & June 3 & 46.09 & Sept. 1 & 47.24 & Nov. 30 & 46.73 \\
\hline
\end{tabular}

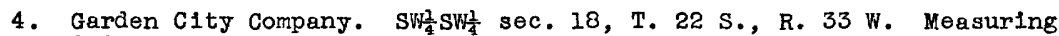
point, 2,912.5 feet above sea level.

Water level, in feet below measuring point, 1941

\begin{tabular}{|rr|lr|lrr|rrr}
\hline Feb. 22 & 34.86 & May & 19 & 34.50 & Aug. & 5 & 34.36 & Nov. 2 & 35.07 \\
Mar. 19 & 34.72 & June & 3 & 34.46 & Sept. 1 & 34.99 & Dec. 11 & 34.70 \\
Apr. 24 & 34.59 & July & 2 & 34.91 & Oct. & 1 & 35.41 & & \\
\hline
\end{tabular}

a Irrigation wells about 200 yards east pumping. 
Finney County--Continued

5. E. Alberta Reeves. SE $\frac{2}{4} N W \frac{1}{4} N W_{4}^{\frac{1}{4}}$ sec. 19, T. 21 S., R. 32 W. Measuring point, 2,894.8 feet above sea level.

Water level, in feet below measuring point, 1941

\begin{tabular}{ll|ll|lll|lr}
\hline Date & $\begin{array}{l}\text { Water } \\
\text { level }\end{array}$ & Date & $\begin{array}{l}\text { Water } \\
\text { level }\end{array}$ & Date & $\begin{array}{l}\text { Water } \\
\text { level }\end{array}$ & Date & $\begin{array}{r}\text { Water } \\
\text { level }\end{array}$ \\
\hline Jan. 29 & 23.24 & Apr. 24 & 23.40 & July 1 & 23.34 & Oct. 1 & 23.15 \\
Feb. 22 & 23.38 & May 15 & 23.35 & Aug. 5 & 23.39 & Nov. 14 & 23.14 \\
Mar. 19 & 23.37 & June 3 & 23.41 & Sept. 5 & 23.18 & Dec. 11 & 23.12 \\
\hline
\end{tabular}

6. T. A. Meakel. NW $\frac{1}{\varepsilon} S W_{\frac{2}{4}} S W \frac{1}{4}$ sec. 36, T. 21 S., R. 29 W. Measuring point, $2,613.1$ feet above sea level.

Water level, in feet below measuring point, 1941

\begin{tabular}{ll|lrr|lll|ll}
\hline Jan. 30 & 18.06 & May & 15 & 19.56 & Aug. 4 & 17.86 & Nov. 13 & 18.96 \\
Mar. 18 & 19.23 & June & 3 & 18.60 & Sept. 4 & 18.30 & Dec. 4 & 19.07 \\
Apr. 24 & 19.65 & July & 1 & 17.62 & Oct. 2 & 18.63 & & \\
\hline
\end{tabular}

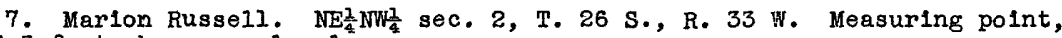
$2,906.3$ feet above sea level.

Water level, in feet below measuring point, 1941

\begin{tabular}{ll|lll|ll|l|r}
\hline Mar. 19 & 77.91 & June & 3 & 77.89 & Sept. 2 & 77.70 & Nov. 1 & 77.59 \\
Apr. 24 & 77.83 & July & 2 & 77.80 & Oct. 10 & 77.70 & Dec. 11 & 77.62 \\
\hline
\end{tabular}

8. 0. G. Reeve. SW $\frac{1}{4} \mathrm{NE}_{\frac{1}{4}} \mathrm{SE}_{\frac{1}{4}}^{\frac{1}{2}}$ sec. 13, T. $25 \mathrm{~S}$, , R. 33 W. Measuring point, $2,899.4$ feet above sea level.

Water level, in feet below measuring point, 1941

\begin{tabular}{ll|ll|ll}
\hline Date & $\begin{array}{l}\text { Water } \\
\text { level }\end{array}$ & Date & Water & Date & Water \\
Jan. 29 & 76.11 & Apr. 24 & 76.23 & Dec. 11 & 76.11 \\
Mar. 19 & 76.19 & Nov. 1 & 76.18 & & \\
\hline
\end{tabular}

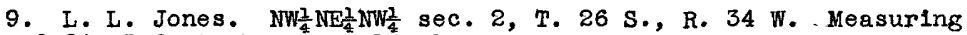
point, $2,945.5$ feet above sea level.

Water level, in feet below measuring point, 1941

\begin{tabular}{ll|lll|ll|ll}
\hline Date & $\begin{array}{l}\text { Water } \\
\text { level }\end{array}$ & Date & $\begin{array}{l}\text { Water } \\
\text { level }\end{array}$ & Date & $\begin{array}{l}\text { Water } \\
\text { level }\end{array}$ & Date & $\begin{array}{r}\text { Water } \\
\text { level }\end{array}$ \\
\hline Mar. 19 & 72.87 & June & 3 & 72.99 & Sept. 2 & 72.92 & Nov. 1 & 72.97 \\
Apr. 24 & 72.89 & July & 2 & 72.91 & Oct. 10 & 72.95 & Dec. 11 & 72.91 \\
May 19 & 72.98 & Aug. & 5 & 72.96 & & & & \\
\hline
\end{tabular}

10. L. R. McBeth. NW $\frac{1}{4} \mathrm{NE}_{\frac{1}{4}}$ sec. 9, T. 24 S., R. 33 W.

Water level, in feet below measuring point, 1941

\begin{tabular}{ll|lrr|lll|lll}
\hline Jan. 29 & 12.88 & May & 19 & 12.22 & Aug. 5 & 11.80 & Oct. & 1 & 12.29 \\
Mar. 19 & 12.33 & June & 3 & 11.98 & Sept. 1 & 11.64 & Nov. & 11.20 \\
Apr. 24 & 12.37 & July & 2 & 11.42 & & & & & \\
\hline
\end{tabular}

12. Nell le Handy. NW $W_{4}^{2} E_{\frac{1}{4}} N_{\frac{1}{4}}^{2}$ sec. 3, T. $25 \mathrm{~S}_{.}, \mathrm{R} .31 \mathrm{~W}$. New measuring point beginning Mar. 19, 1941, top of casing, level'with land surface, 0.17 foot below old measuring point, $2,866.9$ feet above sea level.

Water level, in feet below measuring point, 1941

\begin{tabular}{|c|c|c|c|c|c|c|c|}
\hline $\begin{array}{l}\text { Mar. } \\
\text { Apr. } \\
\text { May }\end{array}$ & $\begin{array}{l}19 \\
24 \\
15\end{array}$ & 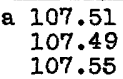 & $\begin{array}{l}\text { June } \\
\text { Aug. }\end{array}$ & $\begin{array}{l}3 \\
4\end{array}$ & $\begin{array}{l}107.58 \\
107.55\end{array}$ & $\begin{array}{ll}\text { Sept. } 2 & 107.81 \\
\text { oct. } 12 \text { b } 109.89\end{array}$ & $\begin{array}{l}\text { Nov. } 3 \\
\text { Dec. } 11\end{array}$ \\
\hline
\end{tabular}

a Below new measuring point.

b Well pumped prior to measurement. 
Finney County--Continued

13. Edwin Wehrley. $\mathrm{NE}_{\frac{1}{4}} \mathrm{SE}_{\frac{1}{4}} \mathrm{SW} \frac{1}{4} \mathrm{sec} .13, \mathrm{~T}, 25 \mathrm{~S}, \mathrm{R}, 31 \mathrm{~W} . \mathrm{Measuring}$ point, $2,743.2$ feet above sea level.

Water level, in feet below measuring point, 1941

\begin{tabular}{lc|lr|lll|lr}
\hline Date & $\begin{array}{c}\text { Water } \\
\text { level }\end{array}$ & \multicolumn{2}{|l|}{ Date } & $\begin{array}{l}\text { Water } \\
\text { level }\end{array}$ & Date & $\begin{array}{r}\text { Water } \\
\text { level }\end{array}$ & Date & $\begin{array}{r}\text { Water } \\
\text { level }\end{array}$ \\
\hline Jan. 29 & 4.22 & May 15 & 3.90 & Aug. 4 & 4.05 & Nov. 3 & 4.34 \\
Mar. 19 & 4.12 & June 3 & 3.39 & Sept. 2 & 4.48 & Dec. 11 & 3.11 \\
Apr. 24 & 4.08 & July 1 & 3.20 & Oct. 12 & 4.27 & & \\
\hline
\end{tabular}

14. John A. Hunter. $\mathrm{NE}_{\frac{1}{4}}^{\frac{1}{2}} \mathrm{SE}_{\frac{1}{4}} \mathrm{NE}_{\frac{1}{4}}^{\frac{1}{2}} \mathrm{sec} .4, \mathrm{~T} .26 \mathrm{~S} ., \mathrm{R} .32$ W. Measuring point, 2,845.9 feet above sea level.

Water level, in feet below measuring point, 1941

\begin{tabular}{ll|lll|ll|ll}
\hline Mar. 19 & 47.24 & June & 3 & 47.30 & Sept. 2 & 46.99 & Nov. I & 46.93 \\
Apr. 24 & 47.27 & JuIy & 2 & 47.17 & Oc. 10 & 46.98 & Dec. II & 46.89 \\
May 15 & 47.37 & Aug. & 4 & 47.02 & & & & \\
\hline
\end{tabular}

15. Flojd A. Edwards, SW $\frac{1}{4} S W_{\frac{1}{4}}$ sec. 2, T. 24 S., R. 33 W.

Water level, in feet below measuring point, 1941

\begin{tabular}{ll|lr|lll|ll|}
\hline Jan. 29 & 15.18 & Apr. 24 & 15.30 & July & 2 & 14.67 & Oct. 1 & 14.13 \\
Feb. 22 & 15.22 & May 19 & 15.37 & Aug. & 5 & 14.23 & Nov. 2 & 13.79 \\
Mar. 19 & 15.25 & June 3 & 14.97 & Sept. 1 & 14.14 & Hov. 30 & 13.62 \\
\hline
\end{tabular}

16. George L. Meeker. $\operatorname{NWT}_{\frac{1}{4}} \mathrm{NE}_{\frac{1}{4}} \mathrm{NE}_{\frac{1}{4}}^{\frac{1}{3}}$ sec. 6, T. 24 S., R. 34 W.

Water level, in feet below measuring point, 1941

\begin{tabular}{ll|l|l|l|ll|ll|}
\hline Jan. 19 & 42.64 & Apr. 24 & 43.32 & July & 2 & 44.21 & Oct. 1 & 43.07 \\
Feb. 22 & 42.88 & May 19 & 43.42 & Aug. 5 & 42.43 & Nov. 16 & 38.28 \\
Mar. 19 & 43.07 & June 3 & 43.04 & Sept. 1 & 42.36 & Dec. 7 & 37.05 \\
\hline
\end{tabular}

17. SE $\frac{1}{4} \mathrm{SWW}_{\frac{1}{4}} \mathrm{NW}_{\frac{1}{4}}$ sec. 7, T. 24 S., R. $33 \mathrm{~W}$. Measuring point, $2,878.5$ feet above sea level.

Water level, in feet below measuring point, 1941

\begin{tabular}{ll|ll|lll|lll}
\hline Jan. 29 & 7.60 & Apr. 24 & 7.19 & July 2 & 5.91 & Oct. & 1 & 6.60 \\
Feb. 22 & 7.40 & May 19 & 6.87 & Aug. 5 & 6.22 & Nov. 2 & 5.73 \\
Mar. 19 & 7.26 & June 3 & 6.42 & Sept. 1 & 6.57 & Dec. & 7 & 5.48 \\
\hline
\end{tabular}

18. A. Finnup. NW $W_{\frac{1}{4}} N W_{\frac{1}{L}}$ sec. 17, T. $24 \mathrm{~S}$, R. 34 W. Measuring point, $2,925.7$ feet above sea level.

Water level, in feet below measuring point, $\cdot 1941$

\begin{tabular}{ll|lll|ll|lll}
\hline Mar. 19 & 12.03 & June & 3 & 12.19 & Sept. 1 & 10.57 & Nov. 2 & 10.63 \\
Apr. 24 & 11.97 & July & 2 & 10.74 & Oct. 1 & 10.61 & Dec. & 7 & 10.37 \\
Mag 19 & 12.22 & Aug. & 5 & 10.32 & & & & & \\
\hline
\end{tabular}

19. H. E. Ramsey. SE $\frac{1}{4} \mathrm{SE}_{\frac{1}{4}}^{\frac{1}{4}}$ sec. 10, T. 23 S., R. 34 W. Measuring point, $2,938.3$ feet above sea level.

Water level, in feet below measuring point, 1941

\begin{tabular}{|c|c|c|c|c|c|c|c|c|}
\hline $\begin{array}{ll}\text { Feb. } 22 \\
\text { Mar. } 19 \\
\text { Apr. } 24\end{array}$ & $\begin{array}{l}32.38 \\
32.51 \\
32.48\end{array}$ & $\begin{array}{l}\text { May I } \\
\text { June } \\
\text { July }\end{array}$ & $\begin{array}{r}19 \\
3 \\
2\end{array}$ & $\begin{array}{l}32.55 \\
32.66 \\
32.56\end{array}$ & $\begin{array}{l}\text { Sept. I } \\
\text { Oct. }\end{array}$ & $\begin{array}{l}32.26 \\
31.91\end{array}$ & $\begin{array}{l}\text { Nov. } 2 \\
\text { Dec. } 11\end{array}$ & $\begin{array}{l}31.59 \\
31.53\end{array}$ \\
\hline
\end{tabular}

20. C. R. RIxon. SW $\frac{1}{4} S W \frac{1}{4}$ sec. 12, T. 23 S., R. 27 W. Measuring point, 2,627.8 feet above sea level.

Water level, in feet below measuring point, 1941

\begin{tabular}{ll|ll|l|lll|l|r}
\hline Jan. 30 & 67.60 & Apr. 24 & 68.86 & June & 3 & 68.94 & Oct. 2 & 68.99 \\
Mar. 18 & 68.89 & May 15 & 68.90 & JuIy & 1 & 68.95 & Nov. 13 & 68.93 \\
\hline
\end{tabular}


FORD COUNTY

By H. A. Walte

Highest and lowest water levels, in feet below measuring point, in 15 wells in Ford County, 1941

\begin{tabular}{|c|c|c|c|c|c|}
\hline WeII & $\begin{array}{l}\text { Highest } \\
\text { recorded } \\
\text { water level } \\
\text { (feet) }\end{array}$ & & Date & $\begin{array}{l}\text { Lowest } \\
\text { recorded } \\
\text { water level } \\
\text { (feet) }\end{array}$ & Date \\
\hline $\begin{array}{r}2 \\
8 \\
11 \\
15 \\
38\end{array}$ & $\begin{array}{r}27.35 \\
5.78 \\
11.31 \\
35.01 \\
39.87\end{array}$ & $\begin{array}{l}\text { Mar. } \\
\text { Aug. } \\
\text { Dec. } \\
\text { Dec. } \\
\text { Oct. }\end{array}$ & $\begin{array}{r}20,1940 \\
1,1941 \\
4,1941 \\
3,1941 \\
6,1941\end{array}$ & $\begin{array}{r}28.78 \\
8.97 \\
13.31 \\
36.92 \\
42.08\end{array}$ & $\begin{array}{l}\text { Sept. 5, } 1939 \\
\text { Nov. 7, } 1939 \\
\text { Jan. 24, } 1940 \\
\text { Sept. 5, } 1939 \\
\text { (Mar. 20, } 1940 \\
\text { (May 16, } 1940\end{array}$ \\
\hline $\begin{array}{l}41 \\
48 \\
57 \\
59 \\
65 \\
79 B \\
79 C \\
96 \\
237 \\
343\end{array}$ & $\begin{array}{r}46.49 \\
6.33 \\
8.29 \\
16.82 \\
17.01 \\
7.45 \\
8.08 \\
9.06 \\
86.29 \\
76.24\end{array}$ & $\begin{array}{l}\text { July } \\
\text { July } \\
\text { Dec. } \\
\text { Dec. } \\
\text { Dec. } \\
\text { Jan. } \\
\text { Dec. } \\
\text { Dec. } \\
\text { Nov. } \\
\text { Dec. }\end{array}$ & $\begin{array}{r}22,1940 \\
11,1941 \\
4,1941 \\
4,1941 \\
3,1941 \\
20,1941 \\
4,1941 \\
4,1941 \\
12,1941 \\
4,1941\end{array}$ & $\begin{array}{l}47.53 \\
10.85 \\
10.93 \\
18.21 \\
18.70 \\
16.09 \\
10.69 \\
11.02 \\
86.92 \\
76.85\end{array}$ & $\begin{array}{l}\text { July } 1,1939 \\
\text { Oct. } 2,1939 \\
\text { Oct. } 2,1939 \\
\text { Sept. } 5,1939 \\
\text { Oct. 2, } 1939 \\
\text { Aug. } 1,1939 \\
\text { Oct. 2, } 1939 \\
\text { Sept. 5, } 1939 \\
\text { Nov. 8, } 1939 \\
\text { Apr. 22, } 1941\end{array}$ \\
\hline
\end{tabular}

Net changes in water level in 1941, and net changes in water level for the period of record in 15 wells in Ford County

\begin{tabular}{|c|c|c|c|c|}
\hline Well & $\begin{array}{l}\text { Length } \\
\text { of record } \\
\text { (years) }\end{array}$ & $\begin{array}{l}\text { Difference } \\
\text { between highest } \\
\text { and lowest } \\
\text { water levels } \\
\text { (feet) }\end{array}$ & $\begin{array}{l}\text { Net rise (+) } \\
\text { or net decline } \\
(-) \text { in } 1941 \\
\text { (feet) }\end{array}$ & $\begin{array}{l}\text { Net rise (+) } \\
\text { or net decline } \\
\text { (-) for period } \\
\text { of record } \\
\text { (feet) }\end{array}$ \\
\hline $\begin{array}{c}2 \\
8 \\
11 \\
15 \\
38 \\
41 \\
48 \\
57 \\
59 \\
65 \\
79 \mathrm{~B} \\
79 \mathrm{C} \\
96 \\
237 \\
343\end{array}$ & $\begin{array}{l}3 \\
3 \\
3 \\
3 \\
3 \\
3 \\
3 \\
3 \\
3 \\
3 \\
3 \\
3 \\
3 \\
3 \\
3\end{array}$ & $\begin{array}{l}1.43 \\
3.19 \\
2.00 \\
1.91 \\
2.21 \\
1.04 \\
4.52 \\
2.64 \\
1.39 \\
1.69 \\
8.64 \\
2.61 \\
1.96 \\
.63 \\
.61\end{array}$ & $\begin{array}{r}+0.80 \\
+2.14 \\
+1.59 \\
+.17 \\
+1.33 \\
+.23 \\
+2.90 \\
+1.20 \\
+1.00 \\
+1.40 \\
a-3.14 \\
+1.60 \\
+1.03 \\
+.31 \\
+.51\end{array}$ & $\begin{array}{r}+0.94 \\
+1.20 \\
+1.47 \\
+.46 \\
+1.47 \\
+.40 \\
+3.27 \\
+.47 \\
+.84 \\
+.98 \\
+1.50 \\
+1.44 \\
+1.40 \\
+.16 \\
+.44\end{array}$ \\
\hline
\end{tabular}

Water-level measurements

2. L. A. Lamb. SW $W_{\frac{1}{4}} S W_{\frac{1}{4}}^{\frac{1}{4}}$ sec. 4, T. 28 S., R. $22 \mathrm{~W}$. Water level, in feet below measuring point, 1941

\begin{tabular}{ll|lr|lr|lrr}
\hline Date & $\begin{array}{l}\text { Water } \\
\text { level }\end{array}$ & Date & $\begin{array}{r}\text { Water } \\
\text { level }\end{array}$ & Date & $\begin{array}{r}\text { Water } \\
\text { level }\end{array}$ & Date & $\begin{array}{r}\text { Water } \\
\text { level }\end{array}$ \\
\hline Jan. 20 & 28.35 & May & 2 & 28.28 & Aug. 2 & 27.85 & Nov. 12 & 27.66 \\
Mar. 17 & 28.26 & June 4 & 28.20 & Sept.11 & 27.71 & Dec. 4 & 27.55 \\
Apr. 22 & 28.20 & July 11 & 27.80 & 0ct. 6 & 27.87 & & \\
\hline
\end{tabular}

a Affected by heavy industrial pumping nearby. 
Ford County--Continued

8. F. H. Diehl. NWl $\mathrm{NW}_{4}^{\frac{2}{4}}$ sec. 34, T. $26 \mathrm{~S}, \mathrm{R}, 25 \mathrm{~W}$.

Water level, in feet below measuring point, 1941

\begin{tabular}{lr|lr|rrr|rr}
\hline Date & $\begin{array}{l}\text { Water } \\
\text { level }\end{array}$ & Date & $\begin{array}{r}\text { Water } \\
\text { level }\end{array}$ & Date & $\begin{array}{r}\text { Water } \\
\text { level }\end{array}$ & Date & $\begin{array}{r}\text { Water } \\
\text { level }\end{array}$ \\
\hline Jan. 21 & 8.13 & May 3 & 7.09 & Aug. 1 & 5.78 & Nov. 12 & 6.27 \\
Mar. 17 & 7.18 & June 6 & 6.89 & 0ct. 6 & 6.79 & Dec. 4 & 5.99 \\
Apr. 22 & 7.62 & July 11 & 6.03 & & & & \\
\hline
\end{tabular}

11. Geo. W. Molitor. SW $\frac{1}{4} W_{\frac{1}{4}}^{\frac{1}{4}}$ sec. 36, T. 21 S., R. 21 W.

Water level, in feet below measuring point, 1941

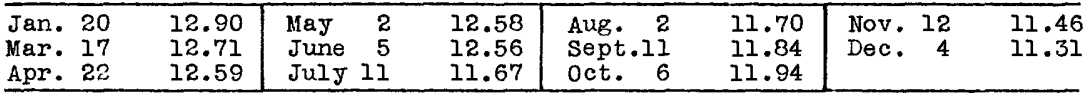

15. George Lutz. SW $\frac{1}{4} N \frac{1}{4}$ sec. 24, T. 29 S., R. 26 W.

Water level, in feet below measuring point, 1941

\begin{tabular}{|c|c|c|c|c|c|c|c|c|c|}
\hline $\begin{array}{ll}\text { Jan. } 21 \\
\text { Mar. } 17 \\
\text { Apr. } 22\end{array}$ & $\begin{array}{l}35.18 \\
35.21 \\
35.16\end{array}$ & $\begin{array}{l}\text { May } \\
\text { June } \\
\text { Aug. }\end{array}$ & 16 & $\begin{array}{l}35.33 \\
35.18 \\
35.21\end{array}$ & $\begin{array}{l}\text { Sept.II } \\
\text { oct. } 9\end{array}$ & $\begin{array}{l}35.27 \\
35.16\end{array}$ & $\begin{array}{l}\text { Nov. } \\
\text { Dec. }\end{array}$ & $\begin{array}{l}7 \\
3\end{array}$ & $\begin{array}{l}35.14 \\
35.01\end{array}$ \\
\hline
\end{tabular}

38. F. Burns. SE $\frac{1}{4} \mathrm{NE} \frac{1}{4}$ sec. 1, T. 26 S., R. $24 \mathrm{~W}$.

Water level, in feet below measuring point, 1941

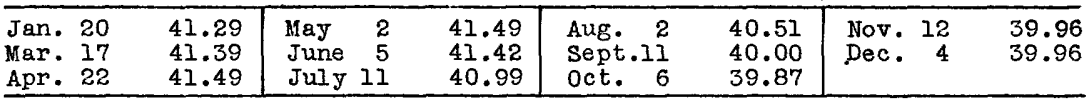

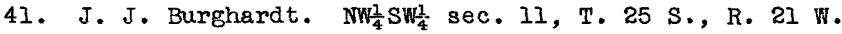

Water level, in feet below measuring point, 1941

\begin{tabular}{ll|lr|r|rr|rr}
\hline Jan. 20 & 46.88 & June 5 & 47.10 & Sept.12 & 46.75 & Nov. 12 & 46.68 \\
Mar. 17 & 47.03 & July 11 & 46.69 & Oct. 6 & 46.78 & Dec. & 4 & 46.65 \\
Apr. 22 & 47.11 & Aug. 2 & 46.67 & & & & & \\
\hline
\end{tabular}

48. G. D. Cochran. $\mathrm{NE}_{\frac{1}{4}}^{\frac{1}{2}} \mathrm{SE} \frac{\mathrm{l}}{4} \mathrm{sec}, 16, \mathrm{~T}, 27 \mathrm{~S} ., \mathrm{R} .23 \mathrm{~W}$.

Water level, in feet below measuring point, 1941

\begin{tabular}{ll|lr|rrr|rr}
\hline Jan. 20 & 9.79 & May & 2 & 9.50 & Aug. 2 & 6.61 & Nov. 12 & 7.11 \\
Mar. 17 & 9.60 & June & 5 & 8.65 & Sept.11 & 8.16 & Dec. 4 & 6.89 \\
Apr. 22 & 9.60 & July 11 & 6.33 & Oct. 6 & 8.70 & & \\
\hline
\end{tabular}

57. Andrew Bogner. NW/ $\mathrm{SE}_{\frac{1}{4}}^{\frac{1}{4}}$ sec. 22, T. 26 S., R. $2 \dot{6}$ W.

Water level, in feet below measuring point, 1941

\begin{tabular}{lr|lr|rrr|rrr}
\hline Jan. 20 & 9.49 & May & 3 & 9.39 & Aug. 1 & 8.37 & Nov. 12 & 8.54 \\
Mar. 17 & 9.38 & June & 5 & 9.18 & Sept.12 & 8.64 & Dec. & 4 & 8.29 \\
Apr. 22 & 9.38 & July 11 & 8.65 & Oct. 6 & 8.80 & & \\
\hline
\end{tabular}

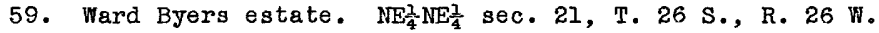

Vhater level, in feet below measuring point, 1941

\begin{tabular}{ll|lrr|lr|rrr}
\hline Jan. 20 & 17.82 & May & 3 & 17.80 & Aug. 1 & 17.10 & Nov. 12 & 17.01 \\
Mar. 17 & 17.83 & June & 5 & 17.57 & Sept.12 & 17.44 & Dec. 4 & 16.82 \\
Apr. 22 & 17.93 & July & 11 & 17.11 & Oct. 6 & 17.42 & & \\
\hline
\end{tabular}

65. John N. Clark. SW $\frac{1}{4}$ SW $\frac{1}{4}$ sec. 33, T. 26 S., R. 25 W.

Water level, in feet below measuring point, 1941

\begin{tabular}{ll|lrr|rr|rr}
\hline Jan. 21 & 18.41 & May & 3 & 18.13 & Aug. 2 & 17.46 & Nov. 12 & 17.22 \\
Mar. 17 & 18.24 & June & 6 & 17.73 & Sept.11 & 17.96 & Dec. 3 & 17.01 \\
Apr. 22 & 18.15 & July & 11 & 17.50 & 0ct. 6 & 17.88 & & \\
\hline
\end{tabular}


Ford County--Continued

79 B. A. N. Nevins. SW $w_{4}^{1}$ SW sec. 23, T. 26 S., R. 24 W. Water level, in feet below measuring point, 1941

\begin{tabular}{lr|lr|lr|lrr}
\hline Date & $\begin{array}{l}\text { Water } \\
\text { level }\end{array}$ & Date & $\begin{array}{l}\text { Water } \\
\text { level }\end{array}$ & Date & $\begin{array}{l}\text { Water } \\
\text { level }\end{array}$ & Date & $\begin{array}{r}\text { Water } \\
\text { level }\end{array}$ \\
\hline Jan. 20 & 7.45 & May & 3 & 10.94 & Aug. 2 & 12.12 & Nov. 12 & 11.19 \\
Mar. 17 & 12.38 & June 6 & 10.83 & Sept.11 & 13.39 & Dec. 4 & 10.59 \\
Apr. 22 & 11.55 & July 11 & 12.32 & 0ct. 6 & 11.87 & & \\
\hline
\end{tabular}

79 C. 0. N. Nevins. SW $w_{4}^{\frac{1}{4}}$ SW sec. 23, T. 26 S., R. 24 W. Water level, in feet below measuring point, 1941

\begin{tabular}{ll|lr|lrr|rr}
\hline Jan. 20 & 9.68 & May & 3 & 9.34 & Aug. 2 & 7.98 & Nov. 12 & 8.26 \\
Mar. 17 & 9.78 & June 6 & 9.30 & Sept.11 & 8.25 & Dec. 4 & 8.08 \\
Apr. 22 & 9.34 & July 11 & 8.20 & Oct. 6 & 8.37 & & \\
\hline
\end{tabular}

96. Henry Hattrup. SE $\frac{1}{2} \mathrm{NE} \frac{1}{4}$ sec. 23, T. $26 \mathrm{~S} ., \mathrm{R} .21 \mathrm{~W}$.

Water level, in feet below measuring point, 1941

\begin{tabular}{|c|c|c|c|c|c|c|c|c|}
\hline $\begin{array}{ll}\text { Jan. } 20 \\
\text { Mar. } 17 \\
\text { Apr. } 22\end{array}$ & $\begin{array}{r}10.09 \\
9.93 \\
9.94\end{array}$ & $\begin{array}{l}\text { May } \\
\text { June } \\
\text { July I }\end{array}$ & $\begin{array}{r}2 \\
5 \\
11\end{array}$ & $\begin{array}{l}9.84 \\
9.82 \\
9.33\end{array}$ & $\begin{array}{l}\text { Aug. } 2 \\
\text { Sept.11 } \\
\text { oct. } 6\end{array}$ & $\begin{array}{l}9.42 \\
9.63 \\
9.67\end{array}$ & $\begin{array}{lr}\text { Nov. } & 12 \\
\text { Dec. } & 4\end{array}$ & $\begin{array}{l}9.17 \\
9.06\end{array}$ \\
\hline
\end{tabular}

237. A. T. and S. F. Ry. NW $\frac{1}{4} N W_{4}^{\frac{1}{4}}$ sec. 28, T. 25 S., R. 22 W.

Water level, in feet below measuring point, 1941

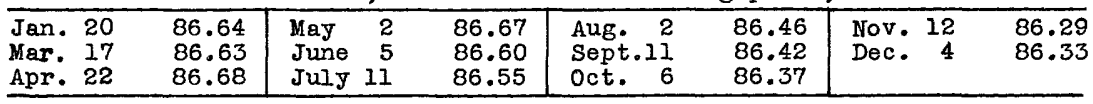

343. B. A. Schuette. $\mathrm{NE}_{\frac{1}{4}} \mathrm{NE} \frac{1}{4} \mathrm{sec} .1, \mathrm{~T} .26 \mathrm{~s} ., \mathrm{R}, 26 \mathrm{~W}$.

Water level, in feet below measuring point, 1941

\begin{tabular}{|c|c|c|c|c|c|c|c|}
\hline $\begin{array}{ll}\text { Jan. } 20 \\
\text { Mar. } 17 \\
\text { Apr. } 22\end{array}$ & $\begin{array}{l}76.75 \\
76.80 \\
76.85\end{array}$ & $\begin{array}{l}\text { May } \\
\text { June } \\
\text { Aug. }\end{array}$ & $\begin{array}{l}3 \\
5 \\
2\end{array}$ & $\begin{array}{l}76.74 \\
76.78 \\
76.75\end{array}$ & $\begin{array}{l}\text { Sept.11 } \\
\text { oct. } 6\end{array}$ & $\begin{array}{l}76.83 \\
76.78\end{array}$ & $\begin{array}{lr}\text { Nov. } & 12 \\
\text { Dec. } & 4\end{array}$ \\
\hline
\end{tabular}

\section{GRANT COUNTY}

By T. G. McLaughlin

A survey of the geology and ground-water resources of Grant and Haskell Count1es, Kansas, was begun in the summer of 1941 by the state Geological Survey of Kansas, and the Federal Geological Survey, in cooperation with the Division of Sanitation of the Kansas State Board of Health, and the Division of Water Resources of the Kansas State Board of Agriculture. 
Grant County--Continued

The investigation was made under the supervision of $S$. W. Lohman, Federal geologist in charge of ground-water investigations in Kansas. Ground-water investigations were made in a part of this area by Haworth in 1897 and by Darton in 1913.

Grant and Haskell are adjacent counties in southwestern Kansas. The area is a relatively flat plain except in local areas of sand dunes, and In the valieys of Cimarron River and its tributaries. Both counties are underlain by thick deposits of sand, silt, and gravel of Tertiary and Quaternary age. All wells in these counties obtain water from these deposits.

Twenty-eight wells were selected for monthly observation of water levels--14 in Grant County and 14 in Haskell County. A total of 190 wettedtape measurements was made in 194l. All water-level measurements were made by Woodrow w. Wilson.

Well descriptions and water-level measurements

1. F. C. WIIllams $\operatorname{SE} \frac{1}{4} \operatorname{SE} \frac{1}{4}$ sec. 30 , T. 27 S., R. 37 W. Unused drilled stock well, diameter 6 inches, depth 57 feet. Measuring point, top of casing, west side, 0.45 foot above land surface, $3,077.9$ feet above sea level. Equipped with lift pump.

Water level, in feet below measuring point, 1941

\begin{tabular}{ll|ll|ll}
\hline Date & $\begin{array}{l}\text { Water } \\
\text { level }\end{array}$ & Date & $\begin{array}{l}\text { Water } \\
\text { level }\end{array}$ & Date & $\begin{array}{r}\text { Water } \\
\text { level }\end{array}$ \\
\hline May 14 & 44.88 & Oct. 16 & 45.48 & Dec. 2 & 44.81 \\
Sept.16 & 45.51 & Nov. 17 & 44.71 & & \\
\hline
\end{tabular}

2. J. B. Shorier. $\mathrm{NE}_{4}^{1} \mathrm{NE}_{4}^{\frac{1}{4}} \mathrm{sec} .26$, T. $27 \mathrm{~S}, \mathrm{R} .38 \mathrm{w}$. Unused drilled domestic and stock well, diameter 6 inches, depth 60 feet. Measuring point, top of casing, north side, level with land surface, $3,096.3$ feet above sea level. Equipped with lift pump and windmill.

\begin{tabular}{lr|ll|llr}
\multicolumn{6}{c}{ Water level, in feet below measuring point, 1941 } \\
\hline May 14 & 46.05 & July 14 & 46.07 & Nov. 17 & 45.68 \\
June 12 & 45.98 & Oct. 16 & 45.75 & Dec. 2 & 45.55 \\
\hline
\end{tabular}

3. A.G. Dyck. SW $\mathrm{SE}_{\frac{1}{4}}^{\frac{1}{4}}$ sec. 8, T. 28 S., R. $38 \mathrm{~W}$. Unused drilled well, diameter 6 inches, depth 52 feet. Measuring point, top of casing, south side, 0.7 foot above land surface, $3,119.35$ feet above sea level. Equipped with lift pump and windmill.

Water level, in feet below measuring point, 1941

\begin{tabular}{llll|ll|ll|l}
\hline Date & $\begin{array}{l}\text { Water } \\
\text { level }\end{array}$ & Date & $\begin{array}{l}\text { Water } \\
\text { level }\end{array}$ & Date & $\begin{array}{l}\text { Water } \\
\text { level }\end{array}$ & Date & $\begin{array}{r}\text { Water } \\
\text { level }\end{array}$ \\
\hline May 14 & 46.30 & July 14 & 46.11 & Sept.16 & 46.50 & Nov. 17 & 46.78 \\
June 12 & 46.14 & Aug. 11 & 46.23 & Dct. 16 & 46.62 & Dec. 2 & 46.29 \\
\hline
\end{tabular}

1/ Haworth, Erasmus, Underground waters of southwestern Kansas: U. S. Geol. Survey Water-Supply Paper 6, 65 pp., 12 pls., 2 figs., 1897.

2/ Darton, N. H., U. S. Geol, Survey Geol. Atlas, Syracuse - Lakin fol1o- (No. 212 ) 10 pp., illus., 1920. 


\section{Grant County--Continued}

4. F. J. Andes. SE $\mathrm{SEE}_{\frac{1}{4}}^{\frac{1}{4}}$ sec. 9 , T. 27 S., R. $38 \mathrm{w}$. Unused drilled domestic and stock well, diameter 8 inches, depth 99 feet. Measuring point, top edge of 8-inch hole in concrete base, south side, 0.7 foot above land surface, 3,150.00 feet above sea level. Equipped with lift pump and windmili.

Water level, in feet below measuring point, 1941

\begin{tabular}{cc|ll|ll|ll}
\hline Date & $\begin{array}{l}\text { Water } \\
\text { level }\end{array}$ & Date & $\begin{array}{l}\text { Water } \\
\text { level }\end{array}$ & Date & $\begin{array}{l}\text { Water } \\
\text { level }\end{array}$ & Date & $\begin{array}{r}\text { Water } \\
\text { level }\end{array}$ \\
\hline May 14 & 88.22 & Aug. 11 & 87.95 & Oct. 16 & 87.55 & Dec. & 87.25 \\
June 12 & 88.15 & Sept.16 & 87.78 & Nov. 17 & 87.34 & & 87 \\
\hline
\end{tabular}

5. C. L. Jury. NE $\frac{1}{4} \mathrm{SE} \frac{2}{4}$ sec. 4, T. 27 S., R. 37 W. Unused drilled domestic and stock well, diameter 6 inches, depth 78 feet. Measuring point, top of casing, 0.4 foot above land surface, $3,070.29$ feet above sea level. Equipped with lift pump and windmill.

Water level, in feet below measuring point, 1941

\begin{tabular}{crr|ll|ll|rr}
\hline May 14 & 67.40 & July 14 & 67.16 & Sept.16 & 67.29 & Nov. 17 & 67.13 \\
June 12 & 67.42 & Aug. II & 67.22 & Oct. 16 & 66.94 & Dec. 2 & 67.19 \\
\hline
\end{tabular}

6. Craig Howard. NW $S W \frac{1}{4}$ sec. 16, T. 27 S., R. 35 W. Unused drilled stock well, diameter 6 inches, depth 182 feet. Measuring point, top of casing, east side, 0.7 foot above land surface, $3,084.89$ feet above sea level. Equipped with lift pump and windmill.

Water level, in feet below measuring point, 1941

\begin{tabular}{lrr|ll|ll|ll}
\hline May 27 & 175.75 & July 14 & 175.69 & Sept.16 & 175.80 & Nov. 17 & 175.70 \\
June 12 & 175.73 & Aug. 11 & 175.65 & Oct. 16 & 175.83 & Dec. & 2 & 175.65 \\
\hline
\end{tabular}

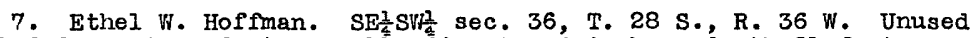
drilled domestic and stock well, diameter 6 inches, depth 91 feet. Measuring point, top of casing, north side, 0.9 foot above land surface, $3,041.94$ feet above sea level. No purp in well.

Water level, in feet below measuring point, 1941

\begin{tabular}{lll|ll|ll|lll}
\hline May 27 & 83.03 & July 14 & 83.14 & Oct. 16 & 83.09 & Dec. & 2 & 83.06 \\
June 12 & 83.14 & Aug. 11 & 83.05 & Nov. 17 & 83.14 & & & \\
\hline
\end{tabular}

8. E. O. Stuart. NWT NE sec. $33, T .29$ S., R. 35 W. Unused drilled stock well, diameter 4 inches, depth 7 feet. Measuring point, top of casing, south side, 0.8 foot above land surface, $2,953.52$ feet above sea level. No pump in well.

Water level, in feet below measuring point, 1941

\begin{tabular}{lll|ll|ll|ll}
\hline May 27 & 59.83 & July 14 & 59.61 & Sept.16 & 59.63 & Nov.17 & 59.73 \\
June 12 & 59.79 & Aug. 11 & 59.59 & Oct. 16 & 59.66 & Dec.2 & 59.71 \\
\hline
\end{tabular}

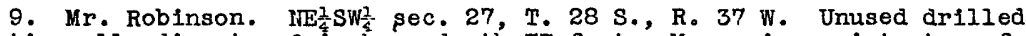
domestic well, diameter 6 inches, depth 77 feet. Measuring point, top of casing, north side, level with land surface, 3,052.69 feet above sea level. No pump in well.

Water level, in feet below measuring point, 1941

\begin{tabular}{lll|ll|ll|lr}
\hline May & 28 & 72.26 & July 14 & 72.35 & Sept.16 & 72.36 & Nov. 17 & 72.49 \\
June 12 & 72.23 & Aug. 11 & 72.42 & Oct. 16 & 72.40 & Dec. & 2 & 72.40 \\
\hline
\end{tabular}

10. E. F. Fowler and Harry Joyce. NW $W_{t}^{\frac{1}{4}}$ SW sec. 27, T. 30 S., R. 37 W., situated 25 feet west of house. Unused drilled domestic and stock well, diameter 6 inches, depth 16 feet. Measuring point, top of casing, levei with land surface, $2,983.54$ feet above sea level. No pump in well.

Water level, in feet below measuring point, 1941

\begin{tabular}{lll|ll|ll|lr}
\hline May & 28 & 11.49 & July 14 & 11.39 & Sept.16 & 12.19 & Nov. 17 & 11.83 \\
June 12 & 11.57 & Aug. 11 & 12.05 & Oct. 16 & 11.62 & Dec. & 2 & 11.88 \\
\hline
\end{tabular}




\section{Grant County--Continued}

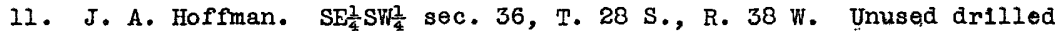
school well, diameter 4 inches, depth 69 feet. Measuring point, top of casing, south side, 0.2 foot above land surface, $3,101.08$ feet above sea level. Equipped with ilf pump.

Water level, in feet below measuring point, 1941

\begin{tabular}{ll|ll|lr}
\hline Date & $\begin{array}{c}\text { Water } \\
\text { level }\end{array}$ & Date & $\begin{array}{l}\text { Water } \\
\text { level }\end{array}$ & Date & $\begin{array}{r}\text { Water } \\
\text { level }\end{array}$ \\
\hline May 28 & 47.43 & July 14 & 47.35 & Nov. 17 & 47.20 \\
June 12 & 47.40 & Oct. 16 & 47.35 & Dec. 2 & 47.27 \\
\hline
\end{tabular}

13. Fred Powell. NW $\frac{1}{4}$ NE⿺ sec. 5, T. 29 S., R. 36 W., situated 200 feet south of U. S. Highway l6̈o. Unused drilled well, diameter 4 inches, depth 119 feet. Measuring point, top of casing, east side, 0.5 foot above land surface, 3,063.99 fe日t above sea level. No pump in well.

Water level, in feet below measuring point, 1941

\begin{tabular}{|c|c|c|c|c|c|c|c|}
\hline Date & $\begin{array}{l}\text { Water } \\
\text { level }\end{array}$ & Date & $\begin{array}{l}\text { Water } \\
\text { level }\end{array}$ & Date & $\begin{array}{l}\text { Water } \\
\text { level }\end{array}$ & Date & $\begin{array}{l}\text { Water } \\
\text { level }\end{array}$ \\
\hline $\begin{array}{ll}\text { May } & 30 \\
\text { June } & 12\end{array}$ & $\begin{array}{l}107.05 \\
107.03\end{array}$ & $\begin{array}{l}\text { July } 14 \\
\text { Aug. } 11\end{array}$ & $\begin{array}{l}107.08 \\
107.02\end{array}$ & $\begin{array}{l}\text { Sept.16 } \\
\text { oct. } 16\end{array}$ & $\begin{array}{l}107.04 \\
106.99\end{array}$ & $\begin{array}{l}\text { Nov. } 17 \\
\text { Dec. } 2\end{array}$ & $\begin{array}{l}106.92 \\
106.88\end{array}$ \\
\hline
\end{tabular}

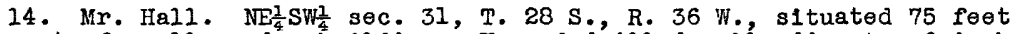
northwest of small wooden building. Unused drilled weli, diameter 6 inches, depth more than 300 feet. Measuring point, top of casing, east side, 1.1 feet above land surface, 3,092.2s feet above sea level. No purp in well.

Water level, in feet below measuring point, 1941

\begin{tabular}{lrr|ll|ll|lr}
\hline May & 30 & 131.57 & July 14 & 131.49 & Sept.16 & 131.46 & Nov. 17 & 131.33 \\
June 12 & 131.56 & Aug. II & 131.43 & oct. 16 & 131.34 & Dec. 2 & 131.33 \\
\hline
\end{tabular}

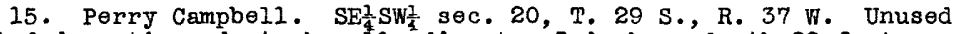
drilled domestic and stock well, diameter' 5 inches, depth 82 feet.

Measuring point, top of casing, southeast side, 0.1 foot above land surface, 3,090.62 feet above sea level. Equipped with ift pump.

Water level, in feet below measuring point, 1941

\begin{tabular}{ccc|cc|ccc|ccc}
\hline May & 30 & 74.16 & Aug. 11 & 74.28 & Oct. 16 & 74.19 & Dec. 2 & 74.17 \\
July 14 & 74.11 & Sept.16 & 74.16 & Nov. 17 & 74.25 & & & \\
\hline
\end{tabular}

GRAY COUNTY

By B. F. Latta

Highest and lowest water levels, in feet below measuring point, in 22 wells in Gray County, 1941

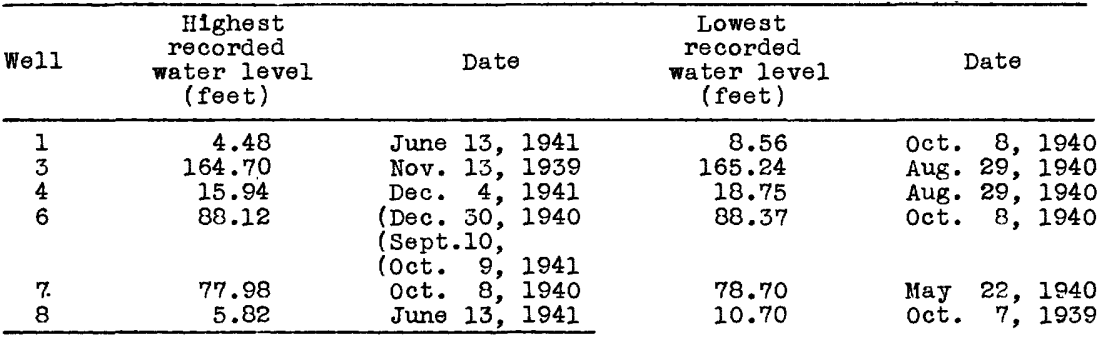


Gray County--Continued

Highest and lowest water levels, in feet below measuring point, in 22 wells in Gray County, 1941--Continued

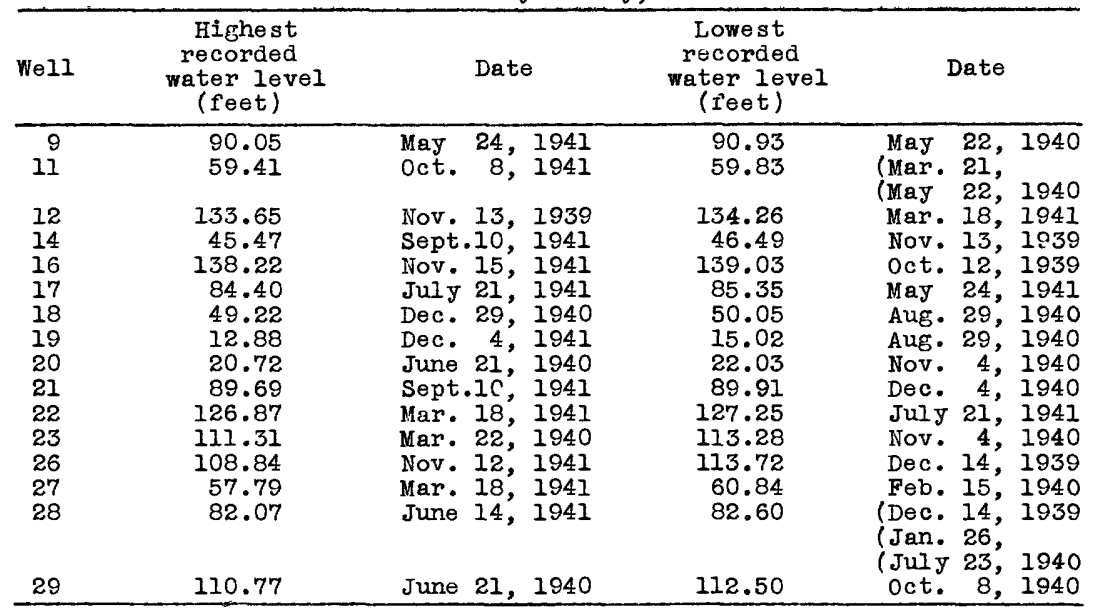

Net changes in water level in 1941, and net changes in water level for the period of record in 22 wells in Gray County

\begin{tabular}{|c|c|c|c|c|}
\hline Well & $\begin{array}{l}\text { Length } \\
\text { of record } \\
\text { (years) }\end{array}$ & $\begin{array}{l}\text { Difference } \\
\text { between highest } \\
\text { and lowest } \\
\text { water levels } \\
\text { (feet) }\end{array}$ & $\begin{array}{l}\text { Net rise (+) } \\
\text { or net decline } \\
(-) \text { in } 1941 \\
\text { (feet) }\end{array}$ & $\begin{array}{l}\text { Net rise (+) } \\
\text { or net decline } \\
\text { (-) for period } \\
\text { of record } \\
\text { (feet) }\end{array}$ \\
\hline $\begin{array}{r}1 \\
3 \\
4 \\
6 \\
7 \\
8 \\
9 \\
11 \\
12 \\
14 \\
16 \\
17 \\
18 \\
19 \\
20 \\
21 \\
22 \\
23 \\
26 \\
27 \\
28 \\
29\end{array}$ & $\begin{array}{l}2 \\
2 \\
2 \\
2 \\
2 \\
2 \\
2 \\
2 \\
2 \\
2 \\
2 \\
2 \\
2 \\
2 \\
2 \\
2 \\
2 \\
2 \\
3 \\
2 \\
2 \\
2\end{array}$ & $\begin{array}{r}4.08 \\
.54 \\
2.81 \\
.25 \\
.72 \\
4.88 \\
.88 \\
.42 \\
.61 \\
1.02 \\
.81 \\
.95 \\
.83 \\
2.14 \\
1.31 \\
.22 \\
.38 \\
1.97 \\
4.88 \\
3.05 \\
.53 \\
1.73\end{array}$ & $\begin{array}{r}+2.38 \\
-.12 \\
+1.8 \\
-.09 \\
-.37 \\
+2.32 \\
-.01 \\
+.15 \\
+.13 \\
+.57 \\
+.11 \\
+.49 \\
-.32 \\
+1.68 \\
+.47 \\
-.02 \\
-.15 \\
+1.47 \\
+1.9 \\
-.86 \\
+.05 \\
+.03\end{array}$ & $\begin{array}{r}+2.91 \\
+.18 \\
+2.43 \\
+.02 \\
+.04 \\
+3.54 \\
+.05 \\
+.21 \\
+.56 \\
+.37 \\
+.74 \\
+.23 \\
+.25 \\
+1.99 \\
+.55 \\
+.02 \\
-.07 \\
+.57 \\
+4.05 \\
+1.61 \\
+.26 \\
+1.27\end{array}$ \\
\hline
\end{tabular}


Gray County--Continued

Water-level measurements

1. G. A. Hard. WW $W_{4}^{\frac{1}{4}} S E \frac{1}{4} S W_{4}^{\frac{1}{4}}$ sec. 20, T. 25 S., R. 29 W. Measuring point, $2,688.3$ feet above sea level.

Water level, in feet below measuring point, 1941

\begin{tabular}{lr|lr|lr|rr}
\hline Date & $\begin{array}{l}\text { Water } \\
\text { level }\end{array}$ & Date & $\begin{array}{l}\text { Water } \\
\text { level }\end{array}$ & Date & $\begin{array}{r}\text { Water } \\
\text { level }\end{array}$ & Date & $\begin{array}{r}\text { Water } \\
\text { level }\end{array}$ \\
\hline Mar. 18 & 7.47 & June 13 & 4.48 & Sept.11 & 7.31 & Nov. 12 & 5.71 \\
Apr. 22 & 7.43 & July 11 & 6.29 & Oct. 6 & 7.14 & Dee. 4 & 5.43 \\
May 22 & 7.16 & Aug. 1 & 6.79 & & & & \\
\hline
\end{tabular}

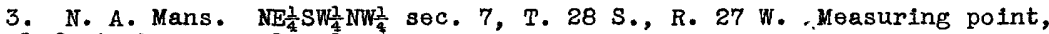
$2,781.1$ feet above sea level.

Water level, in feet below measuring point, 1941

\begin{tabular}{ll|l|l|l|ll|ll}
\hline Apr. 26 & 164.77 & July 21 & 164.73 & Sept.10 & 164.80 & Nov. 12 & 164.81 \\
May 24 & 164.77 & Aug. & 7 & 164.80 & Oct. 9 & 164.90 & Dec. 26 & 164.85 \\
June 14 & 164.92 & & & & & &
\end{tabular}

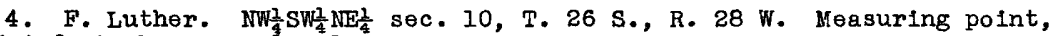
$2,637.4$ feet above sea level.

Water level, in feet below measuring point, 1941

\begin{tabular}{|c|c|c|c|c|c|c|}
\hline $\begin{array}{ll}\text { Mar. } 18 \\
\text { Apr. } \\
\text { May } 22\end{array}$ & $\begin{array}{l}17.68 \\
17.75 \\
17.64\end{array}$ & $\begin{array}{ll}\text { June } 13 \\
\text { July } 11\end{array}$ & $\begin{array}{l}16.96 \\
16.60\end{array}$ & $\begin{array}{l}\text { Sept.11 } \\
\text { oct. } 6\end{array}$ & a & $\begin{array}{l}18.2 \\
17.5\end{array}$ \\
\hline
\end{tabular}

6. S. Dirks. $S E \frac{1}{4} N_{\frac{1}{4}} N E_{\frac{1}{4}}^{\frac{1}{4}} \mathrm{sec} .5, \mathrm{~T}, 28 \mathrm{~S} ., \mathrm{R}, 29 \mathrm{~W}$. Measuring point, $2,773.3$ feet above sea level.

Water level, in feet below measuring point, 1941

\begin{tabular}{|c|c|c|c|c|c|c|c|c|}
\hline $\begin{array}{ll}\text { Mar. } & 18 \\
\text { Apr. } & 26 \\
\text { May } & 24\end{array}$ & $\begin{array}{l}88.16 \\
88.19 \\
88.25\end{array}$ & $\begin{array}{l}\text { June } 1 \\
\text { July } 2 \\
\text { Aug. }\end{array}$ & $\begin{array}{r}14 \\
21 \\
9\end{array}$ & $\begin{array}{l}88.14 \\
88.17 \\
88.35\end{array}$ & $\begin{array}{l}\text { Sept.10 } \\
\text { oct. } 9\end{array}$ & $\begin{array}{l}88.12 \\
88.12\end{array}$ & $\begin{array}{l}\text { Nov. } 15 \\
\text { Dec. } 26\end{array}$ & $\begin{array}{l}88.15 \\
88.21\end{array}$ \\
\hline
\end{tabular}

7. P. Brietenbach et al. $\mathrm{SE}_{\frac{1}{4}} \mathrm{SWW}_{4}^{\frac{1}{4}} \mathrm{SE} \frac{1}{4} \mathrm{sec} .36, \mathrm{~T}, 26 \mathrm{~S} ., \mathrm{R} .29 \mathrm{~W}$. Measuring point, 2,734.7 feet above sea level.

Water level, in feet below measuring point, 1941

\begin{tabular}{lr|lr|rrr|rr}
\hline Mar. 18 & 78.16 & June 14 & 78.33 & Sept.10 & 78.32 & Nov. 15 & 78.35 \\
Apr. 26 & 78.25 & July 21 & 78.26 & oct. 9 & 78.39 & Dec. 26 & 78.44 \\
May 24 & 78.23 & Aug. 9 & 78.30 & & & & & \\
\hline
\end{tabular}

8. NW $\frac{1}{4} \mathrm{NE}_{\frac{1}{4}} \mathrm{SE} \frac{1}{4}$ sec. 11, T. $26 \mathrm{~S} .$, R. $28 \mathrm{~W}$. Measuring point, $2,612.1$ feet above sea level.

Water level, in feet below measuring point, 1941

\begin{tabular}{|c|c|c|c|c|c|c|}
\hline $\begin{array}{ll}\text { Mar. } & 18 \\
\text { Apr. } & 2 \dot{2} \\
\text { May } & 22\end{array}$ & $\begin{array}{l}9.49 \\
9.55 \\
8.55\end{array}$ & $\begin{array}{l}\text { June } 13 \\
\text { July } 11 \\
\text { Aug. }\end{array}$ & $\begin{array}{l}5.82 \\
6.76 \\
6.05\end{array}$ & $\begin{array}{l}\text { Sept.11 } \\
\text { Oct. } 6\end{array}$ & $\begin{array}{l}8.09 \\
7.90\end{array}$ & $\begin{array}{lr}\text { Nov. } & 12 \\
\text { Dec. } & 4\end{array}$ \\
\hline
\end{tabular}

9. L. Naftziger. $\mathrm{NE}_{\frac{1}{4}} \mathrm{SE}_{\frac{1}{4}} \mathrm{NE}_{\frac{1}{4}}^{\frac{1}{4}}$ sec. $24, \mathrm{~T} .24 \mathrm{~S}, \mathrm{R}, 29$ W. Measuring point, $2,761.8$ feet above sea level.

Water level, in feet below measuring point, 1941

\begin{tabular}{lr|lr|lr|rrr}
\hline Mar. 18 & 90.85 & June 13 & 90.78 & Sept.11 & 90.85 & Nov. 12 & 90.84 \\
Apr. 22 & 90.89 & July 11 & 90.90 & Oct. 6 & 90.87 & Dec. 4 & 90.83 \\
May 24 & 90.05 & Aug. 1 & 90.86 & & & & & \\
\hline
\end{tabular}

a Pumped for irrigation during previous month. 


\section{Gray County--Continued}

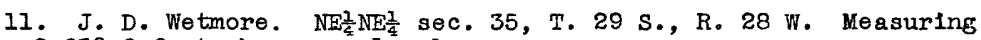
point, $2,630.6$ feet above sea level.

Water level, in feet below measuring point, 1941

\begin{tabular}{ll|ll|ll|lr}
\hline Date & $\begin{array}{l}\text { Water } \\
\text { level }\end{array}$ & Date & $\begin{array}{l}\text { Water } \\
\text { level }\end{array}$ & Date & $\begin{array}{r}\text { Water } \\
\text { level }\end{array}$ & Date & $\begin{array}{r}\text { Water } \\
\text { level }\end{array}$ \\
\hline Mar. 18 & 59.68 & June 14 & 59.79 & Sept. 9 & 59.45 & Nov. 6 & 59.44 \\
Apr. 26 & 59.75 & July 15 & 59.56 & Oct. 8 & 59.41 & Dec. 26 & 59.43 \\
May 24 & 59.78 & Aug. 6 & 59.47 & & & & \\
\hline
\end{tabular}

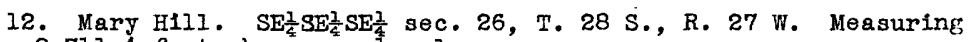
point, $2,711.4$ feet above sea level.

Water level, in feet below measuring point, 1941

\begin{tabular}{|c|c|c|c|c|c|c|c|}
\hline $\begin{array}{ll}\text { Mar. } & 18 \\
\text { Apr. } & 26 \\
\text { May } 24\end{array}$ & $\begin{array}{l}134.26 \\
133.76 \\
133.79\end{array}$ & $\begin{array}{l}\text { June } 1 \\
\text { July } \\
\text { Aug. }\end{array}$ & $\begin{array}{l}133.71 \\
133.81 \\
133.82\end{array}$ & $\begin{array}{l}\text { Sept.10 } \\
\text { oct. } 9\end{array}$ & $\begin{array}{l}133.85 \\
133.93\end{array}$ & $\begin{array}{l}\text { Nov. } 15 \\
\text { Dec. } 26\end{array}$ & $\begin{array}{l}133.87 \\
133.68\end{array}$ \\
\hline
\end{tabular}

13. G. Bowser. SW $N W \frac{1}{4} N W_{\frac{1}{4}}^{\frac{1}{4}}$ sec. $24, T .24$ S., R. 28 W. Measuring point, $2,719.6$ feet above sea level. Measurements discontinued in January 1941.

14. Sarah Marney. SE cor. SW $\frac{1}{4} S E \frac{1}{4}$ sec. 25, T. 29 S., R. 27 W. Measuring point, 2,572.2 feet above sea level.

Water level, in feet below measuring polnt, 1941

\begin{tabular}{ccc|cc|cc|cc|}
\hline Mar. 18 & 46.25 & June 14 & 46.32 & Sept.10 & 45.47 & Nov. 15 & 45.56 \\
Apr. 26 & 46.37 & July 21 & 46.33 & Oct. 9 & 45.89 & Dec. 26 & 45.79 \\
May 24 & 46.42 & Aug. 7 & 46.35 & & & & & \\
\hline
\end{tabular}

16. Ed Wallace. IVE cor. NW sec. 19, T. 29 S., R. 30 W. Measuring point, 2,823.9 feet above sea level.

Water level, in feet below measuring point, 1941

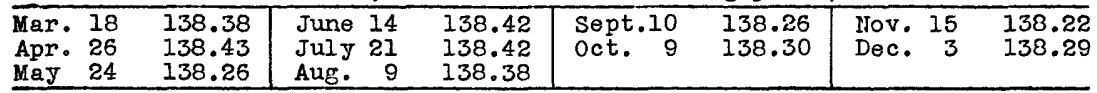

17. V. E. Yeager. NE cor. NW $\frac{1}{4} N E \frac{1}{4}$ sec. 3I, T. 28 S., R. 29 W. Measuring point, $2,762.5$ feet above sea level.

Water level, in feet below measuring point, 1941

\begin{tabular}{lrr|lr|rr|rr}
\hline Mar. 18 & 85.33 & June 14 & 85.01 & Sept.10 & 84.53 & Nov. 15 & 84.60 \\
Apr. 26 & a 86.66 & July 21 & 84.40 & Oct. 9 & 84.61 & Dec. 26 & 84.79 \\
May 24 & 85.35 & Aug. 9 & 84.45 & & & & & \\
\hline
\end{tabular}

18. W. H. Mace. $\operatorname{SE} \frac{1}{4} \mathrm{NE}_{\frac{1}{4}}^{\frac{1}{4}}$ sec. $25, \mathrm{~T} .29 \mathrm{~S} ., \mathrm{R} .29$ W. Measuring point, $2,672.5$ feet above sea level.

Water level, in feet below measuring point, 1941

\begin{tabular}{lll|lr|rr|rr}
\hline Mar. 18 & 49.78 & June 14 & 49.72 & Sept.10 & 49.77 & Nov. 15 & 49.55 \\
Apr. 26 & 49.89 & July 21 & 49.75 & Oct. 9 & 49.59 & Dec. 26 & 49.54 \\
May 24 & 49.76 & Aug. 9 & 49.73 & & & & & \\
\hline
\end{tabular}

19. M. E. Kraushar. NW $W_{4}^{\frac{1}{4}}$ sec. 35, T. 25 S., R. 29 W. Measuring point, $2,671.1$ feet above sea level.

Water level, in feet below measuring point, 1941

\begin{tabular}{ll|lr|rr|rrr}
\hline Mar. 18 & 14.42 & June 13 & 13.10 & Sept.11 & 14.27 & Nov. 12 & 13.18 \\
Apr. 22 & 13.98 & July 11 & 13.57 & Oct. 6 & 14.19 & Dec. 4 & 12.88 \\
May 22 & 14.25 & Aug. 1 & 13.68 & & & & & \\
\hline
\end{tabular}

20. R. and E. Fischer. SE $\frac{1}{4} \mathrm{SW}_{4}^{\frac{1}{4}} \mathrm{NE} \frac{1}{4}$ sec. 23, T. $25 \mathrm{~S}, \mathrm{R}, 30 \mathrm{~W}$. Measuring point, 2,723.2 feet above sea level. Water levels, in feet below measuring point, 1941: Mar. 18, 21.98; Oct. 6, 21.64; Nov. 12, 21.58; Dec. 4, 21.35 .

a Pumped prior to measurement. 
Gray County--Continued

21. C. M. Davis. NW $\frac{1}{4} \mathrm{SW}$ sec. 7, T. 26 S., R. 29 W. Measuring point, $2,785.6$ feet above sea level.

Water level, in feet below measuring point, 1941

\begin{tabular}{ll|ll|ll|ll}
\hline Date & $\begin{array}{l}\text { Water } \\
\text { level }\end{array}$ & Date & $\begin{array}{l}\text { Water } \\
\text { level }\end{array}$ & Date & $\begin{array}{l}\text { Water } \\
\text { level }\end{array}$ & Date & $\begin{array}{r}\text { Water } \\
\text { level }\end{array}$ \\
\hline Mar. 18 & $\mathbf{8 9 . 7 9}$ & June 14 & $\mathbf{8 9 . 8 6}$ & Sept.10 & $\mathbf{8 9 . 6 9}$ & Nov. 15 & 89.76 \\
Apr. 26 & $\mathbf{8 9 . 8 3}$ & July 21 & $\mathbf{8 9 . 8 6}$ & Oct. 9 & $\mathbf{8 9 . 8 2}$ & Dec. 26 & $\mathbf{8 9 . 8 1}$ \\
May 24 & $\mathbf{8 9 . 8 3}$ & Aug. 9 & $\mathbf{8 9 . 8 4}$ & & & & \\
\hline
\end{tabular}

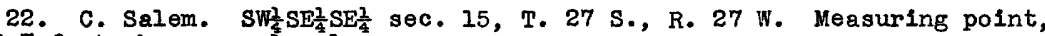
$2,706.7$ feet above sea level.

Water level, in feet below measuring point, 1941

\begin{tabular}{|c|c|c|c|c|c|c|c|}
\hline $\begin{array}{l}\text { Mar. } 18 \\
\text { Apr. } 26 \\
\text { May } 24\end{array}$ & $\begin{array}{l}126.87 \\
126.90 \\
126.93\end{array}$ & $\begin{array}{l}\text { June } 1 \\
\text { July } 2 \\
\text { Aug. }\end{array}$ & $\begin{array}{l}126.96 \\
127.25 \\
126.94\end{array}$ & $\begin{array}{l}\text { Sept.10 } \\
\text { oct. } 9\end{array}$ & & $\begin{array}{l}\text { Nov. } 15 \\
\text { Dec. } 26\end{array}$ & $\begin{array}{l}126 \\
127\end{array}$ \\
\hline
\end{tabular}

23. Pry. NWW SE sec. 24, T. $28 \mathrm{~S}$, , R. 29 W., in Montezuma. Measuring point, $2,783.0$ feet above sea level.

Water level, in feet below measuring point, 1941

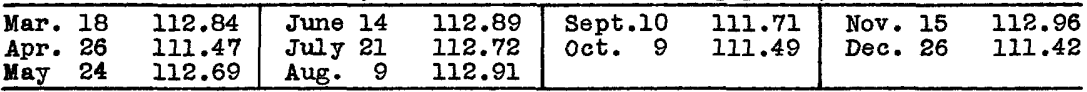

24. J. W. Herb. WW $\frac{1}{4} \mathrm{NE}_{\frac{2}{2}}^{\frac{1}{2}}$ sec. 3, T. $27 \mathrm{~S} ., \mathrm{R} .27 \mathrm{~W}$. Measuring point, $2,656.8$ feet above sea level. Water levels, in feet below measuring point, 1941: Mar. 18, 76.21; Apr. 26, 76.61; May 24, 76.53; Oct. 9, 76.48. Measurements discontinued oct. 9, 1941.

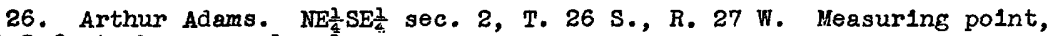
$2,694.3$ feet above sea level.

Water level, in feet below measuring point, 1941

\begin{tabular}{lrr|lr|rr|rr}
\hline Mar. 18 & 111.48 & June 13 & 110.60 & Sept.11 & 109.29 & Nov. 12 & 108.84 \\
Apr. 22 & 111.50 & $\begin{array}{l}\text { July } 11 \\
\text { May } 24\end{array}$ & 111.10 & $\begin{array}{l}109.82 \\
\text { Aug. }\end{array}$ & Oct. 6 & 109.29 & Dec. 4 & 109.31 \\
\hline
\end{tabular}

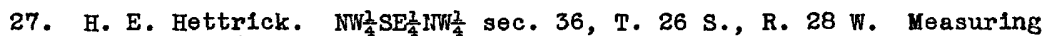
point, $2,674.4$ feet above sea level.

Water level, in feet below measuring point, 1941

\begin{tabular}{|c|c|c|c|c|c|c|c|}
\hline $\begin{array}{ll}\text { Mar. } 18 \\
\text { May } 24 \\
\text { June } 14\end{array}$ & $\begin{array}{l}57.79 \\
58.64 \\
59.16\end{array}$ & $\begin{array}{l}\text { July } 15 \\
\text { Aug. }\end{array}$ & $\begin{array}{l}59.26 \\
59.29\end{array}$ & $\begin{array}{l}\text { Sept. } 9 \\
\text { Oct. } 8\end{array}$ & $\begin{array}{l}59.20 \\
58.34\end{array}$ & $\begin{array}{l}\text { Nov. } 6 \\
\text { Dec. } 26\end{array}$ & $\begin{array}{l}58.55 \\
58.97\end{array}$ \\
\hline
\end{tabular}

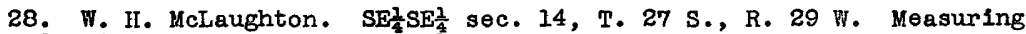
point, $2,750.7$ feet above sea level.

Water level, in feet below measuring point, 1941

\begin{tabular}{|c|c|c|c|c|c|c|c|}
\hline $\begin{array}{ll}\text { Mar. } & 18 \\
\text { Apr. } & 26 \\
\text { May } & 24\end{array}$ & $\begin{array}{l}82.29 \\
82.27 \\
82.16\end{array}$ & $\begin{array}{l}\text { June } 1 \\
\text { July } 2 \\
\text { Aug. }\end{array}$ & $\begin{array}{l}82.07 \\
82.10 \\
82.25\end{array}$ & $\begin{array}{l}\text { Sept.10 } \\
\text { Oct. } 9\end{array}$ & $\begin{array}{l}82.25 \\
82.17\end{array}$ & $\begin{array}{l}\text { Nov. } 15 \\
\text { Dec. } 26\end{array}$ & $\begin{array}{l}82.28 \\
82.30\end{array}$ \\
\hline
\end{tabular}

29. A. F. Hohner. $\mathrm{SE}_{\frac{1}{4}} \mathrm{NW} \frac{\mathrm{T}}{4}$ sec. 2, T. $28 \mathrm{~S} .$, R. $30 \mathrm{~W}$. Measuring point, $2,811.5$ feet above sea level.

Water level, in feet below measuring point, 1941

\begin{tabular}{ll|lll|ll|ll}
\hline Mar. 18 & 112.21 & May 24 & 110.84 & Sept.10 & 111.16 & Nov. 15 & 110.88 \\
Apr. 26 & 110.89 & June 14 & 110.87 & Oct. 9 & 110.90 & Dec. 26 & 110.98 \\
\hline
\end{tabular}


HAM ILTON COUNTY

By T. G. McLaughlin

Highest and lowest water levels, in feet below measuring point, in 16 wells in Hamilton County, 1941

\begin{tabular}{|c|c|c|c|c|c|c|}
\hline Well & $\begin{array}{l}\text { Highest } \\
\text { recorded } \\
\text { water level } \\
\text { (feet) }\end{array}$ & & Date & $\begin{array}{l}\text { Lowest } \\
\text { recorded } \\
\text { water level } \\
\text { (feet) }\end{array}$ & Date & \\
\hline $\begin{array}{l}1 \\
2 \\
3 \\
4 \\
5 \\
6 \\
7 \\
8 \\
9\end{array}$ & $\begin{array}{r}25.64 \\
26.68 \\
12.51 \\
16.88 \\
15.70 \\
52.05 \\
45.18 \\
147.18 \\
190.18\end{array}$ & $\begin{array}{l}\text { Nov. } \\
\text { July } \\
\text { July } \\
\text { June } \\
\text { June } \\
\text { June } \\
\text { Nov. } \\
\text { Dec. } \\
\text { (Dec. } \\
\text { (Nov. }\end{array}$ & $\begin{array}{l}29,1941 \\
18,1941 \\
17,1941 \\
22,1940 \\
22,1940 \\
7,1941 \\
16,1941 \\
23,1940 \\
23,1940) \\
16,1941)\end{array}$ & $\begin{array}{r}26.93 \\
28.14 \\
14.92 \\
21.67 \\
17.88 \\
55.04 \\
46.00 \\
147.97 \\
190.60\end{array}$ & $\begin{array}{l}\text { May 15, } \\
\text { Nov. 22, } \\
\text { Nov. 16, } \\
\text { Dec. 19, } \\
\text { Nov. 16, } \\
\text { Nov. 16, } \\
\text { Nov. 27, } \\
\text { Mar. 15, } \\
\text { Oct. 10, }\end{array}$ & $\begin{array}{l}1940 \\
1940 \\
1939 \\
1939 \\
1939 \\
1939 \\
1940 \\
1941 \\
1939\end{array}$ \\
\hline $\begin{array}{l}13 \\
16 \\
17\end{array}$ & $\begin{array}{l}56.61 \\
84.92 \\
42.86\end{array}$ & $\begin{array}{l}\text { Dec. } \\
\text { Oct. } \\
\text { Aug. }\end{array}$ & $\begin{array}{l}16,1941 \\
28,1941 \\
24,1941\end{array}$ & $\begin{array}{l}57.52 \\
85.83 \\
44.48\end{array}$ & $\begin{array}{l}\text { June 17, } \\
\text { Feb. 19, } \\
\text { (May 15, } \\
\text { (July 18, }\end{array}$ & $\begin{array}{l}1940 \\
1940 \\
1940)\end{array}$ \\
\hline $\begin{array}{l}19 \\
22 \\
27 \\
28\end{array}$ & $\begin{array}{l}128.97 \\
109.18 \\
171.16 \\
221.48\end{array}$ & $\begin{array}{l}\text { May } \\
\text { Oct. } \\
\text { May } \\
\text { Apr. }\end{array}$ & $\begin{aligned} 15, & 1940 \\
20, & 1941 \\
6, & 1941 \\
20, & 1940\end{aligned}$ & $\begin{array}{l}129.23 \\
118.16 \\
171.78 \\
222.46\end{array}$ & $\begin{array}{l}\text { Feb. 19, } \\
\text { May } 1, \\
\text { Nov. } 16, \\
\text { Dec. } 31 \text {, }\end{array}$ & $\begin{array}{l}1940 \\
1941 \\
1939 \\
1941\end{array}$ \\
\hline
\end{tabular}

Net changes in water level in 1941, and net changes in water level for the period of record in 16 wells in Hamilton county

\begin{tabular}{|c|c|c|c|c|}
\hline Well & $\begin{array}{l}\text { Length } \\
\text { of record } \\
\text { (years) }\end{array}$ & $\begin{array}{l}\text { Difference } \\
\text { between highest } \\
\text { and lowest } \\
\text { water levels } \\
\text { (feet) }\end{array}$ & $\begin{array}{l}\text { Net rise (+) } \\
\text { or net decline } \\
(-) \text { in } 1941 \\
(\text { feet })\end{array}$ & $\begin{array}{l}\text { Net rise (t) } \\
\text { or net decline } \\
\text { (-) for period } \\
\text { of record } \\
\text { (feet) }\end{array}$ \\
\hline $\begin{array}{r}1 \\
2 \\
3 \\
4 \\
5 \\
6 \\
7 \\
8 \\
9 \\
13 \\
16 \\
17 \\
19 \\
22 \\
27 \\
28\end{array}$ & $\begin{array}{l}2 \\
2 \\
2 \\
2 \\
2 \\
2 \\
2 \\
2 \\
2 \\
2 \\
2 \\
2 \\
2 \\
2 \\
2 \\
2\end{array}$ & $\begin{array}{r}1.29 \\
1.46 \\
2.41 \\
4.79 \\
2.18 \\
2.99 \\
.82 \\
.79 \\
.42 \\
.91 \\
.91 \\
1.62 \\
.26 \\
8.98 \\
.62 \\
.98\end{array}$ & $\begin{array}{r}+1.14 \\
+.85 \\
+1.93 \\
+1.62 \\
+.02 \\
+.91 \\
+.20 \\
-.19 \\
-.23 \\
+.54 \\
-.04 \\
+1.43 \\
-.11 \\
+1.14 \\
-.03 \\
-.11\end{array}$ & $\begin{array}{r}+0.51 \\
+.51 \\
+1.46 \\
+1.62 \\
+.58 \\
+2.23 \\
+.57 \\
-.25 \\
-.11 \\
+.63 \\
+.80 \\
+1.27 \\
+.02 \\
+6.84 \\
-.01 \\
-.69\end{array}$ \\
\hline
\end{tabular}

Water-level measurements

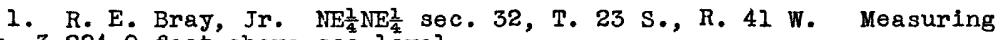
point, $3,284.9$ feet above sea level.

Water level, in feet below measuring point, 1941

\begin{tabular}{|c|c|c|c|c|c|c|c|}
\hline Date & $\begin{array}{l}\text { Water } \\
\text { level } \\
\end{array}$ & Date & $\begin{array}{l}\text { Water } \\
\text { level } \\
\end{array}$ & Date & $\begin{array}{l}\text { Water } \\
\text { level }\end{array}$ & Date & $\begin{array}{l}\text { Water } \\
\text { level } \\
\end{array}$ \\
\hline $\begin{array}{ll}\text { Feb. } 25 \\
\text { Apr. } 18\end{array}$ & $\begin{array}{l}26.78 \\
26.17\end{array}$ & $\begin{array}{lr}\text { May } & 1 \\
\text { Aug. } & 24 \\
\end{array}$ & $\begin{array}{l}26.70 \\
25.63\end{array}$ & $\begin{array}{ll}\text { Oct. } 20 \\
\text { Nov. } 10\end{array}$ & $\begin{array}{l}26.01 \\
25.94\end{array}$ & Nov. 29 & 25.64 \\
\hline
\end{tabular}


Hamilton County--Continued

2. R. Holdren. NW $N W \frac{1}{4}$ sec. $23, T .23$ S., R. 43 W. Measuring point, $3,369.2$ feet above sea level.

Viater level, in feet below measuring point, 1941

\begin{tabular}{ll|ll|ll|ll}
\hline Date & $\begin{array}{l}\text { Water } \\
\text { level }\end{array}$ & Date & $\begin{array}{l}\text { Water } \\
\text { level }\end{array}$ & Date & $\begin{array}{l}\text { Water } \\
\text { level }\end{array}$ & Date & $\begin{array}{r}\text { Water } \\
\text { level }\end{array}$ \\
\hline Jan. 29 & 27.55 & June 12 & 27.16 & Sept.18 & 26.98 & Nov. 10 & 26.36 \\
Feb. 25 & 27.52 & July 18 & 26.68 & Oct.20 & 27.18 & Nov. 29 & 26.70 \\
Apr. 18 & 27.42 & Aug. 24 & 26.95 & & & & \\
\hline
\end{tabular}

3. B. Rees. SW $W_{\frac{1}{4}}$ sec. 8, T. 24 S., R. 40 W. Measuring point, $3,223.6$ feet above sea level.

Water level, in feet below measuring point, 1941

\begin{tabular}{|c|c|c|c|c|c|c|c|}
\hline $\begin{array}{l}\text { Jan. } 29 \\
\text { Feb. } 25 \\
\text { Mar. } 15\end{array}$ & $\begin{array}{l}14.89 \\
14.90 \\
14.89\end{array}$ & $\begin{array}{l}\text { Apr. } 2 \\
\text { May } \\
\text { June }\end{array}$ & $\begin{array}{l}14.89 \\
14.85 \\
12.93\end{array}$ & $\begin{array}{l}\text { July } 17 \\
\text { Aug. } 24 \\
\text { Sept. } 22\end{array}$ & $\begin{array}{l}12.51 \\
13.16 \\
12.79\end{array}$ & $\begin{array}{ll}\text { Oct. } 20 \\
\text { Nov. } 10 \\
\text { Nov. } 29\end{array}$ & $\begin{array}{l}13 \\
13 \\
12\end{array}$ \\
\hline
\end{tabular}

4. Continental Life Insurance Co. Center sec. 14, T. 24 S., R. 40 W. Measuring point, 3,204.7 feet above sea level.

Water level, in feet below measuring point, 1941

\begin{tabular}{ll|ll|l|ll|ll}
\hline Jan. 29 & 21.09 & Apr. 23 & 21.47 & July 17 & 18.81 & Oct. 20 & 18.63 \\
Feb. 25 & 21.27 & May & 1 & 21.60 & Aug. 24 & 19.37 & Nov. 10 & 18.97 \\
Mar. 15 & 21.22 & June & 7 & 18.59 & Sept.22 & 18.99 & Nov. 29 & 19.47 \\
\hline
\end{tabular}

5. W. A. Dunn, $\mathrm{SE}_{4}^{\frac{1}{4}} \mathrm{NE}_{\frac{1}{4}}^{\frac{1}{4}} \mathrm{sec} .20, \mathrm{~T} .24 \mathrm{~S} ., \mathrm{R} .39$ W. Measuring point, $3,172.2$ feet above sea level.

Water level, in feet below measuring point, 1941

\begin{tabular}{ll|lr|lrr|rr}
\hline Feb. 25 & 16.75 & Apr. 23 & 16.80 & Aug. 24 & 16.81 & Nov. 10 & 16.73 \\
Mar. 15 & 16.70 & May & 1 & 16.80 & 0ct. 20 & 16.75 & & \\
\hline
\end{tabular}

6. Bell Heinlein. SW $\frac{1}{4} \mathrm{SE} \frac{1}{4}$ sec. 24, T. 24 S., R. 39 W. Measuring point, $3,181.8$ feet above sea level.

Water level, in feet below measuring point, 1941

\begin{tabular}{lr|lr|lr|lll}
\hline Jan. 29 & 53.42 & Apr. 23 & 53.27 & July 17 & 52.43 & Oct. 20 & 52.97 \\
Feb. 25 & 53.36 & May & 1 & 53.08 & Aug. 24 & 52.80 & Nov. 10 & 52.67 \\
Mar. 15 & 53.35 & June & 7 & 52.05 & Sept.22 & 53.18 & Nov. 29 & 52.51 \\
\hline
\end{tabular}

7. I. E. Martin. NW $\frac{1}{4} W_{\frac{1}{4}}^{\frac{1}{4}}$ sec. 16, T. 23 S., R. 40 W.

Water level, in feet below measuring point, 1941

\begin{tabular}{ll|lrr|lll|ll}
\hline Feb. 25 & 45.40 & May & 5 & 45.50 & Aug. 24 & 45.42 & Nov. 16 & 45.18 \\
Mar. 15 & 45.48 & June 7 & 45.57 & Oct. 28 & 45.43 & Dec. 16 & 45.20 \\
Apr. 23 & 45.52 & July 17 & 45.46 & & & & & \\
\hline
\end{tabular}

8. R. D. Woodman. SW $\frac{1}{2} \mathrm{NW}_{\frac{1}{4}} \mathrm{sec} .21, \mathrm{~T}, 22 \mathrm{~S}, \mathrm{R}, 40 \mathrm{~W}$. Measuring point, $3,513.8$ feet above séa level.

Water level, in feet below measuring point, 1941

\begin{tabular}{ll|lrl|ll|ll}
\hline Feb. 25 & 147.28 & May & 5 & 147.56 & Aug. 24 & 147.33 & Nov. 16 & 147.25 \\
Mar. 15 & 147.97 & June & 7 & 147.35 & Sept.22 & 147.35 & Dec. 16 & 147.47 \\
Apr. 23 & 147.44 & July & 17 & 147.31 & Oct. 28 & 147.53 & &
\end{tabular}

9. Inez Dikeman. $\mathrm{SE}_{\frac{1}{4}} \mathrm{SE} \frac{\mathrm{l}}{4} \mathrm{sec} .21, \mathrm{~T} .21 \mathrm{~S}, \mathrm{R}, 40 \mathrm{~W}$.

Water level, in feet below measuring point, 1941

\begin{tabular}{ll|lr|lll|lll}
\hline Mar. 15 & 190.20 & May 5 & 190.32 & Aug. 24 & 190.31 & Nov. 16 & 190.18 \\
Apr. 23 & 190.44 & July 17 & 190.29 & Oct. 28 & 190.44 & Dec. 16 & 190.43 \\
\hline
\end{tabular}

11. M. Williamson. $N_{\frac{1}{4}}^{\frac{1}{4}} \mathrm{NW}_{\frac{1}{4}} \mathrm{sec} .18, \mathrm{~T}, 26 \mathrm{~S}$, R. $40 \mathrm{~W}$. Measurements discontinued in January 1941 .

12. I. E. Martin. $\mathrm{NE}_{4}^{\frac{1}{4}} \mathrm{SW} \frac{1}{4}$ sec. 2, T. $22 \mathrm{~S}$, R. 41 W. Measurements discontinued in January 1941 . 
Hamilton County--Continued

13. Carl Lewis. SE $\mathrm{SWW}_{\frac{1}{4}}^{\frac{1}{4}}$ sec. 13, T. $21 \mathrm{~S} ., \mathrm{R}, 42 \mathrm{~W}$.

Water level, in feet below measuring point, 1941

\begin{tabular}{lrr|lr|ll|ll}
\hline Date & $\begin{array}{l}\text { Water } \\
\text { level }\end{array}$ & Date & $\begin{array}{l}\text { Water } \\
\text { level }\end{array}$ & Date & $\begin{array}{l}\text { Water } \\
\text { level }\end{array}$ & Date & $\begin{array}{r}\text { Water } \\
\text { level }\end{array}$ \\
\hline Mar. 15 & $\mathbf{5 7 . 1 5}$ & June 7 & $\mathbf{5 7 . 2 1}$ & Aug. 24 & $\mathbf{5 6 . 8 1}$ & Nov. 16 & $\mathbf{5 6 . 6 8}$ \\
Apr. 23 & $\mathbf{5 7 . 2 1}$ & July 17 & $\mathbf{5 6 . 6 8}$ & Oct. 28 & $\mathbf{5 6 . 7 2}$ & Dec. 16 & $\mathbf{5 6 . 6 1}$ \\
May 5 & $\mathbf{5 7 . 2 4}$ & & & & & & & \\
\hline
\end{tabular}

16. Chas. H. Miller. SE $\frac{1}{4} \mathrm{SW}_{4}^{\frac{1}{4}}$ sec. 22, T. 25 S., R. 39 W.

Water level, in feet below measuring point, 1941

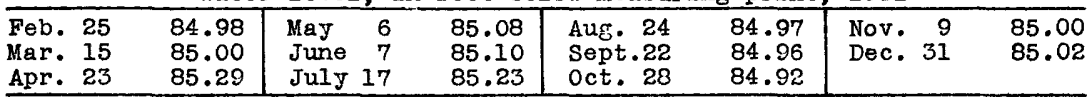

17. Thos. A. Wells. NW $W_{4}^{\frac{1}{4}} W_{\frac{1}{4}}^{\frac{1}{4}}$ sec. 11, T. 25 S., R. 39 W.

Water level, in feet below measuring point, 1941

\begin{tabular}{ll|lll|ll|ll|}
\hline Feb. 25 & 44.42 & May & 6 & 44.44 & Aug. 24 & 42.86 & Nov. 9 & 42.96 \\
Mar. 15 & 44.42 & June & 7 & 44.12 & Oct. 28 & 43.15 & Dec. 31 & 42.99 \\
Apr. 23 & 44.44 & & & & & & & \\
\hline
\end{tabular}

19. W. E. Bereman. NW $\frac{1}{4} \mathrm{NE} \frac{1}{4}$ sec. 26, T. 26 S., R. 39 W. Measuring point, 3,298.6 feet above sea level.

Water level, in feet below measuring point, 1941

\begin{tabular}{rr|lrr|ll|lr}
\hline Feb. 25 & 129.00 & May & 6 & 129.13 & Aug. 24 & 129.11 & Nov. 9 & 129.09 \\
Mar. 15 & 129.03 & June & 7 & 129.18 & Sept.22 & 129.16 & Dec.,31 & 129.11 \\
Apr. 23 & 129.22 & July & 17 & 129.15 & Oct. 28 & 129.12 & & \\
\hline
\end{tabular}

20. Alpha H. Bennett. SW $W_{4}^{2}$ sec. 2, T. 24 S., R. 43 W. Measurements discontinued in January 1941.

22. T. J. Crist. NW $W_{4}^{2} W_{4}^{2}$ sec. 26, T. 24 S., R. 43 W. Measuring point, $3,489.3$ feet above sea level.

Water level, in feet below measuring point, 1941

\begin{tabular}{lr|lr|lr|lll}
\hline Jan. 29 & 110.47 & Apr. 18 & 116.78 & July 18 & 113.25 & Oct. 20 & 109.18 \\
Feb. 25 & 112.41 & May & 1 & 118.16 & Aug. 24 & 113.76 & Nov. 10 & 109.26 \\
Mar. 15 & 114.51 & June & 7 & 117.56 & Sept.18 & 110.48 & Nov. 29 & 109.33 \\
\hline
\end{tabular}

24. Eugene Scherick. NE $\frac{1}{4} N W_{4}^{\frac{1}{4}}$ sec. 5, T. 26 S., R. 42 W. Measurements discontinued in January 1941 .

26. J. C. Kitch. NW $N W_{4}^{2}$ sec. 23 , T. 26 S., R. 42 W. Measurements discontinued in January 1941 .

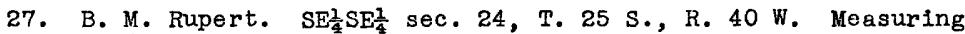
point, $3,361.4$ feet above sea level.

Water level, in feet below measuring point, 1941

\begin{tabular}{ll|lll|ll|lll}
\hline Mar. 15 & 171.28 & May & 6 & 171.16 & July 17 & 171.42 & Nov. 9 & 171.29 \\
Apr. 23 & 171.61 & June & 7 & 171.18 & Oct. 28 & 171.28 & Dec. 31 & 171.31 \\
\hline
\end{tabular}

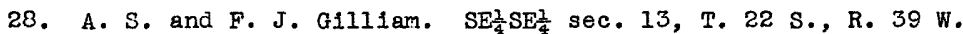
Measuring point, 3,410.5 feet above sea level. Water levels, in feet below measuring point; 1941: Apr. 23, 222.35; Oct. 18, 222.40; Íov. 10, 222.43; Dec. 31, 222.46. 
HARVEY COUNTY

By C. C. Williams and G. H, von Hein

Highest and lowest water levels, in feet below measuring point, in 25 wells in Harvey County that are not affected by pumping, 1941

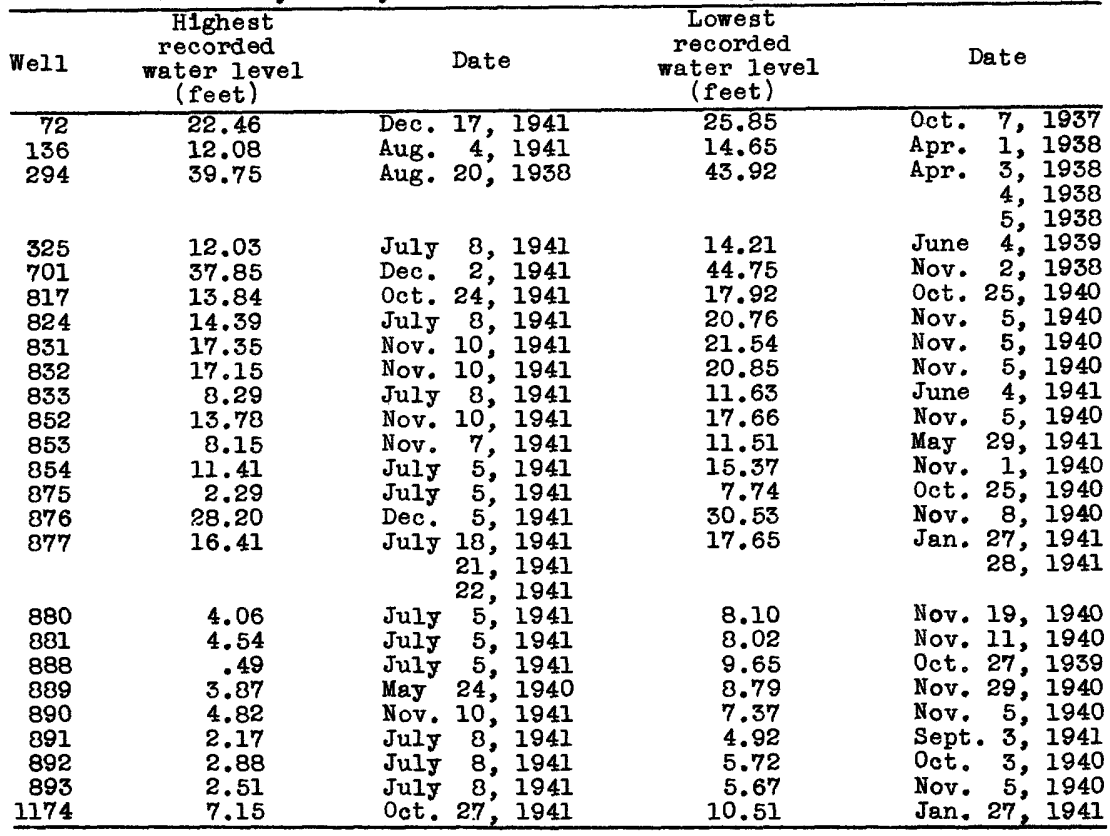

Net changes in water level in 1941, and net changes in water level for the perlod of record in 25 wells in Harvey County that are not affected by pumping

\begin{tabular}{ccccc}
\hline We11 & $\begin{array}{c}\text { Length } \\
\text { of record } \\
\text { (yearg) }\end{array}$ & $\begin{array}{c}\text { Difference } \\
\text { between highest } \\
\text { and lowest } \\
\text { water levels } \\
\text { (feet) }\end{array}$ & $\begin{array}{c}\text { Net rise } \\
\text { or net decline } \\
(-) \text { in 1941 } \\
\text { (feet) }\end{array}$ & $\begin{array}{c}\text { Net rise } \\
\text { or net decline } \\
\text { (-) for period } \\
\text { of record } \\
\text { (feet) }\end{array}$ \\
\hline 72 & 4 & 3.39 & +1.81 & +3.39 \\
136 & 4 & 2.57 & +.79 & +1.93 \\
294 & 4 & 4.17 & +3.06 & +3.81 \\
325 & 4 & 2.18 & +2.04 & +1.85 \\
701 & 3 & 6.90 & +.40 & +2.64 \\
817 & 3 & 4.08 & +2.30 & +1.05 \\
824 & 3 & 6.37 & +5.14 & +1.87 \\
831 & 3 & 4.19 & +2.96 & +1.38 \\
832 & 3 & 3.70 & +2.45 & +.91 \\
833 & 3 & 3.34 & +2.17 & +34 \\
852 & 3 & 3.88 & +2.86 & +1.40 \\
853 & 3 & 3.36 & +2.45 & +1.13 \\
854 & 3 & 3.96 & +3.01 & +1.01 \\
875 & 3 & 5.45 & +1.62 & +62 \\
876 & 3 & 2.33 & +1.01 & +.05 \\
877 & 3 & 1.24 & +.96 & +.95 \\
\hline
\end{tabular}




\section{Harvey County--Continued}

Net changes in water level in 1941, and net changes in water level for the period of record in 25 wells in Harvey County that are not

affected by pumping

\begin{tabular}{|c|c|c|c|c|}
\hline We11 & $\begin{array}{l}\text { Length } \\
\text { of record } \\
\text { (years) }\end{array}$ & $\begin{array}{l}\text { Difference } \\
\text { between highest } \\
\text { and lowest } \\
\text { water levels } \\
\text { (feet) }\end{array}$ & $\begin{array}{l}\text { Net rise (t) } \\
\text { or net decilne } \\
(-) \text { in } 1941 \\
(\text { feet })\end{array}$ & $\begin{array}{l}\text { Net rise (t) } \\
\text { or net decline } \\
\text { (-) for period } \\
\text { of record } \\
\text { (feet) }\end{array}$ \\
\hline $\begin{array}{r}880 \\
881 \\
888 \\
889 \\
890 \\
891 \\
892 \\
893 \\
1174\end{array}$ & $\begin{array}{l}3 \\
3 \\
3 \\
3 \\
3 \\
3 \\
3 \\
3 \\
1\end{array}$ & $\begin{array}{l}4.04 \\
3.48 \\
9.16 \\
4.92 \\
2.55 \\
2.75 \\
2.84 \\
3.16 \\
3.36\end{array}$ & $\begin{array}{l}+1.21 \\
+1.01 \\
+2.89 \\
+2.34 \\
+2.10 \\
+1.36 \\
+1.58 \\
+1.79 \\
+2.04\end{array}$ & $\begin{array}{l}+1.05 \\
+.65 \\
+.86 \\
-.70 \\
+.73 \\
+2.00 \\
+1.99 \\
+1.69 \\
+2.11\end{array}$ \\
\hline
\end{tabular}

Highest and lowest water levels, in feet below measuring point, in 94 wells in Harvey County that are pumped or affected by pumping, 1941

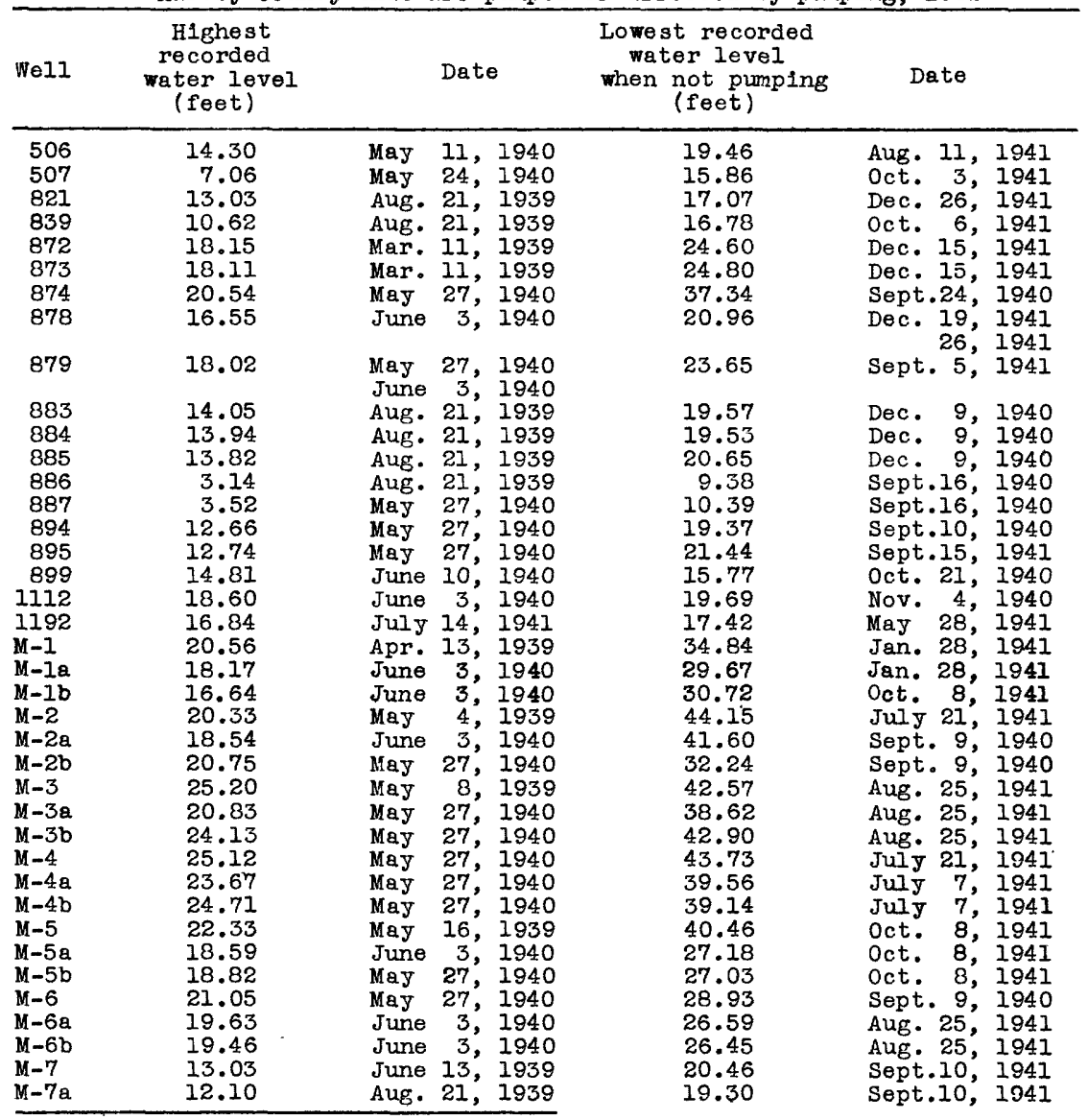


Harvey County--Continued

Highest and lowest water levels, in feet below measuring point, in 94 wells in Harvey County that are pumped or affected by pumpins, 1941--Continued

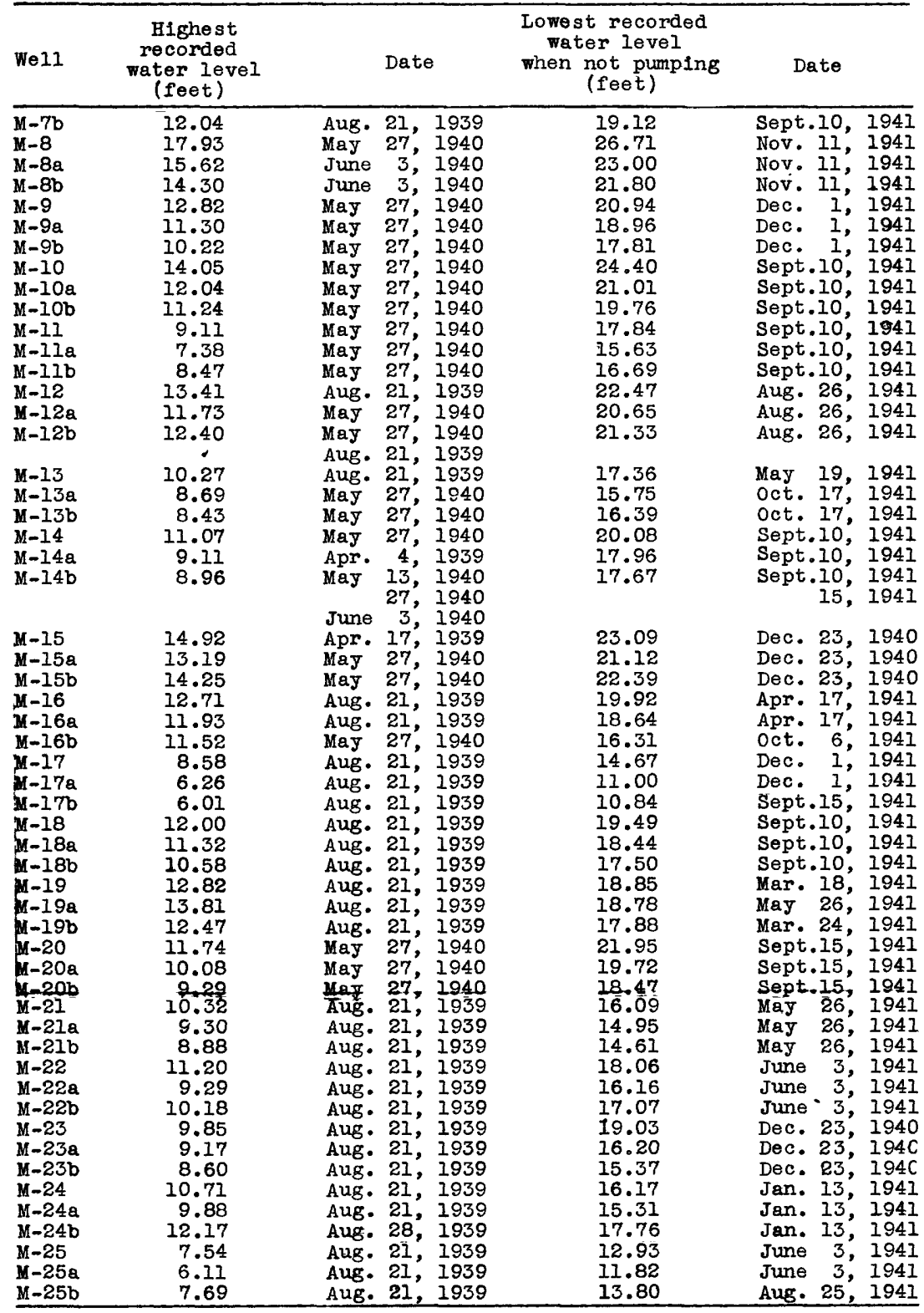




\section{Harvey County--Continued}

Net changes in water level in 1941, and net changes in water level for the period of record in 94 wells in Harvey County that are pumped or affected by pumping--Continued

\begin{tabular}{|c|c|c|c|c|}
\hline Well & $\begin{array}{l}\text { Length } \\
\text { of record } \\
\text { (years) }\end{array}$ & $\begin{array}{l}\text { Difference } \\
\text { between highest } \\
\text { and lowest } \\
\text { water levels } \\
\text { (feet) }\end{array}$ & $\begin{array}{l}\text { Net rise (t) } \\
\text { or net decline } \\
\text { (-) in 1941 } \\
\text { (feet)a }\end{array}$ & $\begin{array}{l}\text { Net rise (t) } \\
\text { or net decline } \\
\text { (-) for period } \\
\text { of record. } \\
\text { (feet)a }\end{array}$ \\
\hline $\begin{array}{c}506 \\
507 \\
821 \\
839 \\
872 \\
873 \\
874 \\
878 \\
879 \\
883 \\
884 \\
885 \\
886 \\
887 \\
894 \\
895 \\
899 \\
1112 \\
1192 \\
M-1 \\
M-1 a \\
M-1 b \\
M-2 \\
M-2 a \\
M-2 b \\
M-3 \\
M-3 a \\
M-3 b \\
M-4 \\
M-4 a \\
M-4 b \\
M-5 \\
M-5 a \\
M-5 b \\
M-6 \\
M-6 a \\
M-6 b \\
M-7 \\
M-11 a \\
M-12 b \\
M-7 b \\
M-8 \\
M-8 a \\
M-8 b \\
M-9 \\
M-9 a \\
M-9 b \\
M-10 \\
M-10 a \\
M-12\end{array}$ & $\begin{array}{l}3 \\
3 \\
3 \\
3 \\
3 \\
3 \\
3 \\
3 \\
3 \\
3 \\
3 \\
3 \\
3 \\
3 \\
3 \\
3 \\
3 \\
2 \\
1 \\
3 \\
3 \\
3 \\
3 \\
3 \\
3 \\
3 \\
3 \\
3 \\
3 \\
3 \\
3 \\
3 \\
3 \\
3 \\
3 \\
3 \\
3 \\
3 \\
3 \\
3 \\
3 \\
3 \\
3 \\
3 \\
3 \\
3 \\
3 \\
3 \\
3 \\
3 \\
3 \\
3\end{array}$ & 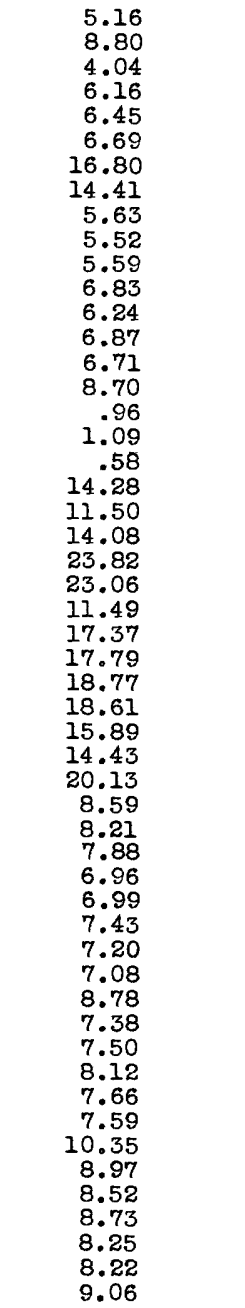 & $\begin{array}{r}+2.22 \\
+2.45 \\
-1.88 \\
+1.12 \\
-2.35 \\
-2.53 \\
-3.29 \\
-1.65 \\
-1.98 \\
-.87 \\
-.95 \\
-2.22 \\
-.42 \\
+.31 \\
-1.67 \\
-2.00 \\
-1.07 \\
+.56 \\
+.52 \\
+10.77 \\
+8.44 \\
+8.14 \\
+7.37 \\
+10.63 \\
+7.21 \\
+4.41 \\
+4.83 \\
+4.58 \\
+5.02 \\
+5.22 \\
+4.81 \\
+1.36 \\
-1.75 \\
-1.82 \\
-1.97 \\
-2.46 \\
-2.29 \\
+.49 \\
+.49 \\
+.50 \\
-3.07 \\
-2.11 \\
-2.56 \\
-3.07 \\
-2.77 \\
-2.71 \\
-1.96 \\
-2.23 \\
-2.39 \\
-2.49 \\
-2.37 \\
-2.47 \\
-. .84\end{array}$ & $\begin{array}{r}+0.92 \\
-1.40 \\
-3.81 \\
-2.24 \\
-6.40 \\
-6.66 \\
-8.62 \\
-4.18 \\
-5.09 \\
-3.76 \\
-3.83 \\
-5.03 \\
-4.27 \\
-4.86 \\
-4.32 \\
-5.41 \\
-.42 \\
+.12 \\
+.52 \\
-3.51 \\
-5.21 \\
-2.56 \\
-9.05 \\
-3.01 \\
-2.70 \\
-5.55 \\
-1.37 \\
-.85 \\
-4.73 \\
-1.50 \\
-3.03 \\
-4.51 \\
-5.19 \\
-5.17 \\
-3.08 \\
-5.91 \\
-5.70 \\
-2.97 \\
-3.01 \\
-3.11 \\
-5.59 \\
-5.43 \\
-5.94 \\
-7.44 \\
-6.77 \\
-6.54 \\
-6.46 \\
-7.08 \\
-7.19 \\
-6.88 \\
-5.94 \\
-5.92 \\
-5.66\end{array}$ \\
\hline
\end{tabular}

a Calculated from first measurement not affected by pumping to last measurement not affected by pumping.

b Through June 2,1941 . 
Harvey County--Continued

Net changes in water level in 1941, and net changes in water level for the period of record in 94 wells in Harvey County that are pumped or affected by pumping--Continued

\begin{tabular}{|c|c|c|c|c|}
\hline Well & $\begin{array}{l}\text { Length } \\
\text { of record } \\
\text { (years) }\end{array}$ & $\begin{array}{l}\text { Difference } \\
\text { between highest } \\
\text { and lowest } \\
\text { water levels } \\
\text { (feet) }\end{array}$ & $\begin{array}{l}\text { Net rise (t) } \\
\text { or net decline } \\
(-) \text { in } 194] \\
(\text { feet }) \text { ] }\end{array}$ & $\begin{array}{l}\text { Wet rise ( }+) \\
\text { or net decline } \\
\text { (-) E period } \\
\text { of record. } \\
\text { (feet) }\end{array}$ \\
\hline $\begin{array}{l}M-12 a \\
M-12 b \\
M-13 \\
M-13 a \\
M-13 b \\
M-14 \\
M-14 a \\
M-14 b \\
M-15 \\
M-15 a \\
M-15 b \\
M-16 \\
M-16 a \\
M-16 b \\
M-17 \\
M-17 a \\
M-17 b \\
M-18 \\
M-18 a \\
M-18 b \\
M-19 \\
M-19 a \\
M-19 b \\
M-20 \\
M-20 a \\
M-20 b \\
M-21 \\
M-21 a \\
M-21 b \\
M-22 \\
M-22 a \\
M-22 b \\
M-23 \\
M-23 a \\
M-23 b \\
M-24 \\
M-24 a \\
M-24 b \\
M-25 \\
M-25 a \\
M-25 b\end{array}$ & $\begin{array}{l}3 \\
3 \\
3 \\
3 \\
3 \\
3 \\
3 \\
3 \\
3 \\
3 \\
3 \\
3 \\
3 \\
3 \\
3 \\
3 \\
3 \\
3 \\
3 \\
3 \\
3 \\
3 \\
3 \\
3 \\
3 \\
3 \\
3 \\
3 \\
3 \\
3 \\
3 \\
3 \\
3 \\
3 \\
3 \\
3 \\
3 \\
3 \\
3 \\
3 \\
3\end{array}$ & $\begin{array}{r}8.92 \\
8.93 \\
7.09 \\
7.06 \\
7.96 \\
9.01 \\
8.85 \\
8.71 \\
8.17 \\
7.93 \\
8.14 \\
7.21 \\
6.71 \\
4.79 \\
6.09 \\
4.74 \\
4.83 \\
7.49 \\
7.12 \\
6.92 \\
6.03 \\
4.97 \\
5.41 \\
10.21 \\
9.64 \\
9.18 \\
5.77 \\
5.65 \\
5.73 \\
6.86 \\
6.87 \\
6.89 \\
9.18 \\
7.03 \\
6.77 \\
5.46 \\
5.43 \\
5.59 \\
5.39 \\
5.71 \\
6.11\end{array}$ & $\begin{array}{r}-.89 \\
-.82 \\
-1.49 \\
-1.74 \\
-2.14 \\
-.56 \\
-.92 \\
-1.40 \\
-1.96 \\
-1.94 \\
-1.92 \\
+.56 \\
+.37 \\
+.07 \\
-.44 \\
-.28 \\
-.41 \\
+.23 \\
+.20 \\
+.40 \\
+.25 \\
+.36 \\
+.31 \\
-2.88 \\
-2.97 \\
-2.83 \\
+.61 \\
+.51 \\
+.43 \\
+.93 \\
+.99 \\
+.96 \\
+1.42 \\
+1.23 \\
+1.29 \\
+1.50 \\
+1.53 \\
+1.42 \\
+1.52 \\
+1.37 \\
+1.34\end{array}$ & $\begin{array}{l}-5.59 \\
-5.57 \\
-5.16 \\
-5.45 \\
-6.23 \\
-4.86 \\
-7.14 \\
-7.56 \\
-6.58 \\
-5.76 \\
-5.75 \\
-4.80 \\
-4.49 \\
-3.32 \\
-3.32 \\
-1.87 \\
-2.49 \\
-3.57 \\
-3.33 \\
-2.95 \\
-3.16 \\
-2.03 \\
-2.29 \\
-7.00 \\
-6.94 \\
-6.74 \\
-2.05 \\
-2.01 \\
-1.98 \\
-1.67 \\
-1.73 \\
-1.85 \\
-1.23 \\
-1.26 \\
-1.26 \\
-1.46 \\
-1.35 \\
-1.54 \\
-.74 \\
-.95 \\
-.98\end{array}$ \\
\hline
\end{tabular}

Well descriptions and water-level measurements

72. Anna Hertzler. SW $W_{4}^{\frac{1}{4}} \mathrm{NE}_{\frac{1}{4}} \mathrm{SE}_{4}^{\mathrm{l}} \mathrm{sec} .16, \mathrm{~T}, 22 \mathrm{~S} ., \mathrm{R} .1 \mathrm{~W}$.

Water level, in feet below measuring point, 1941

\begin{tabular}{lll|lll|ll|ll}
\hline Date & & $\begin{array}{l}\text { Water } \\
\text { level }\end{array}$ & Date & & $\begin{array}{l}\text { Water } \\
\text { level }\end{array}$ & Date & $\begin{array}{l}\text { Water } \\
\text { level }\end{array}$ & Date & $\begin{array}{l}\text { Water } \\
\text { level }\end{array}$ \\
\hline Jan. 3 & 24.27 & Apr & 1 & 24.13 & July 8 & 23.51 & Oct. 13 & 23.54 \\
Feb. 4 & 24.23 & May & 2 & 24.11 & July 23 & 23.71 & Dec. 17 & 22.46 \\
Mar. 4 & 24.34 & June 4 & 24.34 & & & & & \\
\hline
\end{tabular}

136. Ada M. Day. NW cor. sec. 19, T. 23 S., R. 3 W.

Water level, in feet below measuring point, 1941

\begin{tabular}{lll|lll|lll|lr}
\hline Jan. & 2 & 13.13 & Apr. & 1 & 13.21 & July & 8 & 12.25 & Oct. 13 & 12.39 \\
Feb. & 4 & 13.21 & May & 1 & 13.21 & Aug. & 4 & 12.08 & Nov. 10 & 12.37 \\
Mar. & 4 & 13.29 & June & 4 & 13.21 & Sept. 3 & 12.19 & Dec. & 2 & 12.34 \\
\hline
\end{tabular}

a Calculated from first measurement not affected by pumping to last measurement not affected by pumping. 
Harvey County--Continued

294. Owner, J. B. Schmidt; lessee, Hollow 011 Co., SE $\frac{1}{4}$ NW $\frac{1}{4}$ sec. 17, T. $22 \mathrm{~S} ., \mathrm{R}, 3 \mathrm{~W}$.

Lowest daily water level, in feet below measuring point, 1941

\begin{tabular}{|c|c|c|c|c|c|c|c|c|c|c|}
\hline Day & Jan & Feb. & Mar. & Apr. & May & June & July & Sept. & Oct. & Dec \\
\hline 7 & 42.92 & 42.91 & 42.81 & 42.40 & 42.45 & 42.49 & & & & \\
\hline 2 & & 42. & 42.77 & & & 17 & & & & \\
\hline 3 & & 42. & 42.73 & & 12 & 15 & • & - & $\cdots$ & \\
\hline 4 & & 42. & 42.77 & & 2.42 & 42.44 & $\cdots$ & - & $\cdots$ & \\
\hline 5 & & 42.8 & 42.78 & & .40 & 42 & $\ldots$ & $\cdots$ & $\cdots$ & $\bullet$ \\
\hline 6 & & 42 & 42.76 & 42 & 2.39 & 42. & $\cdots \cdots$ & $\cdots$ & $\cdots$ & - \\
\hline 7 & & 42. & 42.75 & & & & $\ddot{0} \ddot{\circ}$ & $\cdots$ & • & \\
\hline 8 & & 42 & & & & & 9 & & & \\
\hline 9 & & 42. & $\ddot{\cdots} \cdot$ & 4 & 2 & & & & & \\
\hline 0 & & $\begin{array}{l}42 . \\
42 .\end{array}$ & $\because$ & & & & & & & \\
\hline 2 & & 42 . & & & & & & & & \\
\hline 3 & & 42. & & 42 & 42 & $4]$ & & & & \\
\hline 4 & & 42. & & 42 & 42 & 41 & & & & \\
\hline & & 42. & & 4 & 42 & 41 & & & & \\
\hline 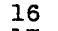 & & 42. & & & & & & $\cdots$ & & \\
\hline 7 & & 42. & 42.56 & & & & & & & \\
\hline 8 & & 42.79 & 42.56 & & & & & & $\cdots$ & \\
\hline & & 42.82 & $\begin{array}{l}42.54 \\
42.51\end{array}$ & & & 41 & - & 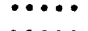 & $\cdots$ & \\
\hline & 48 & 42 & $\begin{array}{l}42.51 \\
42.50\end{array}$ & & 42 & $\mathrm{a} 41.45$ & & $\bullet$ & & \\
\hline & & $\begin{array}{l}42 . \\
42 .\end{array}$ & $\begin{array}{l}42.50 \\
42.50\end{array}$ & & 9 & $\cdots$ & & & & \\
\hline & & 42 & 42.49 & & & & & & & \\
\hline & & & 42.49 & & 42 & b41 & & & & \\
\hline & & & 42.49 & & 42 & •. & & & & \\
\hline & & & 42.48 & & & - & & & & \\
\hline & & 42. & 42.47 & & & $\bullet$ & & & & \\
\hline & & 42.81 & 42.47 & & 42.49 & 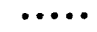 & & - & & \\
\hline & & $\cdots$ & 42.47 & 42 & 42 & $\ldots \ldots$ & & & & \\
\hline & & & $\begin{array}{l}42 \\
42\end{array}$ & . & 42 & $\bullet$ & $\because$ & & $\bullet$ & \\
\hline & & & & & & & & & •・・・ & \\
\hline
\end{tabular}

325. A. I. Gouldner. SW cor. $\mathrm{SE} \frac{1}{4}$ sec. 19, T. $23 \mathrm{~S}, \mathrm{R}, 3 \mathrm{~W}$. New measuring point, beginning June 4, 1941, top of 8 -inch casing southeast side, 1.50 feet below old measuring point, 2.18 feet below bench mark, 1.2 feet above land surface, 1,459.27 feet above sea level. This is 1dentical with the measuring point abandoned June 4, 1939.

Water level, in feet below measuring point, 1941

\begin{tabular}{|c|c|c|c|c|c|c|c|c|c|}
\hline Date & & $\begin{array}{l}\text { Water } \\
\text { level }\end{array}$ & Date & & $\begin{array}{l}\text { Water } \\
\text { level }\end{array}$ & Date & $\begin{array}{l}\text { Water } \\
\text { level }\end{array}$ & Date & $\begin{array}{l}\text { Water } \\
\text { level }\end{array}$ \\
\hline $\begin{array}{l}\text { Jan. } \\
\text { Feb. } \\
\text { Mar. }\end{array}$ & $\begin{array}{l}2 \\
4 \\
4\end{array}$ & $\begin{array}{l}14.16 \\
14.16 \\
14.19\end{array}$ & $\begin{array}{l}\text { Apr. } \\
\text { May } \\
\text { June }\end{array}$ & $\begin{array}{l}1 \\
1 \\
4\end{array}$ & $\begin{array}{r}14.08 \\
14.10 \\
\mathrm{c} 12.88\end{array}$ & $\begin{array}{ll}\text { July } & 8 \\
\text { Aug. } & 4 \\
\text { Sept. } & 3\end{array}$ & $\begin{array}{l}12.03 \\
12.19 \\
12.19\end{array}$ & $\begin{array}{lr}\text { Oct. } 13 \\
\text { Nov. } 10 \\
\text { Dec. } 2\end{array}$ & $\begin{array}{l}12.29 \\
12.11 \\
12.12\end{array}$ \\
\hline
\end{tabular}

506. Owner of well, City of Wichita; owner of property, W.G. Backhaus. NW $\frac{1}{4} \mathrm{NE} \frac{1}{4}$ sec. 28, T. $23 \mathrm{~S}$., R. $2 \mathrm{~W}$.

Lowest daily water level, in feet below measuring point, 1941

Day Jan. Feb. Mar. Apr. May June July Aug. Sept. Oct. Nov. Dec. $\begin{array}{lllllllllllllllllllllll}1 & 18.99 & 18.81 & 18.61 & 18.32 & 18.41 & 18.80 & 17.57 & 19.00 & 19.16 & 18.79 & 15.23 & 17.22\end{array}$ $\begin{array}{lllllllllllllllll}2 & 19.00 & 18.68 & 18.55 & 18.28 & 18.42 & 18.70 & 17.54 & 19.05 & 19.03 & 18.81 & 15.23 & 17.24\end{array}$

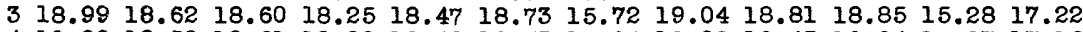
$\begin{array}{lllllllllllllll}4 & 19.00 & 18.52 & 18.61 & 18.28 & 18.48 & 18.73 & 15.04 & 19.09 & 18.43 & 18.84 & 15.27 & 17.16\end{array}$

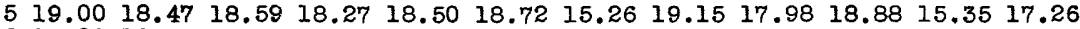
$\begin{array}{llllllllllllllll}6 & 19.00 & 18.53 & 18.55 & 18.21 & 18.57 & 18.68 & 15.26 & 19.13 & 17.83 & 18.88 & 15.45 & 17.26\end{array}$ $\begin{array}{lllllllllllllll}7 & 19.00 & 18.56 & 18.54 & 18.21 & 18.63 & 18.68 & 15.87 & 19.14 & 17.83 & 18.85 & 15.60 & 17.20\end{array}$ $\begin{array}{llllllllllllll}8 & 19.02 & 18.61 & 18.54 & 18.21 & 18.69 & 18.65 & 16.19 & 19.17 & 17.33 & 18.40 & 15.71 & 17.20\end{array}$ $\begin{array}{llllllllllllll}9 & 19.04 & 18.64 & 18.49 & 18.21 & 18.73 & 17.88 & 16.46 & 19.35 & 17.01 & 17.95 & 15.86 & 17.18\end{array}$

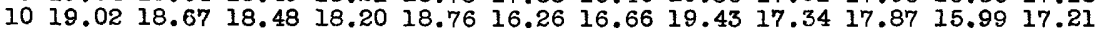

a Automatic recorder discontinued June 20, 1941.

b Wetted-tape measurements beginning this date.

c New measuring point. 


\section{Harvey County--Continued}

506. Owner of well, City of Wichita.--Continued.

Lowest daily water level, in feet below measuring point, 1941

Day Jan. Feb. Mar. Apr. May June July Aug. Sept. Oct. Nov. Dec. $\begin{array}{llllllllllllllllllll}11 & 19.00 & 18.67 & 18.42 & 18.22 & 18.77 & 15.27 & 16.80 & 19.46 & 17.56 & 17.87 & 16.07 & 17.20\end{array}$ $\begin{array}{lllllllllllll}12 & 18.99 & 18.66 & 18.29 & 18.24 & 18.77 & 15.16 & 16.82 & 19.01 & 17.76 & 17.90 & 16.12 & 17.16\end{array}$ $\begin{array}{lllllllllllllll}13 & 18.96 & 18.75 & 18.16 & 18.24 & 18.76 & 15.45 & 16.84 & 18.88 & 17.90 & 18.03 & 16.30 & 17.19\end{array}$ $\begin{array}{lllllllllllllllllll}14 & 18.91 & 18.75 & 18.07 & 18.28 & 18.74 & 15.73 & 16.98 & 18.81 & 18.02 & 18.10 & 16.43 & 17.18\end{array}$ $\begin{array}{llllllllllllll}15 & 18.86 & 18.77 & \ldots & \ldots & 18.28 & 18.72 & 15.97 & 17.21 & 18.78 & 18.12 & 18.08 & 16.52 & 17.17\end{array}$ $16 \quad 18.8318 .77 \ldots \ldots .18 .2618 .72 \quad 16.19 \quad 17.42 \ldots \ldots .18 .2117 .8216 .6117 .16$

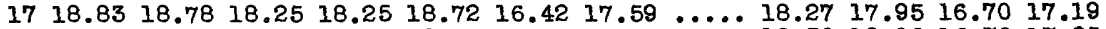

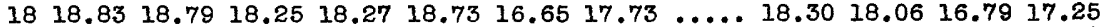

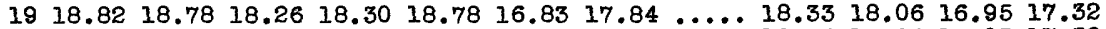
$2018.7918 .7518 .3018 .3318 .8217 .01 \quad 18.01 \ldots \ldots .18 .3417 .9417 .0317 .32$ $21 \quad 18.7918 .73 \quad 18.31 \quad 18.3318 .82 \ldots \ldots .18 .17 \ldots \ldots .18 .3817 .6917 .0317 .32$ $2218.8618 .7018 .31 \quad 18.3218 .79 \ldots \ldots .18 .3018 .73 \quad 18.4016 .5017 .0917 .32$ $23 \quad 18.90 \quad 18.68 \quad 18.30 \quad 18.34 \quad 18.75 \ldots \ldots .18 .38 \quad 18.75 \quad 18.4014 .96 \quad 17.18 \quad 17.16$ $24 \quad 18.92 \quad 18.64 \quad 18.31 \quad 18.3518 .74 \quad 17.51 \quad 18.44 \quad 18.7918 .42 \quad 14.0017 .21 \quad 16.74$ $\begin{array}{lllllllllllllll}25 & 18.96 & 18.64 & 18.32 & 18.36 & 18.73 & 17.59 & 18.54 & 18.84 & 18.72 & 13.52 & 17.20 & 16.66\end{array}$ $\begin{array}{lllllllllllllllll}26 & 19.01 & 18.63 & 18.30 & 18.36 & 18.62 & 17.64 & 18.71 & 18.87 & 18.82 & 14.18 & 17.19 & 16.79\end{array}$ $2719.0418 .63 \quad 18.32 \quad 18.37 \quad 18.64 \quad 17.68 \quad 18.71 \quad 18.88 \quad 18.98 \quad 14.44 \quad 17.18 \quad 16.80$ $\begin{array}{llllllllllllllll}28 & 19.04 & 18.64 & 18.34 & 18.38 & 18.71 & 17.65 & 18.69 & 18.91 & 18.96 & 14.65 & 17.17 & 16.78\end{array}$ $2918.99 \ldots \ldots .18 .3418 .3918 .8017 .3518 .82 \quad 18.95 \quad 18.8414 .91 \quad 17.16 \quad 16.78$

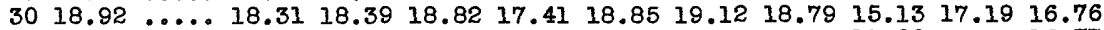

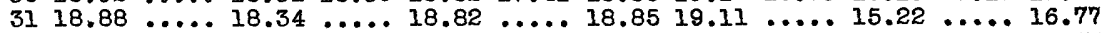

507. Owner of well, city of Wichita; owner of property, W. G. Backhaus. NW $\mathrm{NE}_{\frac{1}{4}}^{\frac{1}{4}}$ sec. $28, \mathrm{~T} .23 \mathrm{~S} ., \mathrm{R} .2 \mathrm{~W}$.

Water level, in feet below measuring point, 1941

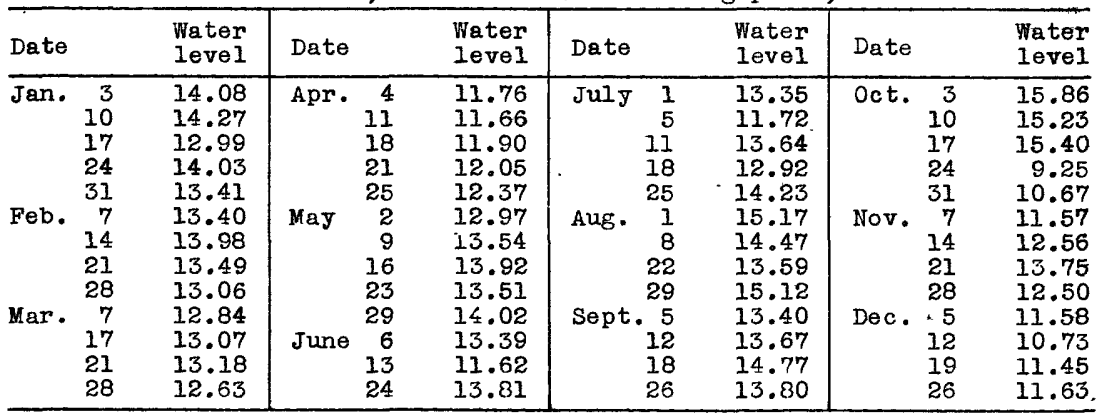

701. Arkansas Valley Interurban Rallway Co., NE cor. NW $\frac{1}{4}$ sec. 3 , T. 23 S., R. 1 W.

Water level, in feet below measuring point, 1941

\begin{tabular}{lll|ll|l|lll|lr}
\hline Jan. & 2 & 38.25 & Apr. & 1 & 38.23 & July & 8 & 38.11 & Oct. 13 & 38.00 \\
Feb. & 4 & 38.18 & May & 1 & 38.17 & Aug. & 4 & 38.04 & Nov. 10 & 37.95 \\
Mar. & 4 & 38.21 & June & 4 & 38.12 & Sept. 3 & 37.92 & Dec. 2 & 37.85 \\
\hline
\end{tabular}

817. City of Wichita. NW cor. sec. 1, T. 24 S., R. 2 W.

Water level, in feet below measuring point, 1941

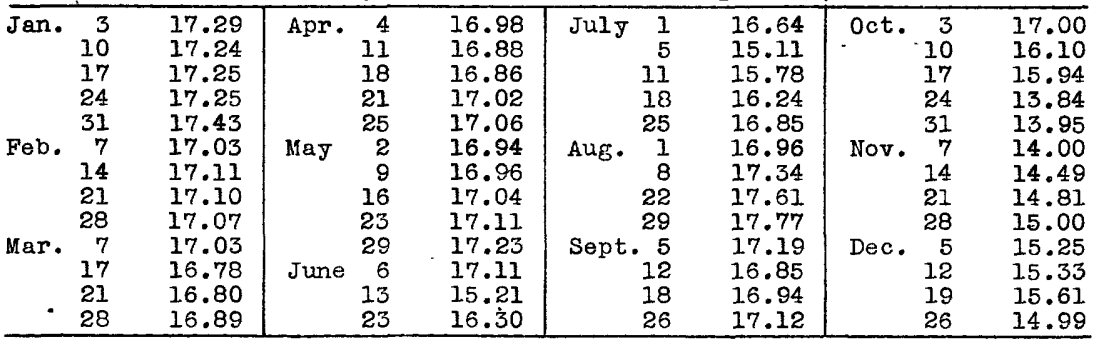


Harvey County--Continued

821. C1ty of Wichita. NW cor. sec. 6, T. 24 S., R. 2 W.

Water level, in feet below measuring point, 1941

\begin{tabular}{|c|c|c|c|c|c|c|c|}
\hline Date & $\begin{array}{l}\text { Water } \\
\text { level }\end{array}$ & Date & $\begin{array}{l}\text { Water } \\
\text { level }\end{array}$ & Date & $\begin{array}{l}\text { Water } \\
\text { level }\end{array}$ & Date & $\begin{array}{l}\text { Water } \\
\text { level } \\
\end{array}$ \\
\hline $\begin{array}{rr}\text { Jan. } & 20 \\
& 28 \\
\text { Feb. } & 10 \\
17 \\
25 \\
\text { Mar. } 3 \\
18 \\
24 \\
31 \\
\text { Apr. } 17 \\
21\end{array}$ & $\begin{array}{l}15.19 \\
15.25 \\
15.39 \\
15.43 \\
15.45 \\
15.50 \\
15.63 \\
15.68 \\
15.74 \\
15.84 \\
15.87\end{array}$ & $\begin{array}{lr}\text { Apr. } & 28 \\
\text { May } & 12 \\
& 19 \\
& 26 \\
\text { June } & 2 \\
& 16 \\
\text { July } & 1 \\
& 5 \\
& 11 \\
18 \\
\\
25\end{array}$ & $\begin{array}{l}15.90 \\
15.98 \\
16.04 \\
16.09 \\
16.16 \\
16.24 \\
16.39 \\
15.59 \\
15.41 \\
15.61 \\
15.83\end{array}$ & $\begin{array}{r}\text { Aug. } \\
8 \\
22 \\
29 \\
\text { Sept. } 5 \\
12 \\
18 \\
26 \\
\text { Oct. } 3 \\
10\end{array}$ & $\begin{array}{l}16.08 \\
16.21 \\
16.45 \\
16.53 \\
16.58 \\
16.61 \\
16.62 \\
16.68 \\
16.71 \\
16.78\end{array}$ & $\begin{array}{l}\text { Oct. } 17 \\
24 \\
\text { Nov. } 7 \\
14 \\
21 \\
28 \\
5 \\
\text { Dec. } \\
12 \\
19 \\
26\end{array}$ & $\begin{array}{l}16.85 \\
16.87 \\
16.91 \\
16.89 \\
16.96 \\
17.00 \\
17.00 \\
17.00 \\
17.01 \\
17.07\end{array}$ \\
\hline
\end{tabular}

824. City of wichita. SE cor. sec. 22, T. 24 S., R. I W.

Water level, in feet below measuring polnt, 1941

\begin{tabular}{lll|ll|llll|ll}
\hline Jan. & 2 & 20.06 & Apr. & 1 & 18.70 & July & 8 & 14.39 & Oct. 13 & 15.92 \\
Feb. & 3 & 19.71 & May & 1 & 18.65 & Aug. 4 & 16.65 & Nov. 10 & 14.54 \\
Mar. & 4 & 19.28 & June & 4 & 18.29 & Sept. 3 & 17.66 & Dec. 2 & 14.92 \\
\hline
\end{tabular}

831. City of Wichita. NE cor. sec. 19, T, 24 S., R. 1 W.

Water level, in feet below measuring point, 1941

\begin{tabular}{lll|lr|lrr|rr}
\hline Jan. & 2 & 21.43 & Apr. 17 & 21.17 & July & 8 & 18.43 & Oct. 13 & 18.27 \\
Feb. & 7 & 21.14 & May & 1 & 21.24 & Aug. 4 & 19.94 & Nov. 10 & 17.35 \\
Mar. & 4 & 21.36 & June & 4 & 21.38 & Sept. 3 & 19.71 & Dec. 2 & 18.47 \\
Apr. & 1 & 21.07 & & & & & & & \\
\hline
\end{tabular}

832. City of Wichlta. NE cor. sec. 19, T. 24 S., R. I W.

Water level, in feet below measuring point, 1941

\begin{tabular}{lll|lr|lrr|rrr}
\hline Jan. & 2 & 20.72 & Apr. 17 & 20.46 & July & 8 & 17.76 & Oct. 13 & 17.74 \\
Feb. & 7 & 20.37 & May & 1 & 20.55 & Aug. 4 & 19.50 & Nov. 10 & 17.15 \\
Mar. & 4 & 20.63 & June & 4 & 20.65 & Sept. 3 & 18.62 & Dec. 2 & 18.27 \\
Apr. & 1 & 20.40 & & & & & & & &
\end{tabular}

833. C1ty of Wichita. SW cor. sec. 19, T. 24 S., R. I W.

Water level, in feet below measuring point, 1941

\begin{tabular}{lll|lr|rrr|rrr}
\hline Jan. & 2 & 11.59 & Apr. 17 & 11.10 & July & 8 & 8.29 & Oct. 13 & 9.05 \\
Feb. & 4 & 11.00 & May & 1 & 11.32 & Aug. 4 & 10.16 & Nov. 10 & 8.41 \\
Mar. 4 & 11.39 & June & 4 & 11.63 & Sept. 3 & 10.95 & Dec. 2 & 9.42 \\
Apr. & 1 & 10.89 & & & & & & & &
\end{tabular}

839. City of Wichita. NE cor. sec. 35, T. 24 S., R. 2 W.

Water level, in feet below measuring polnt, 1941

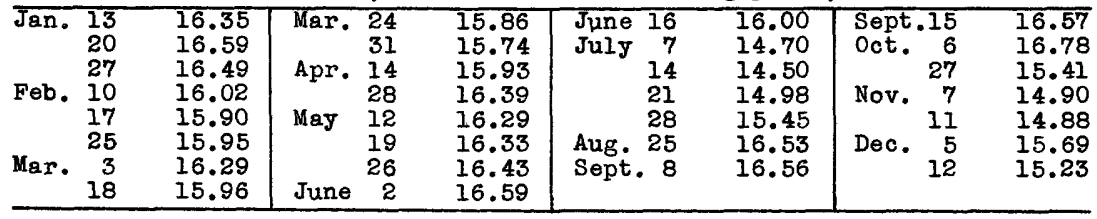

852. City of Wichita. NW cor. sec. 29, T. 24 S., R. 1 W.

Water level, in feet below measuring point, 1941

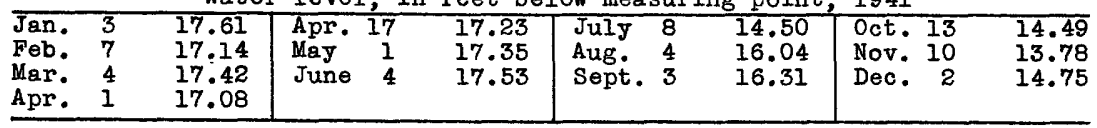


Harvey County--Continued

853. City of Wichita. NW cor. sec. 13, T. 24 S., R. 2 W. Water level, in feet below measuring point, 1941

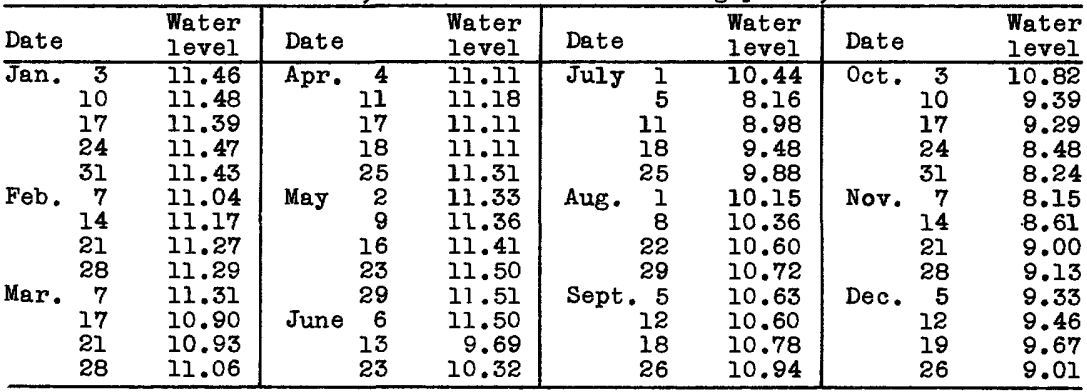

854. City of Wichita. SW cor. sec. 23, T. 23 S., R. 2 W.

Water level, in feet below measuring point, 1941

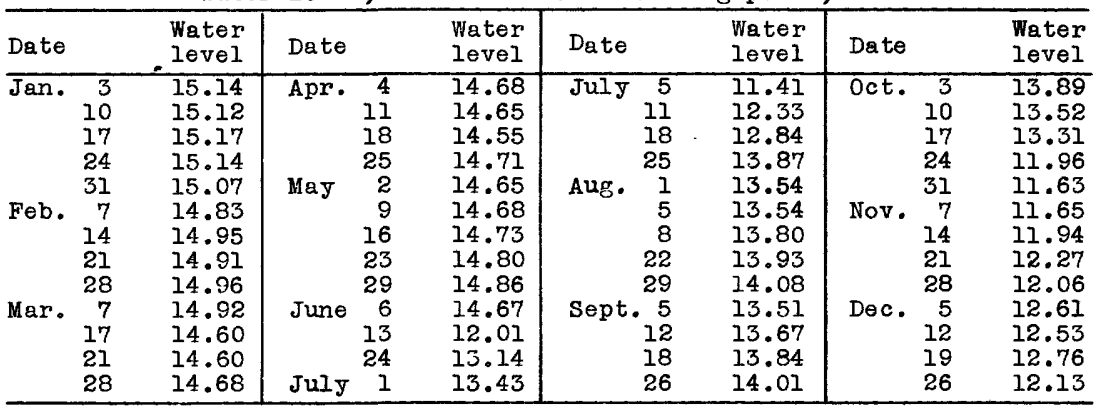

872. Owner of well, City of Wichita; owner of property, D. C. Buller. SE cor. sec. 31, T. $23 \mathrm{~S} ., \mathrm{R} .2 \mathrm{~W}$.

Water level, in feet below measuring point, 1941

\begin{tabular}{|c|c|c|c|c|c|c|c|c|c|c|c|}
\hline Jan. & $\begin{array}{r}13 \\
20 \\
27 \\
3 \\
10 \\
17 \\
25 \\
3\end{array}$ & a & $\begin{array}{l}22.25 \\
21.96 \\
22.05 \\
25.34 \\
22.37 \\
22.35 \\
22.23 \\
23.00\end{array}$ & $\begin{array}{l}\text { Mar. } \\
\text { Apr. } \\
\text { May }\end{array}$ & $\begin{array}{l}18 \\
24 \\
31 \\
21 \\
28 \\
12 \\
19 \\
26\end{array}$ & $\begin{array}{ll}\text { a. } 25.07 \\
\text { a. } 23.94 \\
23.47 \\
22.95 \\
22.90 \\
23.15 \\
23.59 \\
23.64\end{array}$ & 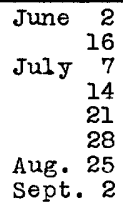 & $\begin{array}{ll}\text { a } & 25.67 \\
\text { a } & 25.91 \\
\text { a } & 24.74 \\
\text { a } & 24.18 \\
\text { a } & 26.71 \\
& 24.40 \\
\text { a } & 24.95 \\
& 24.10\end{array}$ & 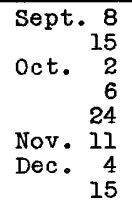 & $\begin{array}{l}a \\
a\end{array}$ & $\begin{array}{l}24.21 \\
24.06 \\
24.20 \\
24.06 \\
24.09 \\
26.46 \\
24.75 \\
24.60\end{array}$ \\
\hline
\end{tabular}

873. Owner of well, City of Wichita; owner of property, D. C. Buller. SE cor. sec. 31, T. $23 \mathrm{~S} .$, R. $2 \mathrm{~W}$.

Water level, in feet below measuring point, 1941

\begin{tabular}{|c|c|c|c|c|c|c|c|c|c|c|c|}
\hline Jan. & $\begin{array}{r}13 \\
20 \\
27 \\
3 \\
10 \\
17 \\
25 \\
3\end{array}$ & $\mathbf{a}$ & $\begin{array}{l}22.27 \\
21.96 \\
22.03 \\
25.45 \\
22.39 \\
22.38 \\
22.25 \\
23.04\end{array}$ & $\begin{array}{l}\text { Mar. } \\
\text { Apr. } \\
\text { May }\end{array}$ & $\begin{array}{l}18 \\
24 \\
31 \\
21 \\
28 \\
12 \\
19 \\
26\end{array}$ & $\begin{array}{l}25.23 \\
\text { a. } 24.06 \\
23.55 \\
23.01 \\
22.97 \\
23.21 \\
23.66 \\
23.72\end{array}$ & $\begin{array}{rr}\text { June } & 2 \\
& 16 \\
\text { July } & 7 \\
14 \\
21 \\
28 \\
\text { Aug. } 25 \\
\text { Sept. } 2\end{array}$ & $\begin{array}{ll}\text { a } & 25.76 \\
\text { a } & 26.10 \\
\text { a } & 24.88 \\
\text { a } & 24.31 \\
\text { a } & 26.96 \\
& 24.55 \\
\text { a } & 25.04 \\
& 24.21\end{array}$ & $\begin{array}{lr}\text { Sept. } 10 \\
15 \\
\text { oct. } \\
2 \\
6 \\
24 \\
\text { Nov. } 11 \\
\text { Dec. } 1 \\
15\end{array}$ & $\begin{array}{l}a \\
a\end{array}$ & $\begin{array}{l}24.31 \\
24.14 \\
24.30 \\
24.17 \\
24.21 \\
26.88 \\
24.87 \\
24.80\end{array}$ \\
\hline
\end{tabular}


Harvey County--Continued

874. Owner of well, City of Wichita; owner of property, D. C. Buller. SE cor. sec. 3I, T. 23 S., R. 2 W.

Water level, in feet below measuring point, 1941

\begin{tabular}{|c|c|c|c|c|c|c|c|c|c|c|c|}
\hline Date & & $\begin{array}{l}\text { Water } \\
\text { level }\end{array}$ & Date & & $\begin{array}{l}\text { Water } \\
\text { level }\end{array}$ & Date & & $\begin{array}{l}\text { Water } \\
\text { level }\end{array}$ & Date & & $\begin{array}{l}\text { Water } \\
\text { level }\end{array}$ \\
\hline $\begin{array}{lr}\text { Jan. } & 13 \\
20 \\
27 \\
\text { Feb. } 3 \\
10 \\
17 \\
\\
25 \\
\text { Mar. } 3\end{array}$ & a & $\begin{array}{l}27.01 \\
26.24 \\
27.31 \\
37.47 \\
27.54 \\
28.48 \\
27.27 \\
29.84\end{array}$ & $\begin{array}{ll}\text { Mar. } & 18 \\
& 24 \\
& 31 \\
\text { Apr. } & 21 \\
& 28 \\
\text { May } 12 \\
19 \\
\\
26\end{array}$ & $\begin{array}{l}\mathrm{a} \\
\mathrm{a}\end{array}$ & $\begin{array}{l}35.26 \\
33.64 \\
29.99 \\
27.91 \\
28.54 \\
29.24 \\
30.86 \\
31.37\end{array}$ & $\begin{array}{r}2 \\
\text { June } \\
16 \\
\text { July } 7 \\
14 \\
21 \\
28 \\
\text { Aug. } 25 \\
\text { Sept. } 2\end{array}$ & $\begin{array}{l}\text { a } \\
\text { a } \\
\text { a } \\
\text { a } \\
\text { a } \\
\text { a }\end{array}$ & $\begin{array}{l}35.35 \\
35.58 \\
35.91 \\
30.63 \\
39.98 \\
32.37 \\
34.36 \\
28.97\end{array}$ & $\begin{array}{lr}\text { Sept. } 10 \\
15 \\
\text { Oct. } \\
6 \\
64 \\
24 \\
\text { Nov. } 11 \\
\text { Dec. } 1 \\
15\end{array}$ & $\begin{array}{l}a \\
a\end{array}$ & $\begin{array}{l}30.12 \\
30.37 \\
30.41 \\
30.35 \\
28.21 \\
35.18 \\
32.12 \\
30.30 \\
\end{array}$ \\
\hline
\end{tabular}

875. Owner of well, City of Wichita; owner of property, A. B. Havely. $\mathrm{SE}$ cor. sec. $17, \mathrm{~T} .23 \mathrm{~S}, \mathrm{R}, 3 \mathrm{~W}$.

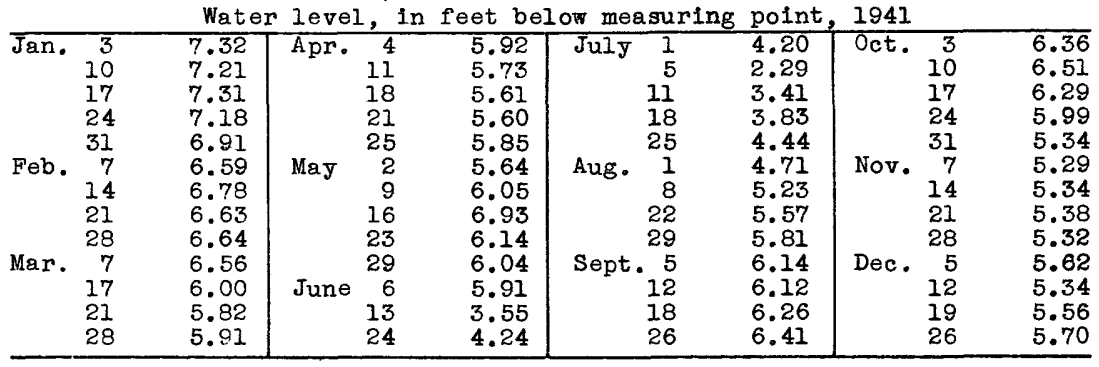

876. Owner of well, City of Wichita; owner of property, A. B. Havely. $\mathrm{SE}$ cor. sec. 17, T. $23 \mathrm{~S}, \mathrm{R}, 3 \mathrm{~W}$.

Water level, in feet below measuring point, 1941

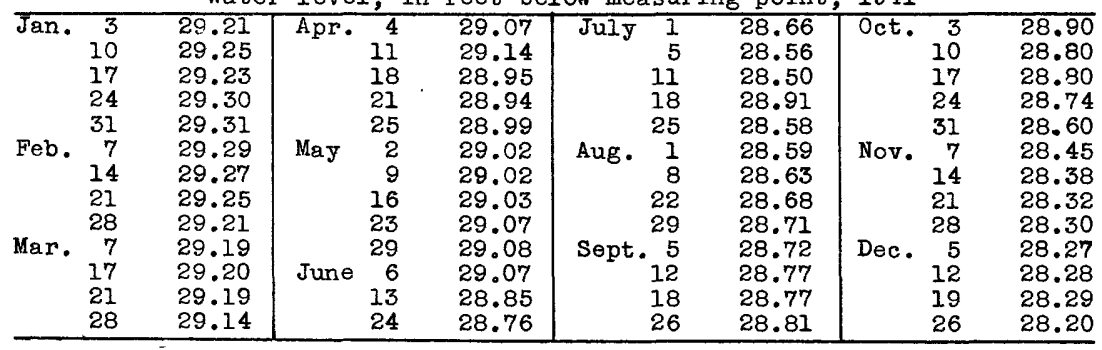

877. Owner of well, Clty of Wichita; owner of property, A. B. Havely. SE cor. sec. 17, T. 23 S., R. 3 W.

Lowest daily water level, in feet below measuring point, 1941

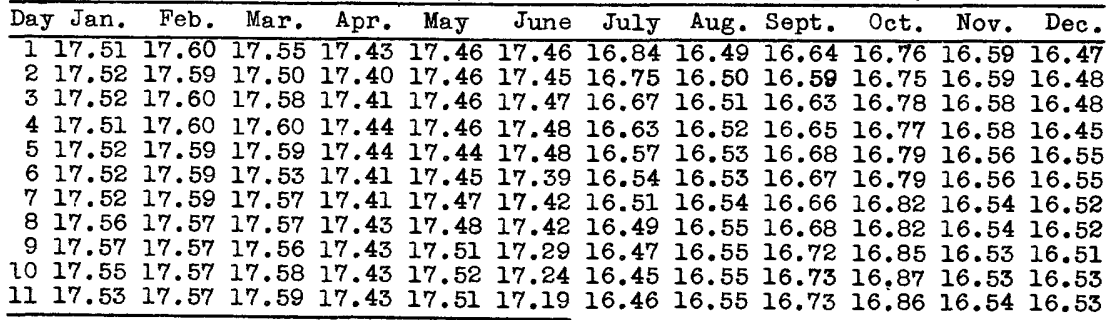

a Nearby well pumping. 
Harvey County--Continued

877. Owner of well, City of Wichita.--Continued.

Lowest daily water level, in feet below measuring point, 1941

Day Jan. Feb. Mar. Apr. May June July Aug. Sept. Oct. Nov. Dec. $\begin{array}{lllllllllllllllll}12 & 17.53 & 17.54 & 17.59 & 17.43 & 17.50 & 17.15 & 16.46 & 16.54 & 16.72 & 16.84 & 16.52 & 16.50\end{array}$

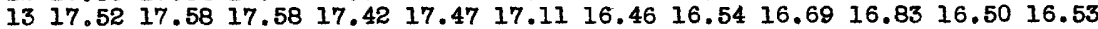
$\begin{array}{llllllllllllll}14 & 17.51 & 17.58 & 17.57 & 17.42 & 17.45 & 17.08 & 16.45 & 16.57 & 16.68 & 16.84 & 16.50 & 16.52\end{array}$ $1517.5017 .58 \ldots \ldots .17 .42 \quad 17.47 \quad 17.0616 .4416 .59 \quad 16.6916 .8616 .49 \quad 16.52$ $\begin{array}{llllllllllllll}16 & 17.53 & 17.56 & \ldots & \ldots & 17.40 & 17.48 & 17.04 & 16.42 & \ldots & 16.71 & 16.85 & 16.47 & 16.51\end{array}$ $17 \quad 17.61 \quad 17.5617 .5417 .4017 .47 \quad 17.0416 .42 \ldots \ldots . .16 .7216 .8216 .4616 .51$

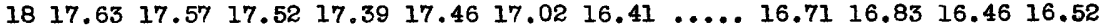

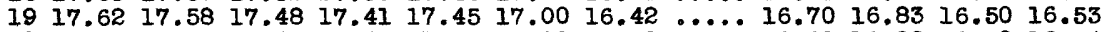
$20 \quad 17.61 \quad 17.56 \quad 17.47 \quad 17.45 \quad 17.47 \quad 16.99 \quad 16.42 \quad \ldots . . .16 .6916 .82 \quad 16.5016 .54$ $2117.6017 .5617 .48 \quad 17.4617 .48 \ldots \ldots .16 .41 \ldots \ldots .16 .7216 .8216 .5016 .51$ $2217.62 \quad 17.56 \quad 17.47 \quad 17.4517 .48 \ldots \ldots .16 .41 \quad 16.61 \quad 16.72 \quad 16.8116 .4716 .48$ $23 \quad 17.62 \quad 17.56 \quad 17.47 \quad 17.46 \quad 17.47 \ldots \ldots .16 .42 \quad 16.6016 .72 \quad 16.8016 .49 \quad 16.48$ $24 \quad 17.62 \quad 17.54 \quad 17.47 \quad 17.46 \quad 17.47 \quad 16.93 \quad 16.44 \quad 16.60 \quad 16.72 \quad 16.79 \quad 16.50 \quad 16.49$ $25 \quad 17.60 \quad 17.54 \quad 17.47 \quad 17.48 \quad 17.46 \quad 16.93 \quad 16.45 \quad 16.59 \quad 16.75 \quad 16.78 \quad 16.49 \quad 16.49$ $26 \quad 17.64 \quad 17.56 \quad 17.46 \quad 17.48 \quad 17.45 \quad 16.92 \quad 16.45 \quad 16.61 \quad 16.76 \quad 16.68 \quad 16.48 \quad 16.56$ $\begin{array}{lllllllllllllllll}27 & 17.65 & 17.57 & 17.47 & 17.47 & 17.45 & 16.91 & 16.45 & 16.62 & 16.76 & 16.73 & 16.48 & 16.57\end{array}$ $28 \quad 17.65 \quad 17.57 \quad 17.48 \quad 17.47 \quad 17.47 \quad 16.83 \quad 16.46 \quad 16.62 \quad 16.8116 .7416 .48 \quad 16.58$ $2917.63 \ldots \ldots .17 .47 \quad 17.47 \quad 17.49 \quad 16.83 \quad 16.47 \quad 16.63 \quad 16.8016 .70 \quad 16.47 \quad 16.58$ $3017.59 \ldots \ldots .17 .4217 .4517 .4916 .8316 .4816 .6316 .7516 .6616 .47 \quad 16.55$

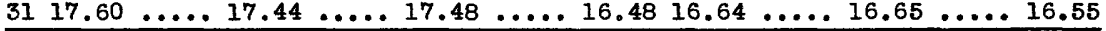

878. Owner of well, City of Wichita; owner of property, C. Cadwell. SE cor. sec. I, T. $24 \mathrm{~S}$, R. $3 \mathrm{~W}$.

Water level, in feet below measuring point, 1941

\begin{tabular}{|c|c|c|c|c|c|c|c|c|}
\hline Date & $\begin{array}{l}\text { Water } \\
\text { level }\end{array}$ & Date & & $\begin{array}{l}\text { Water } \\
\text { level }\end{array}$ & Date & $\begin{array}{l}\text { Water } \\
\text { level }\end{array}$ & Date & $\begin{array}{l}\text { Water } \\
\text { level }\end{array}$ \\
\hline $\begin{array}{l}\text { Jan. } 20 \\
28 \\
\text { Feb. } 10 \\
17 \\
\text { Mar. } 3 \\
18 \\
24 \\
31 \\
31 \\
\text { Apr. } 17 \\
28 \\
\end{array}$ & $\begin{array}{l}19.31 \\
19.35 \\
19.42 \\
19.45 \\
19.54 \\
19.60 \\
19.63 \\
19.70 \\
19.77 \\
19.83\end{array}$ & $\begin{array}{l}\text { May } \\
\text { June } \\
\text { July }\end{array}$ & $\begin{array}{r}12 \\
19 \\
26 \\
2 \\
16 \\
1 \\
5 \\
11 \\
18 \\
25\end{array}$ & $\begin{array}{l}19.92 \\
19.96 \\
20.01 \\
20.05 \\
20.07 \\
20.19 \\
19.98 \\
20.01 \\
20.15 \\
20.22\end{array}$ & $\begin{array}{r}1 \\
\text { Aug. } \\
8 \\
22 \\
29 \\
\text { Sept. } 5 \\
12 \\
18 \\
26 \\
3 \\
\text { Oct. } 10\end{array}$ & $\begin{array}{l}20.27 \\
20.35 \\
20.47 \\
20.51 \\
20.59 \\
20.63 \\
20.78 \\
20.73 \\
20.79 \\
20.78\end{array}$ & $\begin{array}{rr}\text { Oct. } & 17 \\
24 \\
\text { Nov. } 7 \\
14 \\
21 \\
28 \\
5 \\
\text { Dec. } 5 \\
15 \\
19 \\
26\end{array}$ & $\begin{array}{l}20.80 \\
20.80 \\
20.79 \\
20.81 \\
20.83 \\
20.85 \\
20.89 \\
20.90 \\
20.96 \\
20.96\end{array}$ \\
\hline
\end{tabular}

879. Owner of well, City of Wichita; owner of property, C. Cadwell. $\mathrm{SE}$ cor. sec. $1, \mathrm{~T} .24 \mathrm{~S} ., \mathrm{R}, 3 \mathrm{~W}$.

Water level, in feet below measuring point, 1941

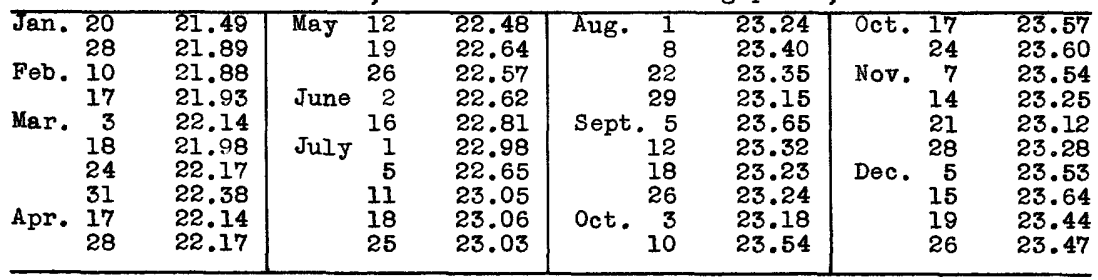




\section{Harvey County--Continued}

880. Owner of well, City of Wichita; owner of property, Peter Miller. SE cor. sec. 11, T. 24 S., R. 3 W.

Water level, in feet below measuring point, 1941

\begin{tabular}{|c|c|c|c|c|c|c|c|c|}
\hline Date & $\begin{array}{l}\text { Water } \\
\text { level }\end{array}$ & Date & & $\begin{array}{l}\text { Water } \\
\text { level }\end{array}$ & Date & $\begin{array}{l}\text { Water } \\
\text { level }\end{array}$ & Date & $\begin{array}{l}\text { Water } \\
\text { level }\end{array}$ \\
\hline $\begin{array}{lr}\text { Jan. } 20 \\
& 28 \\
\text { Feb. } & 10 \\
17 \\
\text { Mar. } 3 \\
18 \\
24 \\
31 \\
31 \\
\text { Apr. } 16 \\
28\end{array}$ & $\begin{array}{l}7.51 \\
7.49 \\
7.15 \\
7.20 \\
7.22 \\
6.83 \\
6.90 \\
6.95 \\
7.02 \\
7.10\end{array}$ & $\begin{array}{l}\text { May } \\
\text { June } \\
\text { July }\end{array}$ & $\begin{array}{r}12 \\
19 \\
26 \\
2 \\
16 \\
1 \\
5 \\
11 \\
18 \\
25\end{array}$ & $\begin{array}{l}7.12 \\
7.23 \\
7.35 \\
7.40 \\
6.12 \\
6.37 \\
4.06 \\
5.36 \\
5.89 \\
6.30\end{array}$ & $\begin{array}{rr}\text { Aug. } & 1 \\
8 \\
22 \\
29 \\
\text { Sept. } 5 \\
12 \\
18 \\
26 \\
\text { Oct. } 3 \\
10\end{array}$ & $\begin{array}{l}6.57 \\
6.83 \\
7.11 \\
7.21 \\
7.35 \\
7.45 \\
7.60 \\
7.63 \\
7.57 \\
7.27\end{array}$ & $\begin{array}{l}\text { Oct. } 17 \\
24 \\
\text { Nov. } 7 \\
14 \\
21 \\
28 \\
\text { Dec. } 5 \\
15 \\
19 \\
26\end{array}$ & $\begin{array}{l}7.15 \\
6.84 \\
6.00 \\
6.20 \\
6.27 \\
6.34 \\
6.43 \\
6.47 \\
6.54 \\
6.30\end{array}$ \\
\hline
\end{tabular}

881. Owner of well, City of Wichita; owner of property, Peter Miller. SE cor. sec. 11, T. $24 \mathrm{~S} ., \mathrm{R} .3 \mathrm{~W}$.

Water level, in feet below measuring point, 1941

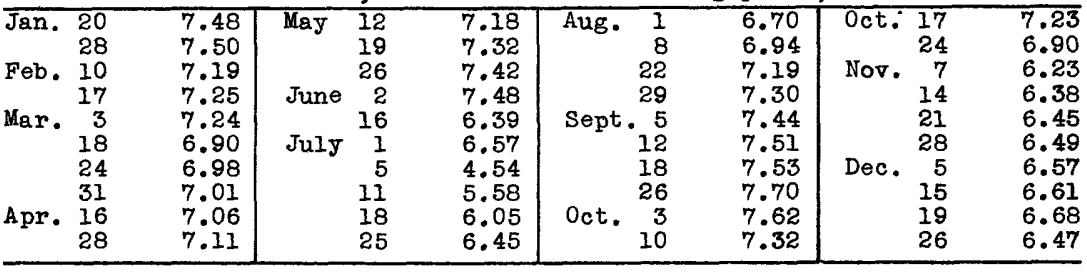

883. Owner of well, City of Wichita; owner of property, Maggie Holle. NW cor. sec.26, T. 24 S., R. 2 W.

Water level, in feet below measuring point, 1941

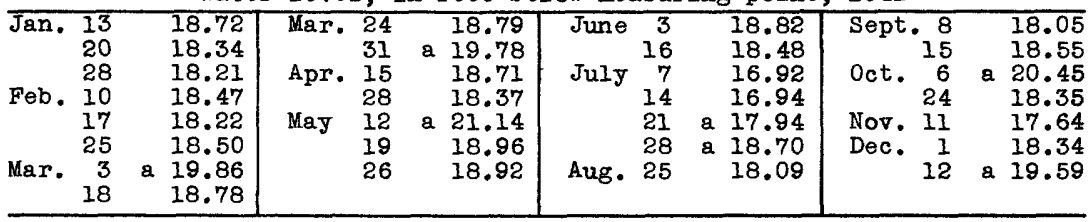

884. Owner of well, City of Wichita; owner of property, Maggie Holle. NW cor. sec. $26, T .24 \mathrm{~S}$, , R. $2 \mathrm{~W}$.

Water level, in feet below measuring point, 1941

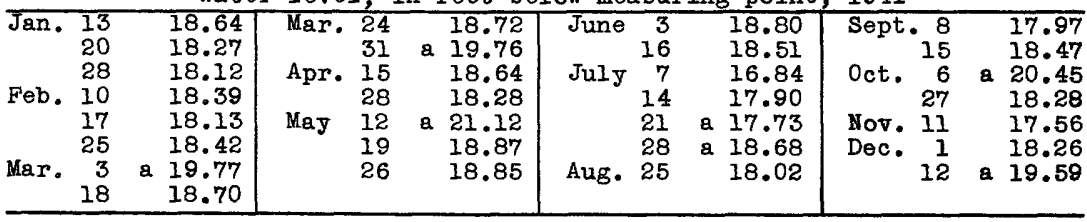

a Nearby well pumping. 
Harvey County--Continued

885. Owner of well, City of Wichita; owner of property, Maggle Holle. NW cor. sec. 26, T. $24 \mathrm{~S}$., R. $2 \mathrm{~W}$.

Water level, in feet below measuring point, 1941

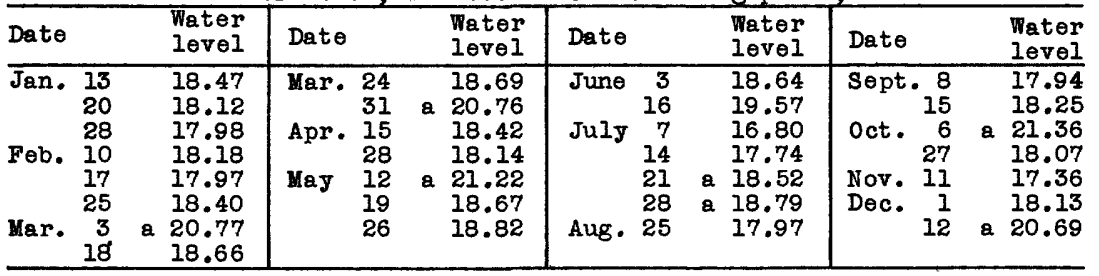

886. Owner of well, City of Wichita; owner of property, F. H. Haiber. NE cor. NWt sec. 16, T. 24 S., R. 2 W.

Water level, in feet below measuring point, 1941

\begin{tabular}{|c|c|c|c|c|c|c|c|c|c|}
\hline $\begin{array}{l}\overline{\text { Jan. }} \\
\text { Feb. } \\
\text { Har. } \\
\text { I }\end{array}$ & $\begin{array}{r}20 \\
28 \\
10 \\
17 \\
3 \\
18 \\
24\end{array}$ & $\begin{array}{r}8.94 \\
8.08 \\
9.22 \\
9.49 \\
8.49 \\
8.13 \\
\text { a } 9.15 \\
\text { a } 8.63\end{array}$ & $\begin{array}{l}\text { Mar. } \\
\text { Apr. } \\
\text { May } \\
\text { June }\end{array}$ & $\begin{array}{l}31 \\
17 \\
28 \\
12 \\
19 \\
26 \\
2\end{array}$ & $\begin{array}{rr}\text { a } & 9.17 \\
\text { a } 9.18 \\
\text { a } 9.46 \\
8.43 \\
\text { a } 10.57 \\
8.79 \\
\text { a } 9.54\end{array}$ & $\begin{array}{rr}\text { June } & 16 \\
\text { July } & 7 \\
14 \\
21 \\
28 \\
\text { Aug. } 26 \\
\text { Sept. } 10\end{array}$ & $\begin{array}{r}8.17 \\
7.68 \\
\text { a } 8.21 \\
8.00 \\
\text { a } 10.09 \\
\text { a } 9.69 \\
\text { a } 12.00\end{array}$ & 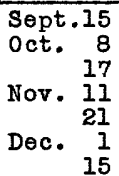 & $\begin{array}{rr}\text { a } & 11.93 \\
\text { a } & 10.75 \\
& 8.10 \\
\text { a } & 8.30 \\
\text { a } & 9.56 \\
& 8.39 \\
& 8.52\end{array}$ \\
\hline
\end{tabular}

887. Owner of well, City of Wichita; owner of property, F. H. Haiber. NE cor. NWt sec. 16, T. 24 S., R. 2 W.

Water level, in feet below measuring point, 1941

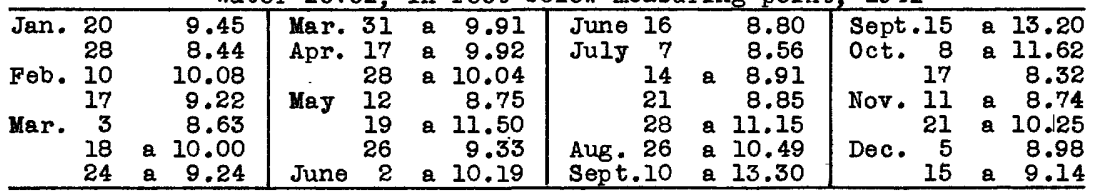

888. Owner of well, City of wichita; owner of property, C. K. Ellis. NW cor. sec. 17, T. $23 \mathrm{~s} ., \mathrm{R} .2 \mathrm{~W}$.

Water level, in feet below measuring point, 1941

\begin{tabular}{|c|c|c|c|c|c|c|c|c|c|c|c|}
\hline Jan. & $\begin{array}{r}3 \\
10 \\
17 \\
24 \\
31 \\
7 \\
14 \\
21 \\
28 \\
7 \\
17 \\
21 \\
28\end{array}$ & $\begin{array}{l}7.76 \\
7.70 \\
7.71 \\
7.50 \\
7.40 \\
6.13 \\
6.29 \\
6.25 \\
6.23 \\
5.91 \\
3.66 \\
3.83 \\
4.11\end{array}$ & $\begin{array}{l}\text { Apr. } \\
\text { May } \\
\text { June }\end{array}$ & $\begin{array}{r}4 \\
11 \\
18 \\
25 \\
2 \\
9 \\
16 \\
23 \\
29 \\
6 \\
11 \\
13 \\
24\end{array}$ & $\begin{array}{l}4.23 \\
3.88 \\
3.10 \\
3.66 \\
3.93 \\
3.27 \\
4.13 \\
4.54 \\
5.16 \\
4.71 \\
.50 \\
.70 \\
2.92\end{array}$ & $\begin{array}{l}\text { July } \\
\text { Aug. } \\
\text { Sept. }\end{array}$ & $\begin{array}{r}1 \\
5 \\
11 \\
18 \\
25 \\
1 \\
8 \\
22 \\
29 \\
5 \\
12 \\
18 \\
26\end{array}$ & $\begin{array}{l}3.32 \\
.49 \\
1.53 \\
2.88 \\
4.06 \\
4.82 \\
5.53 \\
6.50 \\
6.84 \\
7.28 \\
7.42 \\
7.57 \\
7.95\end{array}$ & $\begin{array}{l}\text { Oct. } \\
\text { Nov. } \\
\text { Dec. }\end{array}$ & 2 & $\begin{array}{l}7.89 \\
8.06 \\
7.93 \\
7.38 \\
6.53 \\
6.14 \\
6.09 \\
6.14 \\
6.14 \\
6.25 \\
6.01 \\
6.09 \\
4.87\end{array}$ \\
\hline
\end{tabular}

889. Owner of well, City of Wichita; owner of property, C. K. Ellis. NW cor. sec. 17, T. 23 S., R. 2 W.

Water level, in feet below measuring point, 1941

\begin{tabular}{|c|c|c|c|c|c|c|c|c|c|c|c|}
\hline $\begin{array}{ll}\operatorname{Jan} . & 1 \\
& 1 \\
& \\
& \\
3 & \\
\text { Feb. } & 1\end{array}$ & $\begin{array}{l}3 \\
10 \\
17 \\
24 \\
31 \\
7 \\
14\end{array}$ & $\begin{array}{l}8.44 \\
8.63 \\
8.74 \\
8.57 \\
8.56 \\
8.17 \\
7.88\end{array}$ & $\begin{array}{l}\text { Fob. } \\
\text { Mar. } \\
\text { Apr. }\end{array}$ & $\begin{array}{r}21 \\
28 \\
7 \\
17 \\
21 \\
28 \\
4\end{array}$ & $\begin{array}{l}7.86 \\
7.84 \\
8.19 \\
7.49 \\
7.27 \\
7.29 \\
7.02\end{array}$ & $\begin{array}{l}\text { Apr. } \\
\text { May }\end{array}$ & $\begin{array}{r}11 \\
18 \\
25 \\
2 \\
9 \\
16 \\
23\end{array}$ & $\begin{array}{l}6.72 \\
6.72 \\
7.46 \\
7.78 \\
7.77 \\
7.62 \\
7.74\end{array}$ & $\begin{array}{l}\text { May } \\
\text { June } \\
\text { July }\end{array}$ & $\begin{array}{r}29 \\
6 \\
11 \\
13 \\
24 \\
1 \\
5\end{array}$ & $\begin{array}{l}8.14 \\
8.00 \\
6.61 \\
6.34 \\
6.38 \\
6.45 \\
5.54\end{array}$ \\
\hline
\end{tabular}

a Nearby well pumping. 
Harvey County--Continued

889. - - Continued.

Water level, in feet below measuring point, 1941

\begin{tabular}{|c|c|c|c|c|c|c|c|}
\hline Date & $\begin{array}{l}\text { Water } \\
\text { level }\end{array}$ & Date & $\begin{array}{l}\text { Water } \\
\text { level }\end{array}$ & Date & $\begin{array}{l}\text { Weter } \\
\text { level }\end{array}$ & Date & $\begin{array}{l}\text { Water } \\
\text { level }\end{array}$ \\
\hline $\begin{array}{lr}\text { July } 11 \\
18 \\
\\
25 \\
\text { Aug. } 1 \\
8 \\
22 \\
\end{array}$ & $\begin{array}{l}5.82 \\
5.65 \\
6.93 \\
7.43 \\
7.59 \\
7.39\end{array}$ & $\begin{array}{rr}\text { Aug. } & 29 \\
\text { Sept. } 5 \\
12 \\
18 \\
\\
26 \\
\text { oct. } 3\end{array}$ & $\begin{array}{l}7.78 \\
7.83 \\
7.41 \\
7.53 \\
7.15 \\
8.58\end{array}$ & $\begin{array}{lr}\text { oct. } & 10 \\
17 \\
24 \\
31 \\
\text { Nov. } 7 \\
14\end{array}$ & $\begin{array}{l}8.40 \\
8.00 \\
6.28 \\
5.65 \\
5.89 \\
6.00\end{array}$ & $\begin{array}{r}\text { Nov. } 21 \\
28 \\
\text { Dec. } 5 \\
12 \\
19 \\
26\end{array}$ & $\begin{array}{l}6.33 \\
6.44 \\
6.22 \\
5.88 \\
6.10 \\
6.10\end{array}$ \\
\hline
\end{tabular}

890. Owner of well, city of Wichita; owner of property, J. F. Jorgenson.

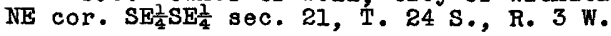

Water level, in feet below measuring point, 1941

\begin{tabular}{|c|c|c|c|c|c|c|c|c|c|}
\hline $\begin{array}{l}\text { Jan. } \\
\text { Feb. } \\
\text { Mar. } \\
\text { Apr. }\end{array}$ & $\begin{array}{l}3 \\
4 \\
4 \\
1\end{array}$ & $\begin{array}{l}7.04 \\
6.71 \\
6.68 \\
6.46\end{array}$ & $\begin{array}{l}\text { Apr. } \\
\text { May } \\
\text { June } \\
\text { July }\end{array}$ & $\begin{array}{r}16 \\
1 \\
4 \\
8\end{array}$ & $\begin{array}{l}6.35 \\
6.38 \\
6.54 \\
5.03\end{array}$ & $\begin{array}{l}\text { Aug. } 4 \\
\text { Sept. } 3 \\
\text { Oct. } 13\end{array}$ & $\begin{array}{l}5.41 \\
5.65 \\
5.64\end{array}$ & $\begin{array}{lr}\text { Oct. } 21 \\
\text { Nov. } 10 \\
\text { Dec. } 2\end{array}$ & $\begin{array}{l}5.51 \\
4.82 \\
4.94\end{array}$ \\
\hline
\end{tabular}

891. Owner of well, C1ty of Wichita; owner of property, Arthur McMurry. SE cor. sec. 31, T. 24 S., R. 3 W.

Water level, in feet below messuring point, 1941

\begin{tabular}{|c|c|c|c|c|c|c|c|c|c|}
\hline $\begin{array}{l}\text { Jan. } \\
\text { Feb. } \\
\text { Mar. }\end{array}$ & $\begin{array}{l}3 \\
4 \\
4\end{array}$ & $\begin{array}{l}3.94 \\
3.40 \\
3.66\end{array}$ & $\begin{array}{l}\text { Apr. } \\
\text { May } \\
\text { June }\end{array}$ & $\begin{array}{l}1 \\
1 \\
4\end{array}$ & $\begin{array}{l}3.49 \\
3.66 \\
4.97\end{array}$ & $\begin{array}{l}\text { July } \\
\text { Aug. } \\
\text { Sept. }\end{array}$ & $\begin{array}{l}8 \\
4 \\
3\end{array}$ & $\begin{array}{l}2.17 \\
3.50 \\
4.92\end{array}$ & $\begin{array}{lr}\text { Oct. } 13 \\
\text { Nov. } 10 \\
\text { Dec. } 2\end{array}$ \\
\hline
\end{tabular}

892. Owner of well, City of Wichita; owner of property, Arthur McMurry. SE cor. sec. 31, T. $24 \mathrm{~S}$., R. $3 \mathrm{~T}$.

Water level, in feet below measuring point, 1941

\begin{tabular}{lll|ll|lll|llr}
\hline Jan. 3 & 5.03 & Apr. & 1 & 4.46 & July & 8 & 2.88 & Oct. 13 & 4.19 \\
Feb. 4 & 4.40 & May & 1 & 4.62 & Aug. & 4 & 4.41 & Nov. 10 & 3.25 \\
Mar. 4 & 4.63 & June & 4 & 4.64 & Sept. 3 & 4.48 & Dec. & 2 & 3.45 \\
\hline
\end{tabular}

893. Owner of well, City of Wichita; owner of property, Arthur McMurry. SE cor. sec. 31 , T. $24 \mathrm{~S}$., R. 3 W.

Water level, in feet below measuring point, 1941

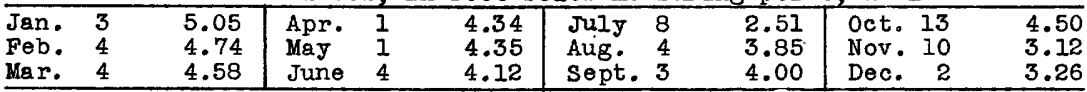

894. Owner of well, Clty of Wichlta; owner of property, H. A. Lawrence. NE cor. sec. 18, T. $24 \mathrm{~s}$., R. $2 \mathrm{~W}$.

Water level, in feet helow measuring point, 1941

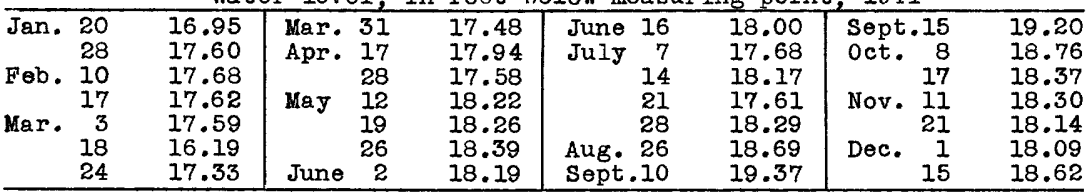

895. Owner of well, City of Wichlta; owner of property, H. A. Lawrence. NE cor. sec. 18, T. 24 S., R 2 W.

Water level, in feet below measuring point, 1941

\begin{tabular}{|c|c|c|c|c|c|c|c|c|c|c|}
\hline $\begin{array}{l}\text { Jan. } \\
\text { Feb. } \\
\text { Mar. }\end{array}$ & $\begin{array}{r}20 \\
28 \\
10 \\
17 \\
3 \\
18 \\
24 \\
31\end{array}$ & $\begin{array}{l}17.31 \\
18.49 \\
18.94 \\
18.27 \\
18.15 \\
16.58 \\
17.88 \\
18.23\end{array}$ & $\begin{array}{l}\text { Apr. } \\
\text { May } \\
\text { June }\end{array}$ & $\begin{array}{r}17 \\
28 \\
12 \\
19 \\
26 \\
2 \\
16\end{array}$ & $\begin{array}{l}19.79 \\
17.98 \\
19.60 \\
19.21 \\
19.77 \\
18.83 \\
18.71\end{array}$ & $\begin{array}{ll}\text { July } & \\
& \\
& 2 \\
& \\
\text { Aug. } & 2 \\
\text { Sept. } & 1\end{array}$ & $\begin{array}{l}19.07 \\
19.70 \\
17.95 \\
20.23 \\
19.65 \\
21.05 \\
21.44\end{array}$ & $\begin{array}{l}\text { Oct. } \\
\text { Nov. } \\
\text { Dec. }\end{array}$ & & $\begin{array}{l}19.39 \\
18.70 \\
18.80 \\
18.51 \\
19.14 \\
19.31\end{array}$ \\
\hline
\end{tabular}


Harvey County--Continued

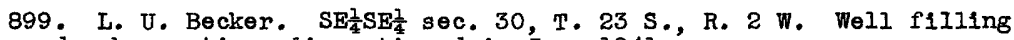
with sand; observations discontinued in June 1941.

Water level, in feet below measuring point, 1941

\begin{tabular}{|c|c|c|c|c|c|c|c|}
\hline Date & $\begin{array}{l}\text { Water } \\
\text { level }\end{array}$ & Date & $\begin{array}{l}\text { Water } \\
\text { level }\end{array}$ & Date & $\begin{array}{l}\text { Water } \\
\text { level }\end{array}$ & Date & $\begin{array}{l}\text { Water } \\
\text { level }\end{array}$ \\
\hline $\begin{aligned} & \text { Jan. } 13 \\
& 20 \\
& 28 \\
& \text { Feb. } 10\end{aligned}$ & $\begin{array}{l}15.66 \\
15.67 \\
15.69 \\
15.65\end{array}$ & $\begin{array}{rr}\text { Feb. } & 17 \\
& 25 \\
& 3 \\
\text { Mar. } & 18\end{array}$ & $\begin{array}{l}15.63 \\
15.68 \\
15.68 \\
15.65\end{array}$ & $\begin{array}{ll}\text { Mar. } & 24 \\
31 \\
\text { Apr. } \\
21 \\
28\end{array}$ & $\begin{array}{l}15.64 \\
15.62 \\
15.62 \\
15.62\end{array}$ & $\begin{array}{lr}\text { May } & 12 \\
& 19 \\
& 26 \\
\text { June } & 2\end{array}$ & $\begin{array}{l}15.61 \\
15.68 \\
15.67 \\
15.73\end{array}$ \\
\hline
\end{tabular}

1112. Owner, M. H. Miller; tenant, A. C. Unmuh. NW cor. NE $\frac{1}{4}$ sec. 31 , T. 23 S., R. 2 W.

Water level, in feet below measuring point, 1941

\begin{tabular}{|c|c|c|c|c|c|c|c|c|}
\hline $\begin{array}{l}\text { Feb. } \\
\text { Mar. }\end{array}$ & $\begin{array}{r}13 \\
20 \\
28 \\
10 \\
17 \\
25 \\
3 \\
18\end{array}$ & $\begin{array}{l}19.64 \\
19.65 \\
19.65 \\
19.64 \\
19.53 \\
19.64 \\
19.25 \\
19.59\end{array}$ & $\begin{array}{ll}\text { Mar. } & 2 \\
& 3 \\
\text { Apr. } & 23 \\
& 28 \\
\text { May } & 12 \\
& 19 \\
& 26\end{array}$ & $\begin{array}{l}19.58 \\
19.57 \\
19.52 \\
19.60 \\
19.62 \\
19.56 \\
19.63\end{array}$ & $\begin{array}{ll}\text { June } & \\
& 1 \\
\text { July } & \\
& 2 \\
& 2 \\
\text { Aug . } & 2\end{array}$ & $\begin{array}{l}19.65 \\
19.34 \\
18.65 \\
18.62 \\
18.74 \\
18.90 \\
19.16\end{array}$ & $\begin{array}{lr}\text { Sept. } 10 \\
\\
15 \\
\text { Oct. } 8 \\
\\
24 \\
\text { Nov. } 11 \\
\text { Dec. } 1 \\
& 15\end{array}$ & $\begin{array}{l}19.28 \\
19.33 \\
19.35 \\
19.35 \\
19.13 \\
19.09 \\
19.08\end{array}$ \\
\hline
\end{tabular}

1174. City of Wichita. Sw cor. sec. 32, T. 24 S., R. 1 W.

Water level, in feet below measu ring point, 1941

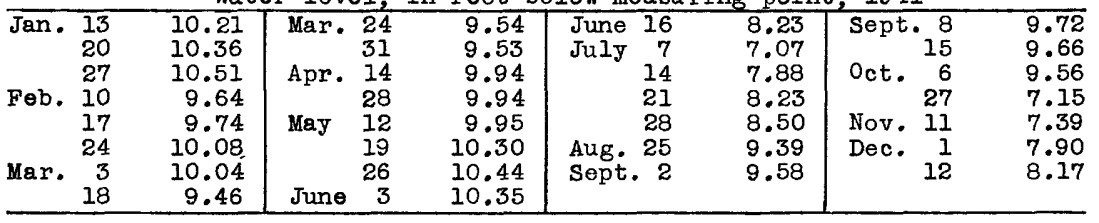

1192. City of Wichita. SE $\frac{1}{2} S E \frac{1}{4}$ sec. 30, T. 23 S., R. 2 W. Situated 1.3 feet west of well M-4a, and 101.3 feet southwest of well M-4. Driven observation well, diameter $1 \frac{1}{2}$ inches, depth 20.5 feet. Measuring point, top of pipe, 1.0 foot above land surface, 0.43 foot above bench mark, $1,432.12$ feet above sea level. Bench mark at city supply well M-4 is a brass plug that protrudes an elghth of an inch above the surface of the concrete floor in the well house, $1,431.69$ feet above sea level.

Water level, in feet below measuring point, 1941

\begin{tabular}{|c|c|c|c|c|c|c|c|c|}
\hline $\begin{array}{l}\text { May } \\
\text { June } \\
\text { July } \\
\end{array}$ & $\begin{array}{r}28 \\
2 \\
16 \\
7 \\
\end{array}$ & $\begin{array}{l}17.42 \\
17.41 \\
17.15 \\
16.91\end{array}$ & $\begin{array}{ll}\text { July } & 1 \\
2 \\
\text { Aug. } 2 \\
\end{array}$ & $\begin{array}{l}16.84 \\
16.87 \\
16.92 \\
17.08\end{array}$ & $\begin{array}{rr}\text { Sept. } 10 \\
15 \\
\text { oct. } 8 \\
24 \\
\end{array}$ & $\begin{array}{l}17.20 \\
17.20 \\
17.22 \\
17.18 \\
\end{array}$ & $\begin{array}{l}\text { Nov. } 11 \\
\text { Dec. } \frac{1}{15}\end{array}$ & $\begin{array}{l}17.00 \\
16.93 \\
16.90\end{array}$ \\
\hline
\end{tabular}

M-1. City of Wichita. NW cor, sec. 29, T. 23 S., R. 2 W.

Water level, in feet below measuring point, 1941

\begin{tabular}{|c|c|c|c|c|c|c|c|c|c|c|c|c|}
\hline Jan. & $\begin{array}{r}13 \\
20 \\
28 \\
10 \\
17 \\
25 \\
3 \\
18\end{array}$ & $\begin{array}{l}\mathbf{a} \\
\mathbf{a}\end{array}$ & $\begin{array}{l}72.57 \\
72.63 \\
34.84 \\
27.30 \\
27.24 \\
29.73 \\
70.11 \\
29.53\end{array}$ & $\begin{array}{l}\text { Mar. } \\
\text { Apr. } \\
\text { May }\end{array}$ & $\begin{array}{l}24 \\
31 \\
21 \\
28 \\
12 \\
19 \\
26\end{array}$ & $\begin{array}{ll} & 27.00 \\
& 27.70 \\
\mathrm{a} & 72.02 \\
\mathrm{a} & 74.00 \\
28.14 \\
\\
27.40 \\
\mathrm{a} 73.59\end{array}$ & $\begin{array}{l}\text { June } \\
\text { July } \\
\text { Aug. }\end{array}$ & $\begin{array}{r}2 \\
16 \\
\% \\
14 \\
21 \\
28 \\
25\end{array}$ & $a$ & $\begin{array}{l}73.78 \\
32.77 \\
29.28 \\
26.46 \\
71.83 \\
73.02 \\
32.63\end{array}$ & $\begin{array}{lr}\text { Sept. } 10 \\
15 \\
\text { Oct. } 8 \\
\\
24 \\
\text { Nov. } \\
11 \\
\text { Dec. } & 1 \\
& 15\end{array}$ & $\begin{array}{l}29.79 \\
29.66 \\
34.03 \\
26.21 \\
29.38 \\
29.04 \\
24.07\end{array}$ \\
\hline
\end{tabular}

M-la. City of Wichita. NW cor. sec. 29, T. 23 S., R. 2 W.

Water level, in feet below measuring point, 1941

\begin{tabular}{rrr|rr|rrr|rrr}
\hline Jan. 13 & b & 36.73 & Feb. 10 & 24.02 & Mar. & 3 & b 36.12 & Mar. 31 & 24.65 \\
20 & b 36.89 & 17 & 23.92 & & 18 & 25.80 & Apr. 21 & b 34.98 \\
28 & 29.67 & 25 & 25.17 & & 24 & 23.91 & & 28 & b 37.72 \\
\hline
\end{tabular}

a Well pumping. $\mathrm{b}$ Well M-I pumping.

$5110050-43-7$ 
Harvey County--Continued

M-1a. City of Wichita.--Continued.

Water level, in feet below measuring point, 1941

\begin{tabular}{|c|c|c|c|c|c|c|c|c|c|}
\hline Date & & & $\begin{array}{l}\text { Water } \\
\text { level }\end{array}$ & Date & $\begin{array}{l}\text { Water } \\
\text { level }\end{array}$ & Date & $\begin{array}{l}\text { Water } \\
\text { level }\end{array}$ & Date & $\begin{array}{l}\text { Water } \\
\text { level }\end{array}$ \\
\hline $\begin{array}{l}\text { May } \\
\text { June }\end{array}$ & $\begin{array}{r}12 \\
19 \\
26 \\
2 \\
16\end{array}$ & $\begin{array}{l}\mathbf{a} \\
\mathbf{a}\end{array}$ & $\begin{array}{l}24.80 \\
24.11 \\
37.64 \\
37.11 \\
29.34\end{array}$ & $\begin{array}{r}\text { July } 7 \\
14 \\
21 \\
28\end{array}$ & $\begin{array}{l}25.55 \\
23.39 \\
\text { a } 36.71 \\
\text { a } 38.33\end{array}$ & $\begin{array}{lr}\text { Aug. } & 25 \\
\text { Sept.10 } & 15 \\
& 15 \\
\text { Oct. } 8\end{array}$ & $\begin{array}{l}23.77 \\
26.55 \\
24.56 \\
30.30\end{array}$ & $\begin{array}{l}\text { Oct. } 24 \\
\text { Nov. } 11 \\
\text { Dec. } \frac{1}{15}\end{array}$ & $\begin{array}{l}23.25 \\
25.73 \\
29.04 \\
21.23\end{array}$ \\
\hline
\end{tabular}

M-1b. City of Wichita. NW cor. sec. 29, T. 23 S., R. 2 W.

Water level, in feet below measuring point, 1941

\begin{tabular}{|c|c|c|c|c|c|c|c|c|c|c|c|c|}
\hline $\mathrm{Feb}$. & $\begin{array}{l}13 \\
20 \\
28 \\
10 \\
17- \\
25 \\
3 \\
18\end{array}$ & a & $\begin{array}{l}33.57 \\
33.75 \\
27.98 \\
22.67 \\
22.61 \\
23.91 \\
33.29 \\
24.58\end{array}$ & $\begin{array}{l}\text { Mar. } \\
\text { Apr. } \\
\text { May }\end{array}$ & $\begin{array}{l}24 \\
31 \\
21 \\
28 \\
12 \\
19 \\
26\end{array}$ & $\begin{array}{l}\mathbf{a} \\
\mathbf{a}\end{array}$ & $\begin{array}{l}22.60 \\
23.30 \\
31.98 \\
34.70 \\
23.45 \\
22.79 \\
34.66\end{array}$ & $\begin{array}{rr}\text { June } & 2 \\
& 16 \\
\text { July } & 7 \\
1 & \\
21 \\
28 \\
\\
\text { Aug. } 25\end{array}$ & $\begin{array}{l}\mathbf{a} \\
\mathbf{a}\end{array}$ & $\begin{array}{l}34.05 \\
28.05 \\
24.36 \\
22.04 \\
33.87 \\
35.42 \\
27.67\end{array}$ & $\begin{array}{l}\text { Sept.1 } \\
\text { Oct. } \\
\text { Nov. } 1 \\
\text { Dec. }\end{array}$ & $\begin{array}{l}25.28 \\
23.27 \\
30.72 \\
21.87 \\
24.55 \\
24.48 \\
19.84\end{array}$ \\
\hline
\end{tabular}

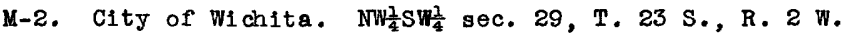

Water level, in feet below measuring point, 1941

\begin{tabular}{|c|c|c|c|c|c|c|c|c|c|c|c|c|c|}
\hline Feb. & $\begin{array}{l}13 \\
20 \\
28 \\
10 \\
17 \\
25 \\
3\end{array}$ & & $\begin{array}{l}36.75 \\
35.16 \\
37.49 \\
36.50 \\
38.57 \\
90.81 \\
37.53\end{array}$ & $\begin{array}{l}\text { Apr. } \\
\text { May }\end{array}$ & $\begin{array}{l}18 \\
24 \\
31 \\
21 \\
28 \\
12 \\
19\end{array}$ & $\begin{array}{r}\text { b } 92.30 \\
34.87 \\
33.79 \\
36.10 \\
39.06 \\
38.69 \\
37.23\end{array}$ & $\begin{array}{l}\text { Ma J } \\
\text { June } \\
\text { July }\end{array}$ & $\begin{array}{r}26 \\
2 \\
16 \\
7 \\
14 \\
21 \\
28\end{array}$ & $b$ & $\begin{array}{l}39.10 \\
36.95 \\
37.03 \\
92.59 \\
32.81 \\
44.15 \\
43.43\end{array}$ & $\begin{array}{l}\text { Sept. } \\
\text { Oct. } \\
\text { Nov. } \\
\text { Dec. }\end{array}$ & $\mathrm{b}$ & $\begin{array}{l}37.73 \\
40.21 \\
44.05 \\
31.59 \\
94.61 \\
38.98 \\
29.38\end{array}$ \\
\hline
\end{tabular}

M-2a. City of W1chita. IW $\frac{1}{4}$ SW $\frac{1}{4}$ sec. 29, T. 23 S., R. 2 W.

Water level, in feet below measuring point, 1941

\begin{tabular}{|c|c|c|c|c|c|c|c|c|c|c|c|}
\hline $\operatorname{Jan}$ & $\begin{array}{r}13 \\
20 \\
28 \\
10 \\
17 \\
25 \\
3 \\
18\end{array}$ & $\begin{array}{r}32.67 \\
32.20 \\
29.86 \\
25.71 \\
26.63 \\
\mathrm{c} 29.60 \\
33.22 \\
\mathrm{c} 30.39\end{array}$ & $\begin{array}{ll}\text { Mar. } & 2 \\
& 3 \\
\text { Apr. } & 2 \\
& 2 \\
\text { May } & 1 \\
& \frac{1}{2}\end{array}$ & $\begin{array}{l}24 \\
31 \\
21 \\
28 \\
12 \\
19 \\
26\end{array}$ & $\begin{array}{l}25.74 \\
25.87 \\
31.55 \\
34.60 \\
26.06 \\
25.79 \\
34.38\end{array}$ & $\begin{array}{l}\text { June } \\
\text { July } \\
\text { Aug. }\end{array}$ & $\begin{array}{r}2 \\
16 \\
7 \\
14 \\
21 \\
28 \\
25\end{array}$ & $\begin{array}{l}33.12 \\
30.31 \\
\text { c } 30.75 \\
24.61 \\
35.20 \\
35.83 \\
\text { c } 34.63\end{array}$ & $\begin{array}{lr}\text { Sept.10 } & 15 \\
& 8 \\
\text { Oct. } & 8 \\
& 24 \\
\text { Nov. } & 11 \\
\text { Dec. } & 1 \\
& 15\end{array}$ & c & $\begin{array}{l}28.47 \\
27.50 \\
32.87 \\
24.11 \\
30.38 \\
28.84 \\
22.04\end{array}$ \\
\hline
\end{tabular}

M-2b. City of Wichita. WW $\mathrm{T} W \frac{1}{4}$ sec. 29, T. 23 S., R. 2 W.

Water level, in feet below measuring point, 1941

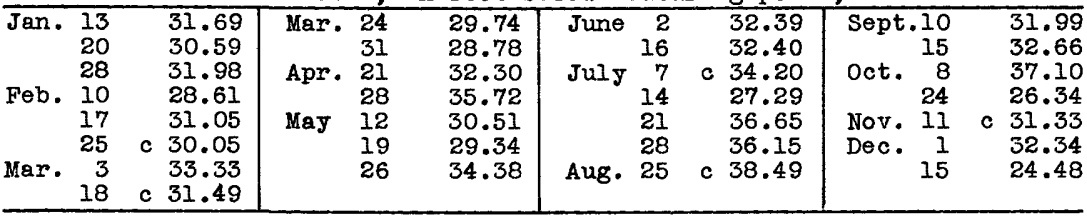

M-3. City of Wichita. $\operatorname{SE}_{\frac{1}{4}} \mathrm{SE}_{\frac{1}{4}} \mathrm{SW} \frac{1}{4}$ sec. $29, \mathrm{~T}, 23 \mathrm{~S}, \mathrm{R}, 2 \mathrm{~W}$.

Water level, in feet below measuring point, 1941

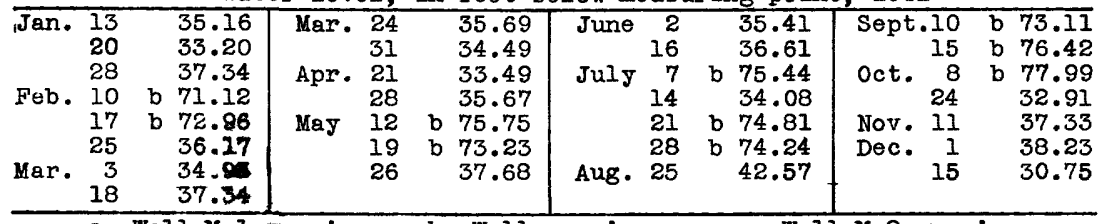


Harvey County--Continued

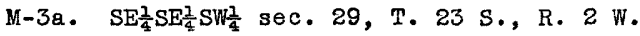

Water level, in feet below measuring point, 1941

\begin{tabular}{|c|c|c|c|c|c|c|c|c|c|c|c|}
\hline Date & & & $\begin{array}{l}\text { Water } \\
\text { level }\end{array}$ & Date & & $\begin{array}{l}\text { Water } \\
\text { level }\end{array}$ & Date & $\begin{array}{l}\text { Water } \\
\text { level }\end{array}$ & Date & & $\begin{array}{l}\text { Water } \\
\text { level }\end{array}$ \\
\hline Jan。 & $\begin{array}{r}13 \\
20 \\
28 \\
10 \\
17 \\
25 \\
3 \\
18\end{array}$ & $\begin{array}{l}\mathbf{a} \\
\mathbf{a}\end{array}$ & $\begin{array}{l}30.20 \\
28.11 \\
32.41 \\
34.64 \\
37.34 \\
30.35 \\
31.01 \\
31.80\end{array}$ & $\begin{array}{ll}\text { Mar. } & 24 \\
& 31 \\
\text { Apr. } & 21 \\
& 28 \\
\text { May } & 12 \\
19 \\
\\
26\end{array}$ & a & $\begin{array}{l}31.45 \\
29.84 \\
29.15 \\
32.22 \\
37.31 \\
35.53 \\
33.68\end{array}$ & $\begin{array}{lr}\text { June } & 2 \\
& 16 \\
\text { July } & 7 \\
14 \\
\\
21 \\
\\
28 \\
\text { Aug. } 25\end{array}$ & $\begin{array}{ll} & 30.52 \\
& 32.50 \\
\text { a } 40.56 \\
\\
28.73 \\
\text { a } 41.12 \\
\text { a } 40.08 \\
& 38.62\end{array}$ & $\begin{array}{lr}\text { Sept. } 10 \\
15 \\
\text { Oct. } 8 \\
24 \\
\text { Nov. } 11 \\
\text { Dec. } 1 \\
15\end{array}$ & $\begin{array}{l}a \\
a \\
a\end{array}$ & $\begin{array}{l}34.88 \\
38.71 \\
42.13 \\
27.46 \\
32.00 \\
33.90 \\
25.37\end{array}$ \\
\hline
\end{tabular}

M-3b. SE $\frac{1}{4} \mathrm{SE} \frac{1}{4} \mathrm{SW} \frac{1}{4}$ sec. 29, T. $23 \mathrm{~S}$, R. $2 \mathrm{~W}$.

Water level, in feet below measuring point, 1941

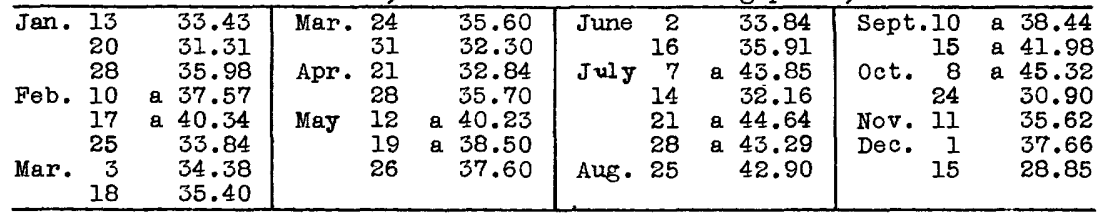

M-4. City of Wichita. SE cor. sec. 30, T. 23 S., R. 2 W.

Water level, in feet below measuring point, 1941

\begin{tabular}{|c|c|c|c|c|c|c|c|c|c|c|c|c|c|}
\hline $\operatorname{Jan}$. & $\begin{array}{l}13 \\
20 \\
28 \\
10 \\
17 \\
25 \\
3 \\
18\end{array}$ & & $\begin{array}{l}34.93 \\
32.80 \\
39.00 \\
34.88 \\
39.44 \\
35.80 \\
68.84 \\
37.54\end{array}$ & $\begin{array}{l}\text { Mar. } \\
\text { Apr. } \\
\text { May }\end{array}$ & $\begin{array}{l}24 \\
31 \\
21 \\
28 \\
12 \\
19 \\
26\end{array}$ & $\begin{array}{l}\mathrm{b} \\
\mathrm{b}\end{array}$ & $\begin{array}{l}37.56 \\
34.47 \\
71.48 \\
73.68 \\
37.33 \\
35.63 \\
40.19\end{array}$ & $\begin{array}{l}\text { June } \\
\text { July } \\
\text { Aug. }\end{array}$ & $\begin{array}{r}2 \\
16 \\
7 \\
14 \\
21 \\
28 \\
25\end{array}$ & & $\begin{array}{l}35.51 \\
37.97 \\
43.48 \\
32.96 \\
43.73 \\
41.49 \\
80.86\end{array}$ & $\begin{array}{lr}\text { Sept. } 10 \\
\\
15 \\
\text { oct. } \\
\\
24 \\
\text { Nov. } \\
11 \\
\text { Dec. } \\
15\end{array}$ & $\begin{array}{l}39.07 \\
\text { b } 79.01 \\
\text { b } 78.16 \\
31.56 \\
37.93 \\
39.90 \\
29.91\end{array}$ \\
\hline
\end{tabular}

M-4a. City of Wichita. SE cor. sec. 30, T, 23 S., R. 2 W.

Water level, in feet below measuring point, 1941

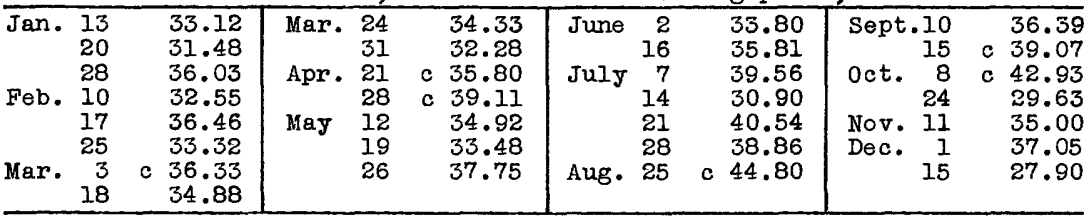

M-4b. City of Wichita. SE cor. sec. 30, T. 23 S., R. 2 W.

Water level, in feet below measuring point, 1941

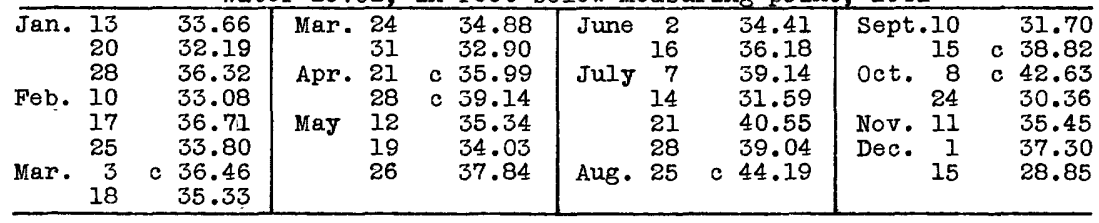

a Well $M-3$ pumping.

b Well pumping.

c Well M-4 pumping. 


\section{Harvey County--Continued}

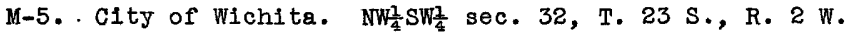

Water level, in feet below measuring point, 1941

\begin{tabular}{|c|c|c|c|c|c|c|c|c|}
\hline Date & $\begin{array}{l}\text { Water } \\
\text { level }\end{array}$ & Date & $\begin{array}{l}\text { Water } \\
\text { level }\end{array}$ & Date & & $\begin{array}{l}\text { Water } \\
\text { level }\end{array}$ & Date & $\begin{array}{l}\text { Water } \\
\text { level }\end{array}$ \\
\hline 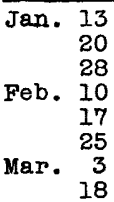 & $\begin{array}{l}28.20 \\
26.85 \\
29.99 \\
27.55 \\
29.06 \\
26.97 \\
28.11 \\
29.15\end{array}$ & $\begin{array}{ll}\text { Mar. } & 24 \\
& 31 \\
\text { Apr. } & 21 \\
& 28 \\
\text { May } & 12 \\
& 19 \\
& 26\end{array}$ & $\begin{array}{r}\text { a. } 92.04 \\
34.30 \\
31.59 \\
31.05 \\
34.08 \\
29.80 \\
\text { a } 90.41\end{array}$ & $\begin{array}{rr}\text { June } & 2 \\
& 16 \\
\text { JuIy } 7 \\
14 \\
21 \\
\text { Aug. } 23 \\
\text { Sept.10 }\end{array}$ & $\begin{array}{l}\mathbf{a} \\
\mathbf{a} \\
\mathbf{a}\end{array}$ & $\begin{array}{l}31.78 \\
37.33 \\
90.41 \\
30.80 \\
93.41 \\
93.41 \\
30.49\end{array}$ & $\begin{array}{lr}\text { Sept.15 } \\
\text { Oct. } \\
2 \\
8 \\
\\
24 \\
\text { Nov. } 11 \\
\text { Dec. } & 1 \\
& 15\end{array}$ & $\begin{array}{l}30.53 \\
30.77 \\
40.46 \\
27.75 \\
31.03 \\
40.03 \\
26.84\end{array}$ \\
\hline
\end{tabular}

M-5a. City of Wichita. NW $\frac{1}{4}$ SW sec. 32, T. 23 S., R. 2 W.

Water level, in feet below measuring point, 1941

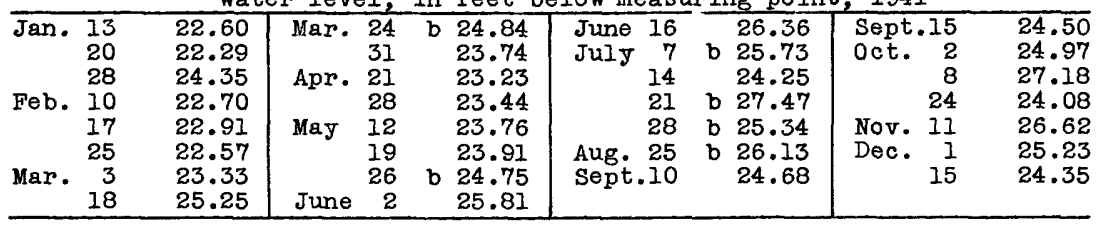

M-5b. City of Wichita. NW $W_{4}^{1} S W_{4}^{1}$ sec. 32, T. 23 S., R. 2 W.

Water level, in feet below measuring point, 1941

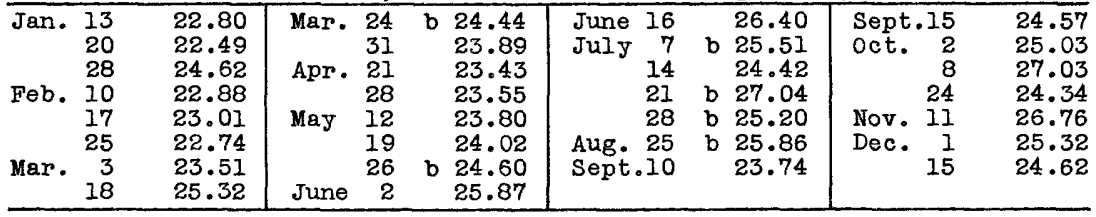

M-6. City of Wichita. SW $\frac{1}{4}$ SW $\frac{1}{4}$ sec. 32, T. 23 S., R. 2 W.

Water level, in feet below measuring point, 1941

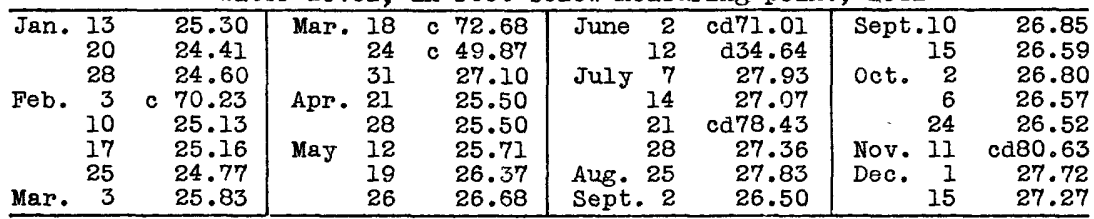

M-6a. City of Wichita. SW $\frac{1}{4} S w_{4}^{\frac{1}{4}}$ sec. 32, T. 23 S., R. 2 W.

Water level, in feet below measuring point, 1941

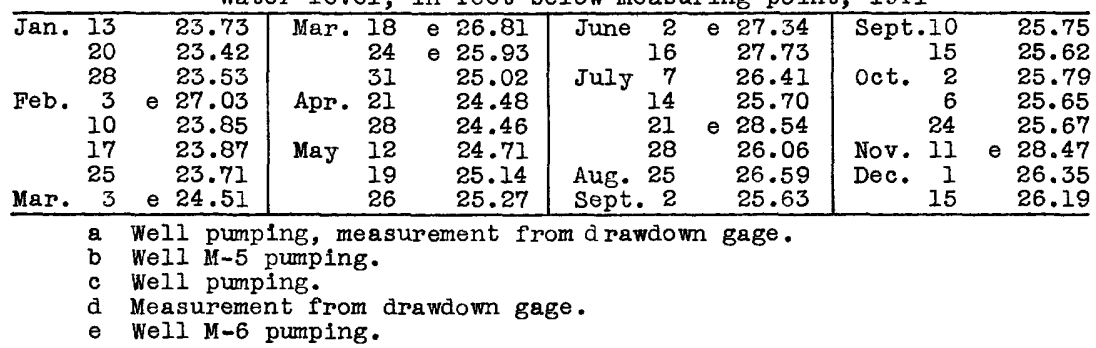


Harvey County--Continued

M-6b. City of Wichita. SW $\frac{1}{4} W_{\frac{1}{4}}$ sec. 32, T. 23 S., R. 2 W.

Water level, in feet below measuring point, 1941

\begin{tabular}{|c|c|c|c|c|c|c|c|c|c|c|c|c|c|c|}
\hline Date & & & $\begin{array}{l}\text { Water } \\
\text { level }\end{array}$ & Date & & & $\begin{array}{l}\text { Water } \\
\text { level }\end{array}$ & Date & & & $\begin{array}{l}\text { Water } \\
\text { level }\end{array}$ & Date & & $\begin{array}{l}\text { Water } \\
\text { level }\end{array}$ \\
\hline Jan. & $\begin{array}{r}13 \\
20 \\
28 \\
3 \\
10 \\
17 \\
25 \\
3\end{array}$ & $a$ & $\begin{array}{l}23.56 \\
23.27 \\
23.33 \\
26.67 \\
23.67 \\
23.68 \\
23.52 \\
24.31\end{array}$ & $\begin{array}{l}\text { Mar. } \\
\text { Apr. } \\
\text { May }\end{array}$ & $\begin{array}{l}18 \\
24 \\
31 \\
21 \\
28 \\
12 \\
19 \\
26\end{array}$ & $\begin{array}{l}a \\
a\end{array}$ & $\begin{array}{l}26.46 \\
25.69 \\
24.80 \\
24.26 \\
24.26 \\
24.48 \\
24.87 \\
25.09\end{array}$ & $\begin{array}{l}\text { June } \\
\text { July } \\
\text { Aug. } \\
\text { Sept }\end{array}$ & $\begin{array}{r}2 \\
16 \\
7 \\
14 \\
21 \\
28 \\
25 \\
2\end{array}$ & $\mathbf{a}$ & $\begin{array}{l}27.08 \\
27.39 \\
26.13 \\
25.44 \\
28.21 \\
25.86 \\
26.45 \\
25.43\end{array}$ & $\begin{array}{lr}\text { Sept. } 10 \\
& 15 \\
\text { Oct. } & 2 \\
& 6 \\
& 24 \\
\text { Nov. } & 11 \\
\text { Dec. } & 1 \\
& 15\end{array}$ & $\mathbf{a}$ & $\begin{array}{l}25.60 \\
25.40 \\
25.62 \\
25.79 \\
25.44 \\
28.10 \\
26.13 \\
25.85\end{array}$ \\
\hline
\end{tabular}

M-7. City of Wichita. NW cor.sW sec. 16, T. 24 S., R. 2 W.

Water level, in feet below measuring point, 1941

\begin{tabular}{|c|c|c|c|c|c|c|c|c|c|c|c|c|c|}
\hline $\begin{array}{l}\text { Jan. } \\
\text { Feb. } \\
\text { Mar. }\end{array}$ & $\begin{array}{r}20 \\
28 \\
10 \\
17 \\
3 \\
18 \\
24\end{array}$ & $\begin{array}{l}17.72 \\
17.57 \\
19.97 \\
18.00 \\
17.36 \\
17.01 \\
17.17\end{array}$ & $\begin{array}{l}\text { Mar. } \\
\text { Apr. } \\
\text { May } \\
\text { June }\end{array}$ & $\begin{array}{r}31 \\
17 \\
28 \\
12 \\
19 \\
26 \\
2\end{array}$ & $b$ & $\begin{array}{l}24.54 \\
17.90 \\
17.86 \\
18.39 \\
18.37 \\
18.18 \\
25.04\end{array}$ & $\begin{array}{l}\text { June } \\
\text { July } \\
\text { Aug. } \\
\text { Sept }\end{array}$ & $\begin{array}{r}16 \\
7 \\
14 \\
21 \\
28 \\
26 \\
10\end{array}$ & $b$ & $\begin{array}{l}24.71 \\
15.89 \\
16.45 \\
16.30 \\
18.00 \\
26.25 \\
20.46\end{array}$ & $\begin{array}{l}\text { Sept.1 } \\
\text { Oct. } \\
\text { Nov. } \frac{1}{2} \\
\text { Dec. }\end{array}$ & & $\begin{array}{l}26.37 \\
19.24 \\
19.11\end{array}$ \\
\hline
\end{tabular}

M-7a. City of Wichita. NW/ $S W \frac{1}{4}$ sec. 16, T. 24 S., R. 2 W.

Water level, in feet below measuring point, 1941

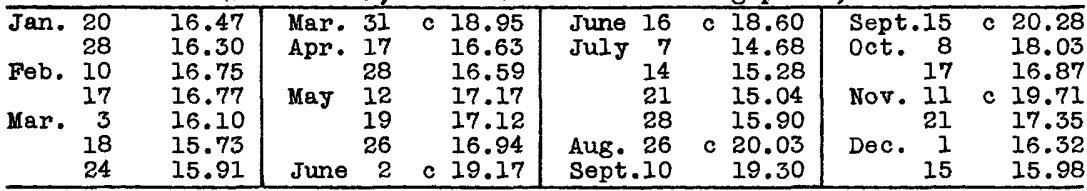

M-Tb. City of Wichita. SW cor. NW sec. 16

Water level, in feet below measuring point, 1941

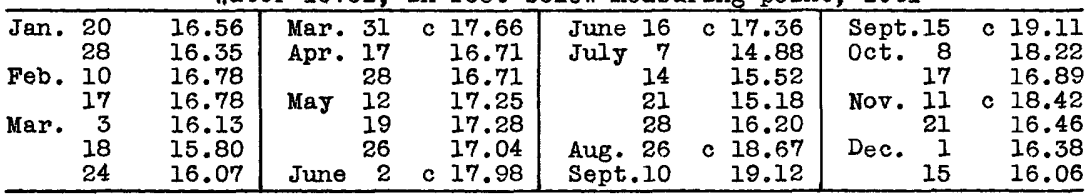

M-8. City of Wichita. $\mathrm{SE}_{\frac{1}{4}} \mathrm{NE} \frac{1}{4} \sec , 6, \mathrm{~T}, 24 \mathrm{~S} ., \mathrm{R} .2 \mathrm{~W}$.

Water level, in feet below measuring point, 1941

\begin{tabular}{|c|c|c|c|c|c|c|c|c|c|c|c|c|c|c|c|}
\hline $\begin{array}{l}\text { Feb. } \\
\text { Mar. }\end{array}$ & $\begin{array}{l}13 \\
20 \\
27 \\
10 \\
17 \\
25 \\
3 \\
18\end{array}$ & & $\begin{array}{l}22.64 \\
22.37 \\
22.58 \\
22.94 \\
22.97 \\
23.05 \\
78.21 \\
24.15\end{array}$ & $\begin{array}{l}\text { Mar. } \\
\text { Apr. } \\
\text { May } \\
\text { June }\end{array}$ & $\begin{array}{r}24 \\
31 \\
17 \\
28 \\
12 \\
19 \\
26 \\
2\end{array}$ & $b$ & $\begin{array}{l}23.77 \\
83.86 \\
23.86 \\
23.88 \\
24.07 \\
81.01 \\
24.50 \\
24.99\end{array}$ & $\begin{array}{l}\text { June } \\
\text { July }\end{array}$ & $\begin{array}{r}16 \\
7 \\
14 \\
21 \\
28 \\
25 \\
10 \\
15\end{array}$ & $\begin{array}{l}b \\
b \\
b\end{array}$ & $\begin{array}{l}25.32 \\
80.12 \\
81.11 \\
81.01 \\
25.26 \\
25.85 \\
25.44 \\
25.08\end{array}$ & $\begin{array}{l}\text { Oct. } \\
\text { Nov. } \\
\text { Dec. }\end{array}$ & & 0 & $\begin{array}{l}24.97 \\
26.71 \\
25.71 \\
81.10 \\
80.71\end{array}$ \\
\hline
\end{tabular}

M-8a. City of Wichita. SE $\frac{1}{4} N E_{\frac{1}{4}}^{\frac{1}{4}}$ sec. 6, T. 24 S., R. 2 W.

Water level, in feet below measuring point, 1941

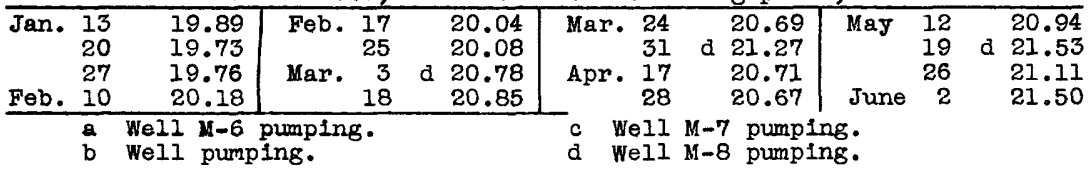


Harvey County--Continued

M-8a.--Continued.

Water level, in feet below measuring point, 1941

\begin{tabular}{|c|c|c|c|c|c|c|c|c|}
\hline Date & $\begin{array}{l}\text { Water } \\
\text { level }\end{array}$ & Date & $\begin{array}{l}\text { Water } \\
\text { level }\end{array}$ & Date & $\begin{array}{l}\text { Water } \\
\text { level }\end{array}$ & Date & & $\begin{array}{l}\text { Water } \\
\text { level }\end{array}$ \\
\hline $\begin{array}{rr}\text { June } & 16 \\
\text { July } & 7 \\
& 14 \\
21\end{array}$ & $\begin{array}{ll} & 21.63 \\
\text { a } & 22.41 \\
\text { a } 22.48 \\
\text { a } 22.87\end{array}$ & $\begin{array}{l}\text { July } 28 \\
\text { Aug. } 25 \\
\text { Sept. } 10\end{array}$ & $\begin{array}{l}22.24 \\
22.64 \\
22.29\end{array}$ & $\begin{array}{l}\text { Sept. } 15 \\
\text { Oct. } 8 \\
\text { Nov. } 11\end{array}$ & $\begin{array}{l}22.11 \\
21.89 \\
23.00\end{array}$ & $\begin{array}{lr}\text { Nov. } 21 \\
\text { Dec. } \\
\\
\\
15\end{array}$ & $\begin{array}{l}a \\
a\end{array}$ & $\begin{array}{l}22.00 \\
22.71 \\
23.26\end{array}$ \\
\hline
\end{tabular}

M-8b. City of Wichita. $\mathrm{SE}_{\frac{1}{4}}^{\frac{1}{2}} \mathrm{NE}_{\frac{1}{4}}^{\frac{1}{4}}$ sec. 6, T. 24 S., R. 2 W.

Water level, in feet below measuring point, 1941

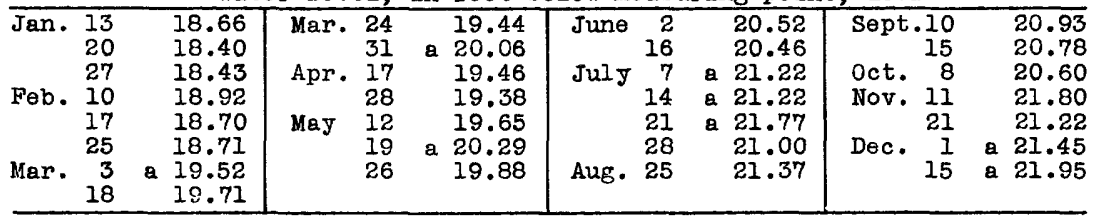

M-9. City of Wichita. NWt $N W_{\frac{1}{4}}^{\frac{1}{4}} \mathrm{sec}, 8$, T. 24 S., R. 2 W.

Water level, in feet below measuring point, 1941

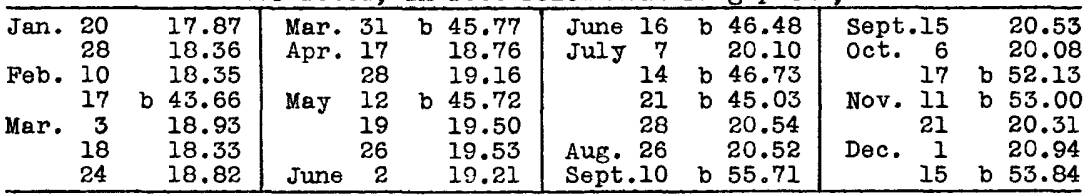

M-9a, City of Wichita. NW $N W \frac{1}{4}$ sec. 8, T. 24 S., R. 2 W.

Water level, in feet below measuring point, 1941

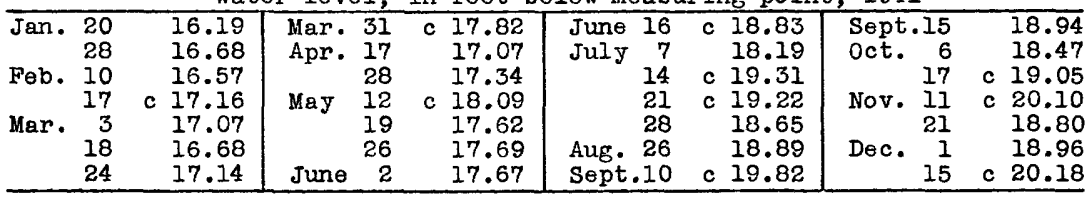

M-9b. City of Wichita. NW $N W_{\frac{1}{4}}^{\frac{1}{4}}$ sec. 8, T. 24 S., R. 2 W.

Water level, in feet below measuring point, 1941

\begin{tabular}{|c|c|c|c|c|c|c|c|c|c|c|c|c|}
\hline $\begin{array}{l}\text { Tan. } \\
\text { Peb. } \\
\text { Iar. }\end{array}$ & $\begin{array}{r}20 \\
28 \\
10 \\
17 \\
3 \\
18 \\
24\end{array}$ & c & $\begin{array}{l}15.10 \\
15.64 \\
15.63 \\
15.94 \\
16.04 \\
15.63 \\
16.04\end{array}$ & $\begin{array}{l}\text { Mar. } \\
\text { Apr. } \\
\text { May } \\
\text { June }\end{array}$ & $\begin{array}{r}31 \\
17 \\
28 \\
12 \\
19 \\
26 \\
2\end{array}$ & $\begin{array}{l}\text { c } 16.35 \\
15.99 \\
16.19 \\
\text { c } 16.71 \\
16.59 \\
16.61 \\
16.60\end{array}$ & $\begin{array}{l}\text { June } \\
\text { July } \\
\text { Aug. } \\
\text { Sept }\end{array}$ & $\begin{array}{r}16 \\
7 \\
14 \\
21 \\
28 \\
26 \\
10\end{array}$ & & $\begin{array}{l}17.44 \\
17.17 \\
17.93 \\
17.83 \\
17.55 \\
17.74 \\
18.54\end{array}$ & $\begin{array}{lr}\text { Sept. } 15 \\
\text { oct. } \\
\\
17 \\
\text { Nov. } \\
11 \\
21 \\
\text { Dec. } \\
15\end{array}$ & $\begin{array}{ll} & 1 \\
& 1 \\
\text { c } & 1 \\
\text { c } & 1 \\
1 \\
\text { c } & 1 \\
\end{array}$ \\
\hline
\end{tabular}

M-10. City of Wichita. NE $\frac{1}{4} N W_{4}^{\frac{1}{4}}$ sec. 8, T. 24 S., R. $2 W$.

Water level, in feet below measuring point, 1941

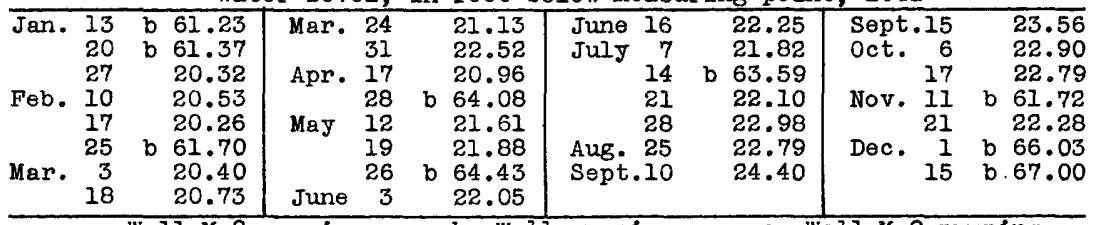

a Well M-8 pumping. b Well pumping. c Well M-9 pumping.


Harvey County--Continued

M-10a. City of Wichita. NE $\frac{1}{4}$ WW $\frac{1}{4}$ sec. 8, T. 24 S., R. 2 W.

Water level, in feet below mesuring point, 1941

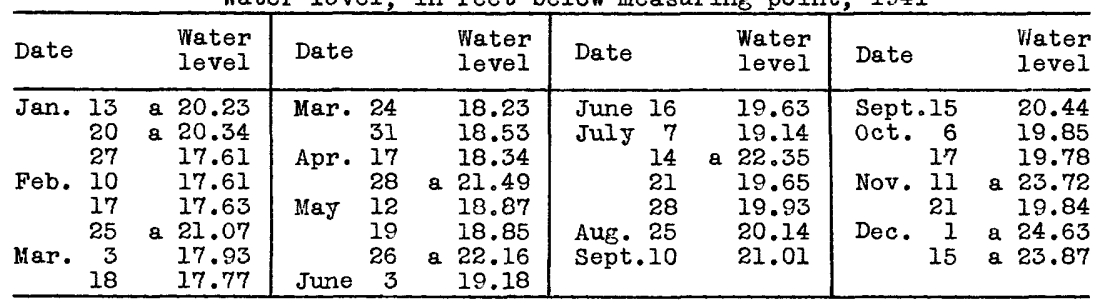

M-1Ob. City of Wichits. $\mathrm{NE}_{\frac{1}{4}}^{\frac{1}{2} \mathrm{NW}} \frac{\mathrm{l}}{4} \mathrm{sec}, 8, \mathrm{~T}, 24 \mathrm{~S}, \mathrm{R}, 2 \mathrm{~W}$.

Water level, in feet below measuring point, 1941

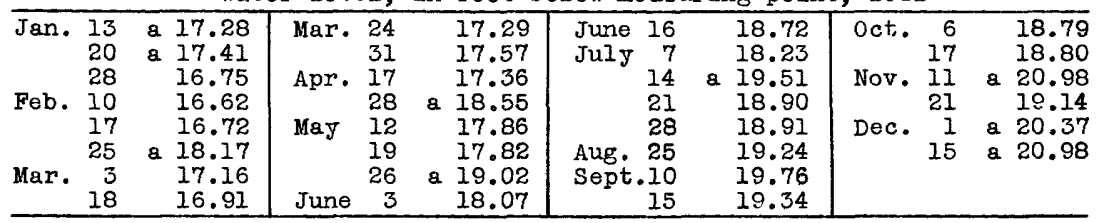

M-11. City of Wichita. SW $\frac{1}{4} \mathrm{NW} \frac{1}{4}$ sec. 8, T. $24 \mathrm{~S} ., \mathrm{R}, 2 \mathrm{~W}$.

Water level, in feet below measuring point, 1941

\begin{tabular}{|c|c|c|c|c|c|c|c|c|c|c|c|c|c|c|c|}
\hline $\begin{array}{l}\text { Jan. } \\
\text { Feb. } \\
\text { Mar. }\end{array}$ & $\begin{array}{r}20 \\
28 \\
10 \\
17 \\
3 \\
18 \\
24\end{array}$ & $\begin{array}{l}b \\
b \\
b\end{array}$ & $\begin{array}{l}14.97 \\
30.11 \\
34.32 \\
32.54 \\
14.60 \\
14.84\end{array}$ & $\begin{array}{l}\text { Mar. } \\
\text { Apr. } \\
\text { May } \\
\text { June }\end{array}$ & $\begin{array}{r}31 \\
17 \\
28 \\
12 \\
19 \\
26 \\
2\end{array}$ & $\mathrm{~b}$ & $\begin{array}{l}15 \\
15 \\
15 \\
15 \\
35 \\
16 \\
15\end{array}$ & $\begin{array}{l}\text { Aug. } \\
\text { Sept }\end{array}$ & $\begin{array}{r}16 \\
7 \\
14 \\
21 \\
28 \\
26 \\
10\end{array}$ & $\mathrm{~b}$ & $\begin{array}{l}16.59 \\
35.93 \\
36.92 \\
16.39 \\
35.93 \\
16.84 \\
17.84\end{array}$ & $\begin{array}{l}\text { Sept. } \\
\text { Oct. } \\
\text { Nov. } \\
\text { Dec. }\end{array}$ & $\begin{array}{r}15 \\
8 \\
17 \\
11 \\
21 \\
1 \\
15\end{array}$ & $\mathrm{~b}$ & \\
\hline
\end{tabular}

M-1la. City of Wichita. SW $\mathrm{NW}_{\frac{1}{4}}^{\frac{1}{4}}$ sec. 8, T. 24 S., R. 2 W.

Water level, in feet below measuring point, 1941

\begin{tabular}{|c|c|c|c|c|c|c|c|c|c|c|c|c|}
\hline $\begin{array}{l}\text { Jan. } \\
\text { Feb. } \\
\text { Mar. }\end{array}$ & $\begin{array}{r}28 \\
28 \\
10 \\
17 \\
3 \\
18 \\
24\end{array}$ & $\begin{array}{l}\mathrm{c} \\
\mathrm{c}\end{array}$ & $\begin{array}{l}12.24 \\
13.29 \\
14.58 \\
14.94 \\
14.85 \\
12.90 \\
13.02\end{array}$ & $\begin{array}{l}\text { Apr. } \\
\text { May }\end{array}$ & $\begin{array}{r}31 \\
17 \\
28 \\
12 \\
19 \\
26 \\
2\end{array}$ & $\begin{array}{r}13.19 \\
13.28 \\
13.26 \\
13.64 \\
\mathrm{c} 15.54 \\
13.98 \\
14.07\end{array}$ & $\begin{array}{l}\text { June } \\
\text { July } \\
\text { Aug. } \\
\text { Sept }\end{array}$ & $\begin{array}{r}16 \\
7 \\
14 \\
21 \\
28 \\
26 \\
10\end{array}$ & & $\begin{array}{l}14 . \\
15 \\
16 . \\
14 \\
16 .\end{array}$ & $\begin{array}{lr}\text { Sept. } 15 \\
\text { Oct. } \\
8 \\
17 \\
\text { Nov. } 11 \\
\\
21 \\
\text { Dec. } 1 \\
15\end{array}$ & $\begin{array}{l}15.21 \\
14.66 \\
14.59 \\
15.24 \\
14.67 \\
14.61 \\
17.35\end{array}$ \\
\hline
\end{tabular}

M-11b. City of Wichita. SW $\frac{1}{2} N W_{4}^{\frac{1}{4}}$ sec. 8, T. 24 S., R. 2 W.

Water level, in feet below measuring point, 1941

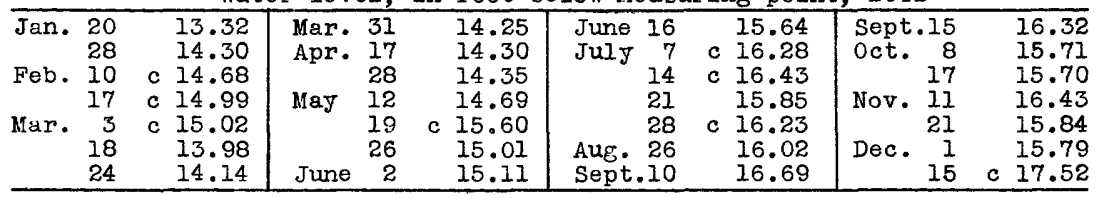

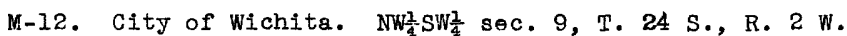

Water level, in feet below measuring point, 1941

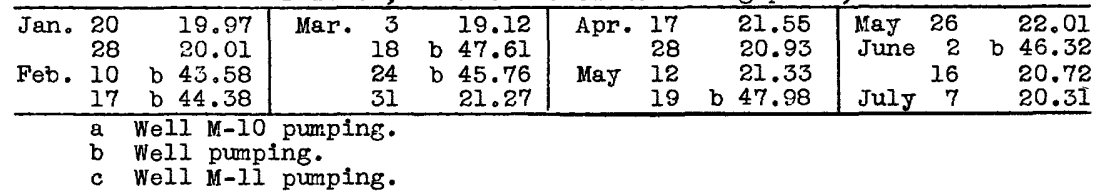


Harvey County--Continued

M-12.--Continued.

Water level, in feet below measuring point, 1941

\begin{tabular}{|c|c|c|c|c|c|c|c|c|c|c|}
\hline Date & & & $\begin{array}{l}\text { Water } \\
\text { level }\end{array}$ & Date & & $\begin{array}{l}\text { Water } \\
\text { level }\end{array}$ & Date & $\begin{array}{l}\text { Water } \\
\text { level }\end{array}$ & Date & $\begin{array}{l}\text { Water } \\
\text { level }\end{array}$ \\
\hline Tuly & $\begin{array}{l}14 \\
21 \\
28\end{array}$ & a & $\begin{array}{l}46.61 \\
19.85 \\
48.51\end{array}$ & $\begin{array}{l}\text { Aug. } 26 \\
\text { Sept. } 10 \\
15\end{array}$ & a & $\begin{array}{l}22.47 \\
51.45 \\
50.63\end{array}$ & $\begin{array}{r}\text { Oct. } 8 \\
17 \\
\text { Nov. } 11\end{array}$ & $\begin{array}{l}50.03 \\
20.00 \\
20.91\end{array}$ & $\begin{array}{l}\text { Nov. } 21 \\
\text { Dec. } \\
15\end{array}$ & $\begin{array}{l}21.19 \\
20.93 \\
20.81\end{array}$ \\
\hline
\end{tabular}

M-12a. City of Wichita. NW $\frac{1}{4} S W \frac{1}{4}$ sec. 9, T. 24 S., R. 2 W.

Water level, in feet below measuring point, 1941

\begin{tabular}{|c|c|c|c|c|c|c|c|c|c|c|c|c|c|}
\hline $\begin{array}{l}\text { Jan. } \\
\text { Feb. } \\
\text { Mar. }\end{array}$ & $\begin{array}{r}20 \\
28 \\
10 \\
17 \\
3 \\
18 \\
24 \\
\end{array}$ & $\begin{array}{l}b \\
b\end{array}$ & $\begin{array}{l}17.95 \\
18.08 \\
23.44 \\
21.31 \\
17.16 \\
24.00 \\
20.91\end{array}$ & $\begin{array}{l}\text { Mar. } \\
\text { Apr. } \\
\text { May } \\
\text { June }\end{array}$ & $\begin{array}{r}31 \\
17 \\
28 \\
12 \\
19 \\
26 \\
2\end{array}$ & $\begin{array}{l}b \\
b\end{array}$ & $\begin{array}{l}19.19 \\
19.61 \\
18.98 \\
19.61 \\
25.20 \\
20.24 \\
23.86\end{array}$ & $\begin{array}{l}\text { June I } \\
\text { July } \\
\\
2 \\
2 \\
\text { Aug. } 2 \\
\text { Sept.I }\end{array}$ & & $\begin{array}{l}1 \\
1 \\
\text { b } \\
2 \\
1 \\
\text { b } 2 \\
2 \\
b 2\end{array}$ & $\begin{array}{l}18.84 \\
18.54 \\
23.16 \\
18.03 \\
26.35 \\
20.65 \\
28.17\end{array}$ & $\begin{array}{lr}\text { Sept. } 15 \\
\text { Oct. } \\
\\
17 \\
\text { Nov. } 11 \\
\\
21 \\
\text { Dec. } 1 \\
15\end{array}$ & $\begin{array}{r}\mathrm{b} 27.20 \\
\mathrm{~b} 26.10 \\
18.39 \\
19.00 \\
19.46 \\
19.21 \\
18.84\end{array}$ \\
\hline
\end{tabular}

M-12b. City of Wichita. NW $W_{\frac{1}{4}}$ SW sec. 9, T. 24 S., R. 2 W.

Water level, in feet below measuring point, 1941

\begin{tabular}{|c|c|c|c|c|c|c|c|c|c|c|c|c|}
\hline $\begin{array}{l}\text { Jan. } \\
\text { Feb. } \\
\text { Mar. }\end{array}$ & $\begin{array}{r}20 \\
28 \\
10 \\
17 \\
3 \\
18 \\
24\end{array}$ & $\mathrm{~b}$ & $\begin{array}{l}18.68 \\
18.77 \\
23.72 \\
21.55 \\
17.81 \\
24.19 \\
22.32\end{array}$ & $\begin{array}{l}\text { Mar. } \\
\text { Apr. } \\
\text { May } \\
\text { June }\end{array}$ & $\begin{array}{r}31 \\
17 \\
28 \\
12 \\
19 \\
26 \\
2\end{array}$ & b & $\begin{array}{l}19.70 \\
20.27 \\
19.72 \\
20.33 \\
25.52 \\
20.91 \\
24.17\end{array}$ & $\begin{array}{lr}\text { June } & 16 \\
\text { JuIy } & 7 \\
& 14 \\
& 21 \\
& 28 \\
& \\
\text { Aug. } & 26 \\
\text { Sept. } 10\end{array}$ & ס & $\begin{array}{l}19.51 \\
19.27 \\
23.47 \\
18.69 \\
26.67 \\
21.33 \\
28.34\end{array}$ & 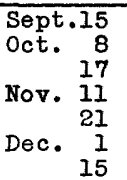 & $\begin{array}{l}\text { b } 27.39 \\
\text { b } 26.47 \\
19.12 \\
19.80 \\
20.24 \\
19.93 \\
19.50\end{array}$ \\
\hline
\end{tabular}

M-13. City of Wichita. NW/ $\mathrm{NE} \frac{1}{4}$ sec. 17, T. 24 S., R. 2 W.

Water level, in feet below measuring point, 1941

\begin{tabular}{|c|c|c|c|c|c|c|c|c|c|c|c|c|}
\hline $\begin{array}{l}\text { Jan. } \\
\text { Feb. } \\
\text { Mar. }\end{array}$ & $\begin{array}{r}20 \\
28 \\
10 \\
17 \\
3 \\
18 \\
24\end{array}$ & $\begin{array}{l}\mathbf{a} \\
\mathbf{a}\end{array}$ & $\begin{array}{l}15.40 \\
37.18 \\
34.94 \\
15.78 \\
15.52 \\
15.86 \\
15.99\end{array}$ & $\begin{array}{l}\text { Mar. } \\
\text { Apr. } \\
\text { May } \\
\text { June }\end{array}$ & $\begin{array}{l}31 \\
17 \\
28 \\
12 \\
19 \\
26 \\
2\end{array}$ & $\begin{array}{l}16.30 \\
\mathrm{a} \quad 38.54 \\
16.08 \\
\mathrm{a} 39.16 \\
17.36 \\
\mathrm{a} \quad 38.99 \\
17.26\end{array}$ & $\begin{array}{l}\text { June } \\
\text { July } \\
\text { Aug. } \\
\text { Sept }\end{array}$ & $\begin{array}{r}16 \\
7 \\
14 \\
21 \\
28 \\
26 \\
10\end{array}$ & & $\begin{array}{l}16.67 \\
37.96 \\
38.80 \\
15.71 \\
38.97 \\
40.26 \\
40.65\end{array}$ & $\begin{array}{lr}\text { Sept. } & 15 \\
\text { Oet. } & 8 \\
& 17 \\
\text { Nov. } & 11 \\
& 21 . \\
\text { Dec. } & 1\end{array}$ & $\begin{array}{ll}\text { a } 40 \\
\text { a } 35 \\
16 \\
16 \\
\\
16 \\
\text { a } 38\end{array}$ \\
\hline
\end{tabular}

M-13a. City of Wichita. NWT $\mathrm{NE}_{\frac{1}{4}}^{\frac{1}{4}}$ sec. 17, T. 24 S., R. 2 W.

Water level, in feet below measuring point, 1941

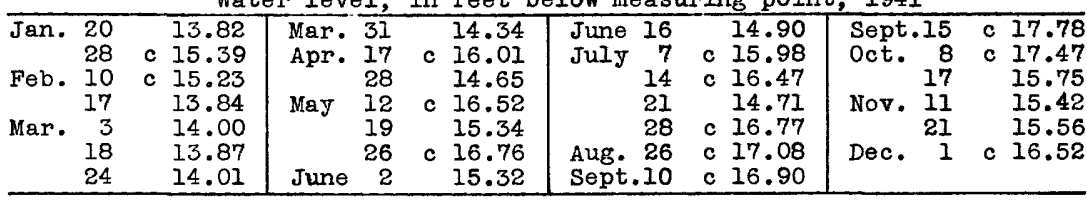

M-13b. City of Wichita. NW $\frac{1}{4} N E \frac{1}{4}$ sec. 17, T. 24 S., R. 2 W.

Water level, in feet below measuring point, 1941

\begin{tabular}{|c|c|c|c|c|c|c|c|c|c|}
\hline $\begin{array}{l}\text { Jan. } \\
\text { Feb. } \\
\text { Mar. }\end{array}$ & $\begin{array}{r}20 \\
28 \\
10 \\
17 \\
3 \\
18 \\
24\end{array}$ & $\begin{aligned} & 14.05 \\
& \text { c } 14.60 \\
& \text { c } 14.42 \\
& 13.97 \\
& 14.26 \\
& 13.87 \\
& 14.20\end{aligned}$ & $\begin{array}{l}\text { Mar. } \\
\text { Apr. } \\
\text { May } \\
\text { June }\end{array}$ & $\begin{array}{l}31 \\
17 \\
28 \\
12 \\
19 \\
26 \\
2\end{array}$ & $\begin{array}{r}14.58 \\
\text { c } 15.10 \\
15.02 \\
\text { c } 15.78 \\
15.63 \\
\text { c } 16.22 \\
15.58\end{array}$ & $\begin{array}{r}\text { June } 1 \\
\text { July } \\
1 \\
2 \\
28 \\
\text { Aug. } 2 \\
\text { Sept. }\end{array}$ & $\begin{array}{ll} & 15.22 \\
\text { c } & 15.77 \\
\text { c } 16.13 \\
\\
\text { c } 15.42 \\
\text { c } 16.40 \\
\text { c } 16.46 \\
17.20\end{array}$ & 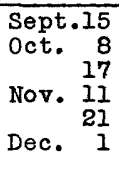 & $\begin{array}{l}\text { c } 17.22 \\
\text { c } 17.09 \\
16.37 \\
15.91 \\
16.19 \\
\text { c } 16.19\end{array}$ \\
\hline
\end{tabular}

a Well pumping. b Well M-I2 pumping. c Well M-13 pumping. 
Harvey County--Continued

M-14. City of Wichita. NW cor. NWt sec. 16, T. 24 S., R. 2 W. Water level, in feet below measuring point, 1941

\begin{tabular}{|c|c|c|c|c|c|c|c|c|c|c|c|c|c|}
\hline Dat the & & & $\begin{array}{l}\text { Weter } \\
\text { level }\end{array}$ & Date & & & $\begin{array}{l}\text { Water } \\
\text { level }\end{array}$ & Date & & $\begin{array}{l}\text { Water } \\
\text { level }\end{array}$ & Date & & $\begin{array}{l}\text { Water } \\
\text { level }\end{array}$ \\
\hline $\begin{array}{l}\text { Jan. } \\
\text { Feb. } \\
\text { Mar. }\end{array}$ & $\begin{array}{r}20 \\
28 \\
10 \\
17 \\
3 \\
18 \\
24\end{array}$ & $a$ & $\begin{array}{l}34.62 \\
17.24 \\
17.40 \\
16.93 \\
17.33 \\
17.08 \\
35.17\end{array}$ & $\begin{array}{l}\text { Mar. } \\
\text { Apr. } \\
\text { May } \\
\text { June }\end{array}$ & $\begin{array}{l}31 \\
17 \\
28 \\
12 \\
19 \\
26 \\
2\end{array}$ & a & $\begin{array}{l}18.11 \\
17.66 \\
36.16 \\
18.21 \\
36.35 \\
18.70 \\
18.57\end{array}$ & $\begin{array}{rr}\text { June } & 16 \\
\text { July } & 7 \\
14 \\
21 \\
28 \\
\text { Aug. } 26 \\
\text { Sept. } 10\end{array}$ & a & $\begin{array}{l}17.72 \\
18.01 \\
36.03 \\
18.01 \\
36.27 \\
18.85 \\
20.08\end{array}$ & $\begin{array}{l}\text { Sept. } 15 \\
\text { oct. } 8 \\
17 \\
17 \\
\text { Nov. } 11 \\
21 \\
\text { Dec. } 1 \\
15\end{array}$ & $a$ & $\begin{array}{l}20.02 \\
38.91 \\
18.72 \\
18.41 \\
37.10 \\
18.50 \\
17.80\end{array}$ \\
\hline
\end{tabular}

M-14a. City of Wichita. NW $W_{4}^{\frac{1}{4}} W_{\frac{2}{4}}$ sec. 16, T. 24 S., R, 2 W.

Water level, in feet below measuring point, 1941

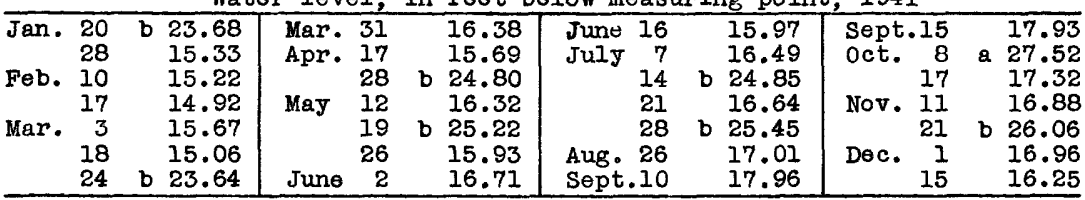

M-14b. Clty of Wichita. SW $W_{4}^{\frac{1}{4}}$ sec. 9, T. 24 S., R. 2 W.

Water level, in feet below measuring point, 1941

\begin{tabular}{|c|c|c|c|c|c|c|c|c|c|c|c|c|c|c|}
\hline $\begin{array}{l}\text { Jan. } \\
\text { Feb. } \\
\text { Mar. }\end{array}$ & $\begin{array}{r}20 \\
28 \\
10 \\
17 \\
3 \\
18 \\
24\end{array}$ & $\begin{array}{r}\text { b } 19.77 \\
15.14 \\
14.90 \\
14.71 \\
15.58 \\
14.84 \\
\text { b } 19.57\end{array}$ & $\begin{array}{l}\text { Mar. } \\
\text { Apr. } \\
\text { May } \\
\text { June }\end{array}$ & $\begin{array}{r}31 \\
17 \\
28 \\
12 \\
19 \\
26 \\
2\end{array}$ & $b$ & $\begin{array}{l}16.18 \\
15.45 \\
20.90 \\
16.20 \\
21.36 \\
16.80\end{array}$ & $\begin{array}{l}\text { June } \\
\text { Julg } \\
\text { Aug. } \\
\text { Sept }\end{array}$ & $\begin{array}{l}16 \\
16 \\
27 \\
28 \\
26\end{array}$ & & $\begin{array}{l}15.88 \\
16.54 \\
21.13 \\
16.73 \\
21.76 \\
16.80 \\
17.67\end{array}$ & $\begin{array}{l}\text { Sept } \\
\text { Oct. } \\
\text { Nov. } \\
\text { Dec. }\end{array}$ & $\begin{array}{r}15 \\
8 \\
17 \\
11 \\
21\end{array}$ & $\mathrm{~b}$ & $\begin{array}{l}17.67 \\
23.57 \\
17.38 \\
16.82 \\
22.25 \\
16.94 \\
16.54\end{array}$ \\
\hline
\end{tabular}

M-15. City of Wichita. SE cor. NE⿺ sec. 9, T. 24 S., R. 2 W.

Water level, in feet below measuring point, 1941

\begin{tabular}{|c|c|c|c|c|c|c|c|c|c|c|c|c|c|}
\hline Jan. & $\begin{array}{r}13 \\
20 \\
27 \\
10 \\
17 \\
25 \\
3 \\
18\end{array}$ & $a$ & $\begin{array}{l}19.54 \\
21.31 \\
38.77 \\
19.69 \\
19.61 \\
18.81 \\
21.00 \\
39.51\end{array}$ & $\begin{array}{l}\text { Mar. } \\
\text { Apr. } \\
\text { May } \\
\text { May }\end{array}$ & $a$ & $\begin{array}{l}19.61 \\
22.53 \\
39.83 \\
21.90 \\
19.59 \\
20.18 \\
19.71\end{array}$ & $\begin{array}{l}\text { June } \\
\text { July } \\
\text { Aug. }\end{array}$ & $\begin{array}{r}3 \\
16 \\
7 \\
14 \\
21 \\
28 \\
25\end{array}$ & & $\begin{array}{l}20.71 \\
21.73 \\
21.44 \\
19.20 \\
21.71 \\
20.66 \\
41.49\end{array}$ & $\begin{array}{lr}\text { Sept. } 10 \\
& 15 \\
\text { oct. } & 8 \\
& 17 \\
\text { Nov. } & 11 \\
\text { Dec. } & 1 \\
& 15\end{array}$ & $\begin{array}{l}a \\
a\end{array}$ & $\begin{array}{l}43.95 \\
43.27 \\
21.30 \\
19.96 \\
39.96 \\
19.62 \\
21.50\end{array}$ \\
\hline
\end{tabular}

M-15a. City of Wichita. SE $\frac{1}{4} \mathrm{NE} \frac{1}{4}$ sec. 9, T. 24 S., R. 2 W.

Water level, in feet below measuring point, 1941

\begin{tabular}{|c|c|c|c|c|c|c|c|c|c|}
\hline $\begin{array}{lr}\text { Jan. } & 13 \\
20 \\
27 \\
27 \\
\text { Feb. } 10 \\
17 \\
25 \\
3 \\
\text { Mar. } 18\end{array}$ & $\begin{array}{r}17.70 \\
19.48 \\
\mathrm{c} 24.64 \\
17.90 \\
17.80 \\
16.99 \\
19.10 \\
\mathrm{c} 25.24\end{array}$ & $\begin{array}{l}\text { Mar. } \\
\text { Apr. } \\
\text { May }\end{array}$ & $\begin{array}{l}24 \\
31 \\
17 \\
28 \\
12 \\
19 \\
26\end{array}$ & $\begin{array}{l}17.82 \\
20.67 \\
\mathrm{c} 25.01 \\
20.09 \\
17.81 \\
18.66 \\
17.91\end{array}$ & $\begin{array}{l}\text { June } \\
\text { July } \\
\text { Aug. }\end{array}$ & $\begin{array}{r}3 \\
16 \\
7 \\
14 \\
21 \\
28 \\
25\end{array}$ & $\begin{array}{r}18.89 \\
19.76 \\
19.55 \\
17.40 \\
19.84 \\
18.86 \\
\mathrm{c} 25.89\end{array}$ & $\begin{array}{lr}\text { Sept. } 10 \\
\\
15 \\
\text { oct. } 6 \\
\\
\text { Nov. } 17 \\
\text { Dec. } 17 \\
& 15\end{array}$ & $\begin{array}{l}\text { c } 28.44 \\
\text { c } 28.79 \\
19.52 \\
18.20 \\
\text { c } 24.55 \\
17.84 \\
19.64\end{array}$ \\
\hline
\end{tabular}

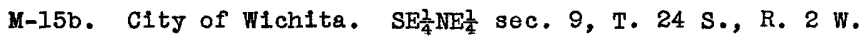

Water level, in feet below measuring point, 1941

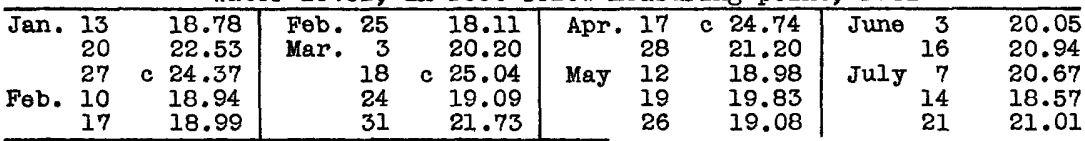

Well pumping. b Well M-14 pumping. c Well M-15 pumping. 


\section{Harvey County--Continued}

M-15b.--Continued.

Water level, in feet below measuring point, 1941

\begin{tabular}{|c|c|c|c|c|c|c|c|}
\hline Date & $\begin{array}{l}\text { Water } \\
\text { level }\end{array}$ & Date & $\begin{array}{l}\text { Water } \\
\text { level }\end{array}$ & Date & $\begin{array}{l}\text { Water } \\
\text { level }\end{array}$ & Date & $\begin{array}{l}\text { Water } \\
\text { level }\end{array}$ \\
\hline $\begin{array}{l}\text { July } 28 \\
\text { Aug. } 25\end{array}$ & $\begin{array}{r}20.05 \\
\mathrm{a} 25.63 \\
\end{array}$ & $\begin{array}{r}\text { Sept. } 10 \\
15\end{array}$ & $\begin{array}{l}\text { a } 28.18 \\
\text { a } 28.62\end{array}$ & $\begin{array}{l}\text { - oct. } 17 \\
\text { Nov. } 11\end{array}$ & $\begin{array}{r}19.37 \\
\mathrm{a} 24.28 \\
\end{array}$ & Dec. $\quad \begin{array}{l}1 \\
15\end{array}$ & $\begin{array}{l}18.91 \\
20.70\end{array}$ \\
\hline
\end{tabular}

M-16. City of W1ch1ta. SE cor. SE $\frac{1}{4}$ sec. 9, T. 24 S., R. 2 W.

Water level, in feet below measuring point, 1941

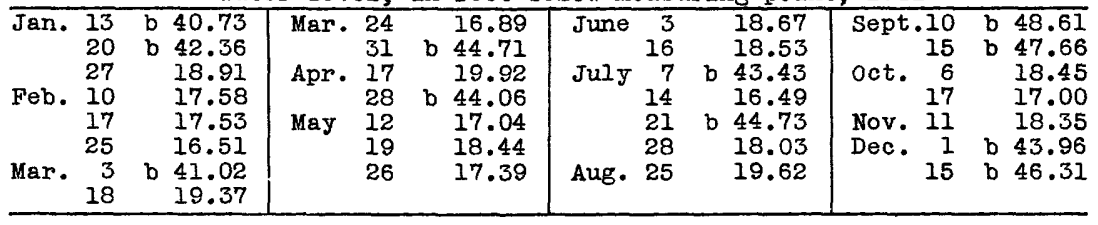

M-16a. C1ty of Wichita. $\mathrm{SE}_{\frac{1}{4}} \mathrm{SE}_{\frac{1}{4}}^{\frac{1}{4}}$ sec. 9, T. $24 \mathrm{~S}, \mathrm{R}, 2 \mathrm{~W}$.

Water level, in feet below measuring point, 1941

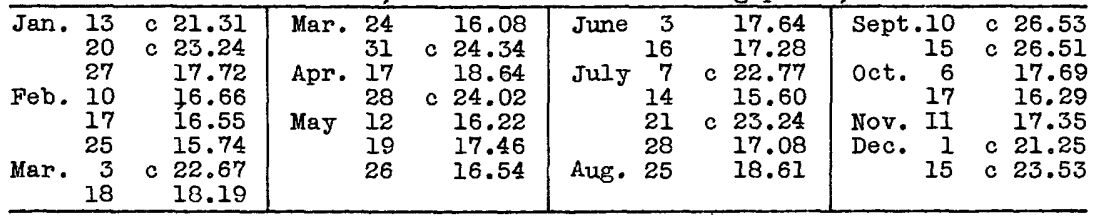

M-16b. City of W1chita. $\mathrm{SE}_{\frac{1}{4}}^{\frac{1}{2} \mathrm{SE}} \frac{1}{4}$ sec. 9, T. $24 \mathrm{~S} ., \mathrm{R}, 2 \mathrm{~W}$.

Water level, in feet below measuring point, 1941

\begin{tabular}{|c|c|c|c|c|c|c|c|c|c|c|c|}
\hline $\operatorname{Jan}$. & $\begin{array}{r}13 \\
20 \\
27 \\
10 \\
17 \\
25 \\
3 \\
18\end{array}$ & $\mathrm{c}$ & $\begin{array}{l}15.18 \\
16.08 \\
15.67 \\
15.09 \\
15.10 \\
14.79 \\
15.97 \\
15.90\end{array}$ & $\begin{array}{l}\text { Mar. } \\
\text { Apr. } \\
\text { May }\end{array}$ & $\begin{array}{l}24 \\
31 \\
17 \\
28 \\
12 \\
19 \\
26\end{array}$ & $\begin{array}{l}15.08 \\
\mathrm{c} 16.62 \\
16.16 \\
\mathrm{c} 16.58 \\
15.28 \\
15.71 \\
15.44\end{array}$ & $\begin{array}{l}\text { June } \\
\text { July }\end{array}$ & $\begin{array}{r}3 \\
16 \\
7 \\
14 \\
21 \\
28 \\
25\end{array}$ & $\begin{array}{l}15.91 \\
15.53 \\
\mathrm{c} \quad 15.87 \\
13.82 \\
\mathrm{c} 16.02 \\
15.45 \\
16.30\end{array}$ & $\begin{array}{lr}\text { Sept.10 } & 15 \\
& 6 \\
\text { Oct. } & 6 \\
& 17 \\
\text { Nov. } & 11 \\
\text { Dec. } & 1 \\
& 15\end{array}$ & $\begin{array}{l}\text { c } 17.77 \\
\text { c } 17.93 \\
16.31 \\
15.59 \\
\\
15.60 \\
\text { c } 15.43 \\
\text { c } 16.44\end{array}$ \\
\hline
\end{tabular}

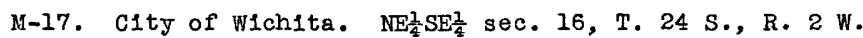

Water level, in feet below measuring point, 1941

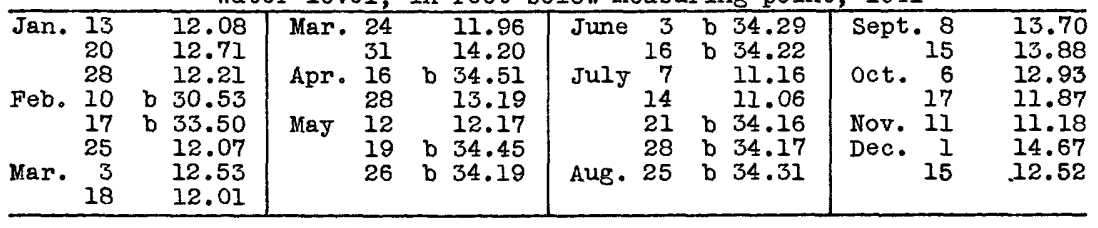

M-17a. Clty of Wichlta. NE $\frac{1}{4} S E \frac{1}{4}$ sec. 16, T. 24 S., R. 2 W.

Water level, in feet below measuring point, 1941

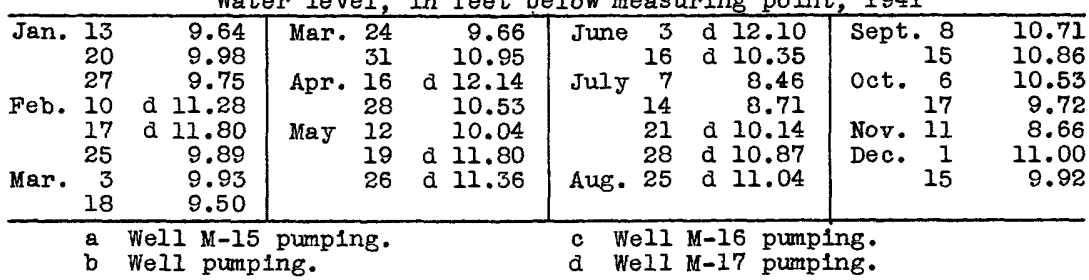


Harvey County--Continued

M-17b. City of Wichita. $\mathrm{NE}_{\frac{1}{4}} \mathrm{SE}_{\frac{1}{4}}^{\frac{1}{2}}$ sec. 16, T. $24 \mathrm{~S} ., \mathrm{R}, 2 \mathrm{~W}$.

Water level, in feet below measuring point, 1941

\begin{tabular}{|c|c|c|c|c|c|c|c|}
\hline Date & $\begin{array}{l}\text { Water } \\
\text { level }\end{array}$ & Date & $\begin{array}{l}\text { Water } \\
\text { level }\end{array}$ & Date & $\begin{array}{l}\text { Water } \\
\text { level }\end{array}$ & Date & $\begin{array}{l}\text { Water } \\
\text { level }\end{array}$ \\
\hline $\begin{array}{l}\text { Jan. } 13 \\
20 \\
27 \\
\text { Feb. } 10 \\
17 \\
25 \\
3 \\
\text { Mar. } 18\end{array}$ & $\begin{array}{rr} & 9.46 \\
& 9.87 \\
& 9.60 \\
\text { a } & 10.38 \\
\text { a } & 10.88 \\
& 9.66 \\
& 9.78 \\
& 9.38\end{array}$ & $\begin{array}{ll}\text { Mar. } & 24 \\
& 31 \\
\text { Apr. } & 16 \\
& 28 \\
\text { May } & 12 \\
19 \\
\\
26\end{array}$ & $\begin{array}{r}9.48 \\
10.67 \\
\text { a } 11.25 \\
10.47 \\
9.78 \\
\text { a } 10.90 \\
\text { a } 10.64\end{array}$ & $\begin{array}{lr}\text { June } & 3 \\
& 16 \\
7 u l y & 7 \\
14 \\
21 \\
28 \\
\text { Aug. } 25\end{array}$ & $\begin{array}{rr}\text { a } & 11.33 \\
\text { a } & 9.69 \\
& 8.59 \\
& 8.74 \\
\text { a } & 9.64 \\
\text { a } & 10.18 \\
\text { a } & 10.44\end{array}$ & $\begin{array}{lr}\text { Sept. } 10 \\
& 15 \\
\text { oct. } & 6 \\
17 \\
\text { Nov. } & 11 \\
\text { Dec. } & \frac{1}{5}\end{array}$ & $\begin{array}{r}10.67 \\
10.84 \\
10.39 \\
9.58 \\
8.53 \\
10.55 \\
9.87\end{array}$ \\
\hline
\end{tabular}

M-18. City of Wichita. SE $\frac{1}{4} \mathrm{SW}_{\frac{1}{4}}$ sec. 22, T. 24 S., R. 2 W.

Water level, in feet below measuring point, 1941

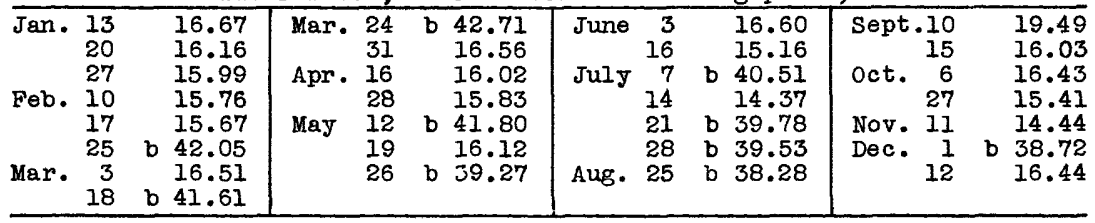

M-18a. City of Wichita. SE $\frac{1}{4} \mathrm{SW}_{4}^{\frac{2}{4}}$ sec. 22, T. 24 S., R. 2 W.

Water level, in feet below measuring point, 1941

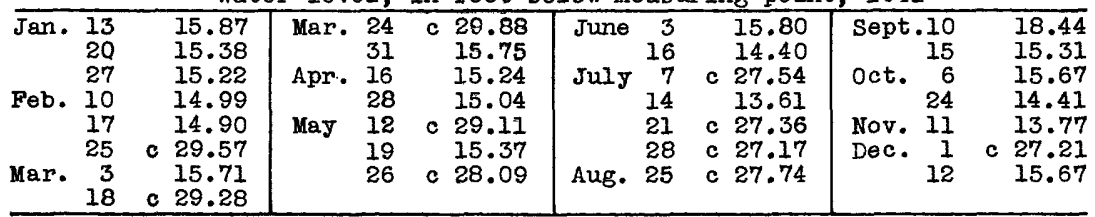

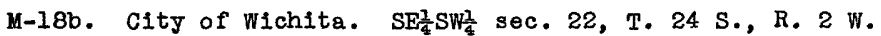

Water level, in feet below measuring point, 1941

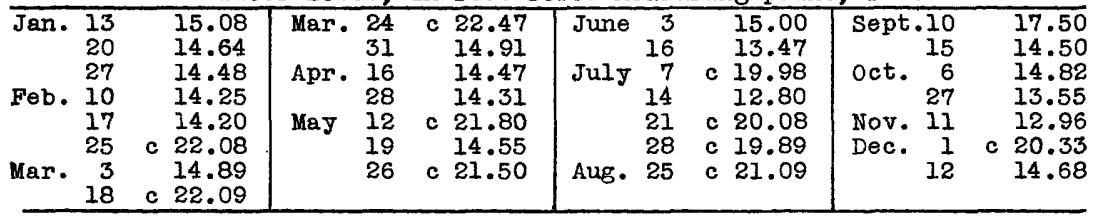

M-19. City of Wichita. $\mathrm{NE}_{\frac{1}{4}} \mathrm{NE} \frac{1}{4}$ sec. 27, T. $24 \mathrm{~S}$, R. 2 W.

Water level, in feet below measuring point, 1941

\begin{tabular}{|c|c|c|c|c|c|c|c|c|c|c|c|c|c|c|}
\hline Feb. & $\begin{array}{l}13 \\
20 \\
27 \\
10 \\
17 \\
25 \\
3 \\
18\end{array}$ & $\begin{array}{l}17.48 \\
17.12 \\
16.98 \\
17.15 \\
16.93 \\
17.61 \\
\text { b } 34.07 \\
18.85\end{array}$ & $\begin{array}{ll}\text { Mar. } & 24 \\
& 31 \\
\text { Apr. } & 15 \\
& 28 \\
\text { May } & 12 \\
& 19 \\
& 26\end{array}$ & b & $\begin{array}{l}17.92 \\
33.73 \\
17.38 \\
17.13 \\
35.30 \\
17.61 \\
18.01\end{array}$ & $\begin{array}{l}\text { June } \\
\text { July } \\
\text { Aug. }\end{array}$ & $\begin{array}{l}28 \\
25\end{array}$ & & $\begin{array}{l}1 \\
3 \\
1 \\
1 \\
2 \\
3 \\
1\end{array}$ & $\begin{array}{l}17.64 \\
32.86 \\
15.97 \\
5.70 \\
28.93 \\
33.29 \\
17.15\end{array}$ & $\begin{array}{l}\text { Sept. } \\
\text { Oct. } \\
\text { Nov. } \\
\text { Dec. }\end{array}$ & $\begin{array}{r}8 \\
15 \\
8 \\
27 \\
11 \\
1 \\
12\end{array}$ & b & $\begin{array}{l}17.12 \\
17.21 \\
33.98 \\
17.02 \\
16.29 \\
17.23 \\
34.91\end{array}$ \\
\hline
\end{tabular}

a Well M-17 pumping. b Well pumping. c Well M-18 pumping. 
Harvey County--Continued

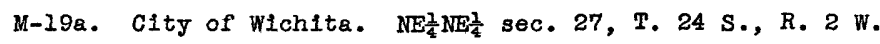

Water level, in feet below measuring point, 1941

\begin{tabular}{|c|c|c|c|c|c|c|c|c|}
\hline Dete & $\begin{array}{l}\text { Water } \\
\text { level }\end{array}$ & Date & $\begin{array}{l}\text { Water } \\
\text { level }\end{array}$ & Date & $\begin{array}{l}\text { Water } \\
\text { level }\end{array}$ & Date & & $\begin{array}{l}\text { Water } \\
\text { level }\end{array}$ \\
\hline $\begin{array}{l}\text { Jan. } 13 \\
20 \\
27 \\
\text { Feb. } 10 \\
17 \\
25 \\
\text { Mar. } 3 \\
18\end{array}$ & $\begin{array}{l}18.49 \\
18.10 \\
17.98 \\
18.21 \\
17.98 \\
18.37 \\
821.27 \\
18.63\end{array}$ & $\begin{array}{ll}\text { Mar. } & 24 \\
& 31 \\
\text { Apr. } & 15 \\
& 28 \\
\text { May } & 12 \\
& 19 \\
& 26\end{array}$ & $\begin{array}{l}18.68 \\
\mathrm{a} 1.22 \\
18.44 \\
18.15 \\
\mathrm{a} 21.71 \\
18.65 \\
18.78\end{array}$ & $\begin{array}{rr}\text { June } & 3 \\
& 16 \\
\text { July } & 7 \\
14 \\
21 \\
\\
28 \\
\text { Aug. } 25\end{array}$ & $\begin{array}{l}18.63 \\
\mathrm{a} 20.05 \\
16.74 \\
16.72 \\
\mathrm{a} 19.05 \\
\mathrm{a} 20.26 \\
17.97\end{array}$ & $\begin{array}{lr}\text { Sept. } 8 \\
& 15 \\
\text { Oct. } & 8 \\
& 27 \\
\text { Nov. } & 11 \\
\text { Dec. } & 1 \\
& 12\end{array}$ & $a$ & $\begin{array}{l}17.91 \\
18.25 \\
21.84 \\
18.06 \\
17.35 \\
18.13 \\
21.18\end{array}$ \\
\hline
\end{tabular}

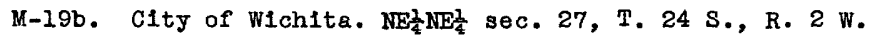

Water level, in feet below measuring point, 1941

\begin{tabular}{|c|c|c|c|c|c|c|c|c|c|c|c|c|}
\hline Jan. & $\begin{array}{r}13 \\
20 \\
27 \\
10 \\
17 \\
27 \\
3 \\
18\end{array}$ & $\begin{array}{r}17.44 \\
17.01 \\
16.86 \\
17.01 \\
16.82 \\
17.54 \\
217.96 \\
17.80\end{array}$ & $\begin{array}{l}\text { Mar. } \\
\text { Apr. } \\
\text { May }\end{array}$ & $\begin{array}{l}24 \\
31 \\
15 \\
28 \\
12 \\
19 \\
26\end{array}$ & $\begin{array}{l}17.88 \\
\text { a } 17.96 \\
17.98 \\
16.99 \\
\text { a } 18.25 \\
17.51 \\
17.92\end{array}$ & $\begin{array}{l}\text { June } \\
\text { July } \\
\text { Aug. }\end{array}$ & $\begin{array}{r}16 \\
7 \\
14 \\
21 \\
28 \\
25\end{array}$ & & $\begin{array}{l}17.63 \\
16.73 \\
15.77 \\
15.54 \\
16.63 \\
17.08 \\
17.16\end{array}$ & $\begin{array}{l}\text { Sept. } \\
\text { Oct. } \\
\text { Nov. I } \\
\text { Dec. }\end{array}$ & 8 & $\begin{array}{l}17.00 \\
17.10 \\
18.32 \\
16.84 \\
16.11 \\
17.13 \\
17.85\end{array}$ \\
\hline
\end{tabular}

M-20. City of Wichita. NW $\frac{1}{4} S E \frac{1}{4}$ sec. 8, T. 24 S., R. 2 W.

Water level, in feet below measuring point, 1941

\begin{tabular}{|c|c|c|c|c|c|c|c|c|c|c|}
\hline $\begin{array}{l}\text { an. } \\
2 \\
28 \\
\text { eb. } \\
1 \\
\text { ar. } \\
1 \\
2 \\
2\end{array}$ & $\begin{array}{r}20 \\
28 \\
10 \\
17 \\
3 \\
18 \\
24\end{array}$ & $\begin{array}{l}17.35 \\
17.00 \\
18.06 \\
18.06 \\
17.78 \\
18.26 \\
18.50\end{array}$ & $\begin{array}{l}\text { Mar. } \\
\text { Apr. } \\
\text { May } \\
\text { June }\end{array}$ & $\begin{array}{r}31 \\
17 \\
28 \\
18 \\
19 \\
26 \\
3\end{array}$ & $\begin{array}{l}\text { b } 43.32 \\
\text { b } 43.34 \\
18.63 \\
\text { b } 44.26 \\
20.02 \\
\text { b } 44.37 \\
\text { b } 43.04\end{array}$ & $\begin{array}{l}\text { June } \\
\text { July } \\
\text { Aug. } \\
\text { Sept }\end{array}$ & $\begin{array}{r}16 \\
7 \\
14 \\
21 \\
28 \\
26 \\
10\end{array}$ & $\begin{array}{l}\text { b } 44.66 \\
19.50 \\
20.63 \\
18.97 \\
\text { b } 44.69 \\
\text { b } 46.22 \\
\text { b } 48.68\end{array}$ & 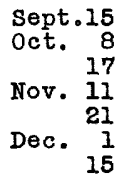 & $\begin{array}{r}2 \\
2 \\
1 \\
1 \\
1 \\
\text { b } 4\end{array}$ \\
\hline
\end{tabular}

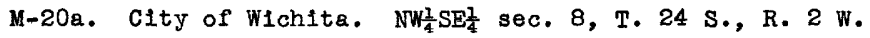

Water level, in feet below measuring point, 1941

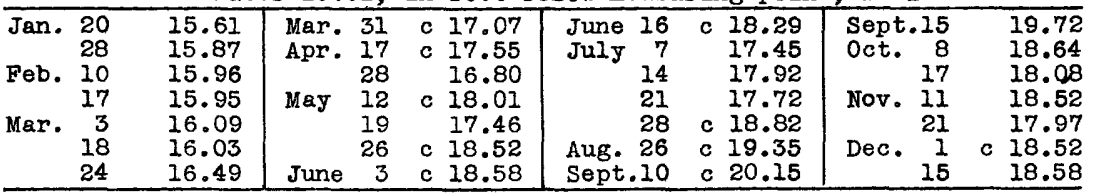

M-2Ob. City of Wichita. NW $\frac{1}{4} S E \frac{1}{4}$ sec. 8, T. 24 S., R. 2 W.

Water level, in feet below measuring point, 1941

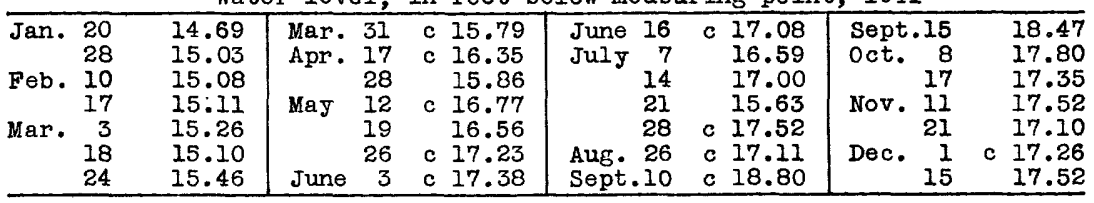

M-21. City of Wichita. SW $\frac{1}{4} \mathrm{SW} \frac{1}{4}$ sec. 26, T. 24 S., R. 2 W.

Water level, in feet below measuring point, 1941

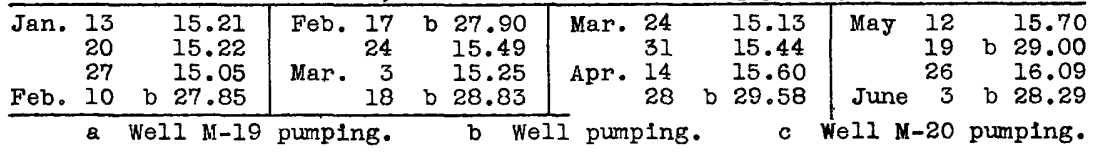


Harvey County--Continued

M-21.--Continued.

Water level, in feet below measuring point, 1941

\begin{tabular}{|c|c|c|c|c|c|c|c|}
\hline Date & $\begin{array}{l}\text { Water } \\
\text { level }\end{array}$ & Date & $\begin{array}{l}\text { Water } \\
\text { level }\end{array}$ & Date & $\begin{array}{l}\text { Water } \\
\text { level }\end{array}$ & Date & \\
\hline $\begin{array}{lr}\text { June } & 16 \\
\text { July } & 7 \\
& 14 \\
& 21\end{array}$ & $\begin{array}{ll} & 15.63 \\
\text { a } & 29.06 \\
\text { a } & 30.13 \\
\text { a } & 28.62\end{array}$ & $\begin{array}{l}\text { July } 28 \\
\text { Aug. } 4 \\
25 \\
\text { Sept. } 2\end{array}$ & $\begin{array}{l}29.73 \\
15.69 \\
15.23 \\
15.43\end{array}$ & $\begin{array}{l}\text { Sept. } 8 \\
15 \\
6 \\
\text { oct. } \\
27\end{array}$ & $\begin{array}{ll} & 15.55 \\
\text { a } & 29.98 \\
\text { a } & 30.13 \\
\text { a } & 30.62\end{array}$ & 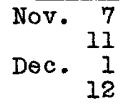 & $\begin{array}{l}15.95 \\
15.62 \\
14.76 \\
14.60\end{array}$ \\
\hline
\end{tabular}

M-2la. City of Wichita. SWT $S W \frac{2}{4}$ sec. 26, T. 24 S., R. 2 W.

Water level, in feet below measuring point, 1941

\begin{tabular}{|c|c|c|c|c|c|c|c|c|c|c|c|c|}
\hline$F \in b$. & $\begin{array}{l}13 \\
20 \\
27 \\
10 \\
17 \\
24 \\
3 \\
18\end{array}$ & $\begin{array}{l}b \\
b\end{array}$ & $\begin{array}{l}14.00 \\
14.04 \\
13.89 \\
19.67 \\
20.00 \\
14.36 \\
14.13 \\
20.33\end{array}$ & $\begin{array}{l}\text { Mar. } \\
\text { Apr. } \\
\text { May } \\
\text { June }\end{array}$ & $\begin{array}{r}24 \\
31 \\
14 \\
28 \\
12 \\
19 \\
26 \\
3\end{array}$ & $b$ & $\begin{array}{l}14.85 \\
14.30 \\
14.41 \\
20.77 \\
14.57 \\
20.49 \\
14.95 \\
20.18\end{array}$ & $\begin{array}{lr}\text { June } & 16 \\
\text { July } & 7 \\
14 \\
21 \\
28 \\
\\
\text { Aug. } 4 \\
\\
25 \\
\text { Sept. } 2\end{array}$ & $\begin{array}{l}b \\
b \\
b \\
b\end{array}$ & $\begin{array}{l}14.54 \\
20.08 \\
20.59 \\
20.34 \\
20.61 \\
14.61 \\
14.12 \\
14.35\end{array}$ & 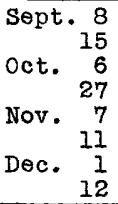 & $\begin{array}{ll} & 14.40 \\
\text { b } & 20.83 \\
\text { b } & 21.48 \\
\text { b } 21.46 \\
14.88 \\
14.53 \\
13.70 \\
13.49\end{array}$ \\
\hline
\end{tabular}

M-2lb. City of Wichita. SW $\frac{1}{4} W_{\frac{1}{4}}$ sec. 26, T. 24 S., R. 2 W.

Water level, in feet below measuring point, 1941

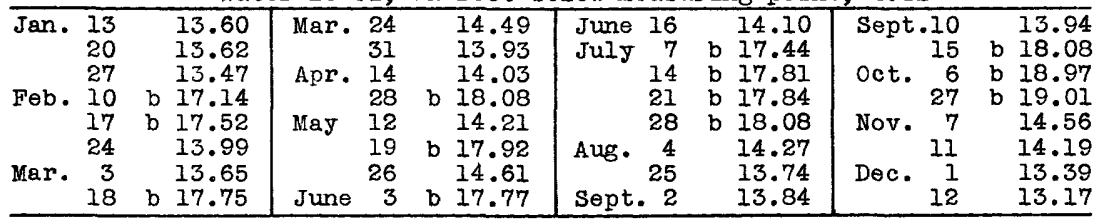

M-22. City of Wichita. SW $\frac{1}{4} S E \frac{7}{4}$ sec. 26, T. 24 S., R. 2 W.

Water level, in feet below measuring point, 1941

\begin{tabular}{|c|c|c|c|c|c|c|c|c|c|c|c|c|}
\hline $\operatorname{Jan}$. & $\begin{array}{r}13 \\
20 \\
27 \\
10 \\
17 \\
24 \\
3 \\
18\end{array}$ & $\begin{array}{l}33.11 \\
16.94 \\
16.71 \\
17.37 \\
17.63 \\
16.84 \\
16.69 \\
17.63\end{array}$ & $\begin{array}{l}\text { Mar. } \\
\text { Apr. } \\
\text { May } \\
\text { June }\end{array}$ & $\begin{array}{l}24 \\
31 \\
14 \\
28 \\
12 \\
19 \\
26 \\
3\end{array}$ & $\begin{array}{l}17.11 \\
16.70 \\
\mathrm{a} \quad 34.02 \\
17.98 \\
17.01 \\
17.90 \\
17.36 \\
18.06\end{array}$ & $\begin{array}{rr}\text { June } & 16 \\
\text { July } 7 \\
14 \\
21 \\
28 \\
\\
\text { Aug. } 25 \\
\text { Sept. } 8\end{array}$ & & $\begin{array}{l}34.23 \\
17.09 \\
17.13 \\
17.60 \\
17.77 \\
17.03 \\
36.61\end{array}$ & $\begin{array}{l}\text { Sept } \\
\text { Oct. } \\
\text { Nov. } \\
\text { Dec. }\end{array}$ & 2 & & $\begin{array}{l}35.97 \\
34.92 \\
36.12 \\
17.23 \\
16.93 \\
16.35 \\
16.01\end{array}$ \\
\hline
\end{tabular}

M-22a. City of Wichita. SW $\frac{1}{4} S E \frac{1}{4}$ sec. 26, T. 24 S., R. 2 W.

Water level, in feet below measuring point, 1941

\begin{tabular}{|c|c|c|c|c|c|c|c|c|c|c|c|}
\hline $\operatorname{Jan}$ & $\begin{array}{r}13 \\
20 \\
27 \\
10 \\
17 \\
24 \\
3 \\
18\end{array}$ & $\begin{array}{r}20.96 \\
15.03 \\
14.71 \\
15.43 \\
15.71 \\
14.88 \\
14.70 \\
15.67\end{array}$ & $\begin{array}{l}\text { Mar. } \\
\text { Apr. } \\
\text { May } \\
\text { June }\end{array}$ & $\begin{array}{r}24 \\
31 \\
14 \\
28 \\
12 \\
19 \\
26 \\
5\end{array}$ & $\begin{array}{r}15.16 \\
14.72 \\
\mathrm{c} 21.61 \\
16.11 \\
15.19 \\
15.98 \\
15.41 \\
16.16\end{array}$ & $\begin{array}{l}\text { June } \\
\text { July } \\
\\
\text { Aug. } \\
\text { Sept. }\end{array}$ & & $\begin{array}{l}21.69 \\
15.23 \\
15.28 \\
15.75 \\
15.86 \\
15.07 \\
22.94\end{array}$ & $\begin{array}{l}\text { Sept. } \\
\text { Oct. } \\
\text { Nov. } \\
\text { Dec. }\end{array}$ & & $\begin{array}{l}23.08 \\
23.23 \\
20.49 \\
15.36 \\
15.04 \\
14.43 \\
14.08\end{array}$ \\
\hline
\end{tabular}

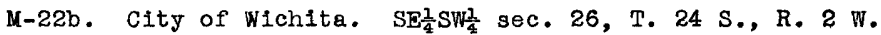

Water level, in feet below measuring point, 1941

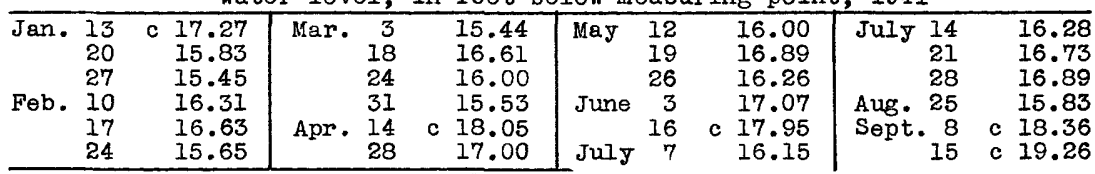

a Well pumping. b Well M-2I pumping. $\quad$ b WelI M-22 pumping. 
Harvey County--Continued.

M-22b.--Continued.

Water level, in feet below measuring point, 1941

\begin{tabular}{rrr|lr|ll|ll}
\hline Date & $\begin{array}{l}\text { Water } \\
\text { level }\end{array}$ & Date & $\begin{array}{l}\text { Water } \\
\text { level }\end{array}$ & Date & $\begin{array}{l}\text { Water } \\
\text { level }\end{array}$ & Date & $\begin{array}{r}\text { Water } \\
\text { level }\end{array}$ \\
\hline Oct. & 6 & a 20.00 \\
27 & a 19.59 & Nov. $\begin{array}{r}7 \\
11\end{array}$ & $\begin{array}{l}16.31 \\
15.95\end{array}$ & Dec. 1 & 15.17 & Dec. 12 & 14.87 \\
\hline
\end{tabular}

M-23. City of Wichita. $\mathrm{SE}_{4}^{\frac{1}{4}} \mathrm{NE}_{\frac{1}{4}}^{\frac{1}{2}}$ sec. 35 , T. $24 \mathrm{~S}$, R. $2 \mathrm{~W}$.

Water level, in feet below measuring point, 1941

\begin{tabular}{|c|c|c|c|c|c|c|c|c|c|c|c|c|c|}
\hline $\begin{array}{l}\text { Jan. } \\
\text { Feb. }\end{array}$ & $\begin{array}{r}13 \\
20 \\
27 \\
10 \\
17 \\
24 \\
3 \\
18\end{array}$ & $\begin{array}{l}\mathrm{b} \\
\mathrm{b}\end{array}$ & $\begin{array}{l}39.61 \\
41.49 \\
16.16 \\
14.97 \\
14.86 \\
15.61 \\
39.85 \\
14.78\end{array}$ & $\begin{array}{l}\text { Mar. } \\
\text { Apr. } \\
\text { May }\end{array}$ & $\begin{array}{l}24 \\
31 \\
14 \\
28 \\
12 \\
19 \\
26\end{array}$ & $\begin{array}{r}14.99 \\
14.88 \\
15.50 \\
\mathrm{~b} 41.60 \\
15.55 \\
16.04 \\
16.98\end{array}$ & $\begin{array}{l}\text { June } \\
\text { July } \\
\text { Aug. }\end{array}$ & $\begin{array}{r}3 \\
16 \\
7 \\
14 \\
21 \\
28 \\
25\end{array}$ & & $\begin{array}{l}16.10 \\
15.15 \\
12.76 \\
13.94 \\
41.01 \\
40.93 \\
40.80\end{array}$ & $\begin{array}{l}\text { Sept. } \\
\text { Oct. } \\
\text { Nov. I } \\
\text { Dec. } \\
\text { I I }\end{array}$ & b & $\begin{array}{l}16.10 \\
15.75 \\
16.28 \\
13.68 \\
14.45 \\
37.60 \\
14.74\end{array}$ \\
\hline
\end{tabular}

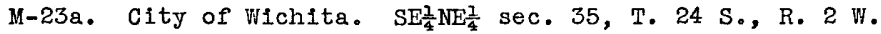

Water level, in feet below measuring point, 1941

\begin{tabular}{|c|c|c|c|c|c|c|c|c|c|c|c|c|}
\hline $\operatorname{Jan}$ & $\begin{array}{r}13 \\
20 \\
27 \\
10 \\
17 \\
24 \\
3 \\
18\end{array}$ & $\begin{array}{ll}\text { c } 17.74 \\
\text { c } 18.27 \\
15.28 \\
14.47 \\
14.32 \\
\\
14.71 \\
\text { c } 17.68 \\
14.25\end{array}$ & $\begin{array}{l}\text { Mar. } \\
\text { Apr. } \\
\text { May }\end{array}$ & $\begin{array}{l}24 \\
31 \\
14 \\
28 \\
12 \\
19 \\
26\end{array}$ & $\begin{array}{l}14.47 \\
14.34 \\
14.67 \\
\mathrm{c} 18.05 \\
15.08 \\
15.15 \\
15.22\end{array}$ & $\begin{array}{l}\text { June } \\
\text { July } \\
\text { Aug. }\end{array}$ & $\begin{array}{l}28 \\
25\end{array}$ & & $\begin{array}{l}15.53 \\
14.62 \\
12.26 \\
13.11 \\
16.85 \\
17.58 \\
18.20\end{array}$ & $\begin{array}{l}\text { Sept. } \\
\text { Oct. } \\
\text { Nov. } 1 \\
\text { Dec. }\end{array}$ & $c$ & $\begin{array}{l}15.32 \\
15.31 \\
15.41 \\
13.42 \\
13.85 \\
16.91 \\
14.05\end{array}$ \\
\hline
\end{tabular}

M-23b. City of Wichita. $\mathrm{SE}_{\frac{1}{4}}^{\frac{1}{4}} \mathrm{NE}_{4}^{\frac{1}{4}}$ sec. 35, T. 24 S., R. $2 \mathrm{~W}$.

Water level, in feet below measuring point, 1941

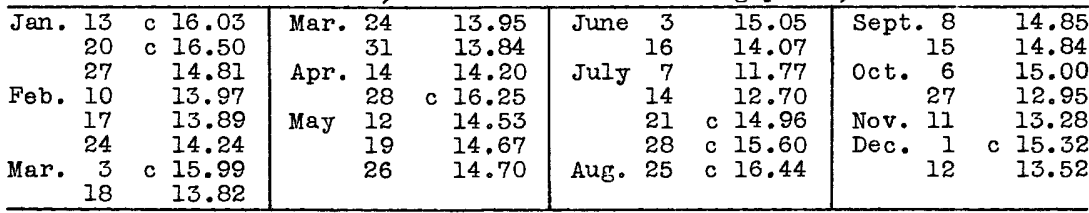

M-24. City of Wichita. $\mathrm{SE}_{\frac{1}{4}}^{\frac{1}{4} \mathrm{SE}} \frac{1}{4} \mathrm{sec}, 35, \mathrm{~T} .24 \mathrm{~S} ., \mathrm{R}, 2$ V.

Water level, in feet below measuring point, 1941

\begin{tabular}{|c|c|c|c|c|c|c|c|c|c|c|c|c|c|c|c|}
\hline $\operatorname{Jan}$. & $\begin{array}{r}13 \\
20 \\
27 \\
10 \\
17 \\
24 \\
3 \\
18\end{array}$ & $\begin{array}{l}16.17 \\
15.94 \\
15.61 \\
15.60 \\
15.19 \\
15.29 \\
15.34 \\
14.81\end{array}$ & $\begin{array}{l}\text { Mar } \\
\text { Apr } \\
\text { May }\end{array}$ & $\begin{array}{l}24 \\
31 \\
14 \\
28 \\
12 \\
19 \\
26\end{array}$ & $\mathrm{~b}$ & $\begin{array}{l}34.48 \\
32.78 \\
15.60 \\
15.43 \\
35.04 \\
16.00 \\
15.71\end{array}$ & $\begin{array}{l}\text { June } \\
\text { July } \\
\text { Aug. }\end{array}$ & $\begin{array}{r}3 \\
16 \\
7 \\
14 \\
21 \\
28 \\
25\end{array}$ & & $\begin{array}{l}1 \\
1 \\
1 \\
1\end{array}$ & $\begin{array}{l}3.17 \\
3.49 \\
3.73 \\
3.91 \\
4.65 \\
5.01 \\
5.53\end{array}$ & $\begin{array}{l}\text { Sept. } \\
\text { oct. } \\
\text { Nov. } \\
\text { Dec. }\end{array}$ & $\begin{array}{r}8 \\
15 \\
27 \\
17 \\
17\end{array}$ & & $\begin{array}{l}15.28 \\
35.62 \\
15.75 \\
14.58 \\
37.10 \\
14.67 \\
35.64\end{array}$ \\
\hline
\end{tabular}

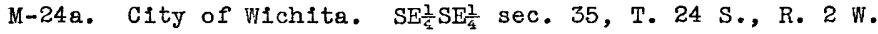

Water level, in feet below measuring point, 1941

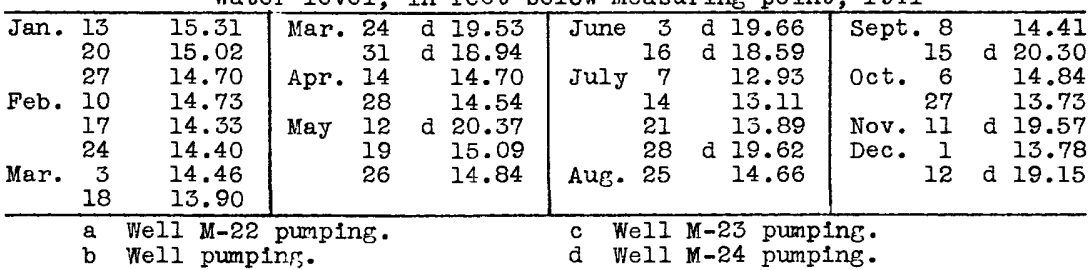


Harvey County--Continued

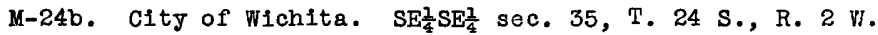
Water level, in feet below measuring point, 1941

\begin{tabular}{|c|c|c|c|c|c|c|c|}
\hline Date & $\begin{array}{l}\text { Water } \\
\text { level }\end{array}$ & Date & $\begin{array}{l}\text { Water } \\
\text { level }\end{array}$ & Date & $\begin{array}{l}\text { Water } \\
\text { level }\end{array}$ & Date & $\begin{array}{l}\text { Water } \\
\text { level }\end{array}$ \\
\hline $\begin{array}{rr}\text { Jan. } & 13 \\
& 20 \\
-\quad 27 \\
\text { Feb. } 10 \\
17 \\
\\
24 \\
\text { Mar. } 3 \\
18\end{array}$ & $\begin{array}{l}17.26 \\
16.93 \\
16.68 \\
16.90 \\
16.57 \\
16.46 \\
16.46 \\
16.15\end{array}$ & $\begin{array}{ll}\text { Mar. } & 24 \\
& 31 \\
\text { Apr. } & 14 \\
& 28 \\
\text { May } & 12 \\
& 19 \\
& 26\end{array}$ & $\begin{array}{ll}\text { a } & 17.77 \\
\text { a } 17.63 \\
16.80 \\
16.61 \\
\text { a } 18.70 \\
17.12 \\
16.85\end{array}$ & $\begin{array}{rr}\text { June } & 3 \\
& 16 \\
7 & \\
\text { July } & 14 \\
21 \\
\\
28 \\
\text { Aug. } 25\end{array}$ & $\begin{array}{ll}\text { a } 18.24 \\
\text { a } 17.32 \\
15.49 \\
15.23 \\
\\
15.89 \\
\text { a } 17.72 \\
16.66\end{array}$ & $\begin{array}{lr}\text { Sept. } 8 \\
& 15 \\
\text { oct. } & 6 \\
& 27 \\
\text { Nov. } & 11 \\
\text { Dec. } & 1 \\
& 12\end{array}$ & $\begin{array}{r}16.37 \\
\mathrm{a} 18.43 \\
16.96 \\
16.14 \\
\mathrm{a} 18.09 \\
15.84 \\
\mathrm{a} 17.66\end{array}$ \\
\hline
\end{tabular}

M-25. City of Wichita. SW $\mathrm{SWE}_{\frac{1}{4}}^{\frac{1}{4}}$ sec. 36, T. 24 S., R. 2 W.

Water level, in feet below measuring point, 1941

\begin{tabular}{|c|c|c|c|c|c|c|c|c|c|c|c|c|}
\hline $\begin{array}{l}\text { Feb. } \\
\text { Mar. }\end{array}$ & $\begin{array}{l}13 \\
20 \\
27 \\
10 \\
17 \\
24 \\
3 \\
18\end{array}$ & & $\begin{array}{l}12.77 \\
33.72 \\
32.71 \\
11.73 \\
11.70 \\
32.43 \\
12.80 \\
11.45\end{array}$ & $\begin{array}{l}\text { Mar. } \\
\text { Apr. } \\
\text { May }\end{array}$ & $\begin{array}{l}24 \\
31 \\
14 \\
28 \\
12 \\
19 \\
26\end{array}$ & $\begin{array}{l}b \\
b \\
b\end{array}$ & $\begin{array}{l}11.84 \\
11.67 \\
33.17 \\
12.65 \\
12.25 \\
33.00 \\
30.67\end{array}$ & $\begin{array}{l}\text { June } \\
\text { July } \\
\text { Aug. }\end{array}$ & $\begin{array}{r}3 \\
16 \\
7 \\
14 \\
21 \\
28 \\
25\end{array}$ & $\begin{array}{r}12.93 \\
11.15 \\
9.20 \\
\mathrm{~b} 36.98 \\
11.45 \\
11.80 \\
12.80\end{array}$ & $\begin{array}{lr}\text { Sept. } 8 \\
15 \\
\text { oct. } \\
67 \\
27 \\
\text { Nov. } 11 \\
\text { Dec. } 1 \\
12\end{array}$ & $\begin{array}{rr}\text { b } 38.97 \\
\text { b } 12.38 \\
\text { b } 36.69 \\
9.73 \\
\text { b } 35.14 \\
\text { b } 11.25 \\
\text { b } 34.56\end{array}$ \\
\hline
\end{tabular}

M-25a. City of Wichita. SW $\frac{7}{4} S E \frac{7}{4}$ sec. 36, T. 24 S., R. 2 W.

Water level, in feet below measuring point, 1941

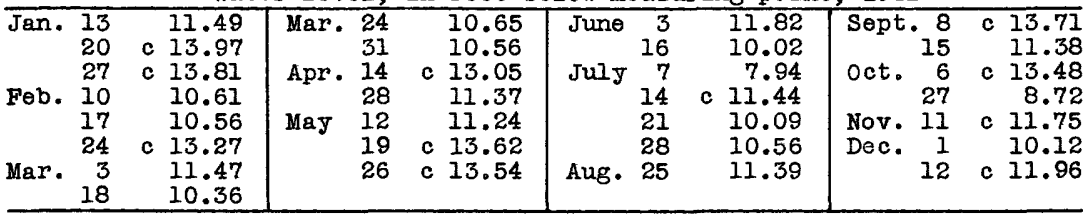

M-25b. City of Wichita. NE $\frac{1}{4} N W \frac{1}{4}$ sec. 1, T. 25 S., R. 2 W.

Water level, in feet below measuring point, 1941

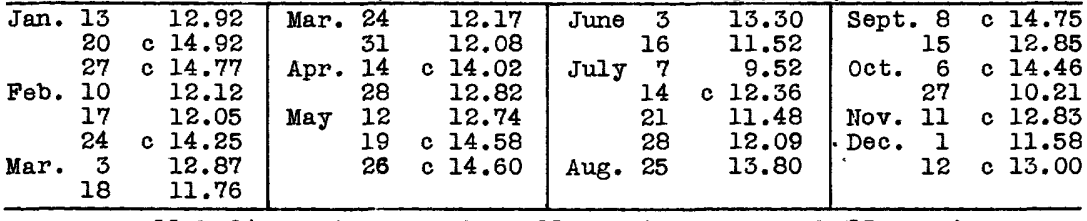

a Well M-24 pumping. b Well pumping. c M-25 pumping. 


\title{
HASKELL COUNTY
}

\author{
By T. G. McLaughlin
}

The observation-well program begun during 1941 in Grant and Haskell Counties is described in the chapter on Grant county.

\section{Well descriptions and water-level measurements}

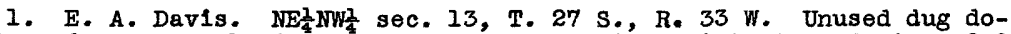
mestic and stock well, depth 113 feet. Measuring point, top of pipe, 0.9 foot above land surface, 2,905.44 feet above sea level. Equipped with lift pump.

Water level, in feet belor measuring point, 1941

\begin{tabular}{lc|lr|lr|lrr}
\hline Date & $\begin{array}{l}\text { Water } \\
\text { level }\end{array}$ & Date & $\begin{array}{r}\text { Water } \\
\text { level }\end{array}$ & Date & $\begin{array}{r}\text { Water } \\
\text { level }\end{array}$ & Date & $\begin{array}{r}\text { Water } \\
\text { level }\end{array}$ \\
\hline June 24 & $\begin{array}{l}109.94 \\
\text { July 26 }\end{array}$ & Aug. 13 & $\begin{array}{l}109.96 \\
\text { 110.01 }\end{array}$ & $\begin{array}{l}\text { Oct. } 13 \\
\text { Sept.17 }\end{array}$ & $\begin{array}{l}109.93 \\
110.15\end{array}$ & Dov. 1 & Dec. & 109.89 \\
\hline
\end{tabular}

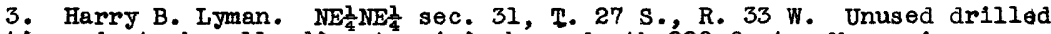
domestic and stock well, dlameter 4 Inches, depth 220 feet. Measuring point, top of casing, northeast side, 1.0 foot above land surface, $2,997.88$ feet above sea level. Equipped with lift pump operated by gasoline englne.

Water level, in feet below measuring point, 1941

\begin{tabular}{|c|c|c|c|c|c|}
\hline Date & $\begin{array}{l}\text { Water } \\
\text { level }\end{array}$ & Date & $\begin{array}{l}\text { Water } \\
\text { level }\end{array}$ & Date & $\begin{array}{l}\text { Water } \\
\text { level }\end{array}$ \\
\hline $\begin{array}{l}\text { June } 26 \\
\text { July } 26\end{array}$ & $\begin{array}{l}191.72 \\
191.83\end{array}$ & $\begin{array}{l}\text { Sept.17 } \\
\text { oct. } 13\end{array}$ & $\begin{array}{l}191.78 \\
191.82\end{array}$ & $\begin{array}{ll}\text { Nov. } & 3 \\
\text { Dec. } & 2\end{array}$ & $\begin{array}{l}191.79 \\
191.64\end{array}$ \\
\hline
\end{tabular}

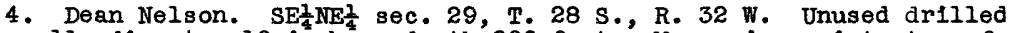
stock well, diemeter 10 inches, depth 206 feet.' Measuring point, top of 6-inch pipe, east side, 0.8 foot above land surface, $2,937.93$ feet above sea level. Equipped with lift pump.

Water level, in feet below measuring point, 1941

\begin{tabular}{|c|c|c|c|c|c|c|c|}
\hline Date & $\begin{array}{l}\text { Water } \\
\text { level }\end{array}$ & Date & $\begin{array}{l}\text { Water } \\
\text { level }\end{array}$ & Date & $\begin{array}{l}\text { Water } \\
\text { level }\end{array}$ & Date & $\begin{array}{l}\text { Water } \\
\text { level }\end{array}$ \\
\hline $\begin{array}{ll}\text { June } & 27 \\
\text { July } & 29\end{array}$ & $\begin{array}{l}198.45 \\
198.41\end{array}$ & $\begin{array}{l}\text { Aug. } 13 \\
\text { Sept. } 17\end{array}$ & $\begin{array}{l}198.40 \\
198.58\end{array}$ & $\begin{array}{l}\text { Oct. } 13 \\
\text { Nov. } 3\end{array}$ & $\begin{array}{l}198.46 \\
198.45\end{array}$ & Dec. 3 & 198.47 \\
\hline
\end{tabular}

5. C. D. Jennings. NE $\frac{1}{4} N E \frac{1}{4}$ sec. 20, T. 29 S., R. 31 W. Unused drilled domestic well, dlameter 4 inches, depth 173 feet. Measuring point, top of casing, east side, 0.2 foot above land surface, $2,060.40$ feet above sea level. No pump in well.

Water level, in feet below measuring point, 1941

\begin{tabular}{|c|c|c|c|c|c|c|}
\hline $\begin{array}{l}\text { June } 27 \\
\text { July } 29\end{array}$ & $\begin{array}{l}161.31 \\
161.26\end{array}$ & $\begin{array}{l}\text { Aug. } 13 \\
\text { Sept.17 }\end{array}$ & $\begin{array}{l}161.22 \\
161.35\end{array}$ & $\begin{array}{lr}\text { Oct. } & 13 \\
\text { Nov. } 3\end{array}$ & $\begin{array}{l}161.32 \\
161.36\end{array}$ & Dec. 3 \\
\hline
\end{tabular}

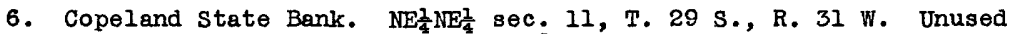
drilled well, diameter 5 inches, depth 178 feet. Measuring point, bottom of hole in casing, east side, 0.6 foot above land surface, $2,854.35$ feet above sea level. No pump in well.

Water level, in feet below measuring point, 1941

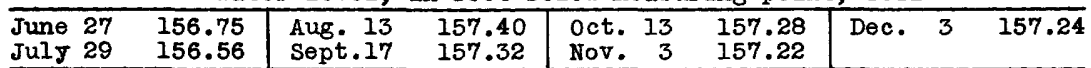


Haskell County--Continued

7. Etta McCoy. NW $\frac{1}{4} N W \frac{2}{4}$ sec. 2, T. $30 \mathrm{~S} .$, R. $32 \mathrm{VI}$. Unused drilled well, diameter 6 inches, depth 196 feet. Measuring point, top of casing, east side, 1.1 feet above land surface, $2,890.29$ feet above ser level. No pump in well.

Water level, in feet below measuring point, 1941

\begin{tabular}{ll|lr|lr|lrr}
\hline Date & $\begin{array}{l}\text { Water } \\
\text { level }\end{array}$ & Date & $\begin{array}{r}\text { Water } \\
\text { level }\end{array}$ & Date & $\begin{array}{r}\text { Water } \\
\text { level }\end{array}$ & Date & $\begin{array}{r}\text { Water } \\
\text { level }\end{array}$ \\
\hline June 28 & 188.60 & Aug. 13 & 188.51 & Oct. 13 & 188.61 & Dec. & 3 & 188.67 \\
July 29 & 188.61 & Sept.17 & 188.62 & Nov. 3 & 188.69 & & \\
\hline
\end{tabular}

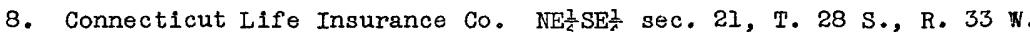
Unused drilled well, diameter 6 inches, depth 221 feet. Measuring point, top of casing, west side, level with land surface, $2,968.83$ feet above sea level. Equipped with lift pump and windmill. Water levels, in feet below measuring point, 1941: Oct. 13, 204.14; Nov. 3, 204.70; Dec. 3, 204.20.

9. Bessie custer. NW $\frac{1}{4} S_{\frac{1}{4}}^{\frac{1}{4}}$ sec. I3, T. $30 \mathrm{~S}$, , R. $34 \mathrm{~W}$. Unused drilled domestic well, depth 219 feet. Measuring point, top of board at west side of pipe, level with land surface, $2,963.08$ feet above sea level. No pump in well.

Water level, in feet below measuring point, 1941

\begin{tabular}{ll|ll|ll|lll}
\hline June & 30 & 207.68 & Aug. 13 & 207.89 & 0ct. 13 & 207.97 & Dec. 3 & 207.75
\end{tabular}

\begin{tabular}{ll|ll|lll} 
July 26 & 207.75 & Sept.17 & 207.89 & Nov. & 1 & 207.79 \\
\hline
\end{tabular}

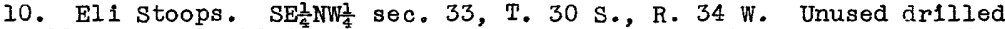
stock well, diameter 10 inches, depth 61 feet. Measuring point, top of pipe, north side, 5.8 feet above land surface, 2,828.94 feet above sea level. Equipped with lift pump and windmill.

Water level, in feet below measuring point, 1941

\begin{tabular}{lll|ll|lr|rrr}
\hline June 30 & 55.67 & Aug. 13 & 56.32 & Oct. 13 & 56.08 & Dec. & 3 & 54.92 \\
July 26 & 56.16 & Sept.17 & 56.53 & Nov. 1 & 54.83 & & & \\
\hline
\end{tabular}

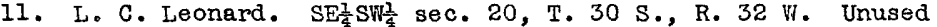
drilled well, diameter 6 inches, depth 198 feet. Measuring point, side of hole in metal. cover over casing, level with land surface, $2,893.02$ feet above sea level. Equipped with lift pump.

Water level, in feet below measuring point, 1941

\begin{tabular}{lr|lr|llr}
\hline Date & $\begin{array}{r}\text { Water } \\
\text { level }\end{array}$ & Date & $\begin{array}{r}\text { Water } \\
\text { level }\end{array}$ & Date & $\begin{array}{r}\text { Water } \\
\text { level }\end{array}$ \\
\hline July 24 & 184.64 & Sept.17 & 184.55 & Nov. 3 & 184.61 \\
Aug. 13 & 184.51 & Oct. 13 & 184.53 & Dec. 3 & 184.57 \\
\hline
\end{tabular}

12. Sybol Smith. SW $W_{\frac{1}{4}}^{\frac{1}{4}}$ sec. II, T. $30 \mathrm{~S}, \mathrm{R}, 32 \mathrm{~W}$. Unused drilled stock well, diameter 6 inches, depth 198 feet. Measuring point, bottom edge of steel pipe clamp at southwest side of pipe, 0.3 foot above land surface, 2,893.02 feet above sea level. Equipped with lift pump.

Water level, in feet below measuring point, 1941

\begin{tabular}{ll|ll|lll}
\hline JuIy 24 & 180.76 & Sept.17 & 180.66 & Nov. & 3 & 179.70 \\
Aug. 13 & 180.64 & Oct. 13 & 180.72 & Dec. & 3 & 180.72 \\
\hline
\end{tabular}

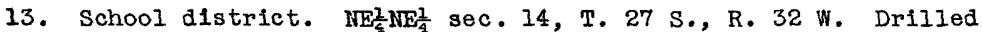
school well, diameter 6 inches, depth 97 feet. Measuring point, top of casing, north side, 0.2 foot above land surface, $2,848.25$ feet above sea level. Equipped with lift pump and windmill.

Water level, in feet below measuring point, 1941

\begin{tabular}{ll|ll|lll}
\hline July 24 & 86.08 & Sept. 17 & 85.85 & Nov. & 3 & 85.90 \\
Aug. 13 & 85.89 & oct. 13 & 86.00 & Dec. 2 & 85.91 \\
\hline
\end{tabular}

$5110050-49-8$ 


\section{Haskell County--Continued}

14. William Dreyer. NW $\frac{1}{4} N \frac{1}{4}$ sec. 2I, T. 27 S., R. 34 W. Unused drilled stock well, diameter $\overline{6}$ inches, depth 167 feet. Measuring point, top of casing, south side, level with land surface, 3,019.89 feet above sea level. Equipped with 1 ift pump and windmill.

Water level, in feet below measuring point, 1941

\begin{tabular}{lr|lr|rrr}
\hline Date & $\begin{array}{r}\text { Water } \\
\text { level }\end{array}$ & Date & $\begin{array}{l}\text { Water } \\
\text { level }\end{array}$ & Date & $\begin{array}{r}\text { Water } \\
\text { level }\end{array}$ \\
\hline July 31 & 151.92 & Sept.17 & 152.02 & Nov. 3 & 151.93 \\
Aug. 13 & 151.92 & Dct. 13 & 151.88 & Dec. 2 & 151.85 \\
\hline
\end{tabular}

15. M. H. Eubank. NW NW $\frac{1}{4}$ sec. 33, T. $28 \mathrm{~S} ., \mathrm{R} .34 \mathrm{~W}$. Unused drilled domestic and stock weli, diameter 6 inches, depth 234 feet. Measuring point, top of casing, east side, 1.2 feet above land surface, 3,015.93 feot above sea level. Equipped with lift pump and windmill.

Water level, in feet below measuring point, 1941

\begin{tabular}{ll|ll|lll}
\hline JuIy 31 & 222.82 & Sept.17 & 222.30 & Nov. & 3 & 222.43 \\
Aug. 13 & 222.82 & Oct. 13 & 222.60 & Dec. & 2 & 222.51 \\
\hline
\end{tabular}

HODGEMAN COUNTY

By H. A. Waite

Highest and Iowest water levels, in feet below measuring point, in 3 wells in Hodgeman County, 1941

\begin{tabular}{|c|c|c|c|c|c|}
\hline Well & $\begin{array}{l}\text { Length of } \\
\text { record } \\
\text { (years) }\end{array}$ & $\begin{array}{l}\text { Highest } \\
\text { recorded } \\
\text { water level } \\
\text { (feet) }\end{array}$ & Date & $\begin{array}{l}\text { Lowest } \\
\text { recorded } \\
\text { water level } \\
\text { (feet) }\end{array}$ & Date \\
\hline $\begin{array}{l}3 \\
4 \\
5\end{array}$ & $\begin{array}{l}I \\
I \\
I\end{array}$ & $\begin{array}{l}33.78 \\
26.27 \\
31.88\end{array}$ & $\begin{array}{l}\text { Aug. 2, } 1941 \\
\text { July 12, } 1941 \\
\text { July I2, } 1941\end{array}$ & $\begin{array}{l}35.77 \\
28.52 \\
33.88\end{array}$ & $\begin{array}{l}\text { Sept.20, } 1940 \\
\text { Oct. 2, } 1941 \\
\text { Oct. 29, } 1940\end{array}$ \\
\hline
\end{tabular}

Net changes in water level in 1941, and net changes in water level for perlod of record in 3 wells in Hodgeman County

\begin{tabular}{|c|c|c|c|}
\hline WeII & $\begin{array}{l}\text { Difference } \\
\text { between highest } \\
\text { and lowest } \\
\text { water levels } \\
\text { (feet) }\end{array}$ & $\begin{array}{l}\text { Net rise (+) } \\
\text { or net decline } \\
(-) \text { in } 1941 \\
\text { (feet) }\end{array}$ & $\begin{array}{l}\text { Net rise (+) } \\
\text { or net decline } \\
\text { (-) for period } \\
\text { of record } \\
\text { (feet) }\end{array}$ \\
\hline $\begin{array}{l}3 \\
4 \\
5\end{array}$ & $\begin{array}{l}1.99 \\
2.25 \\
2.00 \\
\end{array}$ & $\begin{array}{r}+0.27 \\
+\quad .42 \\
+\quad .19 \\
\end{array}$ & $\begin{array}{r}+1.41 \\
+\quad .59 \\
+\quad .05 \\
\end{array}$ \\
\hline
\end{tabular}

Water-Ievel measurements

3. W. J. Fox. SW $\frac{1}{4} N W \frac{1}{4}$ sec. I2, T. $2 I$ S., R. 22 W.

Water level, in feet below measuring point, 1941

\begin{tabular}{|c|c|c|c|c|c|c|c|}
\hline Date & $\begin{array}{l}\text { Water } \\
\text { level }\end{array}$ & Date & $\begin{array}{l}\text { Water } \\
\text { level }\end{array}$ & Date & $\begin{array}{l}\text { Water } \\
\text { level }\end{array}$ & Date & $\begin{array}{l}\text { Water } \\
\text { level }\end{array}$ \\
\hline $\begin{array}{l}\text { Jan. } 20 \\
\text { Mar. } 17 \\
\text { Apr. } 26\end{array}$ & $\begin{array}{l}34.55 \\
34.40 \\
34.25\end{array}$ & $\begin{array}{lr}\text { May } & 10 \\
\text { June } & 6 \\
\text { July } & 12\end{array}$ & $\begin{array}{l}34.23 \\
34.20 \\
33.98\end{array}$ & $\begin{array}{ll}\text { Aug. } & 2 \\
\text { Sept. } & 4 \\
\text { oct. } & 2\end{array}$ & $\begin{array}{l}33.78 \\
34.40 \\
34.91\end{array}$ & $\begin{array}{lr}\text { Nov. } & 13 \\
\text { Dec. } & 4\end{array}$ & $\begin{array}{l}34.47 \\
34.28\end{array}$ \\
\hline
\end{tabular}


Hodgeman County--Continued

4. William Macey. SW $\frac{1}{4} S W \frac{2}{4}$ sec. 13, T. 22 S., R. $22 W$.

Water level, in feet below measuring point, 1941

\begin{tabular}{ll|lr|ll|ll|l}
\hline Date & $\begin{array}{l}\text { Water } \\
\text { leve1 }\end{array}$ & Date & $\begin{array}{l}\text { Water } \\
\text { level }\end{array}$ & Date & $\begin{array}{l}\text { Water } \\
\text { level }\end{array}$ & Date & $\begin{array}{r}\text { Water } \\
\text { level }\end{array}$ \\
\hline Jan. 20 & 27.86 & May 10 & 27.49 & Aug. 2 & 26.47 & Nov. 13 & 27.77 \\
Mar. 17 & 27.61 & June 6 & 27.42 & Sept. 4 & 27.27 & Dec. 14 & 27.44 \\
Apr. 26 & 27.47 & July 12 & 26.27 & Oct. 2 & 28.52 & & & 27 \\
\hline
\end{tabular}

5. Roy Klein. NW $\frac{1}{2} \mathrm{NE}_{\frac{1}{4}}^{\frac{1}{2}}$ sec. 36, T. $21 \mathrm{~S}$, , R. $21 \mathrm{~W}$.

Water level, in feet below measuring point, 1941

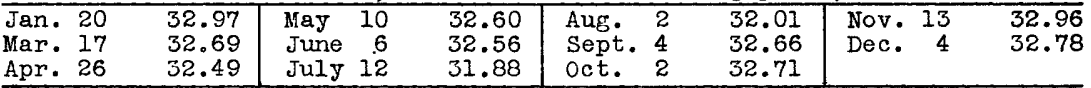

JEWELL COUNTY

By V. C. Fishel

Highest and lowest water levels, in feet above datum in 39 wells in Jewell County, 1941

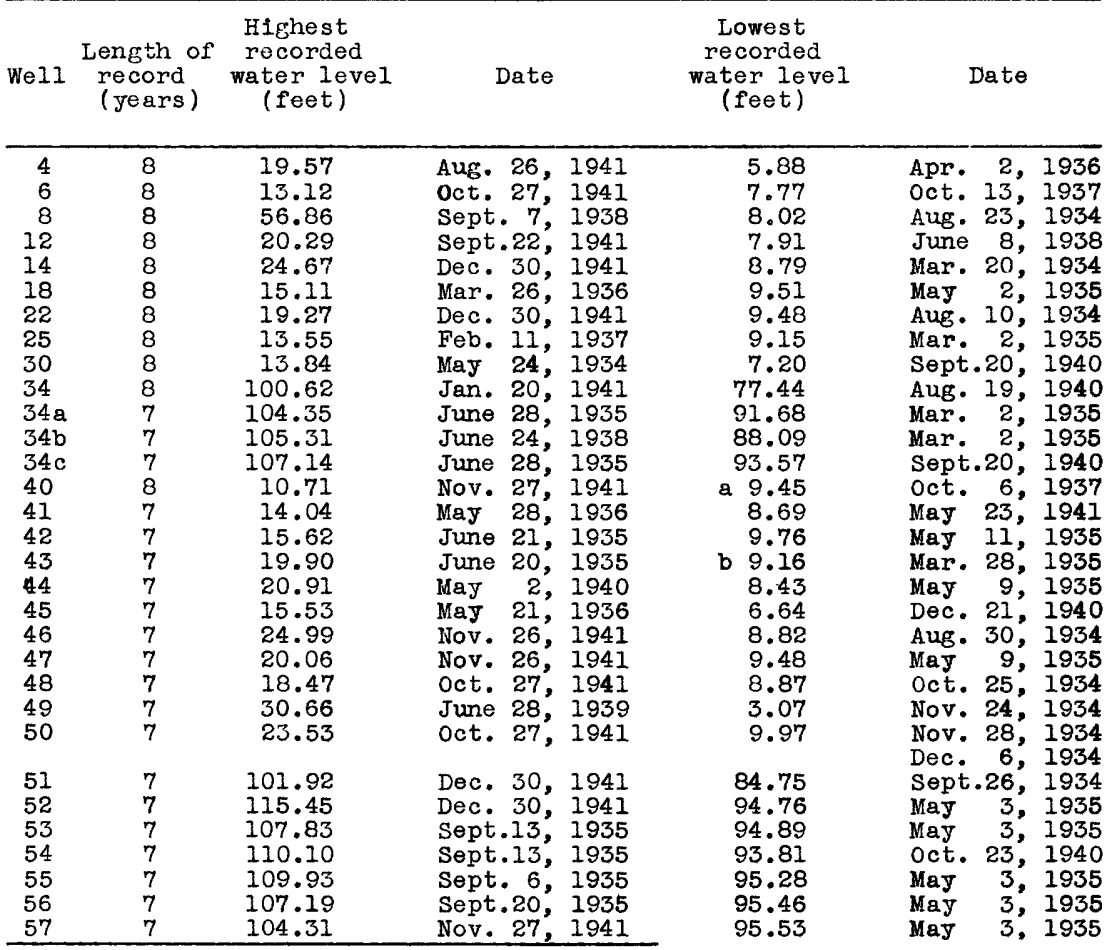

a Water level of 9.30 feet on Mar. 6, 1937, as given in earlier reports, is erroneous.

b Water level of 8.81 feet on Sept.20, 1934, as given in earlier reports, is erroneous. 
Jewell County--Continued

Highest and lowest water levels, in feet above datum in 39 wells in Jewell County, 1941--Continued.

\begin{tabular}{|c|c|c|c|c|c|}
\hline Well & $\begin{array}{l}\text { Length of } \\
\text { record } \\
\text { (years) }\end{array}$ & $\begin{array}{l}\text { Highest } \\
\text { recorded } \\
\text { water level } \\
\text { (feet) }\end{array}$ & Date & $\begin{array}{c}\text { Lowest } \\
\text { recorded } \\
\text { water level } \\
\text { (feet) }\end{array}$ & Date \\
\hline $\begin{array}{l}61 \\
62 \\
63 \\
64 \\
65 \\
66 \\
67\end{array}$ & $\begin{array}{l}7 \\
7 \\
7 \\
6 \\
6 \\
6 \\
7\end{array}$ & $\begin{array}{l}94.44 \\
87.46 \\
95.29 \\
18.27 \\
20.95 \\
16.88 \\
13.22\end{array}$ & $\begin{array}{l}\text { Nov. } 27,1941 \\
\text { Sept.13, } 1935 \\
\text { Dec. 30, } 1941 \\
\text { Oct. 27, } 1941 \\
\text { Dec. 30, } 1941 \\
\text { Dec. 29, } 1941 \\
\text { June 16, } 1937 \\
\text { June } 13,1937\end{array}$ & $\begin{array}{r}83.17 \\
83.10 \\
87.96 \\
10.42 \\
4.04 \\
6.06 \\
4.56\end{array}$ & $\begin{array}{l}\text { Sept.27, } 1935 \\
\text { May 3, } 1935 \\
\text { July 15, } 1935 \\
\text { Jan. 19, } 1938 \\
\text { Aug. 20, } 1940 \\
\text { Oct. 23, } 1940 \\
\text { Dec. 2, } 1940\end{array}$ \\
\hline 69 & 5 & 13.34 & Dec. 29, 1941 & 5.82 & Aug. 19,1940 \\
\hline
\end{tabular}

Net changes in water level in 1941, and net changes in water level for period of record in 39 wells in Jewell County

\begin{tabular}{|c|c|c|c|}
\hline Well & $\begin{array}{c}\text { Difference } \\
\text { between highest } \\
\text { and lowest } \\
\text { water levels } \\
\text { (feet) }\end{array}$ & $\begin{array}{l}\text { Net rise (t) } \\
\text { or net decline } \\
(-) \text { in } 1941 \\
(\text { feet })\end{array}$ & $\begin{array}{l}\text { Net rise (t) } \\
\text { or net decline } \\
(-) \text { for period } \\
\text { of record } \\
\text { (feet) }\end{array}$ \\
\hline $\begin{array}{l}4 \\
6 \\
8 \\
12 \\
14 \\
18 \\
22 \\
25 \\
30 \\
34 \\
34 \mathrm{a} \\
34 \mathrm{~b} \\
34 \mathrm{c} \\
40 \\
41 \\
42 \\
43 \\
44 \\
45 \\
46 \\
47 \\
48 \\
49 \\
50 \\
51 \\
52 \\
53 \\
54 \\
55 \\
56 \\
57 \\
61 \\
62 \\
63 \\
64 \\
65 \\
66 \\
67 \\
69\end{array}$ & $\begin{array}{r}13.69 \\
5.35 \\
48.84 \\
12.38 \\
15.88 \\
5.60 \\
9.79 \\
4.40 \\
6.64 \\
23.18 \\
12.67 \\
17.22 \\
13.57 \\
1.26 \\
5.35 \\
5.86 \\
10.74 \\
12.48 \\
8.89 \\
16.17 \\
10.58 \\
9.60 \\
27.59 \\
13.56 \\
17.17 \\
20.69 \\
12.94 \\
16.29 \\
14.65 \\
11.73 \\
8.78 \\
11.27 \\
4.36 \\
7.33 \\
7.85 \\
16.91 \\
10.82 \\
8.66 \\
7.52\end{array}$ & $\begin{array}{l}+5.54 \\
+3.50 \\
+24.97 \\
+5.90 \\
+13.97 \\
+\quad .74 \\
+7.62 \\
+3.60 \\
+2.30 \\
+15.05 \\
-1.94 \\
+5.71 \\
+8.11 \\
+\quad .35 \\
+(a) \\
+4.60 \\
+(a) \\
+1.43 \\
+4.18 \\
+6.73 \\
+6.63 \\
+6.16 \\
+6.40 \\
+9.86 \\
+5.91 \\
+4.91 \\
+9.21 \\
(a) \\
\quad(a) \\
+(a) \\
+5.34 \\
+4.04 \\
+4.67 \\
+4.81 \\
+4.077 \\
+12.21 \\
+10.28 \\
+4.91 \\
+4.76\end{array}$ & $\begin{array}{l}+8.19 \\
+\quad .21 \\
+33.24 \\
+3.83 \\
+15.88 \\
-1.33 \\
+8.13 \\
+2.55 \\
+\quad .76 \\
+10.61 \\
+6.16 \\
+9.98 \\
+7.86 \\
+\quad .25 \\
+\quad .66 \\
+3.58 \\
+\quad .86 \\
+2.42 \\
+\quad .19 \\
+15.09 \\
+6.75 \\
+7.97 \\
+14.86 \\
+12.06 \\
+17.17 \\
+19.26 \\
+10.90 \\
+7.48 \\
+7.34 \\
+3.37 \\
+8.04 \\
+6.60 \\
+\quad .50 \\
+6.13 \\
+4.59 \\
+7.30 \\
+2.53 \\
-1.13 \\
+1.74\end{array}$ \\
\hline
\end{tabular}

a No measurements made during latter part of 1941 . 
Jewell County--Continued

Average water levels in 12 observation wells $(6,12,18,22,25$, $30,40,41,42,45,48$, and 50) in Jewell County, Kansas, in 1941, in feet above datum

\begin{tabular}{|c|c|c|c|c|c|c|c|}
\hline Date & $\begin{array}{l}\text { Water } \\
\text { level }\end{array}$ & Date & & & $\begin{array}{l}\text { Water } \\
\text { level }\end{array}$ & Date & $\begin{array}{l}\text { Water } \\
\text { level }\end{array}$ \\
\hline $\begin{array}{l}\text { Jan. } 20 \\
\text { Feb. } 20,21 \\
\text { Mar. } 19 \\
\text { Apr. } 21\end{array}$ & $\begin{array}{l}\text { a) } 10.18 \\
\text { a } 10.31 \\
\text { b } 10.35 \\
\text { b } 10.46\end{array}$ & $\begin{array}{l}\text { May } \\
\text { June } \\
\text { July } \\
\text { Aug. }\end{array}$ & $\begin{array}{l}23 \\
25 \\
23,24 \\
26\end{array}$ & $\begin{array}{l}\mathrm{b} \\
\mathrm{c}\end{array}$ & $\begin{array}{l}10.40 \\
12.38 \\
12.25 \\
12.68\end{array}$ & $\begin{array}{l}\text { Sept.22 } \\
\text { Oct. } 27 \\
\text { Nov. } 26,27 \\
\text { Dec. } 29,30\end{array}$ & $\begin{array}{ll}\text { a } & 13.54 \\
\text { a } & 14.38 \\
\text { a } & 14.42 \\
\text { d } & 14.47 \\
\end{array}$ \\
\hline
\end{tabular}

\section{Changes in measuring points}

The original measuring points of all observation wells in Jewell County are described in Water-Supply Paper 845. Changes in measuring points that occurred during 1941 are given below. The old measuring point of well 46 was the top edge of a 2 -inch slot in a square wooden cover resting on the tile casing. The new measuring point is the top edge of the tile casing on the north side. The original measuring points of wells 47 and 51 are still being used, but they have been lowered by settling in the amounts given in the following table. Wells 52, 54, 55, 57, and 61-63 are bored observation wells cased with $1 \frac{1}{2}-$ inch galvanized iron pipe. The casings originally extended above the land surface and were attached to wooden posts for protection. The measuring point at each of these wells remains the top of the casing, but the casings have been damaged or broken by cattle, hence the measuring points are now lower by the amounts given in the table. The elevations of the measuring points were redetermined on october 1, 1941, by H. A. Walte and V. C. Fishel, and are given below.

\begin{tabular}{|c|c|c|c|c|}
\hline el1 & $\begin{array}{l}\text { Distance below } \\
\text { old measuring } \\
\text { point } \\
\text { (feet) }\end{array}$ & $\begin{array}{l}\text { Helght above } \\
\text { land surface } \\
\text { (feet) }\end{array}$ & $\begin{array}{l}\text { Helght above } \\
(+) \text { or below } \\
(-) \text { bench mark } \\
\text { (feet) }\end{array}$ & $\begin{array}{l}\text { Helght above } \\
\text { datum } \\
\text { (feet) }\end{array}$ \\
\hline $\begin{array}{l}46 \\
47 \\
51 \\
52 \\
54 \\
55 \\
57 \\
61 \\
62 \\
63\end{array}$ & $\begin{array}{r}0.20 \\
.25 \\
.02 \\
3.13 \\
2.91 \\
2.93 \\
2.84 \\
3.13 \\
.12 \\
.10\end{array}$ & $\begin{array}{l}2.0 \\
1.2 \\
2.5 \\
.0 \\
.6 \\
.5 \\
.1 \\
.05 \\
4.3 \\
1.3\end{array}$ & $\begin{array}{l}+4.31 \\
-\quad .56 \\
-6.40 \\
+4.21 \\
+\quad .44 \\
+\quad .41 \\
+8.96 \\
-5.37 \\
-13.46 \\
-\quad .92\end{array}$ & $\begin{array}{r}28.56 \\
24.52 \\
104.50 \\
115.11 \\
111.34 \\
111.31 \\
119.86 \\
105.53 \\
97.44 \\
109.98\end{array}$ \\
\hline
\end{tabular}

a Water level for well 41 interpolated in computing average.

$b$ Water level for well 50 interpolated in computing average.

c Water level for wells 20,30 , and 41 interpolated in computing average.

d Average of water levels in 11 wells; well 41 discontinued. 
Jewell County--Continued

Water-level measurements

Water level, in feet above datum, 1941

\begin{tabular}{|c|c|c|c|c|c|c|c|}
\hline Date & 4 & 6 & 8 & 18 & 25 & 41 & 45 \\
\hline $\begin{array}{ll}\text { Jan. } 20 \\
\text { Peb. } 20 \\
\text { Mar. } 19 \\
\text { Apr. } 21 \\
\text { May } 23 \\
\text { June } 25 \\
\text { July } 23 \\
\text { Aug. } 26 \\
\text { Sept. } 22 \\
\text { Oct. } 27 \\
\text { Nov. } 26 \\
\text { Dec. } 29\end{array}$ & $\begin{array}{l}14.22 \\
14.37 \\
14.32 \\
14.76 \\
15.61 \\
18.81 \\
19.01 \\
19.57 \\
17.72 \\
19.63 \\
19.13 \\
18.99\end{array}$ & $\begin{array}{r}9.74 \\
11.17 \\
11.54 \\
11.51 \\
11.24 \\
12.73 \\
12.32 \\
12.10 \\
12.34 \\
13.12 \\
12.34 \\
12.22\end{array}$ & $\begin{array}{l}23.08 \\
23.57 \\
23.10 \\
23.39 \\
23.66 \\
49.27 \\
48.87 \\
39.06 \\
39.87 \\
46.14 \\
47.10 \\
46.15\end{array}$ & $\begin{array}{r}9.97 \\
9.92 \\
9.94 \\
10.07 \\
10.02 \\
10.59 \\
10.73 \\
10.46 \\
10.61 \\
10.47 \\
10.61 \\
10.58\end{array}$ & $\begin{array}{r}9.78 \\
9.73 \\
9.88 \\
10.28 \\
9.96 \\
\ldots \ldots .27 \\
10.27 \\
10.25 \\
12.18 \\
13.01 \\
13.17 \\
12.93\end{array}$ & $\begin{array}{c}\ldots \ldots \\
\ddot{9} . \dot{i} 0 \\
9.36 \\
8.69 \\
\ddot{i} .09 \\
11.50 \\
\text { (a) } \\
\ldots \ldots \\
\ldots \ldots\end{array}$ & $\begin{array}{r}6.68 \\
6.75 \\
6.84 \\
7.01 \\
7.22 \\
8.94 \\
9.17 \\
9.71 \\
8.95 \\
9.76 \\
10.28 \\
10.82\end{array}$ \\
\hline
\end{tabular}

Water level, in feet above datum, 1941

\begin{tabular}{|c|c|c|c|c|c|c|c|c|}
\hline Date & 12 & 14 & 22 & 30 & 40 & 42 & 43 & 44 \\
\hline $\begin{array}{l}\text { Jan. } 20 \\
\text { Feb. } 21 \\
\text { Mar. } 19 \\
\text { Apr. } 21 \\
\text { May } 23 \\
\text { June } 25 \\
\text { July } 24 \\
\text { Aug. } 26 \\
\text { Sept. } 22 \\
\text { Oct. } 27 \\
\text { Nov. } 27 \\
\text { Dec. } 29\end{array}$ & $\begin{array}{r}9.79 \\
9.65 \\
10.09 \\
11.55 \\
12.61 \\
14.14 \\
14.95 \\
15.07 \\
20.29 \\
18.46 \\
17.60 \\
\text { c } 16.39\end{array}$ & $\begin{array}{l}10.71 \\
10.67 \\
11.16 \\
12.40 \\
12.87 \\
14.22 \\
14.76 \\
17.06 \\
17.59 \\
18.81 \\
20.98 \\
\text { c } 24.67\end{array}$ & $\begin{array}{l}11.67 \\
11.87 \\
12.36 \\
12.37 \\
12.35 \\
13.91 \\
13.69 \\
13.19 \\
13.57 \\
18.28 \\
19.25 \\
\text { c } 19.27\end{array}$ & $\begin{array}{r}8.93 \\
8.96 \\
9.29 \\
9.41 \\
9.34 \\
10.9 \\
10.39 \\
8.33 \\
9.76 \\
10.17 \\
10.96 \\
10.84\end{array}$ & $\begin{array}{r}9.97 \\
10.14 \\
10.07 \\
9.80 \\
9.98 \\
10.24 \\
10.46 \mathrm{~b} \\
10.64 \\
10.70 \\
10.62 \\
10.71 \\
10.51\end{array}$ & $\begin{array}{r}9.95 \\
9.90 \\
10.02 \\
10.05 \\
9.92 \\
14.87 \\
13.25 \\
12.58 \\
14.55 \\
14.97 \\
14.87 \\
14.57\end{array}$ & $\begin{array}{c}11.15 \\
11.10 \\
12.74 \\
12.81 \\
12.58 \\
(a) \\
\ldots \ldots \\
\ldots \ldots \\
\ldots \ldots \\
\ldots \ldots\end{array}$ & $\begin{array}{l}16.57 \\
16.77 \\
16.05 \\
16.94 \\
16.20 \\
16.67 \\
16.00 \\
15.18 \\
15.10 \\
16.38 \\
17.65 \\
17.88\end{array}$ \\
\hline
\end{tabular}

Water level, in feet above datum, 1941

\begin{tabular}{lrrrrrr}
\hline Date & 34 & $34 \mathrm{~A}$ & $34 \mathrm{~B}$ & $34 \mathrm{C}$ & $\begin{array}{c}\text { Pond } \\
\text { staff } \\
\text { gage }\end{array}$ & 46 \\
\hline Jan. 20 & 80.31 & 100.62 & 93.79 & 94.38 & 114.70 & 18.40 \\
Feb. 20 & 82.25 & 98.73 & 94.37 & 94.23 & 114.70 & 18.72 \\
Mar. 19 & 84.70 & 98.79 & 96.26 & 94.56 & 118.60 & 20.45 \\
Apr. 21 & 89.71 & 99.66 & 99.84 & 97.48 & 117.50 & 20.14 \\
May 23 & 91.52 & 98.81 & 101.26 & 99.50 & 116.80 & 20.35 \\
June 25 & 96.24 & 100.33 & 100.25 & 101.19 & 118.30 & 22.97 \\
July 23 & 96.12 & 99.64 & 100.67 & 100.63 & 117.90 & 22.14 \\
Aug. 26 & 93.29 & 98.66 & 99.94 & 99.34 & 117.30 & 20.10 \\
Sept.22 & 95.00 & 99.01 & 98.15 & 102.25 & 117.90 & 21.07 \\
Oct. 27 & 94.57 & 98.95 & 98.40 & 102.16 & 117.70 & 24.40 \\
Nov.26 & 95.82 & 98.76 & 98.91 & 102.59 & 118.10 & 24.99 \\
Dec. 29 & 95.37 & 98.80 & 99.24 & 102.44 & 118.20 & 24.88 \\
\hline
\end{tabular}

Water level, In feet above datum, 1941

\begin{tabular}{|c|c|c|c|c|c|c|c|}
\hline Date & 47 & 50 & 51 & 61 & 62 & 63 & $65 \mathrm{~d}$ \\
\hline $\begin{array}{ll}\text { Jan. } 20 \\
\text { Feb. } 21 \\
\text { Mar. } 19 \\
\text { Apr. } 21 \\
\text { May } 23 \\
\text { June } 25 \\
\text { Jul. } 24 \\
\text { Aug. } 26 \\
\text { Sept. } 22 \\
\text { Oct. } 27 \\
\text { Nov. } 27 \\
\text { Dec. } 30\end{array}$ & $\begin{array}{r}11.38 \\
11.32 \\
13.58 \\
12.71 \\
12.36 \\
14.09 \\
14.20 \\
13.41 \\
15.86 \\
19.66 \\
20.06 \\
18.09\end{array}$ & $\begin{array}{c}13.90 \\
13.91 \\
(\mathrm{e}) \\
(\mathrm{e}) \\
\text { (e) } \\
18.28 \\
16.71 \\
23.50 \\
21.07 \\
23.53 \\
23.11 \\
22.89\end{array}$ & $\begin{array}{r}96.13 \\
96.31 \\
101.19 \\
100.89 \\
97.09 \\
96.31 \\
97.05 \\
96.61 \\
97.26 \\
101.43 \\
101.82 \\
101.92\end{array}$ & $\begin{array}{l}88.34 \\
88.43 \\
87.64 \\
87.89 \\
86.72 \\
88.26 \\
87.12 \\
87.24 \\
87.41 \\
88.68 \\
94.44 \\
91.02\end{array}$ & $\begin{array}{l}84.39 \\
83.91 \\
83.95 \\
83.86 \\
83.67 \\
85.37 \\
85.24 \\
85.26 \\
85.37 \\
85.40 \\
85.36 \\
85.52\end{array}$ & $\begin{array}{l}90.56 \\
90.85 \\
91.07 \\
91.84 \\
92.06 \\
92.52 \\
92.69 \\
92.24 \\
92.24 \\
93.57 \\
95.11 \\
95.29\end{array}$ & $\begin{array}{r}8.49 \\
8.47 \\
9.05 \\
9.12 \\
9.51 \\
13.15 \\
12.19 \\
11.57 \\
11.85 \\
14.45 \\
17.63 \\
20.95\end{array}$ \\
\hline $\begin{array}{l}a \\
b \\
c\end{array}$ & easur & $e$ on & 30 & $\begin{array}{ll}d & W e \\
e & W e \\
f & M e\end{array}$ & $\begin{array}{l}\text { yed } \\
\mathrm{y} .\end{array}$ & or & $\begin{array}{l}41 . \\
.26 .\end{array}$ \\
\hline
\end{tabular}


Jewell County--Continued

Water level, in feet above datum, 1941

\begin{tabular}{|c|c|c|c|c|c|c|c|}
\hline Date & 52 & 53 & 54 & $\begin{array}{l}\text { Pond } \\
\text { staff } \\
\text { gage }\end{array}$ & 55 & 56 & 57 \\
\hline $\begin{array}{l}\text { Jan. } 20 \\
\text { Feb. } 21 \\
\text { Mar. } 19 \\
\text { Apr. } 21 \\
\text { May } 23 \\
\text { June } 25 \\
\text { July } 24 \\
\text { Aug. } 26 \\
\text { Sept.22 } \\
\text { Oct. } 27 \\
\text { Nov. } 27 \\
\text { Dec. } 30\end{array}$ & $\begin{array}{r}97.30 \\
97.94 \\
97.70 \\
98.73 \\
97.02 \\
99.35 \\
100.47 \\
101.24 \\
102.75 \\
114.73 \\
115.21 \\
115.45\end{array}$ & $\begin{array}{r}97.41 \\
98.07 \\
97.69 \\
98.59 \\
98.21 \\
101.80 \\
102.73 \\
102.34 \\
106.27 \\
106.72 \\
106.81 \\
106.71\end{array}$ & $\begin{array}{c}98.22 \\
98.56 \\
98.64 \\
99.49 \\
99.21 \\
104.73 \\
104.47 \\
103.58 \\
\text { (b) } \\
\text { (b) } \\
\text { (b) } \\
\text { (b) }\end{array}$ & $\begin{array}{l}103.70 \\
103.60 \\
104.50 \\
104.50 \\
104.20 \\
109.70 \\
108.90 \\
108.20 \\
111.60 \\
111.30 \\
111.20 \\
111.10\end{array}$ & $\begin{array}{r}99.42 \\
99.40 \\
98.51 \\
99.59 \\
99.90 \\
104.73 \\
103.84 \\
\ldots . .0 \\
\text { (b) } \\
\text { (b) } \\
\text { (b) } \\
\text { (b) }\end{array}$ & $\begin{array}{c}99.72 \\
99.75 \\
(a) \\
\ldots \ldots \\
\ldots \ldots \\
\ldots \ldots \\
\ldots \ldots \\
\ldots \ldots \\
\ldots \ldots \\
\ldots \ldots \\
\end{array}$ & $\begin{array}{r}98.72 \\
98.70 \\
99.44 \\
100.49 \\
98.94 \\
100.01 \\
101.05 \\
101.18 \\
102.14 \\
103.98 \\
104.31 \\
104.07 \\
\end{array}$ \\
\hline
\end{tabular}

Water level, in feet above datum, 1941

\begin{tabular}{lcrrrrr}
\hline Date & 48 & 49 & 64 & 66 & $67 \underline{c}$ & $69 \underline{c} /$ \\
\hline Jan. 20 & 12.12 & 22.71 & $\ldots \ldots$. & 6.42 & 5.88 & 8.51 \\
Feb. 20 & 12.33 & 22.57 & 13.9 & 6.40 & 5.90 & 8.53 \\
Mar. 19 & 12.85 & 23.02 & $\mathrm{~d} 13.84$ & 6.58 & 5.93 & 9.21 \\
Apr. 21 & 12.87 & 23.36 & 14.38 & 6.79 & 6.18 & 8.71 \\
May 23 & 13.10 & 24.49 & 14.14 & 6.72 & 6.44 & 8.84 \\
June 25 & 14.05 & 28.79 & 17.49 & 8.15 & 10.60 & 12.73 \\
July 23 & 14.02 & 29.64 & 16.61 & 8.16 & 10.29 & 11.71 \\
Aug. 26 & 14.87 & 28.80 & 15.38 & 7.91 & 8.99 & 10.41 \\
Sept.22 & 16.55 & 28.21 & 17.49 & 14.02 & 7.69 & 11.71 \\
Oct. 27 & 18.47 & 28.66 & 18.27 & 14.93 & 9.15 & 12.68 \\
Nov. 26 & 18.32 & 28.75 & 17.84 & 16.44 & 10.51 & 13.21 \\
Dec. 29 & 18.18 & 28.61 & 17.91 & 16.88 & 10.37 & 13.34 \\
\hline
\end{tabular}

KEARNY COUNTY

By T. G. McLaughlin

Highest and lowest water levels, in feet below measuring point, in 19 wells in Kearny County, 1941

\begin{tabular}{|c|c|c|c|c|c|}
\hline Well & $\begin{array}{l}\text { Length of } \\
\text { record } \\
\text { (Jears) }\end{array}$ & $\begin{array}{l}\text { Highest } \\
\text { recorded } \\
\text { water level } \\
\text { (feet) }\end{array}$ & Date & $\begin{array}{l}\text { Lowest } \\
\text { recorded } \\
\text { water level } \\
\text { (feet) }\end{array}$ & Date \\
\hline $\begin{array}{l}1 \\
2 \\
3 \\
4 \\
6\end{array}$ & $\begin{array}{l}2 \\
2 \\
2 \\
2 \\
2\end{array}$ & $\begin{array}{r}8.57 \\
56.05 \\
92.34 \\
106.81 \\
154.55\end{array}$ & $\begin{array}{l}\text { Dec. } 6,1941 \\
\text { Nov. 10, } 1941 \\
\text { Dec. 20, } 1939 \\
\text { Jan. 28, } 1940 \\
\text { Dec. 20, } 1939 \\
\text { June 22, } 1940\end{array}$ & $\begin{array}{r}12.81 \\
59.94 \\
95.43 \\
107.04 \\
155.85\end{array}$ & $\begin{array}{l}\text { July 20, } 1941 \\
\text { Sept.20, } 1940 \\
\text { Nov. 10, } 1941 \\
\text { Dec. 19, } 1941 \\
\text { Apr. 23, } 1941\end{array}$ \\
\hline $\begin{array}{l}7 \\
11 \\
12 \\
13 \\
14 \\
15 \\
16 \\
17 \\
18\end{array}$ & $\begin{array}{l}2 \\
2 \\
2 \\
2 \\
2 \\
2 \\
2 \\
2 \\
2\end{array}$ & $\begin{array}{r}51.24 \\
14.29 \\
10.53 \\
4.36 \\
226.98 \\
71.05 \\
45.75 \\
89.97 \\
72.17\end{array}$ & $\begin{array}{l}\text { Nov. } 15,1941 \\
\text { Oct. 28, } 1941 \\
\text { Dec. } 6,1941 \\
\text { July } 3,1941 \\
\text { Oct. 25, } 1939 \\
\text { Aug. 22, } 1940 \\
\text { Oct. 25, } 1939 \\
\text { Aug. 22, } 1940 \\
\text { Mar. 25, } 1940\end{array}$ & $\begin{array}{r}53.87 \\
15.87 \\
15.46 \\
8.53 \\
227.98 \\
71.87 \\
48.11 \\
91.99 \\
73.11\end{array}$ & $\begin{array}{l}\text { Oct. 16, } 1939 \\
\text { Mar. 15, } 1941 \\
\text { Nov. 21, } 1940 \\
\text { Dec. 20, } 1939 \\
\text { Apr. 23, } 1941 \\
\text { Sept.20, } 1940 \\
\text { July 3, } 1941 \\
\text { May 23, } 1940 \\
\text { Oct. 18, } 1941\end{array}$ \\
\hline
\end{tabular}

a a PIpe broken off; measurements discontinued.

$b$ b Well surrounded by water.

c Well used throughout 1941.

d Well unsealed; measurements resumed. 


\section{Kearny County--Continued}

Highest and lowest water levels, in feet below measuring point, in 19 wells in Kearny County, 194l--Continued.

\begin{tabular}{|c|c|c|c|c|c|}
\hline WeIl & $\begin{array}{l}\text { Length of } \\
\text { record } \\
\text { (years) }\end{array}$ & $\begin{array}{l}\text { Highest } \\
\text { recorded } \\
\text { water level } \\
\text { (feet) }\end{array}$ & Date & $\begin{array}{l}\text { Lowest } \\
\text { recorded } \\
\text { water level } \\
\text { (feet) }\end{array}$ & Date \\
\hline $\begin{array}{l}19 \\
22 \\
23 \\
26 \\
28\end{array}$ & $\begin{array}{l}2 \\
2 \\
2 \\
2 \\
2\end{array}$ & $\begin{array}{r}130.58 \\
182.85 \\
174.63 \\
86.30 \\
121.93\end{array}$ & $\begin{array}{l}\text { Dec. 23, } 1940 \\
\text { June 5, } 1941 \\
\text { Oct. 24, } 1939 \\
\text { Oct. 24, } 1940 \\
\text { May } 5,1941\end{array}$ & $\begin{array}{r}131.30 \\
183.39 \\
175.20 \\
86.95 \\
124.35\end{array}$ & $\begin{array}{l}\text { Nov. 23, } 1940 \\
\text { Sept. 3, } 1941 \\
\text { July } 17,1941 \\
\text { May } 6,1941 \\
\text { Feb. } 19,1940 \\
\text { Oct. 22, } 1940\end{array}$ \\
\hline
\end{tabular}

Net changes in water level in 1941, and net changes in water level for the period of record in 19 wells in Kearny County

\begin{tabular}{|c|c|c|c|}
\hline Well & $\begin{array}{l}\text { Difference } \\
\text { between highest } \\
\text { and lowest } \\
\text { water levels } \\
\text { (feet) }\end{array}$ & $\begin{array}{l}\text { Net rise (t) } \\
\text { or net decline } \\
\text { (-) in } 1941 \\
(\text { feet) }\end{array}$ & $\begin{array}{l}\text { Net rise (t) } \\
\text { or net decline } \\
\text { (-) for period } \\
\text { of record } \\
\text { (feet) }\end{array}$ \\
\hline $\begin{array}{r}1 \\
2 \\
3 \\
4 \\
6 \\
7 \\
11 \\
12 \\
13 \\
14 \\
15 \\
16 \\
17 \\
18 \\
19 \\
22 \\
23 \\
26 \\
28\end{array}$ & $\begin{array}{l}4.20 \\
3.89 \\
3.09 \\
.23 \\
1.30 \\
2.63 \\
1.58 \\
4.93 \\
4.17 \\
1.00 \\
.82 \\
2.36 \\
2.02 \\
.94 \\
.72 \\
.54 \\
.57 \\
.65 \\
2.42\end{array}$ & $\begin{array}{l}+3.95 \\
+2.31 \\
-.27 \\
-.03 \\
-.09 \\
+1.44 \\
+1.50 \\
+2.76 \\
+.66 \\
-.27 \\
+.26 \\
+.04 \\
-.57 \\
-.85 \\
-.01 \\
-.09 \\
-.10 \\
+.36 \\
-.04\end{array}$ & $\begin{array}{l}+2.84 \\
+2.18 \\
+.53 \\
-.07 \\
+.08 \\
+2.59 \\
+1.31 \\
+4.09 \\
+3.12 \\
-.73 \\
+.45 \\
-1.92 \\
-.23 \\
-.79 \\
-.05 \\
-.15 \\
-.33 \\
-.19 \\
+2.22\end{array}$ \\
\hline
\end{tabular}

Water-level measurements

1. R. T. Beatty. NW $\frac{2}{4} N E \frac{2}{4}$ sec. $34, T .24$ S., R. 36 W. Measuring point, $3,000.94$ feet above sea level.

Water level, in feet below measuring point, 1941

\begin{tabular}{|c|c|c|c|c|c|c|c|}
\hline Date & $\begin{array}{l}\text { Water } \\
\text { level }\end{array}$ & Date & $\begin{array}{l}\text { Water } \\
\text { level }\end{array}$ & Date & $\begin{array}{l}\text { Water } \\
\text { level }\end{array}$ & Date & $\begin{array}{l}\text { Water } \\
\text { level }\end{array}$ \\
\hline $\begin{array}{l}\text { Jan. } 29 \\
\text { Mar. } 15 \\
\text { Apr. } 23\end{array}$ & $\begin{array}{l}11.62 \\
11.52 \\
11.28\end{array}$ & $\begin{array}{lr}\text { May } & 6 \\
\text { June } & 7 \\
\text { July } & 17\end{array}$ & $\begin{array}{r}10.60 \\
9.72 \\
8.89\end{array}$ & $\begin{array}{l}\text { Aug. } 24 \\
\text { Sept.22 } \\
\text { Oct. } 28\end{array}$ & $\begin{array}{r}10.28 \\
10.16 \\
9.19\end{array}$ & $\begin{array}{ll}\text { Nov. } & 9 \\
\text { Dec. } & 6\end{array}$ & $\begin{array}{l}8.85 \\
8.67\end{array}$ \\
\hline
\end{tabular}

2. C. E. Worthen. $\mathrm{SE}_{\frac{1}{4}} \mathrm{SE}_{\frac{2}{4}}^{2} \mathrm{sec}, 16, \mathrm{~T}, 24 \mathrm{~S}, \mathrm{R}, 36 \mathrm{~W}$. Measuring point, 3,064.49 feet above sea level.

Water level, in feet below measuring point, 1941

\begin{tabular}{ll|lll|lll|ll}
\hline Jan. 29 & 58.81 & May & 6 & 58.67 & Aug. 8 & 57.58 & Nov. 10 & 56.05 \\
Mar. 15 & 58.74 & June & 4 & 57.79 & Sept. 3 & 56.33 & Dec. 19 & 56.50 \\
Apr. 23 & 58.89 & July & 3 & 57.66 & Oct. 28 & 56.78 & & \\
\hline
\end{tabular}

3. F. G. Worthen. SW $\frac{1}{4} W_{\frac{1}{4}}^{\frac{3}{4}}$ sec. 10, T. $23 \mathrm{~S}$, , R. $36 \mathrm{~W}$. Measuring point, $3,126.52$ feet above sea level. 
Kearny County--Continued

3.--Continued.

Water level, in feet below measuring point, 1941

\begin{tabular}{|c|c|c|c|c|c|c|c|}
\hline Date & $\begin{array}{l}\text { Water } \\
\text { level }\end{array}$ & Date & $\begin{array}{l}\text { Water } \\
\text { level }\end{array}$ & Date & $\begin{array}{l}\text { Water } \\
\text { level } \\
\end{array}$ & Date & $\begin{array}{l}\text { Water } \\
\text { level } \\
\end{array}$ \\
\hline $\begin{array}{l}\text { Mar. } 19 \\
\text { Apr. } 23 \\
\end{array}$ & $\begin{array}{l}93.83 \\
94.26 \\
\end{array}$ & $\begin{array}{l}\text { May } \\
\text { June }\end{array}$ & $\begin{array}{l}93.84 \\
93.84\end{array}$ & $\begin{array}{ll}\text { Oct. } 18 \\
\text { Nov. } 10 \\
\end{array}$ & $\begin{array}{l}93.95 \\
95.43 \\
\end{array}$ & Dec. 19 & 94.10 \\
\hline
\end{tabular}

4. C. W. Walker. NW $\mathrm{NWW}_{4}^{\frac{1}{4}}$ sec. 11, T. $21 \mathrm{~S} ., \mathrm{R} .37 \mathrm{~W}$. Measuring point, $3,299.18$ feet above sea level.

Water level, in feet below measuring point, 1941

\begin{tabular}{lr|lll|ll|ll}
\hline Mar. 19 & 107.01 & June & 4 & 106.99 & Sept. 3 & 106.97 & Nov. 10 & 106.96 \\
Apr. 23 & 107.00 & July & 3 & 106.99 & Oct. 18 & 106.98 & Dec. 19 & 107.04 \\
May 5 & 106.98 & Aug. & 8 & 106.97 & & & & \\
\hline
\end{tabular}

6. Meta Kettler. NW $\mathrm{NWW}_{\frac{1}{4}}^{\frac{1}{4}}$ sec. 26, T. $24 \mathrm{~S} ., \mathrm{R} .37 \mathrm{~W}$. Measuring point, $3,222.35$ feet above sea level.

Water level, in feet below measuring point, 1941

\begin{tabular}{ll|lll|lll|ll}
\hline Jan. 29 & 154.78 & May & 6 & 154.83 & Aug. 8 & 154.98 & Nov. 10 & 154.80 \\
Mar. 15 & 154.67 & June & 4 & 154.97 & Sept. 3 & 154.86 & Dec. 31 & 154.87 \\
Apr. 23 & 155.85 & July & 3 & 155.08 & Oct. 18 & 155.05 & & & \\
\hline
\end{tabular}

7. C. H. Browne. NW $W_{\frac{1}{4}} W_{\frac{1}{4}}$ sec. 8, T. 25 S., R. 37 W. Measuring point, $3,117.11$ feet above sea level.

Water level, in feet below measuring point, 1941

\begin{tabular}{lr|lll|ll|ll}
\hline Mar. 15 & 52.72 & June & 4 & 51.74 & Sept.13 & 51.83 & Nov. 15 & 51.24 \\
Apr. 23 & 53.67 & July & 3 & 51.89 & Oct. 18 & 51.49 & Dec. 20 & 51.28 \\
May 6 & 52.13 & Aug. & 8 & 51.85 & & & & \\
\hline
\end{tabular}

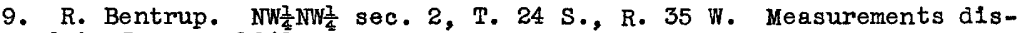
continued in January 1941 .

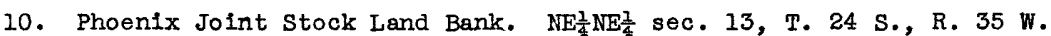
Measurements discontinued in January 1941.

11. P. J. Fichter. SW $\frac{2}{4}$ SW $\frac{3}{4}$ sec. 11, T. 25 S., R. 36 W. Measuring point, 3,005.89 feet above sea level.

Water level, in feet below measuring point, 1941

\begin{tabular}{ll|ll|lll}
\hline Date & $\begin{array}{l}\text { Water } \\
\text { level }\end{array}$ & Date & $\begin{array}{l}\text { Water } \\
\text { level }\end{array}$ & Date & $\begin{array}{r}\text { Water } \\
\text { level }\end{array}$ \\
\hline Mar. 15 & 15.87 & May 6 & 15.85 & Nov. 9 & 14.32 \\
Apr. 23 & 15.81 & Oct. 28 & 14.29 & Dec. 6 & 14.37 \\
\hline
\end{tabular}

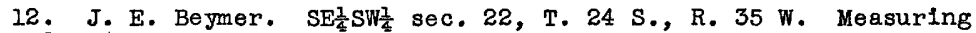
point, 2,954.84 feet above sea level.

Water level, in feet below measuring point, 1941

\begin{tabular}{lr|rr|rrr}
\hline Mar. 19 & 13.29 & Oct. 18 & 11.20 & Dec. 6 & 10.53 \\
Apr. 24 & 14.05 & Nov. 2 & 10.93 & & & \\
\hline
\end{tabular}

13. D. S. N1cholson. NW $N W_{\frac{1}{4}}^{\frac{1}{4}}$ sec. 15, T. 25 S., R. 37 W. Measuring point, 3,049.47 feet above sea level.

Water level, in feet below measuring point, 1941

\begin{tabular}{lr|lr|lr|lr}
\hline Date & $\begin{array}{r}\text { Water } \\
\text { level }\end{array}$ & Date & $\begin{array}{r}\text { Water } \\
\text { level }\end{array}$ & Date & $\begin{array}{r}\text { Water } \\
\text { level }\end{array}$ & Date & $\begin{array}{r}\text { Water } \\
\text { level }\end{array}$ \\
\hline Mar. 15 & 5.70 & June 4 & 4.41 & Sept.13 & 5.82 & Nov. 15 & 4.85 \\
Apr. 23 & 5.44 & July 3 & 4.36 & Oct. 18 & 5.46 & Dec. 20 & 5.04 \\
May 6 & 4.57 & Aug. 8 & 5.21 & & & & \\
\hline
\end{tabular}

14. W. H. Ploeger. SW $\frac{2}{4} \mathrm{SW} \frac{1}{4}$ sec. 32, T. $22 \mathrm{~S}, \mathrm{R}, 38 \mathrm{~W} \cdot$

Water level, in feet below measuring point, 1941

\begin{tabular}{lrr|rr|rrr|rr}
\hline Mar. 15 & 227.58 & June & 5 & 227.73 & Aug. & 8 & 227.93 & Nov. 10 & 227.81 \\
Apr. 23 & 227.98 & July & 3 & 227.95 & Oct. 18 & 227.84 & Dec. 20 & 227.85 \\
May 5 & 227.57 & & & & & & & & \\
\hline
\end{tabular}




\section{Kearny County--Continued}

15. Joseph McNellis. SW $\frac{1}{4} S W_{\frac{1}{4}}^{\frac{1}{2}}$ sec. 36, T. 22 S., R. 35 W. Water level, in feet below measuring point, 1941

\begin{tabular}{|c|c|c|c|c|c|c|c|c|}
\hline Date & $\begin{array}{l}\text { Water } \\
\text { level }\end{array}$ & Date & & $\begin{array}{l}\text { Water } \\
\text { level }\end{array}$ & Date & $\begin{array}{l}\text { Water } \\
\text { level }\end{array}$ & Date & $\begin{array}{l}\text { Water } \\
\text { level }\end{array}$ \\
\hline $\begin{array}{lr}\text { Mar. } & 19 \\
\text { Apr. } & 24 \\
\text { May } & 5 \\
\end{array}$ & $\begin{array}{l}71.54 \\
71.64 \\
71.59\end{array}$ & $\begin{array}{l}\text { June } \\
\text { July } \\
\text { Aug. }\end{array}$ & $\begin{array}{l}4 \\
3 \\
8\end{array}$ & $\begin{array}{l}71.64 \\
71.71 \\
71.54\end{array}$ & $\begin{array}{l}\text { Sept. } 3 \\
\text { oct. } 18\end{array}$ & $\begin{array}{l}71.40 \\
71.33\end{array}$ & $\begin{array}{ll}\text { Nov. } 10 \\
\text { Dec. } 19\end{array}$ & $\begin{array}{l}71.32 \\
71.28\end{array}$ \\
\hline
\end{tabular}

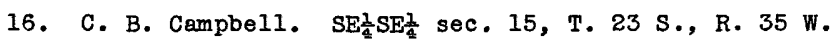

Water level, in feet below measuring point, 1941

\begin{tabular}{lrr|ll|l|ll|ll}
\hline Mar. 19 & 47.74 & June & 4 & 47.80 & Sept. 3 & 47.80 & Nov. 10 & 47.75 \\
Apr. 24 & 47.96 & July & 3 & 48.11 & Oct. 18 & 47.72 & Dec. 19 & 47.70 \\
May 5 & 47.96 & Aug. & 8 & 47.84 & & & & & \\
\hline
\end{tabular}

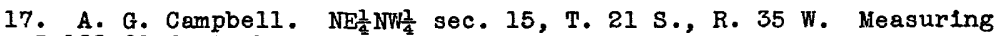
point, $3,129.08$ feet above sea level.

Water level, in feet below measuring point, 1941

\begin{tabular}{lrr|lr|lll|ll}
\hline Mar. 19 & 91.14 & July & 3 & 91.71 & Sept. 3 & 91.71 & Nov. 10 & 91.67 \\
Apr. 24 & 91.44 & Aug. & 8 & 91.72 & Oct. 18 & 91.69 & Dec. 19 & 91.71 \\
May 5 & 91.50 & & & & & & & & \\
\hline
\end{tabular}

18. G. H. Cook. SW SE $\frac{1}{4}$ sec. 28, T. 24 S., R. $38 \mathrm{~W}$. Measuring point, 3,235.70 feet above sea level. Water levels, in feet below measurIng point, 1941: Mar. 15, 72.24; Oct. 18, 73.11; Nov. 10, 73.10; Dec. 31, 73.09 .

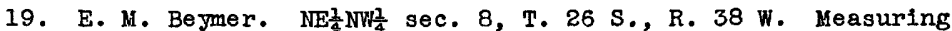
point, $3,247.40$ feet above sea level.

Water level, in feet below measuring point, 1941

\begin{tabular}{|rr|lr|ll|ll|l}
\hline Mar. 15 & 130.91 & June 7 & 130.81 & Sept.22 & 130.81 & Nov. 11 & 130.98 \\
Apr. 23 & 131.25 & July 17 & 131.08 & oct. 28 & 130.76 & Dec. 31 & 130.92 \\
May 6 & 130.80 & Aug. 24 & 130.79 & & & & & \\
\hline
\end{tabular}

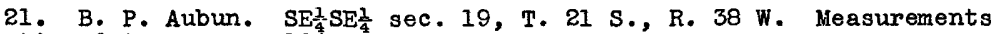
discontinued in January 1941 .

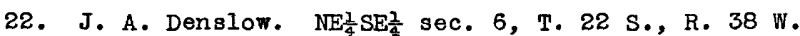

Water level, in feet below measuring point, 1941

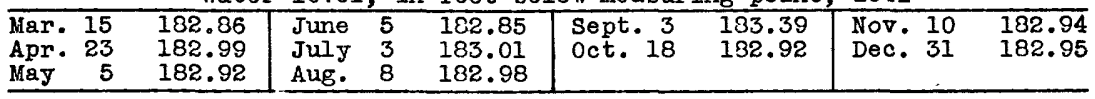

23. James Coghill. NW $N W \frac{1}{4}$ sec. 18, T. $26 \mathrm{~S}$, R. $37 \mathrm{~W}$, Measuring point, 3,203.55 feet above sea level.

Water li vel, in feet below measuring point, 1941

\begin{tabular}{|c|c|c|c|c|c|c|}
\hline $\begin{array}{l}\text { Mar. } 15 \\
\text { Apr. } 23 \\
\text { May }\end{array}$ & $\begin{array}{l}174.89 \\
175.23 \\
175.02\end{array}$ & $\begin{array}{l}\text { June } \\
\text { July } 1 \\
\text { Aug. } 2\end{array}$ & $\begin{array}{l}174.93 \\
175.20 \\
174.99\end{array}$ & $\begin{array}{l}\text { Sept.22 } \\
\text { Oct. } 28\end{array}$ & $\begin{array}{l}174.97 \\
174.95\end{array}$ & $\begin{array}{lrl}\text { Nov. } & 9 & 175.16 \\
\text { Dec. } 31 & 174.99\end{array}$ \\
\hline
\end{tabular}

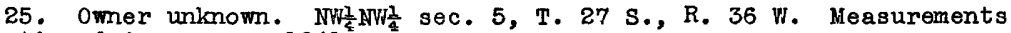
discontinued in January 1941 .

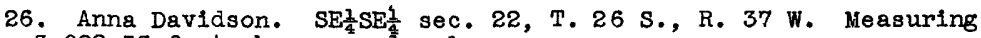
point, 3,092.53 feet above sea level.

Water level, in feet below measuring point, 1941

\begin{tabular}{|c|c|c|c|c|c|c|}
\hline $\begin{array}{lr}\text { Mar. } & 15 \\
\text { Apr. } & 23 \\
\text { May } & 6\end{array}$ & $\begin{array}{l}86.85 \\
86.88 \\
86.95\end{array}$ & $\begin{array}{l}\text { June } \\
\text { July } 1 \\
\text { Aug. } 2\end{array}$ & $\begin{array}{l}86.66 \\
86.57 \\
86.51\end{array}$ & $\begin{array}{l}\text { Sept.22 } \\
\text { oct. } 28\end{array}$ & $\begin{array}{l}86.53 \\
86.48\end{array}$ & $\begin{array}{l}\text { Nov. } 9 \\
\text { Dec. } 31\end{array}$ \\
\hline
\end{tabular}

28. Harry Tate. NW $\frac{1}{4} N W \frac{1}{4}$ sec. 26, T. 22 S., R. 37 W. Measuring point, 3,231.00 feet above sea level.

Water level, in feet below measuring point, 1941

\begin{tabular}{|c|c|c|c|c|c|c|c|c|}
\hline $\begin{array}{lr}\text { Mar. } & 19 \\
\text { Apr. } & 23 \\
\text { May } & 5\end{array}$ & $\begin{array}{l}122.01 \\
121.98 \\
121.93\end{array}$ & $\begin{array}{l}\text { June } \\
\text { July } \\
\text { Aug. }\end{array}$ & $\begin{array}{l}4 \\
3 \\
8\end{array}$ & $\begin{array}{l}122.10 \\
122.01 \\
121.98\end{array}$ & $\begin{array}{l}\text { Sept. } 3 \\
\text { oct. } 18\end{array}$ & $\begin{array}{l}121.99 \\
121.96\end{array}$ & $\begin{array}{ll}\text { Nov. } 10 \\
\text { Dec. } 19\end{array}$ & $\begin{array}{l}121.94 \\
122.05\end{array}$ \\
\hline
\end{tabular}


KIOWA COUNTY

By B. F. Latta

Highest and lowest water levels, in feet below measuring point, in 5 wells in Kiowa county, 1941.

\begin{tabular}{ccccccc}
\hline Well & $\begin{array}{c}\text { Length of } \\
\text { record } \\
\text { (years) }\end{array}$ & $\begin{array}{c}\text { Highest } \\
\text { recorded } \\
\text { wer level } \\
\text { (feet) }\end{array}$ & Date & $\begin{array}{c}\text { Lowest } \\
\text { recorded } \\
\text { water level } \\
\text { (feet) }\end{array}$ & Date \\
\hline 4 & 1 & 76.45 & Dec. 27, 1940 & 76.78 & Oct. 7, 1941 \\
5 & 1 & 41.23 & 0ct. 23, 1940 & a 41.57 & July 28; 1941 \\
7 & 1 & 34.07 & Nov. 20, 1941 & 34.51 & Nov. 20, 1941 \\
8 & 1 & 26.53 & Nov. 20, 1941 & 27.62 & Apr. 28, 1941 \\
10 & 1 & 105.73 & Nov. 4, 1941 & 107.27 & 0ct. 24, 1940 \\
\hline
\end{tabular}

Net changes in water level in 1941, and net changes in water level for the period of record in 5 wells in Kiowa county

\begin{tabular}{cccc}
\hline $\begin{array}{c}\text { Difference } \\
\text { between highest } \\
\text { and lowest } \\
\text { water levels } \\
\text { (feet) }\end{array}$ & $\begin{array}{c}\text { Net rise (t) } \\
\text { or net decline } \\
(-) \text { in } 1941\end{array}$ & $\begin{array}{c}\text { Net rise (t) } \\
\text { or net declire } \\
(-) \text { for period } \\
\text { of record } \\
\text { (feet) }\end{array}$ \\
\hline 4 & 0.33 & -0.18 & -0.11 \\
5 & .34 & -.19 & -.28 \\
7 & .44 & $\mathrm{~b}+.31$ & +.18 \\
8 & 1.09 & +.68 & +.33 \\
10 & 1.54 & +1.19 & +1.46 \\
\hline
\end{tabular}

Water-level measurements

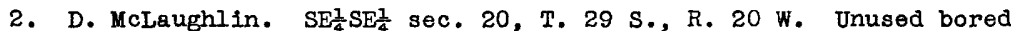
and driven stock well, diameter $1 \frac{1}{2}$ inches, depth 158 feet. Measuring point, top of $1 \frac{1}{2}-1$ inch galvanized-iron pipe, 1.0 foot above land surface. No pump on well.

Water level, in feet below measuring point, 1940-41

\begin{tabular}{|c|c|c|c|c|c|}
\hline Date & $\begin{array}{l}\text { Water } \\
\text { level }\end{array}$ & Date & $\begin{array}{l}\text { Water } \\
\text { level }\end{array}$ & Date & $\begin{array}{l}\text { Water } \\
\text { level }\end{array}$ \\
\hline $\begin{array}{l}\text { oct. } 18,1940 \\
4 ; 1941 \\
\text { Aug. } \\
27\end{array}$ & $\begin{array}{l}145.84 \\
145.53 \\
145.73\end{array}$ & $\begin{array}{l}\text { Sept.12, } 1941 \\
\text { Oct. } 7\end{array}$ & $\begin{array}{l}145.83 \\
145.79\end{array}$ & $\begin{array}{l}\text { Nov. } 4,1941 \\
\text { Dec. } 22\end{array}$ & $\begin{array}{l}145.77 \\
145.73\end{array}$ \\
\hline
\end{tabular}

3. E. M. Pyle. NW $\frac{1}{4} \mathrm{NE} \frac{2}{4}$ sec. 8, T. 29 S., R. 16 W. Well plugged; measurements discontinued in January 1941.

4. H. E. Davis. $\mathrm{SE}_{\frac{1}{4}} \mathrm{NE}_{\frac{1}{4}}^{\frac{1}{3}}$ sec. $4, \mathrm{~T} .28 \mathrm{~S} ., \mathrm{R} .15 \mathrm{~W}$. Measuring point, $2,116.9$ feet above sea level.

Water level, in feet below measuring point, 1941

\begin{tabular}{ll|ll|ll|lr}
\hline Date & $\begin{array}{l}\text { Water } \\
\text { level }\end{array}$ & Date & $\begin{array}{l}\text { Water } \\
\text { level }\end{array}$ & Date & $\begin{array}{r}\text { Water } \\
\text { level }\end{array}$ & Date & $\begin{array}{r}\text { Water } \\
\text { level }\end{array}$ \\
\hline Mar. 22 & 76.56 & June 11 & 76.70 & Sept.12 & 76.68 & Nov. 5 & 76.56 \\
Apr. 28 & 76.59 & JulJ 28 & $\begin{array}{l}76.67 \\
76.56\end{array}$ & Oct. 7 & 76.78 & Dec. 22 & 76.63 \\
May 26 & 76.68 & Aug. 27 & 76.67 & & & & \\
\hline
\end{tabular}

5. I. W. Grimes. $N W_{\frac{1}{4}} \mathrm{NW}_{\frac{2}{4}}$ sec. $34, \mathrm{~T}, 27 \mathrm{~S}, \mathrm{R}, 17 \mathrm{~W}$. Measuring point, $2,132.9$ feet above sea level.

a Messurement of 44.00 feet on Nov. 29, 1940, not included.

b To Nov. 20, 1941 . 
KLowa County--Continued

5.--Continued.

Water level, in feet below measuring point, 1941

\begin{tabular}{ll|lr|ll|ll}
\hline Date & $\begin{array}{l}\text { Water } \\
\text { level }\end{array}$ & Date & $\begin{array}{r}\text { Water } \\
\text { level }\end{array}$ & Date & $\begin{array}{r}\text { Water } \\
\text { level }\end{array}$ & Date & $\begin{array}{r}\text { Water } \\
\text { level }\end{array}$ \\
\hline Mar. 22 & 41.41 & June 11 & 41.49 & Sept.12 & 41.56 & Nov. 20 & 41.57 \\
Apr. 28 & 41.45 & July 28 & 41.57 & Oct. 7 & 41.53 & Dec. 22 & 41.51 \\
May 26 & 41.48 & Aug. 27 & 41.52 & & & &
\end{tabular}

6. Mrs. J. A. Crowe. NWt $S W_{4}^{\frac{1}{4}}$ sec. 16, T. 28 S., R. 18 W. Gasolinepowered pump installed on well by owner; water-level measurements discontinued in January 1941.

7. A. C. Weaver. SW $W_{4}^{1} W_{4}^{\frac{1}{4}}$ sec. 23, T. $27 \mathrm{~S}$, R. 18 W. Measuring point, $2,177.0$ feet above sea level.

Water level, in feet below measuring point, 1941

\begin{tabular}{ll|lll|ll|l|l|}
\hline Mar. 22 & 34.51 & May & 26 & 34.41 & July 28 & 34.23 & Oct. 7 & 34.14 \\
Apr. 28 & 34.43 & June 11 & 34.39 & Aug. 27 & 34.14 & Nov. 20 & 34.07 \\
\hline
\end{tabular}

8. E. E. Miller. $\mathrm{SW}_{\frac{1}{4}} \mathrm{SE}_{\frac{1}{4}}$ sec. 18, T. $27 \mathrm{~S}$, R. $18 \mathrm{~W}$. Measuring point, $2,192.4$ feet above sea level.

Water level, in feet below measuring point, 1941

\begin{tabular}{ll|ll|ll|lll}
\hline Mar. 22 & 27.54 & May 26 & 27.45 & Aug. 27 & 26.75 & Nov: 20 & 26.53 \\
Apr. 28 & 27.62 & July 21 & 26.67 & Sept.12 & 26.95 & Dec. 22 & 26.63 \\
\hline
\end{tabular}

10. J. E. Ely. SW $\frac{1}{4} N W_{4}^{\frac{1}{4}}$ sec. 23, T. 30 S., R. 18 W. Water level, in feet below measuring point, 1941

\begin{tabular}{ll|ll|ll|lrr}
\hline Mar. 22 & 106.55 & June 11 & 106.12 & Sept.12 & 106.09 & Nov. 4 & 105.73 \\
Apr. 28 & 106.42 & July 28 & 106.15 & 0ct. 7 & 106.47 & Dec. 22 & 105.81 \\
May 26 & 106.06 & Sept. 2 & 106.19 & & & & & \\
\hline
\end{tabular}

MCPHERSON COUNTY

By C. C. Williams and G. H. von Hein

Highest and lowest water levels, in feet below measuring point, in 10 wells in McPherson County, 1941

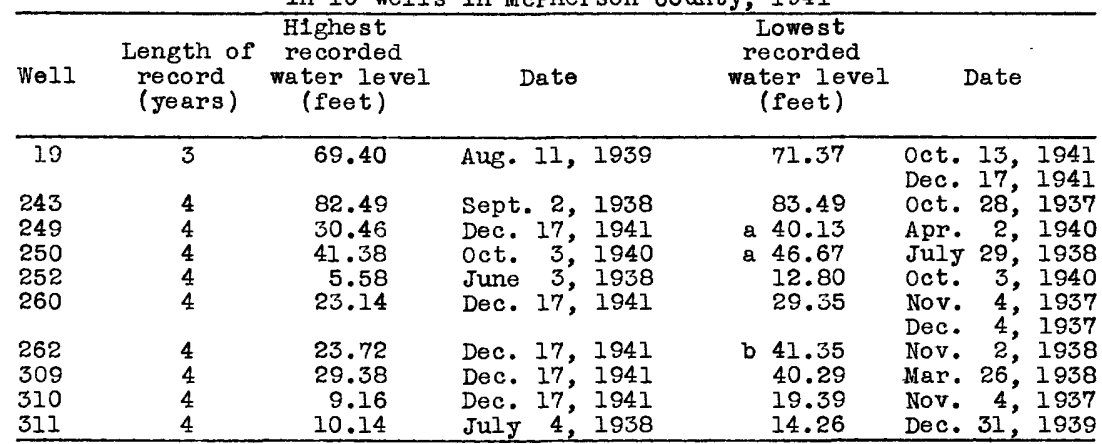

a Measured after well had been pumped.

b Measured while pumping. 
McPherson County--Continued

Net changes in water level in 1941, and net changes in water level for the period of record in 10 wells in McPherson County

\begin{tabular}{lccc}
\hline Well & $\begin{array}{c}\text { Difference } \\
\text { between highest } \\
\text { and lowest } \\
\text { water levels } \\
\text { (feet) }\end{array}$ & $\begin{array}{c}\text { Net rise } \\
\text { or net decline } \\
(-) \text { in } 1941 \\
\text { (feet) }\end{array}$ & $\begin{array}{c}\text { Net rise (+) } \\
\text { or net decline } \\
(-) \text { for period } \\
\text { of record } \\
\text { (feet) }\end{array}$ \\
\hline 19 & 1.97 & -0.72 & -1.77 \\
243 & 1.00 & -.19 & -1.37 \\
249 & 9.67 & +9.21 & +7.23 \\
250 & 5.29 & -.63 & +.92 \\
252 & 7.22 & +2.95 & +2.34 \\
260 & 6.21 & +5.93 & +7.36 \\
262 & 17.63 & +9.91 & +6.31 \\
309 & 10.91 & +6.98 & +10.23 \\
310 & 10.23 & +.83 & +2.36 \\
311 & 4.12 & +1.57 & \\
\hline
\end{tabular}

Water-level measurements

19. Scott Montgomery. NW $\frac{1}{4} \mathrm{NE} \frac{1}{4}$ sec. 29, T. 19 S., R. 3 W. Water level, in feet below measuring point, 1941

\begin{tabular}{|c|c|c|c|c|c|c|c|c|c|c|}
\hline Date & & $\begin{array}{l}\text { Water } \\
\text { level }\end{array}$ & Date & & $\begin{array}{l}\text { Water } \\
\text { level }\end{array}$ & Date & & $\begin{array}{l}\text { Water } \\
\text { level }\end{array}$ & Date & $\begin{array}{l}\text { Water } \\
\text { level }\end{array}$ \\
\hline $\begin{array}{l}\text { Jan. } \\
\text { Feb. } \\
\text { Mar. }\end{array}$ & $\begin{array}{l}3 \\
4 \\
4\end{array}$ & $\begin{array}{l}70.65 \\
70.58 \\
70.56\end{array}$ & $\begin{array}{l}\text { Apr. } \\
\text { May }\end{array}$ & $\frac{1}{2}$ & $\begin{array}{l}70.57 \\
70.79\end{array}$ & $\begin{array}{l}\text { June } \\
\text { JuIf }\end{array}$ & $\begin{array}{l}4 \\
8\end{array}$ & $\begin{array}{l}70.91 \\
71.02\end{array}$ & $\begin{array}{ll}\text { Oct. } 13 \\
\text { Dec. } 17\end{array}$ & $\begin{array}{l}71.37 \\
71.37\end{array}$ \\
\hline
\end{tabular}

243. Emma Bergstrom. $\mathrm{SE}_{\frac{1}{4}}^{\frac{1}{4}} \mathrm{SW}_{\frac{1}{4}} \mathrm{SE}_{\frac{1}{4}}^{\frac{1}{4}}$ sec. 5, T. 19 S., R. 3 W. Water level, in feet below measuring point, 1941

\begin{tabular}{ll|lr|ll}
\hline Date & $\begin{array}{l}\text { Water } \\
\text { level }\end{array}$ & Date & $\begin{array}{l}\text { Water } \\
\text { level }\end{array}$ & Date & $\begin{array}{l}\text { Water } \\
\text { level }\end{array}$ \\
\hline Jan. 3 & 82.93 & July 8 & 83.08 & Dec. 17 & 83.12 \\
Apr. 1 & 83.00 & Oct. I3 & 83.06 & & \\
\hline
\end{tabular}

249. Prudential Life Insurance Co. SE cor. sec. 5, T. 18 S., R. 3 W。 Water level, in feet below measuring point, 1941

\begin{tabular}{lll|lr|lll}
\hline Jan. 3 & 39.67 & July 8 & 33.49 & Dec. 17 & 30.46 \\
Apr. & 1 & 37.44 & Oct. 13 & 32.84 & & \\
\hline
\end{tabular}

250. John Weed. $N E \frac{1}{4} \mathrm{NE} \frac{1}{4} \mathrm{SE} \frac{1}{4}$ sec. $30, \mathrm{~T} .19 \mathrm{~S} ., \mathrm{R} .4 \mathrm{~W}$.

Water level, in feet below measuring point, 1941

\begin{tabular}{|c|c|c|c|c|c|c|}
\hline $\begin{array}{l}\text { Jan. } \\
\text { Apr. }\end{array}$ & $\begin{array}{l}3 \\
1\end{array}$ & $\begin{array}{l}41.95 \\
41.94 \\
\end{array}$ & $\begin{array}{lr}\text { July } 8 \\
\text { Oct. } 13 \\
\end{array}$ & $\begin{array}{l}43.57 \\
43.86 \\
\end{array}$ & Dec. 17 & $\overline{42.58}$ \\
\hline
\end{tabular}

252. David Mills. SE cor. sec. I4, T. 19 S., R. 5 W. Measurements discontinued, July 8, 1941. Water levels, in feet below measuring point, 1941: Jan. 3, 11.56; Apr. 1, 10.07; July 8, 8.61. R. $4 \mathrm{~W}$

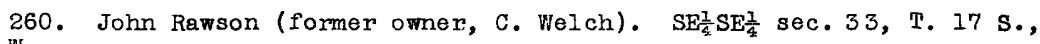

Water level, in feet below measuring point, 1941

\begin{tabular}{lll|ll|ll}
\hline Jan. 3 & 29.07 & JuIy 8 & 26.39 & Dec. 17 & 23.14 \\
Apr. & 1 & 28.98 & Oct. I3 & 24.63 & & \\
\hline
\end{tabular}

262. P. A. Olsen. $\mathrm{NE}_{\frac{1}{4}} \mathrm{NW}_{\frac{1}{4}} \mathrm{NE} \frac{\mathrm{I}}{4} \mathrm{sec} .1, \mathrm{~T} .18 \mathrm{~S}, \mathrm{R}, 5 \mathrm{~W}$.

Water level, in feet below measuring point, 1941

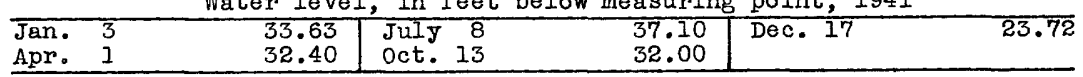

a Through July 8, 1941 . 
McPherson County--Continued

309. Mrs. Ida Tuxhorn. SW $\frac{1}{4}$ SW $\frac{1}{4}$ sec. 9, T. 21 S., R. 4 W. Water level, in feet below measuring point, 1941

\begin{tabular}{|c|c|c|c|c|c|c|c|c|}
\hline Date & & $\begin{array}{l}\text { Water } \\
\text { level }\end{array}$ & Date & $\begin{array}{l}\text { Water } \\
\text { level }\end{array}$ & Dete & $\begin{array}{l}\text { Water } \\
\text { level }\end{array}$ & Date & $\begin{array}{l}\text { Water } \\
\text { level }\end{array}$ \\
\hline Feb. & $\begin{array}{r}3 \\
10 \\
17 \\
24 \\
31 \\
7 \\
14\end{array}$ & $\begin{array}{l}36.36 \\
36.37 \\
36.33 \\
36.37 \\
36.41 \\
36.25 \\
36.40\end{array}$ & $\begin{array}{c}\text { Feb. } 21 \\
28 \\
\text { Mar. } 17 \\
21 \\
28 \\
\\
\text { Apr. } 4 \\
11\end{array}$ & $\begin{array}{l}36.51 \\
36.49 \\
36.54 \\
36.55 \\
36.39 \\
36.09 \\
36.08\end{array}$ & $\begin{array}{rr}\text { Apr. } & 18 \\
& 25 \\
\text { May } \quad 2 \\
\\
\\
\\
16 \\
\\
23 \\
29\end{array}$ & $\begin{array}{l}35.90 \\
36.41 \\
35.71 \\
35.80 \\
35.53 \\
35.66 \\
35.32\end{array}$ & $\begin{array}{lr}\text { June } & 6 \\
& 13 \\
& 24 \\
\text { July } & 7 \\
\text { Oct. } & 13 \\
\text { Dec. } & 17\end{array}$ & $\begin{array}{l}35.05 \\
34.89 \\
34.40 \\
34.14 \\
31.77 \\
29.38\end{array}$ \\
\hline
\end{tabular}

310. City of Moundridge. SW $\frac{1}{4} \mathrm{NE}_{\frac{1}{4}} \mathrm{SW} \frac{1}{4}$ sec. 23, T. 21 S., R. 2 W. Water level, in feet below measuring point, 1941

\begin{tabular}{lll|lll|lll|ll}
\hline Jan. & 3 & 9.99 & Apr. & 1 & 9.68 & June & 4 & 9.81 & Oct. 13 & 9.44 \\
Feb. & 4 & 9.84 & May & 2 & 9.70 & July & 8 & 9.59 & Dec. 17 & 9.16 \\
\hline
\end{tabular}

311. City of Moundridge. SW $\frac{1}{4} \mathrm{SE} \frac{1}{4} \mathrm{SW} \frac{1}{4}$ sec. 33, T. $21 \mathrm{~S}, \mathrm{R}, 2 \mathrm{~W}$. Water level, in feet below measuring point, 1941

\begin{tabular}{|c|c|c|c|c|c|c|c|c|c|c|}
\hline $\begin{array}{l}\text { Jan. } \\
\text { Feb. } \\
\text { Mar. }\end{array}$ & $\begin{array}{l}3 \\
4 \\
4\end{array}$ & $\begin{array}{l}12.61 \\
12.07 \\
11.81\end{array}$ & $\begin{array}{l}\text { Apr. } \\
\text { MaT }\end{array}$ & $\begin{array}{l}1 \\
2\end{array}$ & $\begin{array}{l}11.24 \\
11.22\end{array}$ & $\begin{array}{l}\text { June } \\
\text { July }\end{array}$ & $\begin{array}{l}4 \\
8\end{array}$ & $\begin{array}{l}11.74 \\
11.10\end{array}$ & $\begin{array}{l}\text { Oct. } 1 \\
\text { Dec. } 1\end{array}$ & $\begin{array}{l}12.15 \\
11.04\end{array}$ \\
\hline
\end{tabular}

\section{MEADE COUNTY}

By J. C. Frye

Highest and lowest water levels, in feet below measuring point, in 26 wells in Meade County, 1941

\begin{tabular}{|c|c|c|c|c|c|c|}
\hline Well & $\begin{array}{l}\text { Length of } \\
\text { record } \\
\text { (years) }\end{array}$ & $\begin{array}{l}\text { Highest } \\
\text { recorded } \\
\text { water level } \\
\text { (feet) }\end{array}$ & Date & $\begin{array}{c}\text { Lowest } \\
\text { recorded } \\
\text { water level } \\
\text { (feet) }\end{array}$ & Date & \\
\hline $\begin{array}{r}2 \\
3 \\
10 \\
11 \\
16 \\
23\end{array}$ & $\begin{array}{l}2.5 \\
2.5 \\
2.5 \\
2.5 \\
2.5 \\
2.5\end{array}$ & $\begin{array}{l}20.38 \\
29.27 \\
14.10 \\
10.40 \\
13.23 \\
10.38\end{array}$ & $\begin{array}{l}\text { Dec. } 3,1941 \\
\text { July } 15,1939 \\
\text { June 10; } 1941 \\
\text { July } 17,1939 \\
\text { June 10, } 1941 \\
\text { Aug. } 6,1941\end{array}$ & $\begin{array}{l}22.18 \\
30.68 \\
19.91 \\
14.56 \\
16.90 \\
12.32\end{array}$ & \multirow{2}{*}{\multicolumn{2}{|c|}{$\begin{array}{l}\text { Sept.29, } 1939 \\
\text { Aug. } 17,1940 \\
\text { Aug. 3, } 1939 \\
\text { Sept.20, } 1940 \\
\text { oct. 28, } 1940 \\
\text { oct. 29, } 1940 \\
\text { Jan. 21, } 1941 \\
\text { oct. 29, } 1939 \\
\text { May } 16,1941\end{array}$}} \\
\hline $\begin{array}{l}27 \\
33\end{array}$ & $\begin{array}{l}2.5 \\
2.5\end{array}$ & $\begin{array}{l}19.99 \\
38.14\end{array}$ & $\begin{array}{l}\text { Dec. } 21,1940 \\
\text { Feb. } 14,1940 \\
\text { Dec. } 3,1941\end{array}$ & $\begin{array}{l}20.54 \\
38.48\end{array}$ & & \\
\hline $\begin{array}{l}34 \\
36 \\
37 \\
40 \\
41 \\
42 \\
45 \\
47 \\
55 \\
57 \\
61 \\
62 \\
\end{array}$ & $\begin{array}{l}2.5 \\
2.5 \\
2.5 \\
2.5 \\
2.5 \\
2.5 \\
2.5 \\
2.5 \\
2.5 \\
2.5 \\
2.5 \\
2.5 \\
\end{array}$ & $\begin{array}{r}147.35 \\
157.07 \\
34.31 \\
130.40 \\
157.83 \\
133.13 \\
3.63 \\
43.10 \\
85.47 \\
169.94 \\
60.95 \\
25.66 \\
\end{array}$ & $\begin{array}{l}\text { Feb. } 14,1940 \\
\text { Jan. } 21,1941 \\
\text { July } 20,1939 \\
\text { Feb. } 14,1940 \\
\text { Sept.30, } 1939 \\
\text { Feb. } 14,1940 \\
\text { Oct. 9, } 1941 \\
\text { Jan. } 21,1941 \\
\text { Sept.30, } 1939 \\
\text { Aug. 2, } 1939 \\
\text { Sept. 9, } 1941 \\
\text { Dec. 3, } 1941\end{array}$ & $\begin{array}{r}151.19 \\
160.46 \\
41.72 \\
133.11 \\
158.32 \\
133.72 \\
4.90 \\
44.39 \\
86.52 \\
172.97 \\
61.37 \\
26.66 \\
\end{array}$ & $\begin{array}{l}\text { Oct. 29, } \\
\text { Sept.20, } \\
\text { Sept.29, } \\
\text { Sept.14, } \\
\text { Oct. 8, } \\
\text { Sept. 9, } \\
\text { Aug. 31, } \\
\text { July 22, } \\
\text { Sept.20, } \\
\text { Sept. 9, } \\
\text { May 17, } \\
\text { Aug. 17, }\end{array}$ & $\begin{array}{l}1939 \\
1940 \\
1939 \\
1940 \\
1941 \\
1941 \\
1939 \\
1939 \\
1940 \\
1941 \\
1940 \\
1940 \\
\end{array}$ \\
\hline
\end{tabular}


Meade County--Continued

Highest and lowest water levels, in feet below measuring point, in 26 wells in Meade County, 1941--Continued.

\begin{tabular}{rcccccc}
\hline We11 & $\begin{array}{c}\text { Length of } \\
\text { record } \\
\text { (years) }\end{array}$ & $\begin{array}{c}\text { Highest } \\
\text { wecorded } \\
\text { wer level } \\
\text { (feet) }\end{array}$ & Date & $\begin{array}{c}\text { Lowest } \\
\text { recorded } \\
\text { water level } \\
\text { (feet) }\end{array}$ & Date \\
\hline 73 & 2.5 & 33.18 & Nov. 9, 1939 & 34.55 & Aug. 19, 1940 \\
76 & 2.5 & 27.70 & Sept. 1, 1939 & 38.20 & May 17, 1941 \\
77 & 2.5 & 65.31 & June 20, 1940 & 66.03 & Sept.20, 1940 \\
88 & 2.5 & 42.36 & June 20, 1940 & 44.71 & Sept.20, 1940 \\
101 & 2.5 & 97.38 & Nov. 9, 1939 & 88.47 & Nov. 14, 1940 \\
234 & 2.5 & 14.01 & Nov. 1, 1941 & 15.72 & Aug. 31, 1939 \\
\hline
\end{tabular}

Net changes in water level in 1941, and net changes in water level for the period of record in 26 wells in Meade County

\begin{tabular}{|c|c|c|c|}
\hline Well & $\begin{array}{l}\text { Difference } \\
\text { between highest } \\
\text { and lowest } \\
\text { water levels } \\
\text { (feet) }\end{array}$ & $\begin{array}{l}\text { Net rise (t) } \\
\text { or net decline } \\
(-) \text { in } 1941 \\
\text { (feet) }\end{array}$ & $\begin{array}{l}\text { Net rise }(+) \\
\text { or net deciline } \\
(-) \text { for period } \\
\text { of record } \\
\text { (feet) }\end{array}$ \\
\hline $\begin{array}{r}2 \\
3 \\
10 \\
11 \\
16 \\
23 \\
27 \\
33 \\
34 \\
36 \\
37 \\
40 \\
41 \\
42 \\
45 \\
47 \\
55 \\
57 \\
61 \\
62 \\
73 \\
76 \\
77 \\
88 \\
101 \\
234\end{array}$ & $\begin{array}{l}1.80 \\
1.41 \\
5.81 \\
4.16 \\
3.67 \\
1.94 \\
.55 \\
.34 \\
3.84 \\
2.39 \\
7.41 \\
2.71 \\
.49 \\
.59 \\
1.04 \\
1.29 \\
1.05 \\
3.03 \\
.42 \\
1.00 \\
1.37 \\
9.50 \\
.72 \\
2.33 \\
1.09 \\
1.71\end{array}$ & $\begin{array}{l}+0.88 \\
+\quad .49 \\
+.44 \\
+.71 \\
+1.96 \\
+1.65 \\
+.22 \\
+.17 \\
-.07 \\
-2.57 \\
+.30 \\
-.19 \\
-.01 \\
-.25 \\
+.70 \\
-.56 \\
+.18 \\
-.08 \\
+.15 \\
+.49 \\
+.39 \\
+3.67 \\
-.18 \\
+.73 \\
-.60 \\
+.64\end{array}$ & $\begin{array}{l}+1.02 \\
-.22 \\
+4.50 \\
-.59 \\
-1.22 \\
+.84 \\
+.19 \\
+.15 \\
+.05 \\
-.83 \\
-.75 \\
+.15 \\
-.13 \\
-.10 \\
+.33 \\
+.73 \\
+.10 \\
-2.70 \\
+.20 \\
+.95 \\
+.29 \\
-2.97 \\
-.18 \\
+1.76 \\
-.225 \\
+1.63\end{array}$ \\
\hline
\end{tabular}

Water-level measurements

2. William A. Ellson. NW $\frac{2}{4} \mathrm{SW} \frac{\frac{2}{4}}{4} \mathrm{sec} .5$, T. 30 S., R. 26 W. Water level, in feet below measuring point, 1941

\begin{tabular}{|c|c|c|c|c|c|c|c|}
\hline Date & $\begin{array}{l}\text { Water } \\
\text { level }\end{array}$ & Date & $\begin{array}{l}\text { Water } \\
\text { level }\end{array}$ & Date & $\begin{array}{l}\text { Water } \\
\text { level }\end{array}$ & Date & $\begin{array}{l}\text { Water } \\
\text { level }\end{array}$ \\
\hline $\begin{array}{ll}\text { Jan. } 21 \\
\text { Mar. } 17 \\
\text { Apr. } 21\end{array}$ & $\begin{array}{l}21.26 \\
21.18 \\
21.30\end{array}$ & $\begin{array}{ll}\text { May } & 16 \\
\text { June } & 11 \\
\text { July } & 15\end{array}$ & $\begin{array}{l}21.29 \\
21.13 \\
20.98\end{array}$ & $\begin{array}{l}\text { Aug. } 7 \\
\text { Sept. } 10 \\
\text { oct. } 9\end{array}$ & $\begin{array}{l}20.87 \\
20.67 \\
20.66\end{array}$ & $\begin{array}{ll}\text { Nov. } & 7 \\
\text { Dec. } & 3\end{array}$ & $\begin{array}{l}20.48 \\
20.38\end{array}$ \\
\hline
\end{tabular}

3. H. L. Salmon. NW $\frac{1}{4} S E \frac{1}{4}$ sec. 4, T. 30 S., R. 27 W.

Water level, in feet below measuring point, 1941

\begin{tabular}{ll|lr|rr|rrr}
\hline Jan. 21 & 29.98 & May 16 & 29.90 & Aug. 7 & 29.61 & Nov. & 7 & 29.67 \\
Mar. 18 & 29.98 & June 11 & 29.85 & Sept.10 & 29.69 & Dec. & 3 & 29.49 \\
Apr. 22 & 30.05 & July 15 & 29.71 & Oct. 9 & 29.69 & & & \\
\hline
\end{tabular}




\section{Meade County--Continued}

10. Fred Borchers. SW $\frac{1}{4}$ NW $\frac{1}{4}$ sec. 29, T. 33 S., R. 28 W. Water level, in feet below measuring point, 1941

\begin{tabular}{ll|ll|lll|ll}
\hline Date & $\begin{array}{l}\text { Water } \\
\text { level }\end{array}$ & Date & $\begin{array}{l}\text { Water } \\
\text { level }\end{array}$ & Date & $\begin{array}{l}\text { Water } \\
\text { level }\end{array}$ & Date & $\begin{array}{r}\text { Water } \\
\text { level }\end{array}$ \\
\hline Jan. 21 & 15.09 & May 17 & 14.77 & Aug. 6 & $\begin{array}{l}14.81 \\
\text { Nov. }\end{array}$ & Nov. & 14.18 \\
Mar. 14 & 14.84 & June 10 & 14.10 & Sept. 9 & 15.18 & Dec. 3 & 14.65 \\
Apr. 21 & 14.84 & July 16 & 14.74 & Oct. 8 & 15.09 & & & \\
\hline
\end{tabular}

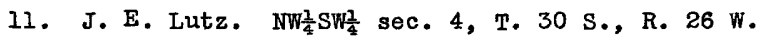
Water level, in feet below measuring point, 1941

\begin{tabular}{|c|c|c|c|c|c|c|c|}
\hline $\begin{array}{l}\text { Jan. } 21 \\
\text { Mar. } 17 \\
\text { Apr. } 21\end{array}$ & $\begin{array}{l}12.70 \\
12.68 \\
12.73\end{array}$ & $\begin{array}{l}\text { May I } \\
\text { June I } \\
\text { July }\end{array}$ & $\begin{array}{l}16 \\
11 \\
15\end{array}$ & $\begin{array}{l}12.82 \\
12.60 \\
12.55\end{array}$ & $\begin{array}{l}\text { Aug. } 6 \\
\text { Sept. } 10 \\
\text { oct. } 9\end{array}$ & $\begin{array}{l}12.52 \\
12.39 \\
12.35\end{array}$ & $\begin{array}{ll}\text { Nov. } & 7 \\
\text { Dec. } & 3\end{array}$ \\
\hline
\end{tabular}

16. B. A. Cordes. SW $\frac{1}{4}$ SW $\frac{1}{4}$ sec. 25, T. 33 S., R. 29 W. Water level, in feet below measuring point, 1941

\begin{tabular}{ll|lr|lrr|lll}
\hline Mar. 14 & 16.57 & June 10 & 13.23 & Sept. 9 & 14.20 & Nov. 6 & 14.58 \\
Apr. 21 & 16.47 & July 16 & 13.99 & Oct. 8 & 14.64 & Dec. & 3 & 14.61 \\
May 17 & 16.49 & Aug. & 6 & 13.42 & & & & & \\
\hline
\end{tabular}

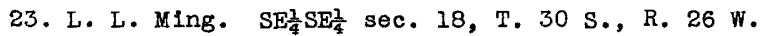
Water level, in feet below measuring point, 1941

\begin{tabular}{ll|lll|ll|lll}
\hline Jan. 21 & 12.32 & May 16 & 11.08 & Aug. 6 & 10.38 & Nov. & 7 & 10.63 \\
Mar. 17 & 11.25 & June 11 & 10.57 & Sept.10 & 10.86 & Dec. & 3 & 10.67 \\
Apr. 21 & 11.17 & July 15 & 10.56 & Oct. 9 & 10.80 & & & \\
\hline
\end{tabular}

27. Ira C. Rees. SW ${ }_{4}^{2} W_{\frac{2}{4}}^{2}$ sec. 9, T. 30 S., R. 26 W. Water level, in feet below measuring point, 1941

\begin{tabular}{ll|ll|lll|lll}
\hline Jan. 21 & 20.49 & May 16 & 20.48 & Aug. 6 & 20.40 & 0ct. 9 & 20.31 \\
Mar. 17 & 20.46 & June 11 & 20.51 & Sept.10 & 20.38 & Nov. & 7 & 20.27 \\
Apr. 21 & 20.45 & July 15 & 20.47 & & & & & \\
\hline
\end{tabular}

33. H. L. Woodruff. $\mathrm{NE}_{\frac{1}{4}}^{\frac{1}{2}} \mathrm{NW}_{4}^{\frac{1}{4}}$ sec. $34, \mathrm{~T}, 33 \mathrm{~S},, \mathrm{R}, 26 \mathrm{~W}$. Water level, in feet below measuring point, 1941

\begin{tabular}{lrl|lr|lrr|lrr}
\hline Mar. 18 & 38.31 & July 15 & 38.25 & Sept. 9 & 38.44 & Nov. & 6 & 38.39 \\
May & 16 & 38.48 & Aug. & 6 & 38.19 & Oct. 8 & 38.41 & Dec. & 3 & 38.14 \\
June 10 & 38.32 & & & & & & & & \\
\hline
\end{tabular}

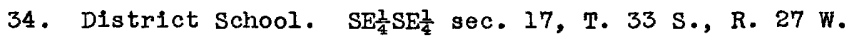
Water level, in feet below measuring point, 1941

\begin{tabular}{ll|lll|lll|lll}
\hline Jan. 21 & 147.36 & May & 16 & 147.81 & Aug. 6 & 147.65 & Nov. & 6 & 147.63 \\
Mar. 18 & 147.70 & June 10 & 147.62 & Sept. 9 & 147.96 & Dec. & 3 & 147.43 \\
Apr. 21 & 148.01 & July 15 & 147.71 & Oct. & 8 & 147.66 & & & \\
\hline
\end{tabular}

36. Tony steinke. NW/ $\mathrm{NE}_{\frac{1}{4}}^{\frac{1}{4}}$ sec. $24, T .32$ S., R. 27 W.

Water level, in feet below measuring point, 1941

\begin{tabular}{ll|ll|lll|lll}
\hline Jan. 21 & 157.07 & Apr. 21 & 159.12 & Oct. & 8 & 160.05 & Dec. & 3 & 159.64 \\
Mar. 18 & 158.27 & May 16 & 160.18 & Nov. & 6 & 160.19 & & & \\
\hline
\end{tabular}

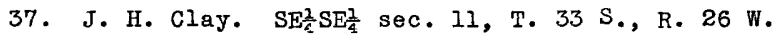

Water level, in feet below measuring point, 1941

\begin{tabular}{ll|ll|ll|lll}
\hline Jan. 21 & 35.36 & Apr. 21 & 35.13 & June 10 & 34.82 & Dec. & 6 & 35.06 \\
Mar. 18 & 35.10 & May & 16 & 34.94 & July & 15 & 34.93 & \\
\hline
\end{tabular}

40. J. A. and D. F. Collingwood. $\mathrm{SE}_{\frac{1}{4}} \mathrm{SE}_{\frac{1}{4}}^{\frac{1}{4}}$ sec. 30, T. 31 S., R. 29 W. Water level, in feet below measuring point, 1941

\begin{tabular}{ll|ll|lll|lll}
\hline Jan. 21 & 130.45 & May 17 & 130.82 & Aug. 6 & 130.70 & Nov. & 6 & 130.80 \\
Mar. 18 & 130.63 & June 10 & 130.82 & Sept. 9 & 131.04 & Dec. & 3 & 130.64 \\
Apr. 22 & 130.77 & July 16 & 130.73 & Oct. & 8 & 130.85 & & & \\
\hline
\end{tabular}


Meade County--Continued

41. D. L. Shranner. NWJ1 $\mathrm{NW}_{\frac{1}{4}}$ sec. 20, T. $30 \mathrm{~S} ., \mathrm{R} .30 \mathrm{~W}$. Water level, in feet below measuring point, 1941

\begin{tabular}{lc|lrr|lr|lrr}
\hline Date & $\begin{array}{l}\text { Water } \\
\text { level }\end{array}$ & Date & $\begin{array}{r}\text { Water } \\
\text { level }\end{array}$ & Date & $\begin{array}{r}\text { Water } \\
\text { level }\end{array}$ & Date & $\begin{array}{r}\text { Water } \\
\text { level }\end{array}$ \\
\hline Jan. 21 & 158.24 & May & 17 & 158.18 & Sept.9 & 158.28 & Nov. & 6 & 158.19 \\
Mar. 18 & 158.22 & June 10 & 158.24 & Oct. 8 & 158.32 & Dec. 3 & 158.25 \\
Apr. 22 & 158.25 & July 16 & 158.15 & & & & & \\
\hline
\end{tabular}

42. H. Jenkinson. SW $\frac{1}{4} \mathrm{SW} / \frac{1}{4} \mathrm{sec} .23$, T. $30 \mathrm{~S} ., \mathrm{R} .29 \mathrm{~W}$.

Water level, in feet below measuring point, 1941

\begin{tabular}{ll|lll|lll|lll}
\hline Jan. 21 & 133.28 & May & 17 & 133.48 & Aug. 6 & 133.48 & Nov. & 6 & 133.45 \\
Mar. 18 & 133.38 & June 10 & 133.55 & Sept. 9 & 133.72 & Dec. & 3 & 133.53 \\
Apr. 22 & 133.45 & July & 16 & 133.52 & Oct. 8 & 133.52 & & & \\
\hline
\end{tabular}

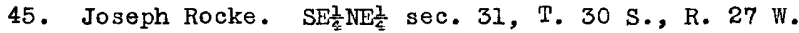

Water level, in feet below measuring point, 1941

\begin{tabular}{ll|ll|lrl|lll}
\hline Jan. 21 & 4.54 & May & 16 & 4.24 & Aug. 7 & 3.97 & Nov. & 7 & 3.87 \\
Mar. 17 & 4.45 & June 11 & 4.13 & Sept.10 & 3.84 & Dec. & 3 & 3.84 \\
Apr. 22 & 4.34 & July & 15 & 3.85 & Oct. 9 & 3.63 & & & \\
\hline
\end{tabular}

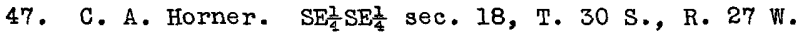
Water level, in feet below measuring point, $194 \mathrm{I}$

\begin{tabular}{lll|ll|lll|lll}
\hline Jan. 21 & 43.10 & July 15 & 44.00 & Oct. & 9 & 43.73 & Dec. 3 & 43.66 \\
June 11 & 43.89 & Sept.10 & 43.80 & Nov. & 7 & 43.69 & & & \\
\hline
\end{tabular}

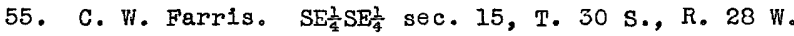
Water level, in feet below measuring point, 1941

\begin{tabular}{ll|lr|lr|lrrr}
\hline Jan. 21 & 86.45 & May 17 & 86.50 & Aug. 9 & 86.32 & 0ct. & 6 & 86.26 \\
Mar. 17 & 86.46 & June 16 & 86.44 & Sept. 8 & 86.28 & Dec. & 3 & 86.27 \\
Apr. 22 & 86.45 & July & 6 & 86.36 & & & & & \\
\hline
\end{tabular}

57. Plains State Bank. $\mathrm{SW}_{4}^{\frac{1}{4}} \mathrm{SE}_{\frac{1}{4}}$ sec. 18, T. 33 S., R. $30 \mathrm{~W}$.

Water level, in feet below measuring point, 1941

\begin{tabular}{ll|lll|lll|lll}
\hline Jan. 7 & 172.60 & May & 17 & 172.60 & Aug. 6 & 172.54 & Nov. 6 & 172.69 \\
Mar. 14 & 172.40 & June & 10 & 172.73 & Sept. 9 & 172.97 & Dec. 3 & 172.68 \\
Apr. 21 & 172.87 & July & 16 & 172.69 & Oct. & 8 & 172.72 & & & \\
\hline
\end{tabular}

61. John Meyer. NW $\frac{1}{4} N W_{4}^{\frac{1}{4}}$ sec. 26, T. 31 S., R. 27 W.

Water level, in feet below measuring point, 1941

\begin{tabular}{ll|ll|lll|lll}
\hline Jan. 21 & 61.11 & May & 16 & 61.05 & Aug. 6 & 61.02 & Nov. & 6 & 61.03 \\
Mar. 17 & 61.07 & June 10 & 61.02 & Sept. 9 & 60.95 & Dec. & 3 & 60.96 \\
Apr. 21 & 61.08 & July 15 & 61.06 & Oct. 8 & 60.97 & & & \\
\hline
\end{tabular}

62. H. L. Salmon. NE $\frac{1}{4} \mathrm{NE} \frac{1}{4}$ sec. 7, T. 31 S., R. $26 \mathrm{~W}$.

Water level, in feet below measuring point, 1941

\begin{tabular}{ll|ll|lll|lll}
\hline $\operatorname{Jan} .21$ & 26.15 & May & 16 & 26.27 & Aug. 6 & 26.12 & Nov. & 6 & 25.87 \\
Mar. 17 & 26.17 & June 10 & 26.19 & Sept. 9 & 26.03 & Dec. & 3 & 25.66 \\
Apr. 21 & 26.20 & July 15 & 26.28 & Oct. 8 & 26.19 & & & \\
\hline
\end{tabular}

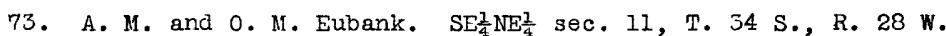

Water levi, in feet below measuring point, 1941

\begin{tabular}{ll|lr|rr|lll}
\hline Jan. 21 & 33.59 & Apr. 21 & 34.08 & JuIy 16 & 33.89 & Nov. & 6 & 33.42 \\
Mar. 14 & 33.53 & June 10 & 33.94 & Oct. 9 & 33.48 & Dec. & 3 & 33.20 \\
\hline
\end{tabular}

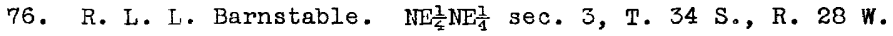
Water level, in feet below measuring point, 1941

\begin{tabular}{lll|lr|rr|lrr}
\hline Apr. 21 & 33.11 & July 16 & 36.48 & Sept. 9 & 36.95 & Nov. & 37.12 \\
May 17 & 38.20 & Aug. 6 & 36.25 & Oct. 8 & 37.49 & Dec. & 3 & 34.44 \\
June 10 & 37.11 & & & & & & & &
\end{tabular}


Meacie County--Continued

77. J. W. Wood. $\mathrm{SE}_{\frac{1}{4}} \mathrm{NE} \frac{\frac{1}{4}}{4} \mathrm{sec} .4, \mathrm{~T} .32 \mathrm{~s} ., \mathrm{R} .28 \mathrm{~W}$.

Water level, in feet below measuring point, 1941

\begin{tabular}{ll|ll|ll|ll|l}
\hline Date & $\begin{array}{l}\text { Water } \\
\text { level }\end{array}$ & Date & $\begin{array}{l}\text { Water } \\
\text { level }\end{array}$ & Date & $\begin{array}{l}\text { Water } \\
\text { level }\end{array}$ & Date & $\begin{array}{r}\text { Water } \\
\text { level }\end{array}$ \\
\hline Jan. 21 & 65.72 & May 16 & 65.69 & Aug. 7 & 65.87 & Nov. 6 & 65.95 \\
Mar. 18 & 65.71 & June 11 & 65.71 & Sept. 9 & 65.89 & Dec. 3 & 65.90 \\
Apr. 22 & 65.82 & July 15 & 65.75 & Oct. 9 & 65.81 & & \\
\hline
\end{tabular}

88. H. V. Gulick. $\mathrm{SE}_{\frac{1}{4}}^{\frac{1}{4}} \mathrm{SE}_{\frac{1}{4}}^{\frac{1}{4}} \mathrm{sec} .14, \mathrm{~T}, 31 \mathrm{~S}, \mathrm{R}, 28 \mathrm{~W}$.

Water level, in feet below measuring point, 1941

\begin{tabular}{ll|l|l|l|lr|lrr}
\hline Jan. 21 & 43.59 & July 15 & 43.07 & Sept.10 & 43.79 & Nov. & 7 & 42.64 \\
Mar. 18 & 43.14 & Aug. & 7 & 43.38 & oct. 9 & 43.62 & Dec. & 3 & 42.68 \\
May 16 & 42.98 & & & & & & & & \\
\hline
\end{tabular}

101. West and Higenbotham. $\mathrm{NE}_{4}^{\frac{1}{4}} \mathrm{NE} \frac{1}{4} \mathrm{sec} .29, \mathrm{~T} .34 \mathrm{~S} ., \mathrm{R} .26 \mathrm{~W}$.

Water level, in feet below measuring point, 1941

\begin{tabular}{ll|ll|lll|lll}
\hline Jan. 21 & 87.57 & May & 16 & 88.10 & Aug. 6 & 87.96 & Nov. & 6 & 88.12 \\
Mar. 18 & 87.97 & June 10 & 87.88 & Sept. & 8 & 88.26 & Dec. & 3 & 88.17 \\
Apr. 21 & 88.29 & July 15 & 88.03 & Oct. & 8 & 88.16 & & & \\
\hline
\end{tabular}

234. Christopher Sobba. NW $\mathrm{NE}_{4}^{\frac{1}{4}}$ sec. 23, T. 30 S., R. $27 \mathrm{~W}$.

Lowest daily water level, in feet below moasuring point, 1941

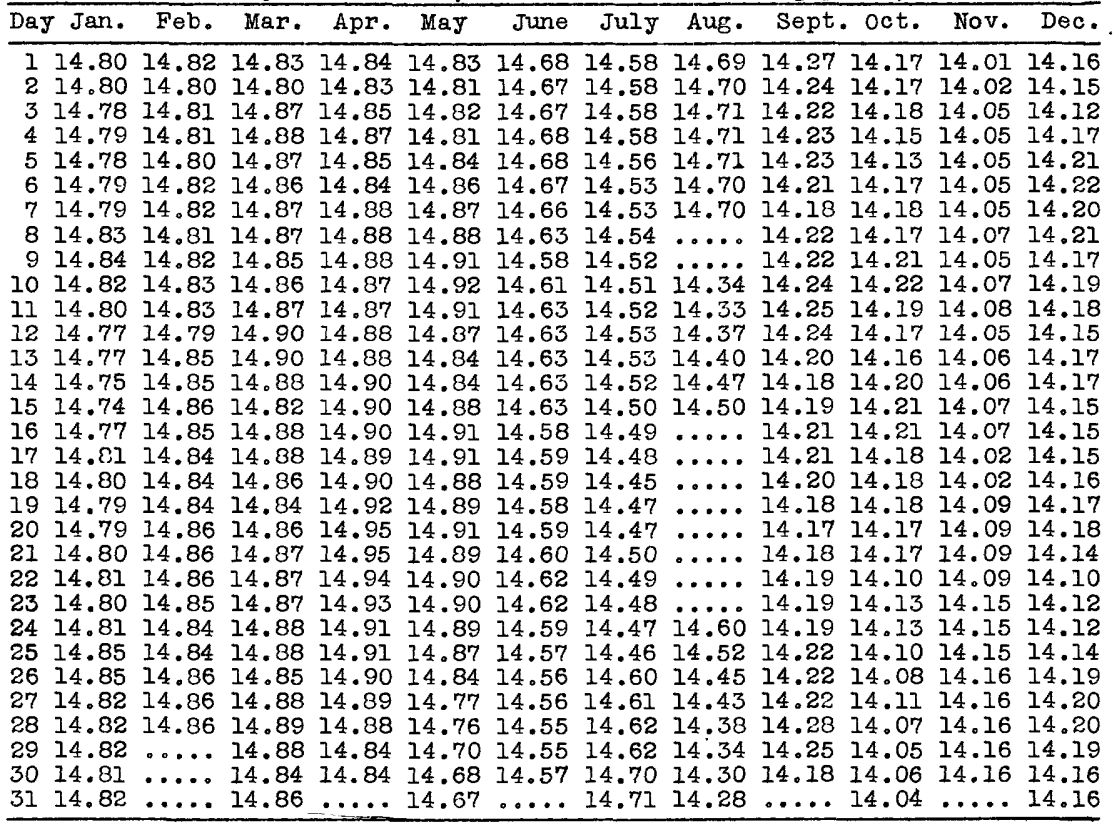

304. A. W. Adams. NW $\mathrm{NWE}_{4}^{\frac{1}{4}}$ sec. 27, T. 34 S., R. 30 W. Water levels, in feet below measuring point, 1941: Mar. 14, 219.91; July 16, 220.34; oct. 8, 220.37 . 
MORTON COUNTY

By T. G. McLaughlin

Highest and lowest water levels, in feet below measuring point, in 6 wells in Morton County, 1941

\begin{tabular}{|c|c|c|c|c|c|c|c|}
\hline Well & $\begin{array}{l}\text { Length } \\
\text { of record } \\
\text { (years) }\end{array}$ & $\begin{array}{c}\text { Highest } \\
\text { recorded } \\
\text { water level } \\
\text { (feet) }\end{array}$ & & Date & $\begin{array}{c}\text { Lowest } \\
\text { recorded } \\
\text { water level } \\
\text { (feet) }\end{array}$ & & Date \\
\hline $\begin{array}{l}22 \\
54 \\
65 \\
93\end{array}$ & $\begin{array}{l}2.5 \\
2.5 \\
2.5 \\
2.5\end{array}$ & $\begin{array}{r}74.33 \\
73.48 \\
53.09 \\
159.31\end{array}$ & $\begin{array}{l}\text { May } \\
\text { July } \\
\text { Oct. } \\
\text { Nov. }\end{array}$ & $\begin{array}{l}12,1941 \\
18,1941 \\
28,1941 \\
18,1941\end{array}$ & $\begin{array}{r}76.65 \\
76.90 \\
54.15 \\
160.14\end{array}$ & \multirow{3}{*}{\multicolumn{2}{|c|}{$\begin{array}{r}\text { Jan. } 6,1941 \\
\text { July } 25,1939 \\
\text { Mar. } 13,1941 \\
\text { Oct. 27, } 1939 \\
\text { (Dec. 16, } 1939 \\
\text { (Apr. 23, } 1940 \\
\text { (Nov. 13, } 1940 \\
\text { July 26, } 1939\end{array}$}} \\
\hline 114 & 2.5 & 226.62 & Aug. & 25,1939 & 226.89 & & \\
\hline 117 & 2.5 & 166.87 & Dec. & 16,1941 & 167.39 & & \\
\hline
\end{tabular}

Net changes in water level in 1941, and net changes in water level for the period of record in 6 wells in Morton County

\begin{tabular}{cccc}
\hline Wel1 & $\begin{array}{c}\text { Difference } \\
\text { between highest } \\
\text { and lowest } \\
\text { water levels } \\
\text { (feet) }\end{array}$ & $\begin{array}{c}\text { Net rise (t) } \\
\text { or net decline } \\
(-) \text { in 1941 } \\
\text { (feet) }\end{array}$ & $\begin{array}{c}\text { Net rise }(+) \\
\text { or net decline } \\
\text { for period } \\
\text { of record } \\
\text { (feet) }\end{array}$ \\
\hline 22 & 2.32 & +1.82 & -0.18 \\
54 & 3.42 & +1.87 & +2.38 \\
65 & 1.06 & +.87 & +.43 \\
93 & .83 & +.30 & +.54 \\
114 & .27 & +.05 & +.07 \\
117 & .52 & +.10 & +.52 \\
\hline
\end{tabular}

Well descriptions and water-level measurements

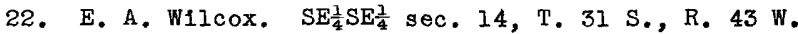
Water level, in feet below measuring point, 1941

\begin{tabular}{lll|ll|ll|ll}
\hline Date & $\begin{array}{l}\text { Water } \\
\text { level }\end{array}$ & Date & $\begin{array}{l}\text { Water } \\
\text { level }\end{array}$ & Date & $\begin{array}{l}\text { Water } \\
\text { level }\end{array}$ & Date & $\begin{array}{l}\text { Water } \\
\text { level }\end{array}$ \\
\hline Jan. 6 & 76.65 & Apr. 18 & 74.64 & July 18 & 74.61 & Oct. 28 & 74.35 \\
Feb. 6 & 74.52 & May 12 & 74.33 & Aug. 14 & 74.58 & Nov. 18 & 74.67 \\
Mar. 13 & 74.59 & June 17 & 74.56 & Sept.18 & 74.43 & Dec. 16 & 74.83 \\
\hline
\end{tabular}

54. V. W. Dickinson. SW $\frac{1}{4}$ SW $\frac{1}{4}$ sec. 13, T. 33 S., R. 40 W. Water level, in feet below measuring point, 1941

\begin{tabular}{lrl|ll|ll|lll}
\hline Jan. & 7 & 76.39 & Apr. 18 & 76.36 & July 18 & 73.48 & Oct. 28 & 74.24 \\
Feb. 5 & 76.33 & May 12 & 76.32 & Aug. 14 & 73.78 & Nov. 18 & 73.89 \\
Mar. 13 & 76.43 & June 17 & 74.10 & Sept.18 & 73.56 & Dec. 16 & 74.52 \\
\hline
\end{tabular}

65. John Hentschel. $S E \frac{1}{4} N E \frac{1}{4}$ sec. 8, T. 33 S., R. 42 W.

Water level, in feet below measuring point, 1941

\begin{tabular}{lrr|ll|ll|lll}
\hline Jan. & 8 & 54.03 & Apr. 18 & 53.16 & July 18 & 53.51 & Oct. 28 & 53.09 \\
Feb. 6 & 54.07 & May 12 & 54.00 & Aug. 14 & 53.29 & Nov. 18 & 53.10 \\
Mar. 13 & 54.15 & June 17 & 53.79 & Sept.18 & 53.28 & Dec. 16 & 53.16 \\
\hline
\end{tabular}

93. Ira Webb. NE: $\frac{1}{4} \mathrm{SE} \frac{1}{4}$ sec. 28, T. $34 \mathrm{~S},, \mathrm{R}, 41$ W.

Water level, in feet below measuring point, 1941

\begin{tabular}{lrl|ll|ll|lll}
\hline Jan. & 7 & 159.70 & Apr. 18 & 159.55 & July 18 & 159.49 & Oct. 28 & 159.48 \\
Feb. 5 & $\mathbf{1 5 9 . 3 8}$ & May 12 & 159.48 & Aug. 14 & 159.51 & Nov. 18 & 159.31 \\
Mar. 13 & 159.48 & June 17 & 159.58 & Sept.18 & 159.52 & Dec. 16 & 159.40 \\
\hline
\end{tabular}


Morton County--Continued

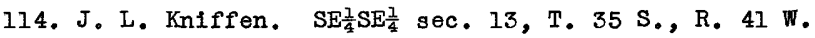
Water level, in feet below measuring point, 1941

\begin{tabular}{lrr|lr|ll|lr}
\hline Date & $\begin{array}{l}\text { Water } \\
\text { level }\end{array}$ & Date & $\begin{array}{l}\text { Water } \\
\text { level }\end{array}$ & Date & $\begin{array}{l}\text { Water } \\
\text { level }\end{array}$ & Date & $\begin{array}{r}\text { Water } \\
\text { level }\end{array}$ \\
\hline Jan. 7 & 226.86 & Apr. 18 & 226.86 & July 18 & 226.81 & Oct. 28 & 226.83 \\
Feb. 5 & 226.72 & May 12 & 226.69 & Aug. 14 & 226.87 & Nov. 18 & 226.87 \\
Mar. 13 & 226.74 & June 17 & 226.81 & Sept.18 & 226.85 & Dec. 16 & 226.81 \\
\hline
\end{tabular}

117. W. C. Washburn. SW $\frac{1}{4} \mathrm{SW} \frac{1}{4}$ sec. 4, T. 35 S., R. 42 W. Water level, in feet bel ow measuring point, 1941

\begin{tabular}{lll|ll|ll|ll}
\hline Jan. & 6 & 166.97 & Apr. 18 & 166.95 & Sept.18 & 167.25 & Nov. 18 & 166.90 \\
Feb. 6 & 166.98 & June 17 & 166.88 & Oct. 28 & 166.88 & Dec. 16 & 166.87 \\
\hline
\end{tabular}

\section{NESS COUNTY}

By H. A. Waite

Highest and lowest water levels, in feet below measuring point, in 2 wells in Ness County, 1941

\begin{tabular}{|c|c|c|c|c|c|}
\hline Well & $\begin{array}{l}\text { Length } \\
\text { of record } \\
\text { (years) }\end{array}$ & $\begin{array}{c}\text { Highest } \\
\text { recorded } \\
\text { water level } \\
\text { (feet) }\end{array}$ & Date & $\begin{array}{l}\text { Lowest } \\
\text { recorded } \\
\text { water level } \\
\text { (feet) }\end{array}$ & Date \\
\hline $\begin{array}{l}1 \\
2\end{array}$ & $\begin{array}{l}1 \\
1\end{array}$ & $\begin{array}{l}35.16 \\
24.76\end{array}$ & $\begin{array}{l}\text { June } 6,1941 \\
\text { Aug. } \\
2,1941\end{array}$ & $\begin{array}{l}36.91 \\
25.90\end{array}$ & $\begin{array}{l}\text { Aug. 27, } 1940 \\
\text { Sept.20, } 1940\end{array}$ \\
\hline
\end{tabular}

Net changes in water level in 1941, and net changes in water level for the period of record in' 2 wells in Ness County

\begin{tabular}{|c|c|c|c|}
\hline Well & $\begin{array}{c}\text { Difference } \\
\text { between highest } \\
\text { and lowest } \\
\text { water levels } \\
\text { (feet) }\end{array}$ & $\begin{array}{l}\text { Net rise (+) } \\
\text { or net decline } \\
(-) \text { in } 1941 \\
(\text { feet })\end{array}$ & $\begin{array}{l}\text { Net rise (+) } \\
\text { or net decline } \\
(-) \text { for period } \\
\text { of record } \\
\text { (feet) }\end{array}$ \\
\hline $\begin{array}{l}1 \\
2\end{array}$ & $\begin{array}{l}1.75 \\
1.14\end{array}$ & $\begin{array}{r}-0.17 \\
+\quad .46\end{array}$ & $\begin{array}{l}+0.69 \\
+\quad .79\end{array}$ \\
\hline
\end{tabular}

Well descriptions and water-level measurements

1. J. E. Flcken. $\mathrm{SE} \frac{\frac{1}{4}}{4} \mathrm{SW} \frac{1}{4}$ sec. 32, T. $20 \mathrm{~S},, \mathrm{R}, 23 \mathrm{~W}$.

Water level, in feet below measuring point, 1941

\begin{tabular}{|c|c|c|c|c|c|c|c|}
\hline Date & $\begin{array}{l}\text { Water } \\
\text { level }\end{array}$ & Date & $\begin{array}{l}\text { Water } \\
\text { level }\end{array}$ & Date & $\begin{array}{l}\text { Water } \\
\text { level }\end{array}$ & Date & $\begin{array}{l}\text { Water } \\
\text { level }\end{array}$ \\
\hline $\begin{array}{ll}\text { Jan. } 20 \\
\text { Mar. } 17 \\
\text { Apr. } 26\end{array}$ & $\begin{array}{l}36.05 \\
36.03 \\
36.02\end{array}$ & $\begin{array}{lr}\text { May } & 10 \\
\text { June } & 6 \\
\text { July } & 12\end{array}$ & $\begin{array}{l}35.30 \\
35.16 \\
35.93\end{array}$ & $\begin{array}{ll}\text { Aug. } & 2 \\
\text { Sept. } & 4 \\
\text { oct. } & 2\end{array}$ & $\begin{array}{l}35.91 \\
36.52 \\
36.40\end{array}$ & $\begin{array}{l}\text { Nov. } 13 \\
\text { Dec. } 4\end{array}$ & $\begin{array}{l}36.53 \\
36.22\end{array}$ \\
\hline
\end{tabular}

2. C. L. Whitley. SW cor. sec. 20 , T. 20 S., R. 22 W.

Wacer level, in feet below measuring point, 1941

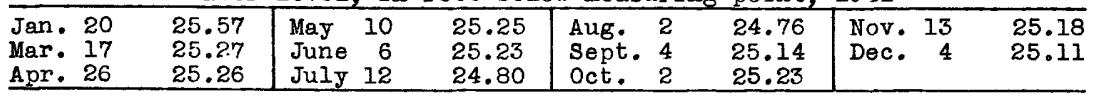


PAWNEE COUNTY

By H. A. Waite

Highest and lowest water levels, in feet below measuring point, in 3 wells in Pawnee County, 1941

\begin{tabular}{ccccccc}
\hline We11 & $\begin{array}{c}\text { Length } \\
\text { of record } \\
\text { (years) }\end{array}$ & $\begin{array}{c}\text { Highest } \\
\text { recorded } \\
\text { water 1evel } \\
\text { (feet) }\end{array}$ & Date & $\begin{array}{c}\text { Lowest } \\
\text { recorded } \\
\text { water level } \\
\text { (feet) }\end{array}$ & Date \\
\hline 6 & 1 & 23.56 & May 10,1941 & 25.02 & Nov. 28, 1940 \\
7 & 1 & 26.13 & Dec. 4, 1941 & 28.00 & Nov. 29, 1940 \\
8 & 1 & 15.83 & (July 12, 1941 & 19.82 & Sept.20, 1940 \\
\hline
\end{tabular}

Net changes in water level in 1941, and net changes in water level for the period of record in 3 wells in Pawnee County

\begin{tabular}{|c|c|c|c|}
\hline We11 & $\begin{array}{c}\text { Difference } \\
\text { between highest } \\
\text { and lowest } \\
\text { water levels } \\
\text { (feet) }\end{array}$ & $\begin{array}{l}\text { Net rise (+) } \\
\text { or net decline } \\
(-) \text { in } 1941 \\
(\text { feet })\end{array}$ & $\begin{array}{l}\text { Net rise (+) } \\
\text { or net decline } \\
(-) \text { for period } \\
\text { of record } \\
\text { (feet) }\end{array}$ \\
\hline $\begin{array}{l}6 \\
7 \\
8\end{array}$ & $\begin{array}{l}1.46 \\
1.87 \\
3.99\end{array}$ & $\begin{array}{l}+0.59 \\
+1.81 \\
+\quad .79\end{array}$ & $\begin{array}{l}+0.44 \\
+1.87 \\
+3.46\end{array}$ \\
\hline
\end{tabular}

Well descriptions and water-level measurements

6. Frank Elmore. SW cor. sec. 27, T. 21 S., R. 19 W.

Water level, in feet below measuring point, 1941

\begin{tabular}{|c|c|c|c|c|c|c|c|}
\hline Date & $\begin{array}{l}\text { Water } \\
\text { level }\end{array}$ & Date & $\begin{array}{l}\text { Water } \\
\text { level }\end{array}$ & Date & $\begin{array}{l}\text { Water } \\
\text { level }\end{array}$ & Date & $\begin{array}{l}\text { Water } \\
\text { level }\end{array}$ \\
\hline $\begin{array}{ll}\text { Jan. } & 20 \\
\text { Mar. } & 17 \\
\text { Apr. } 26\end{array}$ & $\begin{array}{l}24.74 \\
24.69 \\
24.68\end{array}$ & $\begin{array}{lr}\text { May } & 10 \\
\text { June } & 6 \\
\text { July } & 12\end{array}$ & $\begin{array}{l}23.56 \\
24.47 \\
23.84\end{array}$ & $\begin{array}{ll}\text { Aug. } & 2 \\
\text { Sept. } & 4 \\
\text { Oct. } & 2\end{array}$ & $\begin{array}{l}24.00 \\
24.47 \\
24.51\end{array}$ & $\begin{array}{lr}\text { Nov. } & 13 \\
\text { Dec. } & 4\end{array}$ & $\begin{array}{l}24.20 \\
24.15\end{array}$ \\
\hline
\end{tabular}

7. Ralph Lupfer. $\mathrm{NE} \frac{1}{4} \mathrm{NE} \frac{1}{4}$ sec. 18, T. $22 \mathrm{~S}, \mathrm{R}, 17 \mathrm{~W}$.

Water level, in feet below measuring point, 1941

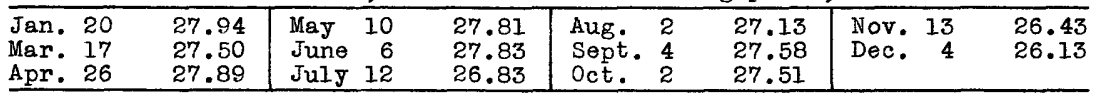

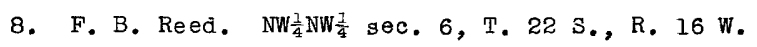

Water level, in feet below measuring point, 1941

\begin{tabular}{ll|lr|lrr|rrr}
\hline Jan. 20 & 16.62 & May & 10 & 16.00 & Sept. 4 & 16.51 & Nov. 13 & 16.03 \\
Mar. 17 & 16.38 & June & 6 & 16.36 & Oct. 2 & 16.38 & Dec. 4 & 15.83 \\
Apr. 26 & 16.20 & July & 12 & 15.83 & & & & & \\
\hline
\end{tabular}


RUSSELL COUNTY

By J. C. Frye

An investigation of the ground-water resources of western and southern Russell County and northeastern Ellis County, Kans., was started in 1941 as described in the introduction to the chapter on Ellis County.

We1l descriptions and water-level measurements

8. F. C. and A. Ptacek. NW $\frac{1}{4}$ NW $\frac{1}{4}$ sec. 16, T. 15 S., R. 12 W. Unused drilled stock well, diameter 6 inches, depth 220.1 feet. Water is obtained from a sandstone of the Dakota formation. Measuring point, top edge of 6Inch galvanized-1ron casing, east slde, 0.3 foot above land surface. No pump in wel?. Water levels, in feet below measuring point, 1941: Aug. 8, 97.00 ; Oct. 3, 94.20; Nov. 7, 118.22; Dec. 1, 117.86.

27. G. M. and A. C. Rogg. NW $\frac{1}{4}$ NW $\frac{1}{4}$ sec. 9, T. 15 S., R. 13 W. Drilled stock well, diameter 6 inches, depth 193.8 feet. Water is obtained from a sandstone of the Dakota formation. Measuring polnt, top of 6-inch $\mathrm{ga}^{7}-$ vanized-iron casing, east side, 0.8 foot above land surface. Equip? witn ift pump and windmill. Water levels, in feet bel ow measuring point, 194l: Aug. 14, 168.10; Oct. 2, 166.95; Nov. 7, 166.15; Dec. 1, 167.20.

45. Jacob Flegler. NW $\frac{1}{4} \mathrm{NW} \frac{1}{4} \mathrm{sec}$. 11, T. $15 \mathrm{~S}, \mathrm{R}, 14 \mathrm{~W}$. Dug stock well, diameter 32 inches, depth 27.2 feet. Water is obtained from alluvium. Measuring point, top of rock curbing, east side, 0.7 foot above land surface. Equipped with hand-operated lift pump. Water levels, in feet below measurIng point, 1941: Aug. 20, 24.98; Oct. 2, 23.35; Nov. 7, 22.25; Dec. 1, 21.77 .

49. Benjamin Boxberger. NW $\frac{1}{4}$ NW $\frac{1}{4}$ sec. 36, T. $15 \mathrm{~S} ., \mathrm{R} .15 \mathrm{~W}$. Drilled domest1c and stock well, dlameter 6 inches, depth 175.9 feet. Water $1 \mathrm{~s}$ obtained from a sandstone of the Dakota formation. Measuring point, top of 6-inch galvanized-1ron casing, west side, 0.5 foot above land surface. Equipped with lift pump and windmill. Water levels, in feet below measurIng polnt, 1941: Aug. 21, 137.99; Oct. 2, 138.45; Nov. 7, 137.95; Dec. 1, 137.87 .

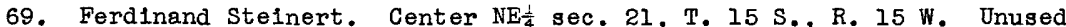
drilled domestic and stock well, diameter 6 inches, depth 17.1 feet. Water is obtained from Pleistocene terrace deposits. Measuring point, top of 6inch galvanized-1ron casing, west side, 0.2 foot above land surface. Equipped with hand-operated'ilft pump. Water levels, in feet below measuring point, 1941: Aug. 27, 13.79; Oct. 2, 13.29; Nov. 7, 13.18; Dec. 1, 13.54 .

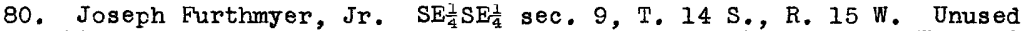
dug domest1c and stock weli, diameter 60 inches, depth 15.1 feet. Water is obtained from Tertiary deposits. Measuring point, top of rock curbing, east side, 0.8 foot above land surface. No pump in weil. Water levels, in feet below measuring point, 1941: Aug. 29, 5.51; 0ct. 2, 6.84; Nov. 7, 5.15; Dec. $1,4.86$.

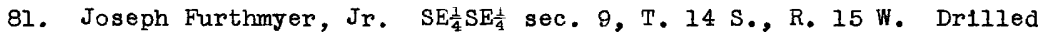
stock well, dlameter 6 lnches, depth 224.1 feet. Water is obtained from a sandstone of the Dakota formation. Measuring point, top of 6-inch galvanized-1rchcasing, west side, 0.3 foot above land surface. Equipped with Iift pump and windmill. Water levels, in feet below measuring point, 1941: Aug. 29, 102.15; 0ct. 2, 128.98; Nov. 7, 131.25; Dec. 1, 127.50 . 
Russell County--Continued

95. George J. Gobleman. SW $\frac{1}{4} \mathrm{NE} \frac{1}{4}$ sec. 28, T. $11 \mathrm{~S}$, , R. $15 \mathrm{~W}$. Unused dug domestic and stock well, diameter 32 inches, depth 12.1 feet. Water is obtained from Carlile shale. Measuring point, top edge of rock curbing, north side, 0.5 foot above land surface. No pump in well. Water levels, in feet below measuring point, 1941: Aug. 30, 9.78; Oct. 3, 8.98; Nov.6, 8.53; Dec. 2, 9.02.

116. George P. Bender. NW $\frac{1}{4} \mathrm{NW} \frac{1}{4}$ sec. 16, T. $13 \mathrm{~S}, \mathrm{R}, 14 \mathrm{~W}$. Unused drilled domestic and stock well, diameter 6 inches, depth 178.9 feet. Water is obtained from a sandstone of the Dakota formation. Measuring point, top edge of 6-inch galvanized-iron casing, west side, 1.0 foot above land surface. No pump in well. Water levels, in feet below measuring point, 1941: Sept. 6, 156.17; Oct. 3, 153.65; Nov. 7, 152.75; Dec. 2, 150.67 .

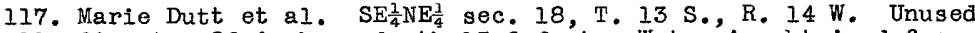
dug well, diameter 26 inches, depth 13.6 feet. Water is obtained from alluvium. Measuring point, top of rock curbing, north side, 0.3 foot above land surface. Equipped with lift pump and windmill. Water levels, in feet below measuring point, 1941: Sept. 8,6.88; Oct. 3, 6.96; Nov. 6, 6.30 ; Dec. 2, 7.22.

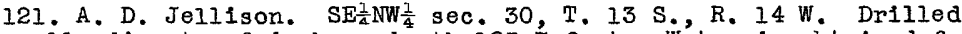
stock well, diameter 6 inches, depth 183.7 feet. Water is obtained from a sandstone of the Dakota formation. Measuring point, top of 6-inch galvanized-iron casing, west side, 0.4 foot below land surface. Equipped with lift pump and windmill. Water levels, in feet below measuring point, 1941: Sept. 8, 163.42; Oct. 2, 162.92; Nov. 7, 163.55; Dec. 2, 164.20.

126. Bertha Dewald. NW $\frac{1}{4}$ SW $\frac{1}{4}$ sec. 19, T. 13 S., R. 13 W. Dug stock well, diameter 30 inches, depth 49.2 feet. Water is obtained from Greenhorn limestone. Measuring point, top edge of rock curbing, south side, 0.2 foot above land surface. Equipped with lift pump and windmill. Water levels, in feet bel ow measuring point, 1941: Sept. 10, 35.32; 0ct. 2 , 34.81 ; Nov. $7,35.05$; Dec. $1,34.70$.

145. Tony Hraik. SE $\frac{1}{4} S W \frac{1}{4}$ sec. 14, T. 14 S., R. 11 W. Drilled stock well, diameter 6 inches, depth 44.2 feet. Water is obtained from Bleistocene terrace deposits. Measuring point, top edge of 6 -inch galvanizedIron casing, east side, 0.4 foot above land surface. Equipped with ift pump and windmill. Water levels, in feet below measuring point, 194l: Sept. 20, 41.46; Oct. 2, 41.67; Nov. 7, 41.30; Dec. 1, 41.69.

146. D. P. Steinle. NW $\frac{1}{4} \mathrm{NE} \frac{1}{4}$ sec. 24, T. $14 \mathrm{~S} ., \mathrm{R}, 12 \mathrm{~W}$. Unused dug well, diameter 28 inches, depth 17.3 feet. Water is obtained from Plelstocene terrace deposits. Measuring point, top of rock curbing, north side, 0.6 foot above land surface. Equipped with hand-operated I1ft pump. Water levels, in feet below measuring point, 1941: Sept. 20, 16.40; 0ct.2, 16.77 ; Nov. $7,16.75$; Dec. 1 , 16.53.

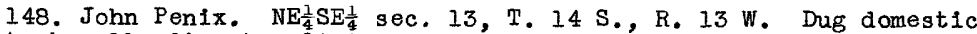
and stock well, diameter 28 inches, depth 11.6 feet. Water $1 \mathrm{~s}$ obtalned from Plelstocene terrace deposits. Measuring point, top of rock curbing, south side, 1.3 feet above land surface. Equipped with lift pump and windmill. Water levels, in feet below measuring point, 194l: Sept. 20, 9.18; oct. 2, 9.22; Nov. 7, 8.74; Dec. 1, 9.15.

149. George Boxberger, Jr. NE $\frac{1}{4} \mathrm{NE} \frac{1}{4}$ sec. 22, T. $14 \mathrm{~S} ., \mathrm{R} .14 \mathrm{~W}$. Unused dug well, dlameter 32 inches, depth 23.1 feet. Water is obtained from Greenhorn limestone. Measuring point, top edge of rock curbing, west side, 1.3 feet above land surface. Equipped with hand-operated lift pump. Water levels, in feet below measuring point, 1941: Sept. 20, 22.16; 0ct. 2, 21.52; Nov. 7, 21.88; Dec. 1, 21.52.

151. D. D. Beisel. SW $\frac{1}{4} \mathrm{SE} \frac{1}{4}$ sec. 10, T. 14 S., R. 12 W. Unused drilled well, diameter 6 inches, depth 183.9 feet. Water is obtalned from a sandstone of the Dakota formation. Measuring point, top of galvanized-iron casing, east side, 0.5 foot below land surface. Equipped with lift pump and windmill. Water levels, in feet below measuring point, 1941: Sept. 22, 170.33; 0ct. 2, 170.63; Nov. 7, 170.47; Dec. 1, 171.02. 


\section{Russell County--Continued}

152. D. D. Beisel. SW $\frac{1}{4} \mathrm{SE} \frac{1}{4} \mathrm{sec}, 10, \mathrm{~T}, 14 \mathrm{~S}, \mathrm{R}, 12 \mathrm{~W}$. Unused dug well, diameter 28 inches, depth 30.8 feet. Water is obtained from Creenhorn limestone. Measuring point, top edge of rock curbing, south side, 0.8 foot above land surface. Equipped with hand-operated iff pump. Water levels, in feet below measuring point, 1941: Sept. 22, 27.25; Oct. 2, 27.12; Nov. 7, 26.94; Dec. 1, 26.92.

154. E. Stielow. SE $\frac{1}{4} \mathrm{SE} \frac{1}{4} \mathrm{sec} .33$, T. 11 S., R. $14 \mathrm{~W}$. Unused dug well, diameter 60 inches, depth 8.9 feet. Water is obtained from alluvium. Measuring point, top edge of rock curbing, west side, 2.5 feet above land surface. No pump in well. Water levels, in feet below measurIng point, 1941: Oct. $3,7.60$; Nov. $6,7.13$; Dec. 2, 7.95 .

\section{SCOTT COUNTY}

By H. A. Waite

Highest and lowest water levels, in feet below measuring point, in 32 wells in Scott County, 1941

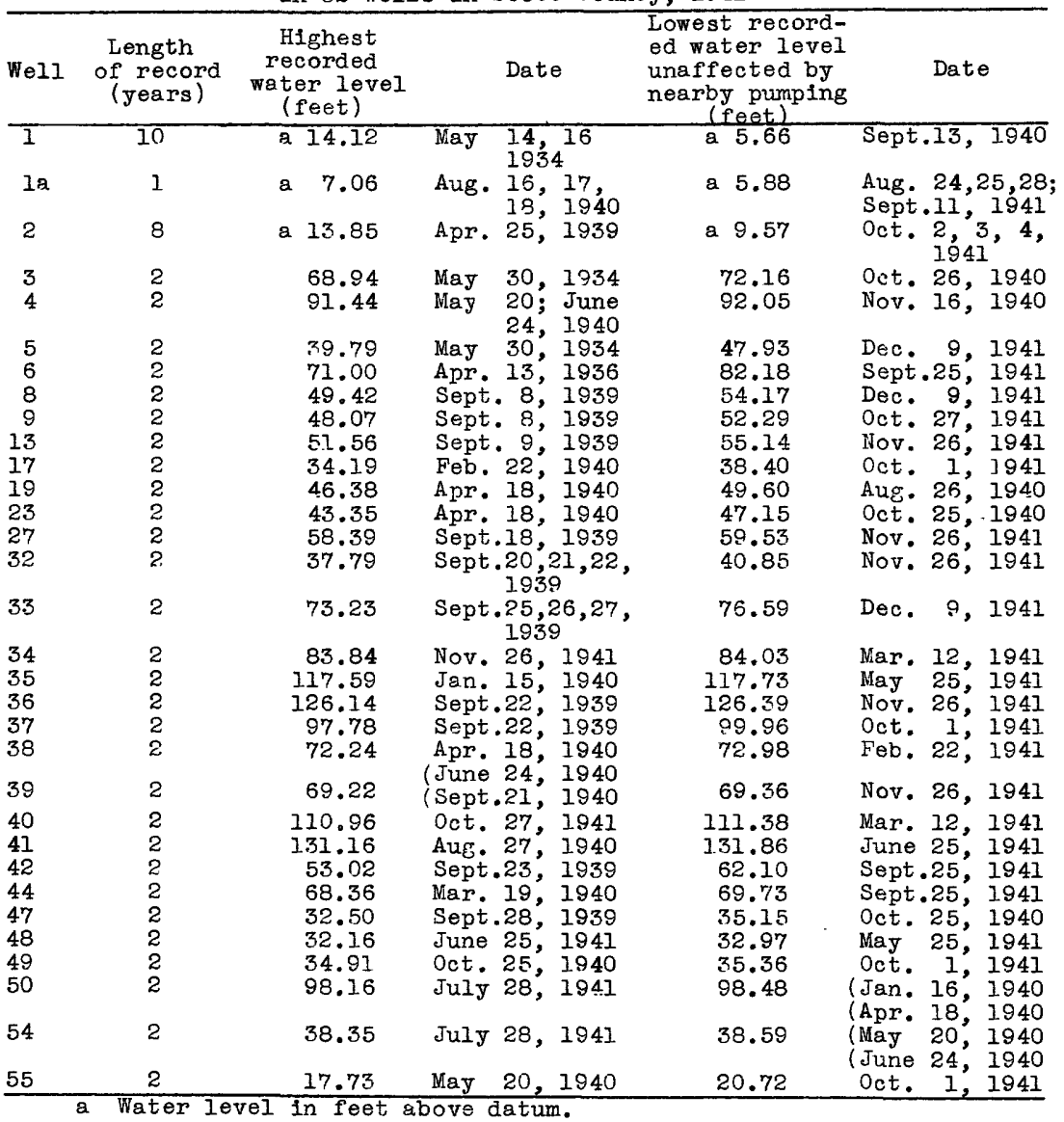


Scott County--Continued

Net changes in water level in 1941, and net changes in water level for the period of record in' 32 wells in Scott County

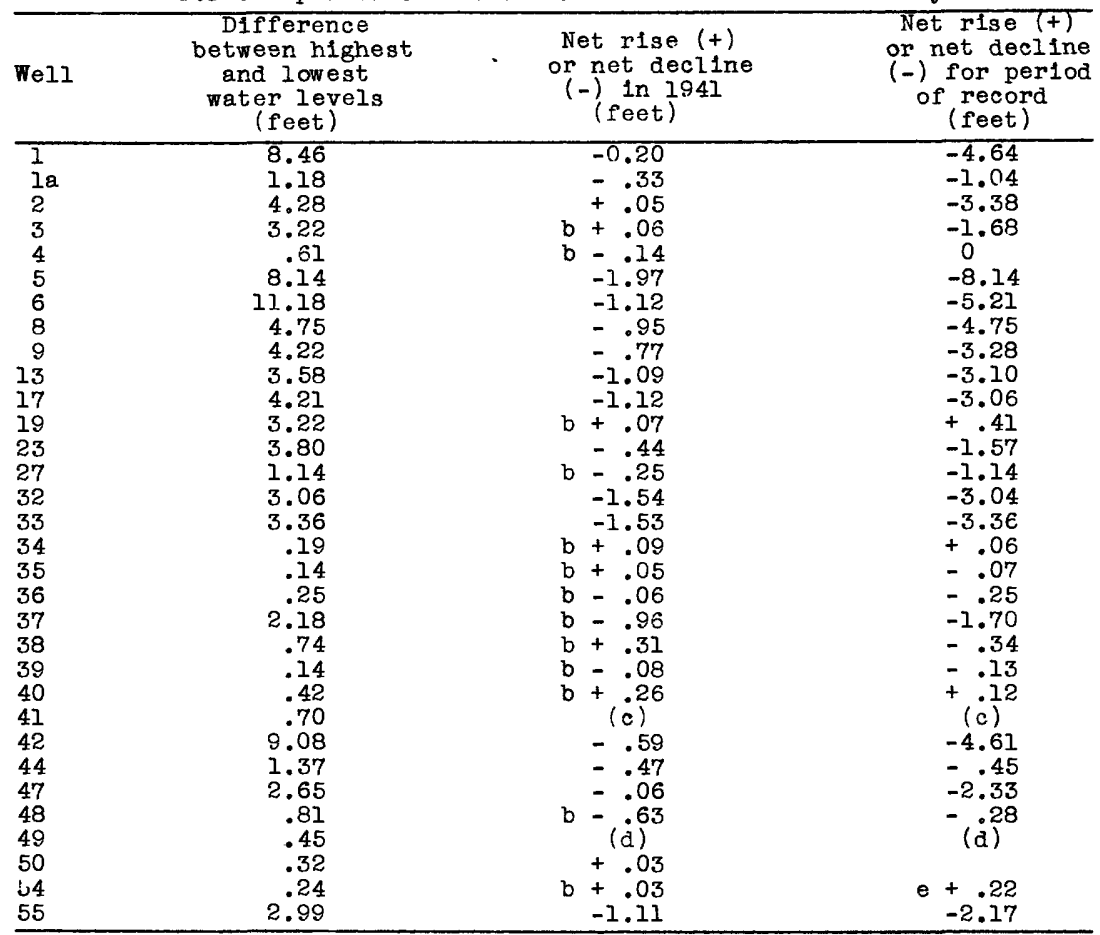

1. Mrs. Rosine Smith. NW cor. sec. 9, T. 20 S., R. 33 W. Mean daily water level, in feet above datum, 1941

\begin{tabular}{ccccccccccccc}
\hline Day & Jan. & Feb. & Mar. & Apr. & May & June & July & Aug. & Sept. & Oct. & Nov. & Dec. \\
\hline 1 & 9.25 & 9.33 & 9.38 & 9.51 & 9.53 & 9.55 & 9.46 & $\ldots$. & 9.04 & 8.27 & 8.87 & 8.95 \\
2 & 9.25 & 9.33 & 9.40 & 9.51 & 9.55 & 9.55 & 9.46 & $\ldots \ldots$ & 9.06 & 8.33 & 8.87 & 8.96 \\
3 & 9.25 & 9.33 & 9.35 & 9.50 & 9.54 & 9.53 & 9.46 & $\ldots \ldots$ & 9.07 & 8.37 & 8.88 & 8.98 \\
4 & 9.27 & 9.33 & 9.34 & 9.48 & 9.55 & 9.54 & 9.47 & $\ldots \ldots$ & 8.65 & 8.42 & 8.90 & 8.99 \\
5 & 9.27 & 9.35 & 9.39 & 9.52 & 9.54 & 9.55 & 9.48 & $\ldots \ldots$ & 7.76 & 8.45 & 8.90 & 8.96 \\
6 & 9.27 & 9.33 & 9.37 & 9.49 & $\ldots \ldots$ & 9.55 & 9.47 & $\ldots \ldots$ & 7.53 & 8.47 & 8.91 & 8.99 \\
7 & 9.27 & 9.33 & 9.37 & 9.47 & $\ldots \ldots .37$ & 9.54 & 9.46 & $\ldots \ldots$ & 7.91 & 8.49 & 8.92 & 9.01 \\
8 & 9.25 & 9.33 & 9.40 & 9.47 & 9.54 & 9.55 & 9.47 & $\ldots \ldots$ & 8.21 & 8.53 & 8.93 & 9.00 \\
9 & 9.25 & 9.34 & 9.41 & 9.47 & 9.51 & 9.55 & 9.48 & $\ldots \ldots$ & 8.39 & 8.54 & 8.94 & 9.03 \\
10 & 9.26 & 9.34 & 9.39 & 9.50 & 9.52 & 9.53 & 9.48 & $\ldots \ldots$ & 8.49 & 8.57 & 8.94 & 9.02 \\
11 & 9.27 & 9.35 & 9.40 & 9.48 & 9.53 & 9.52 & 9.46 & $\ldots \ldots$ & 8.58 & 8.60 & 8.94 & 9.05 \\
12 & 9.27 & 9.38 & 9.38 & 9.48 & 9.55 & 9.52 & 9.46 & $\ldots \ldots$ & 8.66 & 8.62 & 8.97 & 9.03 \\
13 & 9.27 & 9.28 & 9.39 & 9.48 & 9.56 & 9.51 & 9.45 & $\ldots \ldots$ & 8.70 & 8.63 & 8.95 & 9.02 \\
14 & 9.28 & 9.32 & 9.44 & 9.48 & 9.55 & 9.50 & 9.44 & $\ldots \ldots$ & 8.73 & 8.63 & 8.96 & 9.03 \\
15 & 9.28 & 9.31 & 9.41 & 9.48 & 9.54 & 9.50 & 9.43 & $\ldots \ldots$ & 8.49 & 8.65 & 8.97 & 9.04 \\
16 & 9.28 & 9.35 & 9.39 & 9.49 & 9.53 & 9.50 & 9.42 & $\ldots \ldots$ & 7.91 & 8.68 & 8.96 & 9.03 \\
17 & 9.26 & 9.34 & 9.41 & 9.50 & 9.56 & 9.50 & 9.41 & $\ldots \ldots$ & 7.58 & 8.69 & 8.94 & 9.03 \\
18 & 9.27 & 9.35 & 9.43 & 9.50 & 9.58 & 9.50 & 9.42 & $\ldots \ldots$ & 7.39 & 8.69 & 8.93 & 9.02 \\
19 & 9.29 & 9.35 & 9.43 & 9.52 & 9.57 & 9.50 & 9.39 & $\ldots \ldots$ & 7.11 & 8.71 & 8.91 & 9.02 \\
20 & 9.30 & 9.34 & 9.42 & 9.49 & 9.55 & 9.49 & 9.40 & 8.77 & 6.79 & 8.73 & 8.90 & 9.03
\end{tabular}

$\mathrm{b}$ For the period from Feb. 22 to Nov. 26, 1941.

c Accuracy of water-level measurements after June 25, 1941 questionable.

d Measurements discontinued after 0ct. 1, 1941.

e For the period from May 20, 1940 to Nov. 26, 1941 . 
Scott County--Continued

1. Mrs. Rosine Smith--Continued.

Mean daily water level, in feet above datum, 1941

\begin{tabular}{|c|c|c|c|c|c|c|c|c|c|c|c|c|}
\hline $\begin{array}{l}\text { Day } \\
21 \\
22 \\
23 \\
24 \\
25 \\
26 \\
27 \\
28 \\
29 \\
30\end{array}$ & $\begin{array}{l}\text { Jan. } \\
9.31 \\
9.30 \\
9.31 \\
9.33 \\
9.34 \\
9.33 \\
9.31 \\
9.33 \\
9.34 \\
9.34 \\
9.34\end{array}$ & $\begin{array}{l}\text { Feb. } \\
9.35 \\
9.35 \\
9.37 \\
9.36 \\
9.37 \\
9.36 \\
9.35 \\
9.36 \\
\ldots \ldots \\
\ldots \ldots \\
\ldots .\end{array}$ & $\begin{array}{c}\text { Mar. } \\
9.42 \\
9.43 \\
9.45 \\
9.44 \\
9.46 \\
9.47 \\
9.47 \\
9.46 \\
9.50 \\
9.50 \\
9.46\end{array}$ & $\begin{array}{c}\text { Apr. } \\
9.50 \\
9.51 \\
9.51 \\
9.51 \\
9.53 \\
9.53 \\
9.53 \\
9.52 \\
9.54 \\
9.53 \\
. . .\end{array}$ & $\begin{array}{c}\text { May } \\
9.55 \\
9.56 \\
9.54 \\
9.57 \\
9.58 \\
9.58 \\
9.57 \\
9.51 \\
9.52 \\
9.54 \\
9.55\end{array}$ & $\begin{array}{l}\text { June } \\
9.47 \\
9.45 \\
9.46 \\
9.46 \\
9.47 \\
9.47 \\
9.47 \\
9.47 \\
9.46 \\
9.47 \\
\ldots . .\end{array}$ & $\begin{array}{l}\text { July } \\
9.40 \\
9.40 \\
9.40 \\
9.40 \\
9.40 \\
\ldots \ldots \\
\ldots \ldots \\
\ldots \ldots \\
\ldots \ldots \\
\ldots .\end{array}$ & $\begin{array}{l}\text { Aug. } \\
8.81 \\
8.86 \\
8.89 \\
8.94 \\
8.95 \\
8.96 \\
8.98 \\
9.01 \\
9.02 \\
9.03 \\
9.03\end{array}$ & $\begin{array}{l}\text { Sept. } \\
6.93 \\
7.15 \\
6.99 \\
7.27 \\
7.62 \\
7.82 \\
7.96 \\
8.05 \\
8.16 \\
8.22 \\
\ldots \ldots\end{array}$ & $\begin{array}{l}\text { Oct. } \\
8.73 \\
8.74 \\
8.73 \\
8.73 \\
8.77 \\
8.78 \\
8.77 \\
8.81 \\
8.84 \\
8.83 \\
8.86\end{array}$ & $\begin{array}{l}\text { Nov. } \\
8.94 \\
8.94 \\
8.93 \\
8.94 \\
8.94 \\
8.93 \\
8.94 \\
8.95 \\
8.96 \\
8.94 \\
. . .\end{array}$ & $\begin{array}{l}\text { Dec. } \\
9.04 \\
9.04 \\
9.03\end{array}$ \\
\hline
\end{tabular}

1A. Division of Water Resources, Kansas State Board of Agriculture. NW $\frac{1}{4} \mathrm{SW} \frac{1}{4}$ sec. 3, T. 20 S., R. 33 W.

Mean daily water level, in feet above datum, 1941

\begin{tabular}{|c|c|c|c|c|c|c|c|c|c|c|c|c|}
\hline Day & Jan. & $\mathrm{Feb}$. & Mar. & Apr. & May & June & July & Aug. & Sept. & Oct. & Nov. & Dec. \\
\hline 1 & 6.35 & .43 & 6.52 & 6.61 & 6.67 & .42 & 6.07 & 6.08 & 5.88 & 5.95 & 5.96 & .96 \\
\hline 2 & 6.35 & 43 & 6.52 & 6.62 & 6.67 & 6.39 & 6.07 & 6.07 & 88 & 5.95 & .96 & .96 \\
\hline 3 & 6.35 & 44 & 6.52 & 6.62 & 6.67 & 6.36 & 6.07 & 6.06 & & & 5.96 & \\
\hline 4 & 6.36 & 6.44 & 6.52 & 6.62 & 6.67 & 6.34 & 6.08 & 6.03 & 88 & & 5.96 & \\
\hline 5 & 6.36 & 6.45 & 6.53 & 6.63 & 6.67 & 6.30 & 6.08 & 6.02 & 38 & 5. & 96 & \\
\hline 6 & 6.36 & 45 & 6.53 & 6.63 & 6.67 & 6.29 & 08 & 6.02 & & 5 & 96 & \\
\hline $\begin{array}{l}7 \\
8\end{array}$ & $\begin{array}{l}6.36 \\
6.36\end{array}$ & & & & $\begin{array}{l}6.67 \\
6.68\end{array}$ & $\begin{array}{l}6 \\
6\end{array}$ & 8 & $\begin{array}{l}6.01 \\
5.99\end{array}$ & & & & \\
\hline 9 & $\begin{array}{l}0.36 \\
6.37\end{array}$ & & & 0 & $\begin{array}{l}0.08 \\
6.68\end{array}$ & $\begin{array}{l}6 \\
6\end{array}$ & & & & 0 & & \\
\hline 10 & 6.37 & 7 & & & 6.68 & 6. & 9 & 7 & & & & \\
\hline 1 & 6.37 & 47 & & 6. & 6. & 8 & 0 & 6 & & & & \\
\hline 2 & 6.37 & 47 & 6 & 6 & 6.68 & 6 & 0 & 5 & & & & \\
\hline 3 & 6.38 & & 6 & 6 & 6.68 & 5 & 1 & & & & & \\
\hline 4 & 6.38 & & & & 6.69 & 6 & 11 & 5.92 & & & & \\
\hline 5 & 6.38 & & & & & 3 & 1 & & & & & \\
\hline 6 & 6. & & & & 6. & & & & & & & \\
\hline 7 & 6.38 & & 6. & & 6. & & 1 & & & & & \\
\hline Q & 6. & & 6. & 6 & 6. & 6 & 6. & & & & & \\
\hline 9 & 6. & & 6 & & 6. & & & & & & & \\
\hline & & & & & & & & & & & & \\
\hline & & & & & & & & & & & & \\
\hline 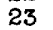 & & & & & & & & & & & & \\
\hline $\mathbf{x}$ & & & & & & & & & & & & \\
\hline 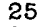 & & & & & & & & & & & & \\
\hline 6 & & & & & & & & & & & & \\
\hline & & & & & & & & & & & & \\
\hline 8 & & & & & & & & & & & & \\
\hline 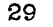 & & & & & & & & & & & & \\
\hline 30 & & & & & & & & & & & & \\
\hline & & & & & & & & & & & & \\
\hline
\end{tabular}

2. E. E. Coffin. NE $\frac{1}{4} S E \frac{1}{4}$ sec. 25, T. 18 S., R. 33 W.

Mean da1ly water level, in feet above datum, 1941

\begin{tabular}{ccccccccccccc}
\hline Day & Jan. & Feb. & Mar. & Apr. & May & June & July & Aug. & Sept. & Oct. & Nov. & Dec. \\
\hline 1 & 9.92 & 10.07 & 10.19 & 10.34 & 10.47 & 10.29 & 10.10 & 10.03 & 9.75 & 9.58 & 9.72 & 9.83 \\
2 & 9.93 & 10.07 & 10.20 & 10.34 & 10.47 & 10.28 & 10.10 & 10.02 & 9.74 & 9.57 & 9.72 & 9.84 \\
3 & 9.93 & 10.08 & 10.20 & 10.35 & 10.48 & 10.26 & 10.10 & 10.01 & 9.73 & 9.57 & 9.73 & 9.84 \\
4 & 9.93 & 10.08 & 10.20 & 10.35 & 10.48 & 10.24 & 10.10 & 10.00 & 9.72 & 9.57 & 9.73 & 9.85 \\
5 & 9.93 & 10.08 & 10.21 & 10.36 & 10.49 & 10.24 & 10.10 & 9.99 & 9.72 & 9.58 & 9.74 & 9.86 \\
6 & 9.94 & 10.09 & 10.21 & 10.36 & 10.49 & 10.22 & 10.11 & 9.98 & 9.71 & 9.59 & 9.74 & 9.86 \\
7 & 9.94 & 10.09 & 10.22 & 10.36 & 10.49 & 10.21 & 10.10 & 9.97 & 9.70 & 9.60 & 9.75 & 9.87 \\
8 & 9.95 & 10.10 & 10.23 & 10.37 & 10.50 & 10.20 & 10.10 & 9.97 & 9.69 & 9.60 & 9.75 & 9.88 \\
9 & 9.95 & 10.10 & 10.23 & 10.38 & 10.50 & 10.19 & 10.11 & 9.96 & 9.69 & 9.61 & 9.76 & 9.88 \\
\hline
\end{tabular}


Scott County--Continued

2. E. E. Coffin--Continued.

Mean da1ly water level, in feet above datum, 1941

\begin{tabular}{|c|c|c|c|c|c|c|c|c|c|c|c|c|}
\hline $9 y$ & $\begin{array}{r}\text { Jan. } \\
9.96 \\
9.96 \\
9.96 \\
9.96 \\
9.96 \\
9.97 \\
9.99 \\
9.99 \\
9.99 \\
10.00 \\
10.00 \\
10.01 \\
10.02 \\
10.03\end{array}$ & $\begin{array}{l}\text { Feb. } \\
10.10 \\
10.11 \\
10.12 \\
10.13 \\
10.13\end{array}$ & $\begin{array}{r}\text { Mar. } \\
10.24 \\
10.24 \\
10.24 \\
10.25 \\
10.25\end{array}$ & $\begin{array}{r}\text { Apr. } \\
10.39 \\
10.39 \\
10.39 \\
10.39 \\
10.40\end{array}$ & $\begin{array}{c}\text { May } \\
10.50 \\
10.51 \\
10.51 \\
10.52 \\
10.53\end{array}$ & $\begin{array}{r}\text { June } \\
10.18 \\
10.17 \\
10.16 \\
10.16 \\
10.15 \\
10.14 \\
10.14 \\
10.13 \\
10.12 \\
10.12 \\
10.11 \\
10.10 \\
10.09 \\
10.09 \\
10.10 \\
10.10 \\
10.09 \\
10.10 \\
10.10 \\
10.10 \\
10.10\end{array}$ & $\begin{array}{r}\text { July } \\
10.10 \\
10.10 \\
10.10 \\
10.10 \\
10.10 \\
10.10 \\
10.09 \\
10.09 \\
10.08 \\
10.08 \\
10.07 \\
10.06 \\
10.06 \\
10.06 \\
10.06 \\
10.05 \\
10.05 \\
10.04 \\
10.03 \\
10.03 \\
10.03\end{array}$ & $\begin{array}{l}\text { Aug. } \\
9.95 \\
9.93\end{array}$ & $\begin{array}{l}\text { Sept. } \\
9.68 \\
9.68 \\
9.67\end{array}$ & $\begin{array}{l}\text { Oct. } \\
9.61 \\
9.61 \\
9.61\end{array}$ & ov. & Dec \\
\hline
\end{tabular}

3. Claude Hughes. SW $\frac{1}{4}$ SW $\frac{1}{4}$ sec. 21, T. 18 S., R. 33 W.

Water level, in feet below measuring polnt, 1941

\begin{tabular}{|c|c|c|c|c|c|c|c|}
\hline Date & $\begin{array}{l}\text { Water } \\
\text { level }\end{array}$ & Date & $\begin{array}{l}\text { Water } \\
\text { level }\end{array}$ & Date & $\begin{array}{l}\text { Water } \\
\text { level }\end{array}$ & Date & $\begin{array}{l}\text { Water } \\
\text { level }\end{array}$ \\
\hline $\begin{array}{l}\text { Feb. } 22 \\
\text { Mar. } 12 \\
\text { Apr. } 25\end{array}$ & $\begin{array}{l}70.68 \\
70.58 \\
70.35\end{array}$ & $\begin{array}{ll}\text { May } & 25 \\
\text { June } & 25 \\
\text { July } & 28\end{array}$ & $\begin{array}{l}70.43 \\
70.52 \\
70.81\end{array}$ & $\begin{array}{lr}\text { Aug. } & 25 \\
\text { Oct. } & 1\end{array}$ & $\begin{array}{l}71.18 \\
71.03\end{array}$ & $\begin{array}{l}\text { Oct. } 27 \\
\text { Nov. } 26\end{array}$ & $\begin{array}{l}70.78 \\
70.62\end{array}$ \\
\hline
\end{tabular}

4. W. N. Robinson. SE $\frac{1}{4} N W \frac{1}{4}$ sec. 31, T. 18 S., R. 34 W.

Water level, in feet below measuring polnt, 1941

\begin{tabular}{ll|lr|lr|lll}
\hline Feb. 22 & 91.70 & May 25 & 91.59 & Aug. 25 & 91.81 & Oct. 27 & 91.99 \\
Mar. 12 & 91.74 & June 25 & 91.64 & Oct. 1 & 91.85 & Nov. 26 & 91.84 \\
Apr. 25 & 91.65 & July 28 & 91.59 & & & & & \\
\hline
\end{tabular}

5. Mrs. Rosine Smith. SW $\frac{1}{4} N W_{\frac{1}{4}}$ sec. 25, T. 19 S., R. 33 W.

Water level, in feet below measuring point, 1941

\begin{tabular}{ll|ll|l|l|ll}
\hline Feb. 22 & 45.96 & May 25 & 46.28 & Aug. 25 & 46.74 & Nov. 26 & 47.25 \\
Mar. 12 & 45.93 & June 25 & 46.39 & Sept.25 & 46.89 & Dec. 9 & 47.93 \\
Apr. 25 & 46.08 & July 28 & $43.5]$ & Oct. 27 & 47.08 & & \\
\hline
\end{tabular}

6. American Life Insurance Co. NW $\frac{1}{4} S W \frac{7}{4}$ sec. 29, T. 19 S., R. 33 W.

Water level, in feet below measuring polnt, 1941

\begin{tabular}{ll|ll|l|l|l|l|}
\hline Feb. 22 & 79.24 & May 25 & 79.00 & Aug. 25 & 80.78 & Nov. 26 & 80.53 \\
Mar. 12 & 79.14 & June 25 & 78.79 & Sept.25 & 82.18 & Dec. 9 & 80.41 \\
Apr. 25 & 79.02 & July 28 & 78.99 & Oct.27 & 80.96 & & \\
\hline
\end{tabular}

8. Mrs. Rosine Smith. NW $\frac{1}{4} N W \frac{1}{4}$ sec. 35, T. 19 S., R. 33 W.

Water level, in feet below measuring point, 1941

\begin{tabular}{|c|c|c|c|c|c|c|c|c|}
\hline $\begin{array}{l}\text { Mar. } \\
\text { Apr. }\end{array}$ & $\begin{array}{l}12 \\
25 \\
\end{array}$ & $\begin{array}{l}53.22 \\
53.34 \\
\end{array}$ & $\begin{array}{l}\text { July } 28 \\
\text { Aug. } 25 \\
\end{array}$ & $\begin{array}{l}53.50 \\
53.44 \\
\end{array}$ & $\begin{array}{l}\text { Sept.25 } \\
\text { Oct. } 27 \\
\end{array}$ & $\begin{array}{l}53.84 \\
53.96 \\
\end{array}$ & $\begin{array}{lr}\text { Nov. } 26 \\
\text { Dec. } 9 \\
\end{array}$ & $\begin{array}{l}54.12 \\
54.17 \\
\end{array}$ \\
\hline & 9 & \multicolumn{2}{|c|}{$\begin{array}{l}\text { Mrs, Rosine Smlth } \\
\text { Water level, }\end{array}$} & $\begin{array}{l}S W \frac{1}{4} S W \\
\text { feet } b\end{array}$ & & & $\begin{array}{c}\text { R. } 33 \\
1941\end{array}$ & \\
\hline $\begin{array}{l}\text { Feb. } \\
\text { Mar. }\end{array}$ & $\begin{array}{l}22 \\
12 \\
25\end{array}$ & $\begin{array}{l}50.58 \\
50.57 \\
50.43\end{array}$ & $\begin{array}{ll}\text { May } & 25 \\
\text { June } & 25 \\
\text { July } & 28 \\
\end{array}$ & $\begin{array}{l}50.60 \\
50.67 \\
50.75\end{array}$ & $\begin{array}{l}\text { Aug. } 25 \\
\text { Sept.25 } \\
\text { Oct. } 27\end{array}$ & $\begin{array}{l}50.82 \\
\text { a } 52.90 \\
52.59\end{array}$ & $\begin{array}{lr}\text { Nov. } & 26 \\
\text { Dec. } & 9\end{array}$ & $\begin{array}{l}51.52 \\
51.35\end{array}$ \\
\hline
\end{tabular}

a Irrigation well 300 yards south pumping. 


\section{Scott County--Continued}

I3. Mrs. Rosine Smith. SW $\frac{1}{4} S W \frac{7}{4}$ sec. 2, T, 20 S, R. 33 W.

Water level, in feet below measuring point, 1941

\begin{tabular}{ll|lr|ll|ll|l}
\hline Date & $\begin{array}{l}\text { Water } \\
\text { level }\end{array}$ & Date & $\begin{array}{r}\text { Water } \\
\text { level }\end{array}$ & Date & $\begin{array}{r}\text { Water } \\
\text { level }\end{array}$ & Date & $\begin{array}{r}\text { Water } \\
\text { level }\end{array}$ \\
\hline Feb. 22 & 53.57 & May 25 & 53.48 & Aug. 25 & 53.72 & Nov. 26 & 55.14 \\
Mar. 12 & 53.49 & June 25 & 53.73 & Sept.25 & a 57.52 & Dec. 9 & 54.66 \\
Apr. 25 & 53.25 & July 28 & 53.66 & Oct.27 & 57.39 & & \\
\hline
\end{tabular}

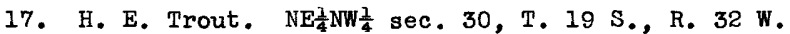

Water level, in feet below measuring point, 1941

\begin{tabular}{ll|ll|ll|ll|l}
\hline Feb. 22 & 36.30 & May 25 & 36.15 & Aug. 25 & 37.85 & Nov. 26 & 38.25 \\
Mar. 12 & 36.11 & June 25 & 36.83 & Oct. 1 & 38.40 & Dec. 9 & 37.42 \\
Apr. 25 & 36.30 & JuIy 28 & 36.74 & & 27 & 38.28 & & \\
\hline
\end{tabular}

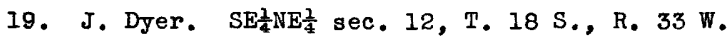

Water level, in feet below measuring point, 1941

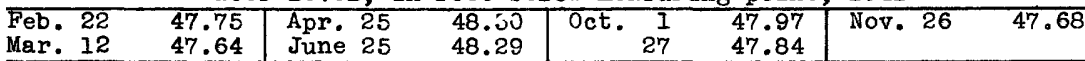

23. $\mathrm{SE} \frac{1}{4} \mathrm{SE} \frac{2}{4} \mathrm{sec} .24, \mathrm{~T}, 18 \mathrm{~S}, \mathrm{R}, 33 \mathrm{~W}$.

Water level, in feet below measuring point, 1941

\begin{tabular}{ll|lll|lr|rrr}
\hline Feb. 22 & 45.12 & May & 25 & 45.44 & Aug. 25 & 46.32 & Nov. 26 & 45.44 \\
Mar. 12 & 45.05 & June 25 & 45.28 & Oct. 1 & 46.25 & Dec. 9 & 45.56 \\
Apr. 25 & 44.84 & July 28 & 45.31 & & 27 & 45.75 & & & \\
\hline
\end{tabular}

27. Anson Mark. NE $\frac{1}{4} \mathrm{NW} \frac{2}{4}$ sec. 15, T. $18 \mathrm{~S}$, R. 33. W.

Water level, in feet below measuring point, 1941

\begin{tabular}{ll|lr|lrr|lr}
\hline Feb. 22 & 59.28 & May 25 & 59.27 & Aug. 25 & 59.34 & Oct. 27 & 59.50 \\
Mar. 12 & 59.29 & June 25 & 59.31 & Oct. 1 & 59.44 & Nov. 26 & 59.53 \\
Apr. 25 & 59.29 & July 28 & 59.31 & & & & & \\
\hline
\end{tabular}

32. E. J. Roark. NW $\frac{1}{4} \mathrm{SE} \frac{1}{4}$ sec. 25, T. 19 S., R. 33 W.

Lowest dally water level, in feet below measuring point, 1941

\begin{tabular}{|c|c|c|c|c|c|c|c|c|c|c|}
\hline Day & Jan. & Feb. & Mar. & Apr. & May & June & Julg & Oct. & Nov. & Dec. \\
\hline$I$ & 39.29 & & & 39.45 & 39.52 & 39.75 & 39.90 & 40.71 & • & \\
\hline 2 & 39.29 & & & 39.46 & 39 & 76 & 90 & .... & & \\
\hline 3 & 39.29 & $\ldots \ldots$ & .... & 39.46 & 39.53 & 39.75 & 39.90 & $\ldots$ & . & \\
\hline 4 & 39.29 & .... & .... & 39.46 & 39 & 39.75 & 39.90 & .... & ..... & .. \\
\hline 5 & 39.29 & .... & ..... & 39.46 & 39.54 & 39.75 & 39.90 & .... & .... & .. \\
\hline 6 & 39.30 & $\cdots \cdots$ & .... & 39.46 & 39 & 39.76 & 39.91 & •... & .... & $\bullet$ \\
\hline 7 & 39 & $\ldots \cdots$ & & 39 & & 39 & & $\cdots \cdots$ & $\cdots \cdots$ & \\
\hline 8 & $\begin{array}{l}39 \\
39\end{array}$ & $\ddot{3} \ddot{\theta} \ddot{0}$ & $\ddot{3} \ddot{0} \dot{6}$ & 39.47 & & 39 & & & & \\
\hline 9 & $\begin{array}{l}39 \\
39\end{array}$ & $\begin{array}{l}39.40 \\
39.40\end{array}$ & $\begin{array}{l}30.46 \\
39.46\end{array}$ & $\begin{array}{l}30.47 \\
39.47\end{array}$ & & $\cdots \cdots$ & $\begin{array}{l}3 \\
3\end{array}$ & $\ldots \ldots$ & $\because \cdots$ & \\
\hline 1 & $\begin{array}{l}39.30 \\
39.30\end{array}$ & 39.40 & $\begin{array}{l}39.46 \\
39.46\end{array}$ & $\begin{array}{l}39.47 \\
39.47\end{array}$ & & $\ddot{3} \ddot{8} \dot{8}$ & $\begin{array}{l}39.92 \\
39.94\end{array}$ & $\ldots \ldots$ & $\cdots \cdots$ & \\
\hline 2 & 39.30 & 39.40 & 39.46 & 39.47 & 39.56 & 39.84 & 39.95 & ..... & .... & \\
\hline$\pi$ & 39. & 39 & 39.46 & 39.47 & 39 & 39.83 & 39 & - & .. & \\
\hline 4 & 39.30 & 40 & 39.46 & 39.48 & 39 & 39.83 & b39 & - & $\cdots$ & \\
\hline .5 & & & 39.46 & 39 & 39 & 39.84 & •.... & .... & .... & $\because$ \\
\hline 6 & & & 39.46 & & 39 & 39.85 & •... & $\bullet$ & - & \\
\hline 7 & 39 & 40 & 39.46 & 39 & 39 & 39.85 & - & 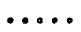 & - & - \\
\hline 8 & 39. & 40 & 39.45 & 39.48 & 39 & 39.86 & & - & . & \\
\hline 9 & 39. & 40 & 39.45 & 39.48 & 39 & 39. & .... & $\ldots \ldots$ & - & - \\
\hline 0 & & & 35 & 38 & 39 & 39. & . . . & $\cdots \cdots$ & $\cdots$ & \\
\hline 1 & & 40 & & & 39 & 39 & -.... & •.. & $\ldots$ & \\
\hline 2 & 39 & & 39.45 & 39 & 30 & 39 & .... & .... & .... & •• \\
\hline 3 & 39 & & 39 & & & & $\bullet$ & $\cdots$ & & \\
\hline 4 & 39 & .. & 39.45 & 39.48 & 39 & 39.88 & ... & $\cdots \cdot$ & $\ldots$ & \\
\hline & & & & 49 & 0 & 39.88 & $\ldots \ldots$ & $\because$ & $\ddot{4} \ddot{8} \ddot{s}$ & \\
\hline & & & & & 3 & & & 40.84 & 40.85 & \\
\hline & & & 35 & & 3 & & & & & \\
\hline & - & & & 52 & 3 & 39 & & $\ldots \ldots$ & . & \\
\hline & & & & & & 39 & & & & \\
\hline & & $\cdots$ & & & & $\ldots \ldots$ & $\cdots$ & .... & •... & $\cdots$ \\
\hline
\end{tabular}

a Irrigation well half a mile north pumping.

b Automatic recorder removed July 14, wetted-tape measurement after this date. 


\section{Scott County--Continued}

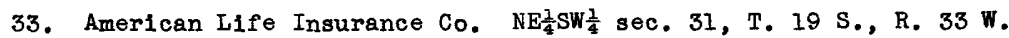

Lowest daily water level, in feet below measuring point, 1941

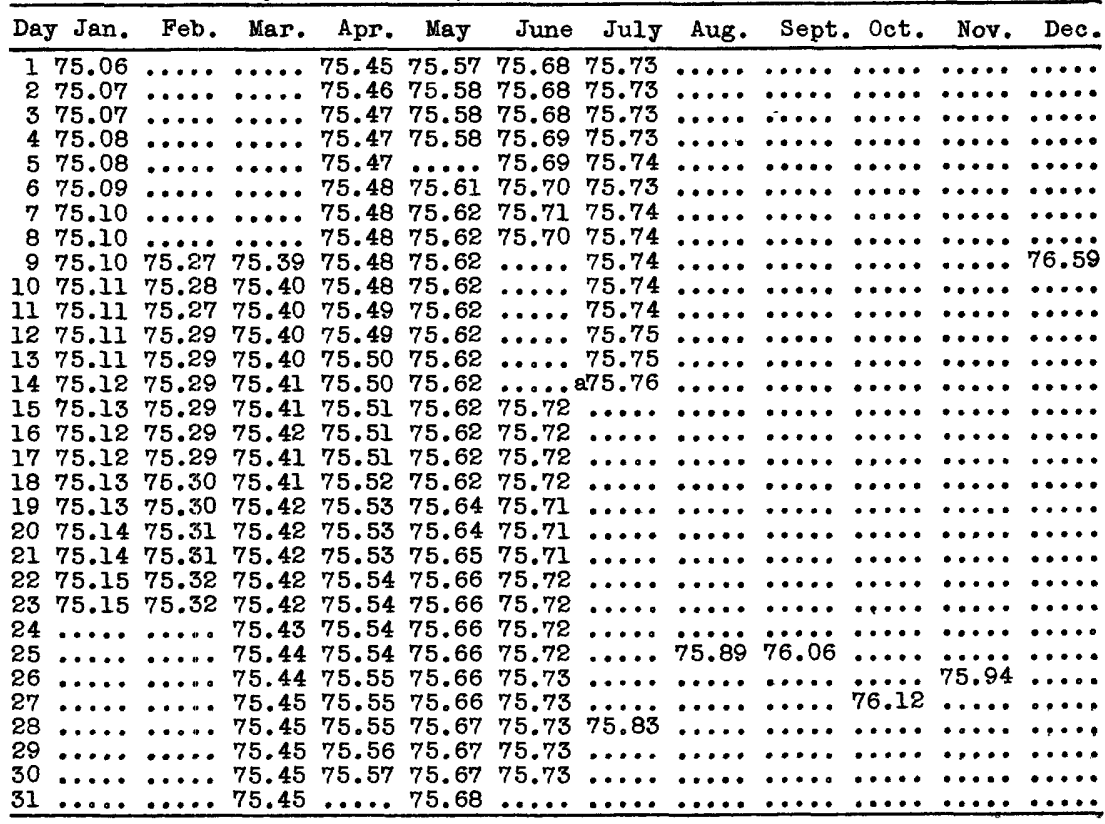

34. H. M. A. Hess et al. SW $\frac{1}{4} \mathrm{SW} \frac{1}{4}$ sec. 19, T. $18 \mathrm{~S} ., \mathrm{R}, 34 \mathrm{~W}$. Water level, in feet below measuring point, 1941

\begin{tabular}{|c|c|c|c|c|c|c|c|}
\hline Date & $\begin{array}{l}\text { Water } \\
\text { level }\end{array}$ & Date & $\begin{array}{l}\text { Water } \\
\text { level }\end{array}$ & Date & $\begin{array}{l}\text { Water } \\
\text { level }\end{array}$ & Date & $\begin{array}{l}\text { Water } \\
\text { level }\end{array}$ \\
\hline $\begin{array}{l}\text { Feb. } 22 \\
\text { Mar. } 12 \\
\text { Apr. } 25\end{array}$ & $\begin{array}{l}83.93 \\
84.03 \\
83.95\end{array}$ & $\begin{array}{ll}\text { May } & 25 \\
\text { June } & 25 \\
\text { July } & 28\end{array}$ & $\begin{array}{l}83.91 \\
83.94 \\
83.89\end{array}$ & $\begin{array}{l}\text { Aug. } 25 \\
\text { Oct. } 1\end{array}$ & $\begin{array}{l}83.85 \\
84.02\end{array}$ & $\begin{array}{l}\text { Oct. } 27 \\
\text { Nov. } 26\end{array}$ & $\begin{array}{l}83.89 \\
83.84\end{array}$ \\
\hline
\end{tabular}

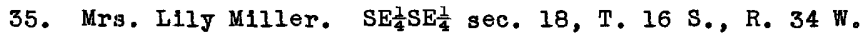

Water level, in feet bel ow measuring point, 1941

\begin{tabular}{ll|ll|lll|ll}
\hline Feb. 22 & 117.82 & Apr. 25 & 117.84 & June 25 & 117.81 & Oct. 27 & 117.85 \\
Mar. 12 & 117.78 & May 25 & 117.73 & Oct. & 1 & 117.88 & Nov. 26 & 117.77 \\
\hline
\end{tabular}

36. Henry S. Mix. SE $\frac{1}{4} N E \frac{1}{4}$ sec. 11, T. 16 S., R. 34 W.

Water level, in feet below measuring point, 1941

\begin{tabular}{ll|ll|lll|ll}
\hline Feb. 22 & 126.33 & Apr. 25 & 126.30 & June 25 & 126.32 & Oct. 27 & 126.18 \\
Mar. 12 & 126.34 & May 25 & 126.25 & Oct. & 1 & 126.38 & Nov. 26 & 126.39 \\
\hline
\end{tabular}

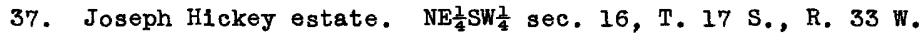

Water level, in feet below measuring point, 1941

\begin{tabular}{ll|lr|lrr|lll}
\hline Feb. 22 & 98.52 \\
Mar. 12 & 98.23 & Juy 25 25 & 98.84 & July 28 & 98.98 & Oct. 27 & 99.67 \\
Apr. 25 & 98.88 & & & & Oct. & 1 & 99.96 & Nov. 26 & 99.48 \\
\hline
\end{tabular}

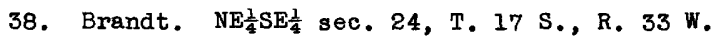

Water level, in feet below measuring point, 1941

\begin{tabular}{ll|lr|ll|lll}
\hline Feb. 22 & 72.98 & Apr. 25 & 72.63 & June 25 & 72.64 & Nov. 26 & 72.67 \\
Mar. 12 & 72.89 & May 25 & 72.64 & Oct. 1 & 72.61 & & & \\
\hline
\end{tabular}

a Automatic recorder removed July 14, wetted-tape measurements after this date. 
Scott County--Continued

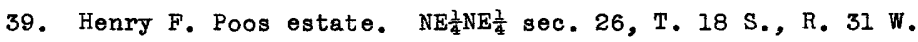
Water level, in feet below measuring point, 1941

\begin{tabular}{|c|c|c|c|c|c|c|c|}
\hline Date & $\begin{array}{l}\text { Water } \\
\text { level }\end{array}$ & Date & $\begin{array}{l}\text { Water } \\
\text { level }\end{array}$ & Date & $\begin{array}{l}\text { Water } \\
\text { level }\end{array}$ & Date & $\begin{array}{l}\text { Water } \\
\text { level }\end{array}$ \\
\hline $\begin{array}{l}\text { Feb. } 18 \\
\text { Mar. } 12 \\
\text { Apr. } 24\end{array}$ & $\begin{array}{l}69.28 \\
69.29 \\
69.29\end{array}$ & $\begin{array}{ll}\text { May } & 25 \\
\text { July } & 28\end{array}$ & $\begin{array}{l}69.31 \\
69.23\end{array}$ & $\begin{array}{l}\text { Aug. } 25 \\
\text { Oct. } 1\end{array}$ & $\begin{array}{l}69.27 \\
69.33\end{array}$ & $\begin{array}{l}\text { Oct. } 27 \\
\text { Nov. } 26\end{array}$ & $\begin{array}{l}69.29 \\
69.36\end{array}$ \\
\hline
\end{tabular}

40. Michael McLaughlin. SW $\frac{1}{4} N W \frac{1}{4}$ sec. 2, T. 17 S., R. 31 W.

Water level, in feet below measuring point, 1941

\begin{tabular}{|c|c|c|c|c|c|c|c|}
\hline $\begin{array}{l}\text { Feb. } 18 \\
\text { Mar: } 12 \\
\text { Apr. } 24\end{array}$ & $\begin{array}{l}111.29 \\
111.38 \\
111.31\end{array}$ & $\begin{array}{l}\text { May 25 } \\
\text { June } 25\end{array}$ & $\begin{array}{l}111.35 \\
111.37\end{array}$ & $\begin{array}{l}\text { Aug. } 25 \\
\text { Oct. } 1\end{array}$ & $\begin{array}{l}111.20 \\
111.25\end{array}$ & $\begin{array}{l}\text { Oct. } 27 \\
\text { Nov. } 26\end{array}$ & $\begin{array}{l}110.96 \\
111.03\end{array}$ \\
\hline
\end{tabular}

41. Almada King. NW $\frac{1}{4} N W \frac{1}{4}$ sec. 12, T. 17 S., R. 32 W.

Water level, in feet below measuring point, 1941

\begin{tabular}{ll|lll|ll|ll}
\hline Feb. 18 & 131.81 & May & 25 & 131.79 & Aug. 25 & al26.91 & 0ct. 27 & a129.07
\end{tabular}

\begin{tabular}{ll|ll|lll|ll} 
Mar. 12 & 131.84 & June 25 & 131.86 & Oct. 1 & al28.42 & Nov. 26 & $\mathbf{0 1 2 8 . 7 9}$
\end{tabular}

Apr. $24 \quad 131.82$

42. Mrs. Rosine Smith. SW $\frac{2}{4} N W \frac{1}{4}$ sec. 26, T. 19 S., R. 33 W.

Water level, in feet below measuring point, 1941

\begin{tabular}{|c|c|c|c|c|c|c|c|}
\hline $\begin{array}{l}\text { Feb. } 22 \\
\text { Mar. } 12 \\
\text { Apr. } 25\end{array}$ & $\begin{array}{l}57.04 \\
57.70 \\
57.21\end{array}$ & $\begin{array}{l}\text { May } 25 \\
\text { June } 25 \\
\text { July } 28\end{array}$ & $\begin{array}{l}57.22 \\
57.25 \\
57.18\end{array}$ & $\begin{array}{l}\text { Sept.25 } \\
\text { Oct. } 27\end{array}$ & $\begin{array}{l}62.10 \\
57.78\end{array}$ & $\begin{array}{l}\text { Nov. } 26 \\
\text { Dec. } 9\end{array}$ & $\begin{array}{l}57.85 \\
57.63\end{array}$ \\
\hline
\end{tabular}

44. Melchior Lang. SW $\frac{1}{4} N E \frac{1}{4} \sec , 2$, T. 20 S., R. 34 W.

Water level, in feet bel ow measuring point, 1941

\begin{tabular}{ll|ll|ll|lll}
\hline Feb. 19 & 68.85 & May 25 & 68.79 & Aug. 25 & 69.46 & Nov. 26 & 69.23 \\
Mar. 12 & 69.50 & June 25 & 69.00 & Sept.25 & 69.73 & Dec. 9 & 69.32 \\
Apr. 25 & 68.83 & July 28 & 69.08 & Oct.27 & 69.34 & & \\
\hline
\end{tabular}

R. $32 \mathrm{w}$.

47. V. M. Harris (Federal Land Bank). SE $\frac{1}{4} \mathrm{NW} \frac{1}{4}$ sec. 29, T. $18 \mathrm{~S}$. ,

Water level, in feet below measuring point, 1941

\begin{tabular}{lr|lr|lrr|lrr}
\hline Feb. 22 & 34.77 & May & 25 & 35.12 & Aug. 25 & 34.80 & Nov. 26 & 34.84 \\
Mar. 12 & 34.65 & June 25 & 34.59 & Oct. & 1 & 35.11 & Dec. 8 & 34.83 \\
Apr. 25 & 34.70 & July 28 & b42.70 & & 27 & 34.89 & & & \\
\hline
\end{tabular}

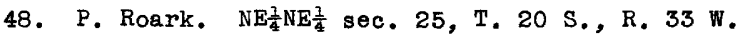

Water level, in feet below measuring point, 1941

\begin{tabular}{ll|ll|ll|lll}
\hline Jan. 29 & 32.17 & Apr. 25 & 32.87 & July 25 & 32.69 & Oct. 27 & 32.72 \\
Feb. 18 & 32.79 & May 25 & 32.97 & Aug. 25 & 32.56 & Nov. 26 & 32.80 \\
Mar. 12 & 32.88 & June 25 & 32.16 & Sept.25 & 32.67 & & & \\
\hline
\end{tabular}

49. Geo, M. Crofton. $\mathrm{SE} \frac{1}{4} \mathrm{NE} \frac{1}{4} \mathrm{sec}, 22, \mathrm{~T}, 20 \mathrm{~S}$, , R. 31 W. Measurements discontinued after 0ct. 1, 1941, because of an obstacle in casing just above water level. Water level, in feet below measuring point, 1941: Mar. 12, 35.05; Oct. 1, 35.36.

50. F. M. Houstin. SW $\frac{1}{4} \mathrm{SE} \frac{1}{4}$ sec. 28, T. 19 S., R. 32 W.

Water level, in feet below measuring point, 1941

\begin{tabular}{|c|c|c|c|c|c|c|c|}
\hline & & & & & & & \\
\hline $\begin{array}{l}\text { Feb. } 18 \\
\text { Mar. } 12 \\
\text { Apr. } 25\end{array}$ & $\begin{array}{l}98.22 \\
98.20 \\
98.19\end{array}$ & $\begin{array}{ll}\text { May } 25 \\
\text { July } 28 \\
\text { Aug. } 25\end{array}$ & $\begin{array}{l}98.23 \\
98.16 \\
98.21\end{array}$ & oct. $\begin{array}{r}1 \\
27\end{array}$ & $\begin{array}{l}98.17 \\
98.24\end{array}$ & $\begin{array}{l}\text { Nov. } 26 \\
\text { Dec. } 9\end{array}$ & $\begin{array}{l}98.19 \\
98.19\end{array}$ \\
\hline
\end{tabular}

54. B. B. Harkness. SE cor. sec. 10, T. 20 S., R. $3 I$ W.

Water level, in feet below measuring point, 1941

\begin{tabular}{ll|l|l|ll|l|ll}
\hline Feb. 18 & 38.40 & May 25 & 38.38 & Aug. 25 & 38.36 & Oct. 27 & 38.38 \\
Mar. 12 & 38.44 & July 28 & 38.35 & Oct. & 38.42 & Nov. 26 & 38.37 \\
Apr. 25 & 38.39
\end{tabular}


Scott Sounty--Continued

55. J. U. Hushaw. NW cor. sec. 7, T. 19 S., R. 32 W. Water level, in feet below measuring point, 1941

\begin{tabular}{|c|c|c|c|c|c|c|c|}
\hline Date & $\begin{array}{l}\text { Water } \\
\text { level }\end{array}$ & Date & $\begin{array}{l}\text { Water } \\
\text { level }\end{array}$ & Date & $\begin{array}{l}\text { Water } \\
\text { level }\end{array}$ & Date & $\begin{array}{l}\text { Water } \\
\text { level }\end{array}$ \\
\hline $\begin{array}{l}\text { Feb. } 22 \\
\text { Mar. } 12\end{array}$ & $\begin{array}{l}18.79 \\
18.46\end{array}$ & $\begin{array}{ll}\text { Apr. } & 25 \\
\text { May } 25\end{array}$ & $\begin{array}{l}19.02 \\
19.19\end{array}$ & oct. $\begin{array}{r}1 \\
27\end{array}$ & $\begin{array}{l}20.72 \\
20.18\end{array}$ & $\begin{array}{lr}\text { Nov. } & 26 \\
\text { Dec. } & 9\end{array}$ & $\begin{array}{l}19.91 \\
19.90\end{array}$ \\
\hline
\end{tabular}

\section{SEDGWICK COUNTY}

By C. C. W11liams and G. H. von Heln

Highest and lowest water levels, in feet below measuring point, in 31 wells in Sedgw 1ck County, 1941

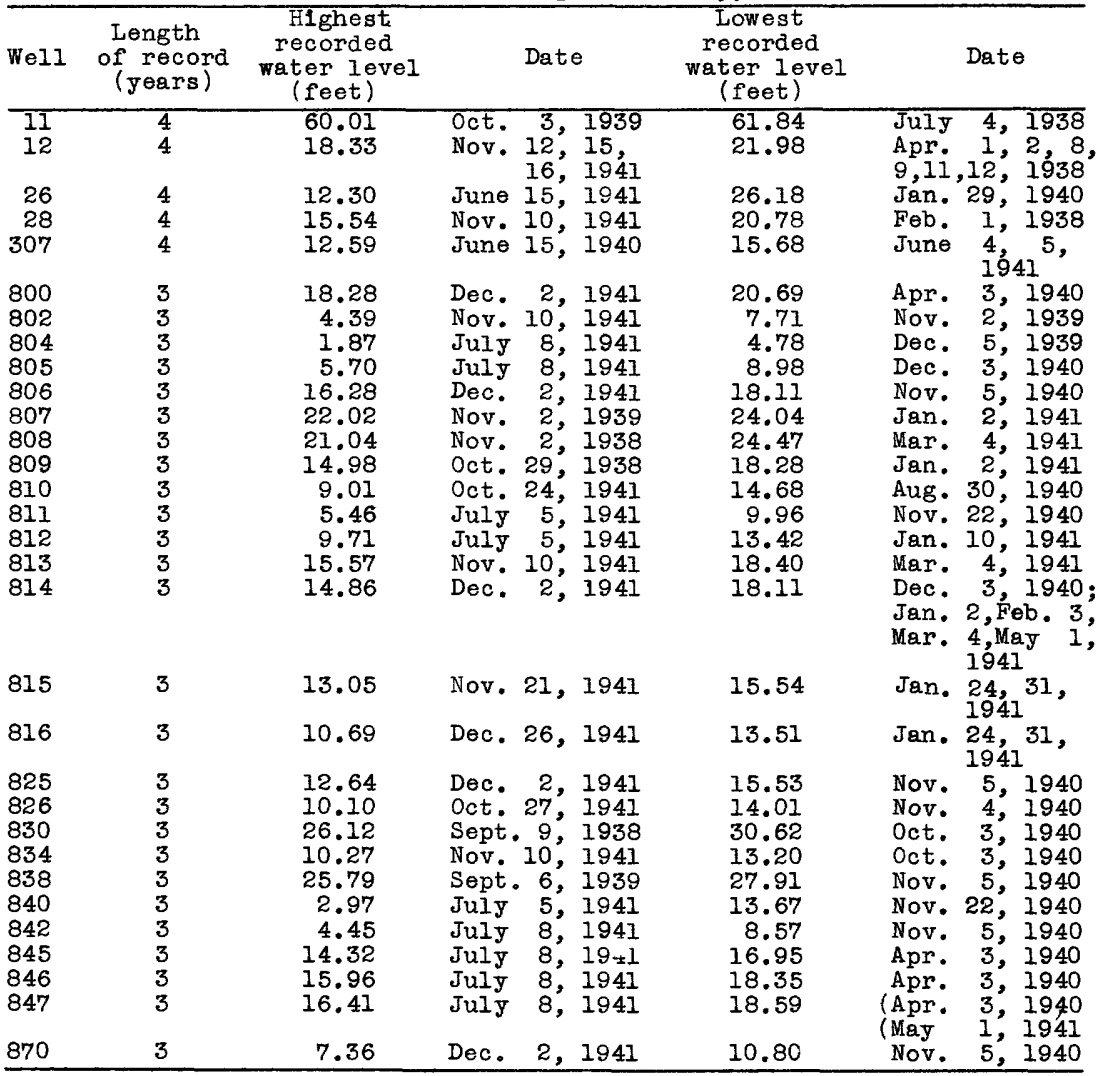




\section{Sedgwick County--Continued}

Net changes in water level in 1941, and net changes in water level for the period of record in $3 i$ wells in Sedgwick County

\begin{tabular}{|c|c|c|c|}
\hline We11 & $\begin{array}{l}\text { Difference } \\
\text { between highest } \\
\text { and lowest } \\
\text { water levels } \\
\text { (feet) }\end{array}$ & $\begin{array}{l}\text { Net rise (+) } \\
\text { or net decline } \\
(-) \text { In } 1941 \\
(\text { feet })\end{array}$ & $\begin{array}{l}\text { Net rise (t) } \\
\text { or net decline } \\
(-) \text { for period } \\
\text { of record } \\
\text { (feet) }\end{array}$ \\
\hline $\begin{array}{l}11 \\
12 \\
26 \\
28 \\
307 \\
800 \\
802 \\
804 \\
805 \\
806 \\
807 \\
808 \\
809 \\
810 \\
811 \\
812 \\
813 \\
814 \\
815 \\
816 \\
825 \\
826 \\
830 \\
834 \\
838 \\
840 \\
842 \\
845 \\
846 \\
847 \\
870\end{array}$ & $\begin{array}{r}1.83 \\
3.65 \\
13.88 \\
5.24 \\
3.09 \\
2.41 \\
3.32 \\
2.91 \\
3.28 \\
1.83 \\
2.02 \\
3.43 \\
3.30 \\
5.67 \\
4.50 \\
3.71 \\
2.83 \\
3.25 \\
2.49 \\
2.82 \\
2.89 \\
3.91 \\
4.50 \\
2.93 \\
2.12 \\
10.70 \\
4.12 \\
2.63 \\
2.39 \\
2.18 \\
3.44\end{array}$ & $\begin{array}{l}+0.42 \\
+2.49 \\
+1.25 \\
+3.73 \\
+1.19 \\
+2.18 \\
+1.12 \\
+1.87 \\
+1.07 \\
+1.78 \\
+1.93 \\
+1.06 \\
+3.14 \\
+2.34 \\
+2.70 \\
+3.32 \\
+2.58 \\
+3.25 \\
+2.38 \\
+2.74 \\
+2.66 \\
+2.23 \\
+2.26 \\
+2.16 \\
+1.98 \\
+7.68 \\
+2.91 \\
+\quad .60 \\
+1.78 \\
+1.07 \\
+2.78\end{array}$ & $\begin{array}{r}+0.35 \\
+2.75 \\
+4.62 \\
+4.05 \\
-1.05 \\
+.48 \\
-.47 \\
+.25 \\
+. .26 \\
+1.52 \\
+1.18 \\
+1.09 \\
+1.12 \\
+.19 \\
+1.45 \\
+2.19 \\
+2.02 \\
+1.97 \\
+1.61 \\
+1.47 \\
+1.56 \\
+.63 \\
-1.96 \\
+1.32 \\
+.46 \\
+6.75 \\
+2.56 \\
+.90 \\
+.02 \\
+. .25 \\
+1.57\end{array}$ \\
\hline
\end{tabular}

11. J. H. Heim. SE cor. sec. 22, T. $26 \mathrm{~S}, \mathrm{R}, 3 \mathrm{~W}$. Water level, in feet below measuring point, 1941

\begin{tabular}{|c|c|c|c|c|c|c|c|c|}
\hline Date & & $\begin{array}{l}\text { Water } \\
\text { level }\end{array}$ & Date & $\begin{array}{l}\text { Water } \\
\text { level }\end{array}$ & Date & $\begin{array}{l}\text { Water } \\
\text { level }\end{array}$ & Date & $\begin{array}{l}\text { Water } \\
\text { level }\end{array}$ \\
\hline $\begin{array}{l}\text { Jan. } \\
\text { Feb. } \\
\text { Mar. }\end{array}$ & $\begin{array}{l}2 \\
4 \\
4\end{array}$ & $\begin{array}{l}60.81 \\
60.88 \\
60.94\end{array}$ & $\begin{array}{ll}\text { Apr. } & 1 \\
\text { May } & 1 \\
\text { June } & 4\end{array}$ & $\begin{array}{l}60.92 \\
60.97 \\
60.96\end{array}$ & $\begin{array}{ll}\text { July } & 8 \\
\text { Aug. } & 4 \\
\text { Sept. } & 3\end{array}$ & $\begin{array}{l}60.81 \\
60.67 \\
60.60\end{array}$ & $\begin{array}{lr}\text { Oct. } 13 \\
\text { Nov. } 10 \\
\text { Dec. } 2\end{array}$ & $\begin{array}{l}60.55 \\
60.40 \\
60.39\end{array}$ \\
\hline
\end{tabular}

12. Dr. A. D. Updegraph. NW $\frac{1}{4} \mathrm{SE} \frac{1}{4}$ sec. 26, T. $25 \mathrm{~S},, \mathrm{R}, 1$ W. Lowest dally water level, in feet below measuring point, 1941

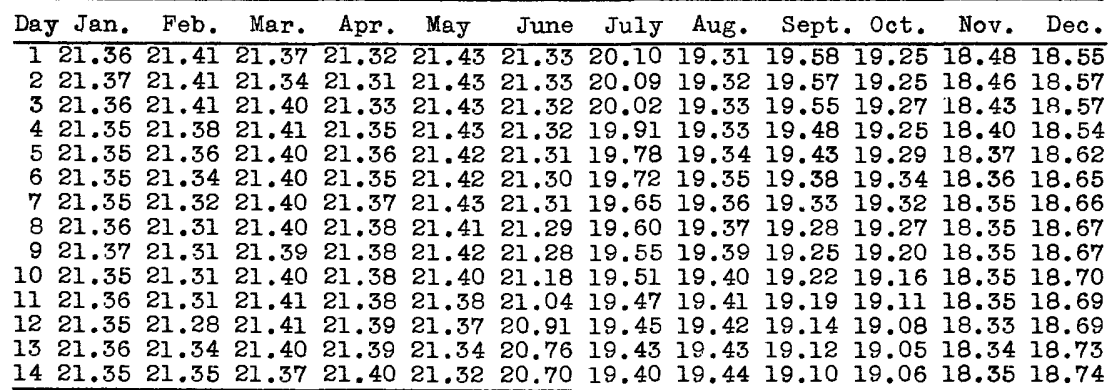
a Drawdown resulting from pumping in nearby well field. 
Sedgwick County--Continued

12. Dr. A. D. Updegraph--Continued.

Lowest dally water level, in feet below measuring point, 1941

Day Jan. Feb. Mar. Apr. May June July Aug. Sept. Oct. Nov. Dec.

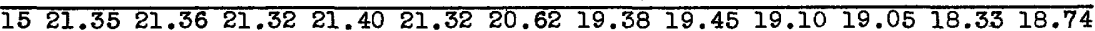

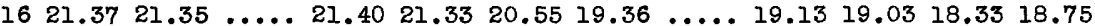
$1721.3921 .3521 .3521 .3821 .3420 .51 \quad 19.34 \ldots \ldots .19 .1319 .0018 .3418 .77$

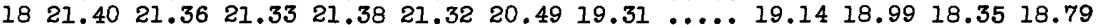

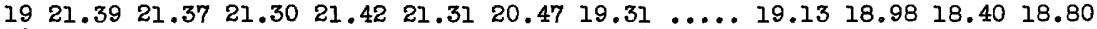

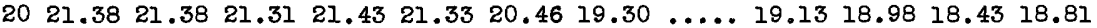
$2121.3821 .3621 .3321 .4421 .34 \ldots \ldots .19 .29 \ldots \ldots .19 .1518 .9818 .41 \quad 18.79$ $22 \quad 21.4021 .3621 .3121 .4321 .34 \ldots \ldots .19 .2719 .5019 .1618 .9818 .4318 .78$ $2321.4021 .35 \quad 21.32 \quad 21.4421 .3520 .22 \quad 19.27 \quad 19.5019 .16 \quad 18.96 \quad 18.45 \quad 18.83$ $2421.4121 .3621 .3321 .4421 .3420 .21 \quad 19.2719 .5019 .1618 .8813 .4618 .83$ $\begin{array}{lllllllllllll}25 & 21.41 & 21.36 & 21.32 & 21.44 & 21.32 & 20.20 & 19.27 & 19.51 & 19.20 & 18.83 & 18.45 & 18.83\end{array}$ $\begin{array}{llllllllllllll}26 & 21.43 & 21.37 & 21.31 & 21.43 & 21.31 & 20.18 & 19.28 & 19.54 & 19.21 & 18.71 & 18.47 & 18.86\end{array}$ $27 \quad 21.43 \quad 21.38 \quad 21.33 \quad 21.43 \quad 21.31 \quad 20.17 \quad 19.28 \quad 19.55 \quad 19.2618 .6718 .4918 .88$ $\begin{array}{lllllllllllll}28 & 21.43 & 21.39 & 21.33 & 21.43 & 21.32 & 20.16 & 19.28 & 19.55 & 19.25 & 18.63 & 18.51 & 18.88\end{array}$ $2921.40 \ldots \ldots .21 .33 \quad 21.42 \quad 21.33 \quad 20.14 \quad 19.28 \quad 19.56 \quad 19.2518 .5818 .5218 .87$

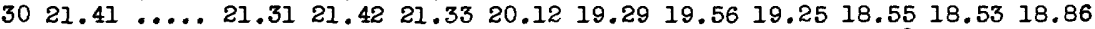

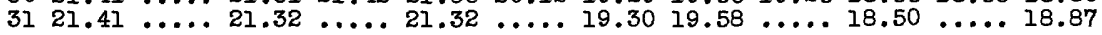

26. Wichita Water Co. SW $\frac{1}{4}$ sec. 18, T. 27 S., R. I W.

Lowest daily water level, in feet below measuring point, 1941

\begin{tabular}{|c|c|c|c|c|c|c|c|c|c|c|c|c|}
\hline & an & & & & & & $\mathbf{y}$ & & . & t. & 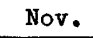 & \\
\hline $\begin{array}{l}1 \\
2 \\
3 \\
4 \\
5 \\
6 \\
7 \\
8 \\
9\end{array}$ & $\begin{array}{l}.02 \\
.04 \\
.03 \\
.06 \\
.07 \\
.06 \\
.07 \\
.00 \\
.00 \\
.99 \\
.00 \\
.98 \\
.97 \\
.97 \\
.99 \\
.06 \\
.08 \\
.06 \\
.97 \\
.96 \\
.91 \\
.90 \\
.93 \\
.97 \\
.00 \\
.98\end{array}$ & $\begin{array}{l}15.83 \\
15.80 \\
15.77 \\
15.75 \\
15.71 \\
15.71 \\
15.72 \\
15.72 \\
15.73 \\
15.72 \\
15.80 \\
15.77 \\
15.77 \\
15.76 \\
15.76 \\
15.76 \\
15.77 \\
15.78 \\
15.76 \\
15.77 \\
15.75 \\
15.72 \\
15.71\end{array}$ & $\begin{array}{l}15.8 \\
15.8 \\
15.7 \\
15.7 \\
15.7 \\
15.7 \\
15.6 \\
15.6 \\
15.6 \\
15.6 \\
15.6 \\
15.5 \\
1 \ldots .6 \\
13.5 \\
15.5 \\
15.5 \\
15.5 \\
15.5 \\
15.5 \\
15.5 \\
15.6 \\
15.6\end{array}$ & $\begin{array}{l}1 \\
1 \\
1 \\
1 \\
1 \\
1 \\
1 \\
1 \\
1 \\
1 \\
1 \\
1 \\
1 \\
1 \\
1 \\
1 \\
1 \\
1 \\
1\end{array}$ & $\begin{array}{l}5 \\
7 \\
5 \\
2 \\
0\end{array}$ & $\begin{array}{l}13.74 \\
13.83 \\
13.91 \\
13.99 \\
14.02 \\
14.02 \\
13.77 \\
13.09\end{array}$ & $\begin{array}{l}1 \\
1 \\
1 \\
1 \\
1 \\
1 \\
1 \\
1 \\
1 \\
1 \\
1 \\
1 \\
1 \\
1 \\
1 \\
1 \\
1 \\
1 \\
1 \\
1\end{array}$ & 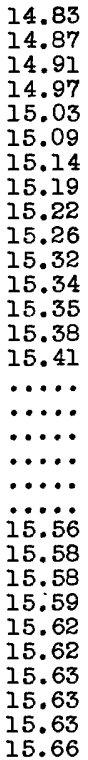 & $\begin{array}{l}5 \\
8 \\
2 \\
3 \\
1 \\
4 \\
8 \\
3\end{array}$ & & $E$ & \\
\hline
\end{tabular}

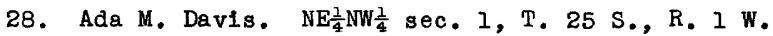

Water level, in feet below measuring point, 1941

\begin{tabular}{lll|lll|lll|lr}
\hline Date & $\begin{array}{c}\text { Water } \\
\text { level }\end{array}$ & Date & $\begin{array}{l}\text { Water } \\
\text { level }\end{array}$ & Date & $\begin{array}{l}\text { Water } \\
\text { level }\end{array}$ & Date & $\begin{array}{r}\text { Water } \\
\text { level }\end{array}$ \\
\hline Jan. & 2 & 19.67 & Apr. & 1 & 18.67 & July 8 & 16.16 & Oct. 13 & 17.01 \\
Feb. 3 & 19.26 & May & 1 & 18.60 & Aug. 4 & 17.42 & Nov. 10 & 15.54 \\
Mar. 4 & 19.23 & June & 4 & 18.71 & Sept. 3 & 17.76 & Dec. 2 & 15.94 \\
\hline
\end{tabular}


Sedgwick County--Continued

307. J. R. Clark. NW $\frac{1}{4} \mathrm{NW}_{\frac{1}{4}} \mathrm{SW} \frac{1}{4} \mathrm{sec} .1, \mathrm{~T} .25 \mathrm{~S} ., \mathrm{R} .2 \mathrm{~W}$.

Lowest daily water level, in feet below measuring point, 1941

Day Jan. Feb. Mar. Apr. May June July Aug. Sept. Oct. Nov. Dec.

$\begin{array}{llllllllllllllll}1 & 15.31 & 15.59 & 15.41 & 15.20 & 15.34 & 15.65 & 14.94 & 14.80 & 15.12 & 15.40 & 14.44 & 14.37\end{array}$

$\begin{array}{lllllllllllllll}2 & 15.32 & 15.57 & 15.39 & 15.23 & 15.38 & 15.66 & 14.94 & 14.34 & 15.10 & 15.40 & 14.43 & 14.38\end{array}$

$\begin{array}{lllllllllllllll}3 & 15.33 & 15.53 & 15.39 & 15.27 & 15.41 & 15.66 & 14.46 & 14.88 & 15.12 & 15.41 & 14.42 & 14.37\end{array}$

$\begin{array}{llllllllllllll}4 & 15.36 & 15.51 & 15.40 & 15.31 & 15.43 & 15.68 & 14.28 & 14.93 & 15.13 & 15.40 & 14.42 & 14.40\end{array}$

$\begin{array}{llllllllllllllll}5 & 15.38 & 15.50 & 15.39 & 15.32 & 15.43 & 15.58 & 14.19 & 14.97 & 15.13 & 15.42 & 14.44 & 14.47\end{array}$

$\begin{array}{lllllllllllllll}6 & 15.39 & 15.45 & 15.37 & 15.35 & 15.45 & 15.66 & 14.13 & 15.01 & 15.13 & 15.42 & 14.45 & 14.47\end{array}$

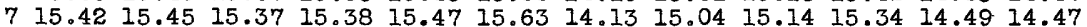

$\begin{array}{llllllllllllll}8 & 15.45 & 15.44 & 15.36 & 15.39 & 15.51 & 15.58 & 14.14 & 15.06 & 15.15 & 15.10 & 14.49 & 14.48\end{array}$

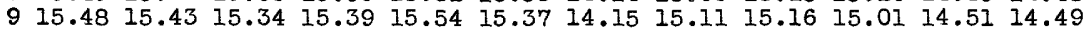

$\begin{array}{lllllllllllllll}10 & 15.49 & 15.43 & 15.30 & 15.39 & 15.56 & 15.02 & 14.16 & 15.12 & 15.18 & 14.90 & 14.54 & 14.50\end{array}$

$1115.5015 .42 \quad 15.21 \quad 15.43 \quad 15.57 \quad 14.8514 .18 \quad 15.16 \quad 15.2014 .82 \quad 14.5414 .50$

$\begin{array}{lllllllllllllllll}12 & 15.47 & 15.39 & 15.17 & 15.43 & 15.58 & 14.75 & 14.18 & 15.16 & 15.22 & 14.80 & 14.54 & 14.56\end{array}$

$13 \quad 15.4715 .40 \quad 15.14 \quad 15.43 \quad 15.6014 .72 \quad 14.18 \quad 15.15 \quad 15.24 \quad 14.8014 .52 \quad 14.59$

$\begin{array}{llllllllllllllllll}14 & 15.46 & 15.40 & 15.11 & 15.42 & 15.59 & 14.66 & 14.18 & 15.14 & 15.28 & 14.84 & 14.52 & 14.61\end{array}$

$\begin{array}{lllllllllllllll}15 & 15.45 & 15.40 & \ldots & 15.42 & 15.59 & 14.67 & 14.18 & 15.14 & 15.31 & 14.85 & 14.49 & 14.63\end{array}$

$16 \quad 15.4515 .38 \ldots \ldots .15 .4015 .6014 .71 \quad 14.18 \ldots \ldots .15 .3414 .8614 .47 \quad 14.64$

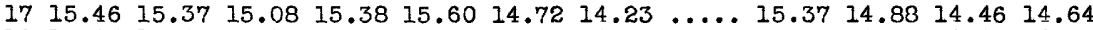

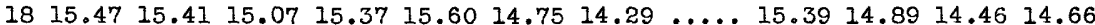

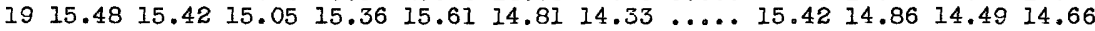

$2015.4915 .43 \quad 15.04 \quad 15.34 \quad 15.61 \quad 14.8514 .34 \ldots \ldots .15 .4414 .8214 .5014 .66$

$21 \quad 15.4915 .4315 .08 \quad 15.3415 .61 \ldots \ldots .14 .36 \ldots \ldots .45 .4814 .8014 .4914 .65$

$22 \quad 15.4915 .43 \quad 15.12 \quad 15.33 \quad 15.61 \ldots \ldots .14 .4015 .0915 .48 \quad 14.77 \quad 14.47 \quad 14.65$

$\begin{array}{lllllllllllllll}23 & 15.49 & 15.43 & 15.16 & 15.32 & 15.62 & 14.94 & 14.44 & 15.09 & 15.44 & 14.70 & 14.48 & 14.57\end{array}$

$24 \quad 15.5015 .43 \quad 15.19 \quad 15.32 \quad 15.62 \quad 14.95 \quad 14.47 \quad 15.08 \quad 15.43 \quad 14.65 \quad 14.46 \quad 14.41$

$\begin{array}{lllllllllllllllll}25 & 15.50 & 15.43 & 15.16 & 15.32 & 15.61 & 14.97 & 14.50 & 15.09 & 15.44 & 14.59 & 14.42 & 14.31\end{array}$

$26 \quad 15.5015 .4415 .13 \quad 15.32 \quad 15.61 \quad 14.98 \quad 14.52 \quad 15.0915 .44 \quad 14.53 \quad 14.3914 .26$

$\begin{array}{llllllllllllllll}27 & 15.53 & 15.44 & 15.12 & 15.31 & 15.61 & 14.99 & 14.54 & 15.09 & 15.45 & 14.48 & 14.38 & 14.24\end{array}$

$\begin{array}{llllllllllllllll}28 & 15.54 & 15.43 & 15.12 & 15.31 & 15.61 & 14.97 & 14.58 & 15.08 & 15.46 & 14.48 & 14.37 & 14.20\end{array}$

$\begin{array}{llllllllllllll}29 & 15.55 & \ldots & 15.10 & 15.31 & 15.62 & 14.94 & 14.65 & 15.09 & 15.44 & 14.48 & 14.36 & 14.17\end{array}$

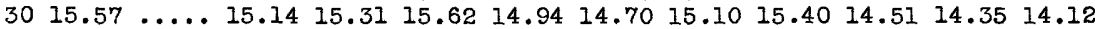

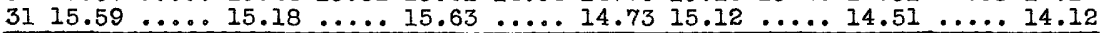

800. City of Wichita. SW cor. sec. 33, T. 26 S., R. I E.

Water level, in feet below measuring point, 1941

\begin{tabular}{|c|c|c|c|c|c|c|c|c|c|}
\hline \multicolumn{2}{|l|}{ Date } & \multirow{2}{*}{$\begin{array}{l}\text { Water } \\
\text { level } \\
20.46 \\
20.36 \\
20.34\end{array}$} & \multicolumn{2}{|l|}{ Date } & \multirow{2}{*}{$\begin{array}{l}\text { Water } \\
\text { level } \\
20.31 \\
20.01 \\
18.71\end{array}$} & \multirow{2}{*}{\multicolumn{2}{|c|}{ 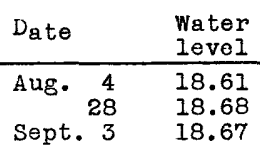 }} & \multirow{2}{*}{$\begin{array}{l}\text { Date } \\
\text { Oct. } 13 \\
\text { Nov. } 10 \\
\text { Dec. } 2\end{array}$} & \multirow{2}{*}{$\begin{array}{l}\text { Water } \\
\text { level } \\
18.57 \\
18.37 \\
18.28\end{array}$} \\
\hline $\begin{array}{l}\text { Jan. } \\
\text { Feb. } \\
\text { Mar. } \\
\text { Apr. }\end{array}$ & $\begin{array}{l}2 \\
3 \\
4 \\
1\end{array}$ & & $\begin{array}{l}\text { May } \\
\text { June } \\
\text { July }\end{array}$ & $\begin{array}{l}1 \\
4 \\
8\end{array}$ & & & & & \\
\hline
\end{tabular}

802. City of Wichita. NW cor. sec. 1, T. 27 S., R. 1 W.

Water level, in feet below measuring point, 1941

\begin{tabular}{lll|ll|lll|lll}
\hline Jan. & 2 & 7.07 & May & 1 & 6.75 & Aug. & 4 & 6.48 & oct. 13 & 6.46 \\
Feb. & 7 & 6.36 & June & 4 & 5.04 & & 8 & 6.47 & Nov. 10 & 4.39 \\
Mar. & 4 & 6.86 & July & 8 & 4.61 & Sept. 3 & 7.20 & Dec. & 2 & 5.95 \\
Apr. & 1 & 6.75 & & & & & & & & \\
\hline
\end{tabular}

804. City of Wichita. SE cor. sec. 16, T. 26 S., R. I W.

Water level, in feet below measuring point, 1941

\begin{tabular}{lll|lll|lll|ll}
\hline Jan. & 2 & 3.31 & Apr. & 1 & 3.09 & July & 8 & 1.87 & Oct. 13 & 3.00 \\
Feb. & 4 & 2.86 & May & 1 & 2.80 & Aug. 4 & 3.38 & Nov. 10 & 2.38 \\
Mar. 4 & 3.13 & June & 4 & 2.65 & Sept. 3 & 3.60 & Dec. 2 & 2.44 \\
\hline
\end{tabular}

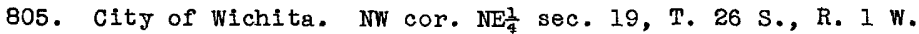

Water level, in feet below measuring point, 1941

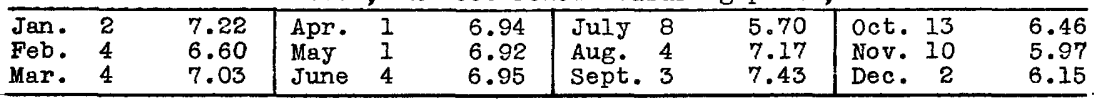


Sedgwick County--Continued

806. City of Wichita. NW cor. SW $\frac{2}{4}$ sec. 16, T. 26 S., R. 2 W. Water level, in feet below measuring point, 1941

\begin{tabular}{lr|ll|lll|lr}
\hline Date & $\begin{array}{l}\text { Water } \\
\text { level }\end{array}$ & Date & $\begin{array}{l}\text { Water } \\
\text { level }\end{array}$ & Date & $\begin{array}{l}\text { Water } \\
\text { level }\end{array}$ & Date & $\begin{array}{r}\text { Water } \\
\text { level }\end{array}$ \\
\hline Jan. 2 & 18.06 & Apr. & 1 & 17.72 & July 8 & 16.39 & Oct. 13 & 16.94 \\
Feb. 14 & 17.91 & May & 1 & 17.78 & Aug. 4 & 16.67 & Nov. 10 & 16.33 \\
Mar. 4 & 17.91 & June 4 & 17.47 & Sept. 3 & 16.97 & Dec. 2 & 16.28 \\
\hline
\end{tabular}

807. City of Wichita. NW cor. sec. 10, T. 26 S., R. 2 W.

Water level, in feet below measuring point, 1941

\begin{tabular}{lrr|lll|lll|lr}
\hline Jan. & 2 & 24.04 & Apr. & 1 & 23.71 & July & 8 & 22.16 & Oct. 13 & 22.87 \\
Feb. 14 & 23.92 & May & 1 & 23.75 & Aug. 4 & 22.25 & Nov. 10 & 22.34 \\
Mar. 4 & 23.85 & June & 4 & 23.39 & Sept. 3 & 22.64 & Dec. 2 & 22.11 \\
\hline
\end{tabular}

808. City of Wichita. SW cor. NW $\frac{1}{4}$ sec. 18, T. 26 S., R. 2 W.

Water level, in feet below measuring point, 1941

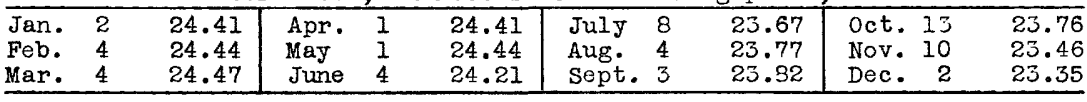

809. City of Wichita. NW cor. sec. 2l, T. 26 S., R. I W.

Water level, in feet below measuring point, 1941

\begin{tabular}{|c|c|c|c|c|c|c|c|c|c|c|}
\hline $\begin{array}{l}\text { Jan. } \\
\text { Feb. } \\
\text { Mar. } \\
\text { Apr. }\end{array}$ & $\begin{array}{l}2 \\
3 \\
4 \\
1\end{array}$ & $\begin{array}{l}18.28 \\
18.13 \\
17.17 \\
17.44\end{array}$ & $\begin{array}{l}\text { May } \\
\text { June } \\
\text { July }\end{array}$ & $\begin{array}{l}1 \\
4 \\
8\end{array}$ & $\begin{array}{l}17.01 \\
16.66 \\
15.43\end{array}$ & $\begin{array}{lr}\text { Aug. } & 4 \\
& 28 \\
\text { Sept. } & 3\end{array}$ & $\begin{array}{l}15.57 \\
15.76 \\
15.84\end{array}$ & $\begin{array}{l}\text { Oct. } \\
\text { Nov. } \\
\text { Dec. }\end{array}$ & 13 & $\begin{array}{l}15.83 \\
15.27 \\
15.14\end{array}$ \\
\hline
\end{tabular}

810. City of Wichita. IJE cor. SE⿺ sec. 35, T. 25 S., R. 1 W.

Water level, in feet below measuring point, 1941

\begin{tabular}{|c|c|c|c|c|c|c|c|c|c|c|}
\hline Jan. & $\begin{array}{r}3 \\
10 \\
17 \\
24 \\
31 \\
7 \\
14 \\
21 \\
28 \\
7 \\
17 \\
21 \\
28\end{array}$ & $\begin{array}{l}14.35 \\
14.37 \\
14.52 \\
14.34 \\
14.36 \\
14.06 \\
14.10 \\
14.14 \\
14.16 \\
14.17 \\
13.91 \\
13.93 \\
14.01\end{array}$ & $\begin{array}{l}\text { Apr. } \\
\text { May } \\
\text { June } \\
\text { July }\end{array}$ & $\begin{array}{r}4 \\
11 \\
18 \\
25 \\
2 \\
9 \\
16 \\
23 \\
29 \\
6 \\
13 \\
1 \\
5\end{array}$ & $\begin{array}{r}14.05 \\
14.08 \\
14.04 \\
14.09 \\
13.90 \\
13.61 \\
13.69 \\
13.75 \\
13.77 \\
13.56 \\
10.46 \\
12.01 \\
9.39\end{array}$ & $\begin{array}{rr}\text { July } 11 \\
18 \\
25 \\
\text { Aug. } \\
1 \\
8 \\
22 \\
29 \\
\text { Sept. } 5 \\
12 \\
18 \\
26 \\
\text { oct. } 3\end{array}$ & $\begin{array}{l}11.00 \\
11.65 \\
12.10 \\
12.43 \\
12.68 \\
12.88 \\
12.94 \\
11.34 \\
11.30 \\
12.03 \\
12.53 \\
12.31\end{array}$ & $\begin{array}{c}\text { Oct. } \\
\text { Nov. } \\
\text { Dec. }\end{array}$ & $\begin{array}{r}10 \\
17 \\
24 \\
31 \\
7 \\
14 \\
21 \\
28 \\
5 \\
12 \\
19 \\
26\end{array}$ & $\begin{array}{r}10.88 \\
11.23 \\
9.01 \\
9.84 \\
10.17 \\
10.94 \\
11.38 \\
11.67 \\
11.92 \\
12.09 \\
12.28 \\
12.01\end{array}$ \\
\hline
\end{tabular}

811. City of Wichita. SE cor. sec. 33, T. 25 S., R. 1 W.

Water level, in feet below measuring point, 1941

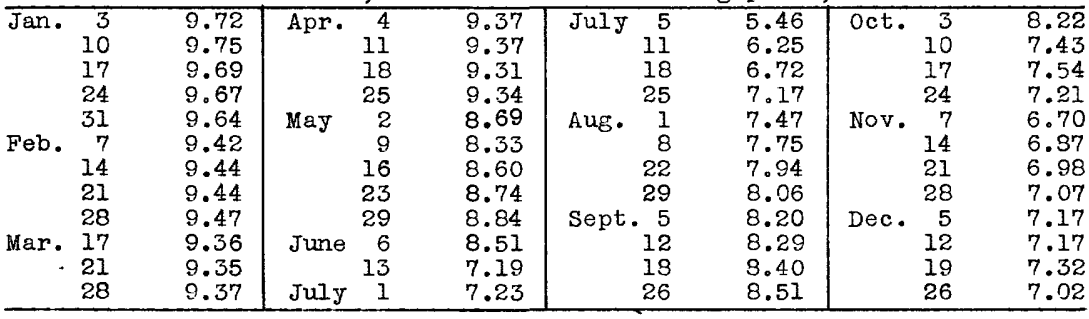


Sedgwick County--Continued

812. City of Wichita. NW cor. sec. 27, T. 25 S., R. 1 W. Water level, in feet below measuring point, 1941

\begin{tabular}{|c|c|c|c|c|c|c|c|c|}
\hline Date & & $\begin{array}{l}\text { Water } \\
\text { level }\end{array}$ & Date & $\begin{array}{l}\text { Water } \\
\text { level }\end{array}$ & Date & $\begin{array}{l}\text { Water } \\
\text { level }\end{array}$ & Date & $\begin{array}{l}\text { Water } \\
\text { level }\end{array}$ \\
\hline Jan. & $\begin{array}{r}3 \\
10 \\
17 \\
24 \\
31 \\
7 \\
14 \\
21 \\
28 \\
7 \\
17 \\
21 \\
28\end{array}$ & $\begin{array}{l}13.40 \\
13.42 \\
13.39 \\
13.41 \\
13.38 \\
13.18 \\
13.25 \\
13.21 \\
13.24 \\
13.24 \\
13.10 \\
13.10 \\
13.11\end{array}$ & $\begin{array}{lr}\text { Apr. } & 4 \\
& 11 \\
& 14 \\
& 18 \\
& 25 \\
\text { May } & 2 \\
& 9 \\
& 16 \\
& 23 \\
& 29 \\
\text { June } & 6 \\
& 13 \\
\text { July } & 1\end{array}$ & $\begin{array}{l}13.09 \\
13.11 \\
13.10 \\
13.05 \\
13.08 \\
13.01 \\
12.64 \\
12.65 \\
12.66 \\
12.66 \\
12.44 \\
11.18 \\
12.00\end{array}$ & $\begin{array}{l}5 \\
\text { July } \\
11 \\
18 \\
25 \\
\text { Aug. } 1 \\
8 \\
22 \\
29 \\
\text { Sept. } 5 \\
12 \\
18 \\
26 \\
\text { oct. } 3\end{array}$ & $\begin{array}{r}9.71 \\
10.02 \\
10.12 \\
10.29 \\
10.39 \\
10.56 \\
10.74 \\
10.82 \\
10.86 \\
10.87 \\
11.00 \\
11.13 \\
11.02\end{array}$ & $\begin{array}{lr}10 \\
17 \\
24 \\
31 \\
\text { Nov. } 7 \\
14 \\
21 \\
28 \\
\text { Dec. } 5 \\
12 \\
19 \\
26\end{array}$ & $\begin{array}{r}10.47 \\
10.47 \\
10.21 \\
9.98 \\
9.80 \\
9.90 \\
9.98 \\
10.04 \\
10.16 \\
10.16 \\
10.31 \\
10.08\end{array}$ \\
\hline
\end{tabular}

813. City of Wichita. SW $\frac{1}{2} S W_{\frac{2}{4}}^{2}$ sec. 24, T. 25 S., R. 1 W.

Water level, in feet below measuring point, 1941

\begin{tabular}{lll|lll|lll|ll}
\hline Jan. & 2 & 18.36 & Apr. & 1 & 18.31 & July & 8 & 15.80 & Oct. 13 & 15.98 \\
Feb. & 3 & 18.39 & May & 1 & 18.35 & Aug. & 4 & 16.20 & Nov. 10 & 15.57 \\
Mar. & 4 & 18.40 & June & 4 & 18.28 & Sept. 3 & 16.40 & Dec. 2 & 15.78 \\
\hline
\end{tabular}

814. City of Wichita. SE cor. sec. 14, T. 25 S., R. I W.

Water level, in feet below measuring point, 1941

\begin{tabular}{lll|lll|lll|lr}
\hline Jan. & 2 & 18.11 & Apr. & 1 & 18.07 & July & 8 & 15.94 & Oct. 13 & 15.41 \\
Feb. & 3 & 18.11 & May & 1 & 18.11 & Aug. & 4 & 15.61 & Nov. 10 & 14.91 \\
Mar. & 4 & 18.11 & June & 4 & 17.95 & Sept. 3 & 15.67 & Dec. 2 & 14.86 \\
\hline
\end{tabular}

815. City of Wichita. NE cor. sec. 17, T. 25 S., R. 1 W.

Vater level, in feet below measuring point, 1941

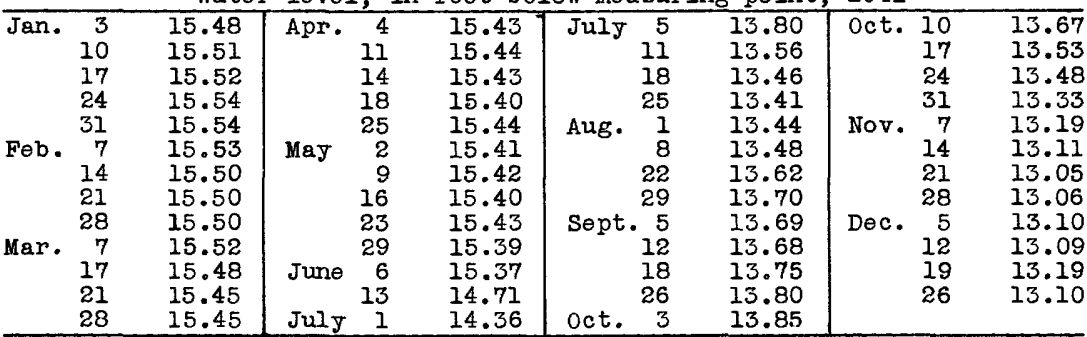

816. C1ty of Wichita. SW cor. sec. 7, T. 25 S., R. I W.

Water level, in feet below measuring point, 1941

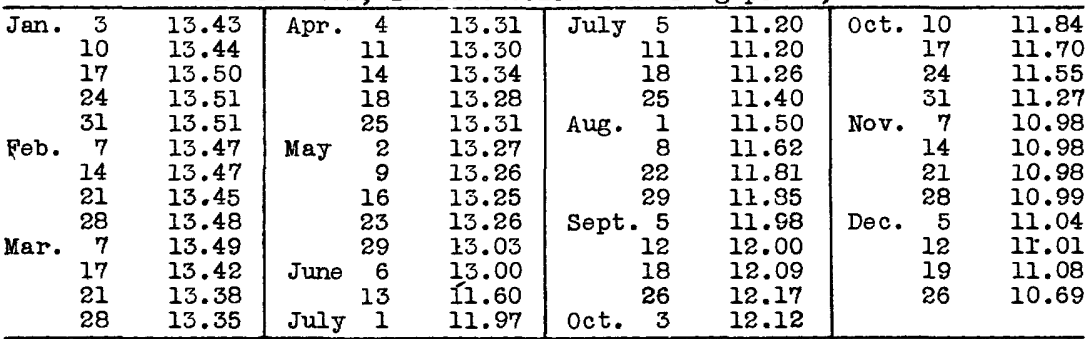


Sedgwick County--Continued

825. City of wichita. NE cor. sec. 3, T. 25 S., R. I W. Water level, in feet below measuring point, 1941

\begin{tabular}{lll|ll|lll|lr}
\hline Date & $\begin{array}{l}\text { Water } \\
\text { level }\end{array}$ & Date & $\begin{array}{l}\text { Water } \\
\text { level }\end{array}$ & Date & $\begin{array}{l}\text { Water } \\
\text { level }\end{array}$ & Date & $\begin{array}{r}\text { Water } \\
\text { level }\end{array}$ \\
\hline Jan. 2 & 15.30 & Apr. 1 & 14.89 & July 8 & 12.82 & Oct. 13 & 13.49 \\
Feb. 3 & 15.17 & May & 1 & 14.77 & Aug. 4 & 13.05 & Nov. 10 & 12.76 \\
Mar. 4 & 15.08 & June 4 & 14.59 & Sept. 3 & 13.41 & Dec. 2 & 12.64 \\
\hline
\end{tabular}

826. City of Wichita. NE cor. sec. 5, T. 25 S., R. I W.

Water level, in feet below measuring point, 1941

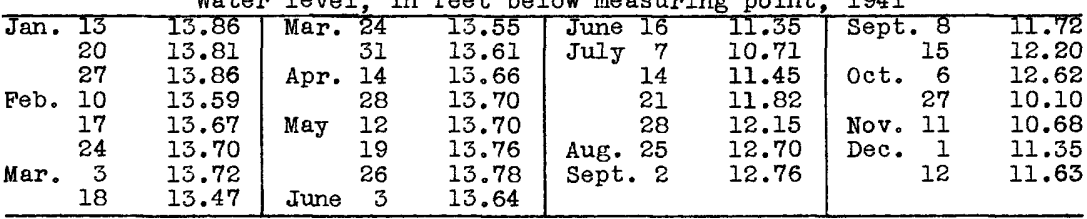

830. City of Wichita. SW cor. sec. 30, T. 25 S., R. 2 W.

Water level, in feet below measuring point, 1941

\begin{tabular}{lll|lll|lll|ll}
\hline Jan. & 2 & 30.34 & Apr. & 1 & 29.81 & July & 8 & 28.18 & Oct. 13 & 29.20 \\
Feb. & 4 & 30.08 & May & 1 & 29.84 & Aug. & 4 & 29.11 & Nov. 10 & 28.07 \\
Mar. & 4 & 30.04 & June & 4 & 29.74 & Sept. 3 & 29.63 & Dec. & 2 & 28.08 \\
\hline
\end{tabular}

834. City of Wichita. SW cor. sec. 9, T. 25 S., R. 3 W.

Water level, in feet below measuring point, 1941

\begin{tabular}{lll|lll|lll|lr}
\hline $\operatorname{Jan}$. & 2 & 12.64 & Apr. & 1 & 12.17 & July & 8 & 10.34 & Oct. 13 & 11.29 \\
Feb. & 4 & 12.39 & May & 1 & 12.28 & Aug. & 4 & 11.57 & Nov. 10 & 10.27 \\
Mar. & 4 & 12.36 & June & 4 & 12.44 & Sept. 3 & 12.20 & Dec. & 2 & 10.48 \\
\hline
\end{tabular}

838. City of Wichita. NE cor. NW

Water level, in feet below measuring point, 1941

\begin{tabular}{lrr|lll|lll|ll}
\hline Jan. & 2 & 27.88 & Apr. & 1 & 27.80 & July & 8 & 26.32 & Oct. 13 & 26.64 \\
Feb. 14 & 27.87 & May & 1 & 27.84 & Aug. 4 & 26.74 & Nov. 10 & 26.00 \\
Mar. 4 & 27.87 & June & 4 & 27.85 & Sept. 3 & 27.06 & Dec. & 2 & 25.90 \\
\hline
\end{tabular}

840. Owner of property, C. A. Berger; owner of well, city of wichita. NE cor. sec. 9, T. 25 S., R. 2 W.

Water level, in feet below measuring point, 1941

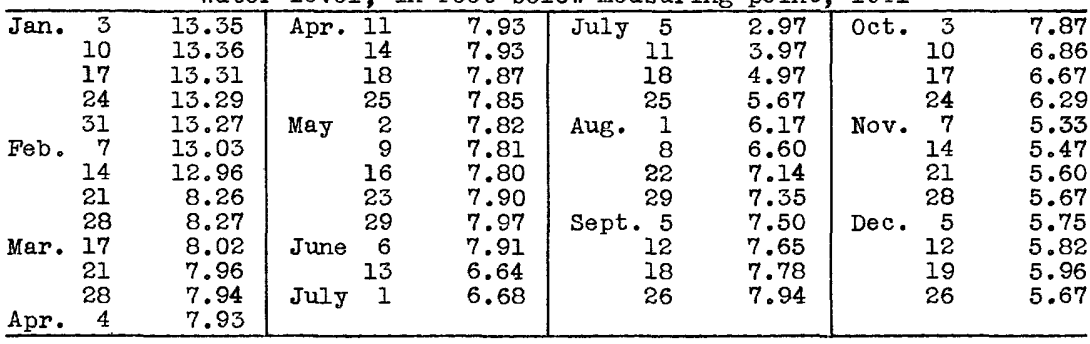

842. City of Wichita. WW cor. sec. 16, T. 25 S., R. 2 W.

Water level, in feet below measuring point, 1941

\begin{tabular}{lll|ll|lll|lll}
\hline Jan. & 2 & 7.90 & Apr. & 1 & 6.88 & July & 8 & 4.45 & Oct. 13 & 6.18 \\
Feb. & 4 & 7.48 & May & 1 & 6.87 & Aug. 4 & 6.40 & Nov. 10 & 4.74 \\
Mar. & 4 & 7.27 & June & 4 & 6.61 & Sept. 3 & 7.22 & Dec. 2 & 4.99 \\
\hline
\end{tabular}


Sedgwick County--Continued

845. City of W1chita. SW Water level, in feet below measuring point, 1941

\begin{tabular}{lll|ll|ll|ll|l}
\hline Date & $\begin{array}{l}\text { Water } \\
\text { level }\end{array}$ & Date & $\begin{array}{l}\text { Water } \\
\text { level }\end{array}$ & Date & $\begin{array}{r}\text { Vater } \\
\text { level }\end{array}$ & Date & $\begin{array}{r}\text { Water } \\
\text { level }\end{array}$ \\
\hline Jan. & 2 & 15.47 & Apr. & 1 & 15.36 & July 8 & 14.32 & Oct. 13 & 14.74 \\
Feb. 4 & 15.23 & May & 1 & 15.38 & Aug. 4 & 15.06 & Nov. 10 & 14.49 \\
Mar. 4 & 15.43 & June 4 & 15.17 & Sept. 3 & 14.37 & Dec. 2 & 14.87 \\
\hline
\end{tabular}

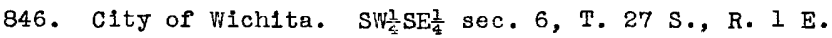
Water level, in feet below measuring point, 1941

\begin{tabular}{|c|c|c|c|c|c|c|c|c|c|c|c|}
\hline $\begin{array}{l}\text { Jan. } \\
\text { Feb. } \\
\text { Mar. }\end{array}$ & $\begin{array}{l}2 \\
4 \\
4\end{array}$ & $\begin{array}{l}17.88 \\
17.43 \\
17.87\end{array}$ & $\begin{array}{l}\text { Apr. } \\
\text { May } \\
\text { June }\end{array}$ & $\begin{array}{l}1 \\
1 \\
4\end{array}$ & $\begin{array}{l}17.81 \\
17.88 \\
17.58\end{array}$ & $\begin{array}{l}\text { July } \\
\text { Aug. } \\
\text { Sept. }\end{array}$ & $\begin{array}{l}8 \\
4 \\
3\end{array}$ & $\begin{array}{l}15.96 \\
17.42 \\
16.46\end{array}$ & $\begin{array}{l}\text { Oct. } \\
\text { Nov. } \\
\text { Dec. }\end{array}$ & $\begin{array}{r}13 \\
10 \\
2\end{array}$ & $\begin{array}{l}16.82 \\
16.44 \\
17.10\end{array}$ \\
\hline
\end{tabular}

847. City of Wichita. SW cor. SE $\frac{1}{4}$ sec. 6, T. 27 S., R. I E. Water level, in feet below measuring point, 1941

\begin{tabular}{lll|ll|lll|llr}
\hline Jan. & 2 & 18.47 & Apr. & 1 & 18.47 & July & 8 & 16.41 & Oct. 13 & 17.84 \\
Feb. & 4 & 18.39 & May & 1 & 18.59 & Aug. & 4 & 17.76 & Nov. 10 & 16.86 \\
Mar. & 4 & 18.50 & June & 4 & 18.27 & Sept. 3 & 18.18 & Dec. & 2 & 17.40 \\
\hline
\end{tabular}

870. W. W1lliams. $\mathrm{NW}_{\frac{1}{4}} \mathrm{NE} \frac{\mathrm{l}}{4}$ sec. 18, T. $25 \mathrm{~S} ., \mathrm{R}, 2 \mathrm{~W}$. Water level, in feet below mea uring point, 1941

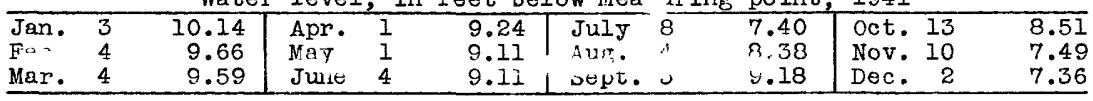

SEWARD COUNTY

By C. C. W111iams

Highest and lowest water levels, in feet below measuring point, in 8 wells in Seward County, 1941

\begin{tabular}{rcclrl}
\hline We11 & $\begin{array}{c}\text { Length of } \\
\text { record } \\
\text { (years) }\end{array}$ & $\begin{array}{c}\text { H1ghest } \\
\text { recorded } \\
\text { wer level } \\
\text { (feet) }\end{array}$ & Date & $\begin{array}{c}\text { Lowest } \\
\text { recorded } \\
\text { water level } \\
\text { (feet) }\end{array}$ & Date \\
\hline 15 & 1 & 17.13 & Nov. 1, 1941 & 18.20 & July 10, 1940 \\
52 & 1 & 207.81 & Mar. 14, 1941 & 209.25 & June 18, 1941 \\
66 & 1 & 216.88 & Nov. 1, 1941 & 218.99 & Dec. 17, 1941 \\
106 & 1 & 208.60 & May 13, 1941 & 209.22 & July 19, 1941 \\
108 & 1 & 108.69 & Dec. 17, 1941 & 111.78 & Apr. 21, 1941 \\
122 & 1 & 204.08 & July 19, 1941 & 205.63 & Aug. 5, 1940 \\
159 & 1 & 96.14 & Jan. 7, 1941 & 97.25 & Dec. 19, 1940 \\
165 & 1 & 147.30 & Nov. 28, 1941 & 168.28 & Dec. 18, 1941 \\
\hline
\end{tabular}

Net changes in water level in 1941, and net changes in water level for the perlod of record in 8 wells in seward county

\begin{tabular}{|c|c|c|c|}
\hline Well & $\begin{array}{l}\text { Difference } \\
\text { between highest } \\
\text { and lowest } \\
\text { water levels } \\
\text { (feet) }\end{array}$ & $\begin{array}{l}\text { Net rise (+) } \\
\text { or net decline } \\
(-) \text { in } 1941 \\
(\text { feet })\end{array}$ & $\begin{array}{l}\text { Net rise (+) } \\
\text { or net decline } \\
\text { (-) for period } \\
\text { of record } \\
(\text { feet) }\end{array}$ \\
\hline $\begin{array}{l}15 \\
52 \\
66\end{array}$ & $\begin{array}{l}1.07 \\
1.44 \\
2.11\end{array}$ & $\begin{array}{l}-0.05 \\
-1.05 \\
-1.71\end{array}$ & $\begin{array}{l}+0.51 \\
-.99 \\
-1.43\end{array}$ \\
\hline
\end{tabular}




\section{Seward County--Continued}

Net changes in water level in 1941, and net changes in water level

for the period of record in 8 welis in Seward County--Continued.

\begin{tabular}{|c|c|c|c|}
\hline WeII & $\begin{array}{l}\text { Difference } \\
\text { between highest } \\
\text { and lowest } \\
\text { water levels } \\
(f e \theta t)\end{array}$ & $\begin{array}{l}\text { Net rise (t) } \\
\text { or net decline } \\
(-) \text { in } 1941 \\
(\text { feet })\end{array}$ & $\begin{array}{l}\text { Net rise (+) } \\
\text { or ret decline } \\
(-) \text { frr period } \\
\text { os iecord } \\
\text { (feet) }\end{array}$ \\
\hline $\begin{array}{l}106 \\
108 \\
122 \\
159 \\
165 \\
\end{array}$ & $\begin{array}{r}0.62 \\
3.09 \\
1.55 \\
1.11 \\
20.98 \\
\end{array}$ & $\begin{array}{r}+0.20 \\
+2.83 \\
+.26 \\
-.80 \\
-17.28 \\
\end{array}$ & $\begin{array}{r}+0.22 \\
+2.64 \\
+.64 \\
+.14 \\
-17.36 \\
\end{array}$ \\
\hline
\end{tabular}

Water-level measurements

8. Liberal Deep Well Company. NW $\frac{1}{4} \mathrm{NE} \frac{1}{4}$ sec. 32, T. 34 S., R. 33 W. Measurements discontinued after May 13, 1941.

Water level, in feet below measuring point, 1941

\begin{tabular}{|c|c|c|c|c|c|}
\hline Date & $\begin{array}{l}\text { Water } \\
\text { level }\end{array}$ & Date & $\begin{array}{l}\text { Water } \\
\text { level }\end{array}$ & Date & $\begin{array}{l}\text { Water } \\
\text { Ievel }\end{array}$ \\
\hline $\begin{array}{ll}\text { Jan. } & 7 \\
\text { Feb. } & 5\end{array}$ & $\begin{array}{l}122.48 \\
122.11\end{array}$ & $\begin{array}{l}\text { Mar. } 14 \\
\text { Apr. } 21\end{array}$ & $\begin{array}{l}122.89 \\
121.94\end{array}$ & May 13 & 121.83 \\
\hline
\end{tabular}

15. R. H. Hitch. SE $\frac{1}{4} \mathrm{SW}_{\frac{1}{4}}$ sec. 2I, T. 32 S., R. 33 W.

Water level, in feet below measuring point, 1941

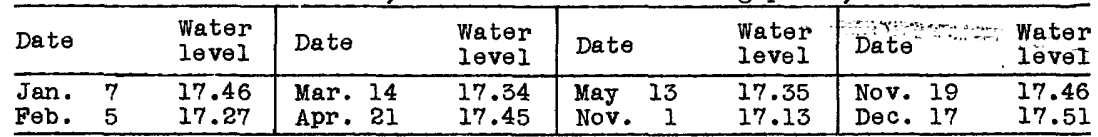

52. Federal Farm Mortgage Co. NW $\frac{1}{4} N W_{4}^{\frac{1}{4}}$ sec. 22, T. 34 S., R. 32 W.

Water level, in feet below measuring point, 1941

\begin{tabular}{|c|c|c|c|c|c|}
\hline $\begin{array}{ll}\text { an. } 7 \\
\text { eb. } 5\end{array}$ & $\begin{array}{l}208.08 \\
209.10\end{array}$ & $\begin{array}{l}\text { Mar. } 14 \\
\text { Apr. } 21\end{array}$ & $\begin{array}{l}207.81 \\
208.22\end{array}$ & $\begin{array}{l}\text { May } \\
\text { June I }\end{array}$ & $\begin{array}{l}13 \\
18\end{array}$ \\
\hline
\end{tabular}

60. Lee Swan. NN $\frac{1}{4} \mathrm{NE} \frac{1}{4}$ sec. 21, T. 33 S., R. 33 W. Measurements discontinued after June 18, 1941. Water levels, in feet below measuring point, 1941: Mar. 14, 182.36; Apr. 21, 182.64; June 18, 182.75.

66. Federal Land Bank. NW $\frac{1}{4}$ SW $\frac{1}{4}$ sec. 35, T. 34 S., R. 31 W.

Water level, in feet below measuring point, 1941

\begin{tabular}{rr|rr|rr|rrr}
\hline Feb. 5 & 217.28 & Apr. 21 & 217.98 & Nov. 1 & 216.88 & Dec. 17 & 218.99 \\
Mar. 14 & 217.48 & June 18 & 216.92 & & 28 & 217.24 & & \\
\hline
\end{tabular}

106. Kansas City L1fe Insurance Company. SW $\frac{1}{4} \mathrm{SW} \frac{1}{4}$ sec. 8, T. $32 \mathrm{~S}$. , R. $34 \mathrm{~W}$.

Water level, in feet below measuring point, 1941

\begin{tabular}{rrr|lr|lr|llr}
\hline Jan. & 7 & 209.02 & Apr. 21 & 209.13 & July 19 & 209.22 & Nov. 1 & 208.86 \\
Feb. 5 & 208.76 & May 13 & 208.60 & Aug. 15 & 209.05 & & 19 & 208.79 \\
Mar. 14 & 208.84 & June & 8 & 208.89 & Sept.19 & 208.89 & Dec. 17 & 208.82 \\
\hline
\end{tabular}

108. C. D. Day. NE $\frac{1}{4} \mathrm{SE} \frac{1}{4}$ sec. 6, T. 31 S., R. 34 W.

Water level, in feet below measuring point, 1941

\begin{tabular}{lr|lr|lr}
\hline Date & $\begin{array}{r}\text { Water } \\
\text { level }\end{array}$ & Date & $\begin{array}{r}\text { Water } \\
\text { level }\end{array}$ & Date & $\begin{array}{r}\text { Water } \\
\text { level }\end{array}$ \\
\hline Jan. 7 & 111.52 & Apr. 21 & 111.78 & Nov. 1 & 108.76 \\
Mar. 14 & III.44 & Aug. 15 & 109.56 & Dec. 17 & 108.69 \\
\hline
\end{tabular}


Seward county--Continued

122. Mrs. Flora Atwell. $\mathrm{NE}_{\frac{4}{4}}^{\frac{1}{4}} \mathrm{SE}_{\frac{1}{4}}^{\frac{1}{4}} \mathrm{sec} .9, \mathrm{~T} .33 \mathrm{~S}, \mathrm{R} .31 \mathrm{~W}$. Water level, in feet below measuring point, 1941

\begin{tabular}{lrr|lr|ll|lrr}
\hline Date & $\begin{array}{l}\text { Watar } \\
\text { level }\end{array}$ & Date & $\begin{array}{l}\text { Water } \\
\text { level }\end{array}$ & Date & $\begin{array}{r}\text { Water } \\
\text { level }\end{array}$ & Date & $\begin{array}{r}\text { Water } \\
\text { level }\end{array}$ \\
\hline Jan. 7 & 205.25 & Apr. 21 & 205.29 & July 19 & 204.08 & Nov. 1 & 204.90 \\
Feb. 5 & 204.97 & May 13 & 204.97 & Aug. 15 & 204.90 & 28 & 204.89 \\
Mar. 14 & 204.86 & June 18 & 204.83 & Sept.19 & 204.94 & Dec. 17 & 204.99 \\
\hline
\end{tabular}

155. Fred Collingwood. $\mathrm{SE}_{\frac{1}{4}}^{\frac{1}{4} \mathrm{SE}} \frac{\mathrm{I}}{4} \mathrm{sec} .11, \mathrm{~T} .31$ S., R. 31 W. Measurements discontinued after Juiy 19,1941 .

Water level, in feet below measuring point, 1941

\begin{tabular}{lrr|l|lr}
\hline Date & $\begin{array}{r}\text { Water } \\
\text { level }\end{array}$ & Date & $\begin{array}{r}\text { Water } \\
\text { level }\end{array}$ & Date & $\begin{array}{r}\text { Water } \\
\text { level }\end{array}$ \\
\hline Jan. 7 & 157.53 & Mar. 14 & 157.34 & May 13 & 157.52 \\
Feb. 5 & 157.39 & Apr. 21 & 157.65 & July 19 & 157.61 \\
\hline
\end{tabular}

159. Liberal Gas Company. $\mathrm{NW}_{\frac{1}{4}} \mathrm{NE} \frac{1}{4} \mathrm{sec}, 3, \mathrm{~T}, 35 \mathrm{~S} ., \mathrm{R} .34 \mathrm{~W}$.

Water level, in feet below measuring point, 1941

\begin{tabular}{|c|c|c|c|c|c|c|c|}
\hline Date & $\begin{array}{l}\text { Water } \\
\text { level }\end{array}$ & Date & $\begin{array}{l}\text { Water } \\
\text { level }\end{array}$ & Date & $\begin{array}{l}\text { Water } \\
\text { level }\end{array}$ & Date & $\begin{array}{l}\text { Water } \\
\text { level }\end{array}$ \\
\hline $\begin{array}{lr}\text { Jan. } & 7 \\
\text { Feb. } & 5 \\
\text { Mar. } & 14\end{array}$ & $\begin{array}{l}96.14 \\
97.03 \\
97.05\end{array}$ & $\begin{array}{ll}\text { Apr. } & 21 \\
\text { May } & 13 \\
\text { June } & 18\end{array}$ & $\begin{array}{l}96.94 \\
96.94 \\
96.94\end{array}$ & $\begin{array}{l}\text { July } 19 \\
\text { Aug. } 15 \\
\text { Sept. } 19\end{array}$ & $\begin{array}{l}96.83 \\
96.98 \\
97.02\end{array}$ & $\begin{array}{l}\text { Nov. } \\
28 \\
\text { Dec. } 17\end{array}$ & $\begin{array}{l}96.84 \\
96.86 \\
96.94\end{array}$ \\
\hline
\end{tabular}

165. Griffith and Baughman. SE $\frac{1}{4} \mathrm{SW}_{\frac{1}{4}}$ sec. 14, T. 31 S., R. 33 W.

Water level, in feet below measuring point, 1941

\begin{tabular}{lrr|ll|ll|ll|l}
\hline Jan. & 7 & 168.03 & Apr. 21 & 168.21 & July 19 & 167.94 & Nov. 1 & 166.79 \\
Feb. 5 & 167.72 & May 13 & 167.78 & Aug. 15 & 167.89 & & 28 & 147.30 \\
Mar. 14 & 167.78 & June 18 & 167.96 & Sept.19 & 166.58 & Dec. 17 & 150.75 \\
\hline
\end{tabular}

STANTON COUNTY

By B. F. Latta

Highest and lowest water levels, in feet below measuring point, in 5 wells in Stanton County, 1941

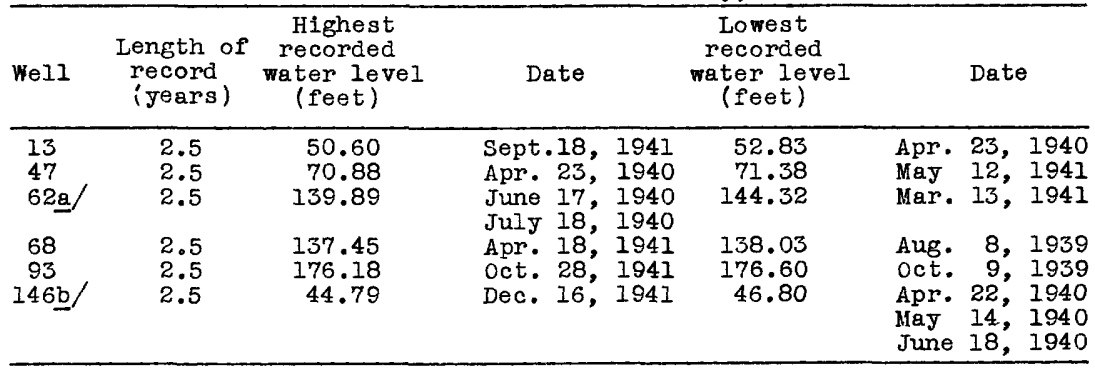

a Pumped considerably since August 1940.

b Messuring point lowered 0.5 foot 0ct. 25, 1940. 
Stanton County--Continued

Net changes in water level in 1941, and net changes in water level for the period of record in 5 wells in Stanton county

\begin{tabular}{|c|c|c|c|}
\hline Well & $\begin{array}{c}\text { Difference } \\
\text { between highest } \\
\text { and lowest } \\
\text { water levels } \\
\text { (feet) }\end{array}$ & $\begin{array}{l}\text { Net rise (t) } \\
\text { or net decline } \\
(-) \text { in } 1941 \\
(\text { feet) }\end{array}$ & $\begin{array}{l}\text { Net rise (t) } \\
\text { or net decline } \\
\text { (-) for period } \\
\text { of record } \\
\text { (feet) }\end{array}$ \\
\hline $\begin{array}{r}13 \\
47 \\
62 \\
68 \\
93 \\
146\end{array}$ & $\begin{array}{l}2.23 \\
.5 \\
4.43 \\
.58 \\
.42 \\
1.51\end{array}$ & $\begin{array}{l}+1.92 \\
-.34 \\
-2.30 \\
+.03 \\
+.1 \\
+1.35\end{array}$ & $\begin{array}{l}+1.64 \\
-.37 \\
-2.81 \\
+.45 \\
+.22 \\
+1.44\end{array}$ \\
\hline
\end{tabular}

Water-level measurements

13. L. Y. Carrithers. NE $\frac{1}{c} S E \frac{1}{4}$ sec. 21, T. 27 S., R. 40 W. Water level, in feet below measuring point, 1941

\begin{tabular}{ll|ll|ll|ll}
\hline Date & $\begin{array}{l}\text { Water } \\
\text { level }\end{array}$ & Date & $\begin{array}{l}\text { Water } \\
\text { level }\end{array}$ & Date & $\begin{array}{l}\text { Water } \\
\text { level }\end{array}$ & Date & $\begin{array}{r}\text { Water } \\
\text { level }\end{array}$ \\
\hline Jan. 6 & 52.61 & Apr. 18 & 52.82 & July 18 & 51.52 & oct. 28 & 50.72 \\
Feb. 6 & 52.65 & May 12 & 52.71 & AuE. 14 & 50.94 & Nov. 18 & 50.76 \\
Mar. 13 & 52.78 & June 17 & 52.20 & Sept.18 & 50.60 & Dec. 16 & 50.69 \\
\hline
\end{tabular}

47. Southwestern College. NW $\frac{1}{4} N E \frac{1}{4}$ sec. 35, T. 28 S., R. 39 W.

Water level, in feet below measuring point, 1941

\begin{tabular}{lrr|ll|ll|r|rr}
\hline Jan. 6 & 70.94 & Apr. 18 & 70.92 & June 17 & 70.94 & Nov. 18 & 71.32 \\
Feb. 6 & 70.92 & May 12 & 71.38 & Oct. 28 & 71.32 & Dec. & 71.28 \\
Mar. 13 & 70.97 & & & & & & & & \\
\hline
\end{tabular}

62. H. Bearman. SW $\frac{1}{4} \mathrm{SW} \frac{1}{4}$ sec. 29, T. 28 S., R. 41 W. Water-level measurements resumed in January 1941. Well pumped considerably during 1941 .

Water level, in feet below measuring point, 1941

\begin{tabular}{lrr|ll|ll|lll}
\hline Jan. 6 & 141.04 & Apr. 18 & 144.07 & AuE. 14 & 143.96 & Nov. 18 & 143.28 \\
Feb. 6 & 141.50 & May 12 & 143.95 & Sept.18 & .143 .80 & Dec. 16 & 143.34 \\
Mar. 13 & 144.32 & June 17 & 143.96 & Oct. 28 & 143.65 & & \\
\hline
\end{tabular}

68. C. D. Wartman. SW $\frac{1}{a}$ SW $\frac{1}{2}$ sec. 29, T. 28 S., R. 42 W.

Water level, in feet below measuring point, 1041

\begin{tabular}{lrr|ll|ll|lll}
\hline Jan. 6 & 137.56 & Apr. 18 & 137.45 & July 18 & 137.81 & Oct. 28 & 137.72 \\
Feb. 6 & 137.54 & May 12 & 137.55 & Aug. 14 & 137.70 & Nov. 18 & 137.48 \\
Mar. 13 & 137.51 & June 17 & 137.99 & Sept.18 & 137.65 & Dec. 16 & 137.53 \\
\hline
\end{tabular}

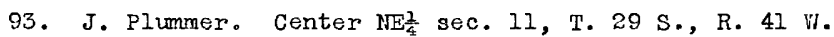

Water level, in feet below measuring point, 1941

\begin{tabular}{lrr|ll|ll|ll}
\hline Jan. 6 & 176.33 & Apr. 18 & 176.31 & July 18 & 176.32 & Oct. 28 & 176.18 \\
Feb. 6 & 176.39 & May 12 & 176.42 & Aug. 14 & 176.28 & Nov. 18 & 176.29 \\
Mar. 13 & 176.34 & June 17 & 176.29 & Sept.18 & 176.27 & Dec. 16 & 176.23 \\
\hline
\end{tabular}

146. C. M. Harrison. SW $\mathrm{SE}_{4}^{\frac{1}{4}}$ sec. $27, \mathrm{~T} .30 \mathrm{~S}, \mathrm{R}^{2} 43$ W.

Vater level, in feet below measuring point, 1941

\begin{tabular}{|rr|rr|ll|lll}
\hline Jan. 6 & 46.14 & July 18 & 45.87 & Sept.18 & 45.44 & Nov. 18 & 44.93 \\
Feb. 6 & 46.22 & Aug. 14 & 45.78 & Oct. 28 & 45.63 & Dec. 16 & 44.79 \\
\hline
\end{tabular}


MINNESOTA

clay county

By A. C. Byers

Periodic measurements of the water levels in 4 wells in Minnesota were begun in July 1940 by the Federal Geological Survey in cooperation with the North Dakota State Geological Survey and the city of Fargo, North Dakota, in connection with an investigation of the ground-water resources of the Fargo area. The descriptions of the wells and the records for 1940 have been published in Water-Supply Paper 908. During 1941 three new wells in Minnesota were added to the program. These are in the locality known as Pleasant Ridge, which is described in following paragraphs. By the end of the jear, water levels were, therefore, being measured weekly in seven wells in Minnesota. About 380 measurements were made by the wetted-tape method by L. K. Wenzel, Ralph Mader, and Alan C. Byers.

The water levels in three of the four wells in which measurements were made throughout the year showed a net decline and the fourth showed a small rise. The average net decline of water level in the 4 wells was 0.61 foot.

The low swell known as Pleasant Ridge extends northward through sections 29 and 32 of T. 139 N., R. 47 W. The material underlying this swell is glacial outwash, the thickness of which is unknown but is at least 100 feet. Cross-bedded sands containing some boulders grade downward into coarse gravel. The superficial Lake Agassiz clay is here very thin, being only 2 to 4 feet thick in some places. The clay has been stripped off in four places where sand pits have been opened. The Fitzgerald, Connelley, and Benedict pits are being worked for sand and gravel; the Wiedemann pit was intended to supply water for irrigation of sugar beets, but proved impractical for the purpose because of sloughing of the sides. The central part of each pit is occupied by a pond, the surface of which is about 20 feet below the surrounding land surface. The groundwater conditions are of the water-table type. 150 
A gravel-packed well, 16 inches in dimeter and 14 feet deep is located near the Fitzgerald and Connelley pits, and is used to irrigate sugar beets. Its capacity is about 4,000 gallons a minute. Some water is also pumped from the Benedict pit for irrigation.

In order to get, data in regard to the drawdown of water levels during the heavy seasonal pumping, 3 driven wells were installed--one each in the Fitzgereld, Connelley, and wiedemann pits on the edges of the ponds. As no measurements of wator levels have been made previously in that vicinity, an arbitrary datum was established for each well by assuming the water level on July 9 to be at an altitude of 100 feet.

No measurements were made of the pumpage from the sand and gravel deposits, but it is estimated that between $60,000,000$ and $80,000,000$ gallons was pumped for irrigation in the period from early July until mid-August. The average maximum drawdown due to this pumpage for the 3 observation wells was 3.80 feet. At the end of 1941 the water levels in the 3 wells averaged 1.14 feet below their levels before pumping began.

3. City of Moorhead. NW $\frac{1}{4}$ SW $\frac{1}{4}$ sec. 32, T. $140 \mathrm{~N}$, , R. 48 W, Water level, Jan. 5, 1941, 36.95 feet below measuring point. (Described in Water-Supply Paper 908 ).

Water leve'. ' 1 feet absve issumed datum, 1942

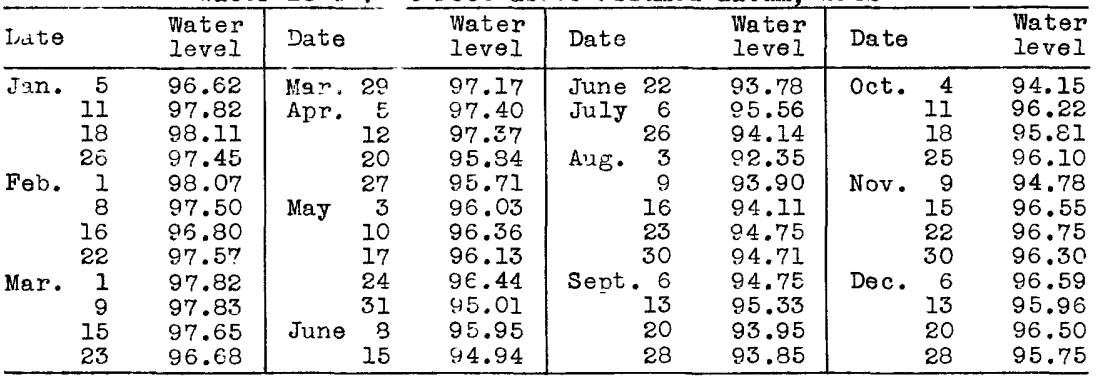

5. W. M. Bailey, 1203 8th St. So., Moorhead. Water level, Jan. 5, 1941, 34.76 feet below measuring point. 'Described in Water-Supply Paper' 908).

Water level, in feet abore assumed datum, 1941

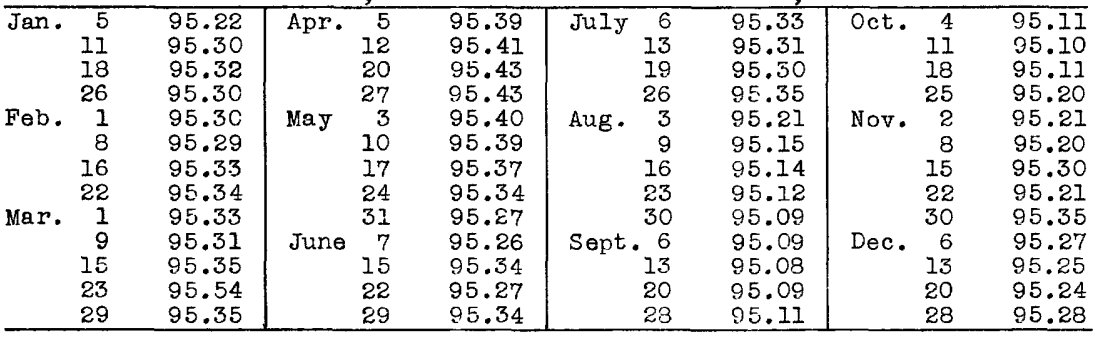


clay county--Continued.

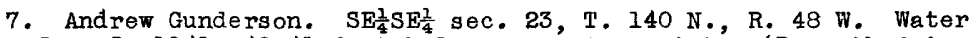
level, Jan. 5, 1941, 42.41 feet below measuring point. (Described in Water-Supply Papor 908 ).

Water level, in feet above assumed datum, 1941

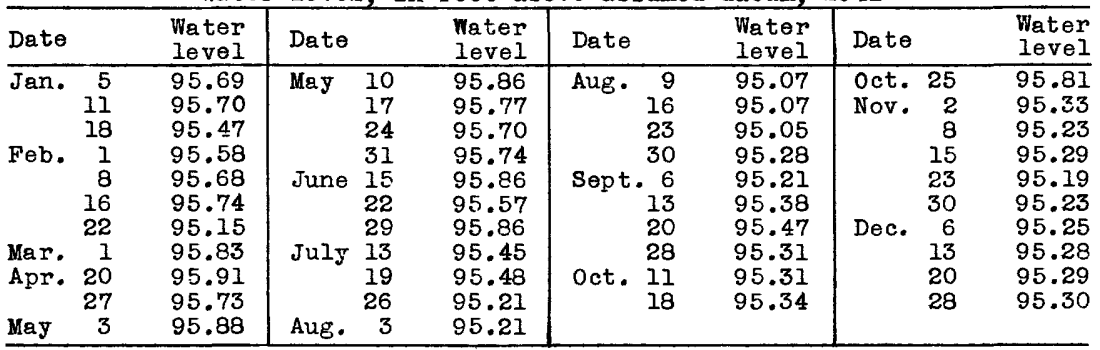

8. NW $\frac{1}{4} \operatorname{SWl} \frac{1}{4}$ sec. 4, T. 140 N., R. 48 W. Water level, Jan. 5, 1941, 21.02 feet below measuring polnt.' (Described in Water-supply Paper 908).

Water level, in feet above assumed datum, 1941

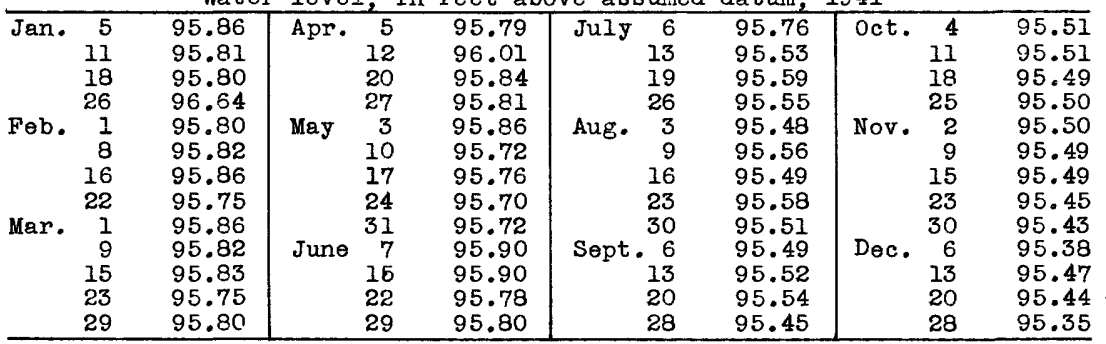

24. SE $\frac{1}{4} S W \frac{1}{4}$ sec. 29, T. 139 N., R. 47 W. In sand pit, on bank of pool. Unused driven observation weil, diameter $1 \frac{1}{4}$ 1nches, depth 7.7 feet below measuring point. Driven June 14, 1941. Measuring point top of coupling on plpe 0.5 foot above pool bank and 101.98 feet above assumed datum. Water level, July 9,1941 , 1.98 feet below measuring point. Water levels affected by pumplng of irrigation well about 3,520 feet south.

Water level, in feet above assumed datum, 1941

\begin{tabular}{|c|c|c|c|c|c|c|c|c|}
\hline $\begin{array}{r}\text { July } \\
1 \\
1 \\
1 \\
1 \\
1 \\
2 \\
2 \\
2 \\
2 \\
2 \\
2 \\
2 \\
2 \\
2 \\
3 \\
3\end{array}$ & $\begin{array}{l}9 \\
12 \\
16 \\
17 \\
18 \\
19 \\
21 \\
22 \\
23 \\
24 \\
25 \\
26 \\
28 \\
29 \\
30 \\
31\end{array}$ & $\begin{array}{r}100.00 \\
99.97 \\
100.00 \\
99.95 \\
99.87 \\
99.81 \\
99.70 \\
99.67 \\
99.64 \\
99.59 \\
99.54 \\
99.46 \\
99.34 \\
99.28 \\
99.08 \\
98.85\end{array}$ & Aug. & $\begin{array}{l}98.58 \\
98.37 \\
98.03 \\
98.01 \\
97.84 \\
97.66 \\
97.53 \\
97.48 \\
97.47 \\
97.55 \\
97.54 \\
97.51 \\
97.44 \\
97.40 \\
97.44 \\
97.35\end{array}$ & $\begin{array}{r}\text { Aug. } 2 \\
2 \\
2 \\
2 \\
2 \\
2 \\
2 \\
2 \\
2 \\
3 \\
\text { Sept. }\end{array}$ & $\begin{array}{l}97.25 \\
97.14 \\
97.04 \\
96.86 \\
97.09 \\
96.97 \\
96.92 \\
96.97 \\
97.11 \\
97.22 \\
97.44 \\
97.51 \\
97.62 \\
97.71 \\
97.77\end{array}$ & $\begin{array}{rr}\text { Sept. } 13 \\
20 \\
28 \\
\text { oct. } \\
4 \\
11 \\
18 \\
-25 \\
\text { Nov. } \\
8 \\
8 \\
15 \\
22 \\
30 \\
13 \\
\text { Dec. } \\
20 \\
28\end{array}$ & $\begin{array}{l}97.88 \\
98.01 \\
98.10 \\
98.09 \\
98.35 \\
98.42 \\
98.45 \\
98.56 \\
98.61 \\
98.64 \\
98.72 \\
98.74 \\
98.81 \\
98.84 \\
98.87\end{array}$ \\
\hline
\end{tabular}


Clay County--Continued.

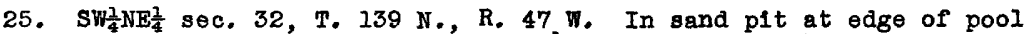
Unused driven observation well, diameter It inches, depth 11.7 feet below measuring point. Driven June 14, 1941. Measuring point top of coupling on top of plpe, 3.50 feet above pool bank and 103.16 feet above assumed datum. Water level July 9, $1941,3.16$ feet below measuring point. Water levels affected by pumping of Irrigation vell about 1,260 feet southwest.

Water level, in feet above assumed datum, 1941

\begin{tabular}{|c|c|c|c|c|c|c|c|c|}
\hline Date & & $\begin{array}{l}\text { Water } \\
\text { level }\end{array}$ & Date & $\begin{array}{l}\text { Water } \\
\text { level }\end{array}$ & Date & $\begin{array}{l}\text { Water } \\
\text { level }\end{array}$ & Date & $\begin{array}{l}\text { Water } \\
\text { level } \\
\end{array}$ \\
\hline July & $\begin{array}{l}9 \\
12 \\
16 \\
17 \\
18 \\
19 \\
21 \\
22 \\
23 \\
24 \\
25 \\
26 \\
28 \\
29 \\
30 \\
31\end{array}$ & $\begin{array}{r}100.00 \\
99.96 \\
99.87 \\
99.40 \\
99.33 \\
99.27 \\
99.23 \\
99.29 \\
99.09 \\
98.98 \\
98.65 \\
98.61 \\
98.57 \\
98.29 \\
97.95 \\
97.61\end{array}$ & Aug. & $\begin{array}{l}97.30 \\
97.02 \\
96.77 \\
96.72 \\
96.51 \\
96.32 \\
96.42 \\
96.17 \\
96.12 \\
96.74 \\
96.45 \\
96.36 \\
96.18 \\
96.20 \\
96.61 \\
96.29\end{array}$ & $\begin{array}{r}\text { Aug. } 20 \\
21 \\
22 \\
23 \\
25 \\
26 \\
27 \\
28 \\
29 \\
30 \\
\text { Sept. } 2 \\
4 \\
6 \\
8 \\
10 \\
13\end{array}$ & $\begin{array}{l}96.10 \\
96.18 \\
96.29 \\
96.17 \\
96.53 \\
96.67 \\
96.77 \\
96.86 \\
96.97 \\
97.06 \\
97.29 \\
97.42 \\
97.51 \\
97.63 \\
97.68 \\
97.79\end{array}$ & $\begin{array}{r}\text { Sept. } 20 \\
28 \\
\text { Oct. } 4 \\
11 \\
18 \\
25 \\
2 \\
\text { Nov. } \\
-8 \\
15 \\
22 \\
30 \\
6 \\
\text { Dec. } \\
13 \\
20 \\
28\end{array}$ & $\begin{array}{l}97.95 \\
98.08 \\
98.25 \\
98.30 \\
98.37 \\
98.44 \\
98.52 \\
98.52 \\
98.63 \\
98.67 \\
98.74 \\
98.76 \\
98.78 \\
98.83 \\
98.84\end{array}$ \\
\hline
\end{tabular}

26a. NW $\frac{1}{4} \mathrm{SE} \frac{1}{4}$ sec. 32, T. $139 \mathrm{~N} ., \mathrm{R} .47 \mathrm{~W}$. In Fitzgerald sand pit, on pool bank. Unused driven observation well, diameter $1 \frac{1}{4}$ inches, depth 9.3 feet below measuring point. Driven June 26, 1941. Measuring point, top of coupling on top of pipe, 0.5 foot above pool bank and 101.04 feet above assumed dátum. Water levei, July 9, 1941, 1.04 feet below measuring point Water levels affected by pumping of' irrigation woll about 330 feet northwest.

Water level, in feet above assumed datum, 1941

\begin{tabular}{|c|c|c|c|c|c|c|c|c|}
\hline July & $\begin{array}{r}9 \\
12 \\
16 \\
17 \\
18 \\
19 \\
21 \\
22 \\
23 \\
24 \\
25 \\
26 \\
28 \\
29 \\
30 \\
31\end{array}$ & $\begin{array}{r}100.00 \\
99.98 \\
99.35 \\
99.28 \\
98.67 \\
99.23 \\
99.22 \\
98.95 \\
98.59 \\
98.47 \\
98.05 \\
98.06 \\
98.02 \\
97.90 \\
97.30 \\
96.99\end{array}$ & Aug. & $\begin{array}{l}96.58 \\
96.36 \\
96.19 \\
96.10 \\
95.87 \\
95.72 \\
95.95 \\
95.59 \\
95.76 \\
96.79 \\
95.97 \\
95.78 \\
95.59 \\
95.63 \\
96.13 \\
95.69\end{array}$ & $\begin{array}{r}\text { Aug. } 20 \\
21 \\
22 \\
23 \\
25 \\
26 \\
27 \\
28 \\
29 \\
30 \\
\text { Sept. } \\
\\
10 \\
8 \\
10\end{array}$ & $\begin{array}{l}95.95 \\
95.75 \\
96.36 \\
95.90 \\
96.61 \\
96.76 \\
96.87 \\
96.98 \\
97.06 \\
97.14 \\
97.36 \\
97.46 \\
97.67 \\
97.68 \\
97.72\end{array}$ & $\begin{array}{r}\text { Sept. } 13 \\
20 \\
28 \\
\text { Oct. } 4 \\
11 \\
18 \\
25 \\
2 \\
\text { Nov. } \\
8 \\
15 \\
22 \\
30 \\
13 \\
\text { Dec. } \\
20 \\
28\end{array}$ & $\begin{array}{l}97.83 \\
98.00 \\
98.16 \\
98.49 \\
98.35 \\
98.44 \\
98.53 \\
98.59 \\
98.62 \\
98.67 \\
98.72 \\
98.85 \\
98.85 \\
98.90 \\
98.88\end{array}$ \\
\hline
\end{tabular}




\title{
NEBRASKA
}

\author{
By H. A. Waite and L. P. Buck
}

The State-wide program of water-level measurements in wells, begun in 1934, was continued in 1941 by the Federal Geological Survey in cooperation with the Conservation and Survey Division of the University of Nebraska. Records of water level and some interpretation of the fluctuations of the water level in the wells from 1934 through 1940 are given in previous annual volumes of the Geological Survey (Water-Supply Papers 777, 817, 840, 845,886 , and 908).

Measurements of water levels made in 375 observation wells are given on the following pages. Included in this group are 46 wells in Hall County and 2 wells in Merrick County that are being observed through informal cooperation with the Grand Island Water Department; 8 wells in Garden ccunty that are being observed through informal cooperation with the Fish and Wildiffe Service, Department of the Interior; and 7 wells in Dawson County, 1 well in Garden County, 1 well in Gosper County, and 30 wells in Keith County that are being observed through informal cooperation with the Central Nebraska Public Power and Irrigation District. Daily tape measurements, furnished by the Nebraska Department of Roads and Iririgation, are given for well 85 in Morrill County. A total of 1,701 individual measurements of water level, most of them made in 1941, are given on the following pages.

The precipitation in Nebraska in 1941 was 24.45 inches, 1.94 inches above normal and 7.20 inches above 1940. As a result, the water levels in most of the observation wells had net rises for the year. The following tables summarize the water-level fluctuations in key wells throughout the State. The average water levels have been recomputed for 1940 using a total of 138 wells instead of 133. The average water levels for $1940 \mathrm{as}$ shown in Water-Supply Paper 908 differ slightly, therefore, from those given in the following table. 
Summary of average water levels, in feet above as sumed datum planes, in observation wells in Nebraska, October-December 1934-41

\begin{tabular}{lccccc}
\hline & $\begin{array}{c}\text { Number } \\
\text { of } \\
\text { wells }\end{array}$ & $\begin{array}{c}\text { 1934 } \\
\text { average } \\
\text { level }\end{array}$ & $\begin{array}{c}1935 \\
\text { average } \\
\text { level }\end{array}$ & $\begin{array}{c}\text { 1936 } \\
\text { average } \\
\text { level }\end{array}$ & $\begin{array}{c}\text { 1937 } \\
\text { average } \\
\text { level }\end{array}$ \\
\hline Northeast & 28 & 99.67 & 99.36 & 98.98 & 99.42 \\
Southeast & 40 & 99.81 & 100.47 & 99.61 & 99.25 \\
North-central & 33 & 99.81 & 100.01 & 99.77 & 99.41 \\
South-central & 38 & 99.84 & 100.11 & 99.68 & 99.66 \\
Northwest & 15 & 99.99 & 100.45 & 100.05 & 99.77 \\
Southwest & 13 & 100.02 & 100.06 & 100.03 & 99.88 \\
Entire State & 167 & 99.84 & 100.08 & 99.62 & 99.50 \\
\hline
\end{tabular}

\begin{tabular}{|c|c|c|c|c|c|c|c|c|}
\hline . & $\begin{array}{c}\text { Number } \\
\text { of } \\
\text { wells }\end{array}$ & $\begin{array}{l}938 \\
\text { Average } \\
\text { level }\end{array}$ & $\begin{array}{c}1 \\
\text { Number } \\
\text { of } \\
\text { wells }\end{array}$ & $\begin{array}{l}939 \\
\text { Average } \\
\text { level }\end{array}$ & $\begin{array}{c}\text { Number } \\
\text { of } \\
\text { wells }\end{array}$ & $\begin{array}{l}940 \\
\text { Average } \\
\text { level }\end{array}$ & $\begin{array}{c}\text { Number } \\
\text { of } \\
\text { wells }\end{array}$ & $\begin{array}{l}941 \\
\text { Average } \\
\text { level }\end{array}$ \\
\hline $\begin{array}{l}\text { Northeast } \\
\text { Southeast } \\
\text { North-central } \\
\text { South-central } \\
\text { Northwest } \\
\text { Southwest } \\
\text { Entire State }\end{array}$ & $\begin{array}{r}28 \\
36 \\
33 \\
36 \\
15 \\
12 \\
160\end{array}$ & $\begin{array}{r}99.74 \\
99.56 \\
99.44 \\
99.66 \\
100.13 \\
100.05 \\
99.68\end{array}$ & $\begin{array}{r}28 \\
40 \\
33 \\
38 \\
15 \\
13 \\
167\end{array}$ & $\begin{array}{l}99.24 \\
99.05 \\
99.59 \\
99.68 \\
99.79 \\
99.88 \\
99.46\end{array}$ & $\begin{array}{l}22 \\
36 \\
27 \\
29 \\
12 \\
12 \\
138\end{array}$ & $\begin{array}{l}99.57 \\
98.80 \\
99.15 \\
99.76 \\
99.68 \\
99.67 \\
99.34\end{array}$ & $\begin{array}{r}22 \\
33 \\
26 \\
32 \\
13 \\
9 \\
135\end{array}$ & $\begin{array}{r}100.31 \\
99.91 \\
99.70 \\
100.05 \\
99.77 \\
100.21 \\
99.98\end{array}$ \\
\hline
\end{tabular}

Summary of changes in average water levels in feet, in observation wells in

\begin{tabular}{|c|c|c|c|c|c|c|c|c|}
\hline & 1935 & 1936 & 1937 & 1938 & 1939 & 1940 & 1941 & $1934-41$ \\
\hline $\begin{array}{l}\text { Northeast } \\
\text { Southeast } \\
\text { North-central } \\
\text { South-central } \\
\text { Northwest } \\
\text { Southwest } \\
\text { Entire State }\end{array}$ & $\begin{array}{l}+0.31 \\
+\quad .66 \\
+.10 \\
+.27 \\
+.46 \\
+.04 \\
+.24\end{array}$ & $\begin{array}{l}-0.38 \\
-.86 \\
-.24 \\
-.43 \\
-.40 \\
-.03 \\
-.46\end{array}$ & $\begin{array}{l}+0.44 \\
-.36 \\
-.36 \\
-.02 \\
-.28 \\
-.15 \\
-.12\end{array}$ & $\begin{array}{l}+0.32 \\
+.31 \\
+.03 \\
+.00 \\
+.36 \\
+.17 \\
+.18\end{array}$ & $\begin{array}{l}-0.50 \\
-.51 \\
+.15 \\
+.02 \\
-.34 \\
-.17 \\
-.22\end{array}$ & $\begin{array}{l}+0.33 \\
-.25 \\
-.44 \\
+.08 \\
-.11 \\
-.21 \\
-.12\end{array}$ & $\begin{array}{l}+0.74 \\
+1.11 \\
+.55 \\
+.29 \\
+.09 \\
+.54 \\
+.64\end{array}$ & $\begin{array}{l}+0.64 \\
+.10 \\
-.21 \\
+.21 \\
-.22 \\
+.19 \\
+.14\end{array}$ \\
\hline
\end{tabular}

The records from 1935 through 1937 and during 1939 are based on 167 wells; those in 1938 on 160 wells; those in 1940 on 138 wells; and those in 1941 on 135 wells. The number of wells included in the averages has diminished during the last 3 years because some of the wells went dry, were destroyed, or were otherwise rendered unfit for observation. The table shows that in 1041 the averages of the water levels in the observation wells rose in all 6 sections of the State. There was an average net rise of 0.64 foot in the 135 wells during the year. At the end of 1941 , the averages in all sections of the State except the north-central and northwest sections were higher than at the end of 1934. In north-central Nebraska there was an average decline in water level in the 7-year period of 0.21 foot, and in northwest Nebraska there was an average decline of 0.22 foot. For the entire state there was an average net rise of 0.14 foot during the 7-year period. 
The records for only 118 of the 135 key wells are complete from 1934 through 1941. Using only the records on these wells, the change in the average water levels for the State in 1841 ' is computed to be a rise of 0.55 foot and the cumulative net change for the State for the period 1934 through 1841 is computed to be a decline of 0.08 foot. If it is assumed that the average net rise of 0.55 foot recorded in the 118 key wells in 1941 represents the average net fluctuations of the water table over the State, and that the specific yleld of the formations in which the water occurs in Nebraska averages 15 percent--that 1s, that each cublc foot of material will jleld or store 0.15 cublc foot of water--then the records Indicate a net increase in ground-water storage in 1941 of about 4,000,000 acre-feet. On the same bas1s, the decrease in ground-water storage in the 7-year perlod ending with 1941 may be computed to be about 600,000 acrefeet. The precipitation in Nebraska during the 7-year period was about 22.5 Inches below average--a deficlency equivalent to about $92,700,000$ acre-feet of water. Since 1934 the decrease in ground-water storage has, therefore, been only about 0.6 percent of the deflclency in the preclpitation.

During 1941, the water levels in 11 of the 135 observation wells reached new high stages and those in 14 of the wells reached new low stages.

Water levels in wells in the vicinity of Grand Island rose during 1941 as a result of an increase in precipitation. The precipitation for the year at Grand Island was 25.23 inches, 1.83 inches below normal, but 13.32 inches more than in 1940. Because the water-levei measurements of the wells in the vicinity of Grand Island are not complete for the last part of the year, the water levels in 1941 in only 22 of the wells are directly comperable to those of 1940. The 22 wells all showed a rise in water level ranging from 0.27 foot to 1.68 feet.

The observation wells, which are listed alphabetically by counties and numerically within each county, have numbers that correspond to those given in Water-Supply Papers 817, 840, 845, 886, and 908. This report gives complete descriptions for only those wells whose records appear for the first time. For most wells the water levels are expressed in feet above an assumed datum, which is 100 feet below the water level on January 1, 1935. The helght of the measuring point above datum for wells that 
have been established since January 1, 1935, has been interpolated from the average water level in a group of similar wells on a selected date. The water levels are directly comparable even though the measuring point has been changed, because the record is given as a helght above a datum that has been referred to one or more bench marks.

\section{Adams gounty}

193. No measurements made in 1941.

448. No measurements made in 1941 .

\section{Antelope County}

111. No measurements made in 1941.

202. Water level, in feet above datum, 1941: 0ct. 17, 99.48.

\section{Arthur County}

250. Water Ievel, in feet above datum, 1941; 0ct. 26, 98.54. 251. No measurements made in 1941.

\section{Banner County}

238. Water Ievel, in feet above datum, 1941: 0ct. 25, 102.79. 354. No measurements made in 1941.

\section{Blaine County}

210. Water level, in feet above datum, 1941: 0ct. 21, 98.95.

211. Water level, in feet above datum, 1941: 0ct. 21, 99.49.

237. Water level, in feet above datum, 1941: 0ct. 21, 98.71.

433. Water level, in feet above datum, 1941: 0ct. 21, 99.76.

434. Water Ievel, in feet above datum, 1941: 0ct. 21, 98.72.

\section{Boone County}

200. Water level, in feet above datum, 1941: 0ct. 14, 99.85.

201. Water Ievel, in feet above datum, 1941: Oct. 14, 99.82.

207. Water level, in feet above datum, 1941: Oct. 14, 100.27.

425. Water level, in feet above datum, 1941: Oct. 14, dry.

426. Water level, in feet above datum, 1941: 0ct. 14, 99.91.

$5110050-43-11$ 


\section{Box Butte County}

129. Water leve1, in feet above datum, 1941: 0ct. 20, a/ 99.46.

338. Water level, in feet above datum, 1941: 0ct. 20, a/ 99.09 .

378. Water level, in feet above datum, 1941: 0ct. 20, 99.32 .

\section{Boyd County}

74. Water level, in feet above datum, 1941: 0ct. 18, 100.28 .

75. Water level, in feet above datum, 1941: 0ct. 18, 98.40.

209. Water level, in feet above datum, 1941: 0ct. 18, 100.09 .

\section{Brown county}

243. No measurements made in 1941.

\section{Buffalo County}

52. No measurements made in 1941.

232. Water level, in feet above datum, 1941: 0ct. 16, 101.02.

262. Measurements discontinued.

263. Water level, in feet above datum, 1941: 0ct. 15, 98.28.

264. No measurements made in 1941.

265. Water level, in feet above datum, 1941: oct. 15, 100.08 .

267. Water level, in feat above datum, 1941: 0ct. 15, a/ 97.42.

268. Water level, in feet above datum, 1941: 0ct. 15, 97.84.

269. No measurements made in 1941.

270. Water level, in feet above datum, 1941: 0ct. 15, a/ 96.43 .

272. Water level, in feet above datum, 1941: oct. 15, a/97.29.

273. No measurements made in 1941.

274. Water leve1, in feet above datum, 1941: 0ct. 15, 101.04 .

278. Water level, in feet above datum, 1941: 0ct. 15, 102.10.

279. Water level, in feet above datum, 1941: 0ct. 15, 97.66.

\section{Surt County}

63. Water level, in feet above datum, 1941: 0ct. 10, 98.46 .

64. Old well destroyed. Altitude of measuring point of new well not yet determined.

402. Water level, in feet above datum, 1941: 0ct. 10, 100.24 .

\section{Butler County}

170. We11 filled in.

a Lowest observed stage in period of record. 


\section{Cass County}

16. Water level, in feet above datum, 1941: 0ct. 6, 97.68.

18. Water level, in feet above datum, 1941: 0ct. 8, a/ 106.42 .

\section{Cedar County}

65. Water level, in feet above datum, 1941: 0ct. 11, 101.72.

66. No measurements made in 1941.

369. Water lovel, in feet above datum, 1941: 0ct. 11, 100.97.

\section{Chase County}

152. No measurements made in 1941.

153. Measuring point disturbed. Altitude of new measuring point not yet determined.

\section{Cherry County}

115. Water level, in feet above datum, 194l: 0ct. 19, b/ 97.71.

116. Water level, in feet above datum, 1941: 0ct. 18, 99.31.

118. Water level, in feet above datum, 1941: 0ct. 19, 98.31.

256. Wacer level, in feet above datum, 194l: Oct. 19, b/ 97.23.

257. Water level, in feet above datum, 194l: oct. 19, 98.92.

312. We ier level, in feet above datum, 1941: 0ct. 21, 98.09.

399. Water level, in feet above datum, 1941: 0ct. 18, 100.22.

431. Water level, in feet above datum, 1941: 0ct. 19, 98.56.

\section{Cheyenne County}

86. Measurements dis continued.

87. Water level, in feet above datum, 1941: 0ct. 25, 100.47 .

90. No measurements made in 1941.

91. Measuring point destroyed. Altitude of new measuring point not yet determined.

92. Water level, in feet above datum, 1941: 0ct. 25, 200.14.

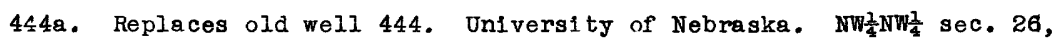
T. 14 N., R. 47 W. Unused driven observation well, diameter 1 inch, depth 29.0 feet. Measuring point, top of pipe, 1.2 feet above land surfaco and 116.6 feet above datum of old well 444. Water level May 29, 1940, 20.72 feet below measuring point. Water levels, in feet above datum: July 28 , $1940,95.12$; Nov. 9 , 1940, 94.58; oct. 25, 1941, 94.24.

a Highest observed stage in period of record.

b Lowest observed stage in period of record. 


\section{Colfax county}

37. Water level, in feet above datum, 1941: 0ct. 9, 98.30.

38. Well destroyed.

332. Water level, in feet above datum, 1941: oct. 10, 96.25.

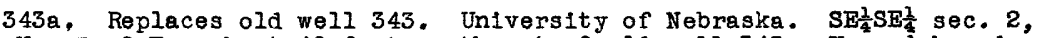
T. $20 \mathrm{~N} .$, R. $^{2}$ F., about 40 feet northeast of old well 343 . Unused bored observation well, dlameter $1 \frac{1}{4}$ Inches, depth 25.0 fret. Measuring point, top of pipe, 2.5 feet above land surface, and 115.21 feet above datum of old well 343. Water levels, in feet above datum: July 11, 1940, 705.65; oct. 23, 1940, 104.13; 0ct. 10, 1941, 103.58.

\section{Cuming county}

61. Water level, in feet above datum, 1941: 0ct. 10,99.55.

69. Water level, in feet above datum, 1941: 0ct. 10, a/ 97.56 .

\section{Custer county}

53. Water level, in feet above datum, 1941: 0ct. 16, 99.57 . 195. Water level, in feet above datum, 1941: 0ct. 16, 98.66. 196. No measurements made in 1941.

219. Water level, in feet above datum, 1941: 0ct. 27, 100.74. 220. No measurements made in 1941.

325. Water level, in feet above datum, 1941: 0ct. 16, 98.78.

435. Water level, in feet above datum, 1941: 0ct. 27, 99.76.

436. Water level, in feet above datum, 1941: 0ct. 27, 99.87.

\section{Dakota County}

104. No measurements made in 1941.

453. Water level, in feet above datum, 1941: oct. 11, 98.61.

\section{Dawes County}

123. Water level, in feet above datum, 1941: 0ct. 20, 101.20.

315. Water level, in feet above datum, 1941: 0ct. 20, 108.20.

396. Water level, in feet above datum, 1941: 0ct. 20, 100.36 .

\section{Daws on County}

99. Water level, in feet above datum, 1941: 0ct. 22, 99.23.

280. Nater level, in feet above datum, 1941: 0ct. 22, 100.86.

283. Water level, in feet above datum, 1941: 0ct. 22, 100.67 .

284. Water level, in feet above datum, 1941: 0ct. 22, 99.66.

285. Water level, in feet above datum, 1941: 0ct. 22, 99.15.

a Lowest observed stage in period of record. 
Dawson County--Continued.

286. Water level, in feet above datum, 194I: 0ct. 22, 98.56.

287. Water level, in feet above datum, 1941: 0ct. 22, 97.97.

288. Water level, in feet above datum, 1941: 0ct. 22, 97.44.

289. Water level, in foet above datum, 1941: 0ct. 22, a/ 97.86.

290. Water level, in foet above datum, 1941: 0ct. 22, 98.42.

291. Water level, in feet above datum, 1941: 0ct. 22, 98.81 .

292. Water level, in feet above datum, 194l: 0ct. 22, 99.03.

293. Water level, in feet above catum, 1941: 0ct. 22, 99.17.

294. Water level, in feet above datum, 1941: 0ct. 22, 99.58.

295. Water level, in feet above datum, 1941: 0ct. 22, 99.64.

296. Water level, in feet above datum, 1941: 0ct. 22, a/ 98.76.

297. Water level, in feet above datum, 1941: 0ct. 22, 101.02.

298. Water level, in feet above datum, 1941: 0ct. 22, 99.82.

299. No measurements made in 1941.

300. Water level, in feet above datum, 1941: 0ct. 22, 99.32 .

301.

Water level, in foot above datum, 1941

\begin{tabular}{|c|c|c|c|c|c|c|c|c|}
\hline Dat & $\begin{array}{l}\text { Water } \\
\text { lovel }\end{array}$ & Date & & $\begin{array}{l}\text { Water } \\
\text { level }\end{array}$ & Date & $\begin{array}{l}\text { Water } \\
\text { level }\end{array}$ & Date & \\
\hline $\begin{array}{r}30 \\
\operatorname{lar} . \\
31 \\
3 I\end{array}$ & $\begin{array}{l}\text { b } 99.06 \\
\text { b } 99.02 \\
\text { b } 98.47\end{array}$ & $\begin{array}{l}\text { May } \\
\text { June } \\
\text { July }\end{array}$ & $\begin{array}{l}1 \\
3 \\
2\end{array}$ & $\begin{array}{l}\text { b } 98.63 \\
\text { b } 98.60 \\
\text { b } 98.02\end{array}$ & $\begin{array}{ll}\text { JuIf } & 30 \\
\text { Sept. } & 2 \\
\text { Oct. } & 6\end{array}$ & $\begin{array}{l}\text { b } 97.42 \\
\text { b } 97.67 \\
\text { b } 98.11\end{array}$ & $\begin{array}{l}\text { Oct. } 22 \\
\text { Nov. } 5 \\
28\end{array}$ & $\begin{array}{l}98.06 \\
\text { b } 98.27 \\
\text { b } 98.37\end{array}$ \\
\hline
\end{tabular}

302 .

Water level, in feet above datum, 1941

\begin{tabular}{|c|c|c|c|c|c|c|c|c|c|c|c|}
\hline $\begin{array}{l}\text { Jan. } 30 \\
\text { lar. }\end{array}$ & $\begin{array}{r}30 \\
4 \\
30\end{array}$ & $\begin{array}{l}\text { b } 99 \\
\text { b } 99 \\
\text { b } 98\end{array}$ & $\begin{array}{l}\text { May } \\
\text { June } \\
\text { July }\end{array}$ & $\begin{array}{l}1 \\
3 \\
2\end{array}$ & $\begin{array}{l}\text { b } 98 \\
\text { b } 98 \\
\text { b } 98\end{array}$ & $\begin{array}{ll}\text { July } & 30 \\
\text { Sept. } & 2 \\
\text { Oct. } & 6\end{array}$ & $\begin{array}{l}\mathrm{b} 9 \varepsilon \\
\mathrm{b} 9 \varepsilon \\
\mathrm{b} 9 \varepsilon\end{array}$ & $\begin{array}{l}\text { Oct. } \\
\text { Nov. }\end{array}$ & & & \\
\hline
\end{tabular}

303.

Water level, in feet above datum, 1941

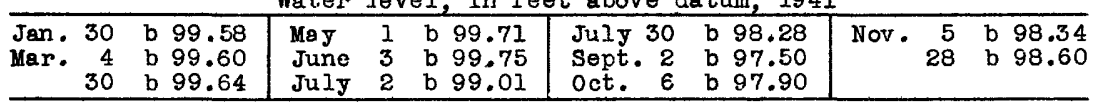

304.

Water level, in feet above datum, 1941

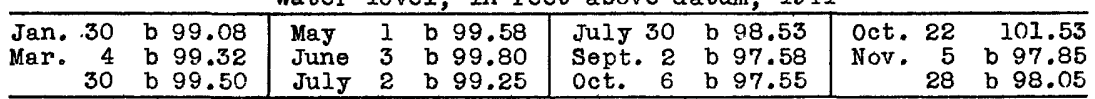

305.

Water level, in feet above datum, 1941

\begin{tabular}{rr|llll|lll|rr}
\hline Jan. 30 & b 99.08 & May & 1 & b 99.49 & July 30 & (c) & Oct. 22 & 99.18 \\
Mar. & 4 & b 99.11 & June & 3 & b 99.57 & Sept. 2 & (c) & Nov. 5 & b 98.44 \\
30 & b 99.33 & July & 2 & b 99.57 & Oct. & 6 & b 98.22 & & 28 & b 98.57 \\
\hline
\end{tabular}

306.

Water level, in feet above datum, 1941

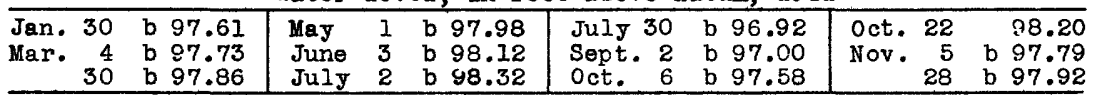

a Lowest observed stage in period of record.

b Heasurement supplied through courtesy of Central Nebraska Public Power and Irrigation District.

c Dry. 
Dawson County--Continued.

308. Water level, in feet above datum, 1941: 0ct. 22, 100.45.

309. Water level, in feet above datum, 1941: 0ct. 22, 102.13.

310. No measurements made in 1941.

311. Water level, in feet above datum, 1941: 0ct. 22, 102.48.

314. No measurements made in 1941.

317. Water level, in feet above datum, 1941: oct. 22, 100.77. 318.

Water level, in feet above dutum, 1941

\begin{tabular}{|c|c|c|c|c|c|c|c|c|c|c|}
\hline Date & & $\begin{array}{l}\text { Water } \\
\text { level }\end{array}$ & Date & & $\begin{array}{l}\text { Water } \\
\text { level }\end{array}$ & Date & & $\begin{array}{l}\text { Water } \\
\text { level }\end{array}$ & Date & $\begin{array}{l}\text { Water } \\
\text { level }\end{array}$ \\
\hline $\begin{array}{l}\text { Jan. } \\
\text { Feb. } \\
\text { Mar. }\end{array}$ & $\begin{array}{r}3 \\
3 \\
3 \\
31\end{array}$ & $\begin{array}{l}\text { a } 99.73 \\
\text { a } 99.91 \\
\text { a } 101.70 \\
\text { a } 100.13\end{array}$ & $\begin{array}{l}\text { May } \\
\text { June } \\
\text { July }\end{array}$ & $\begin{array}{l}1 \\
2 \\
2\end{array}$ & $\begin{array}{l}\text { a } 100.35 \\
\mathrm{a} 100.10 \\
\mathrm{a} 100.21\end{array}$ & $\begin{array}{l}\text { July } 30 \\
\text { Sept. } 1 \\
\text { Oct. } 6\end{array}$ & $\begin{array}{l}\mathbf{a} \\
\mathbf{a} \\
\mathbf{a}\end{array}$ & $\begin{array}{l}99.85 \\
99.38 \\
99.40\end{array}$ & $\begin{array}{lr}\text { Oct. } & 22 \\
\text { Nov. } & 5 \\
& 28\end{array}$ & $\begin{array}{r}100.35 \\
\text { a } 99.66 \\
\text { a } 99.82\end{array}$ \\
\hline
\end{tabular}

319. Water level, in feet above datum, 1941: 0ct. 22, 99.92 .

\section{Deuel County}

94. No measurements made in 1941 .

130. Obstruction 62 feet below measuring point.

\section{Dixon County}

107. No measurements made in 1941.

333. Water level, in feet above datum, 1941: 0ct. 11, dry.

340. Water level, in f'eet above datum, 1941: oct. 11, dry.

\section{Dodge County}

29. Measurements discontinued.

31. Water level, in feet above datum, 1941: 0ct. 8, 102.30.

34. Water leve1, in feet above datum, 1941: 0ct.9,98.76.

401. Water level, in feet above datum, 1941: 0ct. 8, 99.57 .

420. Water level, in feet above datum, 1941: 0ct. 9, 100.50 .

\section{Douglas County}

24. Water level, in feet above datum, 1941: oct. 8, 100.55.

\section{Dundy County}

177. Water level, in feet above datum, 1941: 0ct. 28, 98.93.

361. No measurements made in 1941.

380. Water level, in feet above datum, 1941: 0ct. 28, 100.19. a Measurement supplied through courtesy of Central Nebraska Public Power and Irrigation District. 
Dundy County--Continued.

445. Water level, in feet above datum, 1941: 0ct. 28, a $/ 101.90$.

\section{Fillmore County}

174. Well filled in to 64 feet below measuring point.

191. Water level, in feet above datum, 1941: 0ct..31, 99.36.

192. Measurements discontinued.

\section{Frankiln County}

156. Water level, in feet above datum, 1941: 0ct. 29, 99.95. 221. Water levels, in feet above datum, 1941: Jan. 21, 99.02; oct. $29,99.87$.

224. No measurements made in 1941.

\section{Frontier County}

135. Measurements discontinued.

136. Water level, in feet above datum, 1941: Jan. 22, 100.05.

\section{Furmas County}

145. Water level, in feet above datum, 1941: 0ct. 28, 99.54.

147. Water level, in feet above datum, 194l: oct. 28, b/99.12.

148. Water level, in feet above dạtum, 1941: 0ct. 28, 101.44 .

149. No measurements made in 1941.

180. Water level, in feet above datum, 1541: 0ct. 29, 99.14.

387. No measurements made in $1941 .$.

388. Water level, in feet above datum, 1941: 0ct. 28, 200.70.

395. Water level, in feet above datum, 194l: 0ct. 28, 100.15.

\section{Gage count y}

199. No meásurements made in 1941.

230. Water level, in feet above datum, 194I: Nov. 2, 101.02.

231. No measurements made in 1941.

\section{Garden County}

3. Well dry throughout 1941.

4. Measurementg mpplied through courtesy of Fish and Wildilfe Service, U. S. Dept. of the Interlor.

a Highest observed stage in perlod of record.

$b$ Lowest observed stage in perlod of record. 
Garden Gounty--Continued.

4.---Continued.

Water level, in feet above sea level minus $3,000,1941$

\begin{tabular}{|c|c|c|c|c|c|c|c|}
\hline Date & $\begin{array}{l}\text { Mater } \\
\text { lovel }\end{array}$ & Date & $\begin{array}{l}\text { Water } \\
\text { level }\end{array}$ & Date & $\begin{array}{l}\text { Water } \\
\text { level }\end{array}$ & Date & $\begin{array}{l}\text { Water } \\
\text { level }\end{array}$ \\
\hline $\begin{array}{lr}\text { Jan. } & 8 \\
21 \\
30 \\
\text { Fob. } 7 \\
13 \\
7 \\
\text { Yar. } 7 \\
15 \\
21 \\
\text { Apr. } 2\end{array}$ & $\begin{array}{l}798.84 \\
798.84 \\
798.94 \\
799.16 \\
799.09 \\
799.16 \\
799.09 \\
799.69 \\
799.69\end{array}$ & $\begin{array}{lr}\text { Apr. } & 24 \\
\text { May } & 1 \\
& 15 \\
& 22 \\
\text { June } & 3 \\
& 16 \\
& 27 \\
\text { July } 11\end{array}$ & $\begin{array}{l}800.24 \\
800.24 \\
799.79 \\
799.69 \\
799.59 \\
799.94 \\
799.24 \\
798.89\end{array}$ & $\begin{array}{l}\text { July } 18 \\
26 \\
\text { Aug. } 11 \\
23 \\
\text { Sept. } 5 \\
\text { oct. } 9 \\
15 \\
22\end{array}$ & $\begin{array}{l}798.84 \\
798.59 \\
798.34 \\
799.34 \\
797.89 \\
799.87 \\
799.89 \\
798.89\end{array}$ & $\begin{array}{lr}\text { Oct. } 28 \\
\text { Nov. } 6 \\
13 \\
24 \\
28 \\
28 \\
\text { Dec. } 4 \\
10 \\
17\end{array}$ & $\begin{array}{l}799.19 \\
799.19 \\
799.21 \\
799.24 \\
799.25 \\
799.29 \\
799.29 \\
799.44\end{array}$ \\
\hline
\end{tabular}

5. Measurements supplied through courtesy of Fish and wildiffe Service, U. S. Dept. of the Interior.

Water level, in feet above sea level minus 3,$800 ; 1941$

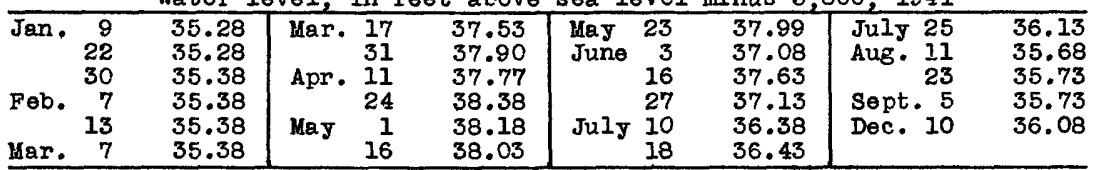

12. Measurements supplied through courtesy of Fish and Wildife

Service, U. S. Dept. of the Interior.

Water level, in feet above sea level minus $3,300,1941$

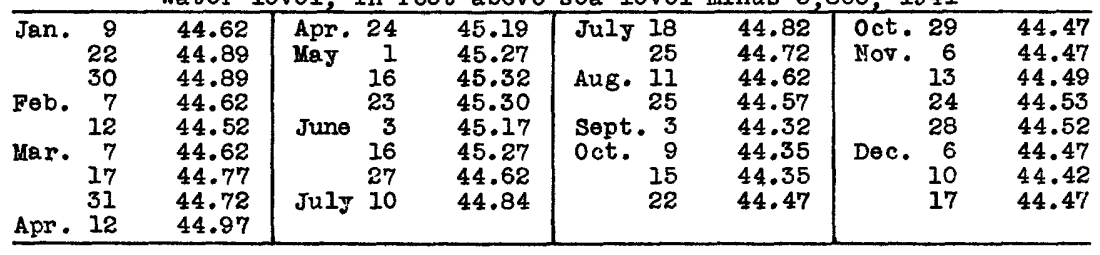

17. Measurements supplied through courtesy of Fish and wildilfe Service, U. S. Dept. of the Interior.

Water level, in foet above sea level minus $3,800,1943$.

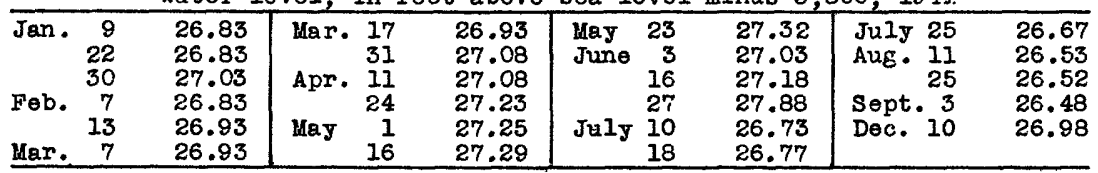

19. Measurements supplied through courtesy of Fish snd wildlife Service, U. S. Dept. of the Interior.

Water level, in feet above sea level minus 3,800, 1941

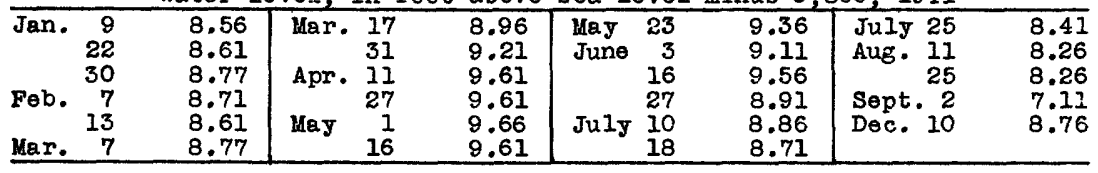

21. Measurements supplied through courtesy of Fish and wildife

Service, U. S. Dept. of the Interior. 
Garden County--Continued.

21.--Continued.

Water level, in feet above sea level minus $3,700,1941$

\begin{tabular}{|c|c|c|c|c|c|c|c|c|c|}
\hline Date & $\begin{array}{l}\text { Water } \\
\text { level }\end{array}$ & Date & $\begin{array}{l}\text { Water } \\
\text { level }\end{array}$ & Date & & $\begin{array}{l}\text { Water } \\
\text { level }\end{array}$ & Date & & $\begin{array}{l}\text { Water } \\
\text { level }\end{array}$ \\
\hline $\begin{array}{lr}\text { Jan. } & 9 \\
22 \\
30 \\
\text { Feb. } \\
73 \\
13 \\
\text { Mar. } 7\end{array}$ & $\begin{array}{l}91.49 \\
91.59 \\
91.69 \\
91.72 \\
91.69 \\
91.72\end{array}$ & $\begin{array}{lr}\text { Mar. } & 17 \\
& 31 \\
\text { Apr. } & 11 \\
& 24 \\
\text { May } & 1 \\
16\end{array}$ & $\begin{array}{l}91.52 \\
91.84 \\
91.91 \\
92.54 \\
92.54 \\
92.61\end{array}$ & $\begin{array}{l}\text { May } \\
\text { June } \\
\text { JuIy }\end{array}$ & $\begin{array}{r}23 \\
3 \\
16 \\
27 \\
10 \\
18\end{array}$ & $\begin{array}{l}92.47 \\
92.29 \\
92.94 \\
92.24 \\
91.89 \\
91.84\end{array}$ & $\begin{array}{l}\text { JuIy } 25 \\
\text { Aug. } 11 \\
25 \\
\text { Sept. } 2 \\
\text { Dec. } 10\end{array}$ & a & $\begin{array}{l}91.54 \\
91.09 \\
91.04 \\
80.99 \\
91.64\end{array}$ \\
\hline
\end{tabular}

25. Messurements supplied through courtesy of Fish and wildilfe Service, U.'S. Dept. of the Interior.

Water level, in feet above sea level minus $3,800,1941$

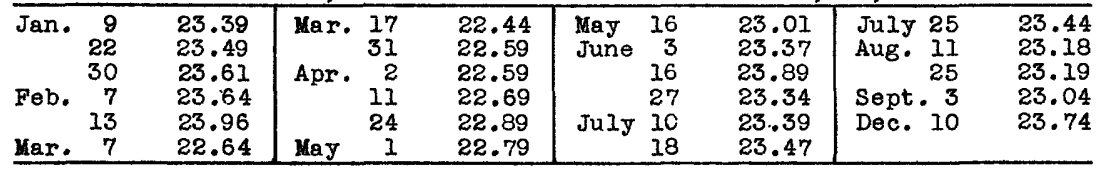

27. Measurements supplied through courtesy of Fish and Wildiffe Service, U. S. Dept. of the Interior.

Water level, in feet above sea level less $3,700,1941$

\begin{tabular}{|c|c|c|c|c|c|c|c|c|c|}
\hline $\begin{array}{lr}\text { Jan. } & 8 \\
21 \\
.30 \\
6 \\
\text { Feb. } 12 \\
12 \\
\text { Mar. } 7 \\
\end{array}$ & $\begin{array}{l}90.87 \\
90.87 \\
91.04 \\
91.04 \\
89.84 \\
89.64\end{array}$ & $\begin{array}{l}\text { Mar. } \\
\text { Apr. I } \\
\text { Kay }\end{array}$ & $\begin{array}{l}17 \\
21 \\
11 \\
24 \\
1 \\
16\end{array}$ & $\begin{array}{l}89.64 \\
89.54 \\
89.49 \\
89.69 \\
89.79 \\
89.69\end{array}$ & $\begin{array}{l}\text { May } \\
\text { June } \\
\text { July }\end{array}$ & $\begin{array}{r}23 \\
3 \\
16 \\
27 \\
10 \\
18\end{array}$ & $\begin{array}{l}89.74 \\
89.69 \\
89.79 \\
89.74 \\
89.69 \\
89.94\end{array}$ & $\begin{array}{l}\text { July } 2 \\
\text { Aug. } \frac{1}{2} \\
2 \\
\text { Sept. } \\
\text { Dec. I }\end{array}$ & $\begin{array}{l}89.79 \\
89.79 \\
89.79 \\
85.69 \\
89.59 \\
89.89\end{array}$ \\
\hline
\end{tabular}

96. Measurements supplied through courtesy of Central Nebraska Public Power and Irrigation District.

Water level, in feet above datum, 1941

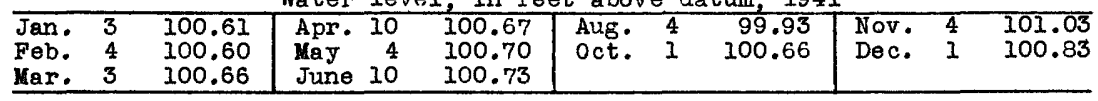

218. Water level, in feet above datum, 1941: oct. 23, 99.83.

326. Water level, in feet above datum, 1941: 0ct. 23, 94.95.

\section{Garfield County}

55. Water level, in feet above datum, 1941: Oct. 16, b/ 106.27 .

\section{Gosper County}

182. Measurements discontinued.

183. Water levels, in feet above datum, 194I: Jan. 22, 100.47; oct. 29, 100.32 .

307.

Water level, in feet above datum, 1941

\begin{tabular}{rrrr|llll|ll|ll}
\hline Jan. 30 & $c$ & 95.50 & May & 1 & $c$ & 95.78 & July 30 & c 96.21 & 0ct. 22 & 96.52 \\
Mar. & 4 & c & 95.60 & June & 3 & c 95.99 & Sept. 2 & c 96.11 & Nov. 5 & c 96.64 \\
30 & c & 95.67 & July & 2 & c 96.21 & oct. & 6 & c 96.41 & & 28 & c 96.81 \\
\hline
\end{tabular}

a Accuracy questionable.

$b$ Highest observed stage in period of record.

c Measurement supplied through courtesy of Central Nebraska Public

Power and Irrigation District. 
Gosper County--Continued.

447. Water level, in feet e.bove datum, 1941: Oct. 29, 100.54 .

\section{Grant County}

215. Water level, in feet above datum, 1941: 0ct. 21, 98.19.

216. Water level, in feet above datum, 1941: 0ct. 21, 99.50.

\section{Greeley County}

206. Water level, in feet above datum, 1941: 0ct. 17, 99,75.

347. Water level, in feet above datum, 1941: 0ct. 17, 97.94.

423. Water level, in feet above datum, 1941: ,0ct. 17, 99.16.

\section{Hall County}

244. Water level, in feet above datum, 1941: 0ct. 15, 99.52. 245 .

Water level, in feet above datum, 1941

\begin{tabular}{lr|lr|lr}
\hline Date & $\begin{array}{r}\text { Water } \\
\text { level }\end{array}$ & Date & $\begin{array}{r}\text { Water } \\
\text { level }\end{array}$ & Date & $\begin{array}{r}\text { Water } \\
\text { level }\end{array}$ \\
\hline Feb. 5 & a 98.97 & June 18 & $\begin{array}{l}\text { al00.77 } \\
\mathbf{9} 100.07\end{array}$ & Oct. 15 & 99.51 \\
Apr. 18 & a 99.42 & Aug. 28 & & \\
\hline
\end{tabular}

246. Water level, in feet above datum, 1941: 0ct. 15, 100.03.

247. No measurements made in 1941.

249. Water level, in feet above datum, 1941: 0ct. 15, 96.87.

258. Water level, in feet above datum, 1941: oct. 15, 96.93.

259. Water level, in feet above datum, 1941: 0ct. 15, 99.54.

260. Water levol, in feet above datum, 1941: 0ct. 15, 99.82 .

261. No measurements made in 1941.

GI202. Water level, in feet above datum, 1941: June 18, a/100.40. GI203. Water levels, in feet above datum, 194l: Mar. 26, a/99.80;

June 18, a $/ 99.90$; July 19 ; a/100.20; Nov. 12, a $/ 100.41$. GI204. Water levels, in feet above datum, 1941: Mar. 26, a /99.02; June 18, a $/ 99.12$; July 19, a $/ 99.37$; Nov. 12, a $/ 99.50$. GI206. Water levels, in feet above datum, 1941: Mar. 26, a/99.40; June 18, 으 $/ 99.75$; Nov. 12, 으/99.56.

GI207, Water levels, in feet above datum, 1941: Feb. 5, a/ /97.15;

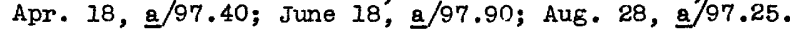

GI208. Water levels, in feet above datum, 1941: Feb. 5, a/ $/$ / $/ 96.65$;

Apr. 18, a/ $/ 98.05$; June 18, a/ $/ 98.20$; Aug. 28, a $/ 97.65$.

GI209. Water level, in feet above datum, 1941: Feb. 5, a/ dry.

GI210. Water Ievels, In feet above datum, 1941: Feb. 5, a/97.40;

Apr. 18, a $/ 97.75$; June 18; a $/ 98.05$; Aug. 28, a $/ \underline{b} / 95.50$.

GI211. Water levels, in feet above datum, 1941; Feb. 5, a/97.42;

Apr. 18, a/97.72; June 18, 으/97.97; Aug. 28, a/b/97.37. ment.

a Measurement supplied through courtesy of Grand Island \#ater Depart.

$\mathrm{b}$ Lowest observed stage in period of record. 
Hall County--Continued.

GI212. Water levels, in feet above datum, 1941: Feb. 5, a/97.80;

Apr. 18, a/98.15; June 18, a/ 98.30; Aug. 28, 으/96.95.

GI214. Water levels, in feet above datum, 1941: Apr. 18, a/94.30;

June 18, a $/ 95.45$; AuE. 28, a $/ 94.65$.

GI215. Water levels, in feet above datum, 1941: Feb. 5, a/97.20;

Apr. 18, 으 $/ 97.75$; June 18, a/ $/ 97.95$; Aug. $28, \underline{a} / \underline{b} / 97.20$.

GI216. Water levels, in feet above datum, 1941: Feb. 5, a/97.62;

Apr. 18, a/98.32; June 18, a/98.32; Aug. 28, a $/ 97.37$.

GI217. Water levels, in feet above datum, 1941: Mar. 26, a/98.65;

June 18, a $/ 98.10$; July 19, a $/ 97.60$; Nov. 12, 의 $/ 98.47$.

GI218. Water levels, In feet abové datum, 1941: Mar. 26, a/98.45;

June 18, a $/ 98.80$; July 19 , a $/ 98.60$; Nov. 12, ㅌ $/ 98.68$.

GI219. Water levels, in feet above datum, 1941: Mar. 26, ㅇ/ 101.32 ;

June 18, a//102.77; July 19, ㅇ/1102.12; Nov. 12, a/101.31.

GI220. Water levels, in feet above datum, 1941: Mar. 26, a/99.75;

June 18, a/100.35; July 19, a /100.55; Nov. 12, a/ $/ 100.14$.

GI221. Water levels, in feet above datum, 1941: Mar. 26, a/99.15;

June $18, \underline{a} / 97.95$; July 19 , a/96.85; Nov. 12, ․․ $/ 99.13$.

GI222. Water levels, in feet above datum, 1941: Mar. 26, a/ $/ 98.50$;

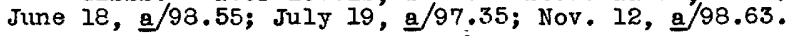

GI223. Water levels, in feet above datum, 1941: Feb. 5, a/99.37;

Apr. 18, a $/ 99.22$.

GI224. No measurements made in 1941.

GI225. Water levels, in feet above datum, 1941: Mar. 26, a/99.30;

June 18, a/100.15; July 19, a/100.35; Nov. 12, ㅌ/ $/ 99.91$.

GI226. Water levels, in feet above datum, 1941: Feb. 5, a/98.50;

Apr. 18, a/ $/ 98.50$; June 18, a $/ 96.60$; Aug. 28, a $/ \underline{b} / 94.40$.

GI227. Water levels, in feet above datum, 1941: Feb. 5, a/97.87;

Apr. 18, a/98.82; June 18, a/98.87; Aug. 28, ㅌ/97.67.

GI2E8. Water level, in feet above datum, 1941: Apr. 18, a/93.59.

GI229. Water levels, in feet above datum, 1941: Apr. 18, a/99.47;

June 18, a/100.92; Aug. 28, a $/ 98.52$.

GI230. Water levels, in feet above datum, 1941: Feb. 5, a $/ 98.57$;

Apr. 18, a $/ 99.27$; June 18, ․ $/ 99.62$; Aug. 28; 트/99.42.

GI231. Water levels, in feet above datum, 1941:

Apr. 13, a/ $/ 98.65$; June 18, a/98.75; Aug. 28, a $/ 97.70$.

GI232. Water levels, in feet above datum, 1941:

Apr. 18, a $/ 97.52$; June 18, a $/ 97.67$; Aug. 28, a $/ 97.49$.

GI233. Water levels, in feet above datum, 1941: Feb. 5, a/97.25;

Apr. 18, a $/ 97.90$; June 18, a $/ 97.95$; Aug. 28, 으. $/ 98.18$.

GI234. Water levels, in feet above datum, 1941: Apr. 18, a/99.60;

Aug. 28, a $/ 99.60$.

GI236. Water levels, in feet above datum, 1941: Feb. 5, a/ㅌ/97.85;

Apr. 18, a $/ 98.95$; June 18, 트/99.35; Aug. 28, 트 $/ 99.80$.

GI237. Water levels, In feet above datum, 1941: Feb. 5, 스으/97.92;

Apr. 18, a $/ 98.77$; June 18, a $/ 99.12$; Aug. 28, a $/ 98.77$.

GI238. Water levels, in feet above datum, 1941: Feb. 5, a/b/97.95;

Apr. 18, a/ $/ 98.75$; June 18, 으/99.20; Aug. 28, 트/98.25.

GI239. Water levels, in feet above datum, 1941: June 18, a/99.38;

July 19, $1 / 99.78 ;$ Nov. 12, a/99.41.

a Measurement supplied through courtesy of Grand Island Water Department.

b Lowest observed stage in period of record. 
Ha11 County--Continued.

GI240. Water levels, in feet above datum, 1941: Mar. 26, a/100.48; June 18, a/101.23; July 19, a/101.58; Nov. 12, a/101.04.

GI241. Water levels, in feet above datum, 1941: June 18, a/99.97; July $19, \underline{a} / 100.42$; Nov. $12, \underline{a} / \underline{b} / 97.21$.

GI242. Vater levels, in feet, above datum, 1941: Mar. 26, a/98.75; June 18, 으/99.50; July 19, a/99.90; Nov. 12, 이요.74.

GI243. No measurements made in 1941.

GI244. Water levels, in feet above datum, 1941: Mar. 26, a/100.02; June 18, a/100.77; July 19, a/101.17; Nov. 12, a/100.55.

GI246. Water levels, in feet above datum, 1941: Mar. 26, a/100.32;

June 18, a//100.97; July 19, a/101.17; Nov. 12, a/100.73.

GI247. Water levels, in feet above datum, 1941: Mar. 26, a/101.33;

June 18, a/102.03; July 19, a/101.68; Nov. 12, a $/ 100.68$.

GI248. Water levels, in feet above datum, 1941: Mar. 26, a/101.15;

June 18, a/101.75; July 19, a/101.65; Nov. 12, a/100.92.

GI249. Water levels, in feet above datum, 1941: Mar. 26, a/101.30;

June 18, a/101.95; July 19, a/101.75; Nov. 12, a/101.01.

GI250. Water levels, in feet above datum, 1941: Mar. 26, a/101.55; June 18, a//101.90; July 19, a/101.50; Nov. 12, 으/100.52.

GI251. Water levels, in feet above datum, 1941: Mar. 26, a/98.15;

June 18, a/98.40; July 19; a $/ 98.95$; Nov. 12, 의 $/ 99.02$.

GI252. Water levels, in feet above datum, 1941: Mar. 26, a/97.60;

June 18, a/97.85; July 19; a/98.65; Nov. 12, a/98.67.

GI253. Water levels, in feet above datum, 1941: Mar. 26, a/b/97.40;

June 15, a/97.75; July 19; a $/ 98.20$; Nov. 12, a/98.32.

Gr254. Water levels, in feet above datum, 1941: Mar. 26, a/97.05;

June 18, a/97.40; July 19, a/98.00; Nov. 12, a/98.07.

GI255. No measurements made in 1941.

\section{Hamilton County}

158. Water levels, in feet above datum, 1941: Jan. 21, b/93.58;

oct. $30,95.40$.

159. Measurements discontinued.

160. Water levels, in feet above datum, 1941: Jan. 21, 97.48;

oct. $30,98.67$.

173. Water level, in feet above datum, 1941: Oct. 30, 100.80.

330. No measurements made in 1941.

\section{Harlan County}

155. Water levels, in feet above datum, 1941: Jan. 21, 100.35; oct. 29, 101.31.

222. No measurements made in 1941.

329. Water level, in feet above datum, 1941: 0ct. 29, 99.76.

389. Water level, in feet above datum, 1941: oct. 29, 99.42 .

a Measurement through courtesy of Grand Island Water Department.

b Lowest observed stage in period of record. 


\section{Heyes County}

141. Water level, in feet above datum, 1941: 0ct. 27, 100.98 . 142. Water levels, in foet above datum, 1941: Jar. 22, 100.95; 0ct. $27,100.97$.

446. Water level, in feet above datum, 1941: 0et. 27, 100.87.

\section{Hitchcock County}

140. Water level, in feet above datum, 1941: 0ct. 27, 98.77.

178. Water level, in feet above datum, 1941: 0ct. 28, 101.35.

362. Water level, in feet above datum, 1941: 0ct. 27, 100.13.

\section{Holt County}

122. No measurements made in 1941.

113. No measurements made in 1941.

203. Water level, in feet above datum, 1941: 0ct. 17,99.62.

373. Water level, in feet above datum, 1941: 0ct. 18, 100.88.

374. Water level, in feet above datum, 1941: 0ct. 18, 98.64.

424. Water level, in feet above datum, 1941: 0ct. 17, 99.26.

428. Water level, in feet above datum, 1941: 0ct. 17, 98.51.

Hooker County

214. Water leveI, in feet above datum, 1941: 0ct. 21, 93.73.

\section{Howard County}

46. Water level, in feet above datum, 1941: 0ct. 17, 100.73.

51. Water level, in feet above datum, 1941: 0ct. 14, 98.30.

59. Water level, in feet above datum, 1941: Oct. 17, 99.09.

98. Water level, in feet above datum, 1941: 0ct. 16, 99.67.

346. Water level, in feet above datum, 1941: 0ct. 16, 100.59.

\section{Jefferson county}

226. Water level, in feet above datum, 1941: Nov. 1, a/ 97.74 . 227. No measurements made in 1941.

228. Water level, in feet above datum, 1941: Nov. 1, 98.82.

229. Water leve1, in feet above datum, 1941: Nov. 1,99.17.

\section{Johnson County}

2. Water level, in feet above datum, 1941: 0ct. 7, 99.84.

3. No measurements made in 1941.

a Lowest observed stage in period of record. 


\section{Kearney County}

181. Water level, in feet above datum, 1941: 0ct. 29, a/102.57.

266. Water level, in feet above datum, 1941: Nov. 1, b/97.74.

Keith County

93.

Water level, in feet above detum, 1941

\begin{tabular}{|c|c|c|c|c|c|c|c|c|}
\hline to & $\begin{array}{l}\text { Water } \\
\text { level }\end{array}$ & Date & & $\begin{array}{l}\text { Water } \\
\text { level }\end{array}$ & Date & $\begin{array}{l}\text { Water } \\
\text { level }\end{array}$ & Date & $\begin{array}{l}\text { Weter } \\
\text { level }\end{array}$ \\
\hline $\begin{array}{l}\text { feb. } \\
\text { Apr. } 1\end{array}$ & $\begin{array}{l}\text { c100.96 } \\
\text { c101.04 } \\
\text { c101.52 }\end{array}$ & $\begin{array}{l}\text { May } \\
\text { June } \\
\text { July }\end{array}$ & $\begin{array}{l}6 \\
1\end{array}$ & $\begin{array}{l}c 101.21 \\
c 101.06 \\
c 101.06\end{array}$ & $\begin{array}{l}\text { Aug. } \\
\text { Sept. } \\
\text { Oct. }\end{array}$ & $\begin{array}{l}c 100.90 \\
c 100.70 \\
c 100.55\end{array}$ & $\begin{array}{lr}\text { Oct. } & 22 \\
\text { Nov. } & 4 \\
\text { Dec. } & 1\end{array}$ & $\begin{array}{r}100.72 \\
c 100.81 \\
c 100.98\end{array}$ \\
\hline
\end{tabular}

255. Water level, in feet above datum, 1941: 0ct. 26, 98.79 .

348. No measurements made in 1941.

350 .

Water level, in feet above datum, 1941

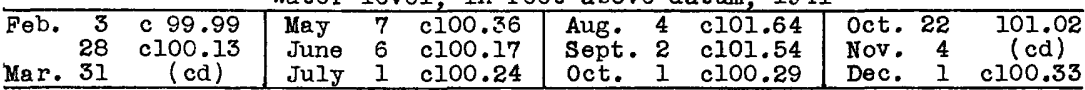

351.

Water level, in feet above datum, 1941

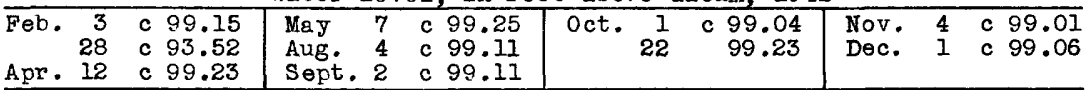

358. Water leveI, in feet above datum, 1941: 0ct. 25, 97.72.

360. Water level, in feet above datum, 1941: 0ct. 26, 99.72.

Nl. Water levels, in feet below measuring point, 1941: Jan. 31, c/11.80; Feb. 26, c/11.00; Apr. 4, c/ well submerged.

N4. Measurements supplied through courtesy of Central Nebraska Public Power and Irrigation District.

Water level, in feet below measuring point, 1941

\begin{tabular}{ll|lll|lll|lll}
\hline Jan. 31 & 18.92 & May & 1 & 19.05 & Aug. & 1 & 19.04 & Nov. & 6 & 19.18 \\
Feb. 26 & 18.97 & June & 5 & 18.65 & Sept. 4 & 19.11 & Dec. & 2 & 19.34 \\
Apr. 12 & 18.66 & July & 2 & 19.02 & Oct. & 3 & 19.12 & & & \\
\hline
\end{tabular}

N5. Measurements supplied through courtesy of Central Nebraska Publia Power and Irrigation District. Water level, in feet below measuring point, 1941

\begin{tabular}{|c|c|c|c|c|c|c|c|}
\hline $\begin{array}{l}\text { Jan. } 31 \\
\text { Peb. } 26 \\
\text { pr. } 12\end{array}$ & $\begin{array}{l}9.60 \\
9.62 \\
9.66\end{array}$ & $\begin{array}{l}\text { May } \\
\text { June } \\
\text { July }\end{array}$ & $\begin{array}{l}1 \\
5 \\
1\end{array}$ & $\begin{array}{l}9.60 \\
9.48 \\
9.55\end{array}$ & $\begin{array}{l}\text { Aug. } 1 \\
\text { Sept. } 4 \\
\text { oct. } 3\end{array}$ & $\begin{array}{l}9.77 \\
9.90 \\
9.93\end{array}$ & $\begin{array}{llr}\text { Nov. } & 6 & 9.89 \\
\text { Dec. } & 2 & \text { b } 10.20\end{array}$ \\
\hline
\end{tabular}

N6. Measurements supplied through courtesy of Central Nebraska Publia Power and Irrigation District.

Water level, in feet below measuring point, 1941

\begin{tabular}{ll|lll|ll|llll}
\hline Jan. 31 & 5.29 & May & 1 & 5.03 & Aug. & 1 & 5.75 & Nov. & 6 & 5.89 \\
Feb. 26 & 5.60 & June & 5 & 4.78 & Sept. 4 & 5.90 & Dec. & 2 & b 5.95 \\
Apr. 12 & 5.60 & July & 2 & 5.24 & Oct. 3 & b 5.95 & & & \\
\hline
\end{tabular}

N7. Measurements supplied through courtesy of Central Nebraska Publio Power and Irrigation District.

a Highest observed stage in period of record.

$b$ Lowest observed stage in perlod of record.

c Measurement supplied through courtesy of Central Nebraska Public Power and Irrigation District.

d Pump operating in well. 
Keith County--Continued.

N7.--Continued.

Water level, in feet below measuring point, 1941

\begin{tabular}{|c|c|c|c|c|c|c|c|c|c|c|}
\hline Date & $\begin{array}{l}\text { Water } \\
\text { Ievel }\end{array}$ & Dete & & $\begin{array}{l}\text { Water } \\
\text { leveI }\end{array}$ & Date & $\begin{array}{l}\text { Water } \\
\text { level }\end{array}$ & Date & & & $\begin{array}{l}\text { Water } \\
\text { level }\end{array}$ \\
\hline $\begin{array}{ll}\text { Jan. } & 31 \\
\text { Feb: } 26 \\
\text { Apr. } 12\end{array}$ & $\begin{array}{l}10.15 \\
10.85 \\
10.77\end{array}$ & $\begin{array}{l}\text { May } \\
\text { June } \\
\text { JuIy }\end{array}$ & $\begin{array}{l}1 \\
5 \\
2\end{array}$ & $\begin{array}{l}10.75 \\
10.70 \\
10.68\end{array}$ & $\begin{array}{l}\text { Aug. } 1 \\
\text { Sept. } 4\end{array}$ & $\begin{array}{l}10.82 \\
10.90\end{array}$ & $\begin{array}{l}\text { Oct. } \\
\text { Dec. }\end{array}$ & $\begin{array}{l}3 \\
2\end{array}$ & $a$ & $\begin{array}{l}10.92 \\
10.81\end{array}$ \\
\hline
\end{tabular}

N9. Measurements supplied through courtesy of Central Nebraska Publia Power and Irrigation District.

Water level, in feet below measuring point, $194 \mathrm{l}$

\begin{tabular}{ll|lll|lll|lll}
\hline Jan. 31 & 13.98 & June & 5 & 13.39 & Sept. 4 & 14.32 & Nov. & 6 & 14.02 \\
Feb. 26 & 13.92 & July & 2 & 13.41 & Oct. & 3 & 14.20 & Dec. & 2 & 13.98 \\
May & 1 & 13.33 & Aug. & 1 & 13.92 & & & & & \\
\hline
\end{tabular}

N1I. Measurements supplied through courtesy of Central Nebraska Public Power and Irrigation District.

Water level, in feet below measuring point, 1941

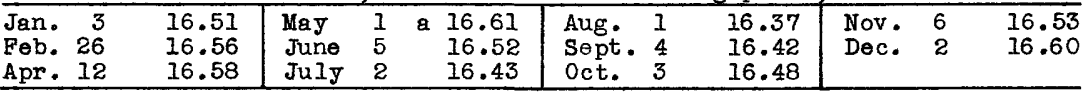

N18. Measurements supplied through courtesy of Central Nebraska Public Power and Irrigation District.

Water level, in feet below measuring point, 194.1

\begin{tabular}{ll|lll|lll|lll}
\hline Jan. 31 & (b) & May & 1 & 34.01 & Aug. & 2 & 34.17 & Nov. & 6 & 34.09 \\
Feb. 26 & 34.10 & June & 6 & 33.99 & Sept. 4 & a & 35.19 & Dec. & 2 & 34.09 \\
Apr. 12 & 34.00 & July & 2 & 34.12 & Oct. & 3 & 34.57 & & & \\
\hline
\end{tabular}

N25. Measurements supplied through courtesy of Central Nebraska Public Power and Irrigation District.

Water level, in feet below measuring point, 1941

\begin{tabular}{lll|ll|lll|lll}
\hline Jan. & 2 & 36.26 & Apr. 7 & 36.34 & July & 5 & 36.40 & Oct. & 1 & 36.37 \\
Feb. & 4 & 36.31 & May 1 & 36.35 & Aug. & 4 & 36.41 & Nov. & 4 & 36.50 \\
Mar. & 3 & 36.31 & June.10 & 36.38 & Sept. 2 & 36.43 & Dec. & 1 & a & 36.51 \\
\hline
\end{tabular}

N35. Measurements supplied through courtesy of Central Nebraska Public Power and Irrigation District.

Water level, in feet below measuring point, 1941

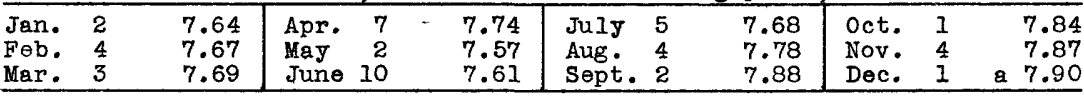

N37. Measurements supplied through courtesy of Central Nebraska Public Power and Irrigation District

Water level, in feet below measuring point, 1941

\begin{tabular}{lll|lrr|lll|llll}
\hline Jan. & 2 & 15.64 & Apr & 9 & 10.67 & July & 5 & 11.55 & Oct. & 1 & 12.82 \\
Feb. & 4 & 14.54 & May & 2 & c 10.44 & Aug. & 4 & 12.95 & Nov. & 4 & 12.32 \\
Mar. & 3 & 12.08 & June & 10 & 10.60 & Sept. & 2 & 14.05 & Dec. & 1 & 11.56 \\
\hline
\end{tabular}

N43. Measurements supplied through courtesy of Central Nebraska Public Power and Irrigation District.

Water level, in feet below measuring point, 1941

\begin{tabular}{lrr|lll|lll|llll}
\hline Jan. 31 & 83.24 & May & 1 & 83.29 & Aug. & 1 & 83.39 & Nov. & 6 & 83.36 \\
Feb. 26 & 83.29 & June & 5 & 83.34 & Sept. 4 & a 83.40 & Dec. & 2 & 83.34 \\
Apr. 4 & 83.33 & July & 1 & 83.37 & Oct. & 3 & 83.38 & & & \\
\hline
\end{tabular}

a Lowest observed stage in perlod of record.

b Well recently submerged.

c Highest observed stage in period of record. 
Keith County--Continued.

N42. Measurements supplied through courtesy of Central Nebraska Public Power and Irrigation District.

Water level, in feet below measuring point, 1941

\begin{tabular}{|c|c|c|c|c|c|c|c|c|c|}
\hline Date & $\begin{array}{l}\text { Water } \\
\text { level }\end{array}$ & Date & & $\begin{array}{l}\text { Water } \\
\text { level }\end{array}$ & Dete & $\begin{array}{l}\text { Water } \\
\text { level }\end{array}$ & Date & & $\begin{array}{l}\text { Water } \\
\text { level }\end{array}$ \\
\hline $\begin{array}{lr}\text { Feb. } & 4 \\
& 26 \\
\text { Apr. } & 12\end{array}$ & $\begin{array}{l}89.38 \\
89.52 \\
91.50\end{array}$ & $\begin{array}{l}\text { May } \\
\text { June } \\
\text { July }\end{array}$ & $\begin{array}{l}1 \\
5 \\
2\end{array}$ & $\begin{array}{l}91.50 \\
92.33 \\
92.32\end{array}$ & $\begin{array}{ll}\text { Aug. } & 2 \\
\text { Sept. } 4 \\
\text { oct. } 3\end{array}$ & $\begin{array}{l}92.45 \\
92.32 \\
92.00\end{array}$ & $\begin{array}{l}\text { Nov. } \\
\text { Dec. }\end{array}$ & $\begin{array}{l}6 \\
2\end{array}$ & $\begin{array}{l}92.03 \\
92.02\end{array}$ \\
\hline
\end{tabular}

S10. Measurements supplied through courtesy of Central Nebraska Public Power and Irrigation District.

Water level, in feet below measuring point, 1941

\begin{tabular}{ll|lll|lll|lll}
\hline Jan. 31 & 22.76 & May & 5 & 22.59 & Aug. & 1 & 22.27 & Nov. 5 & (a) \\
Feb. 26 & 22.91 & June & 6 & 21.55 & Sept. 4 & 22.79 & Dec. & 2 & (a) \\
Apr. 12 & 22.76 & July & 2 & 22.05 & Oct. & 3 & 23.05 & & & \\
\hline
\end{tabular}

S16. Measurements supplied through courtesy of Central Nebraska Public Power and Irrigation District.

Water level, in feet below measuring point, 1841

\begin{tabular}{lrr|lll|lll|lll}
\hline Feb. & 5 & 188.39 & May & 5 & 188.58 & Aug. & 1 & 188.70 & Nov. & 5 & 188.80 \\
& 26 & 188.81 & June & 6 & 188.60 & Sept. & 4 & 188.71 & Dec. & 1 & 188.81 \\
Apr. 12 & 188.70 & July & 2 & 188.85 & Oct. & 3 & 188.65 & & & \\
\hline
\end{tabular}

S18. Measurements supplied through courtesy of Central Nebraska Public Power and Irrigation District.

Water level, in feet below measuring point, 1941

\begin{tabular}{|rr|lll|l|l|l|lll}
\hline Jan. 31 & 162.78 & May & 1 & 162.70 & Aug. & 1 & 162.82 & Nov. & 5 & 162.85 \\
Feb. 26 & 162.79 & Jurie & 5 & 162.69 & Sept. 4 & 163.78 & Dec. & 1 & 162.82 \\
Apr. 4 & 162.82 & July & 2 & 162.98 & Oct. & 3 & bl63.79 & & & \\
\hline
\end{tabular}

S19. Measurements supplied through courtesy of Central Nebraska Public Power and Irrigation District.

Water level, in feet below measuring point, 1941

\begin{tabular}{ll|lll|lll|lll}
\hline Jan. 31 & 167.92 & May & 1 & 167.95 & Aug. & 1 & 168.06 & Nov. & 5 & 168.05 \\
Feb. 26 & 167.93 & June & 5 & 167.93 & Sept. 4 & 168.04 & Dec. & 1 & 168.00 \\
Apr. 4 & 168.12 & July & 2 & 167.95 & Oct. & 1 & 168.00 & & & \\
\hline
\end{tabular}

S20. Measurements supplied through courtesy of Central Nebraska Public Power and Irrigation District.

Water level, in feet below measuring point, 1941

\begin{tabular}{lrr|lrr|lll|lll}
\hline Jan. & 31 & 183.27 & May & 2 & 183.08 & Aug. 4 & 183.34 & Nov. & 4 & 183.16 \\
Mar. & 3 & 183.22 & June & 10 & 183.09 & Sept. 2 & 183.04 & Dec. & 1 & 183.34 \\
Apr. & 7 & 183.37 & July & 3 & 183.40 & Oct. & 1 & 183.05 & & & \\
\hline
\end{tabular}

S21. Measurements supplied through courtesy of Central Nebraska Public Power and Irrigation District.

Water level, in feet below measuring point, 1941

\begin{tabular}{lll|lrr|lll|lll}
\hline Jan. & 2 & 105.62 & Apr. & 7 & 105.70 & July & 3 & 105.70 & Oct. & 1 & 105.46 \\
Feb. & 4 & 105.61 & May & 2 & c104.36 & Aug. & 4 & 105.69 & Nov. & 4 & 105.60 \\
Mar. & 3 & 105.69 & June & 10 & 105.60 & Sept. 2 & 105.48 & Dec. & 1 & 105.67 \\
\hline
\end{tabular}

S22. Measurements supplied through courtesy of Central Nebraska Public Power and Irrigati on District.

Water level, in foet bel ow measuring point, 1941

\begin{tabular}{lrr|lr|lll|lll}
\hline Jan. & 2 & 107.80 & Apr. 10 & 107.74 & July & 3 & 107.97 & Oct. & 1 & 107.79 \\
Feb. & 4 & 107.79 & May 2 & 107.79 & Aug. 4 & 107.85 & Noy. & 4 & 107.76 \\
Mar. & 3 & b108.15 & June 12 & 107.88 & Sept. 2 & 107.73 & Doc. & 1 & 107.97 \\
\hline
\end{tabular}

a Dry.

$b$ Lowest observed stage in period of record.

c Highest observed stage in period of record. 
Keith County--Continued.

S23. Measurements supplied through courtesy of Central Nebraska Public Power and Irrigation District.

Water level, in feet below measuring point, 1941

\begin{tabular}{|c|c|c|c|c|c|c|c|c|c|c|}
\hline Date & & $\begin{array}{l}\text { Water } \\
\text { level }\end{array}$ & Date & $\begin{array}{l}\text { Water } \\
\text { level }\end{array}$ & Date & & $\begin{array}{l}\text { Water } \\
\text { level }\end{array}$ & Date & & $\begin{array}{l}\text { Water } \\
\text { level }\end{array}$ \\
\hline $\begin{array}{l}\text { Jan. } \\
\text { Feb. } \\
\text { Mar. }\end{array}$ & $\begin{array}{l}2 \\
4 \\
3\end{array}$ & $\begin{array}{r}113.65 \\
113.58 \\
\mathrm{a} 113.79\end{array}$ & $\begin{array}{lr}\text { Apr. } & 9 \\
\text { May } & 5 \\
\text { June } & 12\end{array}$ & $\begin{array}{l}113.42 \\
113.66 \\
113.68\end{array}$ & $\begin{array}{l}\text { July } \\
\text { Aug. } \\
\text { Sept. }\end{array}$ & $\begin{array}{l}3 \\
4 \\
2\end{array}$ & $\begin{array}{l}113.71 \\
113.67 \\
113.52\end{array}$ & $\begin{array}{l}\text { Oct. } \\
\text { Nov. } \\
\text { Dec. }\end{array}$ & $\begin{array}{l}1 \\
4 \\
1\end{array}$ & $\begin{array}{l}113.57 \\
113.49 \\
113.39\end{array}$ \\
\hline
\end{tabular}

S24. Measurements supplied through courtesy of Central Nebraska Public Power and Irrigation District.

Water level, in feet below measuring point, 1941

\begin{tabular}{lll|ll|lll|lll}
\hline Jan. & 2 & 60.50 & Apr. 10 & 60.67 & July 3 & 61.05 & Oct. & 1 & 61.23 \\
Feb. & 4 & 60.48 & May 5 & 60.75 & Aug. 4 & 61.11 & Nov. & 4 & 61.35 \\
Mar. & 3 & 60.78 & June 12 & 60.80 & Sept. 2 & 61.15 & Dec. & 1 & a & 61.46 \\
\hline
\end{tabular}

S26. Measurements supplied through courtesy of Central Nebraska Public Power and Irrigation District.

Water level, in feet below measuring point, 1941

\begin{tabular}{lrl|lll|lll|lll}
\hline Feb. 3 & 16.37 & May & 6 & 15.91 & Aug. 4 & 17.20 & Nov. 4 & 16.54 \\
& 28 & 16.16 & June & 6 & 16.46 & Sept. 2 & a 17.48 & Dec. & 1 & 16.25 \\
Apr. 12 & 16.14 & July & 1 & 16.76 & Oct. & 1 & 16.85 & & & \\
\hline
\end{tabular}

s27. Measurements supplied through courtesy of Central Nebraska Public Power and Irrigation District.

Water level, in feet below measu ring point, 1941

\begin{tabular}{lrl|lll|lll|ll}
\hline Feb. & 3 & 13.55 & May & 6 & 13.50 & Aug. 4 & 13.67 & Nov. 4 & 13.31 \\
& 28 & 13.33 & June & 6 & 13.70 & Sept. 2 & 13.82 & Dec. & 1 & 13.86 \\
Apr. 12 & 13.60 & July & 1 & 13.55 & Oct. 1 & 13.75 & & & \\
\hline
\end{tabular}

S31. Measurements supplied through courtesy of Central Nebraska Public Power and Irrigation District.

Water level, in feet below measuring point, 1941

\begin{tabular}{|c|c|c|c|c|c|}
\hline Date & $\begin{array}{l}\text { Water } \\
\text { level }\end{array}$ & Date & $\begin{array}{l}\text { Water } \\
\text { level }\end{array}$ & Date & $\begin{array}{l}\text { Water } \\
\text { level }\end{array}$ \\
\hline $\begin{array}{ll}\text { Jan. } & 2 \\
\mathrm{Feb} . & 4 \\
\end{array}$ & $\begin{array}{r}94.54 \\
\mathrm{a} 95.53 \\
\end{array}$ & $\begin{array}{lr}\text { Mar. } 3 \\
\text { Apr. } 10 \\
\end{array}$ & $\begin{array}{l}95.50 \\
95.40 \\
\end{array}$ & May 2 & 95.40 \\
\hline
\end{tabular}

S32. Measurements supplied through courtesy of Central Nebraska Public Power and Irrigation District.

Water level, in feet below measuring point, 1941

\begin{tabular}{|c|c|c|c|c|c|c|c|c|c|c|c|}
\hline Date & & & $\begin{array}{l}\text { Water } \\
\text { level }\end{array}$ & Date & $\begin{array}{l}\text { Water } \\
\text { level }\end{array}$ & Date & & $\begin{array}{l}\text { Water } \\
\text { level }\end{array}$ & Date & & $\begin{array}{l}\text { Water } \\
\text { level }\end{array}$ \\
\hline $\begin{array}{l}\text { Jan. } \\
\text { Feb. } \\
\text { Mar. }\end{array}$ & $\begin{array}{l}2 \\
4 \\
3\end{array}$ & $\begin{array}{l}a \\
a\end{array}$ & $\begin{array}{l}59.19 \\
59.19 \\
59.18\end{array}$ & $\begin{array}{l}\text { Apr. } \\
\text { May } \\
\text { June }\end{array}$ & $\begin{array}{l}59.14 \\
59.08 \\
59.15\end{array}$ & $\begin{array}{l}\text { July } \\
\text { Aug. } \\
\text { Sept. }\end{array}$ & $\begin{array}{ll}3 & a \\
4 & \\
2 & \end{array}$ & $\begin{array}{l}59.19 \\
59.12 \\
59.00\end{array}$ & $\begin{array}{l}\text { Oct. } \\
\text { Nov: } \\
\text { Dec. }\end{array}$ & $\begin{array}{l}1 \\
4 \\
1\end{array}$ & $\begin{array}{l}59.05 \\
59.16 \\
59.18\end{array}$ \\
\hline
\end{tabular}

S35. Measurements supplied through courtesy of Central Nebraska Public Power and Irrigation District.

Water level, in feet below measuring point, 1941

\begin{tabular}{lrr|ll|lll|lll}
\hline Apr. 12 & 167.01 & Aug. 2 & 167.10 & Oct. & 3 & 167.09 & Dec. & 1 & 167.09 \\
May & 5 & 166.89 & Sept. 5 & 167.12 & Nov. & 4 & 167.10 & & & \\
\hline
\end{tabular}

a Lowest observed stage in perlod of record.

$5110050-49-12$ 


\section{Keyapaha County}

375. Water level, in feet above datum, 1941: 0ct. 18, 99.30.

\section{Kimball County}

88. Water levels, in feet above datum, 1941: 0ct. 25, 99.68; Nov. 28, a $/ 99.80 ;$ Dec. 23, a/99.82.

89. Water level, in feet above datum, 1941: oct. 25, 100.10.

327. Water level, in feet above datum, 1941: oct. 25, b/98.16.

344. Measurements discontinued.

\section{Knox County}

67. Water level, in feet above datum, 1941: 0ct. 11, 98.46.

71. Water level, in feet above datum, 1941: oct. 18, 99.87 :

335. Water leve1, in feet above datum, 1941: 0ct. 18, 97.29.

336. Water level, in feet above datum, 1941: Oct. 18, 101.51.

370. Water level, in feet above datum, 1941: 0̣ct. 11,97.42. ment.

429. Oct. 18, 194I: Insufficient water in well to permit measure-

\section{Laneaster County}

1. No measurements made in 1941.

13. Water level, in feet above datum, 1941: 0ct. 4, 98.19.

14. Water level, in feet above datum, 1941: 0ct. 6, 98.67.

360. Water level, in feet above datum, 1941: Nov. 3, c/104.17.

367. No measurements made in 1941.

\section{Iincoln County}

131. Water level, in feet above datum, 1941: 0ct. 22, 101.73.

133. No measurements made in 1941.

134. Water levels, in feet above datum, 1941: Jan. 22, 100.55; oct. $27,100.61$.

143. Water levels, in feet above datum, 1941: Jan. 22, 101.12; oct. $27, \mathrm{c} / 101.16$.

144. Water levels, in feet above datum, 1941: Jan. 22, b/ $/ 98.97$; oct. $27,99.74$.

241 .

Water level, in feet above datum, 1941

\begin{tabular}{|c|c|c|c|c|c|c|c|c|c|c|c|c|}
\hline get & & & $\begin{array}{l}\text { Water } \\
\text { level }\end{array}$ & Date & & & $\begin{array}{l}\text { Water } \\
\text { level }\end{array}$ & Date & $\begin{array}{l}\text { We } \\
\text { le }\end{array}$ & Date & & $\begin{array}{l}\text { Wa } \\
\text { le }\end{array}$ \\
\hline $\mathrm{eb}$ & $\begin{array}{r}2 \\
1 \\
29\end{array}$ & $\begin{array}{l}\mathrm{d} \\
\mathrm{d} \\
\mathrm{d}\end{array}$ & $\begin{array}{l}99.46 \\
99.40 \\
99.39\end{array}$ & $\begin{array}{l}\text { Apr. } \\
\text { May } \\
\text { June }\end{array}$ & $\begin{array}{l}30 \\
30 \\
30\end{array}$ & $\begin{array}{l}\mathrm{d} \\
\mathrm{d} \\
\mathrm{d}\end{array}$ & $\begin{array}{l}89 \\
32 \\
84\end{array}$ & $\begin{array}{lrr}\text { July } & 28 & d \\
\text { Aug. } & 30 & d b \\
\text { oct. } & 7 & d\end{array}$ & $\begin{array}{l}98 \\
98 \\
98\end{array}$ & $\begin{array}{l}\text { Oct. } 22 \\
\text { Nov. } \\
29\end{array}$ & $\mathrm{~d}$ & $\begin{array}{l}98.92 \\
98.91 \\
98.94\end{array}$ \\
\hline
\end{tabular}

a Measurements supplied by F. C. Foley, U. S. Geological Survey.

$b$ Lowest observed stage in period of record.

c Highest observed stage in period of record.

d Measurement supplied through courtesy of Central Nebraska Public Power and Irrigation District. 
Iincoln County--Continued.

242 .

\begin{tabular}{|c|c|c|c|c|c|c|c|c|}
\hline Date & $\begin{array}{l}\text { Water } \\
\text { level }\end{array}$ & Date & $\begin{array}{l}\text { Water } \\
\text { level }\end{array}$ & Date & & $\begin{array}{l}\text { Water } \\
\text { level }\end{array}$ & Date & $\begin{array}{l}\text { Water } \\
\text { level }\end{array}$ \\
\hline $\begin{array}{lr}\text { Feb. } & 3 \\
\text { Mar. } & 3 \\
30\end{array}$ & $\begin{array}{l}0101.93 \\
2102.23 \\
0.102 .36\end{array}$ & $\begin{array}{ll}\text { Apr. } & 30 \\
\text { May } & 31 \\
\text { June } & 30\end{array}$ & $\begin{array}{l}0.102 .67 \\
0102.38 \\
0102.35\end{array}$ & $\begin{array}{l}\text { July } \\
\text { Aug. } \\
\text { Oct. }\end{array}$ & $\begin{array}{r}28 \\
29 \\
3\end{array}$ & $\begin{array}{r}\text { ac102.99 } \\
\text { a } 102.12 \\
\text { a } 102.26\end{array}$ & $\begin{array}{ll}\text { Oct. } & 27 \\
\text { Nov. } & 1 \\
\text { Dec. } & 1\end{array}$ & $\begin{array}{r}102.42 \\
\mathrm{a} 102.54 \\
\mathrm{a} 102.85\end{array}$ \\
\hline
\end{tabular}

252. Water level, in feet above datum, 1941: Oct. 27, b/99.00.

253. Water level, in feet above datum, 1941: 0ct. 27, b/99.07.

383. No measurements made in 1941.

384. Water level, in feet above datum, 1941: 0ct. 27, 100.59.

385. Water level, in feet above datum, 1941: Oct. 27, 99.48 .

405 .

Water level, in feet above datum, 1941

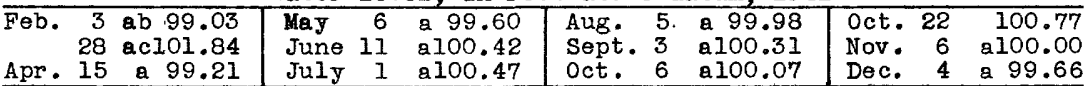

406. Water level, in feet above datum, 1941: Oct. 22, 99.91.

E26. Measurements supplied through courtesy of Central Nebraska Public Power and Irrigation District.

Water level, in feet below measuring point, 1941

\begin{tabular}{rrr|lrr|lll|lll}
\hline Feb. 3 & b 11.30 & May & 7 & 11.03 & Aug. & 5 & 10.29 & Nov. & 5 & 10.58 \\
28 & 11.22 & June 7.1 & 10.75 & Sept. 3 & 10.44 & Dec. & 4 & 10.79 \\
Apr. 11 & 11.26 & July & 1 & 10.48 & Oct. & 6 & 10.18 & & & \\
\hline
\end{tabular}

E27. Measurements supplied through courtesy of Central Nebraska Public Power and Irrigation District.

Water level, in feet below measuring point, 1941

\begin{tabular}{lrr|lr|lll|lrr}
\hline Feb. & 3 & 10.79 & May & 7 & 10.59 & Aug. 5 & 9.91 & Nov. & 5 & 9.91 \\
& 28 & 10.77 & June 11 & 10.57 & Sept. 3 & 10.18 & Dec. & 4 & 10.02 \\
Apr. 11 & 10.81 & July & 1 & 10.26 & Oct. & 6 & c 9.78 & & & \\
\hline
\end{tabular}

E38. Measurements supplied through courtesy of Central Nebraska F iblic Power and Irrigation District.

Water level, in feet below measuring point, 1941

\begin{tabular}{rr|lll|lll|ll}
\hline Feb. & 3 & 13.39 & May & 7 & 13.13 & Aug. 5 & 13.57 & Nov. 4 & 13.33 \\
28 & 13.27 & June & 6 & 13.31 & Sept. 3 & 13.71 & Dec. & 4 & 13.12 \\
Apr. 11 & 13.20 & July & 1 & 13.38 & Oct. & 1 & 13.68 & & \\
\hline
\end{tabular}

JS-1. Measurements supplied through courtesy of Central Nebraska Public Power and Irrigation District.

Water level, in feet below measuring point, 1941

\begin{tabular}{lll|ll|ll|llr}
\hline Jan. & 3 & 35.12 & Mar. 30 & 32.61 & July 29 & 30.81 & Nov. 4 & 30.00 \\
Feb. & 3 & 34.03 & May 31 & 31.37 & Aug. 30 & 30.77 & & 29 & 29.91 \\
Mar. & 3 & 33.26 & July 1 & 30.91 & Oct. 7 & 30.32 & Dec. 31 & c 29.88 \\
\hline
\end{tabular}

JS-2. Measurements supplied through courtesy of Central Nebraska Public Power and Irrigation District.

Water level, in feet below measuring point, 1941

\begin{tabular}{|c|c|c|c|c|c|c|c|c|c|}
\hline $\begin{array}{l}\text { Jan. } \\
\text { Feb. } \\
\text { Mar. }\end{array}$ & $\begin{array}{r}3 \\
3 \\
3 \\
30\end{array}$ & $\begin{array}{l}27.38 \\
22.55 \\
21.84 \\
21.17\end{array}$ & $\begin{array}{l}\text { May } \\
\text { July }\end{array}$ & $\begin{array}{r}1 \\
31 \\
1\end{array}$ & $\begin{array}{l}20.52 \\
20.14 \\
19.97\end{array}$ & $\begin{array}{l}\text { July } 29 \\
\text { Aug. } 30 \\
\text { Oct. } 7\end{array}$ & $\begin{array}{l}19.93 \\
20.32 \\
19.63\end{array}$ & $\begin{array}{l}\text { Nov. } \\
\text { Dec. } 3\end{array}$ & $\begin{array}{r}19.29 \\
\mathrm{c} \quad 19.24 \\
19.25\end{array}$ \\
\hline
\end{tabular}

a Measurements supplied through courtesy of Central Nebraska Public Power and Irrigation District.

$b$ Lowest observed stage in period of record.

c Highest observed stage in period of record. 
Lincoln County--Continued.

JS-3. Measurements supplied through courtesy of Central Nebraska Public Power and Irrigation District.

Water level, in feet below measuring point, 1941

\begin{tabular}{|c|c|c|c|c|c|c|c|c|c|}
\hline Date & $\begin{array}{l}\text { Water } \\
\text { level }\end{array}$ & Date & & $\begin{array}{l}\text { Water } \\
\text { level }\end{array}$ & Date & $\begin{array}{l}\text { Water } \\
\text { level }\end{array}$ & Date & & $\begin{array}{l}\text { Water } \\
\text { level }\end{array}$ \\
\hline $\begin{array}{l}\text { Jan. } \\
\text { Feb: } 3 \\
\text { Mar. } \\
\\
\\
31\end{array}$ & $\begin{array}{l}29.91 \\
29.15 \\
28.74 \\
28.30\end{array}$ & $\begin{array}{l}\text { May } \\
\text { June } \\
\text { July }\end{array}$ & $\begin{array}{l}1 \\
2 \\
1\end{array}$ & $\begin{array}{l}27.71 \\
27.35 \\
27.06\end{array}$ & $\begin{array}{ll}\text { July } 29 \\
\text { Aug. } 30 \\
\text { Oct. } \quad 7\end{array}$ & $\begin{array}{l}27.23 \\
27.45 \\
27.19\end{array}$ & $\begin{array}{lr}\text { Nov. } & 4 \\
& 29 \\
\text { Dec. } & 31\end{array}$ & $\begin{array}{l}a \\
a\end{array}$ & $\begin{array}{l}27.02 \\
27.01 \\
27.01\end{array}$ \\
\hline
\end{tabular}

JS-4. Measurements supplied through courtesy of Central Nebraska Public Power and Irrigation District.

Water level, in feet below measuring point, 1941

\begin{tabular}{lrr|lrr|rrr|rrr}
\hline Jan. & 3 & 19.69 & May & 1 & 17.66 & July & 29 & 17.44 & Nov. 4 & 17.25 \\
Feb. & 3 & 18.79 & June & 2 & 17.28 & Aug. & 30 & 17.88 & & 29 & 17.26 \\
Mar. & 3 & 13.48 & July & 1 & a & 17.09 & Oct. & 7 & 17.45 & Dec. 31 & 17.32 \\
\hline
\end{tabular}

U12. Measurements supplied through courtesy of Central Nebraska Public Power and Irrigation District.

Water level, in feet below measuring point, 1941

\begin{tabular}{|c|c|c|c|c|c|c|c|c|c|c|c|}
\hline $\begin{array}{l}\text { Jan. } \\
\text { Feb. } \\
\text { Mar. }\end{array}$ & $\begin{array}{r}3 \\
3 \\
3 \\
30\end{array}$ & $\begin{array}{l}15.14 \\
14.54 \\
14.05 \\
13.68\end{array}$ & $\begin{array}{l}\text { Apr. } \\
\text { May } \\
\text { June }\end{array}$ & $\begin{array}{l}30 \\
31 \\
30\end{array}$ & $\begin{array}{l}13.31 \\
13.70 \\
13.99\end{array}$ & $\begin{array}{lr}\text { July } 28 \\
\text { Aug. } 29 \\
\text { Oct. } 3\end{array}$ & $\begin{array}{l}13.59 \\
15.33 \\
14.26\end{array}$ & $\begin{array}{l}\text { Nov. } \\
\text { Dec. }\end{array}$ & $\begin{array}{r}1 \\
1 \\
31\end{array}$ & 8 & $\begin{array}{l}13.62 \\
13.23 \\
13.05\end{array}$ \\
\hline
\end{tabular}

U14. Measurements supplied through courtesy of Central Nebraska Public Power and Irrigation District.

Water level, in feet below measuring point, 1941

\begin{tabular}{|c|c|c|c|c|c|c|c|c|c|c|c|}
\hline $\begin{array}{l}\text { Jan. } \\
\text { Feb. } \\
\text { Mar. }\end{array}$ & $\begin{array}{r}3 \\
3 \\
3 \\
30\end{array}$ & $\begin{array}{l}9.14 \\
8.65 \\
8.16 \\
8.03\end{array}$ & $\begin{array}{ll}\text { Apr. } & 3 \\
\text { May } & 3 \\
\text { June } & \end{array}$ & $\begin{array}{l}30 \\
31 \\
30\end{array}$ & $\begin{array}{l}7.52 \\
7.52 \\
7.60\end{array}$ & $\begin{array}{l}\text { July } 2 \\
\text { Aug. } 2 \\
\text { oct. }\end{array}$ & a & $\begin{array}{l}7.18 \\
7.13 \\
8.11\end{array}$ & $\begin{array}{l}\text { Nov. } \\
\text { Dec. }\end{array}$ & 3 & $\begin{array}{l}7.86 \\
7.73 \\
7.60\end{array}$ \\
\hline
\end{tabular}

U21. Measurements supplied through courtesy of Central Nebraska Public Power and Irrigation District.

Water level, in feet below measuring point, 1941

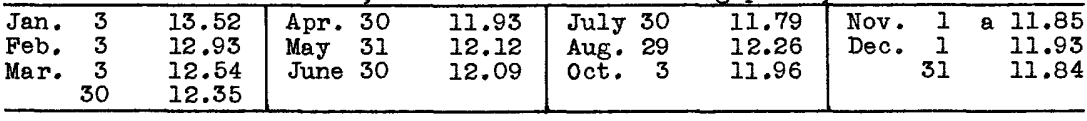

U22. Measurements supplied through courtesy of Central Nebraska Public Power and Irrigation District.

Water level, in feet below measuring point, 1941

\begin{tabular}{|c|c|c|c|c|c|c|c|c|}
\hline $\begin{array}{l}\text { Jan. } \\
\text { Feb. } \\
\text { Mar. }\end{array}$ & $\begin{array}{r}3 \\
3 \\
3 \\
30\end{array}$ & $\begin{array}{l}8.98 \\
7.93 \\
6.99 \\
6.35\end{array}$ & $\begin{array}{ll}\text { Apr. } & 3 \\
\text { May } & 3 \\
\text { June } & 3\end{array}$ & $\begin{array}{l}5.73 \\
5.88 \\
6.08\end{array}$ & $\begin{array}{lr}\text { July } & 30 \\
\text { Aug. } & 29 \\
\text { Oct. } & 3\end{array}$ & $\begin{array}{l}5.50 \\
6.23 \\
6.03\end{array}$ & $\begin{array}{l}\text { Nov. } \frac{1}{1} \\
\text { Dec: } \\
31\end{array}$ & $\begin{array}{l}6.60 \\
6.91 \\
6.97\end{array}$ \\
\hline
\end{tabular}

U32. Measurements supplied through courtesy of Central Nebraska Public Power and Irrigation District.

Water level, in feet below measuring point, 1941

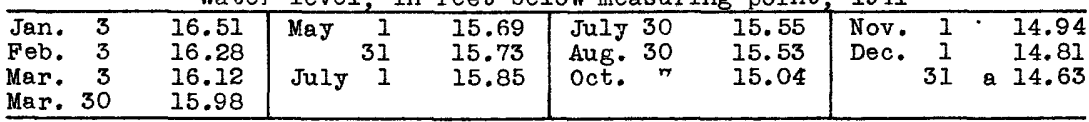

a Highest observed stage in period of record. 
Lincoln County--Continued.

U33. Measurements supplied through courtesy of Central Nebraska Public Power and Irrigation District.

Water level, in feet below measuring point, 1941

\begin{tabular}{|c|c|c|c|c|c|c|c|}
\hline Date & $\begin{array}{l}\text { Water } \\
\text { level }\end{array}$ & Date & $\begin{array}{l}\text { Water } \\
\text { level }\end{array}$ & Date & $\begin{array}{l}\text { Water } \\
\text { level }\end{array}$ & Date & $\begin{array}{l}\text { Water } \\
\text { level }\end{array}$ \\
\hline $\begin{array}{lr}\text { Jan. } & 3 \\
\text { Feb. } & 3 \\
\text { Mar. } & 3 \\
& 30\end{array}$ & $\begin{array}{l}37.72 \\
37.43 \\
37.12 \\
36.75\end{array}$ & $\begin{array}{lr}\text { May } & 1 \\
\text { July } & 1\end{array}$ & $\begin{array}{l}36.25 \\
35.96 \\
35.66\end{array}$ & $\begin{array}{lr}\text { July } 30 \\
\text { Aug. } 30 \\
\text { oct. } \quad 7\end{array}$ & $\begin{array}{l}35.40 \\
35.19 \\
34.65\end{array}$ & $\begin{array}{l}\text { Nov. } 1 \\
\text { Dec. } \\
31\end{array}$ & $\begin{array}{r}34.35 \\
34.03 \\
2 \quad 33.72\end{array}$ \\
\hline
\end{tabular}

034. Measurements supplied through courtesy of Central Nebraska Public Power and Irrigation District.

Water level, in feet below measuring point, 1941

\begin{tabular}{rrr|lrr|rrr|rrr}
\hline Jan. & 3 & 18.37 & May & 1 & 17.98 & July & 30 & 17.95 & Nov. & 1 & 17.42 \\
Feb. & 3 & 18.32 & & 31 & 18.05 & Aug. 30 & 17.84 & Dec. 1 & 17.36 \\
Mar. 3 & 18.25 & July & 1 & 18.00 & Oct. 7 & 17.44 & & 31 & a 17.23 \\
30 & 18.17 & & & & & & & & & & \\
\hline
\end{tabular}

035. Measurements supplied through courtesy of Central Nebraska Public Power and Irrigation District.

Water level, in feet below measuring point, 1941

\begin{tabular}{|c|c|c|c|c|c|c|c|c|c|c|}
\hline $\begin{array}{l}\text { Jan. } \\
\text { Feb. } \\
\text { Mar. }\end{array}$ & $\begin{array}{r}3 \\
3 \\
3 \\
30\end{array}$ & $\begin{array}{r}10.49 \\
9.92 \\
9.50 \\
9.03\end{array}$ & $\begin{array}{l}\text { May } \\
\text { July }\end{array}$ & $3 \frac{1}{1}$ & $\begin{array}{l}8.44 \\
8.16 \\
7.80\end{array}$ & $\begin{array}{lr}\text { July } & 30 \\
\text { Aug. } & 30 \\
\text { oct. } & 7\end{array}$ & $\begin{array}{l}7.71 \\
7.78 \\
7.30\end{array}$ & $\begin{array}{l}\text { Nov. } \\
\text { Dec. }\end{array}$ & & $\begin{array}{r}7.05 \\
6.82 \\
\mathrm{a} 6.57\end{array}$ \\
\hline
\end{tabular}

U38. Measurements supplied through courtesy of Central Nebraska Public Power and Irrigation District.

Water level, in feet below measuring point, 1941

\begin{tabular}{|c|c|c|c|c|c|c|c|c|c|}
\hline Jan. & $\begin{array}{l}3 \\
3 \\
3\end{array}$ & $\begin{array}{l}11.06 \\
10.37 \\
10.00\end{array}$ & $\begin{array}{l}\text { Mar. } 3 \\
\text { May }\end{array}$ & $\begin{array}{l}31 \\
31\end{array}$ & $\begin{array}{l}9.62 \\
9.13 \\
8.92\end{array}$ & $\begin{array}{l}\text { July } \\
\text { Aug. } 3 \\
\text { oct. }\end{array}$ & $\begin{array}{r}1 \\
30 \\
7\end{array}$ & $\begin{array}{l}5 \\
4 \\
0\end{array}$ & $\begin{array}{rr}\text { Nov. } & 4 \\
& 29 \\
\text { Dec. } & 31\end{array}$ \\
\hline
\end{tabular}

040. Heasurements supplied through courtesy of Central Nebraska Public Power and Irrigation District.

Water level, in feet below measuring point, 1941

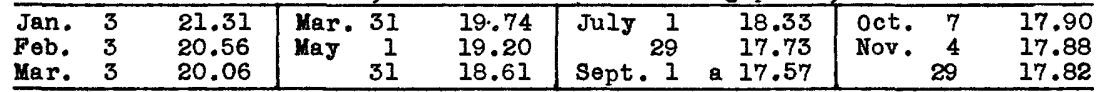

042. Measurements supplied through courtesy of Central Nebraska Public Power and Irrigation District.

Water level, in feet below measuring point, 1941

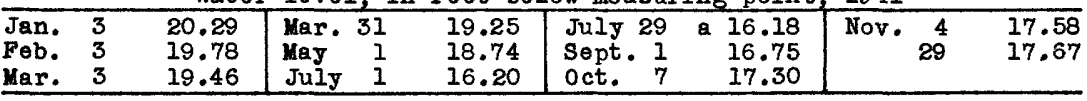

043. Measurements supplied through courtesy of Central Nebraska Public Power and Irrigation District.

Water level, in feet below measuming point, 1941

\begin{tabular}{|c|c|c|c|c|c|c|c|c|}
\hline $\begin{array}{l}\text { Jan. } \\
\text { Feb. } \\
\text { Mar. }\end{array}$ & $\begin{array}{l}3 \\
3 \\
3\end{array}$ & $\begin{array}{l}41.22 \\
39.59 \\
38.95\end{array}$ & $\begin{array}{l}\text { Mar. } 3 \\
\text { May } \\
\text { June }\end{array}$ & $\begin{array}{ll}31 & 38.63 \\
1 & 38.22 \\
2 & 38.15\end{array}$ & 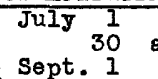 & $\begin{array}{l}37.80 \\
37.19 \\
37.67\end{array}$ & $\begin{array}{l}\text { Oct. } \\
\text { Nov. }\end{array}$ & $\begin{array}{l}37.83 \\
37.47 \\
37.28\end{array}$ \\
\hline
\end{tabular}

050. Measurements supplied through courtesy of Central Nebraska Public Power and Irrigation District.

a Highest observed stage in period of record. 
Lincoln County--Continued.

U50.---Continued.

Water level, in feet below measuring point, 1941

\begin{tabular}{|c|c|c|c|c|c|c|c|}
\hline Date & $\begin{array}{l}\text { Water } \\
\text { level }\end{array}$ & Date & $\begin{array}{l}\text { Water } \\
\text { level }\end{array}$ & Date & $\begin{array}{l}\text { Water } \\
\text { level }\end{array}$ & Date & $\begin{array}{l}\text { Water } \\
\text { level }\end{array}$ \\
\hline $\begin{array}{lr}\text { Jan. } & 3 \\
\text { Feb. } & 3 \\
\text { Mar. } & 3 \\
& 31\end{array}$ & $\begin{array}{r}10.49 \\
9.74 \\
9.42 \\
9.02\end{array}$ & $\begin{array}{lr}\text { May } & 1 \\
& 31 \\
\text { July } & 1\end{array}$ & $\begin{array}{l}8.40 \\
8.30 \\
8.26\end{array}$ & $\begin{array}{lr}\text { July } 29 \\
\text { Aug. } 30 \\
\text { Oct. } 7\end{array}$ & $\begin{array}{l}8.85 \\
8.79 \\
8.11\end{array}$ & $\begin{array}{l}\text { Nov. } 4 \\
29 \\
\text { Dec. } 31\end{array}$ & $\begin{array}{ll} & 7.83 \\
\text { a } & 7.79 \\
\text { a } & 7.79\end{array}$ \\
\hline
\end{tabular}

\section{Logan County}

404. Water level, in feet above datum, 1941: 0ct. 27, 99.90.

\section{Loup County}

234. No measurements made in 1941.

345. Water level, in feet above datum, 1941: 0ct. 16,99.99.

422. Water level, in feet above datum, 1941: oct. 16, a/103.72.

\section{McPherson county}

254. Water level, in feet above datum, 1941: oct. 27, b/99.30.

\section{Madison County}

108. Water level, in feet above datum, 1941: Oct. 17, 99.71.

109. Water level, in feet above datum, 1941: oct. 17, 99.84.

110. Water level, in feet above datum, 1941: 0ct. 14, 99.76.

334. Water level, in feet above datum, 1941: 0ct. 14, 99.74.

\section{Merrick County}

42. Water level, in feet above datum, 194I: Oct. 9, 100.78.

48. Water level, in feet above datum, 1941: oct. 14, 99.56 .

49. Water level, in feet above datum, 1941: 0ct. 14, 99.67.

50. Water level, in feet above datum, 1941: Oct. 14, 100.30.

GI200. Water levels, in feet above datum, 1941: Mar. 26,c/101.80;

June 18, c/103.10; July 19, c/102.25; Nov. 12, c/101.67.

GI201. Water levels, in feet above datum, 1941: Mar. 26, $c / 101.67$; July $19, \mathrm{c} / 102.27$; Nov. I2, c/101.21.

\section{Morrill County}

84. Water level, in feet above datum, 1941: 0ct. 23, 100.43.

85. Measurements supplied through courtesy of Nebraska Department of Roads and Irrigation.

a Highest observed stage in period of record.

$b$ Lowest observed stage in period of record. ment.

Measurement supplied through courtesy of Grand Island Water Depart- 
Morri11. County--Continued.

35.--Continued.

Water level, in feet above datum, 1941

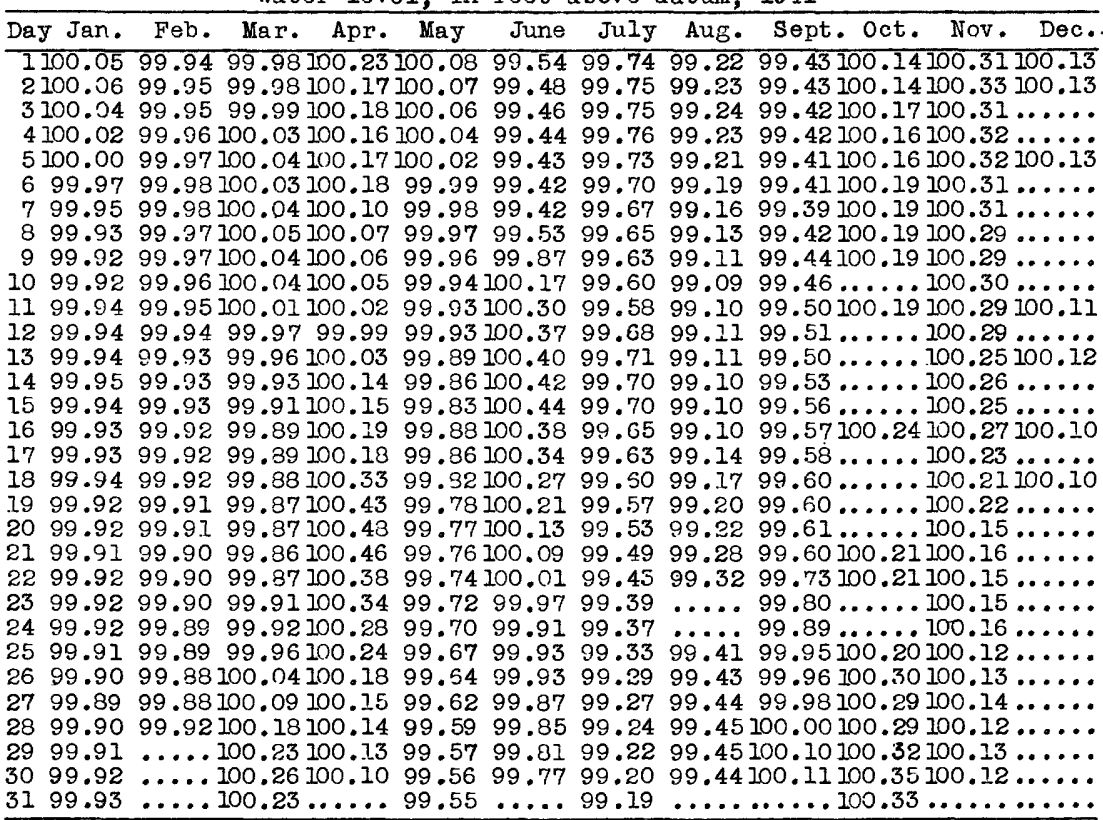

97. Water level, in feet above datum, 1941: 0ct. 23, 99.31.

\section{Nance. County}

43. Water level, in feet above datum, 1941: 0ct. 9, 100.49.

44. Measurements discontinued.

45. Measurements discontinued.

371. ".ater level, in feet above datum, 1941: Oct. 14, 98.43.

\section{Nemaha County}

11. Water level, in feet above datum, 1941: oct. 7, 104.44.

\section{Nuckolls County}

164. Water levels, in feet above datum, 1941: Jan. 21, 99.27; oct. $31,100.69$.

155. Water level, in feet above datum, 1941: Nov. 1, 95.33.

393. Water level, in feet above datum, 1941: - oct. 31, 99.34.

407. No measurements made in 1941. 
otoe County

8. Water level, in feet above datum, 1941: 0ct. 7, 101.77.

9. Water level, in feet above datum, 1941: 0ct. 7, 101.50.

10. Water level, in feet above datum, 1941: 0ct. 7, 97.71.

\section{Pawnee County}

4. No measurements made in 1941.

\section{Perkins County}

151. Water levels, in feet above datum, 1941: Jan. 22, 102.09; oct. $26,102.03$.

\section{Phelps County}

157. Water levels, in feet above datum, 1941: Jan. 22, 98.75; oct. $29,98.92$.

275. Water level, in feet above datum, 1941: 0ct. 16, 99.32.

276. Water level, in feet above datum, 1941: 0ct. 16, 99.09.

277. No measurements made in 1941.

\section{Plorce County}

70. Water level, in feet above datum, 1941: 0ct. 18, 99.58 .

\section{Platte County}

39. Water level, in feet above datum, 1941: 0ct.9, 102.07.

40. Water level, in feet above datum, 1941: 0ct. 9, 100.09.

41. Water level, in feet above datum, 1941: oct. 9, 99.65.

342. Water level, in feet above datum, 1941: oct. 10, 98.07.

368. Water level, in feet above datum, 1941: oct. 10, 105.38.

\section{Redwllow County}

137. Water level, in feet above datum, 1941: 0ct. 28, 101.04.

139. Water level, in feet above datum, 1941: 0ct. 28, 99.51.

179. Water level, in feet above datum, 1941: 0ct. 28, a/99.26.

\section{Richardson County}

No measurements were made on the following wells in Richardson County In 1941: $1,2,3,4,6,8,9,10,11,12,13,14,15,410$, and 417.

a Lowest observed stage in period of record. 
Richardson County--Continued.

5. Water level, in feet above datum, 1941 : 0ct. 6, 100.86.

7. Water level, in feet above datum, 1941: 0ct. 7, 103.02 . 408. Water level, in feet above detum, 1941: 0ct. 7, 98.19. 416. Water level, in feet above datum, 1941: 0ct. 7, 109.99. 418. Water level, in feet above datum, 1941: 0ct. 7, 101.09. 419. Water level, in feet above datum, 1941: 0ct. 7, 99.60.

\section{Rock County}

117. Water level, in feet above datum, 1941: 0ct. 18, 100.67. 198. No measurements made in 1941.

\section{Saline County}

194. Water level, in feet above datum, 1941: 0ct. 4, 101.24.

341. Water level, in feet above datum, 1941: 0ct. 4, 97.09 .

\section{Sarpy County}

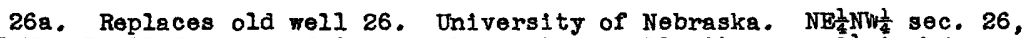
T. $13 \mathrm{~N}$., R. $13 \mathrm{k}$. Unused driven observation well, diameter li inches, depth 27 feet. Measuring point, top of pipe, 2.0 feet above land surface and 115.47 feet above datum. Wáter level May 6, 1940, 17.03 feet below measuring point. Water levels, in feet above dátum: July 9, 1940, 103.85; 0ct. 18, 1940, 103.02; Oct. 8, 1941, 205.61.

27. No measurements made in 1941.

323. Water level, in feet above datum, 2941: 0ct. 8, a/92.15.

\section{Saunders County}

19. Water level, in feet above datum, 1941: 0ct. 6, 100.18 .

21. Water level, in feet above datum, 1941: 0ct. 6, 100.19.

22: Water level, in feet above datum, 194l: Oct. 6, 99.74.

331. Water level, in feet above datum, 1941: 0ct. 6, 102.04.

\section{Scotts Bluff County}

240. Measurements discontinued.

438. Water level, in feet above datum, 1941: Oct. 24, 101.15.

439. Water level, in feet above datum, 1941: 0ct. 24, 100.70.

440. Water level, in feet above datum, 194l: 0ct. 24, 100.45.

441. No measurements made in 1941.

442. Water level, in feet above datum, 1941: 0ct. 24, 100.88.

a Lowest recorded stage in period of record. 


\section{Seward County}

171. Water level, in feet above datum, 1941: 0ct. 4, 99.58.

172. Water level, in feet above datum, 1941: Oct. 4, 100.32.

\section{Sheridan County}

120. Water level, in feet above datum, 1941: 0ct. 20,99.53.

217. Water level, in feet above datum, 1941: oct. 21, a/97.36.

376. Water level, in feet above datum, 1941: 0ct. 20, 100.15.

379. Water level, in feet above datum, 1941: 0ct. 20, 99.32

432. Water level, in feet above datum, 1941: oct. 20, 100.57.

\section{Sherman County}

58. Water level, in feet above datum, 1941: 0ct. 16, 100.64 .

\section{Sioux County}

81. Water level, in feet above detum, 1941: 0ct. 20, 99.69.

125. No measurements made in 1941.

377. Water level, in feet above datum, 1941: 0ct. 20, 101.68 .

\section{Stanton County}

208. Measurements discontinued.

421. No measurements in 1841.

\section{Thayer County}

166. Water level, in feet above datum, 1941: Nov. 1, a/99.32. 187. Water level, in feet above detum, 1941: oct. 31, 99.29. 452. Water level, in feet above detum, 1941: 0ct. 31, 99.77.

\section{Thomas County}

212. No measurements made in 1941.

213. Water level, in feet above datum, 1941: 0ct. 21, 99.77.

\section{Thurston County}

60. Water level, in feet above datum, 1941: oct. 10, 100.64. 102. No measurements made in 1941.

103. Water level, in feet above datum, 1941: Oct. 10,98.03.

a Lowest observed stage in period of record. 
Valley County

54. Water level, in feet above datum, 1941: 0ct. 16, 102.01.

56. Water level, in feet above datum, 194I: oct. 16, 102.86.

57. Water Ievel, in feet above datum, 1941: 0ct. 17, 104.16.

\section{Washington County}

32. Water level, in feet above datum, 1941: Oct. 8, 102.68.

33. Water level, in feet above datum, 1941: oct. 8, 98.87.

Wayne County

100. Water level, in feet above datum, 1941: 0ct. 11, a /99.10.

\section{Webster Count y}

161. Water level, in feet above datum, 1941: 0ct. 29, 99.22.

162. No measurements made in 1941.

163. Water levels, in feet above datum, 1941: Jan. 21, 98.88; oct. $29,100.19$.

\section{Wheeler County}

204. Water level, In feet above datum, 1941: 0ct. 17, 99.18.

205. Water level, in feet above datum, 1941: 0ct. 17, 99.74.

\section{York County}

167. Water levels, in feet above datum, 1941: Jan. 21, a//98.15; oct. 30, 98.29 .

225. Water level, in feet above datum, 1941: Nov. 1, a/97.74.

a Lowest observed stage in period of recora. 


\section{NORTH DAKOTA}

\section{STATE-WIDE PROJECT}

By W. C. Rasmussen

The program of water-level measurements in wells in North Dakota was continued in 1941 by the Federal Geological survey in cooperation with the North Dakota Geological Survey. Fourteen wells were dropped from the program and 11 wells were added. At the end of the year water levels in 173 wells were under observation.

Water levels in 62 wells were measured weekly by 37 local observers. Water levels in 13 wells were measured weekly and 7 wells were measured occasionally through the courtesy of city, State, and Federal agencies. The remaining 91 wells were measured by the writer once, twice, or several times during the year. About 5,500 individual measurements of water level were made in 1941. Eight automatic water-stage recorders were operated on wells during the year.

The ground-water investigation of the vicinity of Fargo was continued by the Federal Geological Survey in cooperation with the North Dakota Geological Survey and the city of Fargo. A discussion of this project is given under the heading of Cass County. A detailed investigation was made of the ground water in an area south of Oakes, in Dickey County. Copies of a preziminary report based on this investigation are filed in typewritten form in the office of the Geological Survey, United states Department of the Interior, Washington, D. C., and in the office of the state Geological Survey, Grand Forks, N. Dak., where they may be consulted by interested persons. A ground-water investigation of Pembina county was begun in 194l. All of this work was done in cooperation with the North Dakota Geological Survey.

I/ See Geological Survey Water-Supply Papers 840, 845, 886, and 908. 
The following table gives average monthly water levels from september 1937 to December 1940 based on records of 10 to 42 wells located in different parts of the state. Records for 22 wells were used to compute average water levels in 1941. In the first $3 \frac{1}{2}$ years of the record the average water levels in corresponding months show, in general, decreases in each succeeding year, but in May 1941 the water levels began a recovery which brought them to a new high average by october. The year closed with the average water level above that at the close of any previous year since the observations were begun in 1937. The preclpitation in North Dakota in 1941, as reported by the United States Weather Bureau, was 37 percent above normal. Excessive rains fell in April, May, June, August, September, and october, with the september rainfall almost 3 times 1 ts 50-year average. In July the rainfall was only 72 percent of normal. These varlations In rainfall may explain the fluctuations shown in the water-level table, with the sag in August due to the subnormal July rainfall, and the record high in october due to the thrice normal september rain.

Average monthly water levels, In feet above assumed datum planes, In observation wells in North Dakota, 1937-41

Year Jan. Feb. Mar. Apr. May June July Aug. Sept. Oct. Nov. Dec.

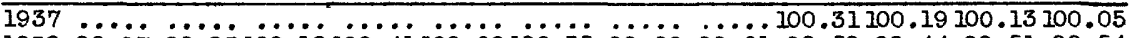
$193899.9799 .93100 .12100 .41100 .68100 .3599 .99 \quad 99.6199 .5999 .4499 .5199 .54$

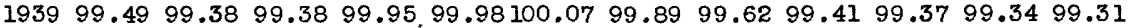

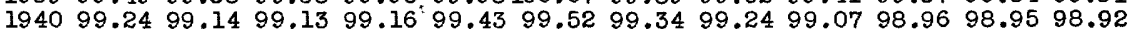
194198.8498 .7498 .8399 .7699 .97100 .43100 .3999 .89100 .16100 .73100 .64100 .26

Average water levels for the perlod of record are shown graphicaliy in the accompanying figure. This figure also shows the average water levela from 1933 to 1941 of $\mathbf{s 1 x}$ water-table wells at Denbigh, N. Dak. A fair correlation of the state average with the Denbigh average is observable, with good agreement of the trends, so that a comparable extrapolation of average water levels is available back to 1933.

Records for 187 observation wells are included in this report. The wells are listed alphabetically by county and numerically within each county. Periodic measurements have been made in wells in every county in the state, and in 1941 measurements were made on wells in every county except Grand Forks, where two wells that were previously measured have gone dry. Complete descriptions are given only for those wells whose descriptions are not included in Water-Supply Papers $840,845,386$, or 908 . In the absence of a description, reference is made to the volume in which it may be found. 
Average water level, in feet

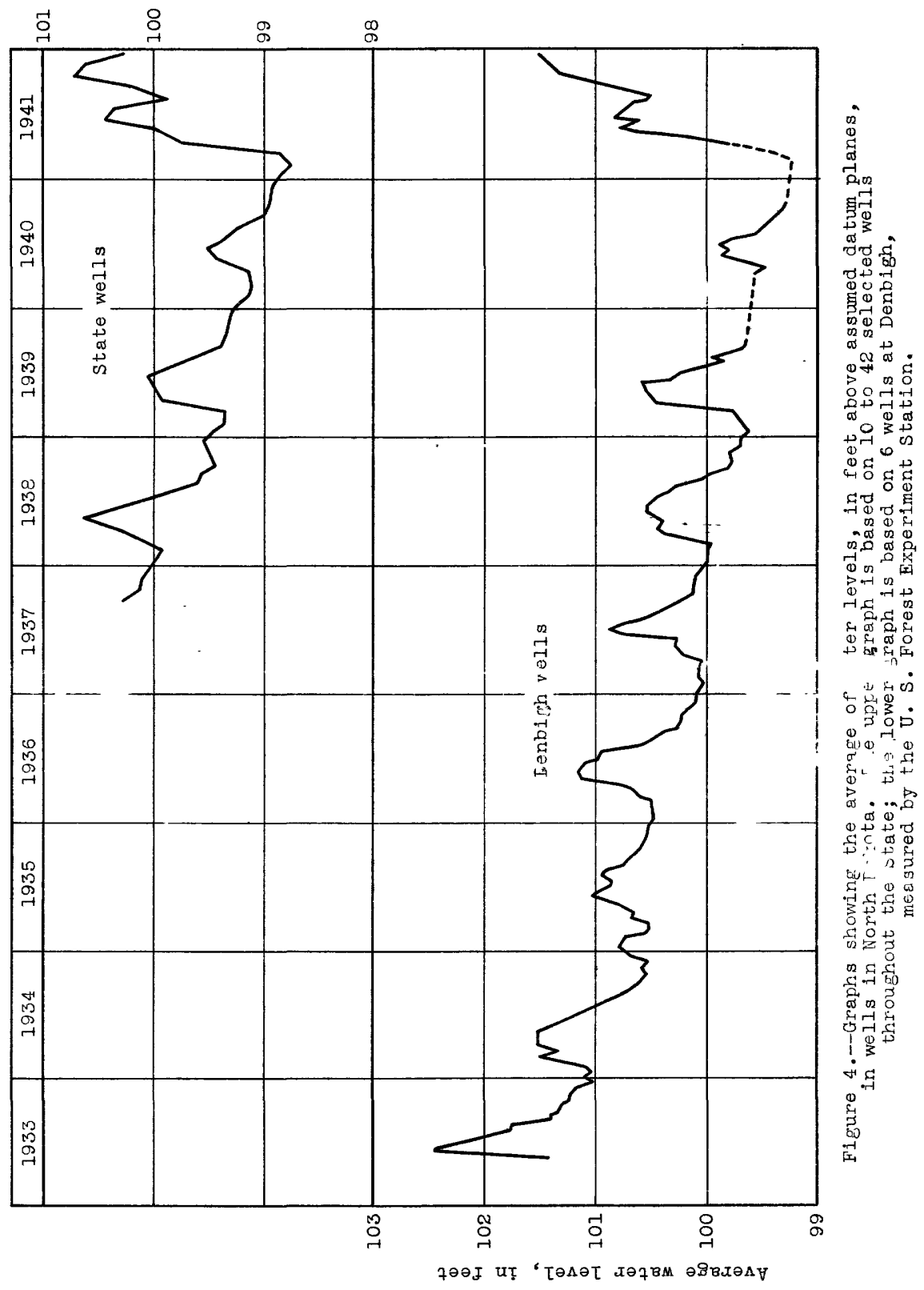


Except where otherwise noted, the water level in each well is expressed in feet above an assumed datum plane, which is 100 feet below the water level in that well on January 1, 1938, or on the nearest date of measurement. In each observation well that has been established since January I, 1938, the water level on the first day of measurement is generally taken to be the same as the average of a group of selected representative wells; that is, if the average is 100.26 feet, the datum plane for that well is 100.26 feet below the water level on that day. The depth of the datum plane below the measuring point is then 100.26 feet plus the depth to the water level below the measuring point. To compute the depth to water level below the measuring point subtract the recorded height of water level above the datum plane from the recorded altitude of the measuring point. Water levels for any one well are directly comparable, even though the measuring point may have been changed, because the record is given in height above a datum plane that has been referred to one or more bench marks near the well.

\section{Adams County}

1. Hirs. Fialvorsen. SW $\frac{1}{4} S W_{4}^{\frac{1}{4}}$ sec. 14, T. 130.N., R. 97 W. (Described in Water-Supply Paper 908). Water levels, in feet below measuring point, 1941: Apr. 16, 53.59; 0ct. 23, 52.39.

\section{Barnes County}

$9^{r}$ 4. - Wilkins. NW $W_{4}^{1} S W \frac{1}{4}$ sec. 5, T. 138 N., R. 57 W: (Described in Vatei-Suwply Paper 886, p. 347). Error in figures for Dec. 14,21 and 28, 19 , lutted in water-Supuly paper 908. Corrected water level, in feet a ve dat.m, 1940: Dec. 14, 98.68; Dec. 21, 98.67; Dec. 28, 98.69.

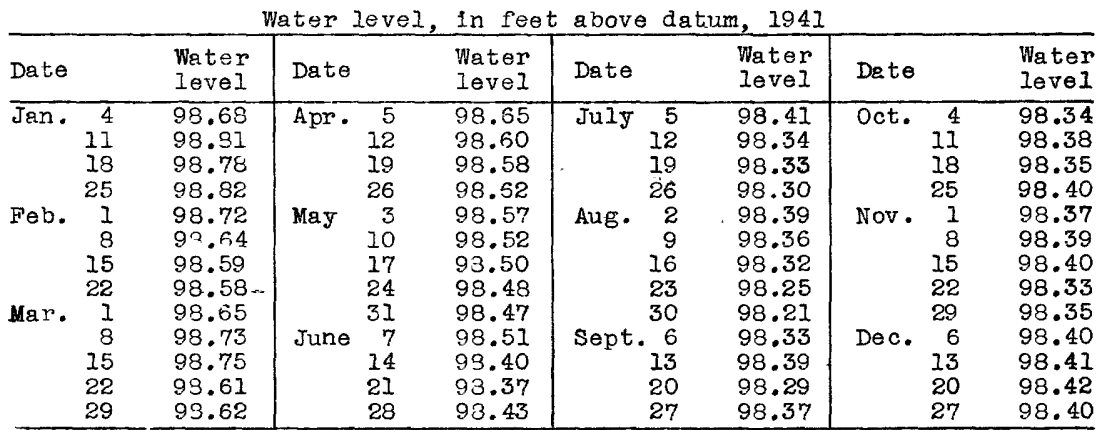


Bames County--Continued

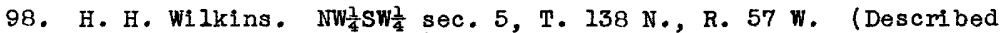
in Water-Supply Paper 886, p. 531).

Water level, in feet above datum, 1941

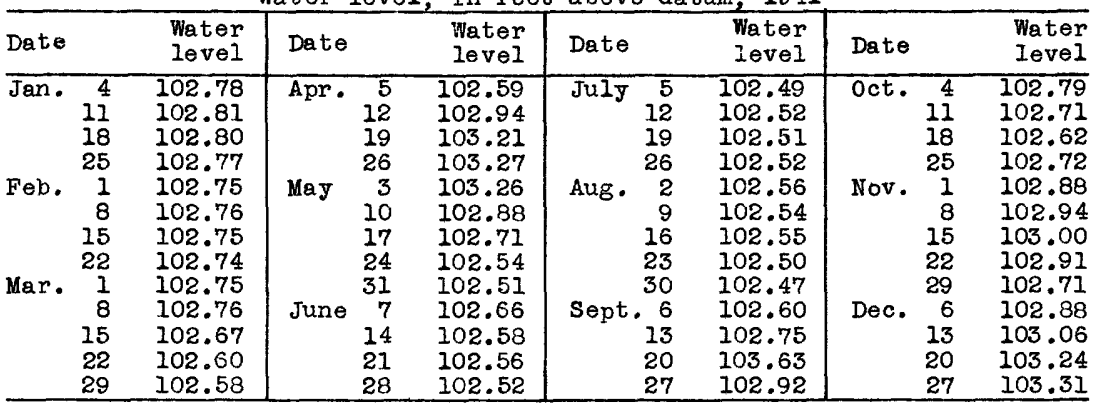

\section{Berson County}

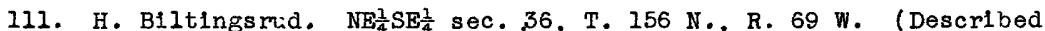
in Water-Supply Paper 908). Correction, Water-Supply Paper 908, measurement was made Nov. 8 , not Nov. 3, 20.72. Water levels, in feet below measuring point, 1941: Apr. 24, 20.50; 0ct. 29, 18.94.

\section{Blilings County}

88. Roosevelt National Park. SW $\frac{1}{4} \mathrm{SE}_{4}^{\frac{2}{4}}$ sec. 32, T. 140 N., R. 100 W. (Described in Water-Supply Papers 845 , p. 347 , and'908).

Water level, in feet above datum, 1941

\begin{tabular}{|c|c|c|c|c|c|c|c|c|c|c|}
\hline \\
\hline Jan. & $\begin{array}{r}4 \\
11 \\
18 \\
25\end{array}$ & $\begin{array}{l}99.62 \\
99.62 \\
99.62 \\
99\end{array}$ & Apr. & $\begin{array}{r}13 \\
19 \\
26 \\
3\end{array}$ & $\begin{array}{l}99.59 \\
99.59 \\
99.60 \\
99.60\end{array}$ & $\begin{array}{ll}\text { July } 12 \\
19 \\
\\
26\end{array}$ & $\begin{array}{l}99.92 \\
99.92 \\
99.92 \\
99.92\end{array}$ & oct. & 11 & $\begin{array}{l}99.89 \\
99.89 \\
99.89 \\
99.92\end{array}$ \\
\hline$F \in b$. & $\begin{array}{r}1 \\
8 \\
15 \\
22\end{array}$ & $\begin{array}{r}99.60 \\
99.60 \\
99.60 \\
99.60\end{array}$ & & $\begin{array}{l}10 \\
17 \\
24 \\
31\end{array}$ & $\begin{array}{r}99.60 \\
99.60 \\
99.60 \\
99.60\end{array}$ & $\begin{array}{r}9 \\
16 \\
23 \\
30\end{array}$ & $\begin{array}{l}99.92 \\
99.92 \\
99.91 \\
99.91\end{array}$ & Nov. & $\begin{array}{l}15 \\
22\end{array}$ & $\begin{array}{l}99.93 \\
99.92 \\
99.93 \\
99.93\end{array}$ \\
\hline Mar. & $\begin{array}{r}1 \\
8 \\
15 \\
22\end{array}$ & $\begin{array}{l}99.60 \\
99.61 \\
99.61 \\
99.59\end{array}$ & June & $\begin{array}{r}7 \\
14 \\
21 \\
28\end{array}$ & $\begin{array}{l}99.61 \\
99.78 \\
99.86 \\
99.90\end{array}$ & $\begin{array}{r}6 \\
13 \\
20 \\
27\end{array}$ & $\begin{array}{l}99.91 \\
99.89 \\
99.89 \\
99.87\end{array}$ & Dec. & $\begin{array}{r}29 \\
6\end{array}$ & $\begin{array}{l}99.93 \\
99.91 \\
99.91 \\
99.92\end{array}$ \\
\hline Apr. & $\begin{array}{r}29 \\
5\end{array}$ & $\begin{array}{l}99.60 \\
99.60\end{array}$ & July & 5 & & oct. & & & 27 & \\
\hline
\end{tabular}

\section{Bottineau County}

60. Federal Land Bank. SE⿺ $\mathrm{NE}_{4} \frac{1}{4}$ sec. $23, \mathrm{~T}, 160 \mathrm{~N}, \mathrm{R}, 76 \mathrm{~W}$. (Described in Water-Suprly Paper 340 , p. 320).

Water level, in feet above datum, 1941

\begin{tabular}{|c|c|c|c|c|c|c|c|c|c|c|}
\hline Jan. & $\begin{array}{l}4 \\
11 \\
18 \\
25\end{array}$ & $\begin{array}{l}99.59 \\
99.59 \\
99.58 \\
99.58\end{array}$ & $\mathrm{Feb}$ & $\begin{array}{r}1 \\
8 \\
15 \\
22\end{array}$ & $\begin{array}{l}99.58 \\
99.57 \\
99.57 \\
99.57\end{array}$ & Mar. & $\begin{array}{r}1 \\
8 \\
15 \\
22\end{array}$ & $\begin{array}{l}99.56 \\
99.55 \\
99.54 \\
99.54\end{array}$ & $\begin{array}{l}\text { Mar. } 29 \\
\text { Apr. } 5 \\
\text { Oct. } 29\end{array}$ & $\begin{array}{r}99.32 \\
99.55 \\
100.73\end{array}$ \\
\hline
\end{tabular}

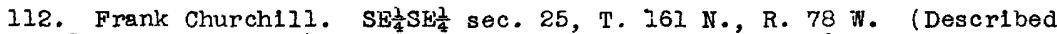
in Water-Supply Paper 908). Correction, Water-Supply Paper 908 , measurement was made Nov. 9, not Nov. 11, 24.07. Water levels, in feet below measuring point, 194i: Apr. 23, 23.56; Oct. 28, 21.54. 
Bowman Count $\mathbf{y}$

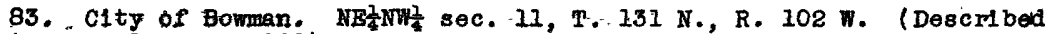
in: Nater-Supply Paper 908).

Nater level, in foet above datum, 1941

\begin{tabular}{|c|c|c|c|c|c|c|c|}
\hline Dato. & $\begin{array}{l}\text { Water } \\
\text { level }\end{array}$ & Date & $\begin{array}{l}\text { Water } \\
\text { level }\end{array}$ & Dato : & $\begin{array}{l}\text { Water } \\
\text { lovel }\end{array}$ & Date & $\begin{array}{l}\text { Water } \\
\text { level }\end{array}$ \\
\hline $\begin{array}{lr}\text { Apr. } & 16 \\
& 19 \\
& 26 \\
\text { May } \quad 3\end{array}$ & $\begin{array}{r}101.34 \\
101.36 \\
298.62 \\
101.39\end{array}$ & $\begin{array}{r}\text { ct. } 23 \\
26 \\
\text { Nov. } \quad 2 \\
9\end{array}$ & $\begin{array}{l}102.42 \\
102.41 \\
102.42 \\
102.39\end{array}$ & $\begin{array}{r}\text { Nov. } 16 \\
23 \\
30 \\
\text { Dec. } 7\end{array}$ & $\begin{array}{l}102.41 \\
102.41 \\
101.93 \\
101.91\end{array}$ & $\begin{array}{r}\text { Dec. } 14 \\
21 \\
28\end{array}$ & $\begin{array}{l}101.97 \\
101.99 \\
101.97\end{array}$ \\
\hline
\end{tabular}

84. City of Bowman. $\mathrm{NE}_{\frac{1}{4}} \mathrm{~S} W_{4}^{\frac{2}{4}}$ sec. 11, T. 131 N., R. 102 W. (Described in Water-Supply Paper 908).

Water level, in feet above datum, 1941

\begin{tabular}{rrr|rr|rrr|rr}
\hline Apr. 16 & 101.40 & Oct. 23 & 102.38 & Nov. 16 & 102.35 & Dec. 14 & 102.38 \\
& 19 & 101.41 & 26 & 102.39 & & 23 & 102.34 & 21 & 102.38 \\
26 & b 99.44 & Nov. 2 & 102.39 & & 30 & 102.35 & 28 & 102.36 \\
May 3 & 101.40 & & 9 & 102.33 & Dec. & 7 & 102.36 & & \\
\hline
\end{tabular}

85. C1ty of Bowman. NE $\frac{1}{4} S W_{4}^{\frac{1}{4}}$ sec. 11, T. 131 N., R. 102 W. (Described in Water-Supply Papor 908).

Water level, in feet above datum, 1941

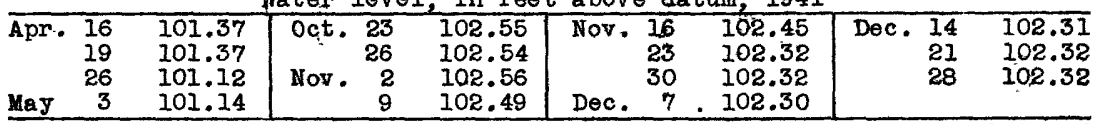

Burike County

52. Fish and Wildilfe Service, U. S. Department of the Interior. $\mathrm{SW}_{4} \mathrm{SE}_{\frac{1}{4}}$ sec. 1 , T. $163 \mathrm{~N} .$, R. $89 \mathrm{~W}$. Unused dus well, diameter 36 inches, depth 19.2 feet. Measuring point, top of wood curb south s1de, 1.4. feet above land surface.

Water level, in feet jolow measuring point, 1937-38, 1941

\begin{tabular}{|c|c|c|c|c|c|c|}
\hline Date & $\begin{array}{l}\text { Water } \\
\text { level }\end{array}$ & Date & . & $\begin{array}{l}\text { Water } \\
\text { level }\end{array}$ & Date & $\begin{array}{l}\text { Water } \\
\text { level }\end{array}$ \\
\hline $\begin{array}{l}\text { Oct. } 3 \frac{1}{7}, 1937 \\
\text { Nov. } 7, \\
\text { Apr. } 19,1938 \\
\end{array}$ & $\begin{array}{l}16.50 \\
16.50 \\
15.29 \\
\end{array}$ & $\begin{array}{l}\text { May } \\
\text { June }\end{array}$ & $\begin{array}{l}6,1938 \cdot \\
15 \\
13\end{array}$ & $\begin{array}{r}14.70 \\
14.47 \\
14.60 \\
\end{array}$ & $\begin{array}{l}\text { Apr. } 24,1941 \\
\text { Oct. } 25\end{array}$ & $\begin{array}{l}16.00 \\
16.04\end{array}$ \\
\hline
\end{tabular}

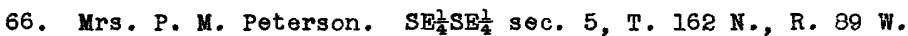

(Described in Water-Supply Paper $840, \mathrm{p} .66$ ).

Water level, in feet above datum, 1941

\begin{tabular}{|c|c|c|c|c|c|c|c|c|c|c|c|}
\hline \multicolumn{2}{|l|}{ Date } & $\begin{array}{l}\text { Water } \\
\text { level }\end{array}$ & \multicolumn{2}{|l|}{ Date } & $\begin{array}{l}\text { Water } \\
\text { level }\end{array}$ & \multicolumn{2}{|l|}{ Date } & $\begin{array}{l}\text { Water } \\
\text { level }\end{array}$ & \multicolumn{2}{|l|}{ Date } & $\begin{array}{l}\text { Water } \\
\text { level }\end{array}$ \\
\hline Jan. & $\begin{array}{l}4 \\
11 \\
18 \\
25\end{array}$ & $\begin{array}{l}100.19 \\
100.19 \\
100.19 \\
100.20\end{array}$ & Apr. & $\begin{array}{r}5 \\
12 \\
19 \\
26\end{array}$ & $\begin{array}{l}100.38 \\
100.38 \\
100.38 \\
100.37\end{array}$ & July & $\begin{array}{r}5 \\
12 \\
19 \\
26\end{array}$ & $\begin{array}{l}100.86 \\
100.75 \\
100.69 \\
100.58\end{array}$ & oct. & $\begin{array}{r}4 \\
11 \\
18 \\
25\end{array}$ & $\begin{array}{l}100.52 \\
100.52 \\
100.53 \\
100.51\end{array}$ \\
\hline $\mathrm{Feb}$ & $\begin{array}{r}1 \\
8 \\
15 \\
22 \\
1\end{array}$ & $\begin{array}{l}100.20 \\
100.23 \\
100.24 \\
100.23\end{array}$ & May & $\begin{array}{r}3 \\
10 \\
17 \\
24\end{array}$ & $\begin{array}{l}100.53 \\
100.55 \\
100.55 \\
100.53\end{array}$ & Aug. & $\begin{array}{r}2 \\
9 \\
16 \\
23\end{array}$ & $\begin{array}{l}100.53 \\
100.48 \\
100.51 \\
100.34\end{array}$ & Nov. & $\begin{array}{r}1 \\
10 \\
15 \\
22\end{array}$ & $\begin{array}{l}100.51 \\
100.49 \\
100.49 \\
100.54\end{array}$ \\
\hline lar. & $\begin{array}{r}1 \\
8 \\
15 \\
22 \\
29\end{array}$ & $\begin{array}{l}100.24 \\
100.24 \\
100.24 \\
100.63 \\
100.28\end{array}$ & June & $\begin{array}{r}7 \\
14 \\
21 \\
28\end{array}$ & $\begin{array}{l}100.59 \\
100.77 \\
100.94 \\
101.05 \\
100.88\end{array}$ & Sept & $\begin{array}{r}30 \\
+6 \\
13 \\
20 \\
27\end{array}$ & $\begin{array}{l}100 . \\
100 . \\
100 \\
100 . \\
100\end{array}$ & Dec. & $\begin{array}{r}29 \\
6 \\
13 \\
20 \\
27\end{array}$ & $\begin{array}{l}100.53 \\
100.53 \\
100.53 \\
100.54 \\
100.54\end{array}$ \\
\hline
\end{tabular}

a Pumped dry 20 hours before.

b Influenced by No. 83, pumped dry 20 hours before.

$511000-0-13$ 
Burke County--Contimed

115. Fish and Wildilfe Service, J. S. Department of the Interlor. SET SW $\frac{1}{4}$ sec. 21, T. 160 N., R. 91 W. (Described in Water-Supply Faper. 908 ): Water level, in feet above datum, 1941

\begin{tabular}{|c|c|c|c|c|c|c|c|}
\hline Date & $\begin{array}{l}\text { Weter } \\
\text { level }\end{array}$ & Date & $\begin{array}{l}\text { Water } \\
\text { level }\end{array}$ & Dat $\theta$ & $\begin{array}{l}\text { Water } \\
\text { level }\end{array}$ & Date & $\begin{array}{l}\text { Weter } \\
\text { level }\end{array}$ \\
\hline $\begin{array}{lr}\text { Apr. } & 12 \\
& 19 \\
\text { May } & 3 \\
& 10 \\
17 \\
\\
24 \\
\\
\text { June } & 71 \\
\end{array}$ & $\begin{array}{l}99.63 \\
99.47 \\
99.70 \\
99.32 \\
99.65 \\
99.70 \\
99.57 \\
99.50\end{array}$ & $\begin{array}{lr}\text { June } & 14 \\
& 21 \\
\text { July } & 5 \\
12 \\
\\
19 \\
\text { Aug. } 2 \\
9 \\
16\end{array}$ & $\begin{array}{l}99.47 \\
99.45 \\
99.44 \\
99.42 \\
99.44 \\
99.47 \\
99.44 \\
99.43\end{array}$ & 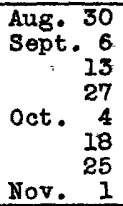 & $\begin{array}{l}99.49 \\
99.46 \\
99.56 \\
99.39 \\
99.40 \\
99.38 \\
99.42 \\
99.44\end{array}$ & $\begin{array}{rr}\text { Nor . } \\
\\
15 \\
29 \\
\text { Dec. } 6 \\
13 \\
20 \\
27 \\
27\end{array}$ & $\begin{array}{l}99.48 \\
99.52 \\
99.54 \\
99.55 \\
99.57 \\
99.45 \\
99.36\end{array}$ \\
\hline
\end{tabular}

116. Fish and Wildilfe Service, U. S. Department of the Interior. (Described in Water-supply Paper 908).

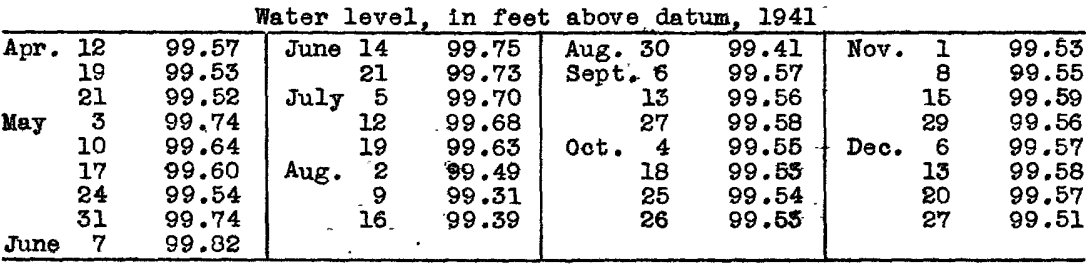

\section{Burleligh county}

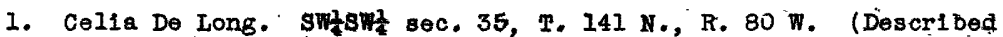
in Water-Supply Paper 908). Incorrectiy located in sec. 25 in Water-sipply Paper 908. Water leve1s, in feet below measuring point, 1941: Apr. 4 ,
15.35 ; Nov. $20,15.67$.

\section{Cass Oounty \\ Fargo area}

By A. C. Byerg

The investigation of the ground-water resources of the Fargo area, begun in 1940 in cooperation with the North Dakota State Coological Survey and the city of Pargo, was continued in 1941. Field work on the problem was completied in the fall of 194l, but measurements of the observation wells are being continued. Of the wells reported under cass county, all except wells 8, 10 , and 29 are measured as part of the Fargo area program, and during 1941 about 1,000 individual measurements of water levels in these 17 wells were made by the wetted-tape method. Automatic water-level recorders were maintained on 6 wells, and about 1,800 water leveis from the charts are recorded in this report. The measurements were made by $L_{0}-\mathrm{K}$ : Wenzel, W. C. Rasmussen, Ralph Mader, and Alan C. Byers. Measurement of

2f See U. s. Geol. Survey Water-Supply Paper 908. 
six wells within the limits of Fargo were made by H. G. Palmer, by courtesy of the city of Fargo. Measurements were alscontinued on 2 wells, so that at the end of 1941 a total of 18 wells were being measured in Cass County, 15 of these being part of the Fargo area program.

In order to provide information concerming the effect of pumping the well used by the c1ty of Fargo to supplement 1ts surface water supply, the well was pumped more strenuousiy in 1941 than in provious years. Pumping was begun on Jane 2. for 8 hours each day; early in August the duration of pumping was increased to 10 hours each-day. The pump was shut down at the ond of.pumping on september 30, after pumpling about 50,000,000 gallons during the season. The water levels in wells 28, 12, 3, 5, and 127 showed effects of the pumping, and the water level in well 67 gave a amall indication. At the ond of 1941 the water levels in these 6 wells averaged 8.83 feet below their average level at the end of 1940 .

phe water level in well 58 was affected by the pumping of well 57 , and at the end of 1941 It was 3.91 feet below thelevel at the end of 1940 . The average water level in wells $8,10,29,43$, and 122, which are shallow water-table we 13 in the lake Agass $1 \ddot{z}$ silt, was 0.83 foot above the ayerage level at the end of 1940 .

1. H. Benson '201 16th st.So. Fargo: (Described in Water-supply Paper 908). Water level, Jan. 4, 1941 ; 27.18 feet below measuring point. Water level, in feet a bove assumed datum, 1941

\begin{tabular}{|c|c|c|c|c|c|c|c|c|}
\hline Date & . & $\begin{array}{l}\text { Water } \\
\text { level }\end{array}$ & Date & $\begin{array}{l}\text { Water } \\
\text { level }\end{array}$ & Date & $\begin{array}{l}\text { Water } \\
\text { level }\end{array}$ & Date & $\begin{array}{l}\text { Water } \\
\text { level }\end{array}$ \\
\hline $\begin{array}{l}\text { Jan. } \\
\therefore \\
\therefore \\
\text { Feb. }\end{array}$ & $\begin{array}{r}4 \\
11 \\
18 \\
26 \\
1 \\
8 \\
16 \\
22 \\
1 \\
9 \\
15 \\
23 \\
29\end{array}$ & $\begin{array}{r}95.34 \\
95.41 \\
95.40 \\
95.48 \\
95.44 \\
95.47 \\
.95 .54 \\
95.63 \\
95.47 \\
95.43 \\
94.60 \\
95.05 \\
94.86\end{array}$ & $\begin{array}{cr}\text { Apr. } & 5 \\
& 12 \\
& 19 \\
& 26 \\
\text { May } & 3 \\
& 10 \\
& 17 \\
& 24 \\
& 31 \\
& 31 \\
\text { June } & 7 \\
& 14 \\
& 28 \\
\text { July } & 6\end{array}$ & $\begin{array}{l}94.23 \\
93.94 \\
94.62 \\
93.54 \\
93.58 \\
93.94 \\
93.95 \\
93.75 \\
.93 .80 \\
93.81 . \\
91.50 \\
91.28 \\
90.38\end{array}$ & 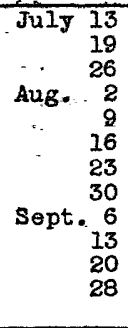 & $\begin{array}{l}89.79 \\
89.28 \\
88.87 \\
90.46 \\
88.13 \\
87.93 \\
87.71 \\
87.45 \\
87.19 \\
87.09 \\
86.92 \\
86.69\end{array}$ & $\begin{array}{rr}\text { oct. } & 4 \\
11 \\
18 \\
25 \\
1 \\
\text { Nov. } 8 \\
15 \\
15 \\
22 \\
30 \\
\text { Dec. } 6 \\
13 \\
20\end{array}$ & $\begin{array}{r}86.79 \\
87.08 \\
87.33 \\
87.68 \\
87.79 \\
87.80 \\
88.13 \\
88.15 \\
88.35 \\
88.46 \\
88.57 \\
88.76\end{array}$ \\
\hline
\end{tabular}

3. The Plerce Co., 1019 Ist Ave. Ho., Fargo. (Described In WaterSupply Paper 90̊): Watér level, Jan. 1, 1941, 24.57 foet below messuring point.

Lowest dally water level, in feet above asamed datum, 1941 (from recorder charts)

Day Jan. Feb. Har. Apr. May June July. Aug. Sept. Oct. Nov. Dec.
1.94.22 94.5694.78.94.8795.0795.09 89.36 85.73 83.14 81.41 86.0988.66

294.2294 .5594 .7594 .8795 .08995 .0989 .1785 .68 83.07 $91.40 \quad 86.2688 .73$

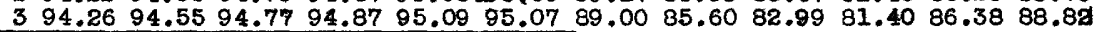
a-Pumping of weII 14 bogan. 


\section{Cass County--Continued}

3. The Pierce Co.--Continued.-

Lowest dally water level, in feet above assumed datum, 1941 (from recorder charts)

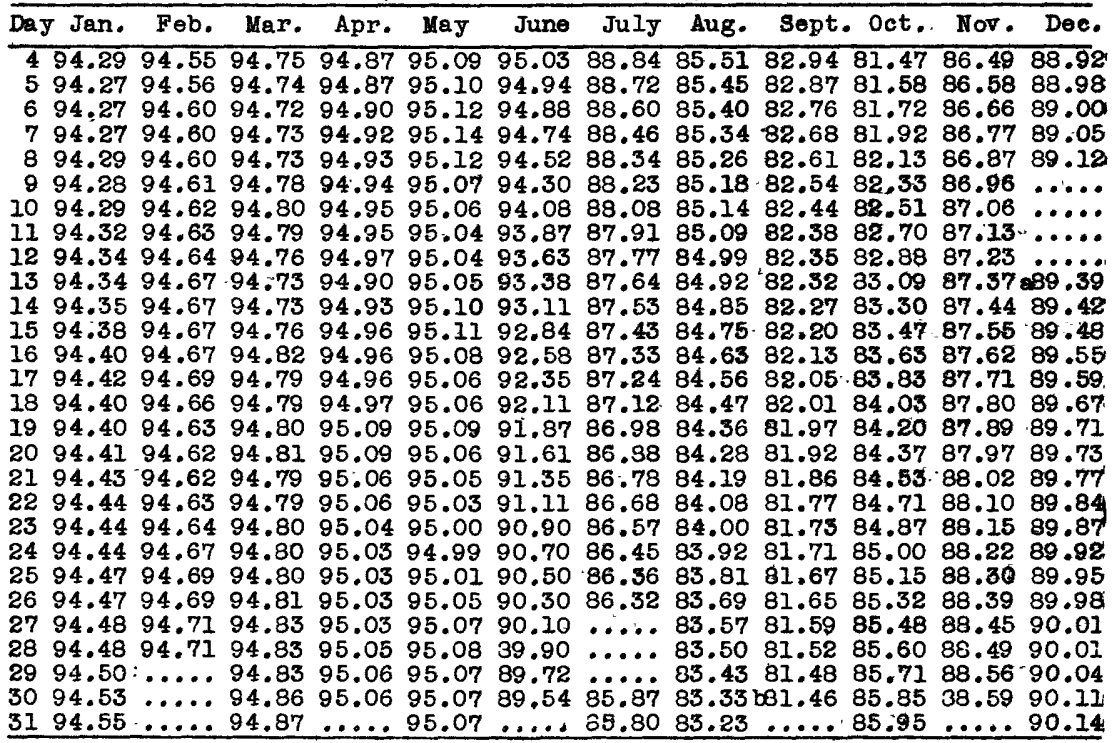

4. City of Fargo, Igland Park. (Described in Water-Supply Paper 908).

Water level, Jan. $1,1941,40.18$ feet below measuring point.

Lowest da1ly water level, in feet above assumed datum, 1941

(from recordér charts)

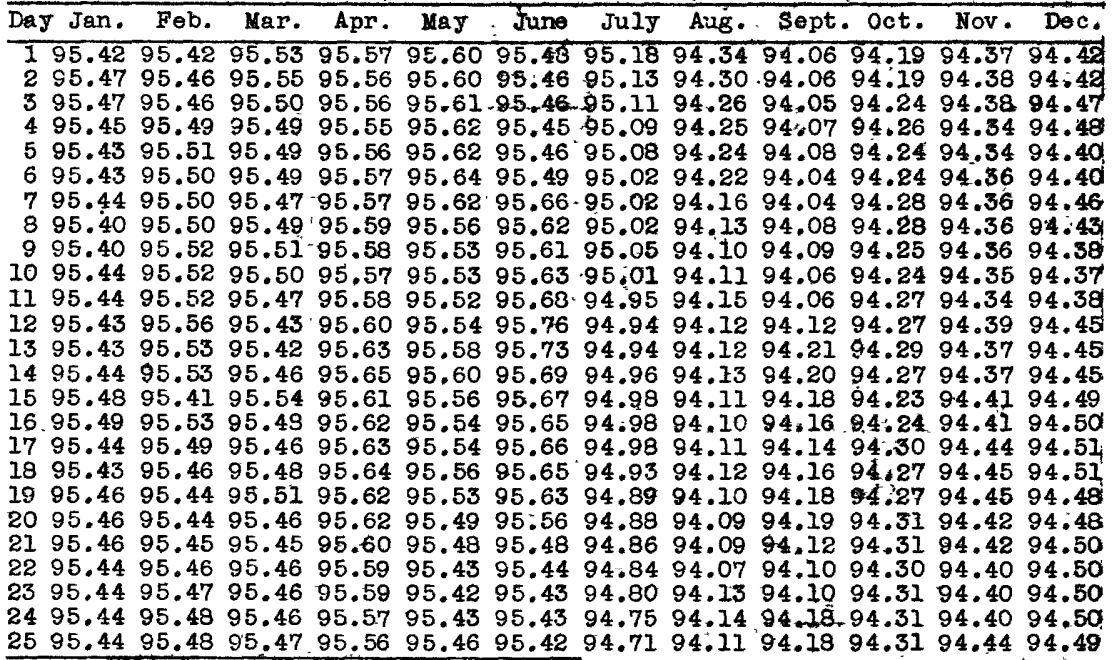

a Tape measurement.

$\mathrm{b}$ Pumping of ne11.14 stopped. 
Cass County--Continued

4. City of Fargo, Island Park--Contlnued.

Lowest daily water level, in feet above assumed datum, .1941

(from recorder charts)

\begin{tabular}{|c|c|c|c|c|c|c|c|c|c|c|c|}
\hline Jan. & Feb. & Mar. & Apr. & $\overline{\text { May }}$ & June & July & Aug. & Sept. & . oct. & Nov. & \\
\hline $\begin{array}{ll}26 & 95.45 \\
27 & 95.43 \\
28 & 95.45 \\
29 & 95.47 \\
30 & 95.50 \\
31 & 95.50\end{array}$ & $\begin{array}{l}95.48 \\
95.48 \\
95.48 \\
\ldots \ldots \\
\ldots \ldots \\
\ldots \ldots\end{array}$ & $\begin{array}{l}95.48 \\
95.47 \\
95.50 \\
95.57 \\
95.55\end{array}$ & $\begin{array}{l}95.57 \\
95.59 \\
95.59 \\
95.59 \\
95.60 \\
\ldots . . .\end{array}$ & $\begin{array}{l}95.50 \\
95.50 \\
95.49 \\
95.45 \\
95.46 \\
95.48\end{array}$ & $\begin{array}{l}95.38 \\
95.35 \\
95.36 \\
95.36 \\
95.29 \\
\ldots . . .\end{array}$ & $\begin{array}{l}94.66 \\
94.60 \\
94.51 \\
94.46 \\
94.39 \\
94.37\end{array}$ & $\begin{array}{l}94.04 \\
94.02 \\
94.02 \\
94.06 \\
94.07 \\
94.07\end{array}$ & $\begin{array}{l}94.18 \\
94.18 \\
94.15 \\
94.15 \\
94.20 \\
\ldots \ldots\end{array}$ & $\begin{array}{l}94.33 \\
94.31 \\
94.30 \\
94.30 \\
94.30 \\
94.30\end{array}$ & $\begin{array}{l}94.43 \\
94.41 \\
94.41 \\
94.39 \\
94.39 \\
\ldots . . .\end{array}$ & $\begin{array}{l}94.44 \\
94.45 \\
94.45\end{array}$ \\
\hline
\end{tabular}

5. Gardner Hotel, First St. No. and Roberts St., Fargo. (Described in Water-supply Paper 908). Water level, Jan. 4, 1941, 22.99 feet below measuring point.

Lowest dally water level, in feet above assumed datum, 1941

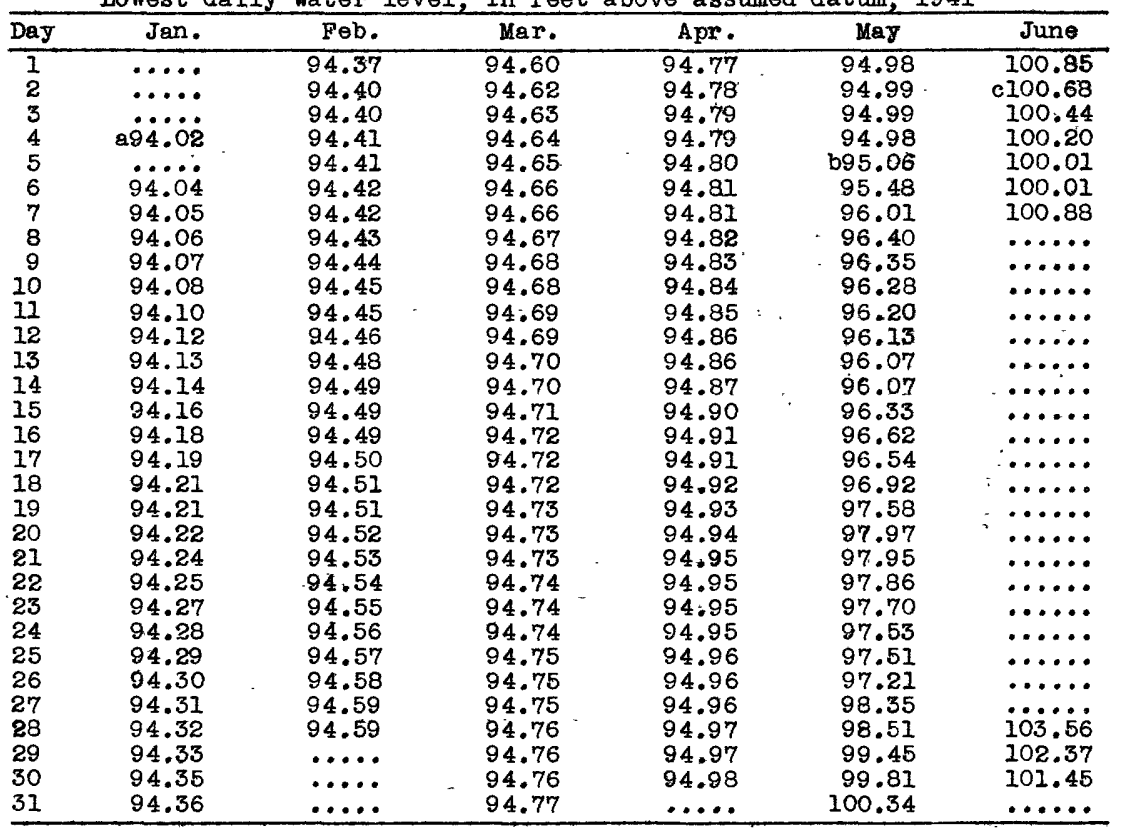

Lowest dally water level, in feet above assumed datum, 1941

\begin{tabular}{crrrrrr}
\hline Day & JuIy & Aug. & Sept. & oct. & Nov. & Dec. \\
\hline 1 & 100.61 & 88.64 & 85.49 & 83.57 & 85.33 & 88.11 \\
2 & 99.78 & 88.48 & 85.41 & 83.50 & 85.45 & 88.18 \\
3 & 99.32 & 88.34 & 85.33 & 83.45 & 85.56 & 88.25 \\
4 & 98.53 & 88.20 & 85.25 & 83.42 & 85.66 & 88.33 \\
5 & 97.76 & 88.08 & 85.17 & 83.39 & 86.78 & 88.40 \\
6 & 97.07 & 87.95 & 85.09 & 83.38 & 85.89 & 88.46 \\
7 & 96.41 & 87.84 & 85.01 & 83.37 & 85.99 & 88.54 \\
8 & 95.80 & 87.72 & 84.93 & 83.37 & 86.10 & 88.60 \\
9 & 95.24 & 87.60 & 84.86 & 83.38 & 86.20 & 88.66 \\
10 & 94.71 & 87.49 & 84.78 & 83.39 & 86.30 & 88.72 \\
11 & 94.23 & 87.39 & 84.69 & 83.40 & 86.40 & 88.77 \\
12 & 93.79 & 87.29 & 84.65 & 83.43 & 86.50 & 88.83 \\
\hline
\end{tabular}

a Tape measurement. b Water from rising water table flooding well.

c Punping of well 14 began. 
Cass Count $y--$ Continued

5. Garaner Hotel--Continued.

Lowest da1ly water level, in feet above assumed datum, 1941

(from recorder charts)

\begin{tabular}{|c|c|c|c|c|c|c|}
\hline Day & July & Aug. & Sept. & oct. & Nov. & Dec. \\
\hline $\begin{array}{l}13 \\
14 \\
15 \\
16 \\
17 \\
18 \\
19 \\
20 \\
21 \\
22 \\
23 \\
24 \\
25 \\
26 \\
27 \\
28 \\
29 \\
30 \\
31 \\
\end{array}$ & $\begin{array}{l}92.09 \\
92.58 \\
92.23 \\
91.91 \\
91.60 \\
91.31 \\
91.04 \\
90.78 \\
90.54 \\
90.30 \\
90.08 \\
39.86 \\
89.67 \\
89.48 \\
89.30 \\
89.12 \\
88.95 \\
88.79\end{array}$ & $\begin{array}{l}87.19 \\
37.10 \\
87.01 \\
86.92 \\
86.82 \\
86.73 \\
86.64 \\
86.55 \\
86.46 \\
86.37 \\
86.28 \\
86.20 \\
86.11 \\
86.01 \\
85.92 \\
85.83 \\
85.74 \\
85.66 \\
85.58\end{array}$ & $\begin{array}{l}84.73 \\
85.00 \\
84.89 \\
84.79 \\
84.68 \\
84.58 \\
84.49 \\
84.40 \\
84.31 \\
84.22 \\
84.13 \\
84.06 \\
83.98 \\
83.91 \\
83.34 \\
83.77 \\
83.70 \\
83.64 \\
\ldots . . .0\end{array}$ & $\begin{array}{l}83.49 \\
83.54 \\
83.61 \\
83.68 \\
83.76 \\
83.85 \\
83.94 \\
84.03 \\
84.13 \\
84.23 \\
84.35 \\
84.45 \\
84.55 \\
84.65 \\
84.77 \\
84.88 \\
84.99 \\
85.11 \\
85.22\end{array}$ & $\begin{array}{l}86.60 \\
86.70 \\
86.80 \\
86.89 \\
86.98 \\
87.08 \\
87.17 \\
87.26 \\
87.35 \\
87.43 \\
87.51 \\
87.59 \\
37.66 \\
87.74 \\
87.82 \\
87.90 \\
87.97 \\
88.05 \\
\ldots . . .0\end{array}$ & $\begin{array}{l}88.89 \\
88.95 \\
89.01 \\
89.06 \\
89.12 \\
89.18 \\
89.23 \\
89.28 \\
89.33 \\
89.39 \\
89.43 \\
89.48 \\
89.53 \\
89.57 \\
89.62 \\
89.66 \\
89.69 \\
89.73 \\
89.77\end{array}$ \\
\hline
\end{tabular}

6. Merehants National Bank \& Trust Co., Elghth Ave. So., and 17 th st., Fargo. (Described in Water-Supply Paper 908). Water level, Jan. 4, $1941,38.15$ feet below measuring point.

Water level; in feet above assumed datum, 1941

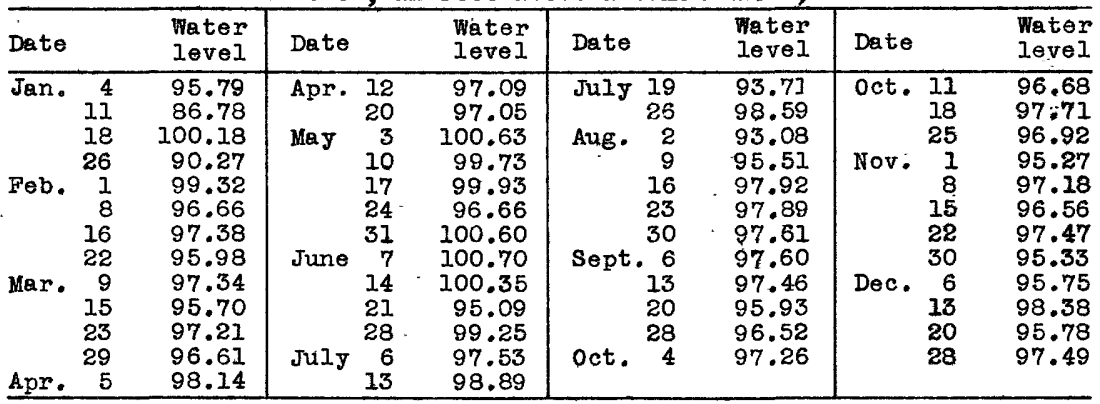

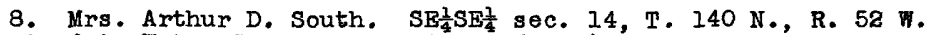
(Described in Water-Supply Paper $840, p_{0}: 321$ ).

Water level, in feet above datum, 1941

\begin{tabular}{|c|c|c|c|c|c|c|c|}
\hline $\begin{array}{rr}\operatorname{Jan} . & 1 \\
& 4 \\
11 \\
18\end{array}$ & $\begin{array}{l}99.52 \\
99.52 \\
99.53 \\
99.53\end{array}$ & $\begin{array}{rr}\text { Jan. } 25 \\
\text { Feb. } \\
1 \\
8 \\
\\
15\end{array}$ & $\begin{array}{l}99.51 \\
99.52 \\
99.52 \\
99.52\end{array}$ & $\begin{array}{lr}\text { Feb. } & 22 \\
\text { Mar. } & 1 \\
& 8 \\
& 15\end{array}$ & $\begin{array}{l}99.53 \\
99.38 \\
99.38 \\
99.37\end{array}$ & $\begin{array}{lr}\text { Mar. } 22 \\
& 2 \hat{9} \\
\text { Apr. } 5 \\
\text { oct. } 14\end{array}$ & $\begin{array}{l}99.36 \\
99.36 \\
99.37 \\
99.57\end{array}$ \\
\hline
\end{tabular}

10. Mrs: Arthur D. South. SEl SEl sec, 14, T. 140 N., R. 52 T.

(Described in Water-Supply Paper 840, p. 321). Water level, in feet above datum, 7941

\begin{tabular}{|c|c|c|c|c|c|c|c|c|}
\hline $\begin{array}{r}\text { Jan. } \\
1 \\
-1\end{array}$ & $\begin{array}{r}1 \\
4 \\
11 \\
18\end{array}$ & $\begin{array}{l}98.58 \\
98.58 \\
98.58 \\
98.58\end{array}$ & $\begin{array}{lr}\text { Jan. } & 25 \\
\text { Feb. } & 1 \\
& 8 \\
& 15\end{array}$ & $\begin{array}{l}98.67 \\
99.65 \\
98.72 \\
98.67\end{array}$ & $\begin{array}{lr}\text { Feb. } 22 \\
\text { Mar. } 1 \\
\\
\\
\\
15\end{array}$ & $\begin{array}{l}98.67 \\
98.67 \\
98.96 \\
98.93\end{array}$ & $\begin{array}{l}\text { Mar. } 22 \\
\\
29 \\
\text { Apr. } 5 \\
\text { oct. } 14\end{array}$ & $\begin{array}{l}98.90 \\
98.89 \\
98.90 \\
98.16\end{array}$ \\
\hline
\end{tabular}

a Pumping of well 14 stopped. 


\section{Cass County--Continued}

12. C1ty of Fargo. Swl sw sec. 1, T. 139 N, R. 49 W. (Described in Water-Supply Paper 840, p. 321). Measuring point changed May 31, 1941, to top of coupling on casting flueh with land surface and 2.14 feet below old measuring point. Automatic water-stage recorder installed May 31 ;

1941. Water level, Jan. 4, 1941, 35.54 feet below old measuring point. Lowest daily water level, with reference to assumed datum, 1941 (from recorder charts)

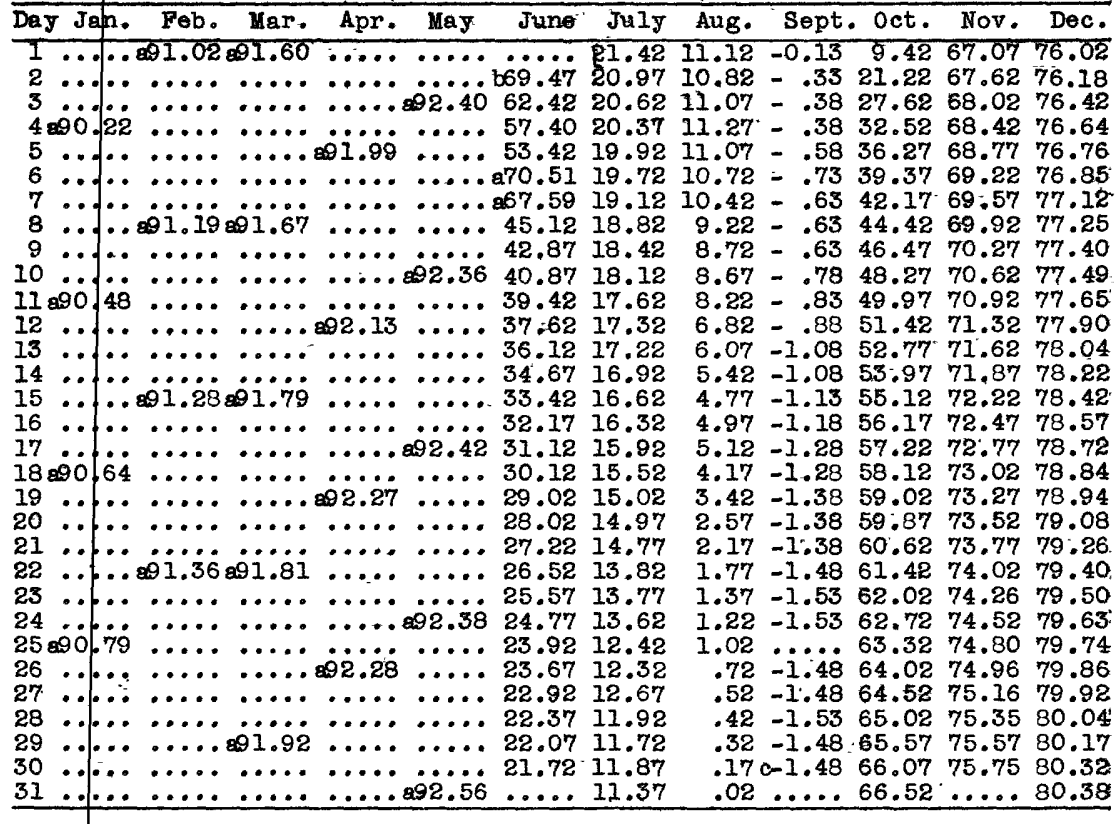

17. David Bossart, 1014 27th st. No., Fargo. (Described in WaterSupply Paper 908). Measurements discontinued after May 1, 1941. Water level|, Jan. 4, 1941, 29.88 feet below measuring point.

\begin{tabular}{|c|c|c|c|c|c|c|c|c|}
\hline Date & & $\begin{array}{l}\text { Water } \\
\text { level }\end{array}$ & Date & $\begin{array}{l}\text { Water } \\
\text { level }\end{array}$ & Date & $\begin{array}{l}\text { Water } \\
\text { level }\end{array}$ & Date & $\begin{array}{l}\text { Water } \\
\text { level }\end{array}$ \\
\hline Jan. & $\begin{array}{r}4 \\
11 \\
18 \\
26 \\
1 \\
8\end{array}$ & $\begin{array}{l}96.02 \\
90.31 \\
92.19 \\
88.50 \\
91.30 \\
93.19\end{array}$ & $\begin{array}{rr}\text { Feb. } & 16 \\
& 22 \\
\text { Mar. } & 1 \\
9 \\
15 \\
15 \\
23\end{array}$ & $\begin{array}{l}90.78 \\
93.26 \\
92.78 \\
93.06 \\
91.99 \\
92.89\end{array}$ & $\begin{array}{rr}\text { Mar. } & 29 \\
\text { Apr. } & 5 \\
& 12 \\
19 \\
\\
26\end{array}$ & $\begin{array}{l}91.75 \\
93.24 \\
93.70 \\
93.48 \\
93.73\end{array}$ & $\begin{array}{rr}\text { May } & 3 \\
10 \\
17 \\
-24 \\
31 \\
34\end{array}$ & $\begin{array}{l}92.62 \\
91.66 \\
93.87 \\
93.72 \\
92.58\end{array}$ \\
\hline
\end{tabular}

28. City of Fargo. NW SW $\frac{1}{4}$ sec. 1, T. $139 \mathrm{~N}, \mathrm{R}, 49 \mathrm{~W}$. (Described In Water-Supply Papers 840 , p. 321 , and 845, p. 350 ). Water level, Jan. 4, 1941 34.30 feet below measuring point.

Water level, in feet above as sumed datum, 1941

\begin{tabular}{|c|c|c|c|c|c|c|c|c|c|c|c|c|}
\hline$\overline{\mathrm{Day}}$ & an. & Feb. & Mar. & Apr. & May & June & July & Aug. & Sept. & oct. & Nov. & Dec. \\
\hline $\begin{array}{l}1 \\
2 \\
3\end{array}$ & & & & & & $\begin{array}{l}0 \\
9\end{array}$ & $\begin{array}{l}39 \\
39\end{array}$ & $\begin{array}{l}30.05 \\
29.53\end{array}$ & $\begin{array}{l}18.36 \\
18.10 \\
17.94\end{array}$ & & & \\
\hline
\end{tabular}

a Tape measurement.

b Pumping of well 14 began. 


\section{Cass County--Continued}

28. City of Fargo--Continued.

Water level, in feet above assumed datur, 1941

\begin{tabular}{|c|c|c|c|c|c|c|c|c|c|c|c|c|}
\hline & an. & reo. & dar. & pr. & lay & 3 & $21 \mathrm{y}$ & BS. & t. & Uet. & Hov. & \\
\hline $\begin{array}{l}4 \\
5 \\
6\end{array}$ & $\begin{array}{l}0.21 \\
\ldots \ldots \\
\ldots \ldots\end{array}$ & $\because$ & $\cdots$ & 01 & . & 4 & & $\begin{array}{l}29.19 \\
29.24 \\
29.09\end{array}$ & $\begin{array}{l}17.82 \\
17.66 \\
17.48\end{array}$ & $\begin{array}{l}34.23 \\
\ldots \ldots \\
\ldots \ldots\end{array}$ & & \\
\hline $\begin{array}{l}7 \\
8\end{array}$ & $\ldots$ & & $\ddot{9} \ddot{1} . \dot{6}$ & $\cdots$ & .... & & $\begin{array}{l}37 \\
37\end{array}$ & $\begin{array}{l}29.10 \\
28.63\end{array}$ & & $\ldots \ldots$ & $\ddot{70.07}$ & - \\
\hline $\begin{array}{r}9 \\
10 \\
11\end{array}$ & $\ddot{0} \ddot{0} \ddot{48}$ & $\because$ & 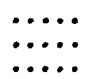 & $\ldots \ldots$ & 皮 & & & $\begin{array}{l}27.86 \\
\ddot{27 .} \ddot{10}\end{array}$ & & 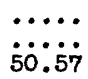 & 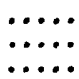 & \\
\hline 2 & & & & & & & & $\ddot{25.04}$ & & $\ldots \ldots$ & & \\
\hline & & & $\ddot{9} \ddot{i} \ddot{8}$ & & & & & $\begin{array}{l}24.88 \\
24.81\end{array}$ & $\ddot{i 6}$ & $\ldots \ldots$ & $\ddot{7}$ & \\
\hline 6 & & $\bullet$ & $\ldots \ldots$ & & & & & 67 & & $\because$ & & \\
\hline 7 & $\ddot{9} \ddot{0} \dot{63}$ & & & & & & 34 & $\ddot{23} \ddot{6} \dot{6}$ & & $\ddot{58} \ddot{8} \dot{5}$ & & \\
\hline & $\cdots$ & & & & & & & $\ddot{2} \ddot{2} . \ddot{3} \dot{5}$ & & 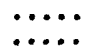 & $\ldots \ldots$ & \\
\hline & . & & & & & & & $\begin{array}{l}21.42 \\
21.11\end{array}$ & • & 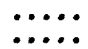 & $\ddot{7}$ & \\
\hline & & & & & & & & & & & & \\
\hline & $\ddot{8 i}$ & & & & & & $\begin{array}{l}32 \\
32\end{array}$ & & & $\ddot{63.64}$ & & \\
\hline & & & & & & & & & 73 & & & \\
\hline & & & & & & & & & & & & \\
\hline & & & & & & & & & & & & \\
\hline & & & & & & & & & & $\cdots \cdots$ & & \\
\hline & & & & & & & & & & & & \\
\hline
\end{tabular}

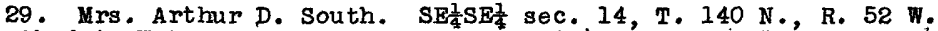
(Described in Water-Supply Paper 840, p. 321). Used da11y for stock and house.

Water level, in feet above datum, 1941

\begin{tabular}{|c|c|c|c|c|c|c|c|}
\hline Date & $\begin{array}{l}\text { Water } \\
\text { level }\end{array}$ & Date & $\begin{array}{l}\text { Water } \\
\text { level }\end{array}$ & Date & $\begin{array}{l}\text { Water } \\
\text { level }\end{array}$ & Date & $\begin{array}{l}\text { Water } \\
\text { level }\end{array}$ \\
\hline $\begin{array}{rr}\text { Jan. } & 1 \\
4 \\
11 \\
18 \\
\end{array}$ & $\begin{array}{l}99.74 \\
99.47 \\
99.79 \\
99.46\end{array}$ & $\begin{array}{rr}\text { Jan. } & 25 \\
\text { Feb. } & 1 \\
8 \\
15\end{array}$ & $\begin{array}{r}99.78 \\
99.00 \\
100.84 \\
100.78\end{array}$ & $\begin{array}{rr}\text { Feb. } 22 \\
\text { Mar. } \\
1 \\
8 \\
15 \\
\end{array}$ & $\begin{array}{r}100.75 \\
100.61 \\
95.67 \\
100.05 \\
\end{array}$ & $\begin{array}{l}\text { Mar. } 22 \\
\\
29 \\
\text { Apr. } 5 \\
\text { oct. } 14\end{array}$ & $\begin{array}{l}100.52 \\
100.43 \\
100.59 \\
100.42\end{array}$ \\
\hline
\end{tabular}

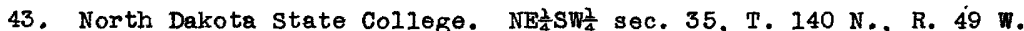
(Described in Water-Supply Paper 908). Water level, Jan. 4, 1941, 7.53 feet below measuring point.

Water level, in feet above assumed datum, 1941

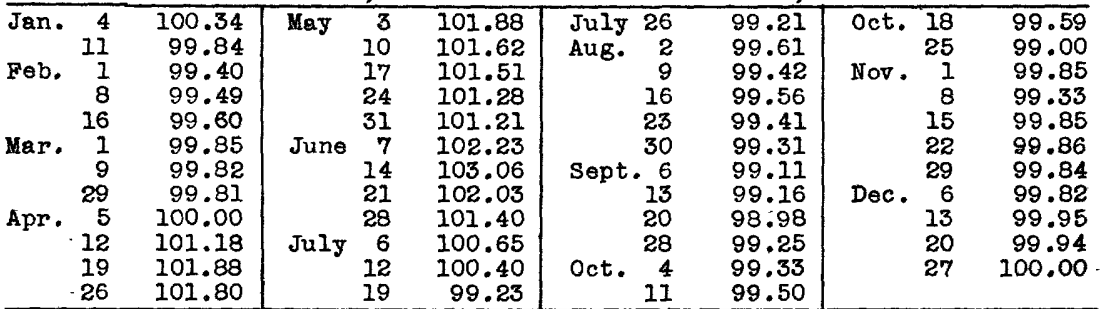

a Pumping of well 14 stopped. 
Cass County--Cont 1nued

57. Union Stockyards. NW $\mathrm{NEE}_{\frac{2}{4}}^{\frac{1}{4}}$ sec..6, T. $139 \mathrm{H}$; R. 49 W. (Described In Water-supply Paper 8̧45, p. 351). Water level, Jan. 4, 1941, 21.15 feet: below measuring point.

Water level, in feet above essumed datum, 19.41

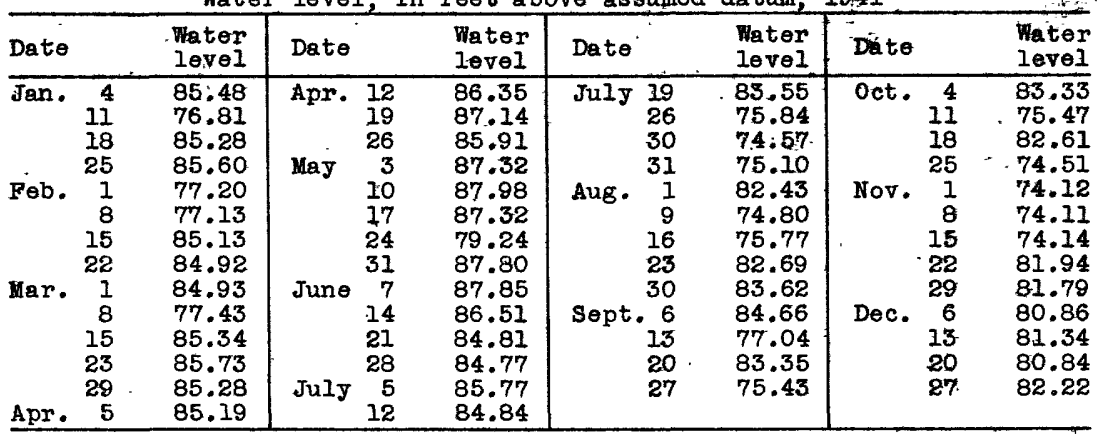

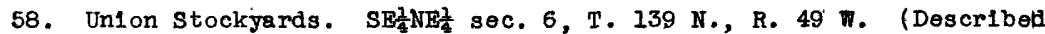
In Water-Supply Paper 845, p. 351). Water level, Jan. 4, 1941, 40.65 feet below measuring point.

Lowest dally water level, in feet above assumed datum, 1941

(from recorder charts)

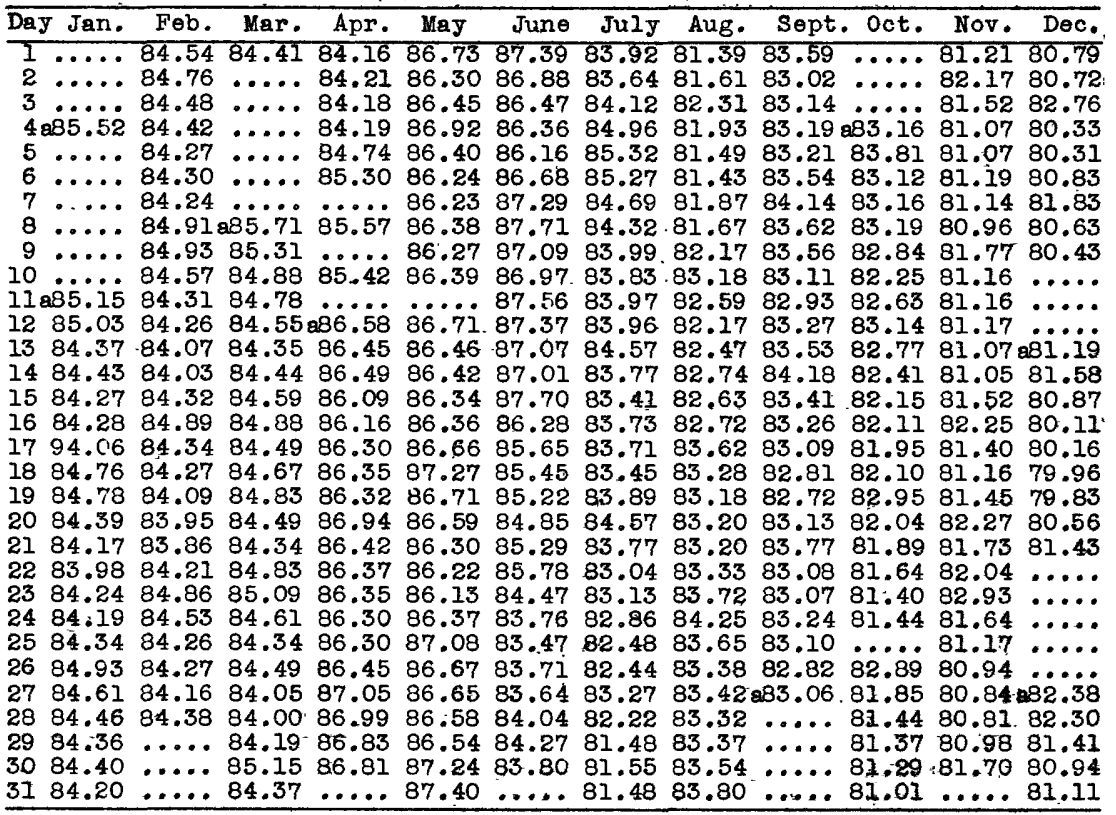

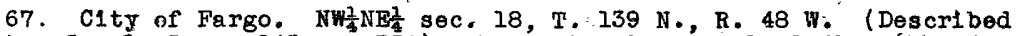
In Water-Supply Paper 845, p. 352). Water Ievel, Ján. 1, 1941, 32́.76 feet below measuring point.

a Tape measurement. 
Cass Gounty--Continued.

67.: Gity of Fargo--Continued.

Lowest daily water level, in feet above assuined datum, 1941 :

(from recorder charts)

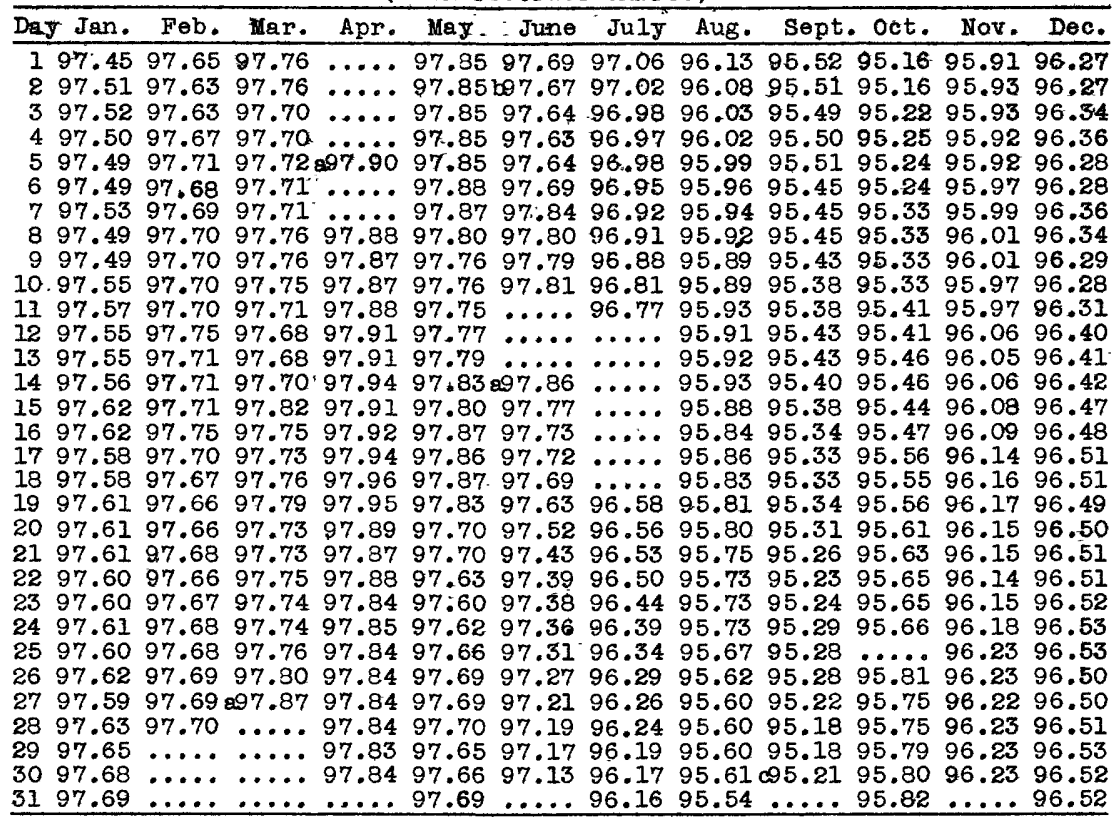

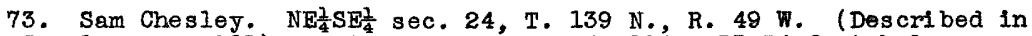
Water-Supply Paper 908). Water level, Jan. 4, 1941, 33.34 feet below measuring point.

Water level, in feet above assumed datum, 1941

\begin{tabular}{|c|c|c|c|c|c|c|c|}
\hline Date & $\begin{array}{l}\text { Water } \\
\text { level }\end{array}$ & Date & $\begin{array}{l}\text { Water } \\
\text { level }\end{array}$ & Date & $\begin{array}{l}\text { Water } \\
\text { level }\end{array}$ & Date & $\begin{array}{l}\text { Water } \\
\text { leve] }\end{array}$ \\
\hline $\begin{array}{rr}\text { Jan. } & 4 \\
11 \\
18 \\
26 \\
\text { Feb. } 1 \\
8 \\
16 \\
22 \\
\text { Mar. } 1 \\
9 \\
15 \\
25 \\
29\end{array}$ & $\begin{array}{l}95.18 \\
95.23 \\
95.25 \\
95.48 \\
95.45 \\
95.57 \\
95.57 \\
95.49 \\
95.29 \\
95.45 \\
95.68 \\
95.69 \\
95.74\end{array}$ & $\begin{array}{cc}\text { Apr. } \\
\text { May } \\
\text { June } \\
\text { Jun } \\
\end{array}$ & $\begin{array}{l}95.73 \\
95.76 \\
95.71 \\
96.40 \\
95.70 \\
95.46 \\
95.54 \\
95.56 \\
95.79 \\
95.83 \\
95.93 \\
95.69\end{array}$ & $\begin{array}{r}\text { July } 6 \\
13 \\
19 \\
26 \\
\text { Aug. } 2 \\
9 \\
9 \\
16 \\
23 \\
30 \\
30 p t .6 \\
13 \\
20 \\
28\end{array}$ & $\begin{array}{l}95.30 \\
95.04 \\
95.28 \\
94.39 \\
95.21 \\
94.10 \\
94.19 \\
94.04 \\
93.93 \\
94.00 \\
93.73 \\
93.61 \\
93.30\end{array}$ & $\begin{array}{rr}\text { Oct. } & 4 \\
11 \\
18 \\
25 \\
\text { Nov. } 1 \\
8 \\
15 \\
22 \\
30 \\
\text { Dec. } 6 \\
13 \\
-20 \\
28 \\
28\end{array}$ & $\begin{array}{l}93.50 \\
93.37 \\
93.34 \\
93.33 \\
93.30 \\
93.69 \\
92.75 \\
93.71 \\
93.88 \\
93.85 \\
93.692 \\
94.04 \\
93.98\end{array}$ \\
\hline
\end{tabular}

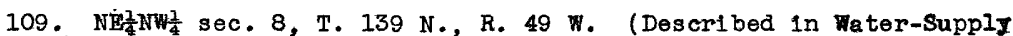
Paper 908) In use as domestic weli' after Nov. 22, 1941. Water level, Jan. 4, 1941, 33.02 feet below measuring point.

Water level, in feet above assumed datum, 1941

\begin{tabular}{|c|c|c|c|c|c|c|c|}
\hline an. 18 & $\begin{array}{r}4 \\
11 \\
18\end{array}$ & $\begin{array}{l}97.13 \\
97.18 \\
96.69\end{array}$ & 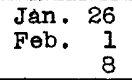 & $\begin{array}{l}96 \\
95 \\
95\end{array}$ & $\begin{array}{l}\text { Feb. } \frac{1}{2} \\
\text { Mar. }\end{array}$ & & $\begin{array}{r}8 \\
15 \\
23\end{array}$ \\
\hline
\end{tabular}

a Tape measurement. b Pumping of well 14 began.

c Pumping of well 14 stopped. 
Cass County--Continuted

109.--Continued.

Water level, in feet above assumed datum, 1941

\begin{tabular}{|c|c|c|c|c|c|c|c|}
\hline Date... & $\begin{array}{l}\text { Water } \\
\text { level }\end{array}$ & Dete & $\begin{array}{l}\text { Water } \\
\text { level }\end{array}$ & Date & $\begin{array}{l}\text { Water } \\
\text { Level }\end{array}$ & Date & $\begin{array}{l}\text { Water } \\
\text { Ievel }\end{array}$ \\
\hline 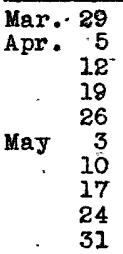 & $\begin{array}{l}94.27 \\
94: 20 \\
94.31 \\
94.60 \\
94.72 \\
94.82 \\
94.79 \\
95.11 \\
95.10 \\
92.76\end{array}$ & $\begin{array}{lr}\text { June } & 7 \\
& 14 \\
& 21 \\
& 28 \\
\text { July } & 5 \\
& 12 \\
& 19 \\
& 26 \\
\text { Aug. } & 2 \\
& 9\end{array}$ & $\begin{array}{l}97.39 \\
96.55 \\
97.24 \\
96.72 \\
96.23 \\
95.77 \\
95.24 \\
94.92 \\
94.06 \\
94.22\end{array}$ & 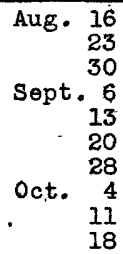 & $\begin{array}{l}94.04 \\
93.94 \\
93.81 \\
93.73 \\
93.74 \\
93.64 \\
93.62 \\
93.39 \\
92.30 \\
92.48\end{array}$ & $\begin{array}{rr}\text { Oct. } & 25 \\
\text { Nov. } & -1 \\
8 \\
\\
15 \\
22 \\
\\
29 \\
\text { Dec. } \\
6 \\
13 \\
20 \\
27 \\
27\end{array}$ & $\begin{array}{l}94.87 \\
94.89 \\
93.67 \\
93.68 \\
90.56 \\
89.99 \\
89.64 \\
89.43 \\
89.38 \\
89.39\end{array}$ \\
\hline
\end{tabular}

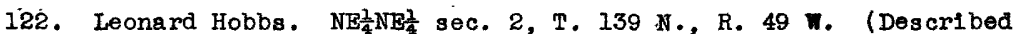
In Water-Supply Papor 908). Water level, Jan. 4, 1941, 17.37 feet below measuring point.

Water level, in feet above assumed datum, 1941

\begin{tabular}{|c|c|c|c|c|c|c|c|c|c|c|c|}
\hline Jan. & $\begin{array}{r}4 \\
11 \\
18 \\
26 \\
1 \\
8 \\
16 \\
22 \\
1 \\
9 \\
15 \\
23 \\
29\end{array}$ & $\begin{array}{l}99.33 \\
99.30 \\
99.22 \\
99.18 \\
99.14 \\
99.10 \\
99.06 \\
98.98 \\
99.00 \\
98.92 \\
98.97 \\
99.10 \\
99.30\end{array}$ & Apr. & $\begin{array}{r}5 \\
12 \\
19 \\
26 \\
3 \\
10 \\
17 \\
24 \\
31 \\
77 \\
14 \\
21 \\
28\end{array}$ & $\begin{array}{r}99.37 \\
99.84 \\
100.38 \\
100.81 \\
101.21 \\
101.11 \\
101.24 \\
101.11 \\
101.25 \\
101.72 \\
103.66 \\
104.55 \\
104.96\end{array}$ & $\begin{array}{l}\text { July } \\
\text { Aug. } \\
\text { Sept. }\end{array}$ & $\begin{array}{r}6 \\
12 \\
19 \\
26 \\
2 \\
9 \\
16 \\
23 \\
30 \\
6 \\
13 \\
20 \\
28 \\
\end{array}$ & $\begin{array}{l}104.97 \\
104.90 \\
104.80 \\
104.56 \\
104.27 \\
104.05 \\
104.08 \\
104.01 \\
104.06 \\
103.81 \\
103.81 \\
103.80 \\
103.81\end{array}$ & oet. & $\begin{array}{l}15 \\
22 \\
29\end{array}$ & $\begin{array}{l}104.03 \\
104.17 \\
104.16 \\
104.15 \\
104.20 \\
104.00 \\
103.96 \\
103.84 \\
103.85 \\
103.68 \\
103.62 \\
103.58 \\
103.37\end{array}$ \\
\hline
\end{tabular}

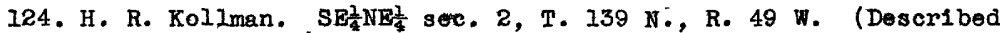
in Water-supply Paper 808 ). Equipped with pump and placed in use for domestic supply Mar. 23, 1941. Measurements discontinued. Water level, Jan. 4, 1941, 32.84 féet below measuring point.

Water level, in feet above assumed datum, 1941

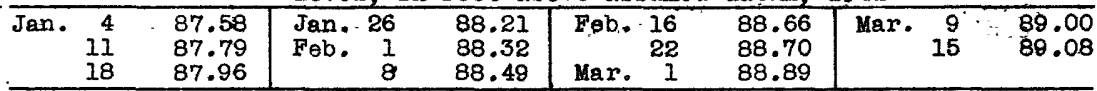

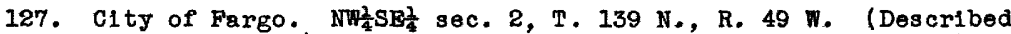
in Water-Supply Paper 908). Water level, Jan. 4, 1941, 19.06 feet below moasuring point.

Water level, in feet above assumed datum, 1941

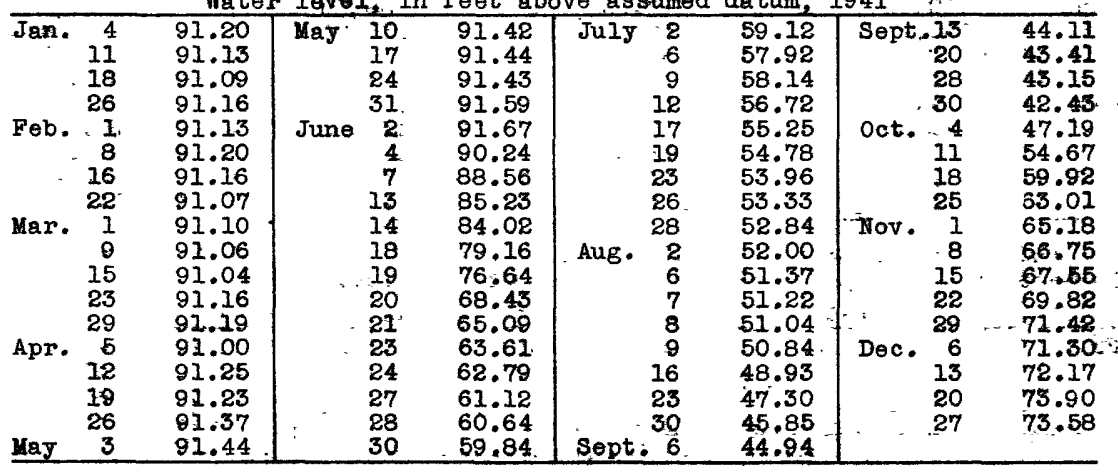




\section{Cavalior County}

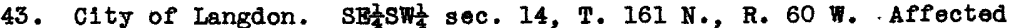
by impounded water. (Described in Water-supply Papers 840 , p. 322, and 845 , p. 352).

Water level, in feet above datum, 1941

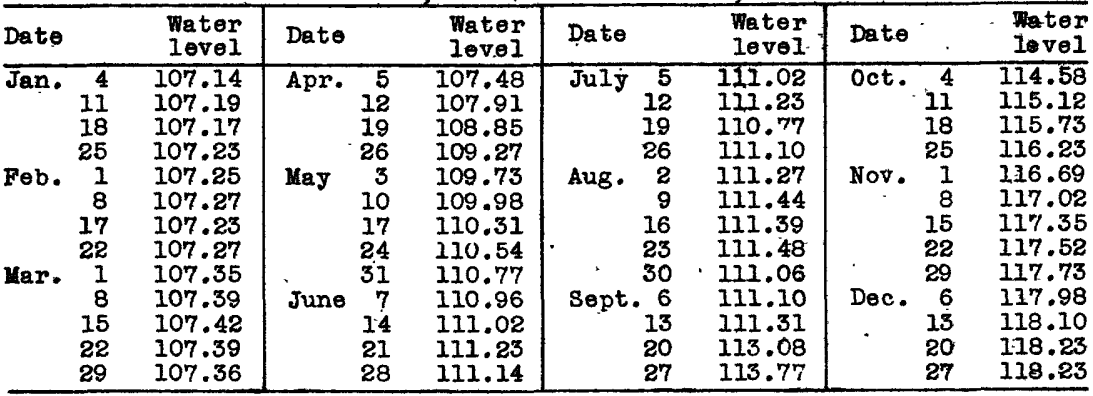

44. City of Langdon. SW $\mathrm{SWE}_{\frac{1}{4}}$ sec. 14, T. $161 \mathrm{~N} .$, R. $60 \mathrm{~W}$. Affected by impounded water. (Described in Water-suppiy Papers 840 , p. 322 , and 845, p. 353).

Water level, in feet above datum, 1941

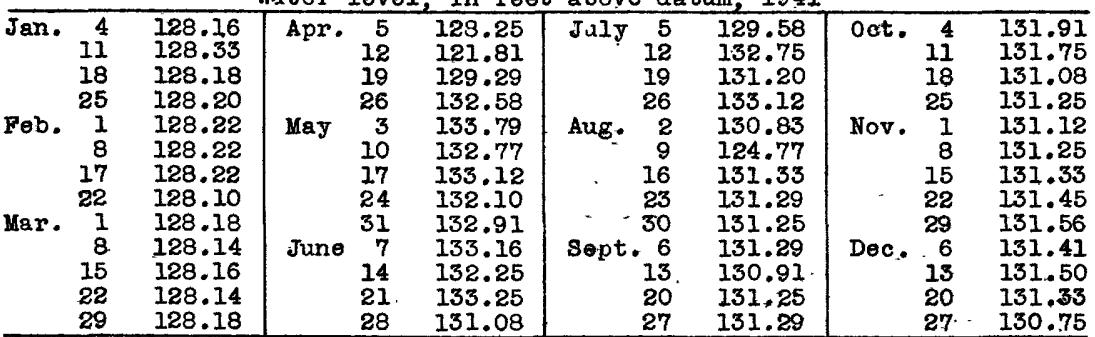

45. C1ty of Langdon. Sim $\frac{1}{4} N W_{4}^{\frac{1}{4}}$ sec. 23, T. $161 \mathrm{~N} .$, R. 60 W. Affected by impounded water. (Described in Water-Supply Papers 840 , p. 322, and 845 , p. 3537 .

Water level, in feet above datum, 1941

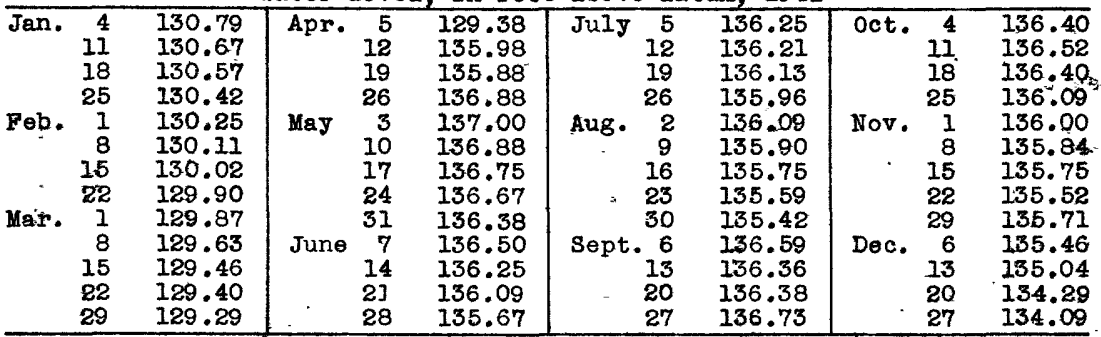

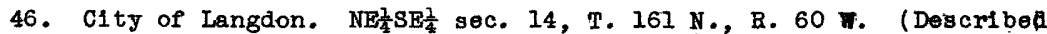
in Water-Supply Papers 840 , p. 322 , and 845, p. 354).

Water level, in feet above datum, 1941

\begin{tabular}{|c|c|c|c|c|c|c|c|c|c|c|}
\hline $\operatorname{Jan}$. & $\begin{array}{l}4 \\
11 \\
18 \\
25\end{array}$ & $\begin{array}{l}98.00 \\
97.92 \\
97.75 \\
97.60\end{array}$ & Feb. & $\begin{array}{r}1 \\
8 \\
15 \\
22\end{array}$ & $\begin{array}{l}97.56 \\
97.47 \\
97.35 \\
97.25\end{array}$ & Mar: & $\begin{array}{r}1 \\
8 \\
15 \\
22\end{array}$ & $\begin{array}{l}97.25 \\
97.21 \\
97.10 \\
97.00\end{array}$ & $\begin{array}{l}\text { Mar. } \\
\text { Apr. }\end{array}$ & $\begin{array}{l}96.92 \\
96.92 \\
98.54 \\
99.75\end{array}$ \\
\hline
\end{tabular}


Cavalier County--Continued

46. City of Langdon.--Continued:-..

Water level, in feet above datum, 1941

\begin{tabular}{|c|c|c|c|c|c|c|c|c|c|c|}
\hline \multicolumn{2}{|l|}{ Date } & $\begin{array}{l}\text { Water } \\
\text { level }\end{array}$ & \multicolumn{2}{|l|}{ Daté } & $\begin{array}{l}\text { Water } \\
\text { level }\end{array}$ & Daté & $\begin{array}{l}\text { Water } \\
\text { level }\end{array}$ & \multicolumn{2}{|c|}{ Date } & $\begin{array}{l}\text { Water } \\
\text { level }\end{array}$ \\
\hline May. & $\begin{array}{r}26 \\
3 \\
10 \\
17 \\
24 \\
31 \\
7 \\
14 \\
21\end{array}$ & $\begin{array}{l}101.64 \\
102.42 \\
102.65 \\
102.94 \\
102.83 \\
102.77 \\
102.98 \\
103.83 \\
104.08\end{array}$ & Aug. & $\begin{array}{r}28 \\
5 \\
12 \\
19 \\
26 \\
2 \\
9 \\
16 \\
23\end{array}$ & $\begin{array}{l}103.75 \\
102.50 \\
102.21 \\
102.96 \\
102.94 \\
102.58 \\
102.33 \\
102.00 \\
101.81\end{array}$ & $\begin{array}{rr}\text { Aug. } & 30 \\
\text { Sept. } 6 \\
13 \\
\\
20 \\
\\
27 \\
\text { oct. } 4 \\
\\
11 \\
18 \\
\\
25\end{array}$ & $\begin{array}{l}101.67 \\
103.54 \\
104.63 \\
104.04 \\
106.65 \\
106.44 \\
707.10 \\
106.54 \\
106.58\end{array}$ & Nov. & $\begin{array}{r}1 \\
8 \\
15 \\
22 \\
29 \\
6 \\
13 \\
20 \\
27\end{array}$ & $\begin{array}{l}106.54 \\
106.17 \\
106.25 \\
105.95 \\
105.33 \\
105.19 \\
104.71 \\
104.25\end{array}$ \\
\hline
\end{tabular}

Dickey County

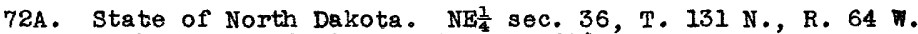

(Described in Water-Supply Paper 886, p. 535).

Water level, in feet above datum, 1941

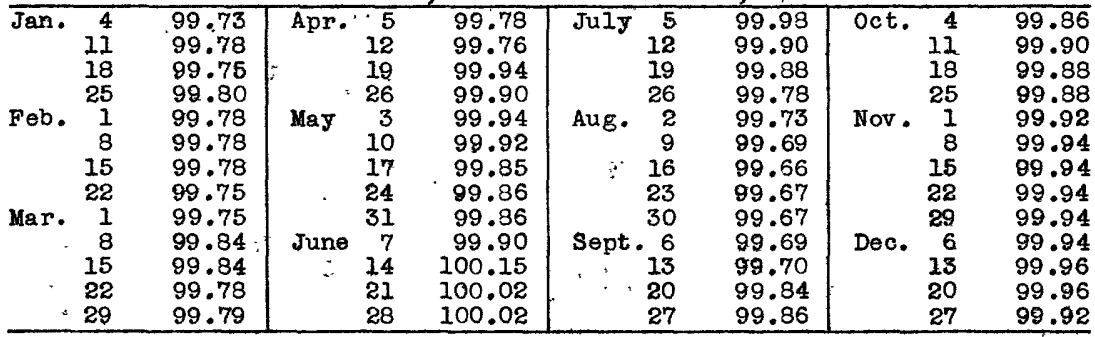

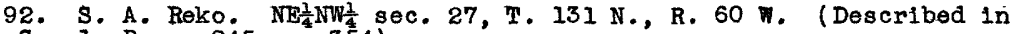
Water-Supply Paper $845, \mathrm{p} .354)$.

Water level, in feet above datum, 1941

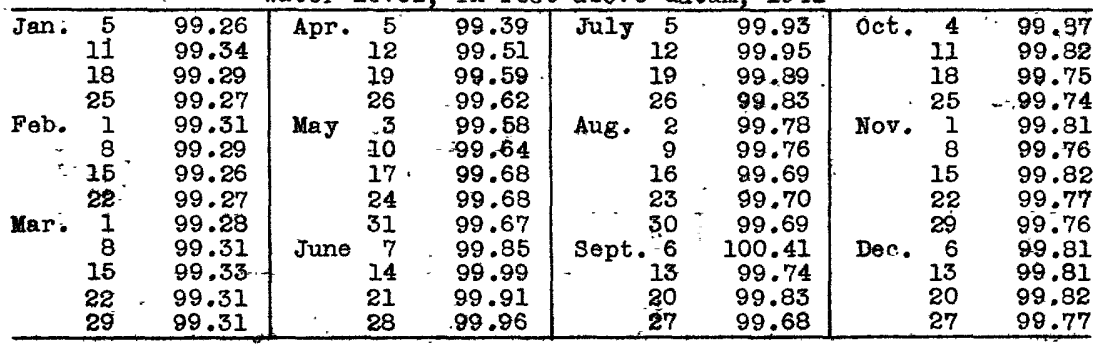

93. Measurements discontinued:

95. Standard 011 Co. $\mathrm{NB}_{4} \mathrm{NE}_{4}^{\frac{3}{4}}$ sec. 29, T. $131 \mathrm{~N}$, R. 59 W. (Described in Water-Supply Paper 908). Measurements discontinued after July 1941 when pipe was pulled. Water levels; in feet below measuring point, 1941: May 8, 19.25; July 25, 19.15:

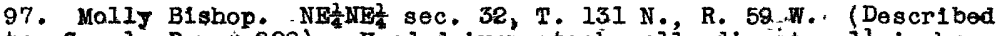
in Water-Supply Paper 908). Used driven stock weil, diameter li inches, depth 21 feet. Measuring point, joint in pitcher pump, 1.1 feet above. land surface, 1,314.3 feet above mean sea level. Water levels, in feet below measuring point: Aug; 8, 1940, 15.11; May 8, 1941, 14.12; July 25, $1941,13.67$; 0ct. 15, 1941, 13.95.

98. Albert M. Schmit. NEt NEt sec. 20, T. 131 N., R. 59 W. (Described In Water-Supply Paper 908). Now unused. Influenced by domestic weIl 5 feet away. Water levels, in feet below measuring point, 1941: May 8, 21.67; oct. 15, 21.32 . 
Dickey County--Continued

90. No measurements made in 1941: ...

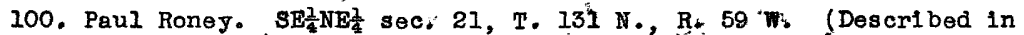
Water-Supply Paper 908). Measuring point. $1,326.06$ feet abové mean sea level.

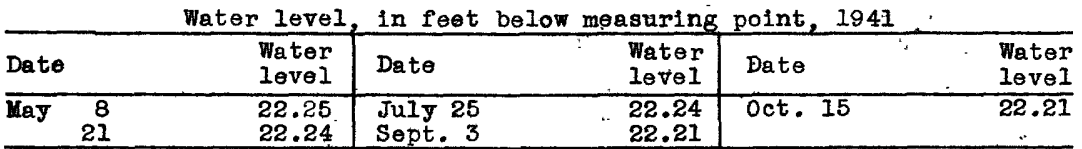

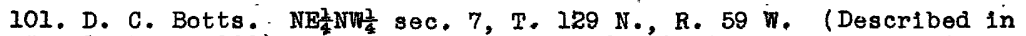
Water-Supply Paper 908). Old well pipe pulled óct. 1941. New pipe driven on site of old well oct. 16, 1941. Unused observation well, diameter $1 \frac{1}{4}$ inches, depth 18 feet. New measuring point, top of casing, 0.34 foot above old one, and 113.72 feet above datum. observer, Art Hendrickson, Ludden.

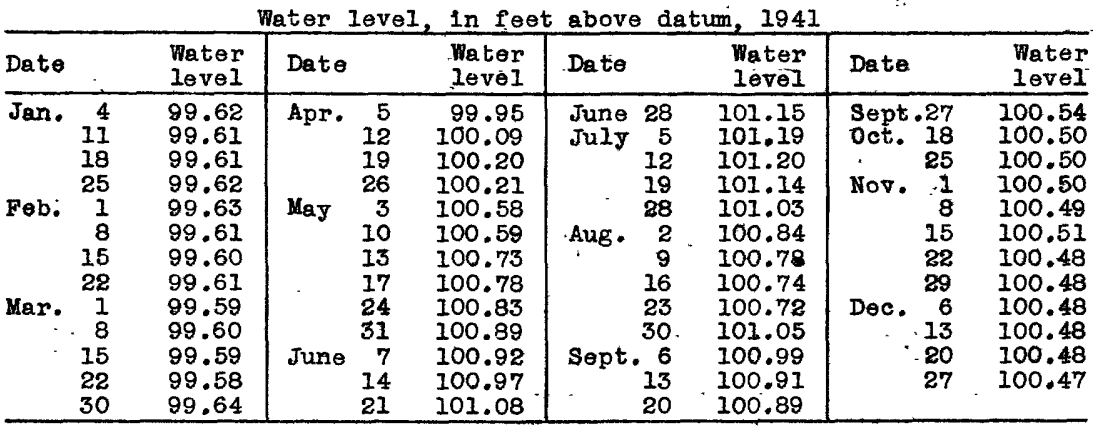

102. State of North Dakota. $S E_{4}^{\frac{1}{4}} S E_{\frac{1}{4}}^{1} \mathrm{sec} .16, \mathrm{~T}, 131 \mathrm{~N}$; R. 59 . (Described in Water-Supply Paper 908). Water levels, in feet below measuring point, 1941: May 8, 24.45; July 25, a/27.79; Aug, 30, 24.06; 0ct. 15, 25.76 .

103. Floyd Ferguson, W7 in Water-Supply Paper 908).

Water level, in feet below measuring point; 1941

\begin{tabular}{lll|l|lll}
\hline Date & $\begin{array}{l}\text { Water } \\
\text { level }\end{array}$ & Dete & $\begin{array}{l}\text { Water } \\
\text { level }\end{array}$ & Date & $\begin{array}{r}\text { Water } \\
\text { leqel }\end{array}$ \\
\hline May 8 & 11.75 & July 25 & 11.60 & 0ct. 15 & 11.85 \\
July 12 & 11.46 & Sept. 3 & 11.92 & & \\
\hline
\end{tabular}

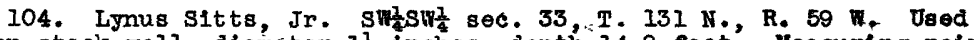
driven stock well, diameter $1 \frac{1}{4}$ inches, depth 14.9 feet. Heasuring point, Joint of pitcher pump, 2.3 feet above land surface. Water level.Sept. 7, 1940, 12.58 feet below measuring point.

Water lovel, in feet below measuring point, 1940-4I

\begin{tabular}{lll|lll|lll}
\hline Sept. 7, 1940 & 12.58 & July 25, 1941 & 11.02 & Oct. 15, 1941 & 11.31 \\
May & 8, 1941. & 11.19 & Sept. 3 & 11.56 & & \\
\hline
\end{tabular}

105. H. G. Martin, administrator. SW NWt sec. 9, T. $130 \mathrm{~N}:$, R. 59 w (Described in Water-Supply Paper 908). Water levela, in feet below measuring polnt, 1941: May 8, 13.58; July 21, 12.92; 0et. 15, 13.22.

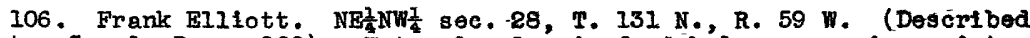
in Water-Supply Paper 908). Water levels, in feet below measuring point, 1941: May 8, 14.03; July 25, 13.92; Sept. 3, 14.22; 0ct. 16, 13.97.

a Probably local drawdown. 


\section{Dlckey County--Contimued:}

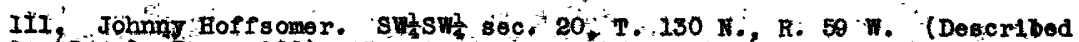
In Water-süpiy paper 908). Previous nessurting point, top of pitcher pump, 1,304.46 feet above mean sea level. Alternate measuring potnt, top af casing, $l, 303.6$ feet above mean sea level. Water levels, in feet abóro sod lovel, 1941 : May 8, 1,292.6; Ju1y 25, 3.202.9; 0ct. 15, 1, 2092.24.

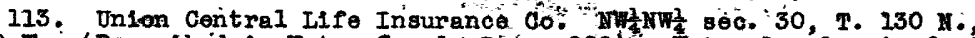
R. 59 F. (Described in Nater-Supply Páper 908). Water levels, in feet below measuring point, 1941: Yay 8, 12.75, 0et. 15, 13.58.

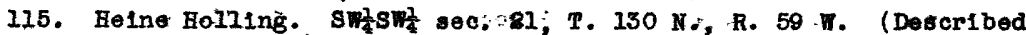
in Water-supply Paper 908). Water levels, in feet below measuring point, 1941: May 8, 10.48; July 25, 9.79; pet. 15, 10.35.

117. B. P. Wilson. NW/2Net sec. 22, T. $130 \mathrm{~F}$, R. 59 W. (Described in Water-8upply Paper 908). Water levels, in feet below measuring point, 1941: 19y 8, 11.27; Juiy $25,10.59$; 0tt: $16,11.21 . \cdots$

120. Esterby Bstate. Swher sec. 3, T. 129 N.; R. 59. W.- Used driven garden vell, dianeter $1 \frac{1}{4}$ inches, depth 13.2 feet. Measuring point, top of casing, $-1,308.3$ fest a bove sea level, and 2.6 feet above land surface. Water levels, in feet below measuring polnt, 1941. May-15, 6.33; July 21, 7.63 ; 0ct. $15,8.38$.

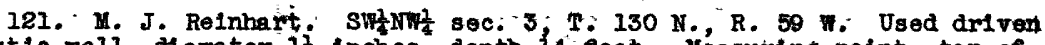
homestic weli, diameter if inches, depth 14 feet. Yeasuring point, top of pitcher pump, $1,314.0$ feot above sea leve1, and 3.3 foet abote land surface Tater levels, in feet below measuring point, 1941: uay 19, 12.23; July 25, $11.81 ;$ Sept.'3, 12:24; oct. 15, 12.28.

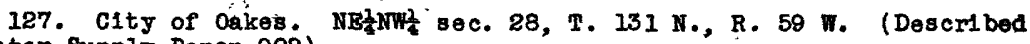
in Water-supply Paper 908).

viater level, in feet above datum, 1941

\begin{tabular}{|c|c|c|c|c|c|c|c|c|c|c|}
\hline Date & & $\begin{array}{l}\text { Water } \\
\text { level }\end{array}$ & Date & & $\begin{array}{l}\text { Water } \\
\text { level }\end{array}$ & Date & $\begin{array}{l}\text { Water } \\
\text { level }\end{array}$ & Date & & $\begin{array}{l}\text { Water } \\
\text { level }\end{array}$ \\
\hline Jan. & $\begin{array}{r}4 \\
11 \\
18 \\
25 \\
1 \\
8 \\
15 \\
22 \\
1 \\
8 \\
15 \\
22 \\
29\end{array}$ & $\begin{array}{l}100.28 \\
100.27 \\
100.27 \\
100.27 \\
100.29 \\
100.29 \\
100.29 \\
100.29 \\
100.29 \\
100.29 \\
100.30 \\
100.41 \\
101.05\end{array}$ & Apr. & $\begin{array}{r}5 \\
12 \\
19 \\
26 \\
3 \\
8 \\
17 \\
24 \\
31 \\
7 \\
14 \\
21 \\
28\end{array}$ & $\begin{array}{l}100.18 \\
100.21 \\
100.33 \\
100.33 \\
100.31 \\
100.39 \\
100.35 \\
100.65 \\
100.65 \\
100.51 \\
100.65 \\
100.89 \\
100.93\end{array}$ & $\begin{array}{rr}\text { fuly } & 5 \\
12 \\
19 \\
26 \\
\text { Aug. } 2 \\
9 \\
16 \\
23 \\
30 \\
\text { sept. } 6 \\
13 \\
20 \\
27 \\
\end{array}$ & $\begin{array}{l}100.97 \\
100.81 \\
100.71 \\
100.56 \\
100.46 \\
100.39 \\
100.23 \\
100.18 \\
100.10 \\
100.10 \\
100.10 \\
100.21 \\
100.27\end{array}$ & $\begin{array}{l}\text { Oct. } \\
\text { Nor. }\end{array}$ & $\begin{array}{r}11 \\
11 \\
18 \\
25 \\
1 \\
8 \\
15 \\
22 \\
29 \\
6 \\
13 \\
20 \\
27\end{array}$ & $\begin{array}{l}100 \\
100 \\
100 \\
100 \\
100 \\
100 \\
100 \\
100 \\
100 \\
100 \\
100 \\
100 \\
100\end{array}$ \\
\hline
\end{tabular}

128. City of Dakes. NHzitz sec. 28, T. 131 N., R. 59 N. (Described in Water-supply Paper g08):

Water level, in feet eböre datum, 1941

\begin{tabular}{|c|c|c|c|c|c|c|c|c|c|c|}
\hline $\begin{array}{l}\text { Jan. } \\
\therefore \\
\text { Feb. }\end{array}$ & $\begin{array}{r}4 \\
11 \\
18 \\
25 \\
1 \\
8 \\
15 \\
22 \\
1 \\
8 \\
15 \\
22\end{array}$ & $\begin{array}{l}99 \\
99 \\
99 \\
99 \\
99 \\
99 \\
99 \\
99 \\
99 \\
99 \\
99 \\
99 \\
99\end{array}$ & $\begin{array}{c}\text { Apr. } \\
\text { May }\end{array}$ & $\begin{array}{r}5 \\
12 \\
19 \\
26 \\
3 \\
-8 \\
87 \\
24 \\
31 \\
7 \\
-14 \\
31\end{array}$ & $\begin{array}{l}100 . \\
100 \\
100 \\
100 \\
100 \\
100 \\
100 \\
100 \\
100 \\
100 \\
100 \\
100\end{array}$ & 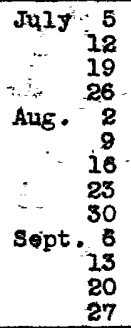 & $\begin{array}{l}10 \\
10 \\
10 \\
10 \\
10 \\
10 \\
10 \\
10 \\
10 \\
10 \\
10 \\
10\end{array}$ & Dev. & & \\
\hline
\end{tabular}


Dickey County--Continued.

129. A. M. Dahlbeck. SWt NWt sec. 21, T. $131 \mathrm{~N} .$, R. 59 W. (Described in Water-Supply Paper 908). Now unused. Influened by aditestic south. Water levelf, in foct below measuring point, 1941 . Yay, $14.16 ;$ oct. 16, 13.96

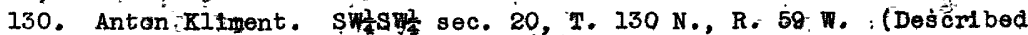
in Water-Supply Paper 908)...Measurements dis continued.

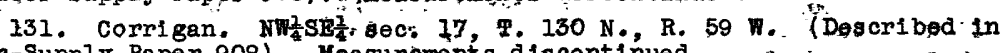
Water-Supply Paper 908). Measurements discontinued.

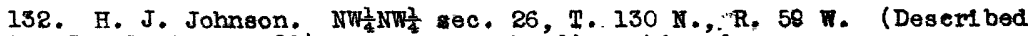
in Water-Supply-Paper 008)." Measurements discont Inued.

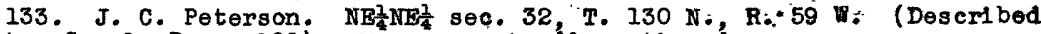
in Water-Supply Paper 908). Messurements discontinued.

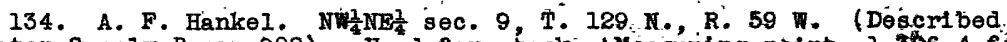
in Water-Supply Paper 908). Used for stock. "Heasuring point, 1,306.4 foet above sea level. Hater levels, in feet belo measuring point, 1941, May $\$$, 6.55 ; July 21,6.96; 0et. 16,8.75.

135 V. S. Dóyen. sthw sec. 24, T. 129 Kr. R. 60 W. (Described. in Water-Supply-Paper 908). Water level, in feet above datum, 1941

\begin{tabular}{|c|c|c|c|c|c|c|c|}
\hline Date & $\begin{array}{l}\text { Water } \\
\text { level }\end{array}$ & Date & $\begin{array}{l}\text { Water } \\
\text { level }\end{array}$ & Date & $\begin{array}{l}\text { Water } \\
\text { level }\end{array}$ & Date & $\begin{array}{l}\text { Water } \\
\text { level }\end{array}$ \\
\hline $\begin{array}{lr}\text { Jan. } & 4 \\
& 11 \\
\text { Feb. } & 1 \\
16 \\
\\
22 \\
\text { Mar. } \\
1 \\
\end{array}$ & $\begin{array}{l}99.35 \\
99.53 \\
99.35 \\
98.35 \\
99.52 \\
99.36 \\
99.34\end{array}$ & $\begin{array}{lr}\text { Har. } & 15 \\
22 \\
29 \\
\text { Apr. } 5 \\
12 \\
\\
26 \\
\text { May } 10\end{array}$ & $\begin{array}{l}99.35 \\
99.35 \\
99.36 \\
99.37 \\
98.89 \\
99.37 \\
99.39\end{array}$ & $\begin{array}{r}\text { Aug. } \\
21 \\
30 \\
\text { Sept. } 6 \\
13 \\
20 \\
\text { oct. } 4 \\
11\end{array}$ & $\begin{array}{l}99.23 \\
99.43 \\
99.36 \\
99.35 \\
99.36 \\
99.37 \\
99.37\end{array}$ & $\begin{array}{r}\text { Hov. } \\
8 \\
\\
15 \\
\text { Dec. } 6 \\
13 \\
20 \\
27 \\
\end{array}$ & $\begin{array}{l}99.37 \\
99.38 \\
99.38 \\
99.37 \\
99.37 \\
99.37 \\
99.37\end{array}$ \\
\hline
\end{tabular}

170. Axel Daniels. SW $\mathrm{SWE}_{\frac{1}{4}} \cdot \mathrm{sec} .34$, T. $129 \mathrm{~N} .$, R. $60 \mathrm{~W}$. .. (Described in Water-Supply Paper 908). No measurements made in 1941.

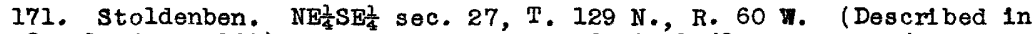
Water-Supply Paper 908). No measurements made in 1941.

\section{Divide County}

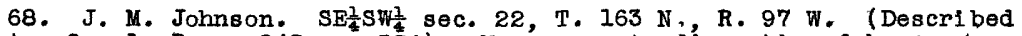
in Vater-supply Paper 845, p. 354).. Measurements discontinued because well went dry Nov. 1940. It was still dry on 0ct. 25, 1941.

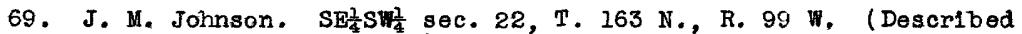
in Water-Supply Paper 845, p. 355). Weli found plugged at 15.9 feet on oct. 25, 1941 .

Water level, in feet below measuining point, 1941 .

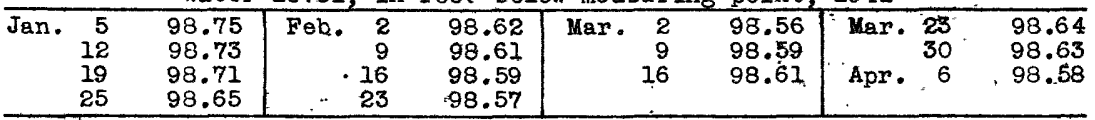

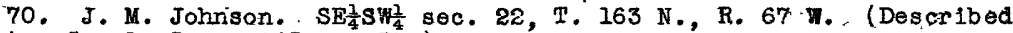
in Water-Supply Paper 845, p.: 355).

Water level, in feet below measuring point, 1941

\begin{tabular}{|c|c|c|c|c|c|c|c|c|c|c|}
\hline Jan. $\frac{1}{\frac{1}{2}}$ & $\begin{array}{r}5 \\
12 \\
19 \\
25\end{array}$ & $\begin{array}{l}97.75 \\
97.72 \\
97.69 \\
97.66\end{array}$ & Feb. & $\begin{array}{r}2 \\
9 \\
16 \\
23\end{array}$ & $\begin{array}{r}97.63 \\
97.56 \\
.97 .54 \\
997.52\end{array}$ & Mar. & $\begin{array}{r}2 \\
9 \\
16 \\
23\end{array}$ & $\begin{array}{r}97.49 \\
2115.49 \\
115.49 \\
115.49\end{array}$ & $\begin{array}{lr}\text { Mar: } & 30 \\
\text { Apr: } & 6 \\
\text { Oct. } 25\end{array}$ & $\begin{array}{r}115.49 \\
106.29 \\
97.85\end{array}$ \\
\hline
\end{tabular}

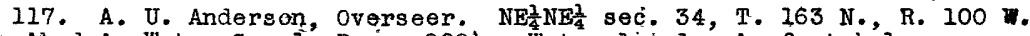
(Described in Water-Supply Paper 908). Water-levels, in feet below measurIng point, 1941: Apr. 20, 18.04; 0ct. 25, 18.08.

a Flooded by thaw water from slough. 
Durin County'

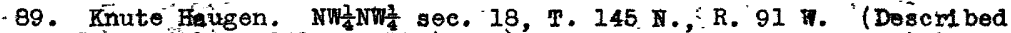
in Water-supply per 845, p. 355$)$ :

Water lovel, in foet above datum, 1941

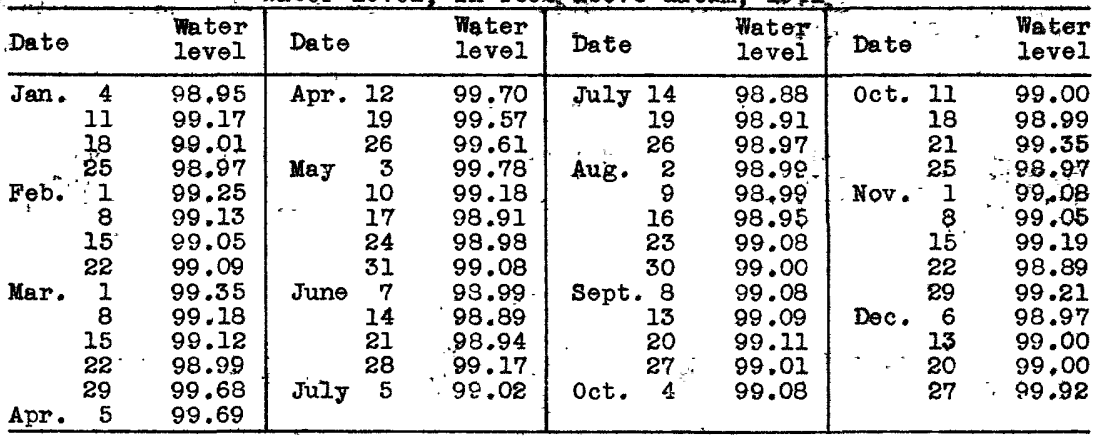

\section{Eddy County}

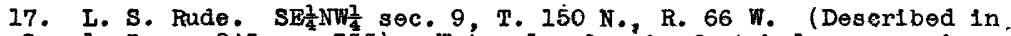
Water-Supply Paper 845, p. 355). Water lovels, in feot below messuring point, 1941: Apr. 4, 10.39; 0ct. 18, 9.14.

18. Stockyards. NW/ $S W_{4}^{\frac{2}{4}}$ sec. 9, T. $150 \mathrm{~N}$, R. 66 W. (Described in Water-Supply Paper 840, p. 323).

Water level, in foet above datum, 1941

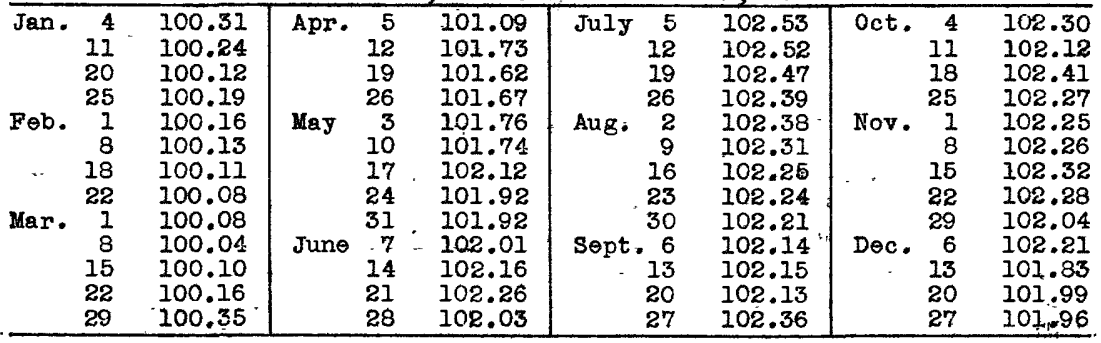

-19. Gilbert 01son. SE NW sec. 9, T. 150.N., R. 66 W. (Described in Tater-Supply Paper 845, p. 356). Water Jevels, in feet below measuring point, 1941: Apr. 4, 14.57; 0ct. 18, 14.57.

20. Fnute Egger. SE $\frac{2}{4} N W_{4}^{2}$ sec. 9, T, 150 N., R. 66 W. (Described in Water-Supply Peper 845, p. 356). Water levels, in feet below measuring point, 1941: Apr. 4,20.51; 0ct. 18, 18.95 .

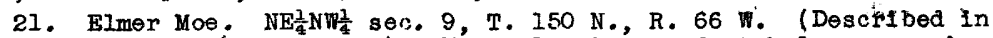
Water-Supply Paper. 845 , p. 356). Water levels, in feet below measuring point, 1947: Apr. 4, 22.13; 0ct. 18, 20.62.

22. John R. Warsing. SRl NWt sec. 9, T. 150 N., R. 66 W. (Described in Water-Supply Paper 845, p. 356). Water levels, in feet below measuring point, 1941: Apr. 4, a / below 16.6; 0ct. 18, 14.91.

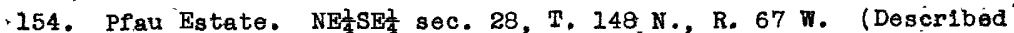
in Water-Supply Paper 908). Unreduced flow, in galions: a minute, 1941: Apr. $4,5.9 ;$ oct. $18,7.8$.

a Well partly plugged.

$6110050-43-14$ 


\section{Bemons County}

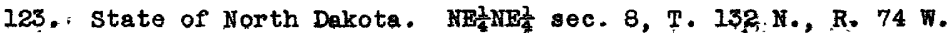
Abandoned dug farm well, dianeter 36 inches, depth 20 foets Heasuring :. point, painted arrow on rood platform, 1.3 feet above land surface. Water levels, in feet bolow measuring polit: JulJ.1, 1940, 14.44; Nov. 23, 15.69; Apr: 11, 1941, 15.58; Oct. 17, 1941, 14.36.

\section{Foster count y}

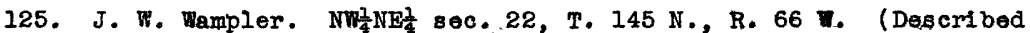
in Mater-Supply Paper 908). On Oct. 19, 1941, well found with platform destroyed, curbing stove in, measuring point destroyed. Water levels, in feet below measuring point, 1941: Apr. 4, 15.71; Oct. 19, about 15.3.

\section{Golden Valiey County}

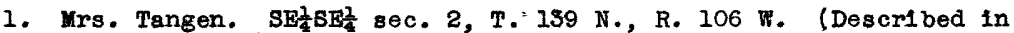
Water-Supply Paper 908). Water levels, in feet below measuring point, 1941: Apr. 17, 31.37; 0ct. 23, between 71 ind 72 feet.

\section{Grant County}

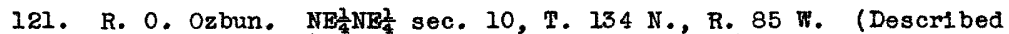
in Water-Supply Paper 908). Water levels, in feet below measuring point, 1941: Apr: 16, 23.57; 0ct. 22, 23.40.

\section{Griggs county}

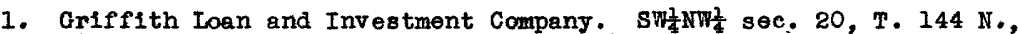
R. 59 W. (Described in Water-Supply Paper 908). Water levels, in feet below measuring point, 1941: Apr. 6, 27.95; 0ct. 14, 26.23 .

\section{Hettinger County}

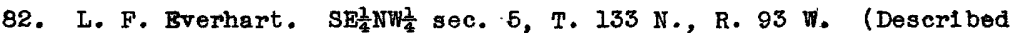
in Water-Supply Paper 845, p. 357 ).

Water level, in feet above datum, 1941

\begin{tabular}{|c|c|c|c|c|c|c|c|c|}
\hline Date & & $\begin{array}{l}\text { Water } \\
\text { level }\end{array}$ & Date & $\begin{array}{l}\text { Water } \\
\text { level }\end{array}$ & Date & $\begin{array}{l}\text { Water } \\
\text { level }\end{array}$ & Dat $\theta_{i}$ & $\begin{array}{l}\text { Water } \\
\text { leve1 }\end{array}$ \\
\hline $\begin{array}{r}\text { Jan. } \\
1 \\
1\end{array}$ & $\begin{array}{l}4 \\
11 \\
18\end{array}$ & $\begin{array}{l}99.73 \\
99.73 \\
99.73\end{array}$ & 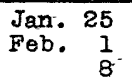 & $\begin{array}{l}99.74 \\
99.73 \\
99.72\end{array}$ & $\begin{array}{rr}\text { Feb. } 15 \\
22 \\
\text { Mar. } 1\end{array}$ & $\begin{array}{l}99.70 \\
99.70 \\
99.71\end{array}$ & $\begin{array}{l}\text { Mar. } 8 \\
\text { Apr: } 16 \\
\text { Oct: } 22\end{array}$ & $\begin{array}{r}99.72 \\
99.97 \\
100.22\end{array}$ \\
\hline
\end{tabular}

\section{KIdder county}

50. Herman Petersen. Sw $\mathrm{SWR}_{\frac{2}{4}}^{\frac{2}{3}}$ sec. 9, T. $138 \mathrm{~N}, \mathrm{R}, 73 \mathrm{~W}$. (Described in Water-\$upply Paper 840, p. 323).

Water lovel, in feet above datum, 1941

\begin{tabular}{|c|c|c|c|c|c|c|c|c|c|c|}
\hline $\begin{array}{l}\text { Apr. } \\
\text { May }\end{array}$ & $\begin{array}{r}10 \\
12 \\
19 \\
26 \\
3 \\
10 \\
17 \\
24 \\
31 \\
7\end{array}$ & $\begin{array}{r}99.96 \\
99.96 \\
99.95 \\
100.06 \\
99.92 \\
99.98 \\
99.99 \\
99.98 \\
100.21 \\
100.29\end{array}$ & June & $\begin{array}{r}14 \\
21 \\
28 \\
5 \\
12 \\
19 \\
26 \\
2 \\
9 \\
16\end{array}$ & $\begin{array}{l}10 \\
10 \\
10 \\
10 \\
10 \\
10 \\
10 \\
10\end{array}$ & $\begin{array}{l}\text { Aug. } \\
\text { Sept. } \\
\text { Oct. }\end{array}$ & & $\begin{array}{l}\text { Oet. } \\
\text { Nov. } \\
\text { Dec. }\end{array}$ & $\begin{array}{r}25 \\
1 \\
10 \\
19 \\
22 \\
29 \\
8 \\
13 \\
20 \\
27\end{array}$ & \\
\hline
\end{tabular}


Kidder County--Continued

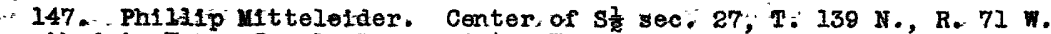
(Deseribed In Waterisupply Paper 908). Water levels, in feet below measurIng point, 1941: Apr. 10, 9.33; 0ct. 18, 5.35.

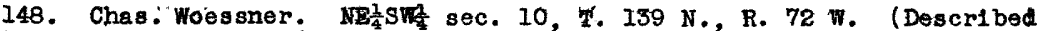
in water-sapply Paper 908). Water levels, in feet below measuring point, 1941: Apr. 10, 18.19; Oct. 19, 16.22.

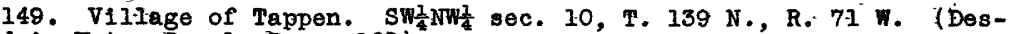
cribed in Water-supply Paper 908).

Fater level, in feet above datum, 1941

\begin{tabular}{|c|c|c|c|c|c|c|c|}
\hline Date & $\begin{array}{l}\text { Weter } \\
\text { level }\end{array}$ & Dete & $\begin{array}{l}\text { Water } \\
\text { level }\end{array}$ & Date & $\begin{array}{l}\text { Water } \\
\text { level }\end{array}$ & Date & $\begin{array}{l}\text { Water } \\
\text { lovel }\end{array}$ \\
\hline 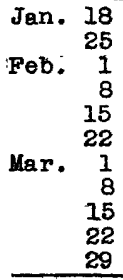 & $\begin{array}{l}99 \cdot 18 \\
99.21 \\
99.11 \\
99.12 \\
99.18 \\
99.18 \\
99.18 \\
99.14 \\
99.30 \\
99.35 \\
99.47 \\
\end{array}$ & $\begin{array}{lr}\text { Apr. } & 5 \\
12 \\
\\
19 \\
\\
26 \\
\text { May } 3 \\
\\
10 \\
17 \\
\\
24 \\
\\
\text { June } \\
31 \\
7 \\
14\end{array}$ & $\begin{array}{r}99.46 \\
99.55 \\
99.59 \\
99.66 \\
99.71 \\
99.77 \\
99.83 \\
99.77 \\
99.81 \\
100.11 \\
100.19\end{array}$ & $\begin{array}{r}\text { July. } 5 \\
12 \\
19 \\
26 \\
\text { Aug. } 2 \\
9 \\
16 \\
23 \\
30 \\
6 \\
\text { Sept. } 6 \\
27\end{array}$ & $\begin{array}{l}100.59 \\
100.84 \\
100.75 \\
100.59 \\
100.67 \\
100.59 \\
100.55 \\
100.59 \\
100.42 \\
100.59 \\
100.77\end{array}$ & 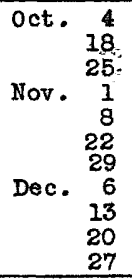 & $\begin{array}{l}100.77 \\
100.75 \\
100.75 \\
100.75 \\
100.84 \\
100.67 \\
100.60 \\
100.68 \\
100.67 \\
100.58 \\
100.62 \\
\end{array}$ \\
\hline
\end{tabular}

150. Ramon Grimm. SW $1 W_{4}^{\frac{1}{4}}$ sec. 14, T. 142 N., R. 70 N. (Desaribed in Water-supply Paper 908). Whter leveis, in feet below meesuring point, 1941: Apr:9, 39.31; Oct. $19,38.62$.

151. Mrs. Fagereng. NWt NB $\frac{1}{4}$ sec. 23, T. 142 N., R. 70 m. (Described in Water-Supply Paper 908): Water levels, in feet, below moasuring point, 1941:...Apr. 9, 20.62; 0ct. 19, 20.84.

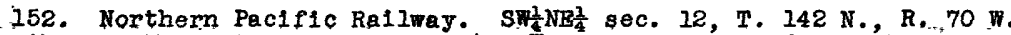
(Described in Water-Supply Paper 908): Water levels, in feet below measuring point, 1941: Apr. 9, 37.35; 0ct. 19, 37.23.

166. Jake Schaurer (formerly Chr1s Werre). Swsitit sec, 3, T. 139 N. R. 71 W: (Described In Water-Supply Paper 908). Water levels, in feet below measuring point, 1941: Apr.9, 17.80; Oct: 19, 16.75 .

\section{La Moure County}

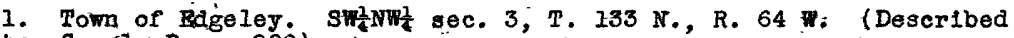
in Water-Supply Paper 908).

Water level, in feet above datum, 1941

\begin{tabular}{|c|c|c|c|c|c|c|c|c|c|}
\hline $\begin{array}{l}\text { Jan. } \\
\text { Eeb. }\end{array}$ & $\begin{array}{r}4 \\
11 \\
1 \\
8 \\
15 \\
22 \\
8 \\
22 \\
29\end{array}$ & $\begin{array}{r}99.83 \\
99.09 \\
100.21 \\
100.12 \\
100.19 \\
98.51 \\
99.03 \\
100.12 \\
99.10\end{array}$ & $\begin{array}{l}\text { Apr. } \\
\text { May } \\
\text { June }\end{array}$ & $\begin{array}{r}5 \\
19 \\
26 \\
3 \\
10 \\
17 \\
24 \\
7 \\
14\end{array}$ & $\begin{array}{r}99.39 \\
100.12 \\
99.79 \\
100.15 \\
100.46 \\
99.23 \\
98.48 \\
99.53 \\
99.01\end{array}$ & $\begin{array}{rr}\text { June } & 21 \\
\text { July } & 5 \\
19 \\
26 \\
\text { Aug. } 2 \\
99 \\
16 \\
30 \\
\text { Sept. } 20\end{array}$ & $\begin{array}{r}99.17 \\
100.52 \\
100.60 \\
98.19 \\
98.76 \\
98.74 \\
98.93 \\
98.91 \\
100.85\end{array}$ & 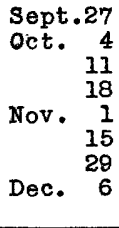 & $\begin{array}{r}101.95 \\
101.96 \\
102.00 \\
102.00 \\
99.63 \\
99.75 \\
99.59 \\
100.91\end{array}$ \\
\hline
\end{tabular}

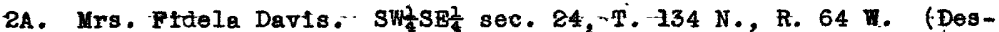
cribed in Water-Supply Paper 886, p. 538).

Water level, in feet above datum, 1941

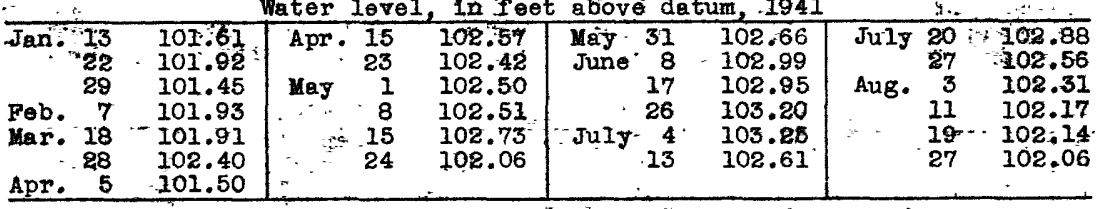




\section{Iogan County}

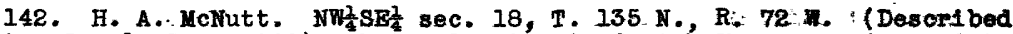
in Water-supply Paper 908). Water levels, in feet below, wesauring point, 1941: Apr. 10, 6.56; 0ct. 17, 6.70.

143. 0scar France, whsth sec. 17, T. 135 N., R. 72 M.c. (Described in Water-supply Paper 908). Water leveis, in foet below measuring polnt, 1941: Apr. 10, 13.75; Oct. 17, 13.77.

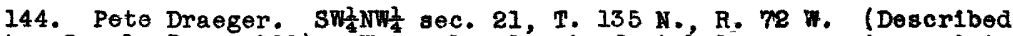
in Water-Supply Paper 908). Water levels, in feet below measuring point, 1941: Apr. 10, 11.18; Oct. 17, 11.33.

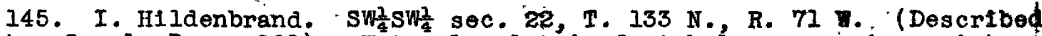
in Water-Supply Paper 908). Water. levels; in feet below measuring point,.: 1941: Apr. 10,9.17; Oct. 17, 9.77.

146. George Dummland. NW $\mathrm{NW}_{4}^{\frac{7}{4}}$ sec. 27, T. $135 \mathrm{~N}$, R. 72 F. (Describod in Water-Supply Paper 908). Water lovels, in feet below measuring point, 1941: Apr. 10, 32.63; Dct. 17, 32.33.

\section{McHenry County}

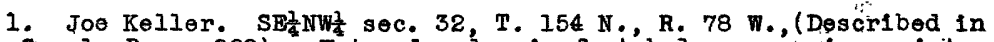
Water-Supply Paper 908). Water levels, in feet below measuring point, 1941: Apr. 22, 27.43; Oct. 28, 27.17.

101. Denbigh Forest Experiment Station weli l. United States Forest

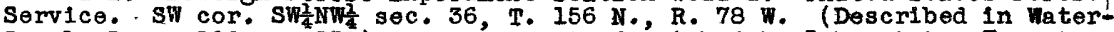
Supply Paper 886, p. 539). Neasurements fumished by Lake statee Foreat Experiment Station, United States Forest Service, University Farm, St. Raul, Ninn.

Water level, in feet above detum, 1940-41

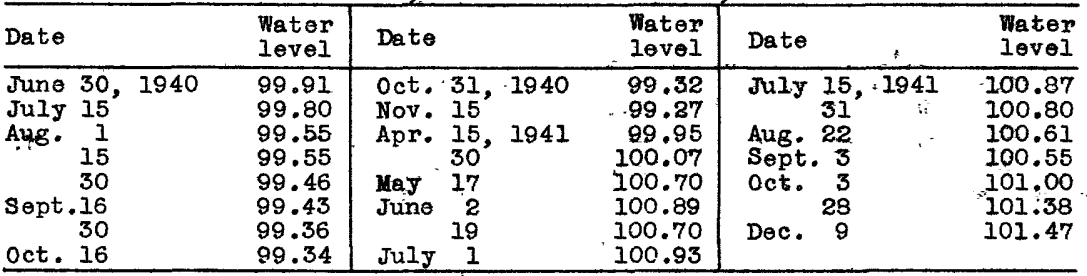

102. Denbigh Forest Experiment Station well 2. United states Forest Service. NW cor. SW $\mathrm{NWW}_{\frac{1}{4}}$ sec. 36, T. $156 \mathrm{~N}, \mathrm{R}, 78$ T. (Described in WaterSupply Paper 886, p. 540). Messurements furmished by Laks States Forest Experiment Station, United States Forest Sorvice, University Farm, st. Pau1, Minn.

Water level, in feet above datum, 1940-41

\begin{tabular}{|c|c|c|c|c|c|c|c|c|c|}
\hline $\begin{array}{r}\text { June } 30 \\
\text { July } 15 \\
\text { Aug. } 1 \\
15 \\
30 \\
\text { sept. } 16 \\
30 \\
\text { oct. } 16\end{array}$ & 1940 & $\begin{array}{r}100.07 \\
99.94 \\
99.66 \\
99.61 \\
99.56 \\
99.53 \\
99.43 \\
99.41\end{array}$ & $\begin{array}{l}\text { Oct. } \\
\text { Nov. } \\
\text { Apr. } \\
\text { May } \\
\text { June } \\
\text { Juily }\end{array}$ & $\begin{array}{r}31, \\
15 \\
15, \\
30 \\
17 \\
2 \\
19 \\
1\end{array}$ & $\begin{array}{l}1940 \\
1941\end{array}$ & $\begin{array}{r}99.37 \\
99.30 \\
99.84 \\
99.98 \\
100.92 \\
101.13 \\
100.96 \\
101.13\end{array}$ & $\begin{array}{rr}\text { July } 15, & 22 \\
& \\
\text { Aug. } 22 \\
\text { Sept. } 3 \\
\text { Oct. } 3 \\
\text { Dec. } 28 \\
\text { Dec. } 9\end{array}$ & 1941 & $\begin{array}{l}101.06 \\
100.95 \\
100.77 \\
100.72 \\
101.28 \\
101.63 \\
101.74\end{array}$ \\
\hline
\end{tabular}

103. Denbigh Forest Experiment station well 3. United stätes Forest Service. NE cor. SW $\frac{2}{4} N W \frac{2}{4}$ sec. 36, T. $156 \mathrm{~N}, \mathrm{R} .78 \mathrm{~W}$. (Described in WaterSupply :Paper 886, p. 54I). Measurements furnished by Lake states Forést. Experiment station, Uni ted states Forest Service, Unlversity. Farm, St: Paul, Minn:

\begin{tabular}{|c|c|c|c|c|}
\hline $\begin{array}{l}\text { June. } 30,1940 \\
\text { July } 15 \\
\text { Aug. } 1\end{array}$ & $\begin{array}{l}99.98 \\
99.87 \\
99.58\end{array}$ & $\begin{array}{l}\text { Aug. } 15,1940 \\
30 \\
\text { Sept. } 16\end{array}$ & $\begin{array}{l}99.54 \\
99.49 \\
99.46\end{array}$ & $\begin{array}{l}\text { Sept.30, } 1940 \\
\text { oct. } 16 \\
\quad \quad 31\end{array}$ \\
\hline
\end{tabular}


McHenry County--Continued

103. Denbigh Forest Experiment Station we11 3,--Contimad. Water level, In feet above datum, 1940-41

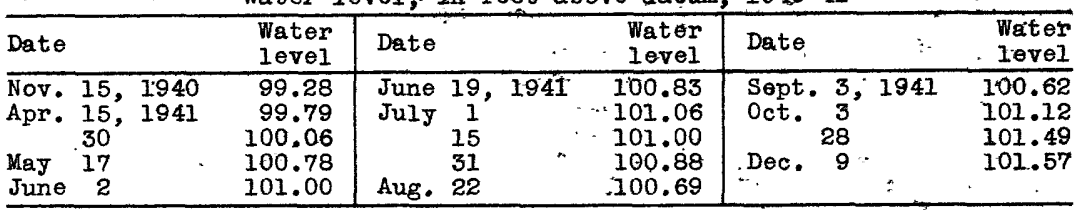

104. Denbigh Forest Bxperiment Station well 4. United States Forest Service. SE cor. SW $\frac{1}{4} \mathrm{NW}_{\frac{1}{4}}$ sec. 36, T. $156 \mathrm{~N}, \mathrm{~N}^{*} \mathrm{R}$. $78 \mathrm{H}$. (Described in WaterSupply Paper 886, p. 542). Measurements furnished by Lake States Forest Experiment Station, United States Forest Service, University Farm; St. Paul, Minn.

Water level, in feet-above datum, 1940-41

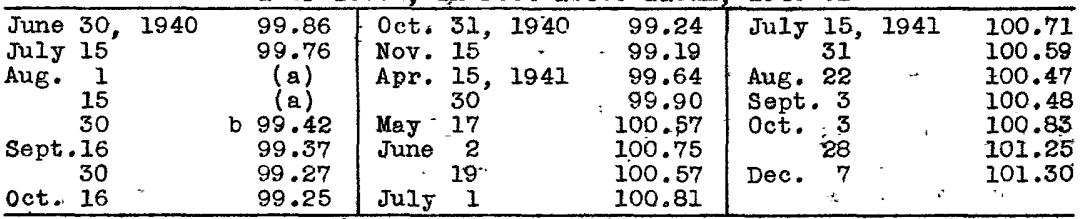

105. Denbigh Forest Experiment Station well 5. "United States Forest

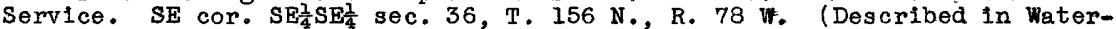
Supply Paper 886, p. 543). Measurements furnished by Lake states Forest Experiment Station, United States Forest Sorvice, University Farm, St. Paul, Minn.

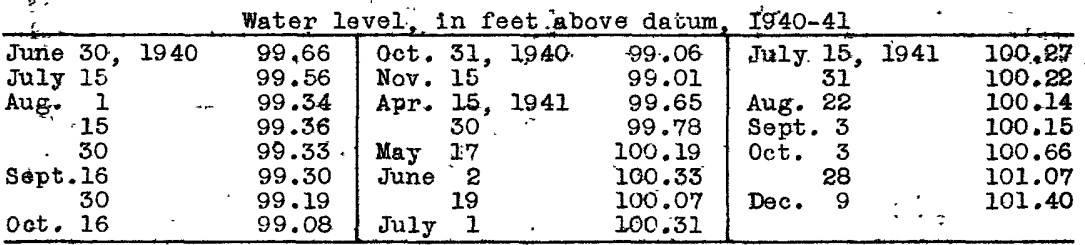

106. Denbigh Forest Bxperiment Station well point 1. United States Forest Service. SW cor. NW $\frac{1}{4} \mathrm{SW}_{\frac{2}{4}} \mathrm{sec} .36$, T. $156 \mathrm{~N}$, , R. 78, W. (Described in Tater-Supply Paper 886, p. 544).

Water level, in feet above datum, 1940-41

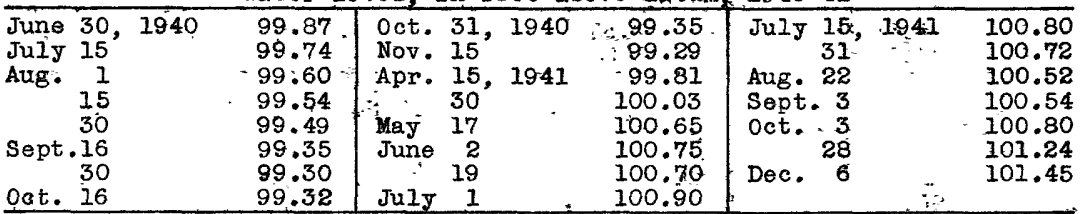

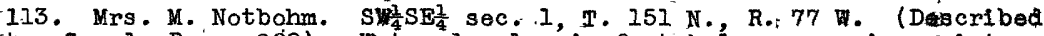
in. Wáter-Supply Paper 908). Water levels, in feet below measurlng point, 1941: Apr. 22, 15.72; Oct. 28, 14.62.

156. Minneapolis, St. Paul, and Sault Sainte Marie Ra1lway. SW $\mathrm{NW}_{4}^{\frac{1}{4}}$ sec. 6, T. 152 N., R. 79 W. (Described In Water-Supply Paper 908). Erronequsly located in SW sec. 6 in Water-Supply Paper 908. Water levels, in feet below measuring point, 1941: Agx. 22, 21.27; May 10, 20.3; 0ct. 28, 22.42.

a Dry. $\quad$ b , Well deepened. 


\section{McHenry County--Continued.}

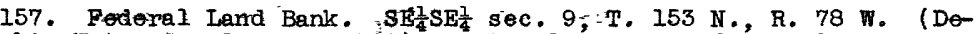
scribed in Water-Supply Paper.908). Water levels, in feet bolow measuring point; 1941: Apr. 22, 25.40; Oct. 28, 25.39:

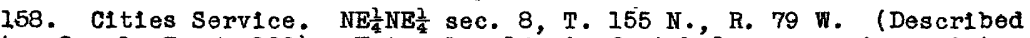
in Water-Supply Paper 908 ). Water levels, in feet below measuring point, 1941: Apr. 23, 4.54; 0ct. 28, 3.29.

159. Harold H. Sullwold. NW $\frac{1}{4} \mathrm{SE} \frac{1}{4}$ sec. 33, T. $156 \mathrm{~N}$, R. 79 W. (Described in Water-Supply. Paper 908). Water levels, in feet"below measuring point, 1941: Apr. 23, 13.83; 0ct. 28, 11.79.

160. United States Forest Service. SEl SW sec. 31, T. 157 N., R. 75 W. (Described in Water-Supply Paper 903).

Water level, in feet below measuring point, 1941

\begin{tabular}{|c|c|c|c|c|c|c|c|}
\hline Dete & $\begin{array}{l}\text { Water } \\
\text { level }\end{array}$ & Date & $\begin{array}{l}\text { Water } \\
\text { level }\end{array}$ & Date & $\begin{array}{l}\text { Water } \\
\text { level }\end{array}$ & Date & $\begin{array}{l}\text { Water } \\
\text { level }\end{array}$ \\
\hline $\begin{array}{lr}\text { Feb. } & 12 \\
& 21 \\
\text { Mar. } & 3\end{array}$ & $\begin{array}{l}7.62 \\
7.62 \\
7.59\end{array}$ & $\begin{array}{ll}\text { Mar. } 21 \\
28 \\
\text { Apr. } 11\end{array}$ & $\begin{array}{l}7.61 \\
7.56 \\
7.29\end{array}$ & $\begin{array}{l}\text { Apr. } 23 \\
\text { Oct. } 29 \\
\text { Dec. } 2\end{array}$ & $\begin{array}{l}7.26 \\
6.68 \\
6.46\end{array}$ & $\begin{array}{r}11 \\
23 \\
30 \\
\end{array}$ & $\begin{array}{l}6.46 \\
6.42 \\
6.50 \\
\end{array}$ \\
\hline
\end{tabular}

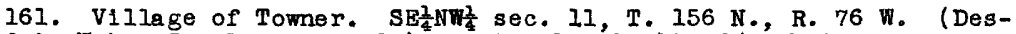
cribed in Water-Supply Paper 908). Water levels, in feet below measuring point, 1941: Apr. 23, 12.99; 0ct. 28, 12.17.

162. Walter Arneson. Sw $\frac{1}{4} \mathrm{SB}_{\frac{1}{4}}$ sec. 3, T. $158 \mathrm{~N} .$, R. $78 \mathrm{~W}$. (Described. in Water-supply Paper 908). Water level, in feet below measuring point, 1941: Qct. 28, $28.76^{2}$.

\section{MeIntosh County}

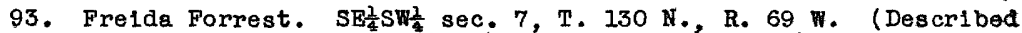
in Water-Supply Paper 845, p. 357). Water levels to December not reliabio because of changes-in weli platform causing unstable measuring point. if

Water level, in feet above datum, 1941

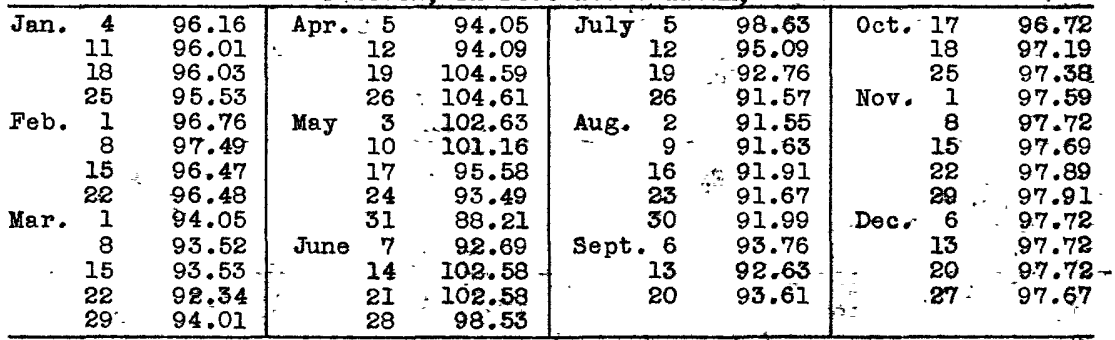

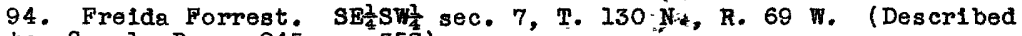
in Water-Supply Paper 845, p. 358 ).

Weter lever, in feet above datum, 1941

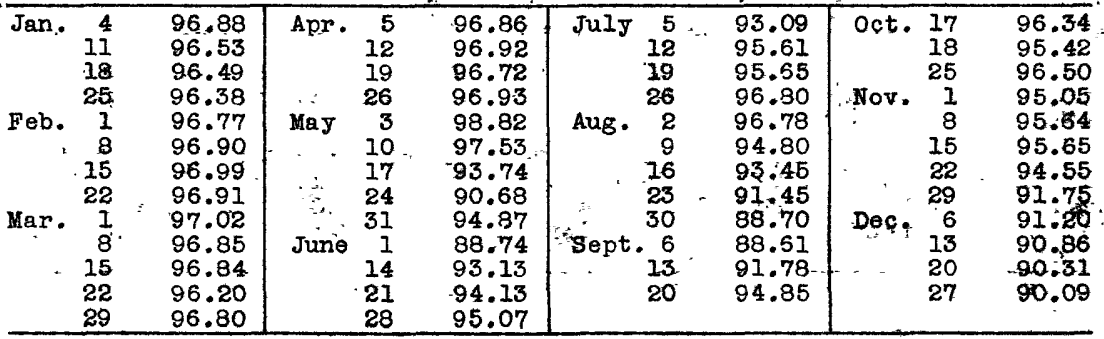




\section{McIntosh County--Continued}

136. State of North Dakota. SFlww/s sec. 36, T. 132 H., R. 7I M. (Described in Water-Supply Paper 908 ). Water levéri, in' feet belor méasurt Ing point, 1941: Apr. 11; 5.21;-0et. 17, 6.35.

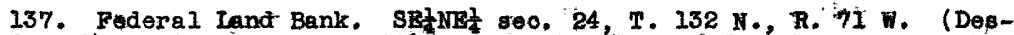
cribea in Water-Supply Paper 908). Water levells, in feet belor wasuring paint, 1941: Apr. 11, 11.48; -Oat. 17, 11.42.

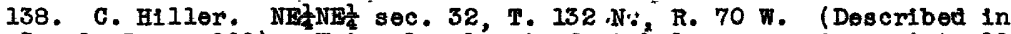
Water-Supply Paper 908). Water levels, in feet below measuring point, 1947: Apr. 11, 19.02; 0ct. 11, a/20.59.

139. Dan Nigisch. NEZSPE sec. 28, T. 132 N., R. 71 T. (Described in Water-supply Paper 908) Water levels, in feet below measuring point, 1941: Apr. 11, 18:33; Oct. 17; 18.46.

140. Jacob Groshanz. NWT/4 sec. 29, T. 131 W. R. 70 W. (Described in Water-Supply Paper 908). Well caved in, summer 1941. Water level, in feet below measuring point, 1941: Apr. 11, 26.79.

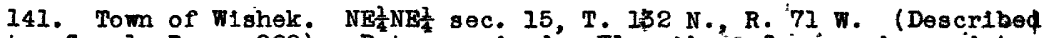
(in Water-Supply Paper 908). Datum revised. Blevation of mésuring point. 121.54 feet above datum. All levels appearing in Water-Supply Paper 908 should be reduced by 0.95 inot. Previous datum was computed on basis of first measurement, which proved to be abnormally low, probably as an effect of local drawdown.

Water level, in feet above datum, 1941

\begin{tabular}{|c|c|c|c|c|c|c|c|}
\hline Date & $\begin{array}{l}\text { Water } \\
\text { level }\end{array}$ & Date & $\begin{array}{l}\text { Water } \\
\text { level }\end{array}$ & Dete & $\begin{array}{l}\text { Water } \\
\text { level }\end{array}$ & Date & $\begin{array}{l}\text { Water } \\
\text { level }\end{array}$ \\
\hline $\begin{array}{rr}J a n . & 4 \\
11 \\
18 \\
25 \\
\text { Feb. } 1 \\
7 \\
22 \\
\text { Mar. } 1 \\
8 \\
15 \\
22 \\
29\end{array}$ & $\begin{array}{l}99.33 \\
99.15 \\
99.15 \\
99.15 \\
99.15 \\
99.24 \\
99.25 \\
99.25 \\
99.25 \\
99.24 \\
99.33 \\
99.46\end{array}$ & $\begin{array}{lr}\text { Apr. } & 5 \\
& 12 \\
& 19 \\
& 26 \\
\text { Hay } & 3 \\
10 \\
\\
17 \\
& 24 \\
& 31 \\
\text { June } & 7 \\
& 14 \\
& 21\end{array}$ & $\begin{array}{l}99.48 \\
99.51 \\
99.48 \\
99.51 \\
99.52 \\
99.48 \\
99.52 \\
99.53 \\
99.53 \\
99.38 \\
99.54 \\
99.55\end{array}$ & $\begin{array}{rr}\text { Jun } & 28 \\
\text { July } & 5 \\
12 \\
19 \\
\\
26 \\
\text { Aug. } 2 \\
8 \\
23 \\
30 \\
\text { Sept. } 13 \\
20 \\
27\end{array}$ & $\begin{array}{l}99.63 \\
99.65 \\
99.71 \\
99.71 \\
99.54 \\
99.62 \\
99.68 \\
99.60 \\
99.59 \\
99.62 \\
99.66 \\
99.60\end{array}$ & $\begin{array}{lr}\text { Oct. } & 18 \\
25 \\
\text { Nov. } \\
1 \\
8 \\
15 \\
22 \\
29 \\
\text { Deo. } \\
6 \\
13 \\
20 \\
27 \\
27\end{array}$ & $\begin{array}{l}99.60 \\
99.57 \\
99.58 \\
99.57 \\
99.58 \\
99.58 \\
99.65 \\
99.42 \\
99.56 \\
89.58 \\
99.57\end{array}$ \\
\hline
\end{tabular}

\section{McKenzie County}

81. Chas.E. Fleck. SW SW $_{4}^{\frac{7}{4}}$ sec. 12, T. 150 N., R. 100 W. (Descrlbed in Water-Supply Paper 845, p. 358).

Water level, in feet above datum, 1941

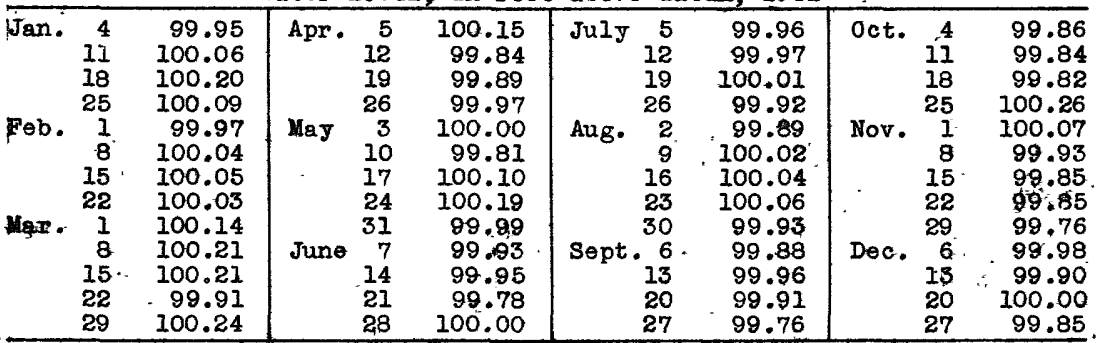

119. Federal Land Bank. NEt⿱s NWt sec. 8, T. $145 \mathrm{~N}$, , R. 98 W. (Described in Water-Supply Paper 908). Water levels, in feet below measuring point, 1941: Apr. 17, 97.46; Oct. 23, 97.62.

a After 40 minutes recovery from pumping, level still rising. 


\section{McLas county}

27. State of North Dakota. SW $w_{4}^{\frac{1}{4}} N W_{4}^{\frac{1}{4}}$ sec. 15, T. 149 N.,. R. 84 v. Described in Water-Supply Paper 840, p. 323).

Wator level, in feet above datum, 1941

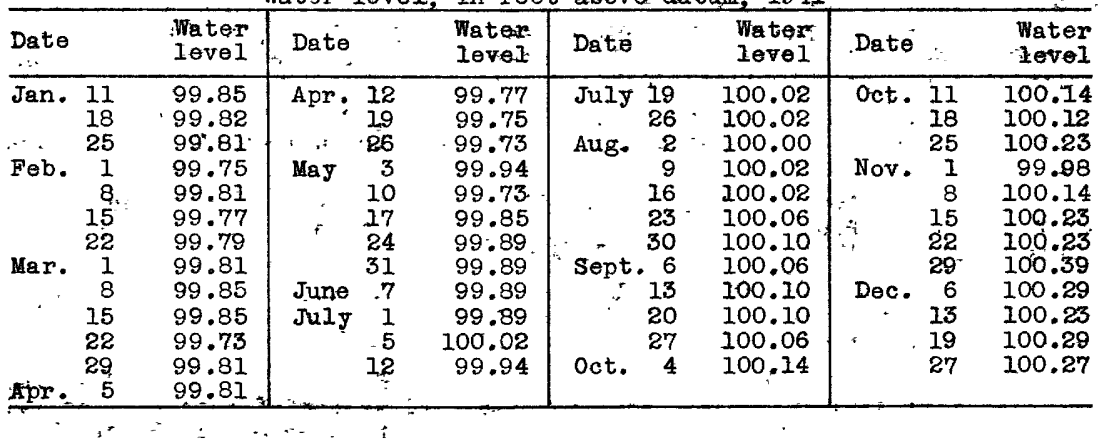

\section{Mercer County}

118. Malchel Bros. SER $\mathrm{NE}_{4}^{\frac{1}{4}}$ sec. 22, T. $144 \mathrm{~N}$, , R. 85 \%. (Described in Water-Supply Paper 908). Water level, in feet below measuring point, 1941: Apr. 13, 27.62; Oct. 18, 27.30.

\section{Morton County}

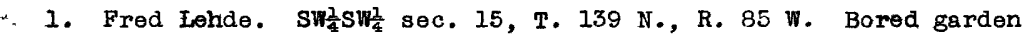
well, dlameter 24 to 16 inches, depth 72.3 feet. Measuring point, north point of wood curbing, 0.4 feet above land surface, and 137.46 feet above datum. Bench mark no. I, nail and washer in the northwest corner of barm 1.4 feet above land surface and 143.95 feet above datum, 130 feet eastnor theast of well. Bench mark no. 2, nall and washer in the southwest side of light pole, 160 feet northeast of well, 1.2 feet above land surface, and 143.94 feet above detum. Water level, Apr. 14, 1941, 37.36 feet below measuring point. Observer, Herbert Lehde.

Water lovel, in feet above datum, 1941

\begin{tabular}{|c|c|c|c|c|c|c|c|c|c|c|}
\hline Apr. & $\begin{array}{r}14 \\
19 \\
26 \\
3 \\
10 . \\
17 \\
24 \\
31 \\
7 \\
14\end{array}$ & $\begin{array}{r}100.10 \\
100.05 \\
99.89 \\
100.02 \\
99.97 \\
99.94 \\
99.79 \\
99.77 \\
101.42 \\
100.50\end{array}$ & $\begin{array}{l}\text { June } \\
\text { July }\end{array}$ & $\begin{array}{r}21 \\
28 \\
5 \\
12 \\
19 \\
26 \\
-2 \\
9 \\
16 \\
23\end{array}$ & $\begin{array}{l}106.17 \\
106.42 \\
106.42 \\
106.08 \\
106.15 \\
106.02 \\
105.93 \\
106.38 \\
105.75 \\
105.72\end{array}$ & $\begin{array}{rr}\text { Aug. } & 30 \\
\text { Sept. } & 6 \\
13 \\
20 \\
20 \\
29 \\
\text { oct. } 4 \\
11 \\
19 \\
21 \\
25 \\
21\end{array}$ & $\begin{array}{l}105.65 \\
105.58 \\
105.50 \\
105.63 \\
105.67 \\
105.58 \\
105.53 \\
105.51 \\
105.52 \\
105.54\end{array}$ & Nov. & $\begin{array}{r}1 \\
8 \\
15 \\
22 \\
1 \\
6 \\
13 \\
20 \\
27\end{array}$ & $\begin{array}{l}105.27 \\
105.19 \\
105.17 \\
105.09 \\
105.05 \\
105.01 \\
104.96 \\
104.89 \\
104.80\end{array}$ \\
\hline
\end{tabular}

2. Henry Polenberg. NW $\frac{2}{4} \mathrm{NE}_{4}^{\frac{2}{4}}$ sec. $32, \mathrm{~T} .139 \mathrm{~N} ., \mathrm{R} .88$ w. Drilled domestic and stock well, diameter 6 inches, depth 59.5 feet. Measuring point, top of casing in pit, 6.2 feet below land surface. Nater levels, In feet below measuring point, 1941: Apr. 14, 3.18; Oct. 21, 2.98 .

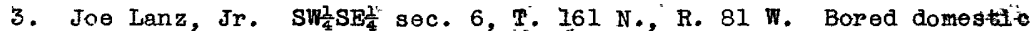
wel1, diameter 24 inches, depth 66.5 feet. Measuring point, top of wood curb, south side, 0.5 foot above land surfece. Water levels, in feet below measuring point, 1941: Apr. 15, 25.73; 0ct. 22, 25.13.

4. Albrecht and Johnson. NW $S W_{\frac{1}{4}}$ sec. 36, T. $134 \mathrm{~N} ., \mathrm{R} .82-\mathrm{W}$. Bored domestic well, diameter 16 inches, depth 20.5 feet. Measuring point, top of curb, east side, 0.7 foot above land surface. Water levels, in feet below measuring point, 1941: Apr. 15, 18.04; Oct. 22, 17.57." 


\section{Morton County--Continued}

49. U. S. Department of Agriculture, Soll Conservation Service. NW $\frac{1}{4}$ NW $\frac{1}{4}$ sec. 4, T. $138 \mathrm{~N}, \mathrm{R}$. 81 W. (Described in Water-Supply Papers 840 , p. 324 , and $\left.845, p_{0}^{-3} 359\right)$ ?

Water level, in feet above datum, 1941

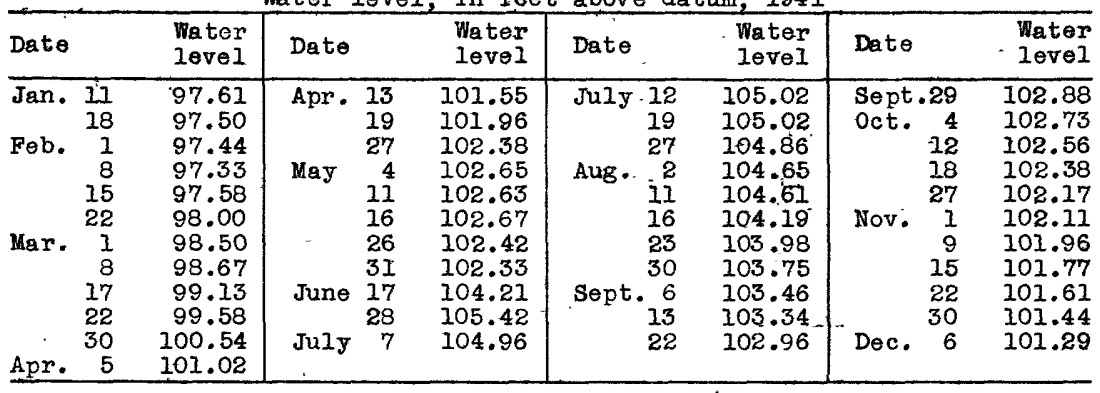

\section{Mountrail County}

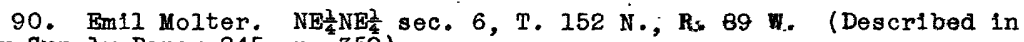
Vater-Supply Paper 845, p. 359).

Water level, in feet above datum, 1941

\begin{tabular}{|c|c|c|c|c|c|c|c|c|c|c|}
\hline $\operatorname{Jan}$ & $\begin{array}{r}4 \\
11 \\
19 \\
25 \\
1\end{array}$ & $\begin{array}{l}99.67 \\
99.67 \\
99.66 \\
99.66 \\
99.65\end{array}$ & $\begin{array}{l}\text { Feb. } \\
\text { Mar. }\end{array}$ & $\begin{array}{r}8 \\
15 \\
22 \\
7 \\
8\end{array}$ & $\begin{array}{l}99.65 \\
99.65 \\
99.62 \\
99.62 \\
99.63\end{array}$ & $\begin{array}{l}\text { Mar. } \\
\text { Apr. } \\
\text { May. }\end{array}$ & $\begin{array}{r}15 \\
22 \\
29 \\
2 \\
2\end{array}$ & $\begin{array}{l}99.62 \\
99.61 \\
89.61 \\
99.60 \\
99.59\end{array}$ & $\begin{array}{l}\text { July } 13 \\
\text { Sept. } 9 \\
\text { Oct. } 26 \\
\text { Dec. } 20\end{array}$ & $\begin{array}{r}99.70 \\
99.98 \\
100.11 \\
200.24\end{array}$ \\
\hline
\end{tabular}

\section{Nelson Count $y$}

47. Tom Miller (formerly State of North Dakota.) (Dessribed in Water

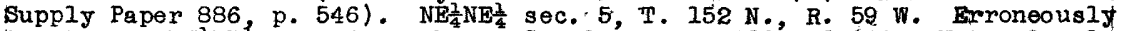
located in NW $\frac{1}{4}$ NW $\frac{1}{4}$ sec. 4, in Water-Supply Papers 886 and 908 . Water levels, in feet below measuring point, 1941: Apr. 4, 19.63 ; Oct. 30, 12.33 .

\section{OLiver County}

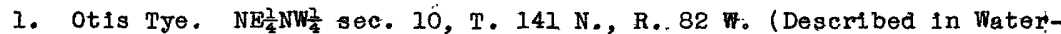
Supply Paper 908). Water levels, in feet below measuring point, 1941: Apr. 13, 19.24; Oct. 21, 18.73.

\section{Pembina, County}

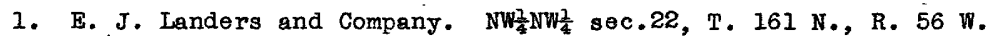
pug domestie and stock we1l, diameter 60 inches, depth 14.1 feet. MeasurIng point, edge of small hole in shed floor, 0.5 foot above land surface, and 112.31 feot above datum. Bench mark iron nail in southeast side of tree trunk, 107 feet northwest of well, 5 feet above land surface and 115 . \& 1 feet above datum. Bench mark iron nail in southwest side of tree trunk, 130 feet north-northwest of well, 3.4 feet above land surface, and 112.88 feet above datum. Water level, Apr. 26, 1941, 12.26 feet below measuring point. observer, Barl Veum.

Water level, in feet above datum, 1941

\begin{tabular}{|c|c|c|c|c|c|c|c|c|c|c|}
\hline $\begin{array}{l}\text { Apr. } \\
\text { May }\end{array}$ & $\begin{array}{r}26 \\
3 \\
10 \\
17 \\
24\end{array}$ & $\begin{array}{l}100.05 \\
100.30 \\
100.51 \\
100.69 \\
100.90\end{array}$ & $\begin{array}{l}\text { May } \\
\text { June }\end{array}$ & $\begin{array}{r}31 \\
7 \\
14 \\
21 \\
28\end{array}$ & $\begin{array}{l}100.93 \\
101.00 \\
101.04 \\
101.10 \\
101.21\end{array}$ & July & $\begin{array}{r}5 \\
12 \\
19 \\
26 \\
2\end{array}$ & $\begin{array}{l}101.39 \\
101.40 \\
101.41 \\
101.09 \\
100.96\end{array}$ & $\begin{array}{r}9 \\
\text { Aug. } \\
16 \\
23 \\
30 \\
\text { sept. } 6\end{array}$ & $\begin{array}{l}100.75 \\
100.65 \\
100.63 \\
100.58 \\
100.80\end{array}$ \\
\hline
\end{tabular}




\section{Pembina County--Continued}

1. E. J. Landers and Company.--Continued.

Water level, in feet above datum, 1941

\begin{tabular}{|c|c|c|c|c|c|c|c|c|c|}
\hline Date & $\begin{array}{l}\text { Water } \\
\text { level }\end{array}$ & Date & $\begin{array}{l}\text { Water } \\
\text { level }\end{array}$ & Date & & $\begin{array}{l}\text { Water } \\
\text { level }\end{array}$ & Date & & $\begin{array}{l}\text { Water } \\
\text { Ievel }\end{array}$ \\
\hline $\begin{array}{r}\text { Sept. } 13 \\
20 \\
27 \\
\text { oct. } 4\end{array}$ & $\begin{array}{l}101.07 \\
101.22 \\
101.35 \\
102.07\end{array}$ & $\begin{array}{lr}\text { oct. } & 13 \\
18 \\
\\
29 \\
\text { Nov. } 1\end{array}$ & $\begin{array}{l}102.36 \\
102.79 \\
102.91 \\
102.95\end{array}$ & Nov. & $\begin{array}{r}8 \\
15 \\
22 \\
29 \\
\end{array}$ & $\begin{array}{l}103.03 \\
102.98 \\
102.97 \\
102.88\end{array}$ & Dec. & $\begin{array}{r}6 \\
13 \\
20 \\
27\end{array}$ & $\begin{array}{l}102.98 \\
102.98 \\
102.98 \\
102.98\end{array}$ \\
\hline
\end{tabular}

5. Garnett A. Snell. SW $S W_{\frac{1}{4}}^{\frac{1}{4}}$ sec. 31, T. 162 N., R. 53 W. Dug domestic and stock well, 48 inches square, depth 17.4 feet. Heasuring point, edge of northeast curb support, a vertical 2 by 4-inch plank, 1.5 feet above land surface and 115.97 feet above datum. Vater level, Sept. 1q, 1941, 16.46 feet below measuring point. Observer, Garnett A. Sneil. Water level, in feet below measuring point, 1941

\begin{tabular}{rr|rr|rr|rrr}
\hline Sept.12 & 99.51 & Oct. 11 & 101.26 & Nov. 8 & 101.97 & Dec. & 6 & 103.38 \\
13 & 100.12 & & 18 & 101.74 & 15 & 102.14 & 13 & 103.61 \\
ioct. 3 & 101.24 & & 25 & 102.14 & 22 & 102.84 & 20 & 103.30 \\
& 4 & 101.24 & Nov. 1 & 101.86 & 29 & 103.55 & 27 & 103.47 \\
\hline
\end{tabular}

41. George Harris. WW $N W \frac{1}{4}$ sec. 27, T, 163 N., R. 51 W. (Described in Water-Supply Paper 840, p. 324.)

Water level, in feet above datum, 1941

\begin{tabular}{|c|c|c|c|c|c|c|c|c|c|c|c|}
\hline 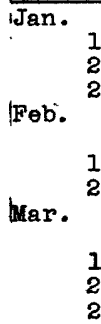 & $\begin{array}{r}6 \\
13 \\
20 \\
27 \\
4 \\
8 \\
15 \\
22 \\
1 \\
8 \\
15 \\
22 \\
29\end{array}$ & $\begin{array}{l}94.52 \\
(a) \\
(a) \\
(a) \\
\text { a) } \\
(a) \\
(a) \\
(a) \\
(a) \\
(a) \\
a) \\
(a) \\
(a)\end{array}$ & $\begin{array}{l}\text { May } \\
\text { June } \\
\text { July }\end{array}$ & $\begin{array}{r}9 \\
12 \\
19 \\
26 \\
3 \\
10 \\
17 \\
24 \\
31 \\
14 \\
21 \\
28 \\
5\end{array}$ & $\begin{array}{c}(a) \\
94.77 \\
97.36 \\
97.15 \\
97.85 \\
95.73 \\
97.29 \\
95.31 \\
97.52 \\
98.61 \\
99.01 \\
99.52 \\
99.61\end{array}$ & $\begin{array}{l}\text { July } \\
\text { Aug. } \\
\text { Sept } \\
\text { Oct. }\end{array}$ & $\begin{array}{r}12 \\
19 \\
26 \\
2 \\
9 \\
16 \\
23 \\
29 \\
6 \\
13 \\
20 \\
27 \\
6\end{array}$ & $\begin{array}{r}99.65 \\
99.56 \\
99.35 \\
99.04 \\
98.83 \\
98.72 \\
98.50 \\
98.35 \\
98.47 \\
98.61 \\
98.71 \\
98.80 \\
101.11\end{array}$ & $\begin{array}{l}\text { Oct. } \\
\text { Nov. } \\
\text { Dec. }\end{array}$ & $\begin{array}{l}22 \\
29\end{array}$ & $\begin{array}{l}101.58 \\
101.56 \\
101.56 \\
101.56 \\
101.56 \\
101.65 \\
101.65 \\
101.61 \\
101.58 \\
101.65 \\
101.61 \\
101.58\end{array}$ \\
\hline
\end{tabular}

42. C. A. Thompsion. SELSW sec. 3, T. 163 N., R. 56 W. (Described in Water-Supply Paper 840, p. 324). Heli caved in. Measurements dis continued. Pembina 72, a half ile west, set up for further measurement.

Water level, in feet above datum, 2941

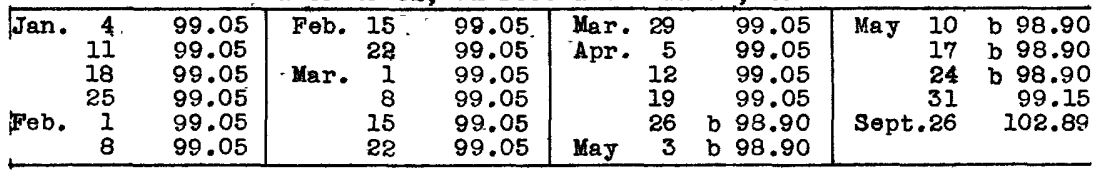

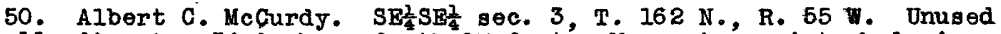
laug weli, diameter 54 inches, depth 17 feet. Measuring point, hole in pecorder platform, 2.8 feet above land supface, and 112.04 feet above datur. Water level, Oct.' 4, 1941, 11.38 feet below measuring point. Automatia recorder put on meli Oct. 11, 1941. Observer, Albert C. McCurdy.

Water level, in feet above datum, 1941

\begin{tabular}{|rr|rr|rr|rrr}
\hline Sept.18 & 99.74 & Oct. 26 & 101.35 & Nov. 22 & 101.39 & Dec. 13 & 101.29 \\
Oct. 4 & 100.66 & Nov. & 1 & 101.39 & & 29 & 101.33 & \\
11 & 100.95 & 8 & 101.40 & Dec. & 6 & 101.22 & 101.14 \\
18 & 101.22 & & 15 & 101.42 & & 27 & 100.87 \\
\hline
\end{tabular}

a Dry below 94.40 .

b Measurements may be accurate only to nearest foot. 


\section{Pembina County--Continued}

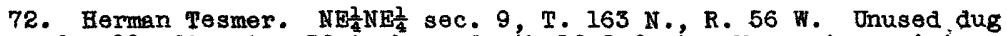
and bored well, diameter 36 inches, depth 16.8 feet. Measuring point, bored hole in well platform, north side of pump, 0.9 foot above land surface and 110.33 feet above datum. Water level, Sept. 26, 1941, 10.33 feet below measuring point. Observer, Herman Tesmer.

Water level, in feet above datum, 1941

\begin{tabular}{|c|c|c|c|c|c|c|c|}
\hline Date & $\begin{array}{l}\text { Water } \\
\text { level }\end{array}$ & Date & $\begin{array}{l}\text { Water } \\
\text { leveI }\end{array}$ & Date & $\begin{array}{l}\text { Watier } \\
\text { level }\end{array}$ & Date & $\begin{array}{l}\text { Water } \\
\text { level }\end{array}$ \\
\hline $\begin{array}{r}\text { Sept.26 } \\
\text { oct. } \\
11 \\
18 \\
\end{array}$ & $\begin{array}{l}100.00 \\
100.66 \\
101.41 \\
101.50\end{array}$ & $\begin{array}{cr}\text { Oct. } & 25 \\
\text { Nov. } & 1 \\
& 8 \\
& 15\end{array}$ & $\begin{array}{l}101.62 \\
101.62 \\
101.58 \\
101.62\end{array}$ & $\begin{array}{l}\text { Nov. } 22 \\
29 \\
\text { Dec. } 6\end{array}$ & $\begin{array}{l}101.41 \\
101.33 \\
101.29\end{array}$ & $\begin{array}{r}\text { Déc. } 13 \\
20 \\
27\end{array}$ & $\begin{array}{l}101.21 \\
101.06 \\
100.87\end{array}$ \\
\hline
\end{tabular}

\section{Plerce County}

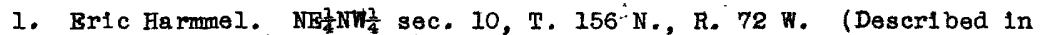
Water-Supply Paper 908). Water levels, in feet below measuring point, 1941a Apr. 23, 26.08; Oct. 29, 26.73 .

\section{Ramsey County}

48. Mrs. Bonnle Boland. SW $\mathrm{NE}_{\frac{1}{4}}^{\frac{1}{4}}$ sec, 14, T. $153 \mathrm{~N},$, R. 65 W. (Described in Water-Supply Paper 840, p. 324).

Water level, in feet above datum, 1941

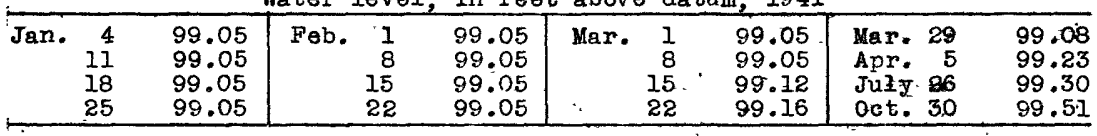

\section{Ransom County}

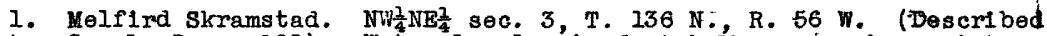
in Water-Supply Paper 908). Water levels, in feet below meásuring point, 1941: Apr. 7, 26.67; Oct. 15, 24.02.

\section{Renville Gounty}

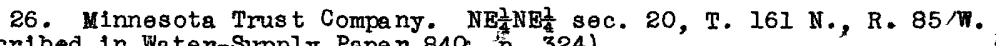
(Described in Water-Supply Paper 840, p. 324).

Water level, in feet above datum, 1941

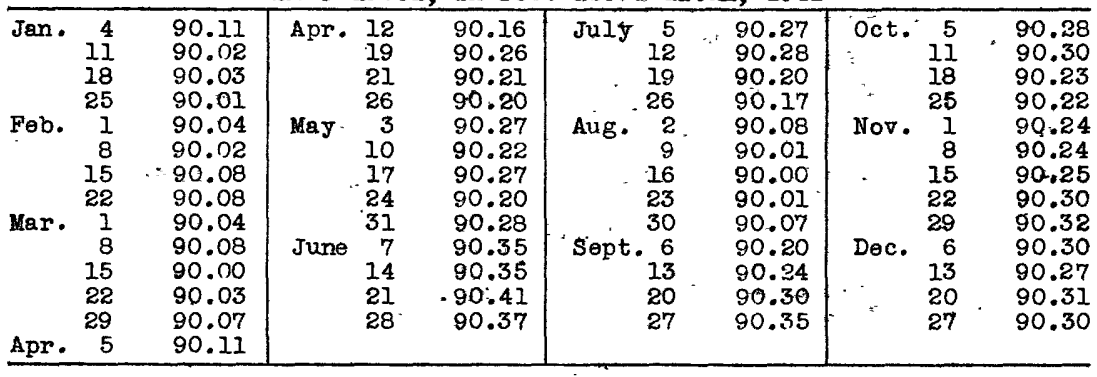




\section{Renville County--Continued}

75. Fish and Wildife service, U. S. Department of the Interior. NW $W_{4}^{\frac{1}{4} N W \frac{2}{4}}$ sec. 31, T. $158 \mathrm{~N} .$, R. 84 W. (Descrlbed in Fater-Supply Paper 840, p. 325$\}$.

Water level, in feet above datum, 1941

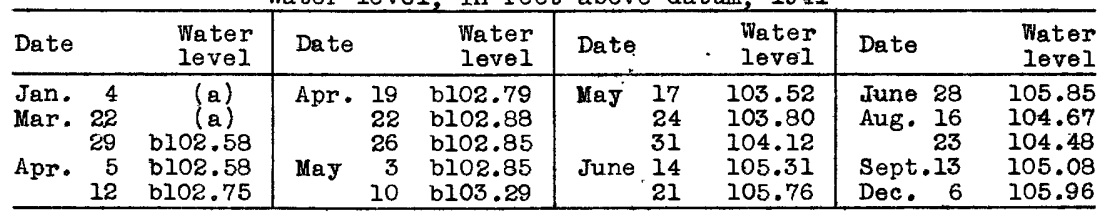

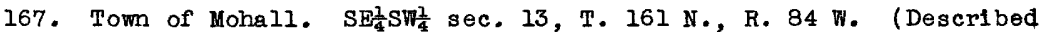
in Water-Supply Paper 908). Water levels, in feet below measuring point, 1941: Apr. 21, 24.11; Oct. 27, 24.15.

168. J. Dighton Taylor. NW $\frac{3}{4} \mathrm{NE}_{\frac{1}{4}}^{\frac{1}{4}}$ sec. 24, T. $161 \mathrm{~N}$, , R. 84 W. (Described in Water-Supply Paper 908). Water levels, in feet below measuring point, 1941: Apr. 21, 11.38; 0ct. 27, 10.86.

169. Fred Paris. SEl SW sec. 13, T. 161 N., R. 84 W. (Described in Water-Supply Paper 908.). Water levels, in feet below measuring point, 1941: Apr. 21, 12.22; 0ct. 27, 7.08.

\section{Richland County}

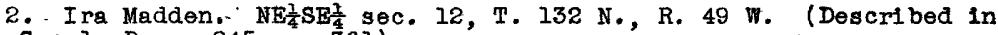
Water-Supply Paper 845, p. 361).

Water level, in feet above datum, 1941

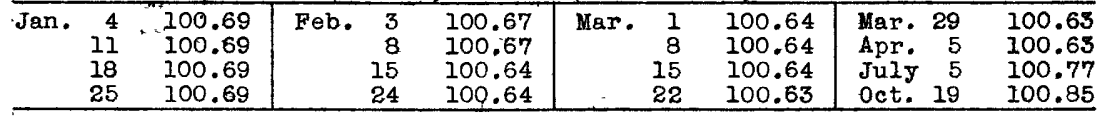

5. John L1ljemark. SW SET sec. 33, T. 133 N., R. 52 W. (Described in Nater-Supply Paper 840, p. 325). Erroneously located in sec. 23 in Water-Supply Papers 886 and 908.

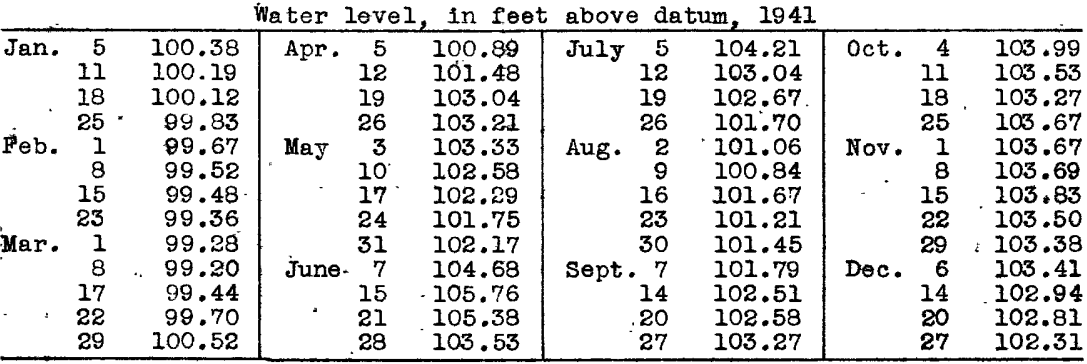

\section{Roletté County}

164. Owner's number 3. Town of Rolla. NE⿺ sec. 17, T. 162 N., R. 69 W. (Described in Water-Supply Paper 908). Measuring point, lo7.82 feet'above datum.

Water level, in feet above datum, 1940-41

\begin{tabular}{|c|c|c|c|c|c|c|c|}
\hline Date & $\begin{array}{l}\text { Water } \\
\text { level }\end{array}$ & Date & & $\begin{array}{l}\text { Water } \\
\text { level }\end{array}$ & Date & & $\begin{array}{l}\text { Water } \\
\text { level }\end{array}$ \\
\hline $\begin{array}{ll}\text { July } 17, & 1940 \\
\text { Nov. } 8 & \\
\text { Apr. } 24, & 1941 \\
26 & \end{array}$ & $\begin{array}{l}99.51 \\
99.27 \\
99.60 \\
99.60\end{array}$ & May & $\begin{array}{l}5,1941 \\
10 \\
19 \\
26\end{array}$ & $\begin{array}{l}99.60 \\
99.60 \\
99.61 \\
99.60\end{array}$ & $\begin{array}{l}\text { May } \\
\text { June }\end{array}$ & $\begin{array}{l}31,1941 \\
9 \\
16 \\
21\end{array}$ & $\begin{array}{l}99.60 \\
99.57 \\
99.60 \\
99.60\end{array}$ \\
\hline
\end{tabular}

a Well frozen and drifted with snow between dates shown

b To ice. 
Rolette County--Continued

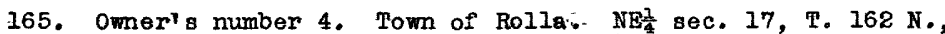
R. 69 W. (Described in Water-Supply Paper.008). Measuring polnt, 12́1.19 feet above datum. Hater level, Nov. 18, 1941, 20.51 feet below measuring point (datum established for this date because previous measurements were affected by local drawdown). Observer, Lucien N. Gautihier.

Water level, in feet above datum, 194Q-4I

\begin{tabular}{|c|c|c|c|c|c|}
\hline Date & $\begin{array}{l}\text { Water } \\
\text { Ievel }\end{array}$ & Date & $\begin{array}{l}\text { Water } \\
\text { level }\end{array}$ & Date & $\therefore$ Hater \\
\hline $\begin{array}{l}\text { July 17, } 1940 \\
\text { Nov. } 8, \\
\text { Apr. 24, } 1941\end{array}$ & $\begin{array}{ll}\text { a } & 86.59 \\
\text { a } & 85.63 \\
\text { b } & 90.38\end{array}$ & $\begin{array}{l}\text { Apr. } 26 ; 1941 \\
\text { Nov. } 18 \\
24\end{array}$ & $\begin{array}{r}90.59 \\
100.68 \\
100.89\end{array}$ & Dec. $\frac{1,1941}{22}$ & $\begin{array}{l}100.92 \\
101.35\end{array}$ \\
\hline
\end{tabular}

\section{Sargent county}

i. Nick Klinkheimer. SW $W_{4}^{2} W_{4}^{2}$ sec. 25, T. I3I N., R: 58 W. (Described in Water-Supply Paper 908). Water levels, in feet below measuring point, 1941: Apr. 7, 28.98; 0ct. 15, 28.58 .

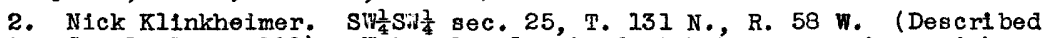
in-Water-Supply Paper 908). Water levels, in feet below measuring point, 1941: Apr. 7, 29.89; Oct. 15, 29.69.

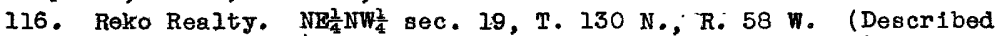
in Fater-Supply Paper 908). New measuring point, top of p1pe, 2.2 feet above land surface, and 0.9 foot below old measuring point. Water levels, In feet bel ow new measuring point, 1941: May 8, 8.62; June 20, 7.93; July 25, 9.40; Oct. 15, 9.52 .

\section{Sheridan County}

95. Bank of North Dakota. NFW NW sec.- 28, T. 145 N., R. 75 w. (Described In Water-supply Paper 845, p. 362). Water level, In feet above datum, 1941

\begin{tabular}{|c|c|c|c|c|c|c|c|}
\hline Date & $\begin{array}{l}\text { Water } \\
\text { level }\end{array}$ & Date & $\begin{aligned} & \text { Water } \\
& \text { : level }\end{aligned}$ & Date & $\begin{array}{l}\text { Water } \\
\text { level }\end{array}$ & Date & $\begin{array}{l}\text { Water } \\
\text { level }\end{array}$ \\
\hline 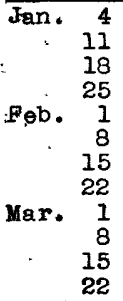 & $\begin{array}{l}99.31 \\
99.32 \\
99.32 \\
99.33 \\
99.32 \\
99.32 \\
99.33 \\
99.33 \\
99.33 \\
99.32 \\
99.33 \\
99.34\end{array}$ & $\begin{array}{rr}\text { Mar. } & 29 \\
\text { Apr. } & 5 \\
& 12 \\
& 19 \\
& 26 \\
\text { Hay } & 3 \\
& 10 \\
& 17 \\
& 25 \\
& 31 \\
\text { June } & 7 \\
& 14\end{array}$ & $\begin{array}{r}99.35 \\
99.35 \\
99.41 \\
99.53 \\
99.61 \\
99.66 \\
99.68 \\
99.97 \\
100.00 \\
100.02 \\
100.14 \\
100.15\end{array}$ & $\begin{array}{rr}\text { June } & 21 \\
& 29 \\
\text { July } & 5 \\
12 \\
19 \\
26 \\
\text { Aug. } 2 \\
9 \\
15 \\
30 \\
\text { Sept. } 6 \\
13\end{array}$ & $\begin{array}{l}100.15 \\
100.23 \\
100.29 \\
100.31 \\
100.27 \\
100.22 \\
100.19 \\
100.19 \\
100.18 \\
100.17 \\
100.18 \\
100.17\end{array}$ & $\begin{array}{rr}\text { oct. } & 18 \\
& 25 \\
\text { Yov. } & 1 \\
& 8 \\
15 \\
\\
22 \\
\\
29 \\
\text { Dec. } \\
6 \\
13 \\
20 \\
27\end{array}$ & $\begin{array}{l}100.11 \\
100.17 \\
100.21 \\
100.23 \\
100.23 \\
100.33 \\
100.31 \\
100.21 \\
100.30 \\
100.29 \\
100.26\end{array}$ \\
\hline
\end{tabular}

\section{Sioux County}

1. Mrs. Looking-out. SW sec. 7, T. $130 \mathrm{~N}$., R. 79 W. (Described in Water-Supply Paper 908). Water levels', in feet bélow measuring point, 1941: Apr. 16, 13.67; 0ct. 22, 12.76.

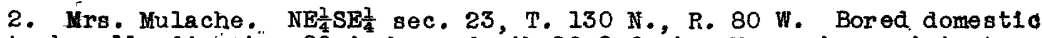
and stock well, diameter 20 Inches, depth 26.8 feet. Measuring point, top of concrete curb, chiseled line, north point, 0.3 foot above land surface. Water levels, in feet below measuring point: Nov. 17, 1940, 25.21; Apr. 16, $1941,25.29$; 0ct. $22,1941,25.43$.
a Pumping.
b After 12 hours recovery. 


\section{Slope County}

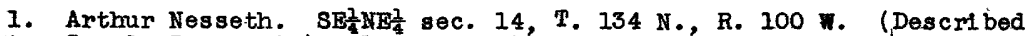
in Water-Supply Paper 908). Water levels, in feet below measuring point, 1941:... Apr. 17, 18.91; 0ct. 23, 18.23.

\section{Stark County}

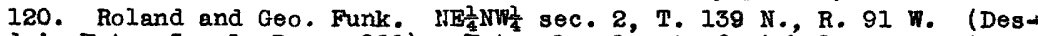
cribed in Water-Supply Paper 908). Water levels, in feet below measuring point, 1941: Apr. 13, 6.06; Oct. 21, 5.11..

\section{Steele County}

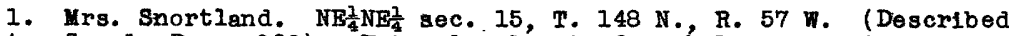
in Water-Supply Paper 908). Water levels, in feet below measuring point, 1941: Apr. 5, 23.20; 0ct. 14, 27.50.

\section{Stutsman County}

124. Union Central Iife Insurance Company. SW SW sec. 33, T. $137 \mathrm{~K}$, R. 64 W. (Described in Water-Supply Paper 908). Erroneously Iisted in T. 7 N. in Water-SuppIy Paper 908). Water levels, in feet below measuring point, 1941: Apr. 8, 53.80; Dct. 6, 53.75.

\section{Towner County}

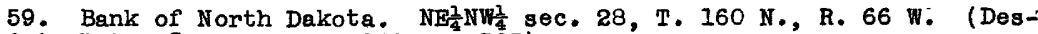
cribed in Water-Supply Paper 840 , p. 325)..

Water level, in foet above datum, 1941

\begin{tabular}{|c|c|c|c|c|c|c|c|c|c|c|c|}
\hline ate & & $\begin{array}{l}\text { Water } \\
\text { level }\end{array}$ & Date & & $\begin{array}{l}\text { Water } \\
\text { lavel }\end{array}$ & Date & & $\begin{array}{l}\text { Water } \\
\text { level }\end{array}$ & Date & & $\begin{array}{l}\text { Water } \\
\text { level }\end{array}$ \\
\hline Tan. & $\begin{array}{r}4 \\
11 \\
18 \\
25 \\
2 \\
8 \\
15 \\
22 \\
1 \\
8 \\
15 \\
22 \\
29\end{array}$ & $\begin{array}{l}100.80 \\
100.83 \\
100.82 \\
100.81 \\
100.81 \\
100.92 \\
101.02 \\
101.13 \\
101.17 \\
101.18 \\
101.22 \\
101.26 \\
101.30\end{array}$ & $\begin{array}{l}\text { Apr. } \\
\text { May }\end{array}$ & $\begin{array}{r}5 \\
12 \\
19 \\
26 \\
3 \\
10 \\
17 \\
24 \\
31 \\
7 \\
14 \\
21 \\
28\end{array}$ & $\begin{array}{l}101.42 \\
101.55 \\
101.59 \\
101.64 \\
101.68 \\
101.73 \\
101.76 \\
101.79 \\
101.79 \\
101.84 \\
101.88 \\
101.85 \\
101.82\end{array}$ & $\begin{array}{r}\text { July } \\
\text { Aug. }\end{array}$ & $\begin{array}{r}5 \\
12 \\
19 \\
26 \\
2 \\
9 \\
16 \\
23 \\
30 \\
6 \\
13 \\
20 \\
27\end{array}$ & $\begin{array}{l}101.80 \\
101.72 \\
101.71 \\
101.68 \\
101.63 \\
101.63 \\
101.63 \\
101.63 \\
101.64 \\
101.84 \\
101.88 \\
101.91 \\
101.98\end{array}$ & $\begin{array}{l}\text { Oct. } \\
\text { Hov. } \\
\text { Dec. }\end{array}$ & $\begin{array}{r}4 \\
11 \\
18 \\
24 \\
1 \\
8 \\
15 \\
22 \\
29 \\
6 \\
13 \\
20 \\
27\end{array}$ & $\begin{array}{l}102.05 \\
100.08 \\
102.12 \\
102.13 \\
102.19 \\
102.22 \\
102.25 \\
102.31 \\
102.29 \\
102.30 \\
102.38 \\
102.37 \\
102.37\end{array}$ \\
\hline
\end{tabular}

\section{Traill county}

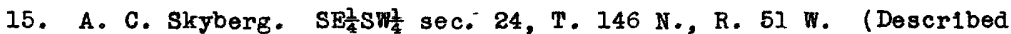
in Water-Supply Paper 840, p. 326).

Water level, in feet above datum, 1941

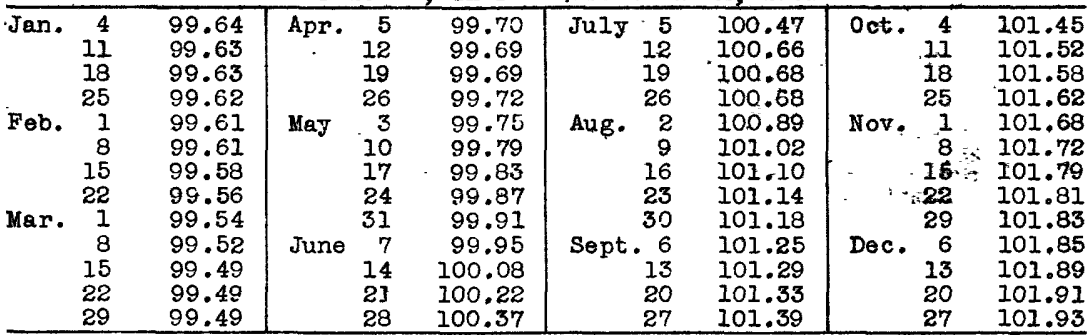


Tra111 County--Continued

31. City of Hetton. Swater sec. 18, T. 148-N., R. 53 W. (Desoribed in Water-supply Paper 845, p. 364).

Water lovel, in feet abovo datum, 1941

\begin{tabular}{|c|c|c|c|c|c|c|c|c|c|}
\hline \multicolumn{2}{|c|}{ Date } & $\begin{array}{l}\text { Water } \\
\text { level }\end{array}$ & \multicolumn{2}{|l|}{ Date } & $\begin{array}{l}\text { Water } \\
\text { level }\end{array}$ & Date & $\begin{array}{l}\text { Water } \\
\text { level }\end{array}$ & Date & $\begin{array}{l}\text { Water } \\
\text { level }\end{array}$ \\
\hline Apr. & $\begin{array}{r}6 \\
19 \\
26 \\
3 \\
10 \\
18 \\
25 \\
31\end{array}$ & $\begin{array}{r}97.88 \\
100.60 \\
100.49 \\
99.88 \\
99.95 \\
100.93 \\
100.77 \\
101.73\end{array}$ & June & $\begin{array}{r}7 \\
14 \\
21 \\
28 \\
5 \\
12 \\
20 \\
26\end{array}$ & $\begin{array}{l}102.65 \\
106.34 \\
106.46 \\
106.60 \\
107.17 \\
107.49 \\
105.62 \\
105.82\end{array}$ & $\begin{array}{rr}\text { Aug. } & 2 \\
9 \\
16 \\
23 \\
30 \\
\text { Sept. } 8 \\
13 \\
20\end{array}$ & $\begin{array}{l}106.59 \\
106.25 \\
106.47 \\
106.60 \\
106.77 \\
107.97 \\
107.89 \\
108.08\end{array}$ & 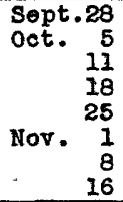 & $\begin{array}{l}108.91 \\
109.64 \\
110.08 \\
110.29 \\
110.54 \\
110.83 \\
110.87 \\
111.12\end{array}$ \\
\hline
\end{tabular}

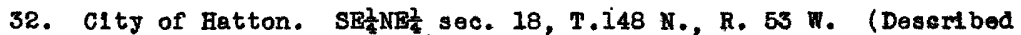
in Water-Supply Paper 845, p. 364).

Water level, in feet above datum, 1941

\begin{tabular}{|c|c|c|c|c|c|c|c|c|c|}
\hline Apr. & $\begin{array}{r}6 \\
19 \\
26 \\
3 \\
10 \\
18 \\
25 \\
31\end{array}$ & $\begin{array}{r}95.56 \\
99.08 \\
100.69 \\
101.23 \\
101.49 \\
102.07 \\
102.11 \\
102.93\end{array}$ & June & $\begin{array}{r}7 \\
14 \\
21 \\
28 \\
5 \\
12 \\
20 \\
26\end{array}$ & $\begin{array}{l}103.54 \\
105.52 \\
106.21 \\
106.50 \\
107.13 \\
107.39 \\
107.29 \\
106.70\end{array}$ & $\begin{array}{r}2 \\
\text { Aug. } \\
10 \\
16 \\
25 \\
30 \\
\text { Sept } 8 \\
13 \\
20\end{array}$ & $\begin{array}{l}106.20 \\
105.63 \\
105.80 \\
105.65 \\
106.65 \\
106.65 \\
107.11 \\
107.33\end{array}$ & 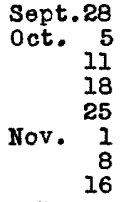 & $\begin{array}{l}107.93 \\
108.47 \\
108.92 \\
109.11 \\
109.36 \\
109.68 \\
109.57 \\
109.84\end{array}$ \\
\hline
\end{tabular}

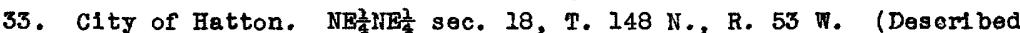
in Vater-Suppis Paper 840, p. 326).

Water level, in foet above datum, 1941

\begin{tabular}{|c|c|c|c|c|c|c|c|c|c|}
\hline $\begin{array}{c}\text { May } \\
\cdot \\
\text { Jane }\end{array}$ & $\begin{array}{r}6 \\
19 \\
26 \\
3 \\
10 \\
18 \\
25 \\
31 \\
7\end{array}$ & $\begin{array}{r}99.53 \\
102.05 \\
100.18 \\
100.14 \\
100.26 \\
100.41 \\
100.51 \\
100.87 \\
100.96\end{array}$ & June & $\begin{array}{r}14 \\
21 \\
28 \\
5 \\
12 \\
20 \\
26 \\
2 \\
10\end{array}$ & $\begin{array}{l}101.56 \\
101.84 \\
102.11 \\
102.84 \\
102.28 \\
103.68 \\
103.44 \\
103.57 \\
103.29\end{array}$ & $\begin{array}{r}\text { Aug. } 16 \\
23 \\
30 \\
\text { Sept. } 8 \\
13 \\
20 \\
28 \\
28 \\
\text { oct. } 5 \\
11\end{array}$ & $\begin{array}{l}105.00 \\
103.99 \\
104.12 \\
105.09 \\
105.55 \\
105.36 \\
106.27 \\
107.04 \\
107.68\end{array}$ & $\begin{array}{lr}\text { oct. } & 18 \\
25 \\
\text { Nov. } 1 \\
8 \\
16 \\
16 \\
22 \\
29 \\
6 \\
\text { Dec. } \\
13\end{array}$ & $\begin{array}{l}108.31 \\
109.12 \\
109.92 \\
110.37 \\
110.96 \\
111.23 \\
111.57 \\
111.87 \\
111.86\end{array}$ \\
\hline
\end{tabular}

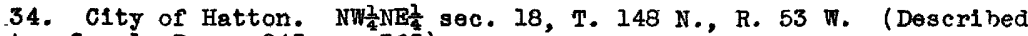
in Water-Supply Paper 845, p. 365).

Water level, in feet above datum, 1941

\begin{tabular}{|c|c|c|c|c|c|c|c|c|c|}
\hline & & & & & & & \\
\hline Apr. & $\begin{array}{r}6 \\
19 \\
26 \\
3 \\
10 \\
18 \\
25 \\
31\end{array}$ & $\begin{array}{r}97.96 \\
101.70 \\
101.71 \\
102.07 \\
102.17 \\
102.81 \\
102.90 \\
103.29\end{array}$ & June & $\begin{array}{r}7 \\
14 \\
21 \\
28 \\
5 \\
12 \\
20 \\
26\end{array}$ & $\begin{array}{l}103.84 \\
105.93 \\
106.74 \\
107.00 \\
107.46 \\
107.54 \\
107.37 \\
106.79\end{array}$ & $\begin{array}{r}\text { Aug. } \\
\text { Sept. } \\
36 \\
23 \\
20\end{array}$ & $\begin{array}{r}107.14 \\
105.42 \\
105.54 \\
104.85 \\
105.49 \\
+105.96 \\
105.99 \\
106.11\end{array}$ & $\begin{array}{lr}\text { Sept.28 } \\
\text { oct. } & 5 \\
& 11 \\
& 18 \\
& 25 \\
\text { Nov. } & 1 \\
& 8 \\
16\end{array}$ & $\begin{array}{l}106.80 \\
107.31 \\
107.71 \\
107.95 \\
108.30 \\
108.64 \\
108.84 \\
109.10\end{array}$ \\
\hline
\end{tabular}

\section{Walsh County}

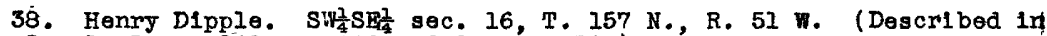
Water-Supply Papers 840, p. 326, and 845, p. 366).

Water level, in feet above datum, 1941

\begin{tabular}{|c|c|c|c|c|c|c|c|c|c|}
\hline Jan. & $\begin{array}{r}4 \\
11 \\
18 \\
25 \\
1\end{array}$ & $\begin{array}{l}99.74 \\
99.80 \\
99.44 \\
99.50 \\
99.46\end{array}$ & $\begin{array}{l}\text { Feb } \\
\text { Mar }\end{array}$ & $\begin{array}{r}8 \\
15 \\
22 \\
1 \\
8\end{array}$ & $\begin{array}{l}99.49 \\
99.48 \\
99.47 \\
99.46 \\
99.64\end{array}$ & Mar. & $\begin{array}{r}99.65 \\
99.56 \\
99.68 \\
99.66 \\
99.48\end{array}$ & $\begin{array}{l}\text { Apr. } \\
\text { May }\end{array}$ & $\begin{array}{r}99.47 \\
99.39 \\
99.40 \\
101.00 \\
101.03\end{array}$ \\
\hline
\end{tabular}


Walsh County--Continued.

38. Henry Dipple.--Continuod.

Water level, in feet above datum, 1941

\begin{tabular}{|c|c|c|c|c|c|c|c|c|c|}
\hline Date & & $\begin{array}{l}\text { Weter } \\
\text { level }\end{array}$ & Date. & $\begin{array}{l}\text { Water } \\
\text { level }\end{array}$ & Date & $\begin{array}{l}\text { Water } \\
\text { level }\end{array}$ & Date & & $\begin{array}{l}\text { Water } \\
\text { level }\end{array}$ \\
\hline $\begin{array}{l}\text { May } \\
\text { June }\end{array}$ & $\begin{array}{r}24 \\
31 \\
7 \\
14 \\
21 \\
29 \\
5 \\
12\end{array}$ & $\begin{array}{l}100.94 \\
100.91 \\
100.98 \\
101.00 \\
100.75 \\
100.74 \\
100.21 \\
100.15\end{array}$ & $\begin{array}{rr}\text { JuIJ } 19 \\
26 \\
\text { Aug. } 2 \\
9 \\
16 \\
23 \\
30 \\
\text { Sept. } 6\end{array}$ & $\begin{array}{r}100.00 \\
100.32 \\
100.20 \\
100.04 \\
99.94 \\
99.99 \\
100.40 \\
100.25\end{array}$ & $\begin{array}{l}\text { Sept. } 13 \\
20 \\
27 \\
\text { Oct. } 4 \\
11 \\
18 \\
\\
25 \\
\text { Nov. } 1\end{array}$ & $\begin{array}{l}100.36 \\
100.38 \\
100.66 \\
100.40 \\
100.49 \\
100.51 \\
100.44 \\
100.37\end{array}$ & $\begin{array}{l}\text { Nov. } \\
\text { Dec. }\end{array}$ & $\begin{array}{r}8 \\
15 \\
22 \\
29 \\
6 \\
13 \\
20 \\
27\end{array}$ & $\begin{array}{r}100.37 \\
100.36 \\
100.36 \\
100.37 \\
100.49 \\
100.48 \\
100.46 \\
99.91\end{array}$ \\
\hline
\end{tabular}

39. Henry Dipple. SW $\frac{1}{4} \mathrm{SE} \frac{1}{4}$ sec. I6, T. 157 N., R. 5I W. (Described in Water-Supply Paper 840 , p. 327).

Water level, in feet above datum, 1941

\begin{tabular}{|c|c|c|c|c|c|c|c|c|c|c|c|}
\hline$F \in b$. & $\begin{array}{r}4 \\
11 \\
18 \\
25 \\
1 \\
8 \\
15 \\
22 \\
1 \\
8 \\
15 \\
22 \\
29\end{array}$ & $\begin{array}{l}98.31 \\
99.32 \\
99.34 \\
99.31 \\
99.38 \\
99.34 \\
99.38 \\
99.32 \\
99.38 \\
97.18 \\
97.75 \\
98.24 \\
99.13\end{array}$ & Apr. & $\begin{array}{r}5 \\
12 \\
19 \\
26 \\
3 \\
10 \\
17 \\
24 \\
31 \\
7 \\
14 \\
21 \\
28\end{array}$ & $\begin{array}{l}101.12 \\
\text { a107.4 } \\
8107.4 \\
104.67 \\
104.55 \\
104.01 \\
103.82 \\
103.77 \\
104.56 \\
104.57 \\
106.17 \\
106.84 \\
105.75\end{array}$ & $\begin{array}{rr}\text { July } & 1 \\
& 1 \\
\text { Aug. } & \\
& 1 \\
2 \\
\text { Sept. } \\
3 \\
\\
\text { I } \\
2 \\
2\end{array}$ & $\begin{array}{r}5 \\
12 \\
17 \\
26 \\
2 \\
9 \\
16 \\
23 \\
30 \\
6 \\
13 \\
20 \\
27\end{array}$ & $\begin{array}{l}104.40 \\
104.29 \\
104.49 \\
102.84 \\
102.11 \\
101.55 \\
101.01 \\
101.04 \\
101.03 \\
105.99 \\
103.98 \\
104.67 \\
105.00\end{array}$ & oet. & $\begin{array}{r}4 \\
11 \\
13 \\
25 \\
1 \\
8 \\
15 \\
22 \\
29 \\
6 \\
13 \\
20 \\
27\end{array}$ & $\begin{array}{l}106.07 \\
106.42 \\
106.40 \\
105.26 \\
105.94 \\
105.99 \\
106.08 \\
106.02 \\
106.26 \\
105.00 \\
105.24 \\
105.40 \\
103.46\end{array}$ \\
\hline
\end{tabular}

40. Henry Dipple: SW $\frac{1}{4} S E_{\frac{1}{4}}^{\frac{1}{4}}$ sec. 16, T. 157 N., R. $5 I$ H. (Described in Water-Supply Paper 840, p. 327).

Water level, In feet above datum, 1941

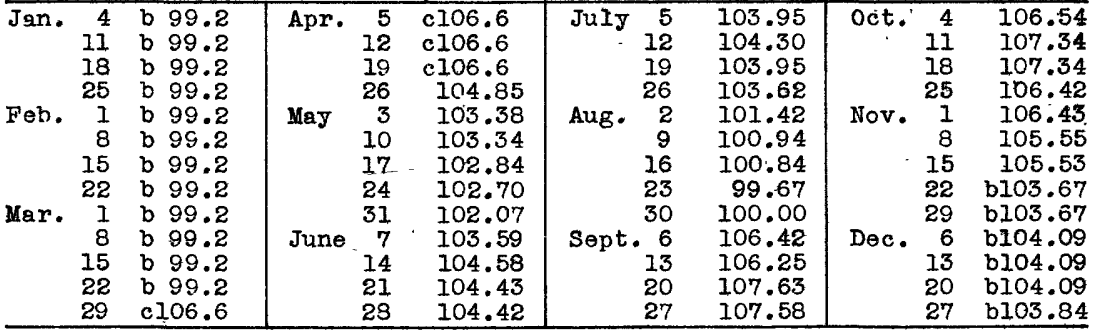

96. C. D. Lewis. SW $\frac{1}{4} S W_{\frac{1}{4}}^{\frac{1}{4}}$ sec. $17, \mathrm{~T} .157 \mathrm{~N} ., \dot{\mathrm{R}} .55 \mathrm{~W}$. (Described in Water-Supply Paper 886, p. 55I).

Water level, in feet above datum, 1941

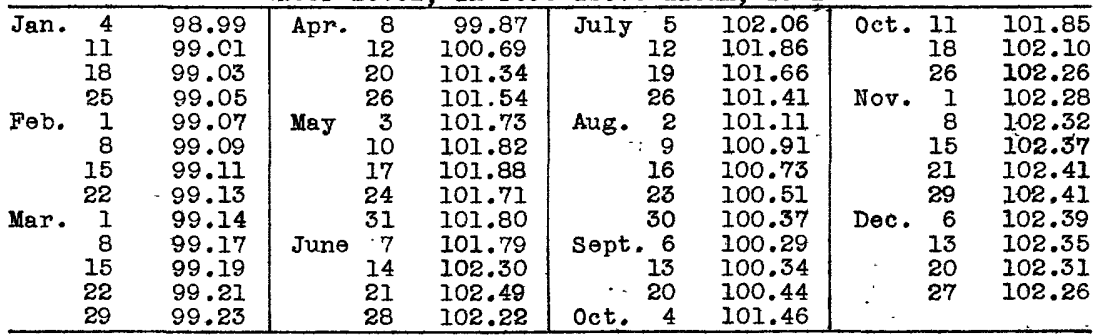

a Filled with water. c Filied with snow-melt water.

$\mathrm{b}$ Top of $1 \mathrm{c} \theta$. 
Ward County

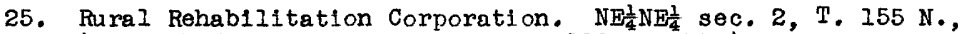
R. 84 W. (Described in Watsr-Supply Paper 386, p. 552).

Water level, in feet above datum, 1941

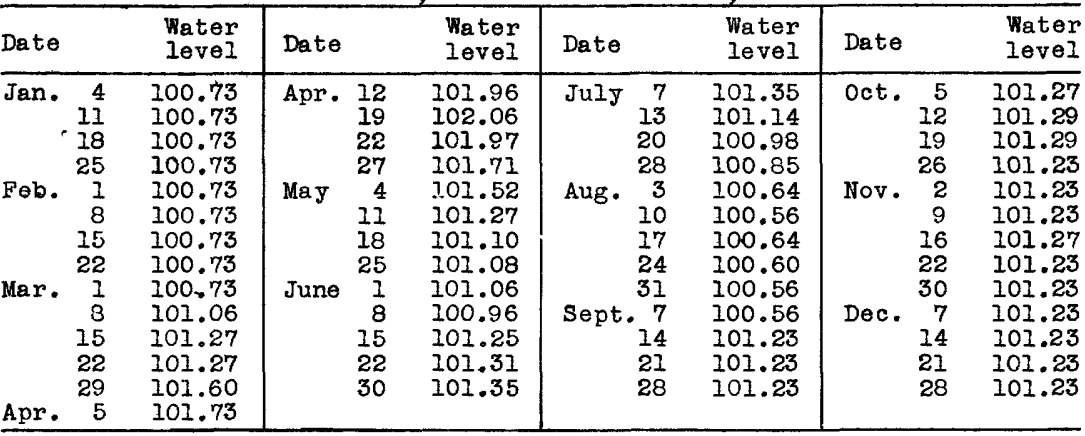

53. Chas. O'Neill. SW $\frac{1}{4} \mathrm{SE} \frac{1}{4}$ sec. 30, T. $160 \mathrm{~N}$, , R. 88 W. (Described in Water-Supply Paper 886 , p. 552).

Water level, in feet above datum, 1941

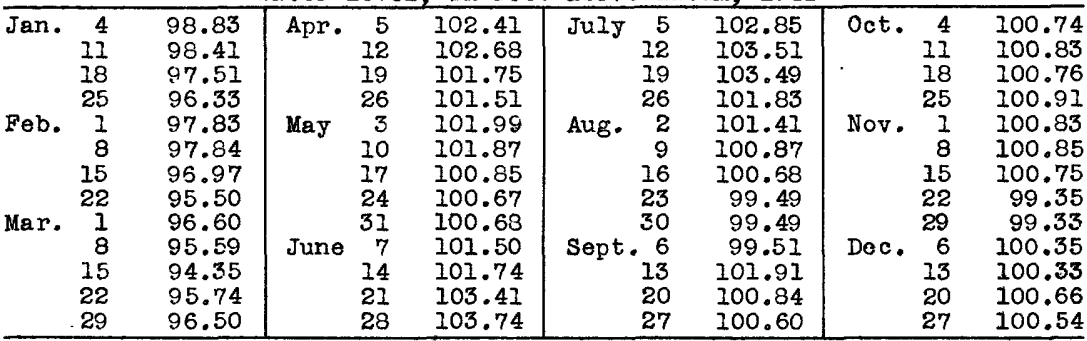

71. FIsh and WIIdlife Service, 0. S. Department of the Interior. SE $\frac{2}{4} \operatorname{SW} \frac{1}{4}$ sec. 5, T. 157 N., R. 84 W. (Described in Water-Supply Paper 840 , p. 327).

Water level, In feet above datum, 1941

\begin{tabular}{|c|c|c|c|c|c|c|c|c|c|c|}
\hline Jan. & $\begin{array}{r}4 \\
11 \\
18 \\
25 \\
1 \\
8 \\
15\end{array}$ & $\begin{array}{l}99.92 \\
99.89 \\
99.87 \\
99.87 \\
99.77 \\
99.79 \\
99.83\end{array}$ & $\begin{array}{l}\text { Mar } \\
\text { Apr. }\end{array}$ & $\begin{array}{r}22 \\
29 \\
5 \\
12 \\
19 \\
22 \\
26\end{array}$ & $\begin{array}{r}99.79 \\
99.39 \\
99.92 \\
99.86 \\
100.08 \\
100.09 \\
100.11\end{array}$ & $\begin{array}{l}\text { May } \\
\text { June }\end{array}$ & $\begin{array}{r}3 \\
10 \\
17 \\
24 \\
31 \\
14\end{array}$ & $\begin{array}{l}100.21 \\
100.22 \\
100.27 \\
100.31 \\
100.31 \\
100.33\end{array}$ & $\begin{array}{l}\text { June } 2 \\
2 \\
\text { Aug. } \\
\\
\text { Sept. } \\
\text { Dec. }\end{array}$ & $\begin{array}{l}100.46 \\
100.54 \\
101.42 \\
101.37 \\
101.46 \\
102.52\end{array}$ \\
\hline
\end{tabular}

73. Fish and Wildilfe Service, U. S. Department of the Interior. SE $\frac{1}{4} \mathrm{SE}_{\frac{1}{4}}^{\frac{1}{4}}$ sec. 2I, T. 157 N., R. 84 W. (Described in Water-Supply Paper 840 , p. 327 ).

Water level, in feet above datum, 1941

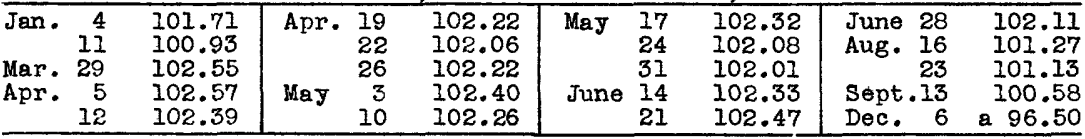

a Unit 96 (a reservolr with water level maintained by dam on

Souris River) drained.

$5110050-43-15$ 


\section{Ward Ccunty--Continued}

74. F1sh and W1ldilfe Service, U. S. Department of the Interior. NE $\frac{1}{4}$ SW sec. 21, T. 157 N., R. 84 W. (Described in Water-Supply Paper 840, p. 328 ).

Water level, in feet above datum, 1941

\begin{tabular}{|c|c|c|c|c|c|c|c|c|c|c|}
\hline Date & & $\begin{array}{l}\text { Water } \\
\text { level }\end{array}$ & Date & & $\begin{array}{l}\text { Water } \\
\text { level }\end{array}$ & Date & & $\begin{array}{l}\text { Water } \\
\text { level. }\end{array}$ & Date & $\begin{array}{l}\text { Water } \\
\text { level }\end{array}$ \\
\hline Jan. & $\begin{array}{r}4 \\
11 \\
18 \\
25 \\
1 \\
8 \\
15\end{array}$ & $\begin{array}{l}101.85 \\
101.87 \\
101.89 \\
101.92 \\
101.96 \\
101.98 \\
101.10\end{array}$ & $\begin{array}{l}\text { Mar. } \\
\text { Apr. }\end{array}$ & $\begin{array}{r}22 \\
29 \\
5 \\
12 \\
19 \\
22 \\
26\end{array}$ & $\begin{array}{l}101.08 \\
101.35 \\
102.44 \\
102.56 \\
102.73 \\
102.77 \\
102.81\end{array}$ & $\begin{array}{l}\text { May } \\
\text { June }\end{array}$ & $\begin{array}{l}3 \\
10 \\
17 \\
24 \\
31 \\
14\end{array}$ & $\begin{array}{l}102.90 \\
102.83 \\
102.90 \\
102.70 \\
102.56 \\
102.87\end{array}$ & $\begin{array}{lr}\text { June } & 21 \\
& 28 \\
\text { Aug. } & 16 \\
& 23 \\
\text { Sept. } 13 \\
\text { Dec. } 6\end{array}$ & $\begin{array}{l}102.88 \\
102.64 \\
101.15 \\
101.02 \\
101.27 \\
101.50\end{array}$ \\
\hline
\end{tabular}

114. Fish and Wilalife Service, U. S. Department of the Interior. SW $\frac{2}{4} \mathrm{NE}_{\frac{2}{4}}^{\frac{1}{2}}$ sec. 32 , T. 160 N., R. 88 W. (Described in Water-Supply Paper 908). Water leveis, in feet below measuring point, 1941: Apr. 21, 8.41; 0ct. 26, 7.42 .

\section{Wells County}

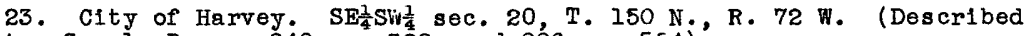
in Water-Supply Papers 840, p. 328 , and 886, p. 554)?

Water level, in feet above datum, 1941

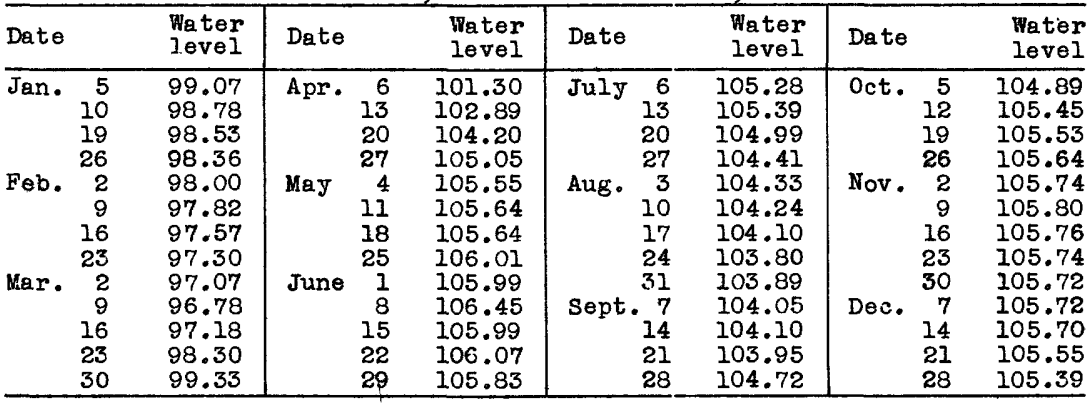

24. City of Harvey. $\mathrm{NE}_{\frac{1}{4}} \mathrm{NW} \frac{3}{4}$ sec. 28, T. $150 \mathrm{~N}, \mathrm{R}, 72 \mathrm{~W}$. (Described in Water-Supply Papers 840, p. 32̌, and 886, p. 554)'

Water level, in feet above datum, 1941

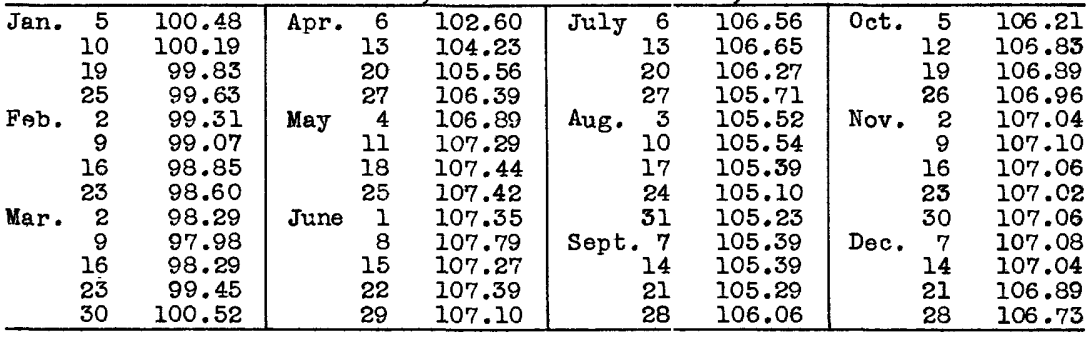

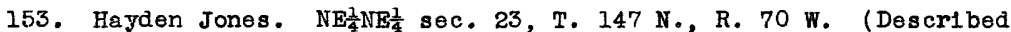
in Water-Suppiy Paper 908). Water level, in feet, below measuring point, 1941: 0ct. 18, 5.95. 


\section{Williams County}

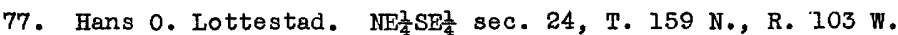
(Described in Water-Supply Paper 886, p. 554). Observer, Henry Hoff, Grenora. Well not used after March.

Water level, in feet below datum, 1941

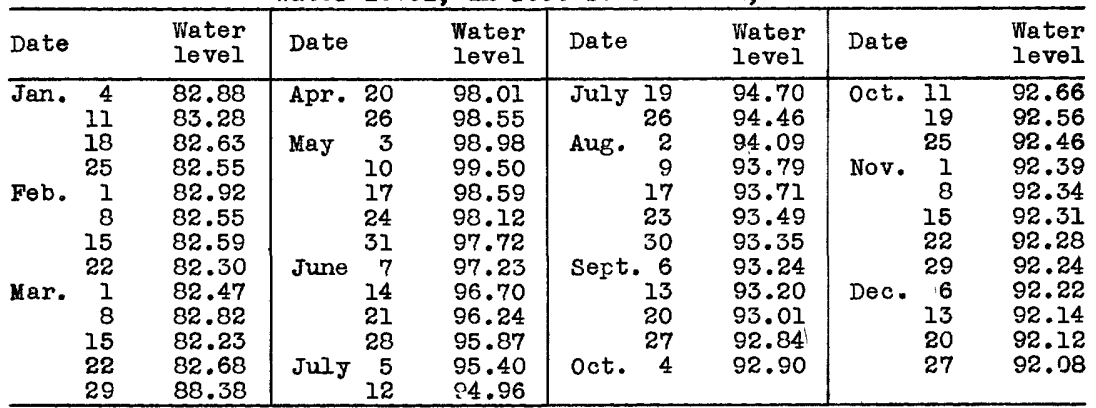

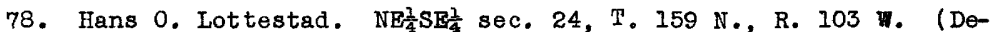
scribes in Fater-Supply .Paper 845, p. 368). Not used after March. Water level, in feet above datum, 1941

\begin{tabular}{|c|c|c|c|c|c|c|c|c|c|c|}
\hline Jan. & $\begin{array}{r}4 \\
11 \\
18 \\
25 \\
1 \\
8 \\
15 \\
22 \\
1 \\
8 \\
15 \\
22 \\
29\end{array}$ & $\begin{array}{l}72.56 \\
75.30 \\
75.53 \\
75.17 \\
76.36 \\
77.55 \\
77.88 \\
77.69 \\
78.00 \\
79.29 \\
77.62 \\
77.98 \\
79.83\end{array}$ & $\begin{array}{l}\text { June } \\
\text { July }\end{array}$ & $\begin{array}{r}20 \\
26 \\
3 \\
10 \\
17 \\
24 \\
31 \\
7 \\
14 \\
21 \\
28 \\
5 \\
12\end{array}$ & $\begin{array}{l}90.62 \\
91.14 \\
92.61 \\
92.34 \\
92.31 \\
91.84 \\
91.47 \\
90.94 \\
90.47 \\
90.13 \\
89.90 \\
89.48 \\
89.13\end{array}$ & 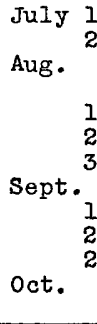 & $\begin{array}{l}88.92 \\
88.74 \\
88.54 \\
88.28 \\
38.02 \\
87.91 \\
87.74 \\
87.62 \\
87.54 \\
87.34 \\
87.09 \\
37.09\end{array}$ & $\begin{array}{l}\text { Oct. } \\
\text { Nov. }\end{array}$ & $\begin{array}{r}11 \\
19 \\
25 \\
1 \\
8 \\
15 \\
22 \\
29 \\
6 \\
13 \\
20 \\
27\end{array}$ & $\begin{array}{l}86.89 \\
86.71 \\
86.63 \\
86.59 \\
86.35 \\
86.39 \\
86.27 \\
86.16 \\
86.12 \\
85.95 \\
85.98 \\
85.79\end{array}$ \\
\hline
\end{tabular}

79. Mrs. Gus B. Swanson Estate. SW (Described in Water-Supply Paper 845, p. 369).

Weter level, in feet above datum, 1941

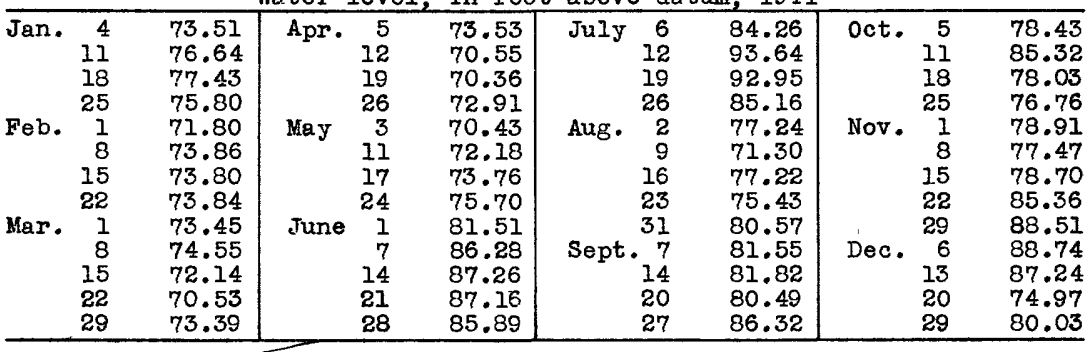


By T. W. Robinson

\section{SOUTHEASTERN SOUTH DAKOTA}

The observation-well program, begun in 1939 in southeastern South Dakota, was continued in 1941 in cooperation with the South Dakota Geological Survey. Measurements of water levels were made monthly in 34 wells and either twice or three times a year in 10 wells. Measurements were discontinued on one well, and one new observation well was added during the year. Two measurements each were made on 5 wells, namely, Nos. 11, 12, 13, 14, and 15, in which no measurements of water level were made in 1940. About 375 individual measurements were made in 1941.

A comparison of the average water levels in a group of 22 wells shows that in 1941 there was a net rise in 7 wells, a net decline in 14 wells and almost no net change in one well. The average change in these wells was, however, a net rise of 0.23 foot. In comparing the water levels in December 1941 with those in December 1939, It is found that there was a net rise in 9 wells, a net decline in 12 wells, and no significant change in 1 well, the average being, however, a net rise of 0.62 foot.

The accompanying figure shows the water-level fluctuations in five water-table wells since December 1939, when the cooperative observationwell program was begun. The topographic situations of the wells is quite different. Well 1 is about 4 miles from the Missourl River on its old flood plain. Wells 5 and 24 are both on the upland, but locally differing in topography. Well 5 is on a rolling upland plain, whereas well 24 is in hilly terrain." The low water level of well 24 in April 1941 may be the result of pumping shortly before the measurement was made. Well 31 is in alluvium about one mile from the Big Sloux River and is probably affected by the stage of the river. Well 42 is on a glacial outwash plain. Wells 1 and 5 are unused, whereas the other three wells are used occaslonally. 


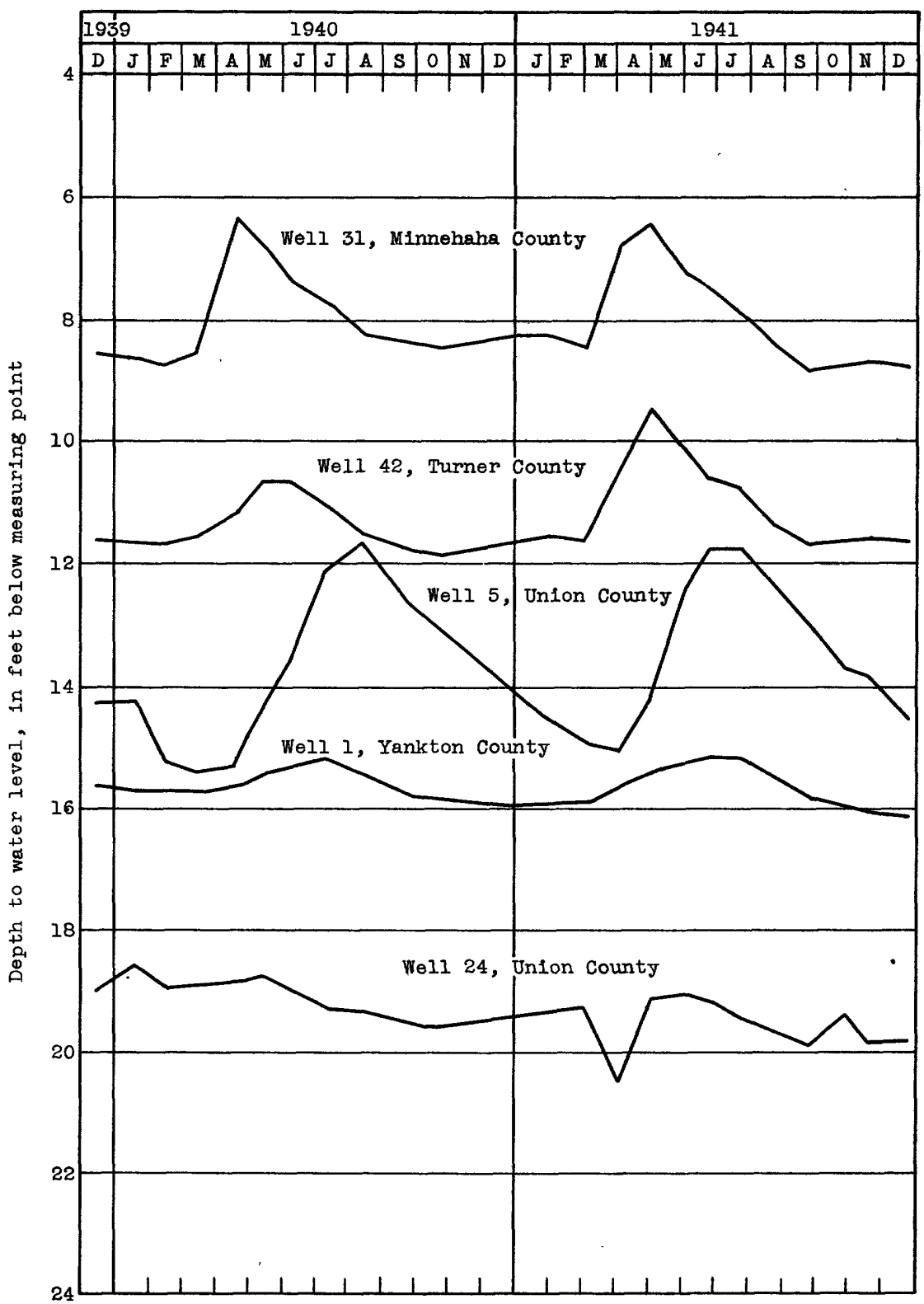

Figure 5.--Graphs showing fluctuations of water level in typical wells in southeasterm South Dakota. 
The precipitation in southeastern South Dakota was below normal during January and February 1941 and nearly normal during March. During April, June, and 0ctober the precipltation was above normal, but during May, July, and August it was below normal. During the remainder' of the year the precipitation was only slightly above or below normal.

on the following pages the observation wells are listed alphabetically by counties, and numerically within each county. The numbers correspond to those given in Water-Supply Papers 886 and 908 . The descriptions of most of the wells and their measuring points are given in Water-Supply Paper 886 and are not repeated in this paper. All the records of water level are expressed in feet below the measuring point. Measurements of water level were made by the Federal Geological Survey in June, July, and August. During the remainder of the year the measurements were made by the State Geological Survey.

\section{Beadie County}

11. Water levels, in feet below measuring point, 1941: Apr. 5, 29.33; June 6, 28.44 .

12. Water levels, in feet below measuring point, 1941: Apr. 5, 33.70; June $6,30.0$.

13. Mrs. Hildur Erickson. Water levels, in feet below measuring point, 1941: Apr. 5, 27.18; June 6, 26.36.

14. Mrs. Ella Johnson. Water levels, in feet below measuring point, 1941: Apr. 5, 30.97; June 6, 35.1.

15. Nels Christensen. Water levels, in feet below measuring point, 1941: Apr. 5, 24.26; June 6, 24.42.

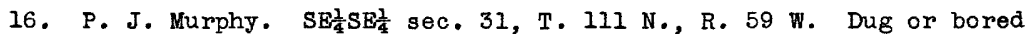
domestic and stock well, diameter 24 inches. Measuring point, inner edge of tile casing at north side. Water levels, in feet below measuring point: Aug. 18, 1940, 17.48; Apr. 5, 1941, 18.07.

\section{Bon Horme County}

7. T. V. Dugovic.

Water level, in feet below measuring point, 1941

\begin{tabular}{|c|c|c|c|c|c|c|c|}
\hline Date & $\begin{array}{l}\text { Water } \\
\text { level }\end{array}$ & Date & $\begin{array}{l}\text { Water } \\
\text { level }\end{array}$ & Date & $\begin{array}{l}\text { Water } \\
\text { level }\end{array}$ & Date & $\begin{array}{l}\text { Water } \\
\text { level }\end{array}$ \\
\hline $\begin{array}{lr}\text { Jan. } 29 \\
\text { Mar. } 9 \\
\text { Apr. } 10\end{array}$ & $\begin{array}{l}25.12 \\
25.18 \\
25.10\end{array}$ & $\begin{array}{lr}\text { May } & 6 \\
\text { July } & 24 \\
\text { Aug. } & 22\end{array}$ & $\begin{array}{l}24.85 \\
22.99 \\
20.80\end{array}$ & $\begin{array}{l}\text { Sept.25 } \\
\text { Oct. } 29\end{array}$ & $\begin{array}{l}20.11 \\
20.3\end{array}$ & $\begin{array}{l}\text { Nov. } 20 \\
\text { Dec. } 18\end{array}$ & $\begin{array}{l}20.21 \\
20.38\end{array}$ \\
\hline
\end{tabular}

8. Jake Berndt.

Water level, in feet below measuring point, 1941

\begin{tabular}{lr|lr|ll|lll}
\hline Jan. 29 & 10.22 & May & 6 & 7.78 & Aug. 22 & 10.23 & Nov. 20 & 11.90 \\
Mar. 9 & 10.37 & June 27 & 8.65 & Sept.25 & 11.30 & Dec. 18 & 12.16 \\
Apr. 10 & 10.55 & July 24 & 9.09 & oct. 29 & 11.59 & & \\
\hline
\end{tabular}


Bon Homme County--Continued.

9.

Water level, in feet below measuring point, 1941

\begin{tabular}{ll|lr|ll|lll}
\hline Date & $\begin{array}{l}\text { Water } \\
\text { level }\end{array}$ & Date & $\begin{array}{l}\text { Water } \\
\text { level }\end{array}$ & Date & $\begin{array}{l}\text { Water } \\
\text { level }\end{array}$ & Date & $\begin{array}{r}\text { Water } \\
\text { level }\end{array}$ \\
\hline Jan. 29 & (a) & May 6 & 5.13 & Aug. 22 & (a) & Nov. 20 & $($ a $)$ \\
Mar. 9 & (a) & June 27 & 9.00 & Sept.25 & (a) & Dec. 18 & (a) \\
Apr. 10 & 7.30 & July 24 & 10.49 & 0ct.29 & 11.20 & & \\
\hline
\end{tabular}

34. Joseph Krejc1.

Water level, in feet below measuring point, 1941

\begin{tabular}{|c|c|c|c|c|c|c|}
\hline $\begin{array}{lr}\text { Jan. } & 29 \\
\text { Mar. } & 9 \\
\text { Apr. } & 10\end{array}$ & $\begin{array}{l}13.02 \\
12.57 \\
11.39\end{array}$ & $\begin{array}{l}\text { May } \\
\text { June } 2 \\
\text { July } 2\end{array}$ & $\begin{array}{r}8.02 \\
9.70 \\
12.14\end{array}$ & $\begin{array}{l}\text { Aug. } 22 \\
\text { Sept. } 25 \\
\text { Oct. } 29\end{array}$ & $\begin{array}{l}12.17 \\
14.51 \\
14.59\end{array}$ & $\begin{array}{l}\text { Nov. } 20 \\
\text { Dec. } 18\end{array}$ \\
\hline
\end{tabular}

\section{Brookings County}

18a. Beginning Apr. 5, 1941, measuring point is top of stove casing, 1.85 feet below previous measuring point. Water levels, in feet below measuring point, 1941: Apr. 5, ㄴ/5.92; June 3, 4.46; Nov. 18, 4.87 .

38. Ed Yusten.

Water level, in feet below measuring point, 1941

\begin{tabular}{lr|lr|ll|lll}
\hline Jan. 29 & 8.24 & May & 2 & 4.80 & Aug. 21 & 7.06 & Nov. 19 & 7.24 \\
Mar. 4 & 7.49 & June 26 & 6.20 & Sept.24 & 7.81 & Dec. 18 & 7.65 \\
Apr. 10 & 5.82 & July 23 & 5.76 & Oct. 29 & 7.45 & & \\
\hline
\end{tabular}

43. University of South Dakota.

Water level, in feet below measuring point, 1941

\begin{tabular}{lrc|cc|cr|rr}
\hline Jan. & 29 & 11.53 & June 27 & 10.20 & Sept.25 & 11.61 & Nov. 20 & 10.45 \\
May & 6 & 9.09 & July 23 & 10.88 & Oct. 29 & 9.04 & Dec. 22 & 11.36 \\
June & 6 & 10.1 & Aug. 21 & 12.37 & & & & \\
\hline
\end{tabular}

47. United States Geological Survey.

Water level, in feet below measuring point, 1941

\begin{tabular}{lr|lr|ll|lll}
\hline Jan. 28 & 9.35 & May & 2 & 7.78 & Aug. 21 & 8.23 & Nov. 19 & 8.84 \\
Mar. 4 & 9.19 & June 26 & 7.80 & Sept.24 & 8.61 & Dec. 18 & 8.90 \\
Apr. 10 & 8.30 & July 23 & 7.74 & Oct. 29 & 8.24 & & \\
\hline
\end{tabular}

48. United States Geological Survey.

Water level, in feet below measuring point, 1941

\begin{tabular}{lr|lr|lll|ll}
\hline Jan. 29 & 18.35 & May & 6 & 17.73 & Aug. 21 & 17.58 & Oct. 29 & 17.75 \\
Mar. 9 & 17.32 & June 25 & 17.43 & Sept.24 & 17.86 & Nov. 19 & 17.80 \\
Apr. 10 & 18.04 & July 23 & 17.29 & & & & \\
\hline
\end{tabular}

\section{Hutchins on County}

35. Herman Krause.

Water level, in feet below measuring point, 1941

\begin{tabular}{lr|ll|lll|ll}
\hline Jan. 29 & 17.42 & June 27 & 11.88 & Sept.25 & 18.06 & Nov. 20 & 22.57 \\
Mar. 9 & 14.55 & July 24 & 13.88 & oct. 29 & 21.62 & Dec. 18 & 21.70 \\
Apr. 10 & 12.98 & Aug. 22 & 16.70 & & & & & \\
\hline
\end{tabular}

a Dry.

b New measuring point. 
Hutchinson County--Continued.

36.

Water level, in feet below measuring point, 1941

\begin{tabular}{lrr|lr|ll|lr}
\hline Date & $\begin{array}{l}\text { Water } \\
\text { level }\end{array}$ & Date & $\begin{array}{l}\text { Water } \\
\text { level }\end{array}$ & Date & $\begin{array}{r}\text { Water } \\
\text { level }\end{array}$ & Date & $\begin{array}{r}\text { Water } \\
\text { level }\end{array}$ \\
\hline Jan. 29 & 6.74 & May 6 & 4.40 & Aug. 22 & 7.80 & Nov. 20 & 7.65 \\
Mar. 9 & a 5.15 & June 26 & 6.77 & Sept.25 & (b) & Dec. 18 & 7.73 \\
Apr. 10 & 3.52 & July 24 & 6.88 & Oct. 29 & (b) & & \\
\hline
\end{tabular}

37. Ed. C. Mettler.

Water level, in feet below measuring point, 1941

\begin{tabular}{lr|lr|lll|ll}
\hline Jan. 29 & 13.42 & May & 6 & 7.50 & Aug. 22 & 12.26 & Nov. 20 & 14.56 \\
Mar. 9 & 12.30 & June 26 & 10.37 & Sept.25 & 13.66 & Dec. 18 & 14.81 \\
Apr. 10 & 10.37 & July 24 & 10.58 & Oct. 29 & 14.44 & & \\
\hline
\end{tabular}

45. Christ. Harnisch.

Water level, in feet below measuring point, 1941

\begin{tabular}{lr|lrr|ll|ll}
\hline Jan. 29 & 15.29 & May & 6 & 14.38 & Aug. 22 & 14.15 & Nov. 20 & 14.10 \\
Mar. 9 & 15.00 & June 26 & 14.20 & Sept.25 & 14.00 & Dec. 18 & 14.20 \\
Apr. 10 & 15.05 & July 24 & 14.14 & Oct. 29 & 14.04 & & & \\
\hline
\end{tabular}

\section{Kingsbury County}

17. Water levels, in feet below measuring point, 1941: Apr. 5, 6.7; June 3, 5.84; Nov. 18, well destroyed; measurements discontinued.

Lincoln County

27. Andrew Lenna.

Water level, in feet below measuring point, 1941

\begin{tabular}{lrr|lrr|lll|ll}
\hline Jan. 28 & c 22.36 & May & 3 & 10.76 & July 23 & 14.43 & Oct. 28 & 19.44 \\
Mar. 4 & 21.36 & June & 3 & 11.54 & Aug. 21 & 16.47 & Nov. 17 & 15.52 \\
Apr. & 4 & 13.30 & & 25 & 13.76 & Sept.24 & 15.49 & Dec. 22 & 14.63 \\
\hline
\end{tabular}

28. H. J. Rolfe.

Water level, in feet below measuring point, 1941

\begin{tabular}{lrl|lrl|ll|l|ll}
\hline Jan. 28 & 20.22 & May & 3 & 20.21 & JuIy 23 & (d) & Oct. 28 & (d) \\
Mar. & 4 & 21.28 & June & 3 & 20.38 & Aug. 21 & (d) & Nov. 17 & (d) \\
Apr. 4 & 20.2 & & 25 & 20.02 & Sept.24 & (d) & Dec. 22 & (d) \\
\hline
\end{tabular}

29. Ed. Devitt.

Water level, in feet below measuring point, 1941

\begin{tabular}{|c|c|c|c|c|c|c|c|c|}
\hline $\begin{array}{lr}\operatorname{Jan} . & 28 \\
\operatorname{Mar} . & 4 \\
\text { Apr. } & 14\end{array}$ & $\begin{array}{l}7.53 \\
6.5 \\
1.49\end{array}$ & $\begin{array}{l}\text { May } \\
\text { June }\end{array}$ & $\begin{array}{r}3 \\
3 \\
25\end{array}$ & $\begin{array}{l}1.94 \\
5.08 \\
6.00\end{array}$ & $\begin{array}{l}\text { July } 23 \\
\text { AuE. } 21 \\
\text { Sept. } 24\end{array}$ & $\begin{array}{l}7.30 \\
8.90 \\
9.69\end{array}$ & $\begin{array}{l}\text { Oct. } 28 \\
\text { Nov. } 17 \\
\text { Dec. } 22\end{array}$ & $\begin{array}{l}9.45 \\
9.61 \\
9.80\end{array}$ \\
\hline
\end{tabular}

44. United States Geological Survey. Measuring point beginning oct. 24, 1941, top of casing, 0.24 foot below previous measuring point.

Water level, in feet below measuring point, 1941

\begin{tabular}{ll|lrl|ll|ll}
\hline Jan. 28 & 6.63 & May & 3 & 4.00 & July 23 & 5.83 & Oct. 28 & 8.4 \\
Mar. 9 & $(\mathrm{e})$ & June & 6 & 5.35 & Aug. 21 & 7.17 & Nov. 17 & 8.79 \\
Apr. 4 & 6.00 & & 25 & 5.18 & Sept.25 & 8.19 & Dec. 22 & 8.65 \\
\hline
\end{tabular}

a Surface water entering well.

b Dry.

c Pumping.

d Well filled or caved in above water level.

e Flooded with surface water. 
Minnehaha County

21. Killeaney.

Water level, in feet below measuring point, 1941

\begin{tabular}{lrl|lc|lc|lr}
\hline Date & $\begin{array}{l}\text { Water } \\
\text { level }\end{array}$ & Date & $\begin{array}{r}\text { Water } \\
\text { level }\end{array}$ & Date & $\begin{array}{r}\text { Water } \\
\text { level }\end{array}$ & Date & $\begin{array}{r}\text { Water } \\
\text { level }\end{array}$ \\
\hline Jan. 28 & 11.12 & May & 3 & 7.8 & July 23 & 8.48 & Oct. 28 & 10.38 \\
Mar. 4 & 11.2 & June & 6 & 7.6 & Aug. 21 & 9.26 & Nov. 19 & 10.65 \\
Apr. 5 & 10.85 & & 25 & 7.93 & Sept.24 & 9.95 & Dec. 22 & 8.72 \\
\hline
\end{tabular}

30. Renner Baseball Park.

Water level, in feet below measuring point, 1941

\begin{tabular}{lrr|lr|ll|l|l|l}
\hline Jan. 28 & 8.91 & May & 2 & 6.33 & July 23 & 7.95 & Oct. 28 & 9.19 \\
Mar. 4 & 8.92 & June & 3 & 7.39 & Aug. 21 & 8.55 & Nov. 17 & 9.16 \\
Apr. 5 & 7.27 & & 25 & 7.38 & Sept.24 & 9.06 & Dec. 22 & 9.22 \\
\hline
\end{tabular}

31.

Water level, in feet below mieasuring point, 1941

\begin{tabular}{lrr|lr|ll|lll}
\hline Jan. 28 & 8.22 & May & 2 & 6.42 & July 23 & 7.90 & Oct. 28 & 8.69 \\
Mar. & 4 & 8.41 & June & 3 & 7.21 & Aug. 21 & 8.37 & Nov. 17 & 8.67 \\
Apr. 5 & 6.74 & & 25 & 7.47 & Sept.24 & 8.79 & Dec. 22 & 8.72 \\
\hline
\end{tabular}

\section{Moody County}

19. Carl B. Jensen. Water levels, in feet below measuring point, 1941: Apr. 5, 37.53; June 3, 37.1; Nov. 17, 37.47.

20. Water levels, in feet below measuring point, 1941: Apr. 5, 4.30; June $3,4.62$; Nov. $17,8.50$.

\section{Turner county}

4. J. H. Shaw.

Water level, in feet below measuring point, 1941

\begin{tabular}{|c|c|c|c|c|c|c|c|}
\hline $\begin{array}{rr}\operatorname{Tan} .29 \\
\text { tar. } 9 \\
\text { tpr. } 10\end{array}$ & $\begin{array}{r}9.70 \\
10.77 \\
9.46\end{array}$ & $\begin{array}{l}\text { May } \\
\text { June } 2 \\
\text { July } 2\end{array}$ & $\begin{array}{l}7.90 \\
8.74 \\
8.60\end{array}$ & $\begin{array}{l}\text { Aug. } 22 \\
\text { Sept. } 25 \\
\text { oct. } 29\end{array}$ & $\begin{array}{r}9.79 \\
10.88 \\
10.32\end{array}$ & $\begin{array}{l}\text { Nov. } 20 \\
\text { Dec. } 18\end{array}$ & $\begin{array}{r}10.80 \\
8.6\end{array}$ \\
\hline
\end{tabular}

22 .

Water level, in feet below measuring point, 1941

\begin{tabular}{lrr|rr|lrr|rr}
\hline Jan. 28 & 9.14 & June & 6 & 7.29 & Aug. 21 & 8.92 & Nov. 19 & 9.84 \\
Apr. & 5 & 6.32 & & 25 & 7.87 & Sept.24 & 9.37 & Dec. 22 & 9.89 \\
May & 2 & 6.17 & July 23 & 8.30 & Oct. 28 & a 13.15 & & \\
\hline
\end{tabular}

32. Otto Kraemer.

Water level, in feet below measuring point, 1941

\begin{tabular}{lrl|lr|ll|ll}
\hline Jan. 28 & 40.73 & May & 2 & 39.65 & Aug. 21 & 40.94 & Nov. 19 & 41.00 \\
Mar. & 4 & 40.4 & June 25 & 40.48 & Sept.24 & 41.04 & Dec. 22 & 41.05 \\
Apr. 5 & 40.00 & July 23 & 40.65 & & & & \\
\hline
\end{tabular}

39. C. E. Johnson.

Water level, in feet below measuring point, 1941

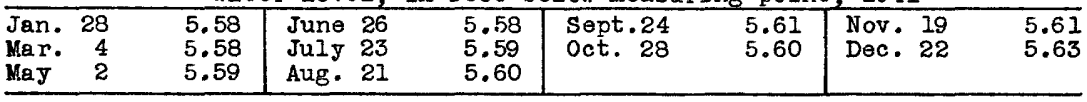

a Pumping. 
Turner County--Continued.

40. W. C. Olson.

Water level, in feet below measuring point, 1941

\begin{tabular}{ll|ll|ll|ll}
\hline Date & $\begin{array}{l}\text { Water } \\
\text { level }\end{array}$ & Date & $\begin{array}{l}\text { Water } \\
\text { level }\end{array}$ & Date & $\begin{array}{l}\text { Water } \\
\text { level }\end{array}$ & Date & $\begin{array}{r}\text { Water } \\
\text { level }\end{array}$ \\
\hline Jan. 28 & 26.27 & July 23 & 26.97 & Sept.24 & 25.17 & Nov. 19 & 26.45 \\
Mar. 4 & 25.3 & Aug. 21 & 27.92 & Oct.28 & 25.02 & Dec. 22 & 26.15 \\
June 26 & 24.94 & & & & & &
\end{tabular}

41. Jorgenson Studio.

Water level, in feet below measuring point, 1941

\begin{tabular}{lrr|lr|l|l|l|l}
\hline Jan. 28 & 16.67 & May & 2 & 13.24 & July 23 & 14.85 & Oct. 28 & 15.49 \\
Mar. 4 & 17.15 & June & 6 & 13.62 & Aug. 21 & 15.56 & Nov. 19 & 15.23 \\
Apr. 5 & 16.34 & & 26 & 14.96 & Sept.24 & 16.81 & Dec. 22 & 15.85 \\
\hline
\end{tabular}

42.

Water level, in feet below measuring point, 1941

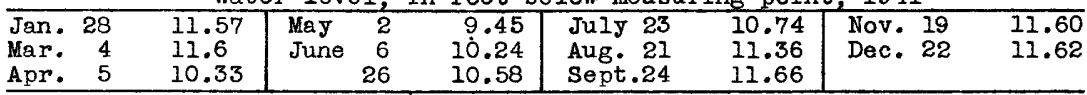

Union County

5. J. J. Dolan.

Water level, in feet below measuring point, 1941

\begin{tabular}{lrr|lr|lll|ll}
\hline Jan. 28 & 14.49 & May & 3 & 14.21 & July 23 & 11.72 & Oct. 28 & 13.7 \\
Mar. 4 & 14.93 & June & 3 & 12.42 & Aug. 21 & 12.30 & Nov. 17 & 13.88 \\
Apr. 4 & 15.08 & & 25 & 11.73 & Sept.24 & 12.92 & Dec. 22 & 14.48 \\
\hline
\end{tabular}

24 .

Water level, in feet below measuring point, 1941

\begin{tabular}{lrl|lrr|ll|lll}
\hline Jan. 28 & 19.37 & May & 3 & 19.15 & July 23 & 19.46 & Oct. 28 & 19.4 \\
Mar. & 4 & 19.3 & June & 3 & 19.05 & Aug. 24 & 19.69 & Nov. 17 & 19.88 \\
Apr. & 4 & 20.5 & & 25 & 19.18 & Sept.24 & 19.89 & Dec. 22 & 19.82 \\
\hline
\end{tabular}

25. A. G. McGuire.

Water level, in feet below measuring point, 1941

\begin{tabular}{lrc|lr|ll|lll}
\hline Jan. 28 & 12.75 & May & 3 & 7.94 & July 23 & 10.51 & Oct. 28 & 12.9 \\
Mar. & 4 & $($ a $)$ & June & 3 & 9.64 & Aug. 21 & 11.88 & Nov. 17 & 13.72 \\
Apr. 4 & 9.27 & & 25 & 8.74 & Sopt.24 & 12.76 & Dec. 22 & 14.31 \\
\hline
\end{tabular}

Yankton County

1. Gayville Cemetery.

Water level, in feet below measuring point, 1941

\begin{tabular}{ll|lrl|ll|lll}
\hline Jan. 29 & 15.96 & May & 6 & 15.37 & Aug. 22 & 15.50 & Nov. 20 & 16.07 \\
Mar. 9 & 15.88 & June 26 & 15.17 & Sept.25 & 15.85 & Dec. 18 & 16.13 \\
Apr. 10 & 15.6 & July 24 & 15.19 & Oct. 29 & 15.99 & & \\
\hline
\end{tabular}

2B. Yankton Golf club.

Water level, in feet below measuring point, 1941

\begin{tabular}{lr|lr|rr|rrr}
\hline Jan. 29 & 10.52 & May & 6 & 7.02 & July 24 & 7.90 & Oct. 29 & 11.40 \\
Apr. 10 & 6.61 & June 26 & 6.68 & Aug. 22 & 10.00 & Nov. 20 & 11.26 \\
\hline
\end{tabular}

a Filled with surface water. 
Yankton County--Continued.

3. Water level, in feet below measuring point, 1941

\begin{tabular}{lr|lr|ll|ll}
\hline Date & $\begin{array}{r}\text { Water } \\
\text { level }\end{array}$ & Date & $\begin{array}{r}\text { Water } \\
\text { level }\end{array}$ & Date & $\begin{array}{l}\text { Water } \\
\text { level }\end{array}$ & Date & $\begin{array}{r}\text { Water } \\
\text { level }\end{array}$ \\
\hline Jan. 29 & 10.32 & May 6 & 5.87 & Aug. 22 & 11.17 & Nov. 20 & $($ a) \\
Mar. 9 & 7.85 & June 26 & 9.85 & Sept.25 & (a) & Dec. 18 & $($ a) \\
Apr. 10 & 6.63 & July 24 & 9.72 & Oct.29 & (a) & & \\
\hline
\end{tabular}

33. Adolph Schoenfeldt.

Water level, in feet below measuring point, 1941

\begin{tabular}{lrr|lr|ll|ll}
\hline Jan. 29 & 18.92 & JuIy 24 & 13.77 & Sept.25 & 16.41 & Nov. 20 & 17.75 \\
May & 6 & 13.94 & Aug. 22 & 15.95 & Oct. 29 & 17.15 & Dec. 18 & 18.31 \\
June 27 & 12.47 & & & & & & & \\
\hline
\end{tabular}

46. Oswald Estate.

Water level, in feet below measuring point, 1941

\begin{tabular}{lrr|lr|ll|ll}
\hline Jan. 29 & 17.46 & July 24 & 13.22 & Sept.25 & 17.47 & Nov. 20 & 18.57 \\
May & 6 & 9.00 & Aug. 22 & 16.15 & Oct. 29 & 18.01 & Dec. 18 & 19.47 \\
June 26 & 11.57 & & & & & & & \\
\hline
\end{tabular}

\section{CITY OF' HURON}

The recording of water-level measurements in gage hole 1 in the city well fleld near Huron, which was begun in 1934, was continued by the city authorities in 1941. There was no pumpage from the well field during the year as the entire c1ty supply of $313,000,000$ gallons was supplied by the James River. This is the first year since 1934 that no water was pumped from the fleld. Records of water levels and pumpage from 1934 through 1940 are given In Water-Supply Papers 817, 840, 845, 886, and 908: The water levels from gage hole 1 for the Huron well field given in this report were furnished by H. M. Plerce, city engineer.

The water level in gage hole 1 was higher in December 1941 than in any December since measurements were begun. The high water level has resulted chiefly from the year of rest from pumping. In December 1941, the water level in gage hole 1 was 12.4 feet higher than on December 31, 1940; 4.7 feet higher than on December 31, 1939; 14.4 feet higher than on December 31, 1938; 11.3 feet higher than on December 25, 1937; 8.8 feet higher than on December 31, 1936; 8.3 feet higher than on December 31, 1935; and 7.7 feet higher than on December 29, 1934.

\begin{tabular}{|c|c|c|c|c|c|c|c|}
\hline $\begin{array}{l}\text { Jan. } \\
\text { Feb. } \\
\text { Mar. }\end{array}$ & $\begin{array}{l}18.12 \\
15.58 \\
14.41\end{array}$ & $\begin{array}{l}\text { Apr. } \\
\text { May } \\
\text { June }\end{array}$ & $\begin{array}{l}13.66 \\
13.70 \\
13.50\end{array}$ & $\begin{array}{l}\text { July } \\
\text { Aug. }\end{array}$ & $\begin{array}{l}13.50 \\
13.79\end{array}$ & $\begin{array}{l}\text { Oct. } \\
\text { Dec. }\end{array}$ & $\begin{array}{l}12.37 \\
11.90\end{array}$ \\
\hline
\end{tabular}

a Dry. 


\section{WISCONSIN}

COON CREEK AREA

By L. K. Wenzel

The observation-well program in the Coon Creek area in Vernon, Monroe, and LaCrosse Counties, Wis., was continued through August 1941 by the Federal Geological Survey. A total of 70 individual measurements of water level was made by Frank J. Fencl in 10 wells during the year.

Measurements in the 10 wells were used for computing average water levels for 1941. The water levels in well 3 for January and February, when the well was dry, were obtained by inspection from the water levels in nearby wells.

The average water level at the beginning of 1941 , as shown by measurements made on Januery 4 and 5 , was 10.62 feet above the assumed datum planes. The average water level declined in January but rose slightly in February and considerably in March, reaching an average stage of 11.61 feet during the first part of April. Recharge was apparentiy greatest in March. The average water level declined 0.15 foot to 11.46 feet by the first week in July and then declined 0.60 foot to 10.86 feet by August 2 to 4 , at which time measurements were discontinued. Water levels in August were at comparable stages with those in August of previous years.

\begin{tabular}{cccccccc}
\multicolumn{7}{c}{ Water levels, in feet above datum planes, 1941} \\
\hline Well & Jan. & Feb. & Mar. & Apr. & May & July & Alug. \\
& $4-5$ & $4-5$ & 5 & 8,10 & 4 & 2,5 & 2,4 \\
\hline 2 & 10.57 & 10.28 & 10.05 & 16.85 & 16.43 & 15.07 & 14.01 \\
3 & $(a)$ & $(2)$ & 10.13 & 11.66 & 11.66 & 12.10 & 12.14 \\
4 & 12.72 & 12.63 & 12.80 & 14.00 & 13.18 & 13.79 & 12.70 \\
8 & 10.67 & 10.55 & 10.79 & 10.89 & 11.74 & 11.15 & 10.59 \\
9 & 11.62 & 11.24 & 10.08 & 10.72 & 10.81 & 10.98 & 10.49 \\
10 & 10.60 & 10.18 & 10.31 & 10.94 & 10.72 & 11.15 & 10.16 \\
11 & 9.83 & 9.48 & 9.61 & 10.59 & 10.09 & 10.52 & 9.62 \\
12 & 10.62 & 10.16 & 11.14 & 10.80 & 10.80 & 10.99 & 10.78 \\
13 & 9.25 & 9.13 & 8.26 & 9.83 & 9.69 & 9.70 & 9.38 \\
14 & 9.73 & 9.33 & 9.44 & 9.85 & 9.44 & 9.19 & 8.68 \\
\hline Average 10.62 & 10.33 & 10.36 & 11.61 & 11.46 & 11.46 & 10.86 \\
\hline
\end{tabular}

1 See Water-Supply Papers $777,817,840,845,886$, and 908. a WeIl dry. 